\title{
Black and White in Ink: \\ Discourses of Resistance in South African Cartooning, 1985-1994
}

by Andrew John Mason

Submitted in partial fulfilment of the requirements for the Masters degree in Culture, Communication and Media Studies at the University of KwaZulu-Natal

Supervisors: Professor Keyan Tomaselli; Professor Lynn Dalrymple 


\section{Acknowledgements}

Acknowledgements are due to my supervisors, Professors Lynn Dalrymple and Keyan Tomaselli, for their guidance and patience.

Possibly the greatest benefit I have derived from this project has been the opportunity to meet and develop friendships with some of South Africa's leading cartoonists and comic art practitioners. In particular, I would like to thank Anton Kannemeyer, Jonathan Shapiro, Rico Schacherl and Len Sak. I have enjoyed many hours of debate and reminiscence with all of them, and they have all made a major contribution to this study. I would also like to thank all the other cartoonists who were interviewed for this study and who gave their time to follow-up discussions.

\section{Declaration}

I declare that the work that appears in this dissertation is my own original work, based on my own independent study and research, and that where the ideas or concepts of other writers have been used, this usage has been properly acknowledged.

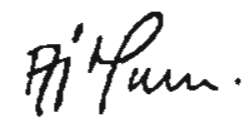

Andrew John Mason

October 2004 


\section{Table of Contents}

Abstract 5

Table of Figures 6

Introduction 11

Key arguments 11

Methodology 14

Chapter One: Theoretical Context and Methodology 21

Key issues in the analysis of cultural products 21

Approaches to ideology in the analysis of symbolic forms 28

Problems specific to the analysis of visual culture 32

The application of Thompson's methodology of interpretation 41

\section{Chapter Two: Defining Cartooning 48}

Introduction $\mathbf{4 8}$

Defining cartooning 51

Form, medium and practice: making the distinctions 54

Towards a workable definition of cartooning 57

Chapter Three: Key Trends in $20^{\text {th }}$ Century Cartooning 73

Introduction 73

The satirical tradition $\mathbf{7 4}$

The evolution of contemporary cartooning 77

The Anglophone and Francophone traditions 79

Mid-century anti-comics campaigns 81

Some significant trends in contemporary cartooning 84

Chapter Four: South African Cartooning before1984: Themes and Directions 88

Introduction $\mathbf{8 8}$

The South African satirical tradition 90 
The contradictions of liberalism 93

The semiotics of racial caricature 94

The challenge of Soweto 102

Chapter Five: Cartooning in the Last Decade of Apartheid 104

Introduction 104

The intellectual milieu 106

Key themes in oppositional cartooning $\mathbf{1 1 6}$

Political cartooning in the mainstream opposition press 117

Chapter Six: From Rebels at the Gate to Icons of the New Mainstream:

South Africa's New Cartooning, 1985-1994 124

Introduction 124

Ink and blood in the alternative press 126

Zapiro: cartooning the transition 133

Madam E Eve: Reinventing social relationships 143

Cartooning for social change: Educational comics and the Storyteller Group 150

Reviling the father: Bitterkomix and the white underground 158

\section{Conclusion 169}

Review of key arguments 169

Theoretical and methodological issues $\mathbf{1 7 1}$

Postscript: The way forward $\mathbf{1 7 3}$

Bibliography 179 


\section{Abstract}

In the last decade of apartheid (1985-1994), South African cartoonists demonstrated a range of responses to the political imperatives of the day. While some worked in support of the status quo, the cartoonists who are the subject of this study opposed it. Like practitioners in other areas of cultural activity during this period, oppositional cartoonists were passionately engaged with the political process and participated in the articulation and dissemination of discourses of resistance.

This study situates South African cartooning both in the context of South African resistance discourse, and in the historical and discursive context of cartooning as a form of international popular culture. It presents an argument as to how cartooning should be defined and studied--as a cluster of signifying practices that produce a range of forms in a variety of media. In terms of this definition, anti-apartheid cartooning in South Africa is identified as a specific historical category, within which distinct streams of cartooning are identified. The study locates the various activities of South African cartooning within these streams, and examines the ideological and educational functions they performed during the 1985-1994 period.

The study positions cartooning within the broad theoretical field of cultural and media studies, and examines some theoretical problems that are specific to the analysis of visual culture. A language of exposition appropriate to the study of cartooning is developed, borrowing terms from the sometimes widely variant traditions of art history, literary criticism and cultural studies. A methodology for the interpretation of symbolic forms is derived from the work of British cultural theorist, John B. Thompson (1990), whereby selected cartooning texts are subjected to a combination of textual interpretation, socio-historical analysis and discursive analysis, reinforced by insights derived from conversations with 15 selected South African cartoonists.

Textual analysis of selected cartooning texts from the 1985-1994 period clearly demonstrates that oppositional cartoonists gave visual expression to discourses of resistance that existed in the anti-apartheid movement, and amongst the broader public, at that time. In so doing, they contributed to the disruption of the hegemony of the apartheid state, to the legitimation of the anti-apartheid struggle and to the provision of symbols and icons that ordinary South Africans were able to utilise in 'rethinking' their own lives in relation to the demands of a rapidly transforming society. 


\section{Table of Figures}

Figure 1. Exhibition catalogue cover: Black \& White in Ink: Under the Skin of South African Cartooning, 2002.

Figure 2. Cartoon by Joe Dog (Anton Kannemeyer), Loslyf, 1995.

Figure 3. (Above) Drawing by Richard Biox, 1921. (Below) Photograph by Alfred

Stieglitz of a sculpture by Marcel Duchamp, 1921.

Figure 4. Cover of The Madiba Years by Zapiro (Jonathan Shapiro), 1996.

Figure 5: Diagram representing the field of cartooning activity.

Figure 6: "Punch and Burn 'em Show". Cartoon by Fred Mouton, Die Oosterlig, 1986.

Figure 7. South African Airways safety instruction card.

Figure 8. Page from Understanding Comics: The Invisible Art, by Scott McCloud, 1990.

Figure 9. Portrait of P.W. Botha by Richard Smith, 1985.

Figure 10. Page from "Museum of Archeology" by Milan Hulsing, Lapin, 1999.

Figure 11. Page from Understanding Comics: The Invisible Art, by Scott McCloud, 1990.

Figure 12. Diagram demonstrating the simplification of the human face.

Figure 13. Page from Akira by Katsuhiro Otomo, 2000.

Figure 14. Caricature of Richard Nixon by Gerald Scarfe, 1993.

Figure 15. (Above) Cartoon of Hoggenheimer by Daniel Boonzaier, circa 1905. (Below)

Drawing from Johannesburg, $2^{\text {nd }}$ Greatest City after Paris, by William Kentridge, 1989.

Figure 16. (Above) Untitled cartoon by David Marais, 1963. (Below) Untitled cartoon by Bob Connolly, 1966.

Figure 17. (Above) Cartoon by Zapiro, 2001. (Below) Examples of Zapiro's caricatures.

Figure 18. (Above) "Death and Time", a 16 $6^{\text {th }}$ century English woodcut; "The Last Drop", an $18^{\text {th }}$ century caricature. (Below) Cartoon by Dov Fedler, 1991.

Figure 19. (Above) "The Brilliant Career of Eugene Terre'blanche". Cartoon by Zapiro,

1997. (Below) Untitled cartoon by Zapiro, 2001.

Figure 20. (Above) "Independent Newspapers". Cartoon by Zapiro, 2000. (Below)

Cartoon by Zapiro, 2001. 
Figure 21. Page from Bonginkosi, a comic book by Rodney Harbour and Rick Andrew, 1987.

Figure 22. "Tortures of St Erasmus", a medieval woodblock print, circa 1460.

Figure 23. "Nightly Visitors at St. Ann's Hill". Cartoon by James Gillray, 1798.

Figure 24. Cartoon by William Schroder, 1884.

Figure 25. Cover of The Complete Maus by Art Spiegelman, 1996.

Figure 26. "Haight-Ashbury, early '68..." Cartoon by Robert Crumb, 1992.

Figure 27. Cartoon by George Cruikshank, 1819.

Figure 28. Photograph of a Drakensberg San rock painting, circa 1836.

Figure 29. Cartoon of Paul Kruger by William Schhroder, 1887.

Figure 30. "Geluk, liewe Maatjie..." Cartoon by T.O.Honiball, 1966.

Figure 31. "Hy Lei... Maar waarheen?" Cartoon by Etam (Eric Thamm), 1948.

Figure 32. (Above) Cartoon by David Marais, 1958. (Below) Cartoon by Len Sak, 1961.

Figure 33. "The Winds of Change". Cartoon by Jock Leyden, 1960.

Figure 34. Cartoons by Jock Leyden, 1967, 1969.

Figure 35. (Above) Phaki, a comic strip by G.L. Norton, circa 1950. (Below) Cartoon by David Marais, 1963.

Figure 36. Cartoon from Abe Berry's South Africa and How it Works, 1989.

Figure 37. Page from "Goombi - Private detective" (anonymous), a serialised comic strip in Drum, 1958.

Figure 38. Cartoon from Jojo's World by Len Sak, 1991.

Figure 39. Cartoon from Jojo's World by Len Sak, 1991.

Figure 40. Cartoon of Richard Nixon by Richard Smith, 1971.

Figure 41. Smith and Abbot Ink cartoon strips by Richard Smith and David Barrit, 1973.

Figure 42. Soweto? Cartoon by Donald Kenyon, 1976.

Figure 43. Cartoons by Fred Mouton, 1975, 1976.

Figure 44. Cover of In the Heart of the Country by J.M.Coetzee, by Richard Smith, 1978.

Figure 45. Staffrider magazine illustration by Dikobe Martins, 1988.

Figure 46. "Workers of South Africa! Unite and fight for a living wage!" Cover of a Cosatu comic book, 1989.

Figure 47. Panel from Equiano: The Slave who Fought to be Free, a comic book illustrated by Rick Andrew, 1988. 
Figure 48. Page from The Big Chillum a comic strip by by N.D.Mazin (Andy Mason), 1986.

Figure 49. Page from Case No. 308 by Joe Dog (Anton Kannemeyer) and Adam Baard (Contrad Botes), 1989.

Figure 50. Cartoon by Len Sak, 1991.

Figure 51. "June 16 - Salute the youth who brave the dragon". Cartoon by Zapiro, 1987.

Figure 52. Cartoon by Andy (Dave Anderson), 1982.

Figure 53. Cartoon by Andy, 1988.

Figure 54. Cartoon by Tony Grogan, 1993.

Figure 55. Cartoon from Grogan's South Africa by Tony Grogan, 1989.

Figure 56. Cartoon by Dov Fedler, 1973.

Figure 57. Cartoon by Dov Fedler, 1990.

Figure 58. Cartoon by Nanda Soobben, 1980 .

Figure 59. "Steve Biko - In Memoriam". Cartoon by Derek Bauer, 1987.

Figure 60. "The Ghosts of Christmas Past, Present and Future". Cartoon by Derek Bauer, 1989.

Figure 61. Cartoon by Derek Bauer, 1986.

Figure 62. "Cheer up guys, I'm here to stay!" Cartoon by Derek Bauer, 1987.

Figure 63. "The dreaded Marxist forces strike again!" Cartoon by Derek Bauer, 1986.

Figure 64. "'Skus Meneer, ons soek 'n genuine black leaderr". Cartoon by Derek Bauer, 1986.

Figure 65. Cartoon by Derek Bauer, 1987.

Figure 66. Page from "Behind the Barbed Wire Curtain", a comic strip by Zapiro, 1988.

Figure 67. UDF Calendar illustration by Zapiro, 1987.

Figure 68. Cartoons by Zapiro from South, 1987.

Figure 69. "Laughter in the Belly of the Beast". Exhibition poster by Zapiro, 1988.

Figure 70. Cover of Roxy: Life, Love and Sex in the Nineties, a photo-comic book by the Story Circle, 1991.

Figure 71. Cover cartoon by Zapiro for Die Suid-Afrikaan, 1991.

Figure 72. Cartoon by Zapiro, 1994.

Figure 73. "Inauguration". Cartoon by Zapiro, 1994.

Figure 74. Madam \& Eve cartoon strip by Stephen Francis, Harry Dugmore \& Rico (Ricardo Schacherl), 1993. 
Figure 75. Madam \& Eve cartoon strip by S. Francis, H. Dugmore \& Rico, 1992. Figure 76. Cartoon from It Takes two To Toyi Toyi by Gus Silber, illustrated by Stidy (Anthony Stidolph), 1991.

Figure 77. Madam \& Eve cartoon strip by S. Francis, H. Dugmore \& Rico, 1993. Figure 78. Madam \& Eve cartoon strip by S. Francis, H. Dugmore \& Rico, 1998. Figure 79. Madam \& Eve cartoon strip by S. Francis, H. Dugmore \& Rico, 1993. Figure 80. Madam \& Eve cartoon strip by S. Francis, H. Dugmore \& Rico, 1993. Figure 81. Madam \& Eve cartoon strip by S. Francis, H. Dugmore \& Rico, 1993. Figure 82. Madam \& Eve cartoon strip by S. Francis, H. Dugmore \& Rico, 1994. Figure 83. "Big Mr. Westernman". Cartoon strip by Olly, 1972.

Figure 84. Cover cartoon by Richard Smith, Wits Student, circa 1972.

Figure 85. Page from Vittoke in Azania, a serialised comic strip by Pooh (Andy Mason), 1979.

Figure 86. Cover of Vusi Goes Back, a comic book written by Dick Cloete and Rob Berold, illustrated by Andy Mason, 1981.

Figure 87. Page from Sloppy, a serialised comic strip by Mogorosi Motshumi, (initially with N.D.Mazin), 1983.

Figure 88. Page from The Instant Poet, a comic strip by Percy Sedumedi, 1987.

Figure 89. Panels from Down Second Avenue, a comic strip based on the book by Ezekiel Mphahlele, illustrated by Mzwakhe Nhlabati, 1981.

Figure 90. Cover of 99 Sharp St. : The River of Our Dreams, a comic book by the Storyteller Group, illustrated by Carlos Carvalho, 1991.

Figure 91. Page from The Collector of Treasures, a comic strip based on a story by Bessie Head, illustrated by Lois Head, 1993.

Figure 92. Cover of Heart to Heart: From Dream Love to True Love, a comic book produced by the Story teller Group, illustrated by Justin Wells, 1994.

Figure 93. Cover of Bitterkomix No. 1, illustrated by Joe Dog and Conrad Botes, 1991. Figure 94. Cover of Pax No. 6, illustrated by N.D.Mazin, 1987.

Figure 95. Page from "Urban Blues", a comic strip by Rick Andrew, 1987.

Figure 96. Cover of Icy Blind: The Final Kak, illustrated by Peter Rapp, circa 1985.

Figure 97. Page from "Sex Then and Now", a comic strip by Wild Beast (Joanne Bloch), 1991. 
Figure 98. Page from "Jeugweerbaarheid" a comic strip by Joe Dog, 1994 (English translation, 1998).

Figure 99. Page from "Siembamba" by Conrad Botes, 1994 (English translation, 1998).

Figure 100. Cover of Bitterkomix No. 3, illustrated by Joe Dog, 1993.

Figure 101: Page from "Liefde en Missiele", a comic strip by Joe Dog, 1994.

Figure 102: Page from "Boetie", a comic strip by Joe Dog, 1995. (English translation, "Sonny", 1998).

Figure 103: Page from "Bloedrivier", a comic strip by Conrad Botes, 1995. (English translation, "Blood River", 1998).

Figure 104. Cartoon of B.J. Vorster by David Marais, 1963.

Figure 105. Cartoon by Sifiso Yalo, 2004.

Figure 106. Majimbos, a comic strip by Themba Siwela, 2004.

Figure 107. Cartoon by Brandan Reynolds, 2004.

Figure 108. Cartoon by Alastair Findlay, 2002.

Figure 109. Cartoon by Stidy (Anthony Stidolph), 2004.

Figure 110. Zapiro's Madiba, 1998. 


\section{Introduction}

\section{Key arguments}

This study argues that in the last decade of apartheid (1985-1994), South African cartoonists gave visual expression to discourses of resistance that existed in the democratic movement at that time, contributing to the disruption of the hegemony of the apartheid state and to the legitimation of the anti-apartheid struggle. In doing so, they contributed to the provision of symbols and icons that ordinary South Africans were able to utilise in 'rethinking' their own lives in relation to the demands of a rapidly transforming society. Cartoons and comics were also used to provide information to ordinary people in peri-urban and rural settings where low levels of literacy were the norm, and, in some cases, to mobilse communities around social and political issues.

Obviously, not all South African cartoonists opposed apartheid, and there were those whose work served the interests of the status quo. But the work of pro-apartheid cartoonists falls outside the scope of this study. Amongst those cartoonists who gave expression to discourses of resistance, a range of responses to the circumstances and political imperatives of the time is evident. In each case, the work of the individual cartoonist was mediated via a publishing process that, in a sense, defined and shaped it. The opportunities for publication within the existing publishing environment were severely constrained, and several cartoonists were involved in attempts to establish alternative publishing avenues. A few chose to produce their own publications rather than to slot their work into existing publications, and there were some who saw no future in cartooning in this country and chose rather to work as fine artists or in advertising and graphic design. And there were some excellent potential cartoonists who, because they were black, were unable to take up cartooning as a career because of the lack of enabling conditions that would have allowed them to do sol.

This study provides a brief survey of anti-apartheid cartooning in South Africa in the last decade of apartheid, based on bibliographical research, the collection and archival viewing of primary research material in the form of comics and cartoons in their

\footnotetext{
${ }^{1}$ In keeping with conventional South African usage, the rubric 'black' is used throughout this dissertation to include people of African, Indian and so-called 'coloured' descent.
} 
original published form ${ }^{2}$, and recorded conversations with selected cartooning practitioners who were active during the period. The practitioners who were interviewed are Len Sak, Richard Smith, Dov Fedler, Jonathan Shapiro (Zapiro), Stephen Francis, Ricardo Schacherl (Rico), Nanda Soobben, Alastair Findlay (Findlay), Neil Napper, Peter Esterhuysen (who has subsequenetly passed away), Mogorosi Motshumi, Joanne Bloch (Wild Beast), Anton Kannemeyer (Joe Dog) and Conrad Botes (Konradski) ${ }^{3}$. Derek Bauer was tragically killed in a car crash in December 2001, and in his absence his widow and a close friend were interviewed. Due to the limitations of this study, it has not been possible to include transcripts of these conversations, but they nevertheless constitute a foundation upon which the discussions of these practitioners and their work is based.

In addition to these selected practitioners, there are several others whose work is important, but who for various reasons have not been included in this study, or have only been mentioned in passing. This study is therefore not all-inclusive and the choice of practitioners to be interviewed depended to a large extent on practical reasons. Cartooning work produced by the present writer is also referred to, but is not analysed in any detailt.

This introduction provides a summary and overview of the structure and methodology of the study as a whole. What cartooning is, and how the various cartooning forms should be grouped and defined, is argued in Chapter Two. In this study, the term 'cartooning', rather than 'comic art', is chosen as the rubric under which the broad range of graphic narrative activities is incorporated. 'Cartooning' is thus viewed as a cluster of

\footnotetext{
${ }^{2}$ Some archival newspaper research was done in the Natal Society Library in Pietermaritzburg and in the Stellenbosch University Library's special collection of South African cartooning, but most of the newspaper cartoons referred to are derived from published collections of the work of individual cartoonists or secondary bibliographical sources.

${ }^{3}$ Most of these conversations were recorded on audio tape and have been transcribed, but at the time of writing the transcriptions had not yet been edited for publication. Some of the conversations were supplemented with second and third telephonic interviews to elaborate on certain issues or points of fact, but these exist only in the form of notes. One of the conversations (with Alastair Findlay) was lost due to tape malfunction.

${ }^{4}$ Following the example of Guy Berger (2000), I have chosen to refer to myself in the third person in the main text of this study. My comics and publications described in this study were produced 20 or more years ago and a retrospective 'distancing' from these texts is accorded by the passage of time. For this reason I have chosen not to use the more engaged and reflexive first person voice in the main text, but to adopt the relatively non-reflexive voice implied in the use of the third-person phrase "the present writer". However, the first person voice is retained in the footnotes.
} 
signifying practices that produce a range of popular culture forms in a variety of media. These include cartoons of various kinds, comic strips and comic books (including underground comix and graphic novels), humorous art and caricature ${ }^{5}$.

In terms of this definition of cartooning, anti-apartheid or 'resistance' cartooning in South Africa is identified as a specific historical category, within which three distinct streams of cartooning are identified:

* cartooning in the mainstream opposition press;

* cartooning in the alternative press; and

* underground cartooning.

These parallel streams are investigated as sites of cartooning activity. Given that some cartoonists worked across more than one stream while others were exclusively situated within one of the streams, attention is paid to the structural conditions through which these streams flowed and which acted upon them, conditioning and influencing the practice of the cartoonists who worked within them. This study locates the various activities of South African cartooning within these streams, and examines the ideological and educational functions they performed during the 1985-1994 period. These functions may be grouped into three categories:

* counter-hegemonic satire and ridicule of the establishment;

* pro-social iconography supportive of the transition; and

* education and conscientisation around social and political issues.

Embedded within these functional categories is an issue that is beyond the scope of this study to investigate, but nevertheless cannot be ignored and needs to be acknowledged as a problem area demanding of further research and analysis. This issue goes to the heart of the functionality of cartooning, and arises out of the relative lack of black (African, Indian and 'Coloured') cartoonists in South Africa during the 20th century. It is a controversial area of analysis, and a degree of reluctance is experienced by the present writer in generalising about it, given the inevitable sensitivities that exist amongst practitioners, editors and publishers around levels of activism or conduct during the apartheid period. However, it is incontrovertible that very little was done by South African publishers and editors during the 1985-1994 period, or prior to the period, to develop black cartooning. As a result, with a few notable exceptions, the work of white cartoonists was usually used to illustrate publications aimed at black readers. In

\footnotetext{
${ }^{5}$ Animated cartooning has been excluded from this definition (see Chapter Three).
} 
addition, the 'structuration' of the South African publishing industry at the time was such that it would have been very difficult for black cartoonists to establish sustainable careers for themselves in the industry $y^{6}$. This is borne out, for instance, in the biography of Mogorosi Motshumi, who in in the late 1970 s was detained by the security police in connection with his cartooning activities and was then fired from the white-owned newspaper where he had been working (see Chapter Six). He subsequently worked in the alternative press, producing an important and long-running comic strip about township life, but never regained a secure foothold in the mainstream press.

It is therefore suggested that the lack of black cartoonists in South Africa during the apartheid period was the result of the absence of an 'enabling environment', but this proposition is not rigorously investigated. It is also suggested that problems of identity, authenticity and credibility experienced by publishers, editors and media activists who attempted to use comics as a tool for the education and conscientisation of the voteless masses' during the last decades of apartheid were related to the lack of black cartoonists ${ }^{7}$.

\section{Methodology}

The methodology utilised in this study is explicated in Chapter One. Cartooning, as a cluster of signifying practices, gives rise to specific kinds of symbolic forms which require an analytic procedure that takes into account their historical origins, their special formal and structural characteristics, their artistic nature, the way in which they are mediated, the economic and social factors that underlie their creation, distribution and reception, and their functionality within the specific social and historical milieux in which they are located. Chapter One doffs its hat in each of these directions and adopts a methodology and set of procedures based on the insights derived from this overview.

The chapter begins by positioning the study of cartooning within the broad theoretical field of cultural and media studies, with an awareness of the historical differences between the 'cultural studies' and 'political economy' approaches, but choosing to work from the point where these two approaches converge. It is observed that the analysis of cultural products is generally gathered at three nodes: political economy, audience

\footnotetext{
${ }^{6}$ The term 'structuration' is used by Vincent Mosco to refer to the consideration of structure as "a duality including constraining rules and enabling resources" (1996: 212).

${ }^{7}$ These problems are identified and discussed in Chapter Six. The phrase 'the voteless masses' was a common 'struggle cliché' during the 1970s and 1980s.
} 
reception and textual analysis. The chapter then looks very briefly at approaches to the problem of ideology in the analysis of symbolic forms, noting the lexical shift from the classic Marxist formulation of ideology to the 'new' approach in left-wing cultural studies, which hinges around the notion of 'signification'.

Some problems specific to the analysis of visual culture are then briefly examined, from both the fine art and cultural studies viewpoints, demonstrating that these different approaches commonly identify a profound paradigm shift in the relationship between text (written and aural) and visual images (photographed and drawn) over the course of the 20 th century. New ways of thinking about the relationship between text and image have consistently lagged behind the technologically driven innovations that have moved modern society away from a predominantly textual base towards an increasingly visual base. This has gone hand in hand with globalisation, the internationalisation of popular culture and the concomitant need for trans-national visual languages.

The chapter notes that the conceptual challenges thrown up by rapid technological and social change were identified by the artistic avant-gardes of the early 20th century, who overturned the prevailing critical paradigms and demanded new ways of looking at visual culture. The chapter very briefly surveys the development of these new ways of looking at and talking about visual images, both as fine art, and as signs. It attempts to find a language of exposition that incorporates terms from the sometimes widely variant traditions of art history, literary criticism and cultural studies.

The chapter ends with an argument for the adoption of a methodology for the interpretation of symbolic forms derived from the work of the British cultural theorist, John B. Thompson (1990). Thompson's methodology arises out of the theory and practice of what he terms 'depth hermeneutics'. Very briefly, symbolic forms are investigated through a synthesis of three procedures:

* socio-historical analysis, in which symbolic forms are placed against their social and historical background;

* discursive analysis, in which the underlying structure of symbolic forms is revealed; and

* interpretation/re-interpretation, in which the information derived from the other two procedures is synthesised with the interpretation of selected primary texts.

Thompson's methodology is adopted, with minor disagreements and adaptations, for the purposes of this study. Chapter Two is based around discursive analysis; Chapters 
Three and Four are based around socio-historical analysis; and Chapters Five and Six are based around the interpretation/re-interpretation of selected primary texts. However, as Thompson points out, these procedures are not mutually exclusive, nor can they be undertaken separately or in any specific order, and therefore the three procedures are interwoven throughout the study. All the chapters contain some discursive analysis, some socio-historical analysis, and some interpretation/reinterpretation.

Following the discursive analysis in Chapter Two, Chapter Three provides a broadly contextualised and very briefly sketched historical overview of the development of cartooning as a form of popular culture in Britain, Europe and the USA, in which several issues of specific relevance to South African cartooning are identified. In Chapter Four, the study focuses specifically on South Africa, covering the period 1884-1984. It begins with a superficial overview of South African cartooning over this period of 100 years, picking up several threads which are then followed through into the 1985-1994 period. The early period has been dealt with in some detail by Murray and Elzabe Schoonraad (1983) and Ken Vernon (2000), and their work has been utilised as the source of material for analysis. Several key cartoonists_William Schroder, Daniel Boonzaier, T.O. Honniball and Jock Leyden-are briefly discussed in order to exemplify trends in South African cartooning, but unfortunately the limitations of this study have proscribed discussion of the work of equally important cartoonists like David Marais, John Jackson and Abe Berry. Afrikaans newspaper cartoonists from this period, other than Boonzaier, Honniball, Eric Thamm and Fred Mouton ${ }^{8}$, have been omitted from the study, since their work was generally congruent with the ideology of apartheid and therefore does not fit into a discussion of discourses of resistance in South African cartooning. The chapter then examines the work of Len Sak and Richard Smith.

Chapters Five and Six bring the discussion into the 1985-1994 period, the focus of the study. Here the insights derived from the discursive analysis and the threads picked up in the preceding socio-historical analysis are woven together with the information derived from the interviews in the interpretation of the selected primary texts. In some cases, the textual interpretation has been informed by answers provided by the practitioners themselves to questions about the intended meanings of their cartoons.

\footnotetext{
${ }^{8}$ Mouton's cartoons, along with those of Eric Thanm and several other pro-apartheid cartoonists, are referred to as examples of cartooning that reproduced and reinforced the ideology of apartheid.
} 
Chapter Five begins with a piece of socio-historical analysis that may initially appear to be external to the topic under study, in that the texts discussed are not 'cartooning' texts per se. But the brief survey of selected literary and critical texts from the period undertaken here performs a crucial function in terms of Thompson's methodology for the interpretation of symbolic forms.

In terms of Thompson's methodology, the analysis of the symbolic forms produced by anti-apartheid cartoonists in South Africa between 1985 and 1994 necessitates some prior understanding of the historical and social context out of which these symbolic forms emerged. This context is itself not a simple thing and comprises several interwoven strands, which may be summarised as:

(i) the historical emergence of cartooning as a cluster of signifying practices expressed in a set of popular culture forms integral to the evolving media of mass communication;

(ii) the history of South African cartooning and its relation to the broader social history of South Africa in the 20th century; and

(iii) the specific social, intellectual and political milieu of the late apartheid period that provided the immediate context in which the cartoonists who are the subjects of this study were embedded and to which they gave expression in their work.

Before proceeding to the primary texts selected for interpretation, it was therefore necessary to pay some attention to the surrounding textual environment through the examination of selected literary and critical texts from the immediate period. Thompson speaks of the 'ethnographic moment' when a symbolic form is released into the public domain, where it is received and appropriated by its original audience. Obviously, we cannot go back in time and re-inhabit this original moment of reception. Therefore the reconstruction of the ethnographic moment, and of the immediate contextual domain from which the symbolic forms we are studying originally sprung, is a necessary precondition for the hermeneutic re-interpretation of previously published texts. Since the normal tools of ethnographic research at the point of reception, such as audience interviews and participant observation, are out of reach, a retrospective method of analysis needs to be followed in order to reconstruct the ethnographic moment. In this study, this method includes conversations with the practitioners themselves, during which they were encouraged to reflect on their experiences of working during the 19851994 period; the present writer's own recollections of the period (a form of participant observation); and other evidence derived from the examination of texts and commentaries produced by other (non-cartooning) artists, writers, commentators and 
cultural practitioners who were actively involved in the propagation of discourses of resistance at that time.

In several cases, textual interpretation has revealed that the originally intended meanings of the cartoons, being related to the political specifics of the day, have been lost or are no longer meaningful, whereas their unconscious or unintended ideological content remains pungently evident and relevant. This process, whereby unintended meanings are revealed, validates Thompson's methodology of 'depth hermeneutics', and his contention that the textual analysis of previously published material involves the re-interpretation of a pre-interpreted domain.

While we have no direct access to the public's responses to the cartoons at the time of their original publication, it seems very likely that, in most cases, the interpretation of these symbolic forms by their respective publics was ideologically congruent with the opinions consciously or unconsciously expressed by the cartoonists. With the benefit of hindsight, and armed with the knowledge of operational semiotic codes and conventions derived from discursive analysis, it is possible to interpret the ideological content embedded in the selected cartoons to reveal important information about public attitudes at the time that the cartoons were originally published. Within the constraints of this study, this has been attempted, although only a few examples are dealt with in much depth. Nevertheless it is hopefully demonstrated that the method works well in uncovering the ideological meanings of cartooning texts.

Similarly, the structural factors that conditioned the practice of the cartoonists themselves come sharply into focus in the light of the socio-historical analysis. These structural factors were generally expressed in terms of the requirements of the publications in which the cartoons first appeared, and in the form of editorial prescription of, or interference in, the content of the cartoons. But structuration also reveals itself in other more subtle ways, resulting in products that often represent some sort of negotiated compromise between the artistic or political intentions of the cartoonists themselves and the prevailing ideological imperatives of the day.

The second half of Chapter Five moves from the discussion of the milieu in which the selected cartooning practitioners found themselves between 1985 and 1994, to a brief examination of some of the key themes in the oppositional cartooning of the period. It distinguishes between political cartooning in the mainstream opposition press, the subject of the remainder of the chapter, and cartooning in the alternative press, dealt 
with in Chapter Six. The cartoonists discussed in Chapter Five are Dov Fedler, Nanda Soobben, Andy (Dave Anderson) and Tony Grogan.

Chapter Six deals with those cartooning practitioners whose work either began in or predominantly appeared in the alternative press. The alternative press is defined to include both the anti-apartheid weekly newspapers of the 1980s and early 1990s, many of which were linked to the United Democratic Front (UDF), and the monthly magazines published by externally funded civil society organisations ${ }^{9}$. Additionally, the definition is widened to include the many publishing projects intitiated by media activists based in NGOs and ecumenical and political organisations who sought to use printed materials in support of their political or educational agendas. Taken together, these alternative publishing initiatives provided an unprecedented range of publishing opportunities for cartoonists and comic strip creators, and most of the important cartooning work accomplished during the 1985-1994 period took place in the context of the broadly defined alternative press.

The most important cartooning practitioners or projects of the 1985-1994 period were Derek Bauer, Zapiro, Madam \& Eve, the Storyteller Group and Bitterkomix. Each of these is discussed in some detail, utilising a combination of textual interpretation, sociohistorical analysis and discursive analysis, reinforced by insights derived from conversations with the practitioners themselves. These five practitioners or projects are also discussed in relation to other practitioners and projects of the period, both in terms of what they gave to and took from each other, and in terms of the profound impact they had on the overall practice of cartooning in South Africa. Undoubtedly, as a result of their pioneering innovations, cartooning in South Africa changed fundamentally in the ten years between the beginning of 1985 and the end of 1994. It also played a not insignificant role in recording, interpreting and inspiring the fundamental social transformation that the country as a whole underwent during this period.

The Conclusion very briefly reiterates the main points of the study as a whole, both in terms of the efficacy of the methodological approach, and in terms of the key themes and insights that have emerged. The key arguments of the study are validated and the

\footnotetext{
${ }^{9}$ The notion of civil society, as it is commonly used in South Africa, is derived from Gramsci (1971) and refers to non-profit-making organisations such as NGOs, churches, trade unions, political organisations and cultural bodies which are generally concerned with transformative interventions in the social or economic realms (see Marais, 1998; Mason, 2001).
} 
most important theoretical and methodological issues are briefly revisited in the light of the insights derived from the analysis of the selected cartooning texts.

The Conclusion ends with a postscript that positions South African cartooning at the end of 1994 in relation to the developments to follow over the next decade, bringing the discussion up to the present. Particularly notable, from the perspective of this study, is the subsidence of the discourses of resistance that had buoyed resistance art in general, and cartooning in particular, before 1994. But while these discourses have subsided, they have not disappeared entirely, and many of the concerns of the 1990s are still with us today. Nevertheless, the role of the oppositional cartoonist in South Africa has changed, from one of unbridled opposition to one of engaged participation, conforming to international practice in democratic societies. This shift is exemplified in the work of Zapiro between 1989 and 2004.

The Conclusion ends by briefly noting the emergence of a post-apartheid popular press in South Africa. The opportunities provided by the new dailies and weekly tabloids have already brought new black cartoonists to the fore, leading to something of a South African cartooning renaissance that at the time of writing is still in its infancy, but shows great potential. 


\section{Chapter One}

\section{Theoretical Context and Methodology}

\section{Key issues in the analysis of cultural products}

While the various forms that cartooning has taken have often resulted in products rich in artistic and literary value, the intention of this study is not to argue for the 'elevation' of cartooning to the status of fine art or serious literature. Since mid-century, the traditional critical distinctions between 'high' and 'popular' art forms have been eroded (Hopkins, 2000) and there are many examples of artists whose work hangs suspended between the categories of cartooning and fine art (eg. Saul Steinberg, Ralph Steadman, Derek Bauer, Richard Smith) ${ }^{10}$, just as there are many fine artists who have delved deeply into the iconography of cartooning (e.g. Picasso, Roy Lichtenstein, William Kentridge, Norman Catherine) ${ }^{11}$. Nevertheless, although the historical barricades erected to protect fine art and literature from the prurient products of populism have long been breached, cartooning does not belong in the echoing halls of the highbrow. Its various forms are clearly situated within the ambit of popular culture, closely entwined with the media of mass communication, and it is as forms of popular culture that cartoons and comics should be studied.

Situating cartooning firmly within the ambit of popular culture necessitates a constant reference to the media via which cartoons and comics are transmitted. In all cases, the technical prerequisites of these media have placed formal problems in the way of cartoonists and publishers, whose responses to the requirements of multiple reproduction, and to the economic factors called into play by the necessities of distribution and sale, are an integral aspect of the story of cartooning.

Thus the study of cartooning is best located within the broad theoretical field of cultural and media studies. This field is itself a contested arena where theorists have historically

\footnotetext{
10 See Saul Steinberg by Harold Rosenberg, 1978; Between the Eyes by Ralph Steadman, 1984; S.A. Flambe and other Recipes for Disaster by Derek Bauer, 1989; and Richard Smith's book and magazine covers (Figures 9 and 44).

11 See Picasso: Artist of the Century by Jean Leymarie, 1971; Lichtenstein by J. Hendrikson, 1994; William Kentridge by Dan Cameron et al, 1999; Norman Catherine by Hazel Friedman et al, 2000.
} 
grappled with "the shifting interplay between the economic and symbolic dynamics of contemporary culture" (Murdock 1997: 86). Much has been achieved in recent writing in this field to settle the feud between the competing 'cultural studies' and 'political economy' approaches (see Ferguson and Golding (eds) 1997) ${ }^{12}$.

The debate often appears to be centred around starting points: culturalists who begin at the point of audience reception are criticised for glossing over the political economies that underlie production and distribution, while sociologists who begin with the relations of production are criticised for their reductionistic and economistic tendencies. In a sense, the debate within cultural and media studies may be seen as endemic to a field that attempts to incorporate discourses that are traditionally separate, the one originating in the arts, the other in the social sciences. It is where academic analysis leans towards political critique that these two fields of discourse find each other, notably in their attempts to identify "the myriad ways in which culture [is] implicated in and shaped by sedimented structures of inequality" (Murdock 1997: 87). The study of cartooning in pre-1994 South Africa provides an excellent opportunity to apply the converging approaches of cultural studies and political economy to a set of cultural products located within, implicated in and shaped by the sedimented structures of apartheid.

According to Douglas Kellner, "the construction of media texts and their reception by audiences is deeply influenced by the systems of production and distribution within which media products circulate and are received" (1997: 104). Kellner provides a welcome corrective to the "artificial" divide between political economy and cultural studies, which he sees as "rooted in an arbitrary academic division of labour" (1997: 104). He locates his argument at the three major sites where the debate has hitherto been concentrated: (i) the political economy of cultural production; (ii) textual analysis; and (iii) audience reception.

(i) The political economy of cultural production

\footnotetext{
${ }^{12} \mathrm{Nevertheless,} \mathrm{proponents} \mathrm{of} \mathrm{the} \mathrm{political} \mathrm{economy} \mathrm{approach} \mathrm{continue} \mathrm{to} \mathrm{berate} \mathrm{'culturalists'} \mathrm{who,} \mathrm{while}$ they are to be congratulated for turning away from "the unabashed elitism of conventional literary and art studies", have unfortunately fallen into "an unabashed populism" that suggests that simply because something is popular, it must be worthy of academic study, and therefore valuable (Gitlin 1997: 30). For Gitlin, the uncritical valorisation of the liberating potential of the media is problematic, particularly in an era of tightening concentration of media ownership.
} 
Kellner notes that the term 'political economy' "links culture to its political and economic context and opens up cultural studies to history and politics" (1997; 105). Cultural production thus occurs within existing relations of domination and subordination and may be seen either to support (reproduce) or challenge (resist) the prevailing structures of power and authority. Cultural products are never innocent of the relations of power that surround their production, and even the most banal products of consumerised media culture reveal themselves as embodiments of the prevailing orthodoxies of the society that has produced them. Numerous studies have demonstrated how media products serve to reproduce the existing relations of domination and subordination in society and at a global level (see, for example, Horkheimer and Adorno, 1972; Dorfmann and Mattelart, 1975; Hall, 1982; McRobbie, 1991; Herman and McChesney, 1997; Botes, $1998)^{13}$. Others have sought to demonstrate how subordinate groupings in society have attempted to resist the hegemony of dominant groupings through the creation of subcultural codes and products, and how these products have often come to signify counter-cultural value systems around which social movements have been able to coalesce (see, for example, Eisen (ed), 1969; Hebdige, 1985; Younge, 1988; Kannemeyer, $1997)^{14}$.

\footnotetext{
${ }^{13}$ Adomo and Horkheimer's analysis of the 'culture industry', written in the 1940's but only published in English in 1972, is the classic statement of Frankfurt School's critique of mass consumption society, later elaborated by Herbert Marcuse in One-dimensional Man (1964). In Honv to Read Donald Duck (1975), their famous diatribe against US imperialism in Chile, Dorfmann and Mattelart demonstrate how Disney comics participate in the propagation of the ideology of the 'American way of life'. Stuart Hall's influential 1982 essay argues for the "re-discovery" of ideology in terms of the insights of structuralism and the politics of signification, providing a sophisticated critique of how a democratic society like Britain relies on "the continuous production of popular consent" by the mass media to ensure its "continuity of existence" (1982: 63). Angela McRobbie's (1981, 1991) analyses of Jackie, the British romance comic for girls, demonstrate how the semiotic codes embodied in the publication-romance, fashion and beauty, pop, and personal and domestic life-combine to prepare girls for their social role as wives, mothers and consumers. Herman and McChesney's (1997) critique of the global media in the late 1990's demonstrates how the global media system serves the globalising market economy, stands in the way of selfdetermination in developing countries and erodes the public sphere. Conrad Botes's 1998 MA thesis demonstrates how South African photo-comics of the 1960s and 1970s propagated stereotypes that reinforced the ideologies of both gender and racial domination.

${ }^{14}$ In The Age of Rock (1969), Jonathan Eisen assembles readings to demonstrate that rock music was a music of revolt, "profoundly involved with the search for new categories of thought and action" (1969:xv). The same is probably true of hip hop music in the 1990s. Dick Hebdige's 1985 comparison between two British magazines of the 1980s, Ten.8 and The Face, demonstrates how these 'alternative' periodicals provide their readers with a body of myth and metaphor as well as "fundamental epistemological categories" in much the same way as the Bible did for previous generations. Gavin Younge (1988) shows that art produced in the South African townships during the 1980s, reproduced on posters and T-shirts and in pamphlets and publications like Staffrider, developed a visual lexicon of struggle and resistance that the apartheid regime found extremely threatening. In his MA dissertation (1997), Anton Kannemeyer demonstrates how the South African underground comix magazine Bitterkomix is located in an iconoclastic subcultural tradition of underground comix publishing.
} 
However, there are also those who argue for a negotiated middle position. For example, in his study of the way that critics and cultural theorists have responded to comics in Britain, Martin Barker (1989) has gone to some lengths to demonstrate that those studies that have imputed ideological motives to the publishers of comics, such as Angela McRobbie's (1981)15 critique of the British girls' romance comic Jackie, have tended to oversimplify the 'contract' between the editors of periodicals and their readers. He argues that the editors of Jackie were very responsive to ideological shifts in British society over the decades of the magazine's production, and shows how the magazine's view of romance shifted progressively from postwar conservatism, through the radicalism of the late 1960s, to the questioning of male superiority in the 1970 s and a decline in confidence in the possibilities of romance after 1975, followed by the rise of anxiety around sexual risk in the early 1980s. The idea of a constantly renewable 'contract' between publishers and their readers is a useful way of conceptualising a more active role for readers while acknowledging the power that media have to shape attitudes ${ }^{16}$.

Clearly, not all cultural products are easily categorised into dominant or oppositional categories. In a society like South Africa, where the dominant political grouping is not necessarily identical to the most powerful economic forces in society (Marais 1999), the relations of domination and subordination are more opaque. In such a context, the media themselves may become a site of struggle between the national political agenda and the hegemonic agenda of the global media marketplace (Barnett 2000). In such a context it may be more difficult to discern the extent to which the products of popular culture serve to reproduce or resist the relations of domination in society, since these relations themselves are to some extent contested.

In addition, it is in the nature of cultural products themselves to resist easy analysis or reduction to simplistic dichotomies like 'dominant' and 'oppositional'. Works of art and

15 Barker responds to two McRobbie 1978 papers and to Feminism for Girls: an adventure story (with Trisha McCabe, 1981). McRobbie's book Feminism and Youth Culture: From Jackie to Just Seventeen, was published in 1991, after the publication of Barker's book.

16 Interestingly, in a more recent study of women's magazines in Britain, McRobbie (1996) argues for a reconceptualisation of production and consumption in the world of consumer magazines to allow a space for greater reflexivity and critique on the part of the reader. The female reader of fackie, previously directed single-mindedly to the pursuit of romance in search of the ideal husband, is now "given a distance from what in the past was presented as a feminine destiny, to find the man of one's dreams" (McRobbie 1996: 188). 
literature, almost by definition, tend towards ambiguity, ambivalence and complexity, and cultural products that embody these characteristics may not easily surrender their meanings in terms of their relation to prevailing structures of power and orthodoxy in society.

\section{(ii) Textual analysis}

The textual analysis of cultural products is therefore an essential component of cultural studies. However, the utilisation of traditional approaches to literary and fine art criticism in the analysis of the products of popular culture is beset with theoretical problems. Most particularly, these problems emerge around the role played by the processes whereby texts are mediated for popular consumption. Moreover, because the mass media through which popular culture is transmitted are by their nature capitalintensive and organisationally or institutionally structured and owned, the form and content of mediated cultural products cannot easily be abstracted from the economic and political relations in which they are embedded.

It is at this point that the notion of ideology unavoidably enters into the project of textual analysis. According to Kellner :

\footnotetext{
The concept of ideology requires analysing texts in terms of specific relations of power and domination and criticizing the ways in which cultural artifacts reproduce or resist oppression and subordination. Ideological analysis thus forces one to situate the text within the context of existing systems of domination and struggles against it (1997: 110).
}

Teasing out the ideological dimensions of a cultural product, without falling into the trap of reductionistic determinism, may require, argues Kellner, a multi-perspectival and transdisciplinary approach, and may need to incorporate a variety of methodologies. These might include a Marxist approach to issues of class, a feminist approach to issues of gender, a semiotic approach to the identification of significatory codes and the production of meaning, or a psychoanalytic investigation of subliminal content (1997: 110), as well as more traditional literary approaches to the analysis of narrative structure, plot and characterisation Likewise, the aesthetic categories of fine art criticism might be employed in the analysis of composition, light, colour and texture, and filmic categories utilised to reveal strategies of framing, viewpoint, timing and so on. 
Textual analysis is by nature preoccupied with the ways in which meanings are 'encoded' in texts, but, as the influential branch of cultural studies led by Stuart Hall (1986) has acknowledged, it cannot be assumed that a predictable set of meanings will automatically be 'decoded' by the audience. Each reading of a text will be one of many possible readings, but it is nevertheless likely, within a specific system of production, distribution and reception, that certain 'preferred readings' will be privileged by the structural conditions that constitute this context. In turn, the expectation of preferred readings is likely to influence the process of encoding at the point of cultural production.

\section{(iii) Audience reception}

Referring to audience reception as the third site of analysis, Kellner acknowledges the contribution of cultural studies in stressing that "all texts are subject to multiple readings depending on the perspectives and subject positions of the reader" (1997: 112). However, he warns that "(w)hile this emphasis... helps cultural studies overcome earlier one-sided textualist orientations, it can also lead to simple affirmations of audience pleasure and fandom, thus promoting, rather than critically analysing, media culture" (1997: 113). A key issue is the extent to which audiences are enculturated within systems of media representation, habituated to textual codes and thus themselves become "constructs of a system of production" (1997: 114) in which preferred readings are reinforced. Kellner, along with Gitlin (1997), opposes the tendency in some cultural studies writing to romanticise the extent to which audiences are able to produce their own meanings and escape the manipulative power of the mass media.

Finally, Kellner suggests that there has been "a tendency in the cultural studies tradition of reception research to dichotomize between dominant and oppositional readings" (1997: 115). As noted earlier, not all texts present themselves unambiguously with respect to the revelation of their meanings. Ambiguity, ambivalence and complexity are often signals of artistic merit. A single text may engender a range of contradictory emotions in the reader, reinforcing certain meanings that correspond with dominant or preferred readings while challenging others, often resulting in a 'negotiated' reading that incorporates both dominant and oppositional elements (See Figure 2) ${ }^{17}$.

\footnotetext{
17 The work of South African underground cartoonist Joe Dog (Anton Kannemeyer) frequently presents images that rupture the distinction between 'dominant' and 'oppositional' readings. In Figure 1, a cartoon published in the Alrikaans soft-porn magazine Loslyf (1995), the exploitative presentation of the female figure as a fantasy objecl for the male gaze is overturned and fears of castration and penile ampulation are wittily conflated with the codes of big game hunting. Kannemeyer's work, widely condemned as pornographic, critiques white male power and makes [requent reference to male fears of sexua] inadequacy.
} 


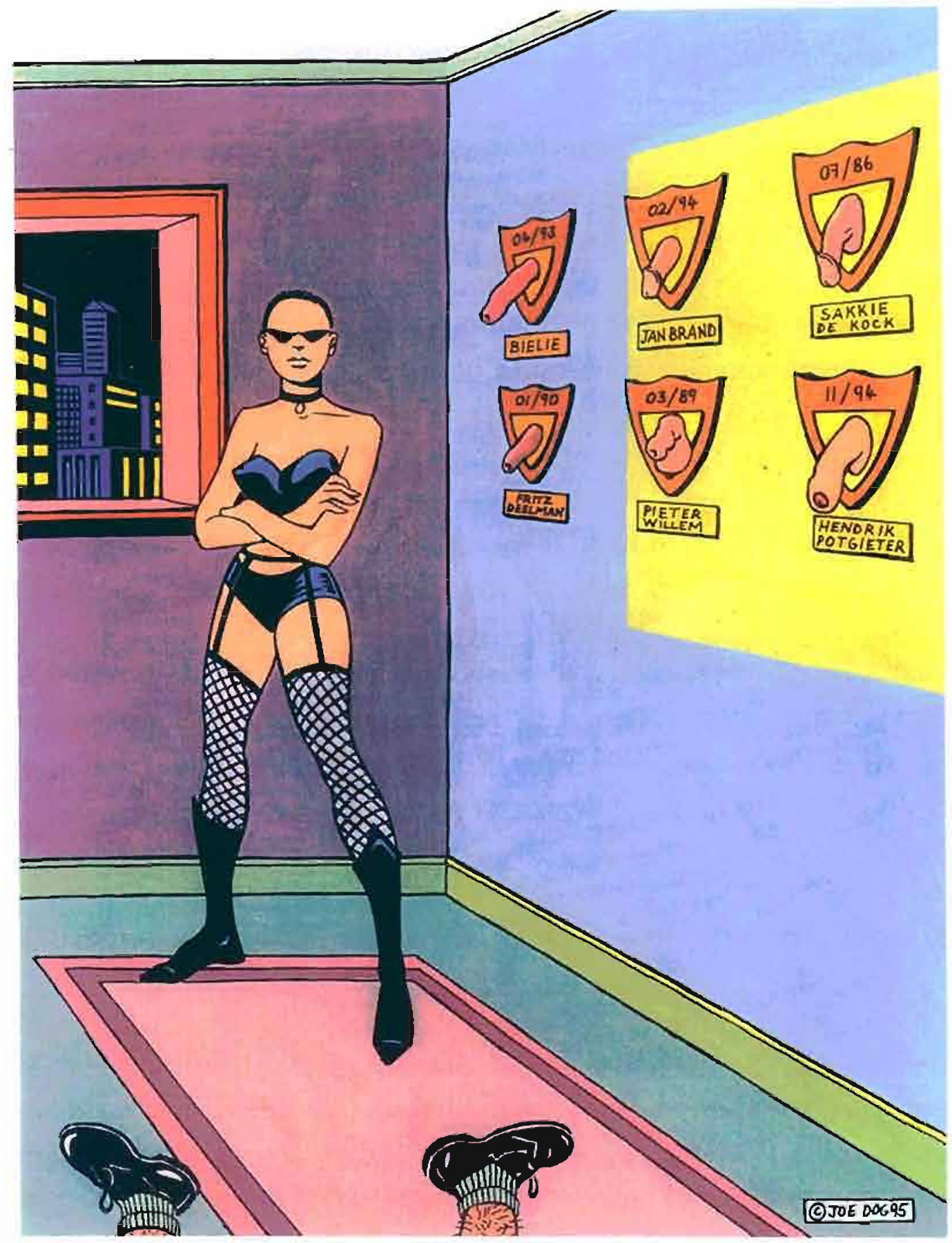

FIGURE 2

The distinction between 'dominant' and 'oppositional' readings of a visual text is ruplured by Bitterkomix cartoonist /publisher Anton Kannemeyer (Joe Dog) in this cartoon, published in the Afrikaans soft-porn magazine Loslyf in 1995. Pornography's conventional presentation of the female figure as a fantasy object constructed for the male gaze is subverted and fears of castration and penile amputation are wittily conflated with the codes of big game hunting. 
A prerequisite of effective critical analysis is the recognition that cultural products are inherently complex at the point of creation and are further complicated in the processes of production, mediation and reception. They involve the metaphorical or symbolic representation of meanings which, in the process of being published or broadcast, are often, if not usually, subjected to collaborative interventions that may cause the original idea to be manipulated and distorted. The editorial and publishing processes bring to bear upon the original idea a range of ideological pressures that relate to the agendas of the organisations that own the means of cultural production and these pressures are further compounded by the technical requirements of the medium. Thus a journalist's story may be sub-edited to remove ambiguity, photographs selected to reinforce the editorial agenda and headlines and captions added to point the reader towards a preferred reading. Furthermore, the requirement for stories to be written to, or shortened to, a specific length dictated by technical requirements may further truncate or distort the original idea. The history of cartooning presents numerous examples of this kind of editorial interference, and attempts by artists and writers to escape these constraints have led to key developments in the field, such as the rise of self-publishing and the underground comix movement of the late 1960s.

However, editorial interference or manipulation is only one factor that has to be dealt with in the analysis of cultural products. The operation of ideology at a deeper level is far more difficult to describe, and the identification of the ideological underpinnings of particular products invariably invokes the critic's own ideological perspective. Ideological analysis is further complicated by the perception that the meanings and potential meanings embedded in a cultural product may not be fully evident to the creators thereof, and the meanings taken from cultural products by audiences may be completely at odds with those intended.

Critical analysis is therefore at best an imperfect art, can never be a science, and is essentially interpretative, bringing to the process of decoding the particular concerns and proclivities of the critic. Recognising these problems, Thompson (1990) has outlined a useful methodology for the interpretation of what he calls 'symbolic forms', which is utilised in this study. But before considering Thompson's methodology, it is first necessary to examine some of the theoretical problems that arise in the analysis of ideology in relation to the production and consumption of symbolic forms in general, and, more specifically, the kinds of issues that arise where symbolic forms are primarily visual. 


\section{Approaches to the problem of ideology in the analysis of symbolic forms}

Thompson's view of ideology (1990), while broadly consistent with that of Kellner (1997), is perhaps idiosyncratic in that he insists that the use of the term 'ideology' should be reserved for those symbolic forms which serve to establish and sustain relations of domination, and are by nature hegemonic, whereas "contestatory symbolic forms" are not ideological in themselves but are "incipient forms of the critique of ideology" (Thompson, 1990: 68-9). Thompson amassed considerable argument in support of this viewpoint in his book Ideology and Modern Culture (1990), but this aspect of his work does not appear to have been as well received by the media studies community as he might have wished.

For the purposes of this study, following Keliner, the term 'ideology' will be used in its broader sense: not merely to refer to those cultural products that reproduce and sustain relations of domination in society, but rather to signify the overall relationship that any text may have to the system of power and domination in which it is located and embedded. The ideological tendency of a particular work may therefore be analysed in terms of the way in which it reproduces, resists or negotiates an ambiguous position in relation to the dominant ideas of the particular society and epoch in which it is located and embedded. In this way, the oversimplifying dichotomy between dominant and oppositional readings can be avoided and the complex and ambiguous nature of cultural products allowed for.

Film and television are two categories of cultural production that have received a lot of attention from cultural theorists, but their combination of aural and visual texts poses different problems from those media in which the visual image is unsupported by sound. The fine arts in turn have generated an extensive body of critical discourse, stretching back over centuries, but the primarily aesthetic discourses of fine art theory and criticism have generally been bypassed in cultural studies writing. The print-based products of cartooning, such as the editorial cartoon or the comic book, pose particular kinds of problems for analysis because they are primarily visual, or involve a combination of visual imagery with text. These problems are of a different order from those that attend animated cartooning, where problems of visual representation are more closely related to those of film than to those of printed texts. In any event, cartooning has not received sustained attention from English cultural studies theorists ${ }^{18}$

\footnotetext{
18 Angela McRobbie (1991), Martin Barker (1989) and Roger Sabin (1988) are amongst the few comics theonsts to have emerged from the British cultural and media studies tradition.
} 
and an examination of the kinds of analytic problems that are raised in the study of cartooning must therefore begin with insights derived from studies of other forms of popular culture.

As Tony Bennet (1982.1) has shown in his presentation of the so-called 'Four Traditions' of media theory, early responses to the media of mass communication focused on their presumably deleterious effects. In the early decades of the 20th century, conservative and Marxist critics alike bemoaned the onset of mass culture and the loss of cultural value. A key remedial text in this respect was Walter Benjamin's celebrated 1936 essay "The Work of Art in the Age of Mechanical Reproduction" (1999), which, while registering the loss of the 'aura' of the original fine art work in reproduction, nevertheless celebrated the exposure of the products of high culture to the masses. In the United States, the pessimism of the Frankfurt School was aggressively countermanded by the pragmatic optimism of the U.S. school of mass communication research, which valorised new forms of popular culture (such as comics) and sought to appropriate them as expressions of a hegemonic Americarism (Witek, 1999).

Bennet suggests that the liberal-pluralist tradition of social theory emerged from within the mass society tradition by means of a critique of it (1982.1: 40). He argues that the younger generation of American sociologists of the post-war years strove to develop a social theory that would enable a clear distinction between the 'positive' expression of mass society found in the Western democracies and the 'negative' expression found in the totalitarian regimes which were defined in the emerging rhetoric of the Cold War as the enemies of democracy. In terms of the liberal-pluralist tradition, the media, far from being mind-numbing agents of totalitarianism, were in fact the healthy expression of a heterogeneous culture in which a diversity of viewpoints was tolerated and even encouraged.

The idea that the mass media, particularly television, could contribute to the upliftment of society as a whole by bringing into the living rooms of the masses cultural experiences and values hitherto reserved for the elite classes of society, provided that proper regulatory measures were in place, lay behind the development of the public broadcasting model of the BBC (Thompson, 1990). While the public broadcasting model pioneered by the BBC's John Reith espoused "a tradition of public service and of devotion to the highest interests of community and nation" (Reith, quoted by Thompson, 1990: 255), it carried the risk, as noted by Thompson, " of a kind of cultural paternalism in which the cultivated sensibilities of particular social groups are institutionalized as the 
norm to be maintained in the production and appreciation of mediated symbolic forms" (1990: 257, original emphasis). As the various struggles over the control of television broadcasting in southern Africa have demonstrated (see Tomaselli \& Durn (eds), 2001), and as Thompson has noted in the British context, "broadcasting institutions have been particularly susceptible to the exercise of power by members of the government and officials of the state" (1990: 258).

Thompson's response to these problems is to espouse "the principle of regulated pluralism" in which a plurality of independent media institutions would be protected by institutional arrangements from erosion by either the state on the one hand or largescale corporate media organisations on the other (1990: 261-263). The controversy about the role of 'embedded journalism' in the 2003 war in Iraq has demonstrated the continuing importance of this debate, pointing to ongoing collaboration in the construction of news between the powerful state institutions of the U.S. and equally powerful media organisations like CNN. In such a context the maintenance of a plurality of independent voices becomes even more difficult, and more vital.

Scepticism towards the ideal of independent media institutions has always been a characteristic of the Marxist tradition in cultural and media studies, which has generally viewed the mass media as primary vehicles for the propogation of hegemonic viewpoints, Since the 1970s, Marxist cultural analysis has shored up its position in response to allegations of mono-causal determinism by incorporating insights derived from psychology, structural anthropology, linguistics, structuralism, semiology and semiotics (see, for example, Bennet, 1982.2; Hall, 1982; Curran, 1996). These innovations have been germinal to the development of the fourth of the traditions identified by Bennet-a more sophisticated Marxist theory of the media in which media messages themselves have been subjected to intense scrutiny, particularly with regard to the way that meanings are embedded (encoded) in them and extracted (decoded) from them, and the ways in which the possible meanings to which individuals have access are structured by the significatory systems of class, gender and race.

The key to this new approach, argues Bennet, has been something of a paradigm shift in the understanding of what is meant by 'ideology'. The classic Marxist formulation is that ideology is the product of forms of consciousness primarily determined in the economic sphere. According to this formulation, the view that ordinary people have of their world is distorted by the action upon their consciousness of unconsciously apprehended social and economic relationships that structure their thinking in ways of 
which they are unaware. In short, "ideology comprises the sphere of representations within which an 'imaginary' relationship to the conditions of existence is produced" (Bennett, 1982.1: 48). In terms of the 'new' approach to ideology, however, "the signifying systems which constitute the sphere of ideology are themselves viewed as the vehicles through which the consciousness of social agents is produced" (1982.1: 51).

The lexical shift from the classic formulation of ideology to the 'new' approach in leftwing cultural studies is subtle but profound. It hinges on the notion of 'signification', a key term in the vigorous discourse that revitalised Marxist thinking in the second half of the 20th century. A common thread that runs through the influential writings of (mainly) French social theorists of the late 1960s and 1970s, such as Louis Althusser (1969), Michel Foucault (1970) and Roland Barthes (1973), is structuralism-the idea that meaning is generated within signifying systems governed by discoverable sets of rules that can be systematically elaborated (O'Sullivan et al, 1994: 302-305). The structuralist project strove to demonstrate that the kind of structuration found in language is also found in all kinds of cultural activity. The concepts of 'sign-system' ('signifying system') and 'signification', common to linguistics, structuralism, semiology and semiotics, were derived from the linguistic theory of Ferdinand de Saussure (1916) and elaborated in the 1930 s by Peirce (1931) and others, who sought to establish a 'general science of signs' ! (McQuail, 1994: 245). These ideas were then re-articulated by the French structuralist project of the 1970s.

The classic theory of 'reflection' or 'mimesis', as exemplified in the literary criticism of Georg Lukacs (1962), insists that symbolic forms should reflect or mirror the society from which they emerge. But, by the end of the 1970s-despite its decades of service to the cause of socialist realism and the social command (Laing, 1978: 20-45)-reflection theory had been rather ignominiously consigned, alongside other exhausted metanarratives, to the dustbins of critical history, and replaced by the more flexible and knowingly postmodern idea of 'signification'.

While the demise of reflection theory should probably be mourned as the loss of an important way of looking at cultural products, the notion of 'signification' is nevertheless preferable to 'reflection' as a critical tool, because it denotes a far more active process whereby meaning is constructed along a continuum which proceeds from the encoding of the media message by its producer(s) to its decoding by individual members of the media audience. At no point along this continuum are meanings fixed, but, as noted above, are always to some extent ambiguous and negotiated. This process 
takes place within signifying systems in which preferred meanings are held in place by enculturated significatory codes and conventions, but these meanings are nevertheless open to some degree of individual interpretation by members of the audience. Possible variations in the meanings constructed by individuals are in turn constrained by the extent to which the social groupings to which they belong will tolerate deviations from normative preferred readings.

This formulation was very important in the development of media theory because it overcame the economic reductionism inherent in the classic Marxist concept of ideology and allowed an element of pluralism, without blunting the critical edge of the Marxist approach. The most influential English theorist in the shift towards the new lexicon of cultural and media studies was Stuart Hall, progenitor of the so-called Birmingham School of media and cultural studies. In a series of celebrated essays (inter alia, 1980; $1982 ; 1985 ; 1986)$, Hall defined the concerns and parameters of the new approach to cultural studies. However, for the purposes of this study, it is the way in which the debate around visual messages has been formulated into a new lexicon of 'visual culture' (Evans and Hall, 1999) that is of primary concern.

\section{Problems specific to the analysis of visual culture}

The notion of 'visual culture' as a field of study that cuts across specific media categories and the disciplines constructed around them is relatively new (Evans and Hall, 1999: 6). Whereas the analysis of cultural products in general throws up a slew of theoretical problems, some of which have been mentioned above, even more complex problems are revealed by the concentration of analysis specifically on those kinds of products where the visual image plays a central role in holding meaning. During the course of the 20th century, the dominant critical approaches to literary studies and the theory of art revealed themselves to be increasingly inadequate in the face of the theoretical demands of photography, film, television, consumer magazines and other aspects of popular visual culture. New approaches to the analysis of visual culture were required.

In the fine arts, the epistemological foundations of art theory and criticism had been shaken at the turn of the 20th century by a series of seismic developments. The neoclassical academicism of European painting with its highly sophisticated techniques aimed at achieving a naturalistic portrayal of the world, refined and finessed over the course of several centuries, was challenged on the one hand by Impressionism, PostImpressionism and Cubism, and on the other by photography and film. The 
documentary function of painting-particularly with regard to portraiture and the representation of accumulated wealth (Berger, 1972)_became increasingly redundant under the onslaught of the new film-based technologies. By the 1890 s it was no longer a privilege reserved for the elite classes of society to possess an accurate visual representation of oneself19. Photography and film asserted their primacy as documentary media, liberating painting and sculpture from what Norman Bryson (1999) has termed 'the natural attitude', the goal of which was the 'perfect copy' of reality.

The intellectual ferment of the early years of the 20th century gave rise to a bewildering proliferation of fine art movements-Post-Impressionism, Symbolism, Fauvism, Expressionism, Cubism, Futurism, Surrealism-as artists began to explore the terra incognito of the new century (Hughes, 1991). From this cacophony of competing claims emerged the thesis of Modernism-championed by the art critic Clement Greenberg and exemplified in the Abstract Expressionism of Jackson Pollock (Hopkins, 2000). In terms of Greenberg's Modernism, representational art was anathema; a painting had to assert its existence purely on its own terms. Questions of subject matter were deemed extraneous to the meaning and function of art, which, in Greenberg's view, should be restricted to self-referential formal concerns that emphasised quality and inviolability (Hopkins, 2000: 40). Modernism thus expressed the antithesis of the 'natural attitude' of Renaissance painting.

Against this humourless Modernism were ranged the oppositional forces that gathered around Dada - the iconoclastic art movement led by proto-Dadaist, Marcel Duchamp (Naumann, 1996). The Dadaists introduced into the refined academic discourses of fine art a noisy, disrespectful and mischievous spirit that demanded new ways of looking at painting and sculpture. Dada strove to disrupt the 'stillness' of the surface of the work of art through a variety of disquieting, absurd or contradictory textual strategies, such as the inclusion in the work of false signatures, or intentionally obscure or contradictory titles. Whereas the Modernism espoused by Greenberg sought to purify the activity of abstraction until all textual elements were eliminated from the work, Dada focused the entire work around its textual meaning. The radicalism of Dada had been anticipated in the Cubist paintings and assemblages of Picasso, Braque, Gris and Leger, who incorporated text elements into their works ${ }^{20}$. For the Dadaists, this incursion of text

\footnotetext{
19 The Kodak box camera was launched onto the market in 1888 (Hughes, 1991).

${ }^{20}$ See Ruhrberg et al, 2000. The inclusion of text in visual images was a feature of medieval painting, but was proscribed by the ascendancy of 'the natural attitude' in Renaissance painting (Bryson, 1999). It was also commonly found in popular woodcuts, chapbooks, caricatures and cartoons from the medieval period onwards (Klingender, 1944; Kunzle, 1973).
} 

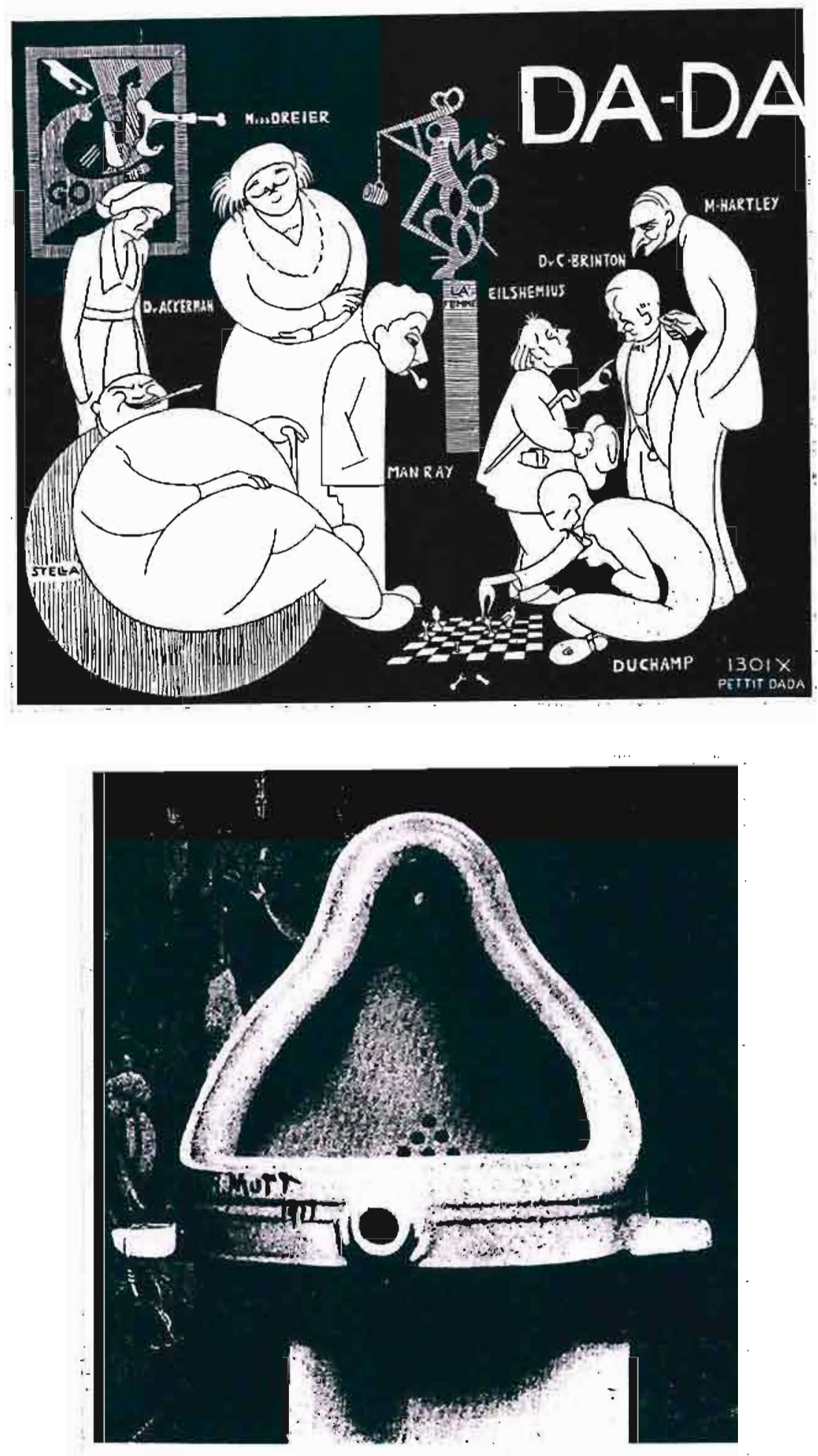

FIGURE 3

(Above) This drawing by Richard Biox describes a seminal Dada meeting in New York on 1 April, 1921, attended by proto-Dadaists Marcel Duchamp and Man Ray. The objects in the background satirise contemporary Dadaist art works.

(Below) A photograph by Alfred Stieglitz of the infamous "Fountain" by Marcel Duchamp (1917), an upturned urinal upon which is inscribed the signature " $R$. Mutt". 
onto the visual surface of the artwork became a crucial strategy, defining the parameters of a debate that was to occupy visual art theorists for the rest of the century.

Francis Naumann (1996) draws attention to one aspect of Dada that has particular relevance to the development of visual culture in relation to the history of cartooning - the use of humour and satire to poke fun at and debunk the dearly-held beliefs of the establishment, be they aesthetic, political or religious. According to Naumann (1996: 19), epater les bourgeois-the highly intellectual kind of humour specifically designed to shock and confuse, employed by Duchamp, Man Ray et al-derived from a time-honoured French tradition. But the bawdy humour of comic theatre, cartoons and caricatures was frowned upon in the context of the serious visual arts. Dada strove to mock and disrupt this highbrow seriousness. Naumann notes that the pseudonym Duchamp chose to adopt in signing his controversial 1917 work Fountain (an upturned urinal) - R. Mutt-alludes to the comic strip Mutt \& Jeff (1996: 20) (Figure 3) ${ }^{21}$. Thus, in Dada, we find the embodiment of two essential ingredients of cartooning: the incursion of text onto the visual surface, and the use of humour and satire.

By legitimating the banal, Dada had an inordinate impact on the development of visual culture in the 20th century and contributed greatly to the collapse of critical distinctions between 'high art' and 'popular culture'. Evans and Hall (1999: 6) note that by the late 20 th century the notion of 'art history' had been displaced by the notion of 'visual culture', denoting a dual shift from 'art' to 'visual' and from 'history' to 'culture'. In this context of disintegrating normative paradigms, a new critical lexicon to replace the embattled vocabularies of literary criticism and art theory was required. This took decades to emerge, and may have been one of the great critical projects of the 20th century. Arguably, this new lexicon is still in the process of being formulated, and may never catch up with the rapid pace of technologically-driven change that is constantly reconfiguring the global media environment. More than a superficial gloss over the development of new ways of talking about words and pictures is impossible here, but it

\footnotetext{
21 Below: A photograph by Alfred Stieglitz of "Fourtain" by Marcel Duchamp (1917), Above: A drawing by Richard Biox, entitled "DA-DA (New York Dada Group) (1921); both reproduced in Francis M. Naumann, Making Mischief: Dada inzades New York, 1996.
} 
is necessary to at least mention some of the major concerns that have emerged over the last 50 years, particularly those that are germinal to the study of cartooning.

In their introductory statement to Visual Culture: The Reader (1999), Jessica Evans and Stuart Hall outline the key terminological parameters of the debate. They argue that "the mechanically or electronically reproduced image is the semantic and technical unit of the modern mass media and at the heart of post-war popular culture" (1999: 2), but observe that the study of the visual image has been a sorely neglected area of media and cultural studies. They advance a number of reasons for this apparent neglect, suggesting that a preoccupation with social practices and relations as signifying systems, and with the social and ideological contexts in which visual texts are used and manipulated, has tended to overshadow analysis of the precise nature of visual images themselves.

The so-called 'linguistic turn' in cultural studies has led to "a privileging of the linguistic model in the study of representation" and this, in turn, has led to "the assumption that visual artefacts are fundamentally the same, and function in just the same way as any other cultural text" (1999: 2). The contrary is in fact the case, as noted by Roland Barthes as early as 1964, in his famous analysis of a magazine advertisement for Panzani Foods, where he pointed out, from the viewpoint of linguistic structuralism and semiology, that the visual image presents a number of special problems:

Thus we find ourselves immediately at the heart of the most important problem facing the semiology of inages: can analogical representation (the 'copy') produce true systems of signs and not merely agglutinations of symbols? [...] We know that linguists refuse the status of language to all communication by analogy [...] Nor are linguists the only ones to be suspicious as to the linguistic nature of the image; general opinion too has a vague conception of the image as an area of resistance to mearing [...] Thus from both sides the image is felt to be weak in respect of meaning; there are those who think that the image is an extremely rudimentary system in comparison with language and those who think that signification cannot exhaust the image's ineffable richness [...] (Barthes, (1973) 1999: 33)

Forty years after it was written, Barthes' analysis of the Panzani advertisement still resonates with conceptual problems. The idea of the visual image being "weak in respect of meaning" is in itself an indication that the semiological theory derived from Saussarian structuralism becomes inadequate or inappropriate the moment it is systematically applied to the analysis of visual images (particularly photographs). The 
problem with photographs, in these terms, is that the signifier (the photograph) often so closely resembles the referent (the object photographed) that the notion of the 'signified'- the mental picture or idea invoked by a physical sign in a given language code that is supposed to stand between the referent and the signifier-begins to slide away. Because it appears to be mechanically created, "stencilled off the real" (Sontag, 1999: 80), the photographic image presents the photographed object as something that has an existence "independent of a viewing subject" (Evans, 1999: 13). Key concepts in Saussarian semiology - of the signifier as an arbitrary image assigned to a particular referent, with no necessary physical resemblance to the referent-are not applicable in the case of the apparently 'purely denotative' photograph.

In his analysis of the visual components of the Panzani advertisement, Barthes was led to conclude that where a photograph is an accurate representation or 'copy' of an object in the real world, it tends to resist semiological analysis: it becomes " a message without a code" (original emphasis) (Barthes, 1999: 35). No special knowledge or access to cultural conventions is required to understand it, and reading it is "a matter of an almost anthropological knowledge" (Barthes, 1999: 36).

This notion of 'anthropological' knowledge is obviously problematic, and Barthes and his contemporaries subsequently debated and elaborated these concepts to the point where it became widely accepted that there is no such thing as a message without a code and that "every sign presupposes a code" (Chandler, 1996). What is important about Barthes' initial reaction to the problem of the apparently "purely denotative" photograph is that it demonstrated the limitations of the semiological lexicon derived from Saussure in its application to the increasingly visual culture of the 20th century.

In order to better understand this crucial moment in the discourse around visual culture, it is necessary to take a few steps back and look at the challenge that photography posed to traditional notions of the relationship between visual images and the reality they were supposed to represent. As Susan Sontag has pointed out, photographic imagery is so authoritative and has become so pervasive and so predominant in contemporary culture that the 'real world' is, in a sense, being replaced by an 'image world':

'Such images are indeed able to usurp reality because first of all a photograph is not only an image (as a painting is an image), an interpretation of the real; it is also a trace, something directly stencilled off the real, tike a footprint or a death mask" (Sontag. 1999: 80). 
A photograph is more than a copy of its subject, "captured mechanically" (Chandler, 1996)-it appears to be a physical outgrowth of reality. Because of its compelling veracity, it adds to or changes the way that reality is experienced. It is also an appropriation of the world-a way of gaining control over it. The notion of reality as something 'out there' and the photograph as a 'copy' of that 'extemal' reality is no longer appropriate in a world where photographic images have increasingly come to 'constitute' reality itself. People use photographs to decipher and appropriate reality by dividing off from the continuous stream of experience discrete moments that are important to them. For many people, a wedding or anniversary is not 'real' until it has been 'fixed' in a photograph. As Barthes has suggested, the photograph 'illogically' conjoins the mutually exclusive categories of "the here-now and the there-then" (original emphasis) (1999: 40).

Turning back to fine art, if one looks behind the notion of the so-called 'perfect copy' that was the goal of the 'natural attitude' in Renaissance painting, it is clear that, as Bryson (1999) has demonstrated, this attitude was itself an expression of the prevailing ideology of the day. And this ideology was, in tum, closely related to the technically determined representational possibilities of the available visual media at that time. Prior to the invention of photography, oil painting was the most convincing method available for the documentation of reality. The ability to provide a 'perfect copy' of reality was made possible by the discovery of single-point perspective and the refinement of the chiaroscuro technique in oil painting. Used together, these techniques allowed a very convincing illusion of reality to be produced on canvas. But, as Robert Hughes (1991) has pointed out, the view of reality propagated in Renaissance and post-Renaissance painting was "an ideal view, imagined as being seen by a one-eyed, motionless person who is clearly detached from what he sees" (1991: 17). In direct opposition to this view, the fine art movements of the early 20th century expressed the realisation that human perception of the world is by its very nature fleeting, momentary, flawed and unstable.

Barthes' 1964 analysis of the Panzani advertisement suggests that, at that time, there was a need for a critical lexicon that acknowledged that people were looking at and responding to visual images in new ways. A crucial key to unlocking the riddle of the 'message without a code' was to be found in the work of the American philosopher Charles Peirce (1931). As Tomaselli (1996) has pointed out, Peirce provided an important key to the understanding of sign systems by allowing semiotics to distance itself from the restrictive parameters of Saussurian linguistic structuralism. From the contested terrain of semiotic terms (Tomaselli, 1996: 29), Peirce's concept of the non-linguistic sign 
emerged as an important tool in the analysis of visual culture. As Evans (1999) explains, the meaning of visual signs, as conceptualised by Peirce, is not dependent on the idea that meaning is assigned by convention to an arbitrary signifier. Nor is Peirce's concept of the sign compromised where the signifier resembles the referent. On the contrary, the signifier may so closely resemble the referent that the signified appears to disappear from the process of signification. This realisation was profoundly important because it liberated semiotics from the linguistic conundrum:

While Saussure argued that linguistics could serve as a model for semiotics because in the case of language [the] arbitrary and conventional nature of the sign is especially clear, Peirce was concerned that non-linguistic signs may be less arbitrary; his extension of the analysis of the sign into the triad of sign, icon and index was an attempt to think about the relationship between signs and referents, an approach explicilly outlawed in Saussure's model in which it is only the conventional relationship between signifier and signified that is relevant. (Evans, 1999: 13)22.

The categories provided by Peirce for the study of signs provide a route into the analysis of visual images that is very useful in the study of cartooning. In terms of Peirce's formulation (Chandler, 1996; Tomaselli, 1996: 30-31), an 'iconic' sign is one which looks like the thing signified, while an 'indexical' sign is one which draws attention to the thing to which it refers without actually representing it A sign may be said to be 'symbolic' if it depends upon meanings that are relatively arbitrary and assigned in terms of generally understood conventions. Thus, in a cartoon, the depiction of a cartoon character would be iconic, the depiction of motion lines to signify rapid movement would be indexical, whereas the depiction of a conventional object assigned to suggest the character's profession, such as a pen behind the ear, a stethoscope around the neck or a certain kind of hat, would be symbolic. The codes of cartooning are therefore a combination, in Peircian terms, of iconic, indexical and symbolic signs.

Another semiotic insight of importance to the study of cartooning, derived from Barthes, is the distinction between 'denotative' and 'connotative' signs (Chandler, 1996). In his analysis of the Panzani advertisement, Barthes identified three kinds of messages: a linguistic message, a coded iconic message and an uncoded iconic message (1999: 36). The difficulties inherent in the notion of the uncoded iconic message have been mentioned above, but the idea behind it is that the uncoded image is essentially

\footnotetext{
22 I have replaced the word 'and' with the word 'the' (bracketed) to correct what appears to be a typographical error in Evans's Introduction to Part 1 of Vissul Culture: The Reader (1999: 13).
} 
denotative - it merely denotes what is already there without commenting on it. Barthes uses the word "utopian" to describe the notion of a purely denotative image, indicating that it is very difficult to imagine an image that does not connote or imply something other than itself. A coded iconic message, on the other hand, is laden, saturated even, with meanings other than those denoted by the physical attributes of the image. The intention of the coded iconic message is not to copy reality, but to comment on it by drawing attention to things that are not immediately evident, and to add to it by referring to other things that are not even in the picture.

These are crucial concepts for the study of cartooning. In addition, Barthes made some very interesting distinctions between photographs and drawings that should be mentioned here. The conceptual difficulties that attend photographs dissolve in the case of drawings: drawings are always coded, connotative messages. The coded nature of the drawing can be seen at three levels. First, it requires "a set of rule-governed transpositions" (original emphasis) which are historical (notably those governing perspective) (1999: 39). Secondly, the drawing is selective in a way that the photograph cannot be. The photographer (or editor), by framing and cropping a photograph, can select some information and omit other information (at the time that Barthes was writing, it was not easy to intervene within the photograph itself 23 ). By contrast, in a drawing, the artist intuitively eliminates or backgrounds insignificant aspects of the picture, while foregrounding and emphasising the connotatively significant elements. In a cartoon, this process is often taken to extremes, and everything except the connotative and indexical information is eliminated from the cartoon. This is why cartoons are often such powerful messages.

The third level of encoding of the drawing, according to Barthes, refers to the fact that, "like all codes, drawing demands an apprenticeship". This is important because "the 'execution' of the drawing itself constitutes a connotation" (1999:39). This is an extraordinarily interesting insight in respect of cartooning, suggesting that the artistic ability (and in particular, the technical proficiency) of the cartoonist is likely to confer some form of authority upon the idea promoted in the cartoon. A poorly drawn cartoon would therefore be less likely to be effective than a well-drawn one.

\footnotetext{
${ }^{23}$ Today it is common practice for editors to interfere with the information within photographs by using Adobe Photoshop or similar pholo-manipulation software.
} 
Another set of insights derived from Barthes that are particularly relevant to cartooning are the notions of 'anchorage' and 'relay'. These are important because they relate to the relationship between the image and the text. Barthes points out that images invariably exist in relation to texts. These may be spoken texts, as in animated cartoons, or written texts, as in cartoons and comics. Even where an image contains no text, it is still contextualised by the text that surrounds it. Barthes makes an interesting suggestion as to why images are so often hemmed in by text. Because images are polysemic (capable of holding more than one meaning) there is a general phobia about them - "a terror of uncertain signs" - which derives from their unfixed nature and their capacity to harbour unintended, subversive or sexual meanings:

Hence in every society various techniques are developed intended to fix the floating chain of signifieds in such a way as to counter the terror of uncertain signs; the linguistic message is one of these techniques. (Barthes, 1999: 37).

Sontag (1999: 31) amplifies this idea by referring to the power that images held in socalled 'primitive' societies where there was no clear distirction between images (which generally had an instrumental, ritual purpose) and real things. In much African art, practical objects are imbued with symbolic significance by the carving of images into them or by being shaped in certain ways. In the study of Bushman paintings, Lewis Williams (2003) and others have shown that the drawing is not so much a representation of reality as a conduit into a shamanic state of consciousness or ritual ecstasy.

In the fine arts, as in poetry, the polysemic nature of images is celebrated and ambiguity is encouraged. But in the news media, this uncertainty is at odds with the need to produce 'objective' messages from which ambiguity has been stripped. Thus, suggests Barthes, the function of the caption below a photograph is to 'anchor' the visual image in a textual message that constrains its polysemic potential. It works as "a kind of vice which holds the connoted meanings from proliferating" (1999: 37). The textual anchorage of visual images is invariably ideological, serving to guide the reader to preferred meanings and avoiding or suppressing oppositional readings.

The function of 'relay' is less common in news, but is very common in cartooning. In fact it is one of the dominant characteristics of cartooning:

Here lext (most often a snatch of dialogue) and image stand in a complementary relationship; the words, in the same way as the images, are fragments of a more general syntagym and the unity of 
the message is realized at a higher level, that of the story, the anecdote, the diegesis (which is ample confirmation that the diegesis must be treated as an autonomous system)." (Barthes, 1999: 38).

The function of relay is not to constrain or repeat the information in the image, but rather to add to and advance it "by setting out, in the sequence of messages, meanings that are not to be found in the image itself" (1999: 38). This is what makes cartoons so economical: the reader is spared all the 'costly' descriptive text; text and image work together to produce meanings without the need for textual description. Scott McCloud (1993) presents a similar idea in his analysis of how comics function at the textual level. This is discussed in more detail in Chapter Two.

\section{The application of Thompson's methodology of interpretation}

The paradigm shift in ways of producing, looking at and talking about visual culture that occurred during the course of the 20th century calls up so many diverse and intriguing problems that, from an analytical point of view, there is a real danger of losing the plot amongst the proliferation of conceptual detail. The methodology for the analysis of symbolic forms provided by John B. Thompson (1990), while it may appear rather pedestrian against the pyrotechnics of postmodernism, has the virtue of providing a theoretical framework that is able to accommodate structuralist and poststructuralist concerns without surrendering to them. Thompson's approach pays more than lip service to the historical legacy of the British New Left school of literary criticism and, true to the British philosophical tradition, is nothing if not pragmatic.

Thompson draws on the intellectual tradition of hermeneutics-"concerning interpretation, especially the interpretation of the Bible or literary texts" (Oxford, 1999)— to develop his theoretical framework. He is concerned to denote the creative, indeterminate nature of interpretation, which he variously describes as "interpretative explication" or "the creative construction of possible meaning". The indeterminacy of explication arises because, unlike the object domains of the natural sciences which lend themsel ves to empirical investigation, the object domains of the social sciences are made up of subjects who are themselves involved in a daily process of interpreting the realities they inhabit and producing meaningful actions and expressions. The object domain presented to the social scientist, according to Thompson, is therefore also a 'subject domain', and the interpretation of the symbolic forms that emerge from this domain is of necessity "the re-interpretation of a pre-interpreted domain" (1990: 20-23; 271-291). 


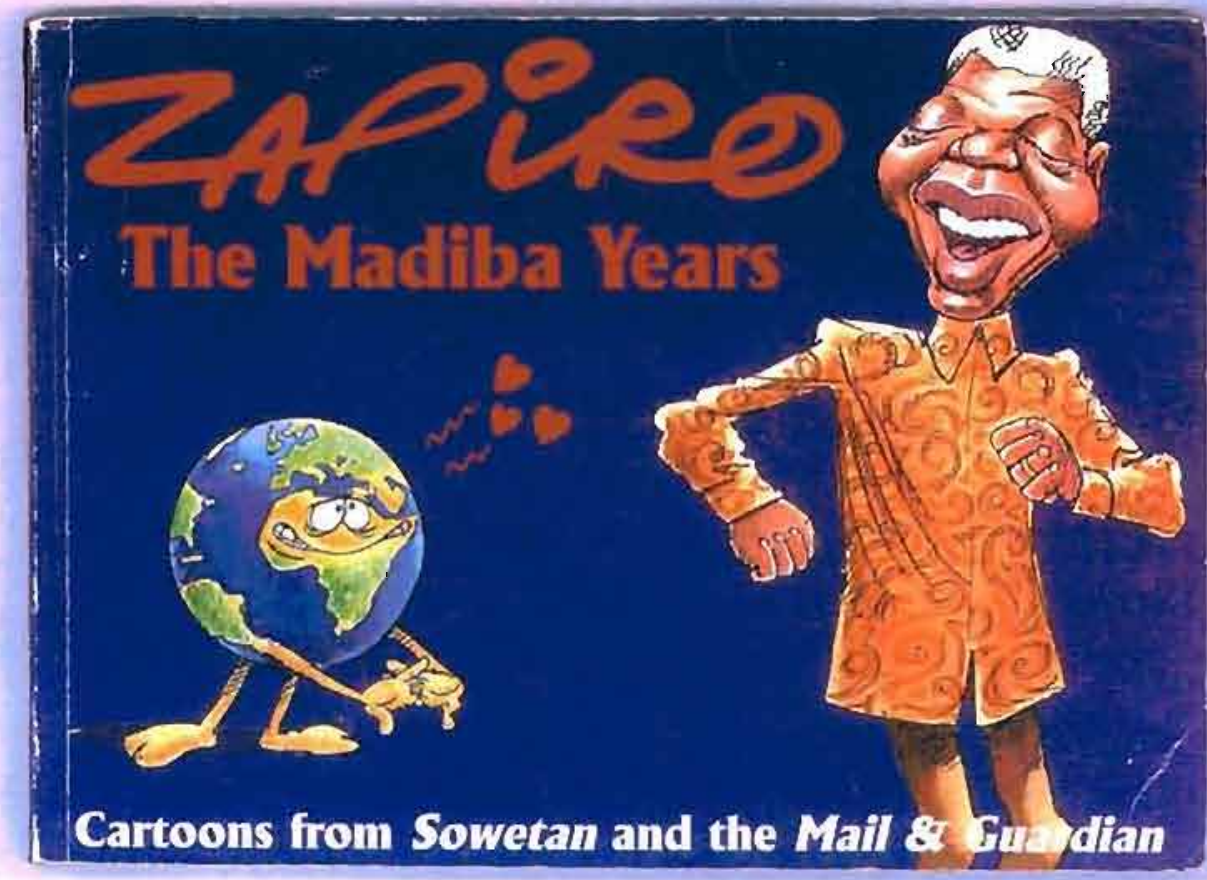

FIGURE 4

Zapiro's much-loved 'Madiba' icon connotes the aspirations and emotions attached to South Africa's euphoric 'national consensus' of 1994. 
Thompson points out that the subjects who in part make up the social world are always embedded in historical traditions. They are part of history, not just observers of it: the complex clusters of meaning and value that they create constitute a part of who and what they are (1990: 276). The subjects of this study, South African cartoonists who opposed apartheid and who participated in the propagation of discourses of resistance between 1985 and 1994, are a special case of what Thompson is talking about. Like the ordinary subjects of social enquiry, they "are constantly involved in understanding themselves and others, and in interpreting the actions, utterances and events which take place around them" (Thompson, 1990: 275). However, cartoonists (who may also be artists, writers and social commentators) are generally (though not always) more deeply and effectively involved in an ongoing act of interpretation of social reality than the ordinary person-this is what their work requires of them. They effectively contribute to the collective understanding of the social groups who are their audiences by interpreting and putting a humorous or satirical spin on news events and social issues. In turn, members of the public utilise the cartoonists' products, in amongst all the other material that they have available to them, to help them interpret the realities they inhabit, and to construct meanings about their own lives and how they fit into the social order.

A good editorial cartoon offers the succinct distillation of a political or social issue in the form of a memorable image, often combined with a pithy text. In producing an intelligible meaning out of a complex situation that might otherwise have an inchoate existence in the minds of the audience, it performs an important ideological function. Occasionally, a particularly brilliant cartoon will appear to capture or symbolise the essence of a historical moment. Such images often become texts that achieve instrumentality within the collective experience and meaning-making processes of social groups - they become visual icons connoting the cluster of meanings and emotions attached to the historical moment that they are interpreting. Zapiro's 'Madiba' icon is a good example of this, connoting as it does the aspirations and emotions attached to South Africa's euphoric 'national consensus' of 1994 (Figure 4) 24 ,

\footnotetext{
24 Of course, the meanings that cartoonists produce are always ideological-the positivist notion of "objectivity" does not apply to cartooning. And it should be noted that a cartoon can never actually 'capture' the 'essence' of a 'historical moment', since such moments are themselves the creations of human beings in the process of interpreting and acting upon the social reality they inhabit. The words 'essence' and historical moment' are used metaphorically-this study attempts to steer dear of essentialism. historicism and positivism.
} 
Thompson's methodology utilises a set of procedures that constitute what he terms 'depth hermeneutics' - the investigation of symbolic forms through a synthesis of sociohistorical, discursive and textual analyses. These procedures are not mutually exclusive, nor can they be undertaken separately or in any specific order, and therefore the three procedures are to some extent interwoven throughout the analysis that follows.

\section{(i) Socio-historical analysis}

For Thompson, the process of interpreting symbolic forms begins with socio-historical analysis-the interpretative reconstruction of the social and historical contexts in which symbolic forms are located and embedded. The purpose of socio-historical analysis is to paint a picture of the milieu out of which the symbolic forms being studied emerged - and of the economic, political, cultural and ideological relations that influenced, conditioned or determined their production, distribution and reception.

In these terms, an analysis of the symbolic forms produced by anti-apartheid cartoonists in South Africa between 1985 and 1994 should begin with some understanding of the historical and social context out of which they emerged. But this context is not a simple or unitary thing. It requires some knowledge of the historical emergence of cartooning as a duster of signifying practices expressed in a set of popular culture forms integral to the evolving media of mass communication (see Chapter Three), as well as an understanding of the social and political history of South Africa in the 20th century.

Also required is a sense of the theoretical context (the subject of this chapter) which in turn requires some understanding of the radical transformation, mainly over the last 50 years, in the way modem urban people read visual texts and what they expect to derive from them, and how academios have developed a critical language to talk about this 0 . And finally - to change the contextual focus from wide-angle to telephoto-the immediate context of the symbolic forms under discussion is constituted in the specific social, intellectual and political milieu that prevailed during the last decade of the

\footnotetext{
25 This is important, especially when it comes to understanding the variety of ways in which visual images are likely to be read in a multi-cultural context like South Africa, where poverty, inequality and social fragmentation produce such a range in people's daily life experiences and the educational and interpretative resources to which they have access.
} 
apartheid period (1985-1994). This is the crucible in which the perceptions, attitudes and ideas of the cartoonists who are the subjects of this study were produced.

The contextual analysis provided in this study is an unavoidably sketchy attempt to provide a brief overview of the broad theoretical, historical and social context, before focusing specifically on the 1985-1994 period. However, because cartooning is such a specific set of activities, operating in a realm of meaning that is predominantly textual and visual (rather than oral and aural), it is necessary to assemble a set of critical tools appropriate to the task of understanding the general characteristics of cartoons and comics, the modes of signification they employ and their relation to the media of mass communication. In terms of Thompson's methodology, this requires discursive analysis. In this study, the chapter dealing with discursive analysis (Chapter Two) is presented before those dealing with socio-historical analysis simply because it is first necessary to assemble an appropriate set of terms and definitions from which to proceed.

\section{(ii) Discursive analysis}

Discursive analysis involves the examination of the internal organisation, structural features, patterns and relationships of the complex symbolic constructions that Thompson calls 'symbolic forms'. In the case of cartoons and comics, this includes examination of the specific formal and structural qualities and stylistic conventions that separate cartoons and comics from other forms of art and literature, and in particular, the constituting role played by the procedures and techniques of production and reproduction required by the media through which they are transmitted.

As symbolic forms, cartoons and comics are open to interrogation in terms of the requirements of discursive analysis. What are the defining characteristics that set the various forms of cartooning apart from other forms of art, literature and popular culture? Is there a specific 'way of signifying' that is unique to cartooning? Is it proper to group the range of activities practised by cartoonists under a single rubric? Is cartooning specifically tied to certain forms of media? Does it require certain specific conditions in order to exist? These questions are considered in Chapter Two.

Thompson suggests a number of possible methods of formal or discursive analysis, citing semiotic analysis, syntactic analysis, conversation analysis, argument analysis and narrative structure analysis, as examples (1990: 284-291). All of these methods have 
some relevance to the study of cartooning, but lack specificity with regard to the particular "way of signifying' that cartooning involves.

Interestingly, in the literature around cartooning, the most influential analytical work has come not from academics but from practitioners themselves. Cartooning practitioners have provided very specific, in-depth analyses of the structural properties and internal organisation of cartooning forms. They have also described in minute detail the actual methods they use, the kinds of problems they encounter, and the ways in which the media environment in which they work defines, restricts and enables their practice. In the analysis of their own practice, cartoonists themselves have provided the most direct route into the discussion of the formal and structural characteristics of cartooning.

'Practitioner analysis' - the discursive analysis by practitioners of their own work, the work of their mentors, their influences and their own particular concerns and obsessions-might contain aspects of the other methods of discursive analysis listed by Thompson, but it is generally conducted in a language that emerges out of practice rather than out of theory. Certainly the most influential writers about the theory and practice of cartooning, such as Will Eisner (1985), Scott McCloud (1993; 2001) and Art Spiegelmann (1998), have been practitioners whose theories have arisen out of practical engagement with actual problems and issues inherent in their work, rather than from externally derived theoretical perspectives. As a result, their writing sometimes demonstrates a semantic naivety that results from an idiosyncratic use of academic terms that is at odds with the vocabulary of academic discourse. However, once this limitation is accommodated, it is clear that practitioner analysis has certain distinct benefits, particularly in terms of its focus and specificity. Practitioners are also generally very well informed about the practical problems and issues surrounding message creation and delivery, as well as the reception by the public of the symbolic forms that emerge from their practice, since their livelihood depends to such a great extent on audience reception.

In the fine arts, music and film, and probably in the theorisation of every form of artistic or cultural practice, central importance is placed upon the findings of those practitioners who have taken pains to study and understand the specific and particular nature of their work and how the products of their labours are distributed to, and received by, their audiences, and there is no reason why cartooning should be any different. All of the interviews with South African cartoonists undertaken for this study touch to a 
greater or lesser extent on issues of practice ${ }^{26}$. In addition, analytic texts written by renowned practitioners in the field of cartooning, notably Eisner, Spiegelman and McCloud, are utilised in the construction of a set of appropriate defiritions and analytic tools for the interpretative analysis of comics and cartoons.

\section{(iii) Interpretation/re-interpretation}

The interpretation of symbolic forms through close textual analysis of selected primary texts is the third component of the methodology of interpretation applied in this study. As Thompson constantly reminds us, interpretation is a creative act, not a scientific process. In the interpretative explication of the text, all the insights and information derived from the socio-historical and discursive analyses are synthesised, but the interpretation remains, for all this, no more than an informed opinion.

In terms of the present application of Thompson's methodology, it is necessary, before proceeding directly to the analysis of the primary texts, to linger awhile in the milieu out of which the work being studied emerged, in order to absorb something of the social and intellectual atmosphere of this milieu. Attempting to gain as much insight as possible into the personal experiences, attitudes and thinking of the creators of the selected primary texts before interrogating the texts themselves is one way of guarding against the common theoretical error of trying to 'read off' the characteristics of an epoch directly from the texts themselves, as though these texts were 'reliable' sources of 'objective' information about their own contexts.

Much as an archeologist would dig and scrape around a buried artifact, analysing the ground and the surrounding fragments in order to reconstruct as accurately as possible the immediate context of the object under study, the study of symbolic forms requires that some attention is first paid to surrounding texts-joumalistic, literary and critical-from the immediate period.

Thompson speaks of "the hermeneutics of everyday life"; and of the "ethnographic moment" when a symbolic form is released into the public domain, where it is received and appropriated by the audience (1990: 279). Both poles of this so-called 'ethnographic moment'-production and reception-are informed by what Thompson calls "doxa"-the prevailing beliefs, opinions and attitudes of the day. In order to approach

26 Unfortunately, transcripts of the interviews with the practitioners are not included in this study. 
the text from an informed position, we first need to examine the so-called 'doxa' of the 'ethnographic moment'. Obviously, in a historical study such as this, ethnographic research cannot be undertaken at the point of reception. Instead, journalistic, literary and critical texts from the immediate contextual environment may provide an important substitute for the lack of primary ethnographic data. In Chapter Five literary and crtitical texts from the period are referred to in order to provide a very brief and impressionistic sketch of the intellectual and cultural milieu of the last decade of apartheid.

Once the immediate context is understood, the camera may move closer, the focus pulled even tighter. Now, the subject is examined at close range: the detail in the viewfinder becomes very sharp and an intimate view is possible. This is where close textual analysis of the selected cartoons and comics, informed by interviews with their creators and all of the other information derived from the socio-historical and discursive analyses, begins in earnest. The benefit of this method is that it steers a path between the competing 'sociological' and 'textualist' paradigms in cultural and media studies.

During the course of the present research, which has involved frequent conversations with some of the cartooning practitioners whose work is the subject of this study, personal friendships have developed which will have unavoidably informed the act of interpretation. As Thompson points out, interpretation is a creative act, and as such reaches below the surface of conscious thought into the psychic wellspring of human experience from which all cultural production ultimately derives. In so doing, the act of interpretation removes itself from the contested domain of so-called 'objectivity'.

The final chapter of this study is therefore the least 'scientific' and 'objective', and the most 'creative' and 'personal' of all the chapters. Hopefully, the cautious approach prescribed by Thompson's methodology of interpretation will mitigate against the implicit surrender of objectivity and the slide into complete subjectivism that so often seems to characterise the practice of art and the criticism thereof. 


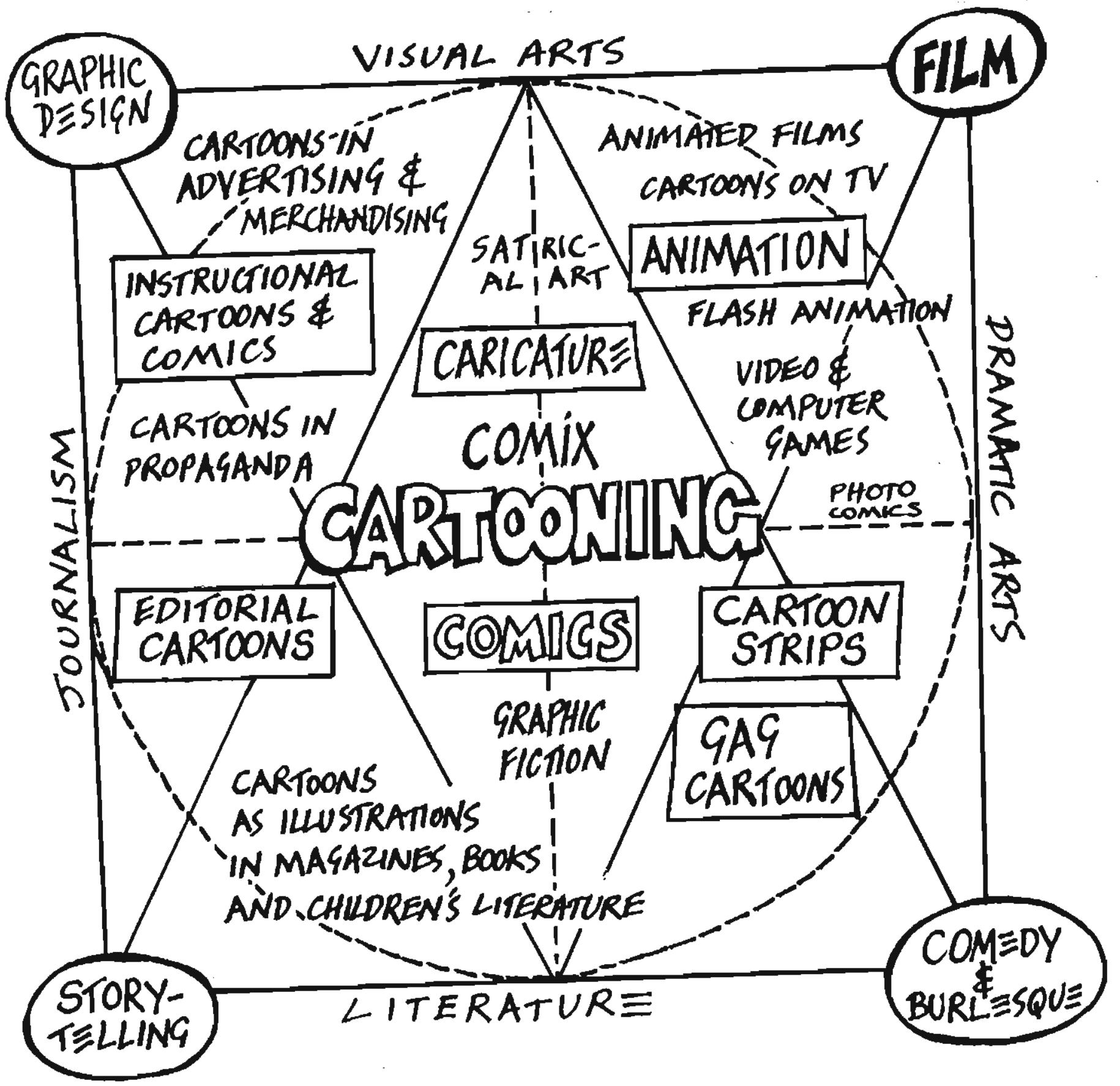

FIGURE 5

The various kinds of cartooning activity are located contiguously in a field of signifying practices where they are clustered with other similar activities that find expression in a variety of styles and genres. (Diagram: N.D. Mazin.) 


\section{Chapter Two}

\section{Defining Cartooning}

\section{Introduction}

'Cartooning' is the term chosen in this study to refer collectively to the making of cartoons, caricatures, comics and comix ${ }^{27}$. An alternative term that might have been used is 'comic art'28. Neither term is ideal, since they both privilege certain notions at the expense of others, but our language does not provide a more suitable portmanteau for this cluster of signifying practices. In French, the tradition of narrative strip story telling is referred to as bande dessinee ( $B D)$, meaning, literally, 'drawn strips', whereas political cartooning is usually referred to as caricature. The activities of narrative strip production (comics) and political cartooning or caricature are generally referred to separately, as though they were entirely different things, but of course they are not. They are located contiguously in a field of signifying practices where they are clustered with other similar activities that find expression in a variety of styles and genres (Figure 5).

As will be argued later in this chapter, the products of cartooning should not be confused with the media in which they appear, although there is a widespread tendency amongst comics scholars to refer to comics as a 'medium'29. Cartoons and comics in various forms appear throughout the entire range of media available in the world today,

\footnotetext{
7 See page 54 for an explanation of the term 'comix'.

${ }^{28}$ The editor and publisher of the International Journal of Comic Art (JJOCA), Dr John Lent, chose the rubric 'comic art' for his publication, which includes scholarly articles on all forms of cartooning and comics production, including "comic books, newspaper and magazine comic strips, caricature, gag and political cartoons, humorous art, and humour/ cartoon magazines", but excludes animation (Lent, JjOCA Vol.1 No. 1, 1999). The division between animation and other cartooning forms is justified by the technological differences between working in film and working in print, although the recent surge in Internet-based Flash animation further complicates this distinction. However, there is a serious argument that distinguishes oral/aural modes of signification (animated cartooning) from visual/textual modes (cartoons, comics and comix).
}

${ }^{29}$ Benjamin F. Towle, writing in JJOCA Vol. 5, No. 2, 2003, declares that "[c]omics is a medium not a genre." And, in the same issue, Joseph Witek talks about comic books and comic strips as "two contrasting narrative approaches in the comics medium". 
and defining comics as a medium just adds to the semantic confusion in which comics scholarship is already enmired. This chapter pursues the notion of an identifiable mode of signification-a certain way of signifying - that can be applied generally to editorial cartoons, gag cartoons, cartoon illustrations, comic strips, comic books and graphic novels, as well as to the use of cartoons and comics in education, advertising and merchandising. This way of signifying is called cartooning in this study, but it might just as well have been called comic art.

The traditions out of which this way of signifying has emerged go back into the distant past. Many of the pictorial and visual narrative conventions that we associate with cartooning today are observable, for instance, in 10th century Chinese prints, preColumbian picture manuscripts, ancient Egyptian paintings and Hellenic vases (see Honour \& Fleming, 1984; McCloud 1993, Johnson 2003). This study, however, limits itself to discussion of the tradition of cartooning and caricature that emerged in the late medieval and early modern period in Europe and Britain (see Chapter Three).

This chapter argues that, generally, the symbolic forms that cluster around cartooning's mode of signification all participate in and use a common set of visual codes and pictorial or graphic conventions. These codes and conventions are widespread and extremely familiar. They are found not only in the most obvious forms of cartooning, but in numerous other applications, including animated cartoon films, press and TV advertising, product merchandising, children's television programmes, children's literature and school textbooks. In this study, the word 'cartooning' is used in a very broad way that allows for the inclusion of the full range of cartoon and comics forms, products and media, and it locates these in a long history of popular and satirical art that is intimately linked to the history of the mass media ${ }^{30}$.

As a site of analysis, cartooning is a field with blurred boundaries. As an activity, it interacts with and is situated amongst similar activities in the plastic arts, graphic design, film, journalism, storytelling and comic theatre. In terms of genres and styles, it is widely invasive and accumulative. It would be difficult to find a corner of contemporary culture that does not display the influence of cartooning in its semiology. Likewise, cartooning has appropriated imagery and stylistic conventions from film, the fine arts, industrial design, advertising, television and street culture.

\footnotetext{
${ }^{30}$ Of the poster and billboard designs selected for inclusion in The 100 best posters of the Century (Campaign, 1999), some 20 per cent include cartoon illustrations. Most of these were produced before 1960. After 1960, photography and photomontage are the main sources of imagery.
} 


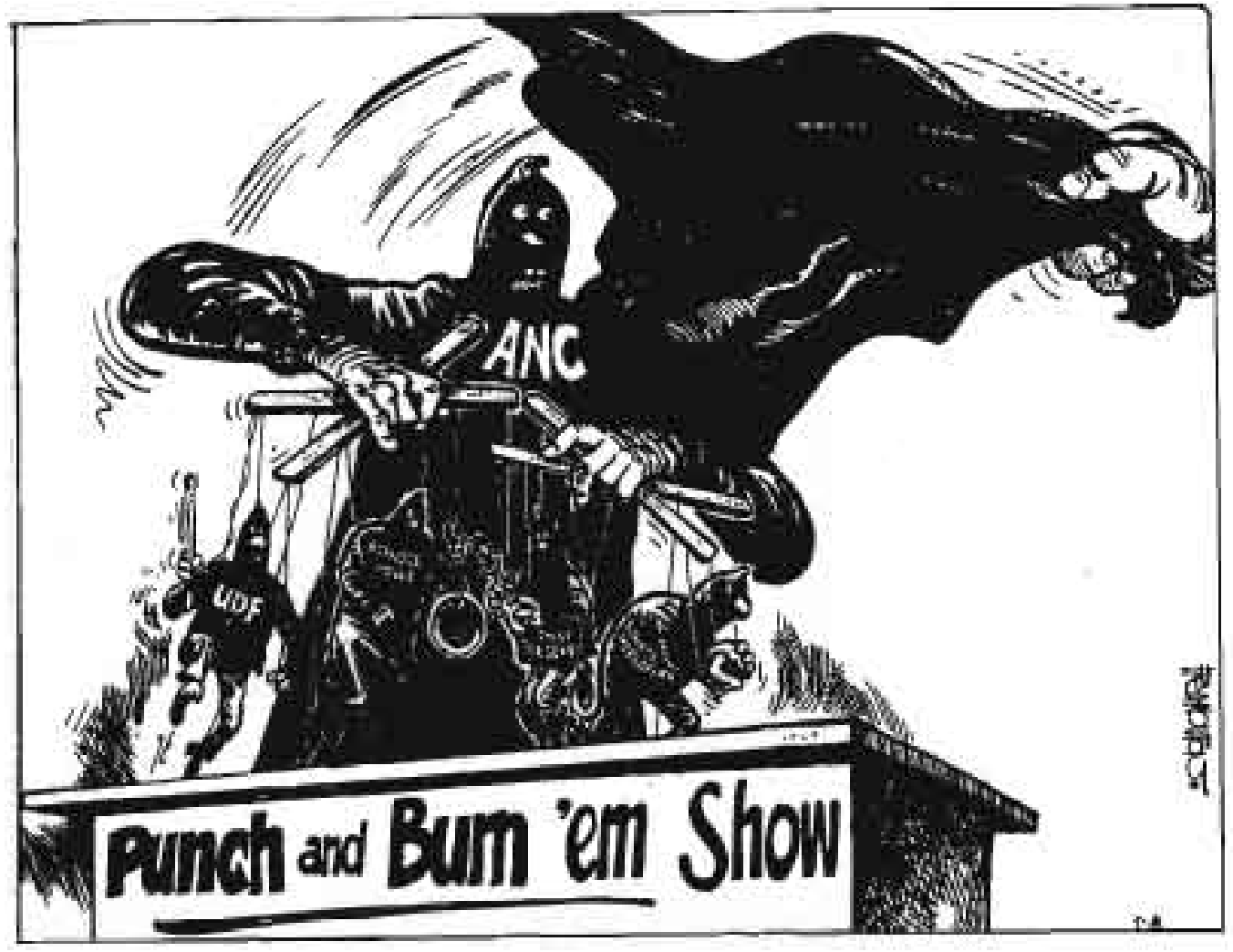

FIGURE 6

Some South African newspapers and magazines were extremely close to the state and the editorial cartoons they carried were unambiguous in their demonisation of the extra-parliamentary iberation movement. In this cartoon by Fred Mouton, published in Die Oosterlig in 1986, the United Democratic Front (UDF), "boendu howe" (kangaroo courts), "onlus plegers" (rioters) and "linkse meerlopers" (leftists) were all shown to be controlled by their master puppeteer, the externally-based ANC (Vemon, 2000). 
While the best-known studies in the field, certainly in English, have tended to focus specifically on comic books and newspaper comic strips, the focus of this study has less to do with specific media forms than with the relation of cartooning in general to the specific social and historical context of South Africa during the last decade of the apartheid period. A broad definition of cartooning is thus combined with a narrow historical focus.

In the period under discussion (1985-1994), a wide range of South African cartooning forms is evident. Some of these appear at first glance to have been politically neutral, such as cartoons and comics used in the advertising and marketing of consumer products, or cartoons and comic strips in popular magazines or newspaper cartoon supplements. But visual texts, however banal they may appear to be, are seldom innocent, and usually can be shown to be loaded with ideological or mythological meanings. Cartoons were certainly utilised for propaganda purposes by the South African state and its ideological apparatuses during the apartheid period. Some newspapers and magazines were extremely close to the state and the editorial cartoons they carried, although often very witty, were unambiguous and undisguised in their vilification and demonisation of the extra-parliamentary liberation movement and its allies (Vernon, 2000)(Figure 6) ${ }^{31}$.

These tendencies are broadly reviewed in the historical overview of South African cartooning in the 20th century (see Chapter Four), but the focus of this study is on those cartooning forms that emerged out of the discourses of resistance that prevailed in the last decade of the apartheid period. Anti-apartheid cartooning took many forms, including:

- editorial cartoons and comic strips in the mainstream opposition press and the alternative press;

- cartoon illustrations and comics in educational publications produced by the non-governmental (NGO) sector;

- cartooning for propaganda purposes by agencies or individuals in the employ of trade unions or political organisations;

- cartooning in the student press; and

- underground comix publishing.

31 Fred Mouton, Die Oosterlig, 1986. Reproduced in Vernon, 2000: 158. 


\section{Defining cartooning}

In the context of contemporary mass media convergence, cartooning properties, whether in the form of cartoon characters or entire comics 'universes' (such as the 'Marvel Comics Universe'), are often presented simultaneously in a variety of media, used in advertising and merchandising, made into animated films, converted into computer and video games, and made into toys. It therefore makes sense to formulate a definition that is broad enough to include the widest possible range of forms and media.

The Concise Oxford Dictionary (1999), provides the following definitions:

comic * adj. causing or meant to cause laughter. ^relating to or in the style of comedy. ${ }^{*} \mathrm{n} .1$ a comedian. 2 a children's periodical containing comic strips. $\wedge$ (comics) N. Amer. comic strips.

-ORIGIN C16: via L. from Gk komikos, from komos 'revel'.

comic strip * $n$. a sequence of drawings in boxes that tell an amusing story.

cartoon * $n .1$ a drawing executed in an exaggerated style for humorous or satirical effect. 2 a film made from a sequence of such images, using animation techniques to produce the appearance of movement. 3 a full-size drawing made as a preliminary design for a painting or other work of art. * $v$. represent in a cartoon. -DERIVATIVES cartooning n. cartoonish adj. cartoonist $\mathrm{n}$. cartoony adj. - ORIGIN C16: from Ital. cartone, from carta, from L. carta, charta .

caricature * $n$. a depiction of a person in which distinguishing characteristics are exaggerated for comic or grotesque effect. * $v$. make a caricature of. DERIVATIVES caricatural adj. caricaturist $\mathrm{n}$. -ORIGIN C18: from Fr., from Ital caricatura, from caricare 'load, exaggerate' from L. carricare.

The definition of 'comic' confirms that the plural form, 'comics', denotes sequential pictorial narrative in the print media and is most commonly used to refer to the U.S. tradition of comic strip and comic book publishing, whereas its adjectival form denotes humorous content and is related to theatrical comedy. The adjectival meaning clings to 
the noun, even where the comic book or strip referred to has no humorous intention ${ }^{32}$ definitions confirm the important semantic distinction between the comic book as a medium and the kinds of texts that comic books contain (comic strips).

The defiritions of the words 'cartoon' and 'caricature' are equally instructive. Although both definitions refer to the activity of exaggeration for humorous or comic effect, 'cartoon' and 'caricature' come from different roots. 'Cartoon' shares the same root as the words 'chart' and 'card', connoting the activity of producing a simplified or diagrammatic image that refers to or stands for another more complex image or set of information. (Interestingly, the outlines produced by Renaissance painters and transferred mechanically to a wall or ceiling prior to embarking on the painting itself are known as cartoons) (Goldscheider, 1975).

'Caricature' is derived from the Latin word carricare, to 'load' onto a wheeled vehicle (carrus), and is similar to the idea of 'heaping up' (the word 'exaggerate' derives from the Latin exaggerare, to 'heap up') ${ }^{33}$

It is noteworthy, therefore, that the characteristics of simplification, exaggeration and humour - key characteristics of modern cartooning in general-are embedded in the Latin or Greek roots of the words 'cartoon', 'caricature' and 'comic' - to chart, to load up, and to revel.

While the word 'cartoon' is generally reserved for single illustrations of a humorous or satirical nature, it also refers to animated films in the comics style, so that a 'comic' in print becomes an 'animated cartoon' when translated into film. Comic strip creators often, if not generally, describe themselves as 'cartoonists' and the work they do as 'cartooning'34.

\footnotetext{
${ }^{32}$ Will Eisner introduced the phrase 'Sequential Art' in the foreword to Comics and Sequential Art (1985: 5), referring to it as an "ancient form of art" and "a discernable discipline alongside filmmaking" that "has generally been ignored as a form worthy of scholarly discussion". It has often been observed that phrases like 'sequential art' and 'graphic novel' may have been employed by cartooning practitioners and publishers in an endeavour to distance 'serious' graphic literature from the pejorative implications inherent in the word 'comic'.

${ }^{33}$ A dispute appears to exist with regard to the origin of the word 'caricature' in that it is held by some art historians to derive not from the Latin root given by the Oxford Dictionary but from the name of the Italian Renaissance painter Annibale Carracci (1560-1609) (Honour \& Fleming 1982: 496; Vemon 2000: 10).

${ }^{34}$ Robert Crumb (1997), Art Spiegelman (1998), G.B. Trudeau (1981) and Eric Drooker (Comics Journal interview by Lanier, 2003) all refer to themselves as 'cartoonists'.
} 
the noun, even where the comic book or strip referred to has no humorous intention ${ }^{32}$. definitions confirm the important semantic distinction between the comic book as a medium and the kinds of texts that comic books contain (comic strips).

The definitions of the words 'cartoon' and 'caricature' are equally instructive. Although both definitions refer to the activity of exaggeration for humorous or comic effect, 'cartoon' and 'caricature' come from different roots. 'Cartoon' shares the same root as the words 'chart' and 'card', connoting the activity of producing a simplified or diagrammatic image that refers to or stands for another more complex image or set of information. (Interestingly, the outlines produced by Renaissance painters and transferred mechanically to a wall or ceiling prior to embarking on the painting itself are known as cartoons) (Goldscheider, 1975).

'Caricature' is derived from the Latin word carricare, to 'load' onto a wheeled vehicle (carrus), and is similar to the idea of 'heaping up' (the word 'exaggerate' derives from the Latin exaggerare, to 'heap up')33.

It is noteworthy, therefore, that the characteristics of simplification, exaggeration and humour - key characteristics of modern cartooning in general-are embedded in the Latin or Greek roots of the words 'cartoon', 'caricature' and 'comic'-to chart, to load up, and to revel.

While the word 'cartoon' is generally reserved for single illustrations of a humorous or satirical nature, it also refers to animated films in the comics style, so that a 'comic' in print becomes an 'animated cartoon' when translated into film. Comic strip creators often, if not generally, describe themselves as 'cartoonists' and the work they do as 'cartooning'34.

\footnotetext{
${ }^{32}$ Will Eisner introduced the phrase 'Sequential Art' in the foreword to Comics and Sequential Art (1985: 5), referring to it as an "ancient form of art" and "a discernable discipline alongside filmmaking" that "has generally been ignored as a form worthy of scholarly discussion". It has often been observed that phrases like 'sequential art' and 'graphic novel' may have been employed by cartooning practitioners and publishers in an endeavour to distance 'serious' graphic literature from the pejorative implications inherent in the word 'comic'.
}

\footnotetext{
${ }^{33}$ A dispute appears to exist with regard to the origin of the word 'caricature' in that it is held by some art historians to derive not from the Latin root given by the Oxford Dictionary but from the name of the Italian Renaissance painter Annibale Carracci (1560-1609) (Honour \& Fleming 1982: 496; Vernon 2000: 10). 34 Robert Crumb (1997), Art Spiegelman (1998), G.B. Trudeau (1981) and Eric Drooker (Comics Journal
interview by Lanier, 2003) all refer to themselves as 'cartoonists'.
} 
Some commentators have suggested that Egyptian hieroglyphics, pre-Columbian friezes or prehistoric cave paintings are antecedents of comics in that they employ pictorial sequencing for narrative purposes (McCloud, 1993: 10-20). That many of the codes and conventions of cartooning appear to have been acquired and adapted from these ancient traditions lends credence to this idea. However, the placement by human beings of pictorial images next to each other in some kind of narrative sequence is so widespread that its utility as a defining characteristic of cartooning is questionable, particularly where no continuity with the tradition of modern cartooning can be demonstrated.

What these examples do tell us is that from the earliest times people have placed stylised pictorial images in sequence to create meaning, often combining them with text. But the definition of cartooning would be dented beyond utility if all forms of graphic narrative were to be included under the rubric 'cartooning'. For example, while they are usually very stylised and often combined with text, the stained glass windows in Christian churches are not a kind of cartooning, even though a direct lineage can be traced from the bold outlines and flat colours of medieval stained glass windows to the stylisations of Art Noveau and from there to the use of simplified forms, heavy outlines and flat colours of modern cartooning (Couperie, 1972).

Other kinds of stylised sequential illustrations combined with text would also stretch the definition of cartooning to breaking point were they to be included. Examples are the sequential illustrations that appear on airline safety instruction cards or in user manuals for video cassette recorders or other hi-tech gadgets. These employ many of the codes and conventions of cartooning, but it is unhelpful to include them under the definition of cartooning because they are purely functional and have no aesthetic intention or purpose (Figure 7).

In his introductory chapter to Understanding Comics: The Invisible Art (1993), the most influential theoretical work on comics of the 1990s, Scott McCloud takes the view that all forms of sequential pictorial narrative, from airline safety instruction cards to stained glass windows, should be included under the rubric 'comics', which he defines as follows:

comics (kom'iks) n. plural in form, used with a singular verb. 1. Juxtaposed pictorial and other images in deliberate sequence, intended to convey information and/or produce an aesthetic response in the viewer. (McCloud 1993: 9) 
McCloud cleverly mimics the conventions of dictionary presentation in an attempt to invest his definition with authority, but this does not conceal the fact that it is fatally flawed. McCloud's definition, predicated on the sequential nature of comics and little else, is the weakest aspect of his otherwise brilliant exposition of how comics work. By emphasising sequence at the expense of other important characteristics that comics share with other forms of cartooning (and in particular by de-emphasising the aesthetic dimension), McCloud fails to come to terms with the idea that comics belong in a larger cluster of signifying practices linked by a common way of signifying.

In this study, the idea that comics and cartoons of various kinds are part of a larger category is considered to be an essential component of their definition. For the purposes of this study, 'cartooning' is regarded as a way of signifying, the defining characteristics of which are constituted in a set of visual codes and stylistic conventions involving simplification, exaggeration and humour that have developed over the course of a long and continuous tradition of popular and satirical art.

Within this broadly defined field, the various forms, characteristics, genres and styles of cartooning may be positioned. For example, 'caricature', the intentional distortion of facial features for comic or satirical effect, found both in cartooning and the fine arts, may be defined both as a form of cartooning in its own right and a frequently-observed characteristic of cartooning, but caricature is not always present in or definitive of cartooning. On the other hand, 'comix', the influential underground, anti-establishment comics that first surfaced in the U.S. in the late 1960s, may be defined as a genre of cartooning with which a certain style of cartooning is associated ${ }^{35}$.

\section{Form, medium and practice: making the distinctions}

As has been observed in Chapter One, it has not been academics as much as cartooning practitioners themselves who have made the most original and lively contributions to the discourse around the processes of signification that characterise the various forms of cartooning. Will Eisner, creator of The Spirit and one of the great practitioners of the 'golden age' of comics, made a valiant attempt in Comics and Sequential Art (1985) to

\footnotetext{
${ }^{35}$ Underground comix emerged in response to the self-imposed censorship under which comics publishing in the U.SA had suffered since the imposition of the Comics Code in 1954. The freedom of expression championed in these comics, expressed both stylistically and in terms of content, had a tremendous impact on the development of comics worldwide and led to the development of derivative genres in many countries, including South Africa (see Chapter Three).
} 
describe the activity of comic strip creation in formal terms. Simultaneously, he was a pioneer of the so-called 'graphic novel', a comic strip form that he saw as aspiring to be free of the constraints imposed by the commodifying relations of mainstream comic strip production.

Eisner was able to apply himself to comics theory from the vantage point of a highly skilled practitioner, tackling such topics as the use of frames, speech balloons, pictorialised lettering and sound effects, and, crucially, the way in which the gutters between the panels of a comic strip signify the dimension of time. Joseph Witek (1999) acknowledges Eisner as the first writer (in English, at least) to provide the foundation for "a common technical vocabulary for the textual analysis of comics" (1999: 14). The phrase "sequential art" is important, argues Witek, because it "allows for the crucial conceptual distinction between the overall medium of visual narrative on the one hand and the specific forms of that medium (that is, comic strips and comic books) on the other" (1999: 14).

In his valorisation of Eisner's contribution to the theroisation of cartooning practice, Witek unfortunately perpetuates the semantic muddles that continue to bedevil the theorisation of cartoons and comics. His claim that 'visual narrative' is a 'medium' and that comic books and comic strips are 'forms' of this medium is spurious. Visual narratives can take many forms and can be conveyed visually via any number of media, including comic books, feature films and television news programmes. A more correct formulation, I would argue, would be to distinguish between the overall signifying practice of cartooning, the forms that this practice takes, such as cartoons or comic strips, and the media through which these forms are transmitted to their publics.

Readings from the first five years (1999-2003) of the International Journal of Comic Art (IJOCA) reveal that critical and theoretical writing about cartooning often fails to make these kinds of distinctions. But this is not an unusual problem. In the field of visual culture generally, the distinction between medium and practice is often blurred. In the fine arts, for instance, the word 'painting' may denote both the medium of painting (the physical application of paint to paper, canvas or board) and the practice of painting (the creative act of applying paint to a surface according to a pre-existent set of conventions).

The term art form is often used in art theory to distinguish one practice or medium from others: painting is a specific art form, distinct from sculpture or print-making. This usage of the word is less concerned with the separation of 'form' from 'content' for the 

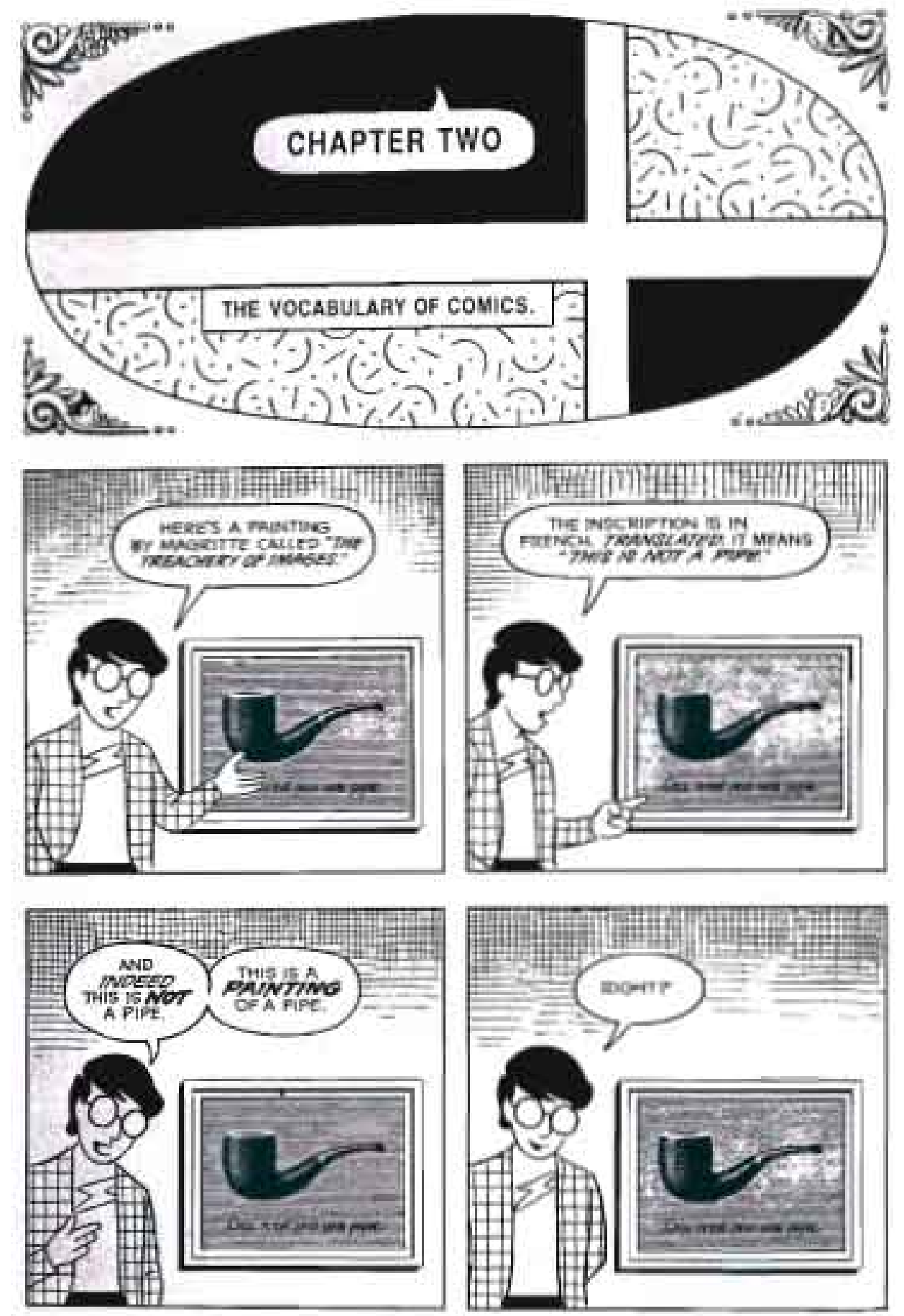

FIGURE 8

In Understanding Comics: The Invisible Ant (1990), Scott MeCloud utilises the comics form to explain how comics work. The text is prodiced entirely as a comic strip, with McCloud's own voice attributed to a cartoon character who purports to be the author. 
purpose of criticism than with distinctions between specific practices and the media they employ. Similarly, within the field of cartooning, there are a number of specific cartooning forms. Although they may share a common way of signifying, each of these forms has its own peculiar textual and stylistic conventions, often conditioned or determined by the technical requirements of the media in which they are carried. These cartooning forms may in turn be grouped into genres, often denoted by particular styles of rendition, each with its own origin and history.

While Marshall McLuhan's famous (1964) dictum that "the medium is the message" is certainly true of comics (what they have to say is usually inextricable from how they say it), I would argue with Raymond Williams for a restrained approach to the use of the terms 'medium' and 'media'. As Williams notes in Keywords (1976), these words carry their own set of problems, and should be used with discretion. Williams notes:

In most modern science and philosophy, and especially in thinking about language, this idea of a medium has been dispensed with; thus language is not a medium but a primary practice, and writing (for print) and speaking or acting (for broadcasting) would be practices. It is then controversial whether print and broadcasting... are media, or, more strictly, material forms and sign systems" (Williams 1976:203).

The terms medium and media should arguably be reserved, therefore, for the physical or technological 'carriers' of cultural forms and sign systems, rather than applied to these forms and sign systems themselves. Thus, cartooning is not a medium but a practice, while newspapers, magazines, comic books, films, TV programmes and video games are the media through which the cartoons and comics which are the products of this practice are delivered and received by the public according to specific relations of production and distribution.

Scott McCloud's insightful attempt to provide a workable definition of what comics are (1993: 2-23) is unfortunately marred by a vague or idiosyncratic use of terms. Himself a comics practitioner, McCloud set out to utilise the comics form to explain how comics work, and the text of Understanding Comics is produced entirely as a comic strip, with his own voice attributed to a cartoon character who purports to be the author (Figure 8). McCloud generously acknowledges his debt to the pioneering work of Eisner and his bibliography demonstrates that, like Eisner, he derived his insights mainly from his own work as a practitioner and comics fan, rather than from exposure to the discourses of cultural and media studies. While many of the concepts he employs are incisive, they 
are sometimes expressed in an idiosyncratic way that is at odds with the conventional usage of terms within the more formal critical discourses. In his attempt to tackle the semantic problems inherent in the definition of what he variously refers to as "the art of comics" (1993: 5) and "the artform-the medium-known as comics" (1993: 6), McCloud immediately runs into trouble as a result of his unformulated use of key terms - and particularly because he does not distinguish between media, forms and practices.

These problems of definition are important. They tend to emerge wherever an overall definition is attempted, particularly at the fuzzy edges where cartooning merges into other cultural practices. McCloud's definition of comics as "juxtaposed pictorial and other images in deliberate sequence" (1993: 9) is derived from Eisner's phrase 'sequential art', but it emphasises 'sequence' while de-emphasising 'art'. It is problematic precisely because it abandons the aesthetic dimension of comics as a defining principle, and fails to position comics as a form of cartooning situated within the broader field of cartooning or comic art.

For theoretical efficacy, it is important that comics are comfortably housed within this broader field. While the juxtaposition of images in sequence is a defining characteristic of comics, this characteristic is not exclusive to comics (juxtaposition of sequential images is characteristic of other kinds of symbolic forms as well), nor is it a necessary characteristic of cartooning in general. In the editorial cartooning of Zapiro, for example, sequential juxtaposition is a frequently observed characteristic. Sequential juxtaposition is therefore one of the characteristics of cartooning - and the definitive characteristic of comics-but is neither necessary nor exclusive to cartooning in general.

\section{Towards a workable definition of cartooning}

As noted in Chapter One, a particularly useful way of identifying and defining the way of signifying that characterises cartooning is to make use of the semiotic categories proposed by Peirce (1931): icon, index and symbol (Chandler 1996). In addition to these categories, it is important to understand how the pictorial and graphic conventions of cartooning are grouped together into the codes that constitute the different styles and genres of cartooning. It is aso important to note that many of these conventions, codes, styles and genres are intertextual-they are derived from or shared with other forms of art and popular culture. 
According to Chandler (1996. Codes: 2), neither Saussure nor Peirce elaborated the theory of codes and codification to any significant extent: this was to be the contribution of the linguistic structuralist Roman Jakobson (1971). According to Chandler, Jakobson insisted that the meaning of a sign will always depends on the code within which it is situated: signs make no sense unless they are situated within the framework of a code. And only very rarely (if at all) are such codes intelligible to an untrained ear or eye $e^{36}$.

In the case of symbolic or idexical signs, where the signifier does not resemble the referent, or does so only obliquely, we rely solely upon learned conventions in order to interpret the sign. But in the case of iconic signs such as cartoon figures, our 'anthropological' response to the visual content of the sign is articulated against other relatively arbitrary meanings that have been attributed to it by conventional usage. And, as in written or spoken language, a process of codification takes place whereby layers of conventional meaning adhere to the naturally apprehended sign over a period of time.

These layers of conventional meaning may be intelligible to some readers but not others, depending on the reader's enculturation to the conventions of a particular code (or membership of a subculture or counter-hegemonic group). For instance, as has often been remarked, Walt Kelly's famous strip Pogo could either be read as a humorous recreational strip about cute animals in the Okefenokee swamp, or as a complex critique of U.S. politics (Perry and Aldridge, 1971). As observed by Dr Lent, cartoonists in repressive political contexts sometimes operate at more than one level of meaning in order to transmit oppositional messages that are intended to be invisible to the authorities ${ }^{37}$.

In the case of complex messages comprising iconic, indexical and symbolic signs expressed in combinations of text and visual images, as in editorial cartoons or comic strips, there is generally a dimension of visual meaning that is transmitted in a primal, pre-linguistic way. As was noted by Barthes (1999) (see Chapter One), linguists tend to become uncomfortable when confronted by the "terror of uncertain signs". This phobia often appears to be shared by parents and other people in positions of social authority. Visual imagery, if unanchored or loosely anchored by text, may lend itself to the

\footnotetext{
${ }^{36}$ Part of the problem of the photographic 'message without a code' that so unsettled Roland Barthes in 1964, was the idea that photographs seem to have an iconic veracity that enables the spontaneous recognition of their 'purely denotative' meaning to seem 'almost anthropological' (see Chapter One).

37 Dr Lent made this observation during an international cartoon journalism workshop in Botswana in 2000 (See Mason, 2001).
} 


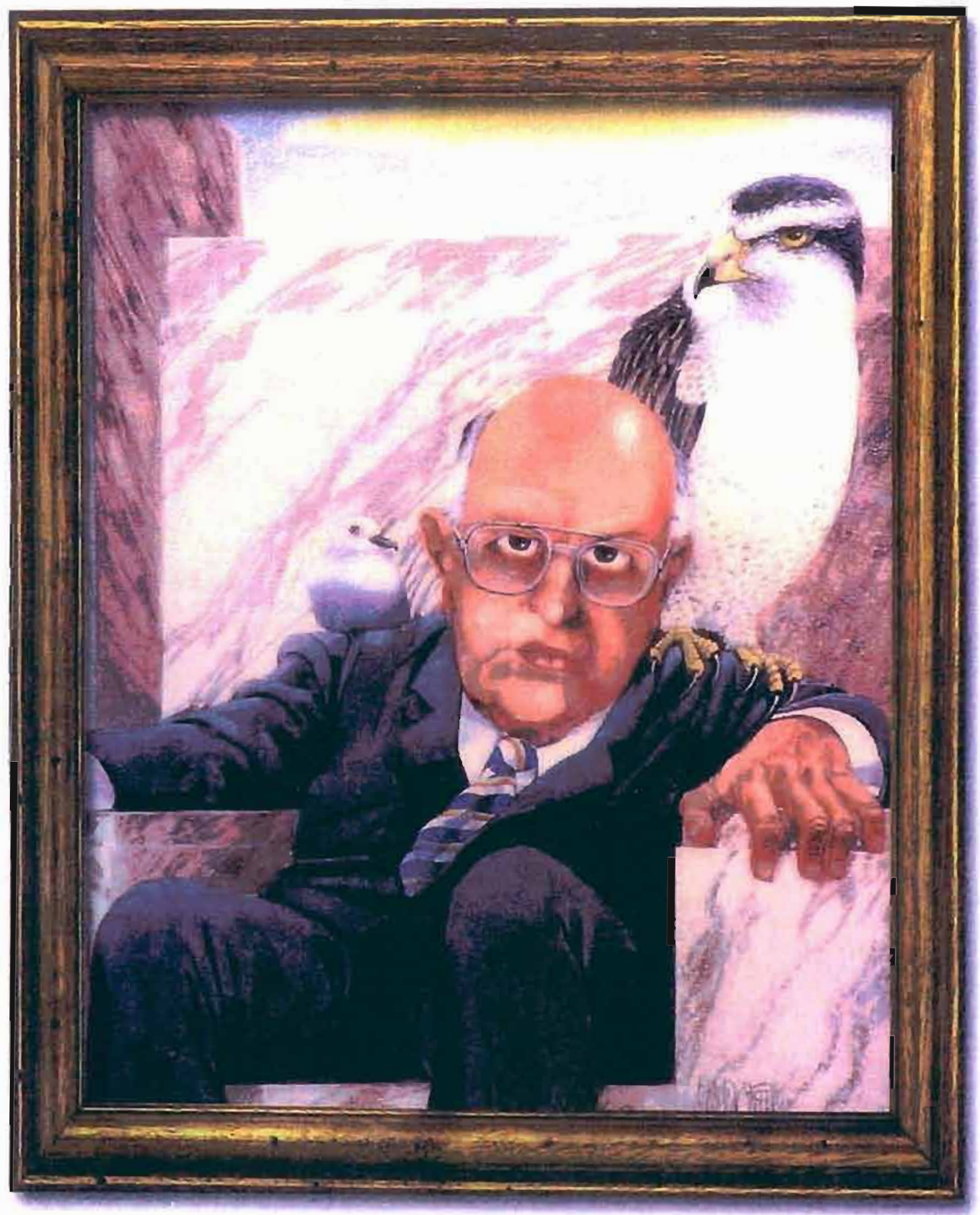

FIGURE 9

Like many other cartoonists who also work as professional illustrators, South Africa's Richard Smith is able to oscillate between a simplified or 'cartoony' style of caricature and a more figurative style which relies on milder levels of exaggeration and caricature. as in this 1985 Leadership Magazine cover illustration. 
accumulation of unintended subliminal meanings, or may result in the relay of a multiplicity of new meanings. Where this happens, according to Barthes' formulation, the sign moves beyond simple denotation (where meaning is relatively fixed), and beyond connotation, where meanings proliferate, into the realm of myth, where culturally shared ideas are brought into play on a grand scale.

Cartoons and comics are strongly connotative, in that they are saturated with symbolic and mythical meanings that are not physically visible in the sign. They are also denotative, in the sense that pictures of objects in cartoons and comics generally look something like the physical objects they represent. Cartoons are therefore not linguistic signs in the Saussurian sense, although they do have linguistic elements, in that their indexical and symbolic meanings are arbitrary and assigned.

The codes of cartooning thus involve a combination of iconic, indexical and symbolic signs, and generally incorporate the following primary characteristics:

(1) pictorial stylisation and simplification;

(2) iconic characterisation and codification; and

(3) visual narrative conventions such as panels, speech balloons, motion lines etc.

In addition to these primary characteristics, cartooning generally, but not always, involves the following secondary attributes:

- exaggeration,

- over-dramatisation,

- visual slapstick,

- pictorial puns,

- incongruous juxtaposition,

- incorporation of text into pictures, and

- the pictorialisation of text.

\section{(i) Pictorial stylisation and simplification}

While the techniques of pictorial stylisation and simplification are endemic to cartooning, neither these nor any other illustrative techniques are unique to cartooning, nor is any one technique definitive of cartooning. Examples of stylised pictorial simplification can be found throughout 'primitive', folk, classical and modern art. It is thus impossible to define cartooning in purely stylistic terms, although it is true to say 

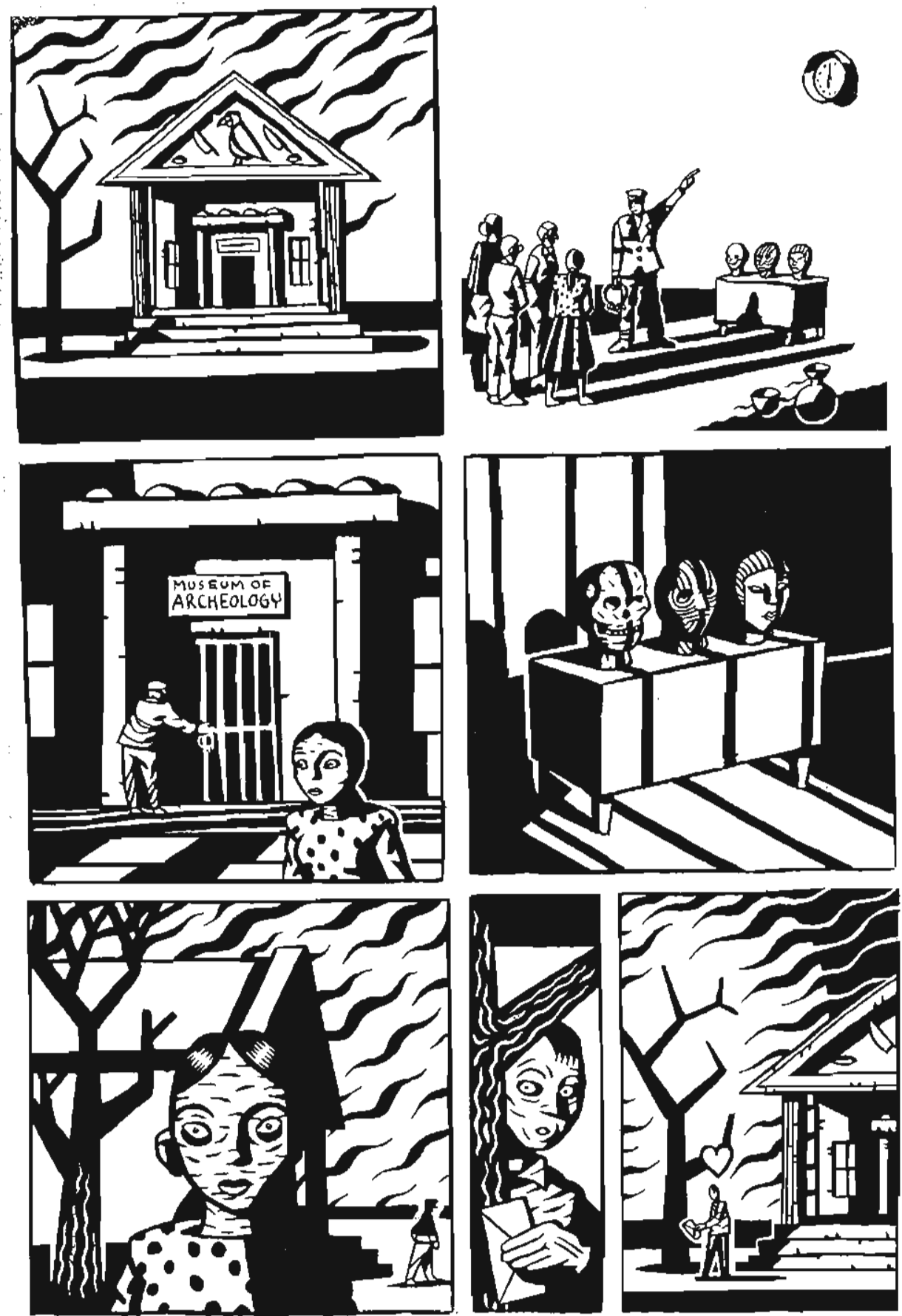

FIGURE 10

A tendency towards crude expressionism, combined with extreme forms of stylisation, is visible in the work of 'serious' contemporary comic strip and bande dessinee practitioners in the USA and Europe, as can be seen in this page from the strip "Museum of Archeology" by Milan Hulsing, published in the October 1999 issue of the French avant garde bande dessinee magazine, Lapin. 
that pictorial simplification is very often characteristic of cartooning. Nevertheless, there are countless cartoonists with excellent portraiture skills, such as Israel's Ori Hofmekler (1982) or South Africa's Richard Smith, who are able to render their work in full colour in a highly detailed, figurative style that relies on mild forms of exaggeration and caricature, rather than simplification, to get their message across (Figure 9) ${ }^{38}$. Likewise, there are numerous examples of comic strips rendered in highly detailed or painterly styles ${ }^{39}$.

Stylised and simplified narrative drawings of the kind normally associated with cartooning are characteristic of centuries-old traditions of narrative illustration in China, Britain, Europe and elsewhere in the world. Modern cartooning draws both from these ancient roots and from contemporary culture, and 20th century cartooning resounds with echoes from the worlds of ancient, traditional and modern art. As the century progressed, cartoonists borrowed freely from the proliferating styles and fashions of fine art and popular culture, often extending and contributing to them. Examples abound of cartooning styles, techniques and approaches drawn from Art Nouveau, Art Deco, Neo-Classicism, Expressionism, Pop Art and Op Art, and from film and fashion design (Couperie, 1972).

More recently, a tendency towards crude expressionism, combined with extreme forms of stylisation, has been visible in the work of 'serious' contemporary comic strip and bande dessinee practitioners in the USA and Europe, as can be seen in the pages of avant garde magazines like RAW (USA) and Lapin (France) (Figure 10)40.

Recent mainstream contemporary comic book art, on the other hand, exhibits highly sophisticated forms of pictorial stylisation, still rooted in classical approaches to the human figure, but heavily influenced by film in terms of framing, composition and pacing, that have developed organically within the various comics genres.

\footnotetext{
38 Richard SEuth, P.W. Botha. Cover illustration, Leadership Magazine, 1985.

39 Neil Gaiman's tecent Sandman anthology, Endless Nights (2003), a lavish hard-covered book, uses a different artist to render the seven stories or 'chapters' that it contains. Each story is rendered in a different style, ranging from classical comic book illustration to postmodern montage, each carefully chosen to resonate with the text in a different way.

40 Milan Hulsing, "Museum of Archeology", Lapin, No. 25, 1999: 51. Also see, for instance, the work of Marjane Satrapi in the same issue, and of Gary Panter and Mark Beyer in Spiegelman and Mouly (eds), Read yourself $R A W, 1987$.
} 
Contemporary superhero comics and the associated genres have benefited enormously from the superb results made possible by state-of-the-art colouring software, digital reproduction and new printing technologies. Exponential advancements in graphic reproduction and printing have freed cartoonists from virtually all the constraints they once worked under, and the digital convergence of media technologies has ensured that the products of cartooning are able to cross freely between media platforms.

Nevertheless, despite this bewildering plurality of forms and surfeit of opportunities, cartooning still continues to hold its own as a distinct and identifiable way of signifying. There is no single key to the definition of this way of signifying, but of all the stylistic approaches associated with cartooning, those styles associated with the 'clear line' are possibly the most definitive. The term 'clear line' (sometimes also called the 'Belgian clear line') is most often used with reference to the style of Herge's Tintin, but it has broad applicability throughout the world of cartooning ${ }^{41}$. The simplified, stylised, uncluttered 'clear' line is characteristic of classic animation, of the entire Disney ouvre, of mainstream U.S. comic books like the Archie Comics range, of contemporary strips like the Hernandez Brothers' Love ERockets and the graphic novelisations of Charles Burns, and of the work of European artists like Ever Muelen and Joost Swarte ${ }^{42}$. In these products, widely divergent stylistic approaches have in common the obvious use of techniques of pictorial simplification and stylisation. In other genres and styles, the simplification and stylisation typical of cartooning may not be so obvious, obscured by layering, cross-hatching, painting, sketchy outlines or the use of halftones. Nevertheless, some form of pictorial simplification or stylisation normally reveals itself on closer inspection.

\section{(ii) Iconic characterisation and codification}

In his chapter on the vocabulary of comics, McCloud (1993: 24-59) begins with the notion of the 'icon'. In conventional (non-semiotic) terms, an icon is a highly stylised or simplified visual representation of an object or concept-normally an object of veneration. The notion of the icon has its origins in very specific forms of religious imagery, but the Oxford Dictionary (1999) also refers to its usage in linguistics as "a sign which has a characteristic in common with the thing it signifies" and in computing as "a

\footnotetext{
41 See www.tintincom.

42 See www. archiecomics.com; Jaime Hernandez's Love and Rockets collection, 1987; Charles Burns's HardBoiled Defective Stories, 1988; Ever Muelen and Joost Swarte's comic strips in Spiegelman \& Mouly's Read Yourself Raw, 1987.
} 

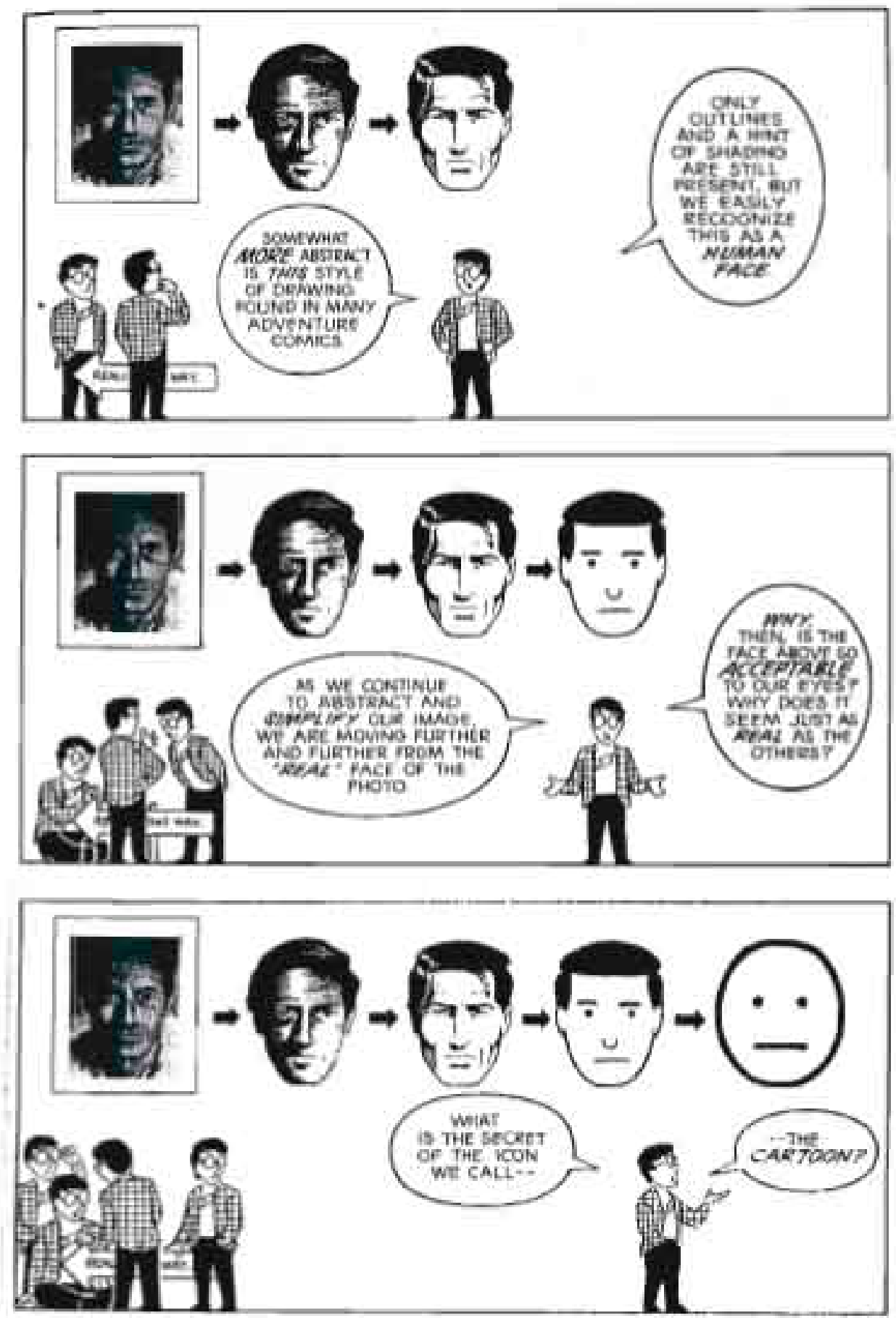

FIGURE 11

McCloud (1993) presents a continuum of images of a human face, beginning on the left with a photograph of a face and moving to an icon of a face on the right. The icon is derived by abstracting the face down to a basic set of lines and dots that signifies a 'universal' face. 
symbol or graphic representation on a VDU screen of a program, option or window". However, the idea that representational paintings or photographs may be considered iconic (O'Sullivan et al, 1994: 138) is a usage peculiar to semiotics, and, as the Oxford definition demonstrates, has not yet been absorbed into conventional English usage. This disjuncture between conventional English usage and the usage in semiotics is a source of semantic confusion.

In his treatment, McCloud reveals by his employment of the conventional usage that he has not grounded his exposition in the language of semiotics, and this makes it difficult to accommodate his insights into the framework provided by semiotics. To complicate matters further, McCloud's employment of conventional usage is also idiosyncratic, particularly where he includes letters of the alphabet, numerals and other nonrepresentational signs under the term 'icon' (he eschews the term 'symbol' on the grounds that it is too 'loaded')(1993: 27). As has been explained above, the term 'iconic' should be reserved for those signs "in which there is a marked physical or perceptual resemblance between the signifier and that for which it stands" (O'Sullivan et al, 1994: 138), whereas letters of the alphabet and mathematical signs are more properly referred to as 'symbols'.

This semantic caveat noted, McCloud's analysis of what he calls 'pictorial icons' is nevertheless extremely illuminating, especially with regard to the way in which the reader participates in the process of signification. In one particularly convincing example (1993: 29-31), MoCloud presents a continuum of images of a human face, beginning on the left with a photograph of a face and moving to an icon of a face on the right. The icon consists of a circle containing two dots and a line, which, he argues, we cannot help but see as a face. He points out that the icon is in fact an extremely simplified cartoon, derived by abstracting the face down to a basic set of lines and dots that signifies a 'universal' face (Figure 11).

Charles Shultz's Charlie Brown (Peanuts) is a classic example of the simplification of the human face down to its basic elements, but there are thousands of other examples in cartoons and comics, each occupying a position on the continuum between pictorial representation and iconic signification (Figure 12). McCloud's most compelling insight here is that reader involvement is increasingly amplified along the continuum as the image becomes more iconic: "By stripping down an image to its essential 'meaning', an artist can amplify that meaning in a way that realistic art can't" (1993: 30). 


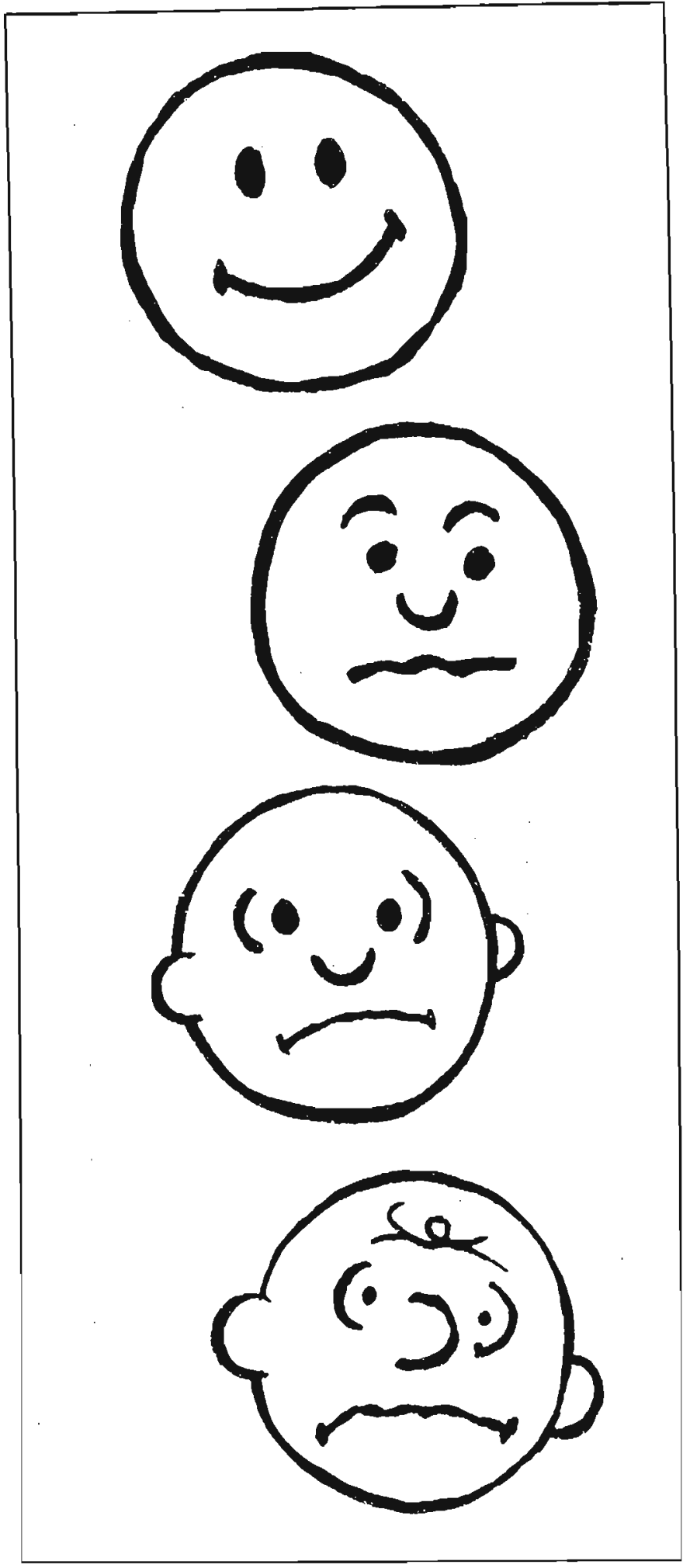

FIGURE 12

Charles Shultz's Charlie Brown (Peanuts) is a classic example of the simplification of the human face down to its basic elements, but there are thousands of other examples in cartoons and comics, each occupying a position on the continuum between pictorial representation and iconic signification. (Diagram: N.D. Mazin.) 
This insight brings to mind the inspiration that Picasso, Braque and other Postimpressionist and Cubist painters derived from exposure to the iconic simplicity of African and Oceanic masks in the early years of the 20th century. These artists were experimenting with processes of simplification and abstraction in an endeavour to reveal, beneath the surface level of pictorial representation, the underlying structures of reality (see Leymarie 1971; Rurhberg et al, 2000). Perhaps the greatest contribution of these artists was the trajectory they pursued, away from the conceptual restrictions of representationalism and the 'natural attitude', towards the 'freedom' of abstraction.

The claustrophobic academicism that still dominated the Western art world at the end of the 19th century demanded an extremely high level of pictorial naturalism, and it was against the requirement that fine art should be mimetic that the artists of the fin de siecle rebelled (Sweetman 1999). Drawings and paintings from that period by artists as varied as Picasso, Henri Toulouse-Lautrec, Aubrey Beardsley, James Ensor, Edvard Munch, Henri Matisse, Raoul Dufy and Emil Nolde (see Ruhrberg et al, 2000) display the kinds of pictorial stylisation, simplification and exaggeration that are characteristic of cartooning.

A key concept in the movement away from mimesis towards simplification and abstraction is the notion of 'closure'. Closure is one of the fundamental principles of perceptual organisation identified by Gestalt psychologists, in terms of which "interpretations which produce 'closed' rather than 'open' figures are favoured" (Chandler, 1996. Codes: 3-9). McCloud uses this term to refer to the process whereby our minds complete and 'fill in' the missing bits to construct meaningful mental pictures-"observing the parts but perceiving the whole" (1993: 63). The idea that the human mind, in its quest for 'closure', is compelled to complete an incomplete image, is, he argues, crucial to an understanding of how comics work, and perhaps provides a clue as to why they are so popular. According to McCloud: "The fact that your mind is capable of taking a circle, two dots and a line and turning them into a face is nothing short of incredible! But still more incredible is the fact that you cannot avoid seeing a face here. Your mind won't let you!"43 (1993: 30).

\footnotetext{
${ }^{43}$ McCloud's suggestion that we can't help amplifying iconic images--that this is not something that we have to learn to do, but something that we can't avoid doing - has important implications for the use of comics in education, particularly in respect of illiterate or semi-literate readers. Even the most cursory study of the African, Oceanic and Oriental artistic traditions and of so-called 'primitive' masks from around the world suggests that the abstraction and simplification of the human face to the level of pure form is archetypal and that iconic simplification is a universal practice.
} 
McCloud's assertions are compelling in that they imply that the simplified cartoon face has the same kind of significatory power as the mask in drama or ritual. By placing mask-like iconic faces on the heads of their characters, cartoonists remove from their characters the 'fixed' meanings that the realistic representation of a face would entail, allowing symbolic and mythical meanings to flow into the space provided by the loss of anchored representation. In so doing, cartoonists invite their readers to 'don' these masks and 'inhabit' the cartoon characters themselves, and thereby to invest the characters with meanings derived from their own experience. This process is even more obvious in the superhero genre where the heroes are usually masked.

McCloud acknowledges Marshall McLuhan (1964) in the formulation of this idea. McLuhar's distinction between 'hot' and 'cool' media relates to the extent to which the reader is required to be actively involved in the processes of signification: "Hot media are [...] low in participation, and cool media are high in participation or completion by the audience" (McLuhan, 1964: 31). For McLuhan, the level of audience participation is related to how much data is presented and whether this data is 'high-definition' or 'lowdefinition':

A hot medium is one that extends one single sense in 'high definition'. High definition is the state of being well filled with data. A photograph is, visually, 'high definition'. A cartoon is 'low definition', simply because very little visual information is provided (McLuhan, 1964: 31).

While many of McLuhan's formulations seem dated today, his argument about audience participation provides a very useful entry point into the discussion about the way of signifying that is peculiar to cartooning. McCloud extends this argument to suggest that iconic forms belong in "the realm of the concept", while representational forms belong in "the realm of the senses" (1993: 39). The higher the level of representational defirition in the artwork, he argues, the less the reader is required to dwell in the realm of the conceptual.

Interestingly, many cartoons and comics employ both high-definition and lowdefinition imagery, often in the same frame. In editorial cartooning and caricature, high definition is often reserved for the face, which may be disproportionately larger than the body, while body and background may be iconically sketched. The opposite is true of 

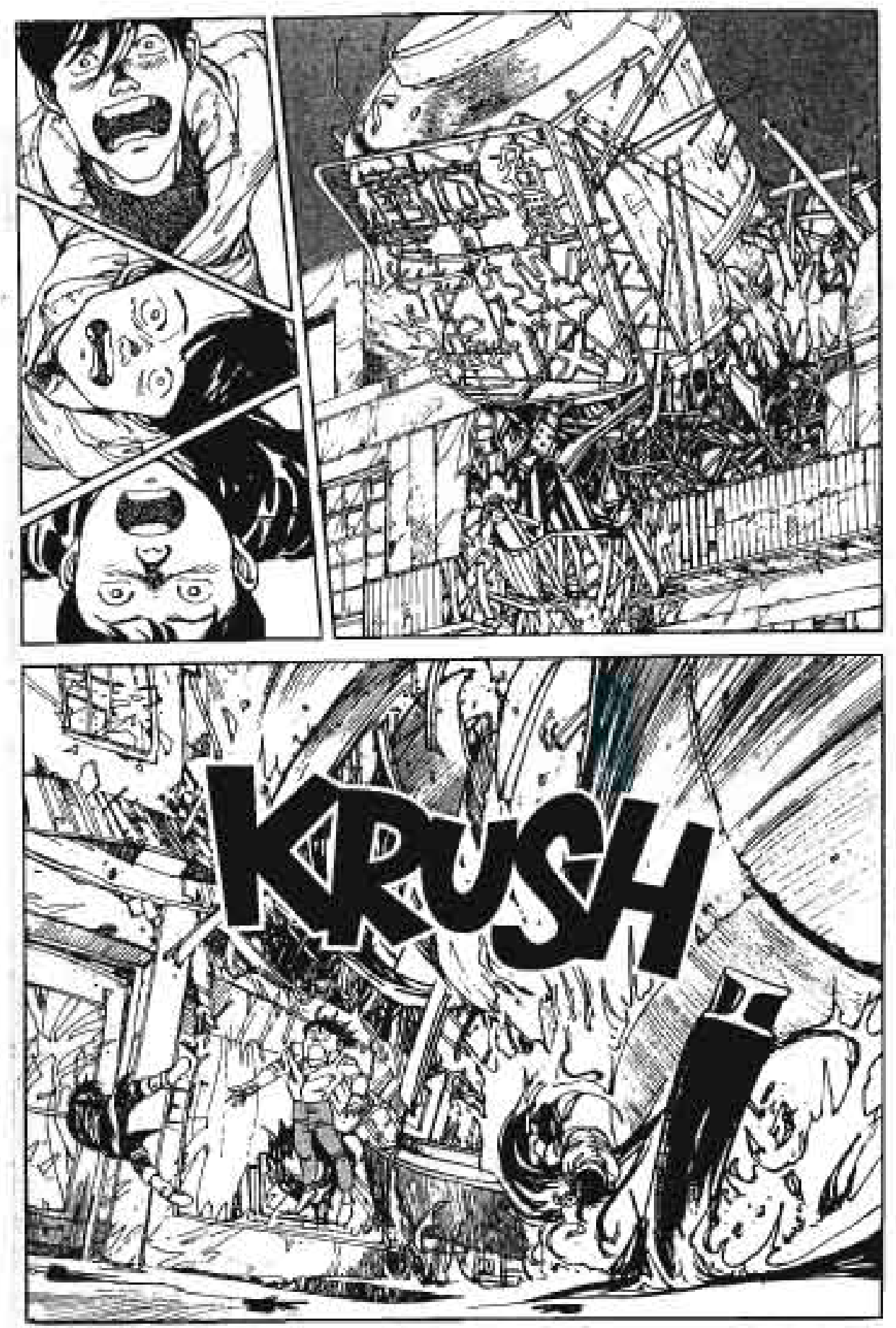

\section{FIGURE 13}

Like Herge's Tintin, the work of Japanese manga artist Katsuhiro Ottorno (2000) places characters with low-definition, cartoony faces into detailed, high-definition environments. This allows the reader to 'inhabit' the low-definition (or iconic) character while experiencing the texture and detail of the high-definition environment in which the character is placed (2000:53). 
comics, where high definition is often reserved for backgrounds and objects, while low definition is employed in the portrayal of characters. In some cases the character might even be given a high-definition body and a low-definition mask for a face.

McCloud's insight into this methodology is that it allows the reader to 'inhabit' the lowdefinition (or iconic) character while experiencing the texture and detail of the highdefinition environment in which the character is placed. A classic example of this method is Herge's Tintin where iconic characters move through environments that are often rendered in a naturalistic way, but more extreme examples are to be found in the work of Japanese manga artists like Katsuhiro Ottomo (2000) (Figure 13).

The placement of iconic characters in realistic settings is also a feature of animated films, where low-definition cartoon characters often inhabit high-definition environments. The difference in the rendering of character and background in animated films is itself a function of the technical requirements of the medium, which require the moving figures to be simplified for the purposes of repetition while the painted or computer-generated backdrops across which they move can be extremely complex. The same applies to interactive computer and video games where iconic characters move through very convincing three-dimensional environments. The need for the characters in comics and animated cartoons to be recognisably portrayed throughout the narrative, and to be simplified to aid the requirements of repetition, is a structural or technical precondition that underlies the tendency towards iconic characterisation.

In editorial cartoons, particularly those that portray public figures, the development of iconic characterisation follows a different path. Here, the need for the public figures portrayed to be instantly recognisable by the readership, is crucial. Caricature, the exaggeration of obvious facial characteristics, is the formal device most often used to achieve this.

However, caricature is also employed with the intention of ridiculing or demeaning these public figures. The simplification of the caricature to an instantly recognisable cartoon face requires the same techniques of iconic characterisation as are used in comics, but for a completely different purpose. Here, the simplified cartoon face is not intended to produce a generalised and inhabitable 'everyman' like Charlie Brown, but rather to identify and tag an actual person. A fine example of iconic characterisation in 


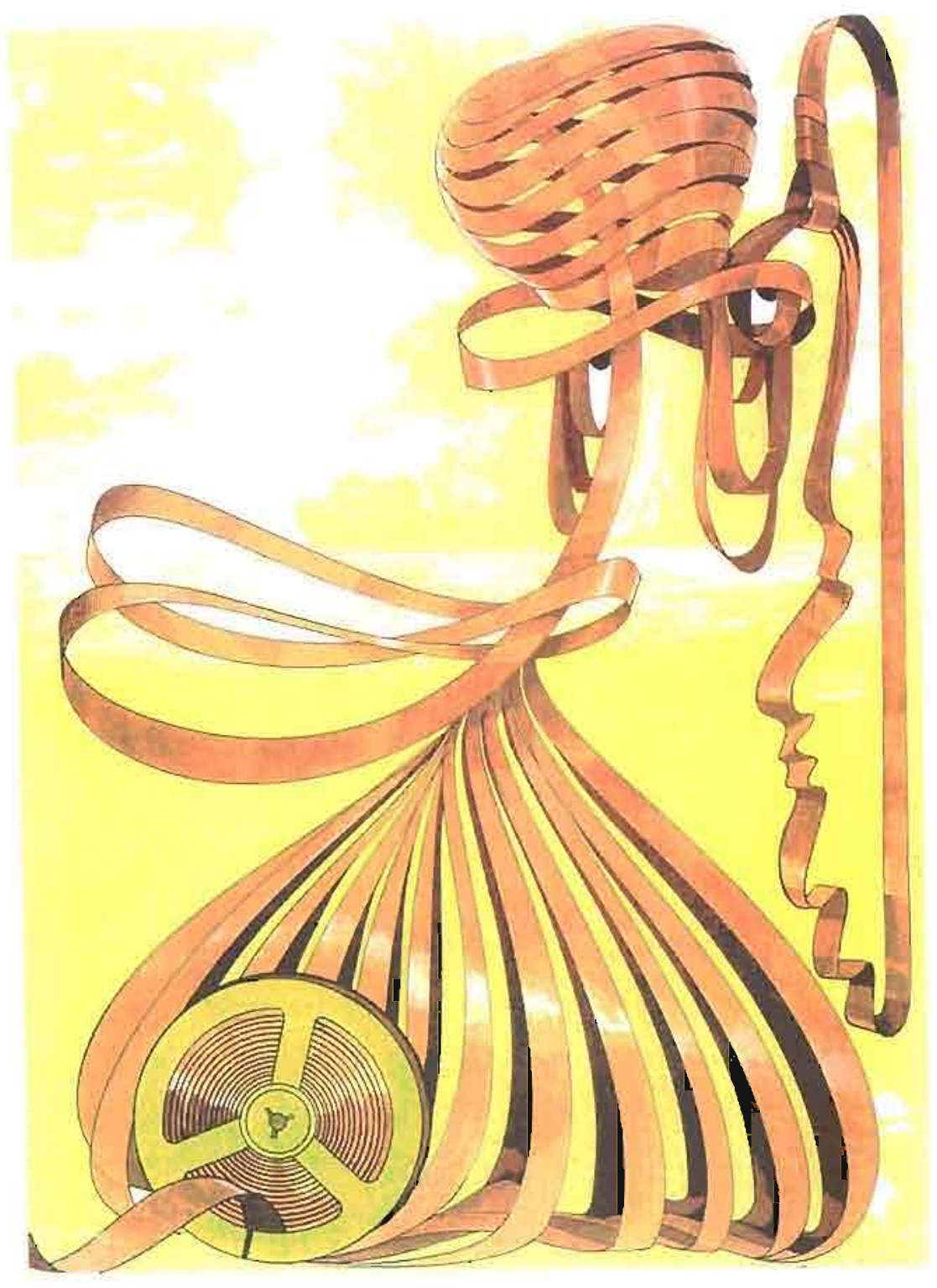

\section{FIGURE 14}

The distortions of facial anatomy that ensure character recognition in editorial cartoons sometimes become so over-elaborated that iconic signification eclipses pictorial representation.

The shape of Richard Nixon's nose is a case in point, represented here as a tangle of Walergate tape by Gerald Scarfe (Scarfe Face, 1993). 
South African cartooning is Richard Smith's cartoon strip Smith and Abbot Ink, which first appeared in the Rand Daily Mail in 1972. In this strip, instantly recognisable caricatures of politicians are placed onto truncated puppet-like bodies in an iconic environment (see Figure 40) ${ }^{44}$.

Usually, the distortions of facial anatomy that ensure character recognition in editorial cartoons also function as encoded political or ideological messages, in that they convey the cartoonist's attitude to the person represented. Often these distortions become so elaborated over the course of time that iconic signification eclipses pictorial representation. The shape of Richard Nixon's nose is a case in point. So many cartoonists participated in the exaggeration of this appendage that its shape became a signifier in the public mind, not only of Nixon as a person, but of governmental corruption and venality in general. Nixon's nose achieved a particularly sublime portrayal in a caricature by Gerald Scarfe where his face is portrayed as a tangle of Watergate tape (Scarfe, 1993: 71) (Figure 14).

Because it is expressed graphically and stylistically, the visual voice of the cartoonist is ideal for the transmission of subtexts that express deep-seated attitudes, ideological tendencies or prevailing mythologies. The use of iconic characterisation and caricature plays an important role in the (sometimes very subtle) portrayal of racial, gender or other stereotypes. In addition, cartoonists employ or develop visual codes representing philosophical or political ideas that are integral to the iconic characterisation of public figures. These codes begin as agglutinations of signs that adhere to the characterisation and, as their meanings become familiar and conventional, they may come to signify more general ideological or political meanings.

One particularly pungent example of this in South African cartooning is the figure of Hoggenheimer, the Jewish arch-capitalist, who from the early 1900's appeared from time to time in the cartoons of the celebrated Afrikaans cartoonist Daniel Boonzaier (Vernon, 2000). Hoggenheimer is a generic character, not intended to represent any single individual, but rather to portray, via the iconic signs of which the characterisation is constituted, one of the most threatening enemies of Afrikanerdom. The agglutination of signs of which Hoggenheimer is composed is in itself a fully-constituted code: the fat belly encased in its waistcoat and adorned with a gold watch chain, the white

\footnotetext{
44 The overlarge heads and dumpy bodies in Smith's strip give the represented politicians a cute, childlike look reinforced by the humour of the text, which is curiously at odds with the crimes against humanity that they are alleged to have perpetrated. (See Chapter Four.)
} 

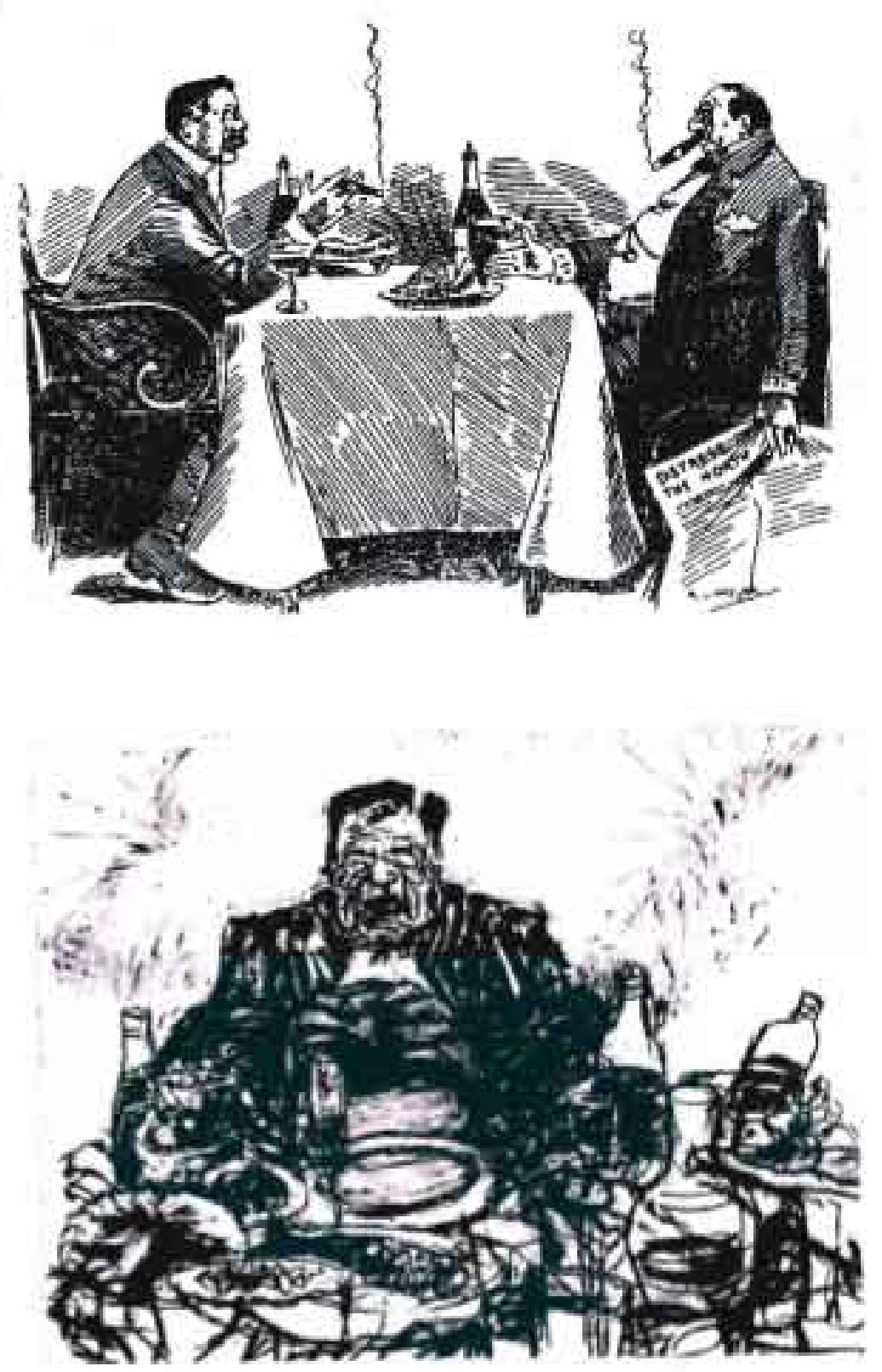

FIGURE 15

The iconic figure of Hoggenheimer, who from the earty 1900 's appeared in the cartoons of the celebrated Arrkaans cartoonitut Daniel Bocnzaier, is an assemblage of signs and symbols that constitute an encoded ideological message: the fat belly encased in its waistcoat and adorned with a gold watch chain, the white handkerchief in the top pocket. the monoclo, the ogar, the stiff collar, the large nose and corpulent jowls, all conspire to signify greed, wealth and contempt for the values of Afrikanerdom. Noarly a century iater, the figure of Hoggenheimer is further elaborated in the animated films of Wiliam Kentridge, in the character Soho Ekstein

(Vemon, 2000, Cameron, 1990). 

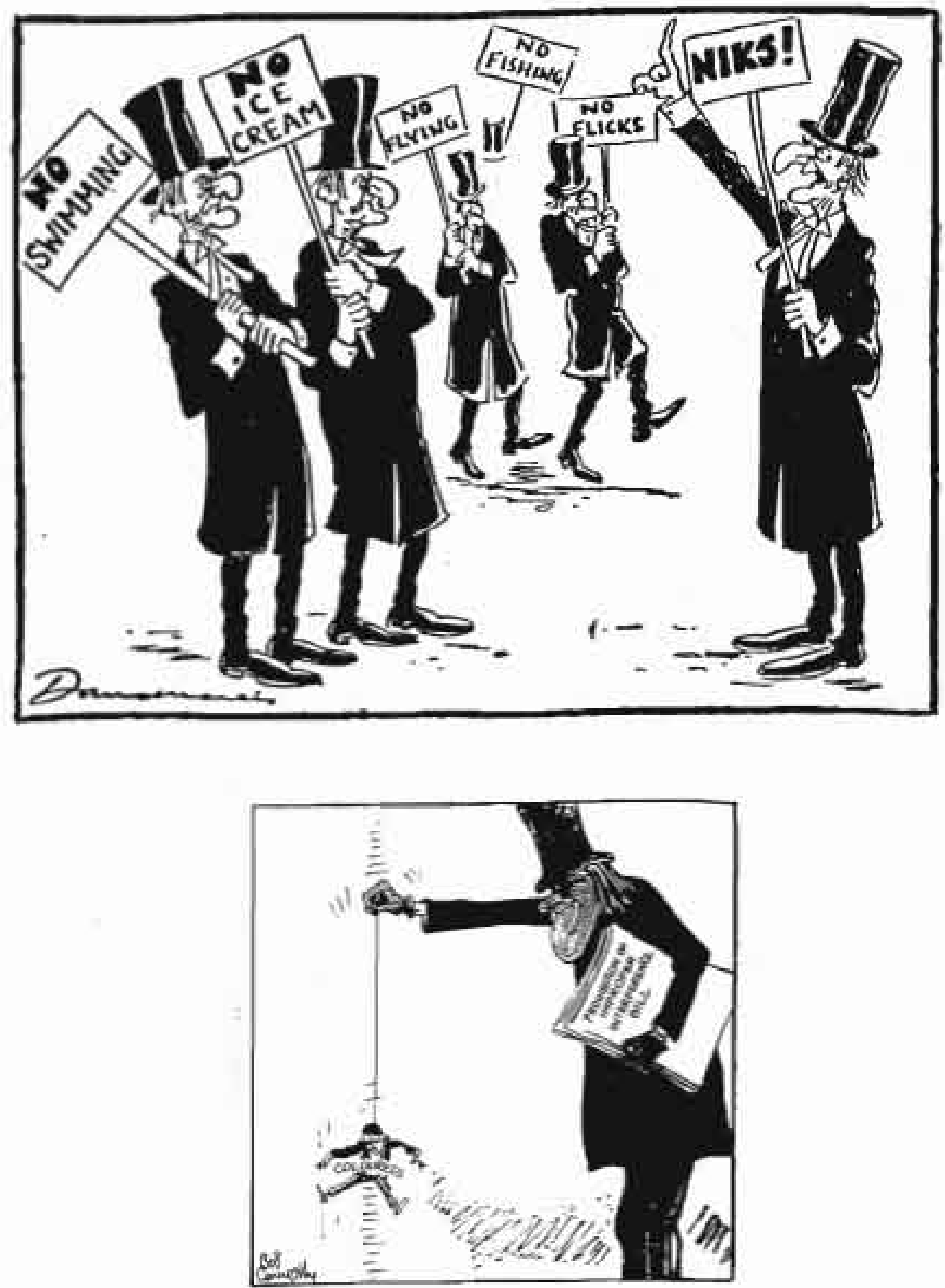

FIGURE 16

An excellent example of iconic codification in South African political cartooning is the figure of the verkrampte Afrikaner Nationalist bogeyman, as portrayed by the Cape Times' David Marais (above) and the Rand Daily Mail's Bob Connolly (below) in the 1960s. The figure is dressed in black, representing the evils of conservatism, with a top hat signifying Afrikanet republican aspirations, and unkempt hair to signify the unsophisticated rural roots of the Atrikaner. In Connolly's figure, sunglasses are added to indicate a lack of vision, and gloves to indicate social ineptitude and lack of grace (Vernon 2000. 119). 

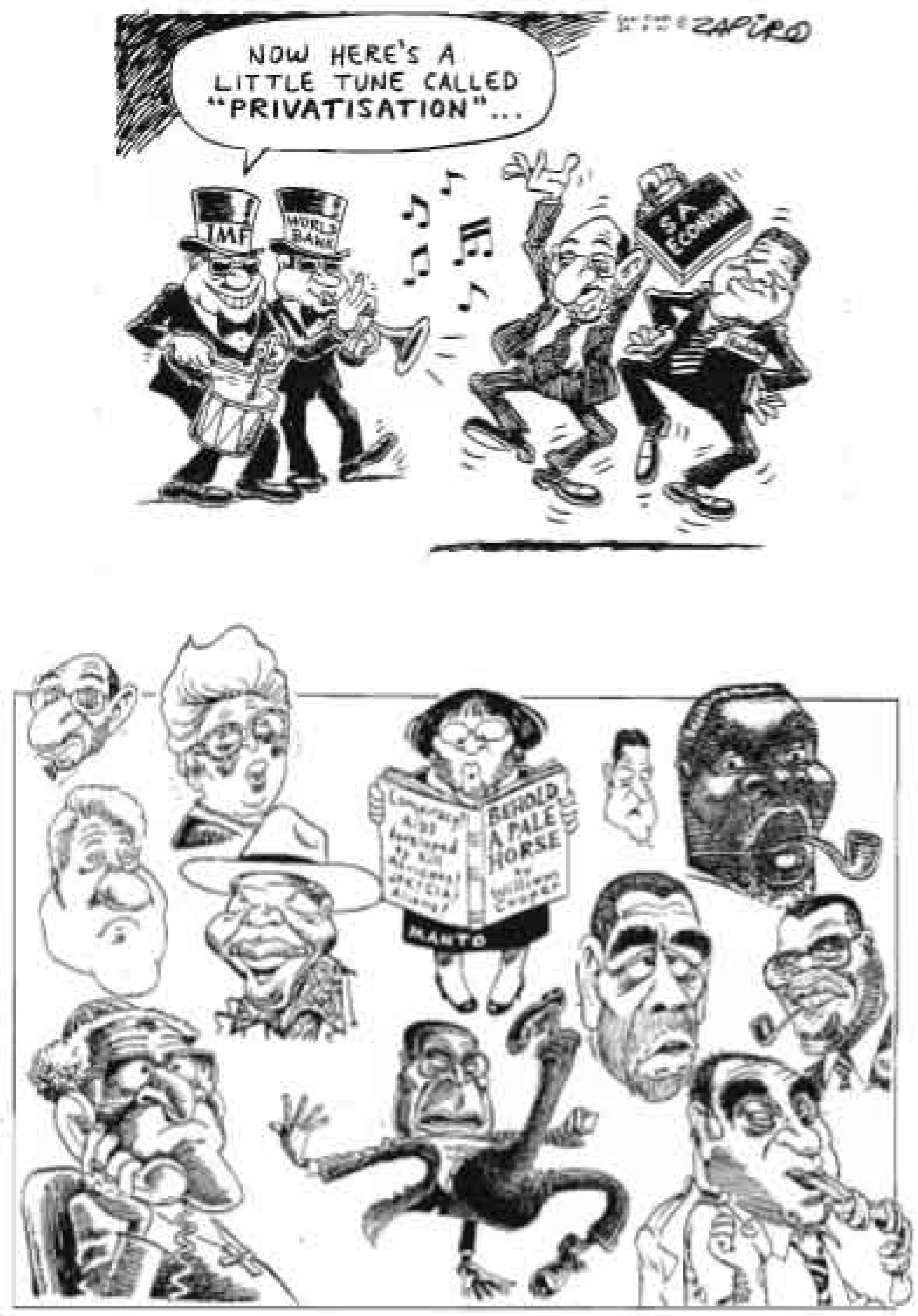

FIGURE17

(Above) Zapiro's work displays a constant use of codes, chen combined with visual wordplay in which idiomatic expressions are visually speit out as in this 2001 cartoon of govemment ministers Trevor Manuel and Jeff Radebe dancing to the tune of the IMF and World Bank. Zapiro's attitude to these two institutions is clearly encoded in the dark glasses, top hats, dress sults and shiny shoes in which he dresses their representatives, who are presented as showmen and by implication, charlatans.

(Beiow) A key attribute of the successful editorial cartoonist is the ability to produce instantly recognisable caricatures - an ability that Zapiro possesses in abundance. 

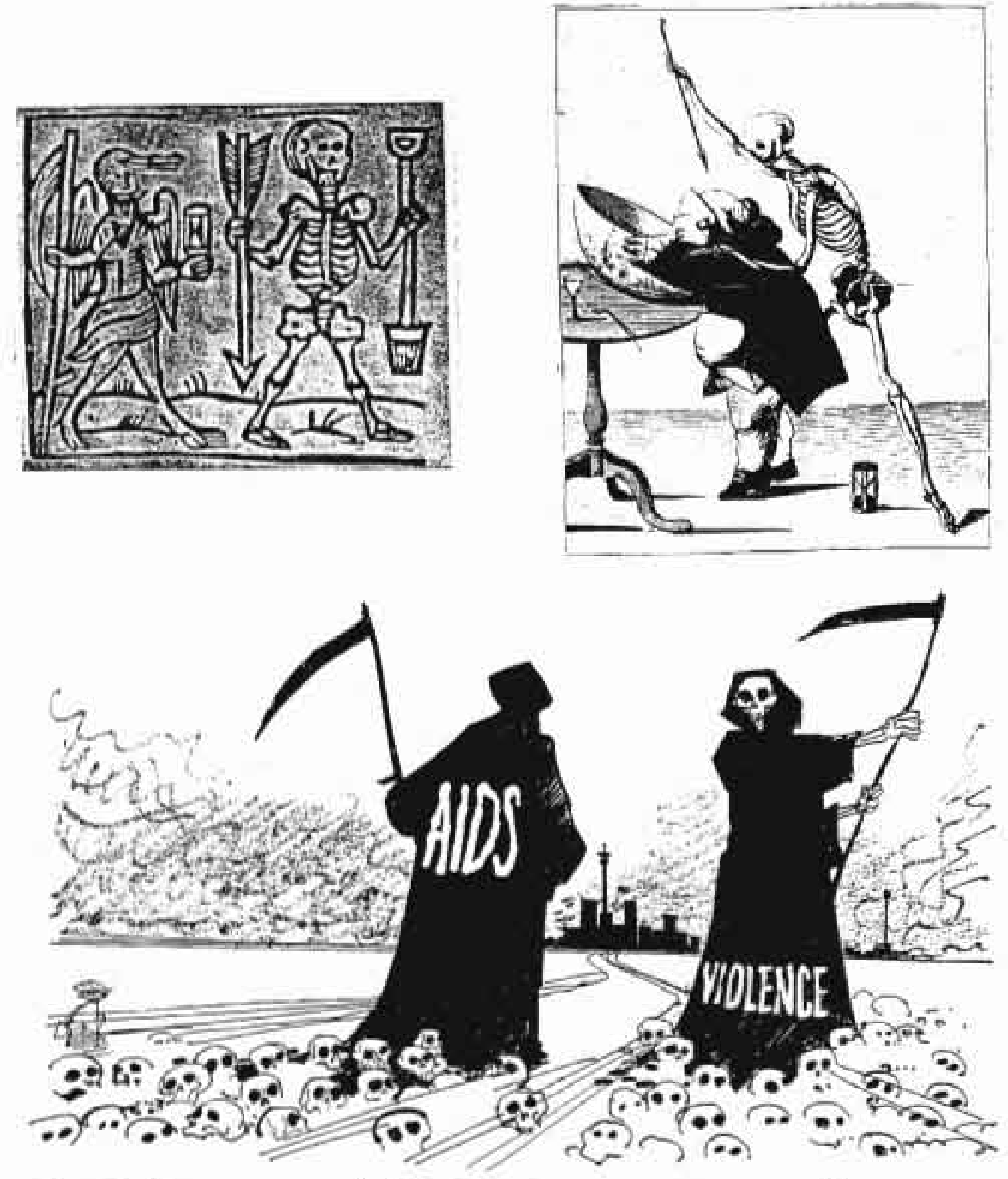

zorferifer

" FANCY MEETING YOU HERE

\section{FIGURE 18}

The figures of Death and Time are a good example of codes developed many centuries ago that have been passed down to us. (Above lef) A chudely rendered 16 th century English woodcut entitled 'Death and Time'. (Above right) An anonymous 18 h century caricature entitled 'The Last Drop'. (Below) A 1991 cartoon by South African cartoonist Dov Fedier. By now the medieva! codes for Death and Time have been conflated into the figure of the Grim Reaper, who makes frequent appearances in Fedler's cartoons (Klingender, 1944, Fedier, 1991.) 
handkerchief in the top pocket, the monocle, the cigar, the stiff collar, the large nose and corpulent jowls, all conspire to signify greed, wealth and contempt for the values of Afrikanerdom. Nearly a century later, the figure of Hoggenheimer is further elaborated in the animated films of William Kentridge, where the iconic code that constitutes the characterisation of the Jewish industrialist is investigated from the 'inside' through Kentridge's portrayal of the character Soho Ekstein (Cameron, 1999: 53)(Figure 15).

Another excellent example of iconic codification in South African political cartooning is the figure of the verkrampte Afrikaner Nationalist bogeyman, as portrayed by the Cape Times' David Marais, the Rand Daily Mail's Bob Connolly, and other liberal cartoonists in the 1950s and 1960s (Figure 16). Ken Vernon (2000: 119) traces the evolution of this figure in Connolly's work, citing the example of a 1966 cartoon:

Gone are the trappings that once signified the Afrikaner element in the NP, such as the rural clothes, unkempt beard, farm boots, pipe and so on. Instead, the figure has evolved into an urban bogeyman, which Connolly used extensively throughout the 1960s. By 1966, the (supposedly) menacing figure was dressed totally in black, to represent evil, having lost his striped pants somewhere along the way. The top hat remains, a vestige of the hat that represented the Republican aspirations of the Afrikaner. The unkempt long hair remains, but the pipe is gone. The figure wears sunglasses to indicate a lack of vision, and gloves to indicate social ineptitude and lack of grace (Vernon 2000: 119).

In the cartoons of Zapiro, the cartoonist's uncanny ability to produce instantly recognisable caricatures is combined with a constantly inventive use of codes, often combined with visual wordplay in which idiomatic expressions are visually spelt out. Thus in a 2001 cartoon government ministers, Trevor Manuel and Jeff Radebe, are shown literally dancing to a tune played by characters representing the IMF and World Bank. Zapiro's attitude to these institutions is clearly codified in their dark glasses, top hats, dress suits and shiny shoes-they are typical showmen, and by implication, charlatans (Zapiro, 2001:152) (Figure 17).

Many of the visual codes and conventions commonly used in cartooning go back a very long way. The figures of Death and Time are a good example of codes developed many centuries ago that have been passed down to us (Figure 18). In a crudely-rendered 16th century English woodcut, entitled 'Death and Time', Death is shown as a simplified skeleton, carrying a spade in the left hand and an arrow in the right, while Time is a winged figure, carrying an hourglass in the left hand and a scythe in the right 

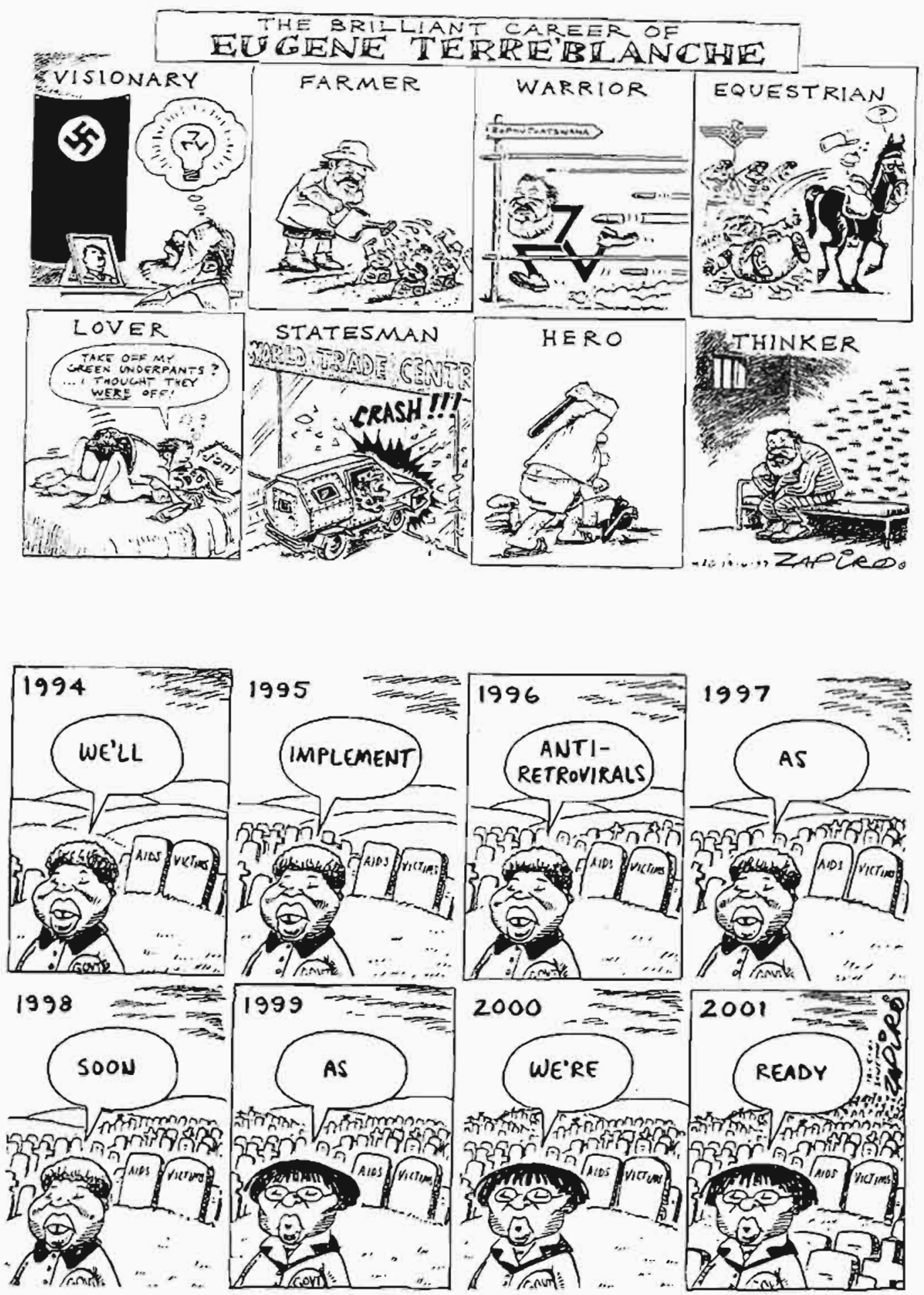

FIGURE 19

Zapiro's editorial cartoons often consist of two or more juxtaposed panels. Sometimes the panel-to-panel transitions are synchronic or comparative, as in this 1997 cartoon parodying the biography of Eugene Terreblanche (above) and sometimes they are diachronic and progressive, as in this 2001 cartoon critiquing the government's tardy approach to the implementation of antiretroviral treatment to people with Aids (below).

(Zapiro, 1997: 2001) 
(Klingender, 1944: 26). Some 200 years later, in an anonymous 18th century caricature, entitled 'The Last Drop', the same figure of Death stands behind a fat glutton who is drinking out of a large bowl (Klingender, 1944: 26). On the table is a wine glass and a tobacco pipe, signifying addiction, while an hourglass stands next to him on the floor. Death is about to plunge his arrow into the glutton's head. Two centuries later again, a 1991 cartoon by South African cartoonist Dov Fedler ${ }^{45}$ shows two black-robed and hooded skeletons wielding scythes in a field of skulls. By now, the medieval codes for Death and Time have been conflated into the figure of the Grim Reaper, who makes frequent appearances in cartoons by Fedler, Zapiro and other South African cartoonists.

\section{(iii) Visual narrative conventions}

In his examination of the way comics tell their stories, McCloud (1993) has dealt in detail with a wide range of formal and structural characteristics that are germinal to comics, and these do not need to be repeated here, although it should be noted that many of them apply across the various forms of cartooning. McCloud is strongest in his evocation of the minutae of the craft of comics making, and particularly in his description of the structural underpinnings of sequential pictorial narrative. He has extended the formal vocabulary pioneered by Eisner, and cemented into comics discourse concepts such as 'closure', 'panel-to -panel transition' and how the dimension of time is signified in the 'invisible' gutters between frames. He has also shown how Japanese manga, which developed largely in isolation from the American, British and European graphic narrative traditions, exhibit significantly different narrative rhythms that are often synchronic and tend to be more akin to Eastern ways of signifying reality than to the goal-orientated, diachronic storytelling traditions of the West (McCloud 1993: Chapters 3 \& 4).

It is worth emphasising that, in cartooning, narratives need not always be linear progressions from one event to another along a single chain of causality. A narrative may proceed along parallel chains of causality; sometimes the notion of causality may even disappear and the only remaining link between images may be their juxtaposition. Philosophically, this relates to the difference between synchronic and diachronic views of the way reality is constituted, and one of the strengths of cartooning in print is that, because it is constituted as a static and therefore simultaneous presentation of images, it

${ }^{45}$ Dov Fedler, undated cartoon, The Star, reproduced in The Season of Violins, 1991. 

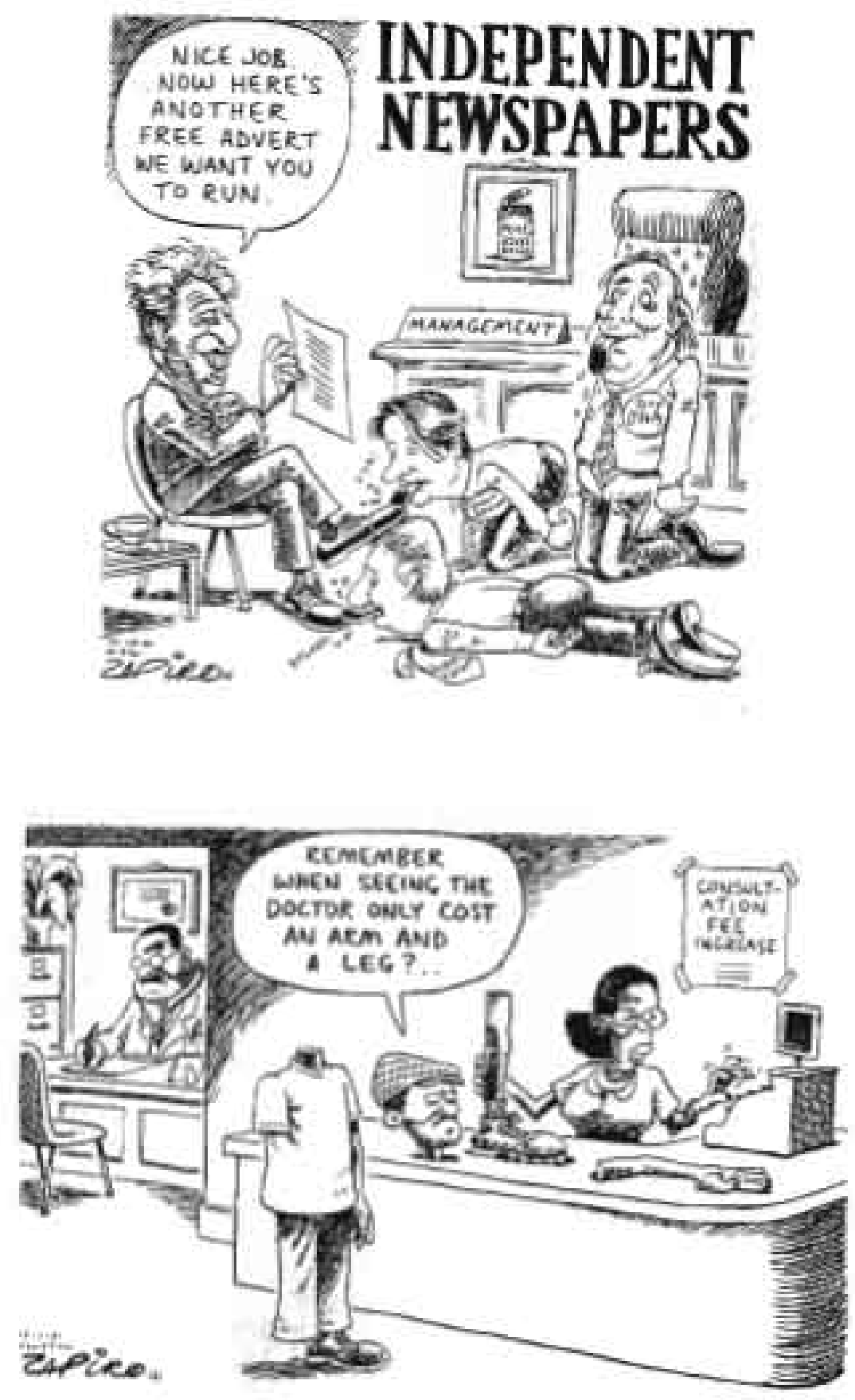

FIGURE 20

Senior press figures are shown licking the shoes of government minister Essop Pahad in a 2000 Zapiro cartoon (above), while in a 2001 cartoon about rising medical expenses a man missing an arm and a leg offers his head in exchange for medical supplies (below). In these cartoons Zapiro exploits one of his favourite sources of humour, the visual representation of an idiomatic expression. (Zapiro, 2000, 2001.) 
can allow synchronic relations between these images. This cannot easily be achieved on film, where the forward movement of the action privileges diachronic presentation.

Editorial cartooning is usually regarded as a single-panel form, but it is common practice for some editorial cartoonists, notably Zapiro, to utilise sequential narrative whenever the message they are trying to convey requires the representation of a sequence. Zapiro's editorial cartoons often consist of two or more juxtaposed panels. Sometimes the sequence serves to record a diachronic progression through time and sometimes the purpose of the juxtaposition is synchronic or comparative (Figure 19).

McCloud's analysis concerns itself exclusively with comics, but his categories of panelto-panel transition are applicable across the range of cartooning forms. They may also be applied to the analysis of scene-to-scene transitions in animated cartoon films. This demonstrates a key argument of this chapter: that the various forms of cartooning share a common set of codes. The corollary to this is that the primary characteristics of cartooning apply to all of its forms, including comics. There is an enormous amount of cross-pollination amongst the various cartooning forms, styles and genres and hence it is no surprise that many of the codes identified by McCloud as characteristic of comics also apply to editorial cartooning. Chapter Three argues that most of the primary characteristics of cartooning were all in place long before the beginning of the $20^{\text {th }}$ century, as were many of its secondary attributes such as exaggeration, overdramatisation, visual slapstick, pictorial puns and incongruous juxtaposition.

In a 2000 Zapiro cartoon, senior press figures are shown licking the shoes of government minister, Essop Pahad (2001: 22), while in a 2001 cartoon about rising medical expenses, a man missing an arm and a leg offers his head in exchange for medical supplies (2001: 51)(Figure 20). In these cartoons Zapiro exploits one of his favourite sources of humour, the visual representation of an idiomatic expression. In doing so, he draws attention to the extent to which visual imagery is incorporated into idiomatic language, and how pungent are some of the expressions that we unthinkingly use. The facility with which cartooning lends itself to this kind of visual pun, and to an over-dramatised or exaggerated rendition at the expense of the dignity of public figures is part of the reason why cartooning is so often associated with counter-hegemonic positions in society. As has been noted above, the structures of authority in society have in the past often demonstrated nervousness about unrestrained visual imagery and it is no coincidence that cartooning flourishes under democratic conditions and is embattled under conditions of authoritarianism. 


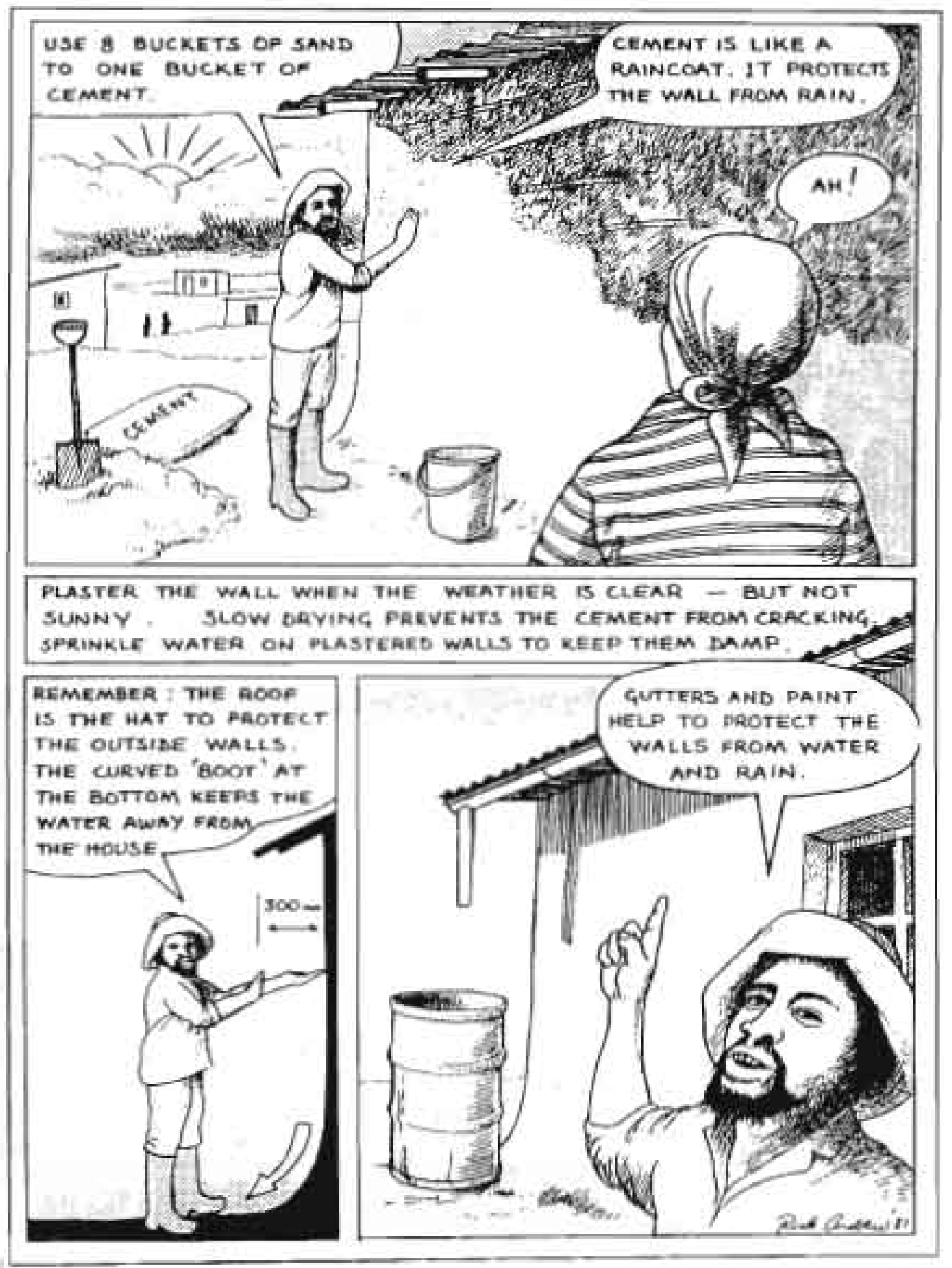

FIGURE 21

Bonginkosi, an instructional comic on informal house building methods written by Rodney Harbour and illustrated by Rick Andrew, combines aesthetically pleasing lliustrations and simply written text to create an extremely effective educational tool aimed at low-literacy readers (University of Natal Appropriate Housing Technology Unit, 1987.) 
Similarly, because cartoons and comics are ideally suited to flights of the imagination and do not require the physical construction of sets or expensive travel arrangements to create environments for the purposes of visual storytelling, they are ideally suited to adventure, fantasy, science fiction and similar storytelling genres. It has only been recently that live action films, through the agency of computerised animation techniques, have been able venture into the fantasy worlds that have for decades been the stock in trade of comics and animated cartoons.

However, not all forms of cartooning are concerned with exaggeration, humour or fantasy. The graphic narrative conventions and codes of cartooning lend themselves to educational tasks, particularly where these entail the communication of technical information. Most textbooks and technical manuals involve some form of illustration and the distinction between cartooning and what is sometimes called instructional design' is not always easy to make.

As noted earlier, instructional booklets such as those used by airlines or the manufacturers of high-tech products constitute a form of graphic narrative that sits uncomfortably on the margins of cartooning. McCloud argues for their inclusion in the definition of comics simply because they involve drawn images in deliberate sequence, while I have chosen to exclude them from my definition of cartooning because they lack aesthetic intention. Arguably then, if aesthetic intention were to be demonstrated in an instructional strip, it would have to be included under the definition of cartooning.

Will Eisner himself produced instructional comics, utilising iconic characterisation and visual narrative conventions to turn mundane subjects into readable visual texts. Eisner identifies two forms of instructional comics: 'technical' and 'attitudinal' (Eisner 1985: 142). The examples he gives (a page from a technical manual produced for the military and a series of booklets on careers) display how he responded artistically to the technical requirements of very mundane subject matter and in particular how he used iconic characterisation to enliven the material.

There are many South African examples of comics produced with the intention to instruct. The need to convey technical or ideological information to people of limited literacy has resulted in various experiments in instructional cartooning, such as a 1987 booklet on informal house building methods, written by Rodney Harbour and 
illustrated by Rick Andrew (Figure 21) ${ }^{46}$. These will be discussed in Chapter Six. However, to anticipate the outcome of that discussion, it may be suggested that where these instructional comics have failed in aesthetic terms, it is likely that they will also have failed in achieving their intention to effectively engage with, and therefore to successfully instruct, their readers.

The necessity for the products of cartooning to exhibit aesthetic qualities thus emerges, perhaps not as a defining characteristic, but certainly as an additional caveat. Unfortunately, as McCloud himself admits in his second book, Reinventing Comics (2000), the aesthetic quality of much of the output of the U.S. comics industry is often doubtful, and this is probably true the world over. In constructing his (1993) definition of comics, McCloud flippantly, although with great consequence, eliminated the word 'art' (derived from Eisner's phrase 'sequential art') from an early draft of his definition. He did so on the grounds that the word 'art' contains a 'value judgement' (McCloud 1993: 8). McCloud's reluctance to include within his definition the notion of aesthetic value was a mistake, since it forced him to include visual narratives with no aesthetic value or intention (such as airline instruction sheets and VCR instruction booklets) within the definition of 'comics'. In this regard, Eisner's use of the term 'sequential art' and Dr Lent's use of the term 'comic art' are both preferable-they are both positive affirmations that the work that cartoonists do is artistic and should be responsive to aesthetic concerns.

Positioned somewhere between art and literature, related directly to film and intimately involved with journalism, cartooning needs to be located, from a critical point of view, within the aesthetic dimension of human productive activity. For Joseph Witek (1999), the reluctance of comics scholars to unreservedly acknowledge the aesthetic dimensions of comics as an art form is one of the reasons why they have remained so marginal in academic discourse.

The propensity of cartooning forms to express themselves in humour, satire and popular storytelling genres may have led to the marginalistion of cartooning from the mainstream of critical discourse in literature and the fine arts. Or, it may simply be that, falling somewhere between art, graphic design, journalism and literature, cartooning has struggled to find a home in the academies where aesthetic theories have traditionally been developed. It may be that the proliferation in recent decades of

\footnotetext{
${ }^{46}$ Rodney Harbour and Rick Andrew, Bonginkosi, 1987.
} 
multidisciplinary cultural and media studies and mass communications courses at tertiary institutions around the world lies behind the recent upsurge in academic conferences, journal articles, textbooks and Internet web sites devoted to the promotion of cartooning and comics scholarship. 


\section{Chapter Three}

\section{Key Trends in $20^{\text {th }}$ Century Cartooning}

\section{Introduction}

As a cluster of signifying practices expressed in a set of popular culture forms integral to the evolving media of mass communication, cartooning presents itself as a quintessentially 20th century phenomenon. However, as Perry (1967), Kunzle (1973) and McCloud (1993) show, cartooning as we know it today has historical antecedents that extend back to the middle ages and beyond. South African cartooning in the $20^{\text {th }}$ century derives from British, European and American traditions, and a clear continuity can be demonstrated between the metropolitan cartooning that originated in London, Paris and New York and the forms of cartooning that later emanated from Johannesburg, Cape Town, and Durban.

This study is, however, about discourses of resistance, and this chapter situates antiapartheid cartooning in a tradition that, while its roots undoubtedly lie much further back in history, developed primarily during the $20^{\text {th }}$ century. Not only have cartoonists around the world presented vivid commentaries on political events, but in many cases, particularly over the last fifty years, have openly sided with oppositional political struggles and anti-hegemonic social movements ${ }^{47}$. As South African comics creator ${ }^{48}$, Anton Kannemeyer, argues in his M.A. dissertation (1997), underground comix have played an important iconoclastic role in western culture by challenging social norms and giving stark visual expression to taboo subjects. This is exemplified in the U.S. underground comix movement of the late 1960s and 1970s, which had a liberating effect on cartooning internationally, and as will be demonstrated, had a particular resonance for South African cartoonists working within the apartheid context.

\footnotetext{
${ }^{47}$ Lent, in a 2000 conference presentation, enumerated instances of "resistance" cartooning in the Soviet bloc, China, Thailand, Cambodia, Indonesia, Malaysia, Syria, Korea, Algeria, Iran, Sri Lanka, and a number of Latin American and African countries. See also Packalen \& Odoi, 1999; Nyamnjoh, IJOCA Vol. 2, No. 2, 2000; Nevins (interview with Joe Sacco) and Kwon (on Korean cartoonists' reactions to Bush), both in IJOCA Vol. 4, No. 2, 2002; and Lanier (interview with Eric Drooker) in The Comics Journal No. 253, 2003.
}

${ }^{48}$ Kannemeyer does not like to be described as a cartoonist (interview, 2000). 
In modern art, and in the field of $20^{\text {th }}$ century visual culture more generally, the codes, conventions and iconography of cartooning were frequently invoked in projects aimed at dismantling the 'ways of seeing' that had dominated Western culture since the Renaissance. Many of the references and strategies used by artists such as Marcel Duchamp, Pablo Picasso, Roy Lichtenstein, Andy Warhol and Saul Steinberg to subvert the time-honoured codes and conventions of officially sanctioned 'high' art ${ }^{49}$, were drawn from cartooning's mode of signification.

\section{The satirical tradition}

Throughout the history of Western art, there is evidently a subterranean lineage of picture making, intimately entwined with the history of cartooning, that has consistently challenged and subverted high art, often targeted towards popular audiences. The way of signifying that characterises cartooning and the kinds of themes that are generally associated with it are already visible in late medieval and renaissance painting ${ }^{50}$, and are especially evident in the satirical prints that were so popular in the early modern period (Klindenger, 1944; Perry and Aldridge, 1967; Kunzle, 1973).

John Berger (1972) has demonstrated, in his analysis of European painting between 1500 and 1900, how specific 'ways of seeing' marked the rise of mercantile capitalism and the ascension of scientific discourses in Renaissance Europe. These ways of seeing achieved formal philosophical expression in Enlightenment philosophy and, bolstered by the grand narratives of logical positivism and notions of supremacy that arose from the conquest of less developed countries, had by the 19th century assumed the grandeur of an imperial vision ${ }^{51}$. The mimetic tradition of the European academies gave visual expression to the instrumentality of Western culture, to the concept of 'genius', to the

\footnotetext{
${ }^{49}$ See Naumann, 1996; Leymarie, 1972; Hughes 1991; Hopkins 2000; Rosenberg, 1978.

${ }^{50}$ For example, the paintings of Hieronymous Bosch introduced elements of the surreal and the forbidden into the precincts of high art, prefiguring the roles that Surrealism and Dada were later to play, while Bosch's contemporary, Peter Breughel the Elder's affectionate portrayal of the lives of medieval villagers and bumpkins anticipated the humorous depictions of ordinary people in early European, British and American cartooning.
}

51 Berger (1972: 83-112) argues that oil painting was an especially suitable medium to convey the impression of solidity required for the realistic portrayal of 'possessions'-material objects, architecture, landscape, and the female body. The inherent properties of the medium, enhanced by a sophistication of 
economics of accumulation, and to the celebration of manners, etiquette and the human form embodied in the revival of the heroic traditions of classical mythology (Berger, 1972: 83-112).

Oil painting, by virtue of the technical possibilities that it embodied as a medium, presented specific opportunities for ideological signification. But while it allowed the apparently accurate representation of reality, oil painting as a medium also imposed a limitation on the distribution of its products, in that, until the 20th century, oil paintings could not be reproduced (except for individually painted copies or the adaptation of paintings into line art for the purposes of engraving and printmaking). The accumulation of easel paintings was restricted to the wealthy classes of society, and it was only through monumental works commissioned by church or state that the paintings and sculptures of the Renaissance could be distributed to a wider public. In this way, the privileged ownership of fine art served to reinforce a class-based distinction between 'high' and 'popular' art, and the public reception of fine art imagery was mediated, as it had been throughout the medieval period, by the context of reception. Exposure to these images occurred within the constrained contexts of public and religious spaces where preferred readings were strongly reinforced.

However, below the level of high art were forms of visual representation expressed at town, village and parish level, in the form of commercially distributed prints, broadsheets and chapbooks containing woodcuts or engravings, representing popular and religious themes ${ }^{52}$. It is common cause that the advancement of printing techniques engineered by Gutenberg in the mid-1400s heralded the birth of the mass media ${ }^{53}$. The combination of printed text and simplified iconic graphics, the use of pictorial shorthand and a choice of subject matter designed to appeal to popular audiences_all

technique that took centuries to perfect, gave rise to what Norman Bryson (1983) has called 'the natural attitude'-the achievement in art of 'the perfect copy' of reality.

52 The technique of making prints from metal plates was first developed in Germany and Italy in the midfifteenth century. It combined two existing techniques-printing from carved woodblocks and engraving silver ornamentally - and was facilitated by improvements in the quality of linen-rag paper. (Honour and Fleming, 1982: 401).

${ }^{53}$ The art of printing from movable type was initially employed to diffuse religious texts and the edition of the Bible printed by Johann Gutenberg at Mainz in 1455 was the first major printed work. Early printers often left spaces at the beginnings of chapters for the insertion of elaborate capital letters printed from woodblocks, mimicking the elaborate illuminations of medieval manuscripts. In addition, some texts combined "typography of unprecedented clarity and illustrations in which forms are fully defined by a few confidently drawn lines" (Honour and Fleming, 1982: 397-8). 


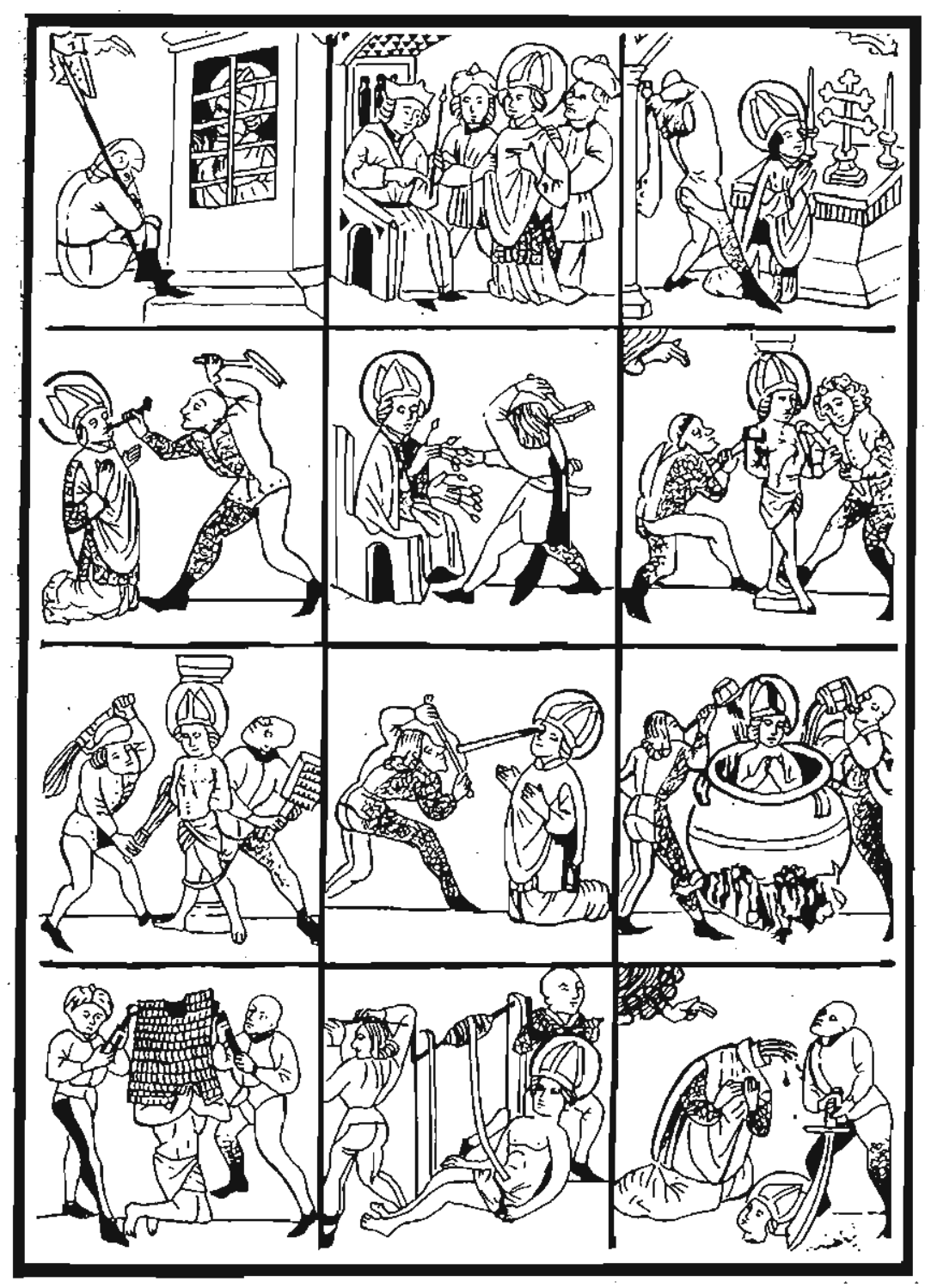

FIGURE 22

"The Tortures of Saint Erasmus" (circa 1460), a pictorial sequence in which the saint is subjected to a variety of heinous tortures, demonstrates two key aspects that link the popular woodblock prints of the late medieval period to modern cartooning-the techniques of pictorial simplification and iconic representation, and an inordinate interest in violent and purient themes.

(McCloud, 1993). 
characteristics of cartooning - were thus integral to the development of the mass media from their earliest beginnings.

The stylistic similarities noticed by Marshall McLuhan (1964:176) between the woodcuts of the 15th century and modern newspaper comics are no coincidence: they relate both to the technical prerequisites of mechanical reproduction and to the enduring themes of the popular tradition ${ }^{54}$. Many early prints display an inordinate interest in violent crimes and even more violent punishments, tortures and martyrdoms (Figure 22)55. But while there is ample evidence of iconic simplification, there appears to be little evidence of facial and anatomical distortion for the purposes of caricature. Kunzle points out that "the humorous style of caricature was simply not available in Northern Europe until the eighteenth century" (1973: 426). While satire and allegory were common features of broadsheet publishing during the $16^{\text {th }}$ and $17^{\text {th }}$ centuries, the subjects of the broadsheets were predominantly documentary or moral tales, realistically drawn within the conventions of the times. From the beginning, broadsheets were produced and distributed for propagandistic reasons, both religious and political, and aimed at all classes of society.

Although European dilettantes had been experimenting with caricature since the late 1500s, when Agostino Carracci of Bologna produced his famous physiognomic sketches entitled "Sheet of caricatures" (1594), the use of caricature did not enter the mainstream of broadsheet publishing for another 150 years, with the arrival of William Hogarth (1697-1764), generally recognised as the founding father of modern satirical caricature (Kunzle, 1973: 356; Sabin 1993: 14). Many of the conventions of modern cartooning were cemented into place over the next 100 years (Figure 23) ${ }^{56}$. The tradition of popular satire

\footnotetext{
${ }^{54} \mathrm{McLuhan}$ would not have seen David Kunzle's authoritative work on this subject, The Early Comic Strip (1973), since he died before it was published, and he might have been startled to see the technical sophistication displayed by the leading broadsheet artists of the $15^{\mathrm{h}}$ and $16^{\mathrm{th}}$ centuries, who worked either in wood or in copper. Nevertheless, there is sufficient evidence in the prints Kunzle has assembled to defend the argument that the technical limitations of woodblock printing would have had the effect of leading to pictorial simplification, and as a corollary to it, iconic representation. However, as Kunzle shows, iconic representation derived primarily from existing religious iconography, adapted for secular usage.

55 This is exemplified in "the Tortures of Saint Erasmus" (circa 1460), a pictorial sequence in which the saint is subjected to a variety of heinous tortures (Kunzle, 1973: 15; McCloud, 1993: 16). An inordinate number of surviving prints from the $15^{\text {th }}$ and $16^{\text {th }}$ centuries depict excruciating punishments and tortures.

56 James Gillray (1757-1815) was undoubtedly one of the most influential innovators. His use of speech balloons was particularly modern, as was his use of facial and anatomical distortion (see Klingender,
$1944)$.
} 


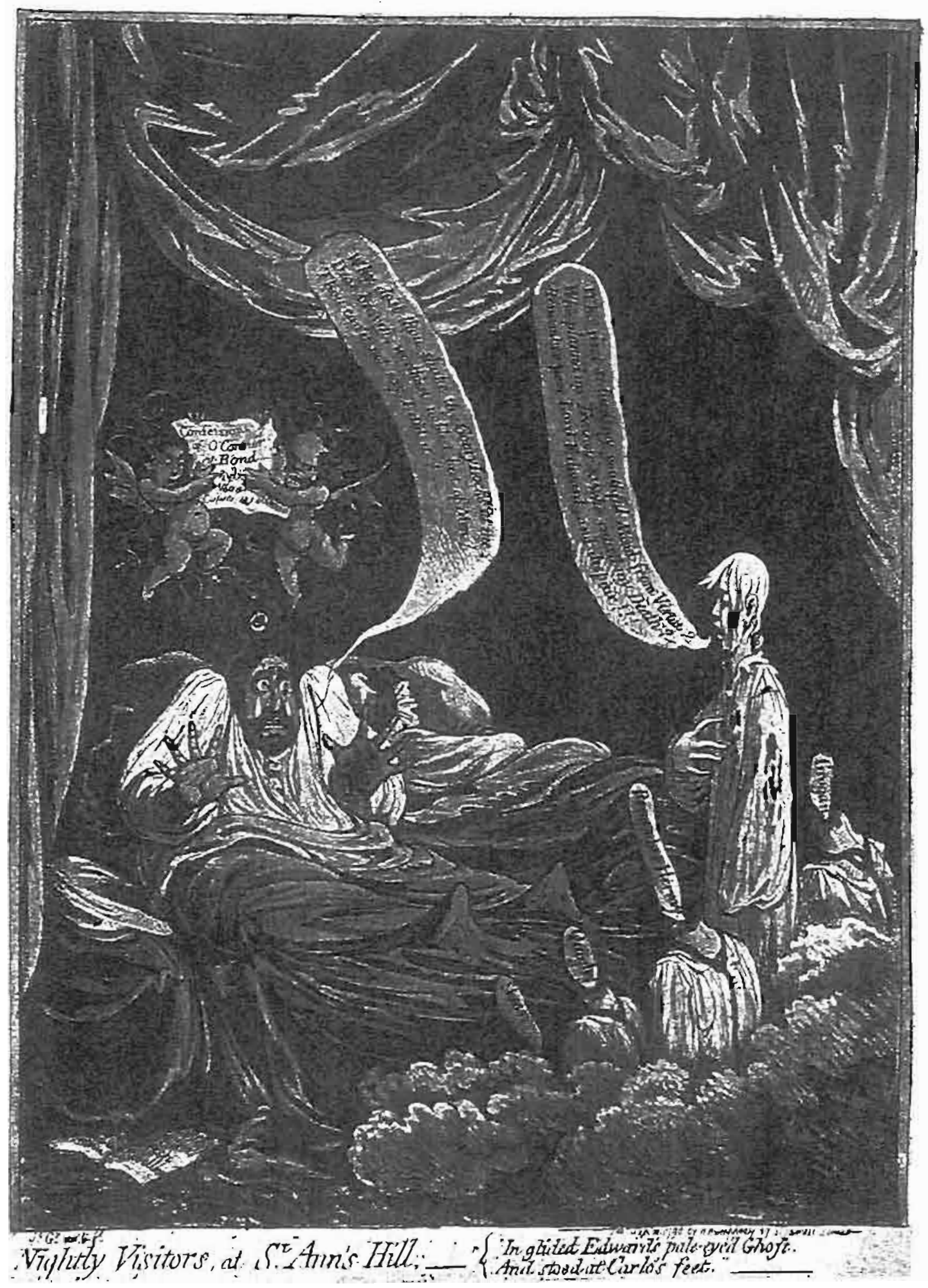

\section{FIGURE 23}

James Gillray (1757-1815) was undoubtedly one of the most influential innovators in the history of English and European cartooning. His use of speech balloons was particularly modern, as was his use of facial and anatomical distortion (Klindinger, 1944). 
pioneered by Hogarth and refined by Rowlandson, Gillray, Cruikshank and others was incorporated into the visual journalism of the illustrated magazines of the 19th century (Klingender, 1944; Kunzle, 1973).

As demonstrated in William Schroder's weekly satirical paper The Knobkerrie (Figure $24)^{57}$, this tradition is evident in the periodicals published in the early years of the Cape Colony, and thus the origins of South African cartooning are demonstrably part of a continuum that extends back, via the $18^{\text {th }}$ and $19^{\text {th }}$ century British tradition of satirical caricature, to the origins of European and British cartooning ${ }^{58}$.

\section{The evolution of contemporary cartooning}

The evolution of contemporary cartooning is a fascinating story: from satirical caricature to modern editorial cartooning; from the incorporation of comic strips into the popular press to the 'golden age' of newspaper comics and the rise of the comic book industry; from the mid-century moral panic about comics and attempts to hobble the industry's independence to the emergence of the underground comix movement; and from the origins of animated cartooning in cinema to the evolution of new forms of HTML-based animation for the Internet. However, only those aspects that can be shown to have impacted directly upon South African cartooning will be mentioned here.

It should have been convincingly demonstrated by now that the evolution of the various forms, styles and genres of cartooning is directly related to the technical possibilities and requirements of the media in which cartoons, caricatures and comics have historically appeared. The development of cartooning as a cluster of mass media forms in the 20th century was contemporaneous with the sophistication of the mass media themselves during the course of the century, and each new direction that the mass media took resulted in the emergence of appropriate forms of cartooning. Thus, the emergence of daily newspapers led to the daily editorial cartoon and the daily cartoon strip; the introduction of colour newspaper pages established the conditions for the celebrated Sunday comics of the so-called 'golden age' of newspaper comics; and the spread of

\footnotetext{
57 Bound copies of The Knobkerrie, self-published by Schroder between 1884 and 1886, are archived in the University of Stellenbosch library.

58 In an important South African link, George Cruikshsank (1792-1878), who followed Gillray, penned at least two important cartoons about the 1820 settlers. See Figure 32, Chapter Four).
} 


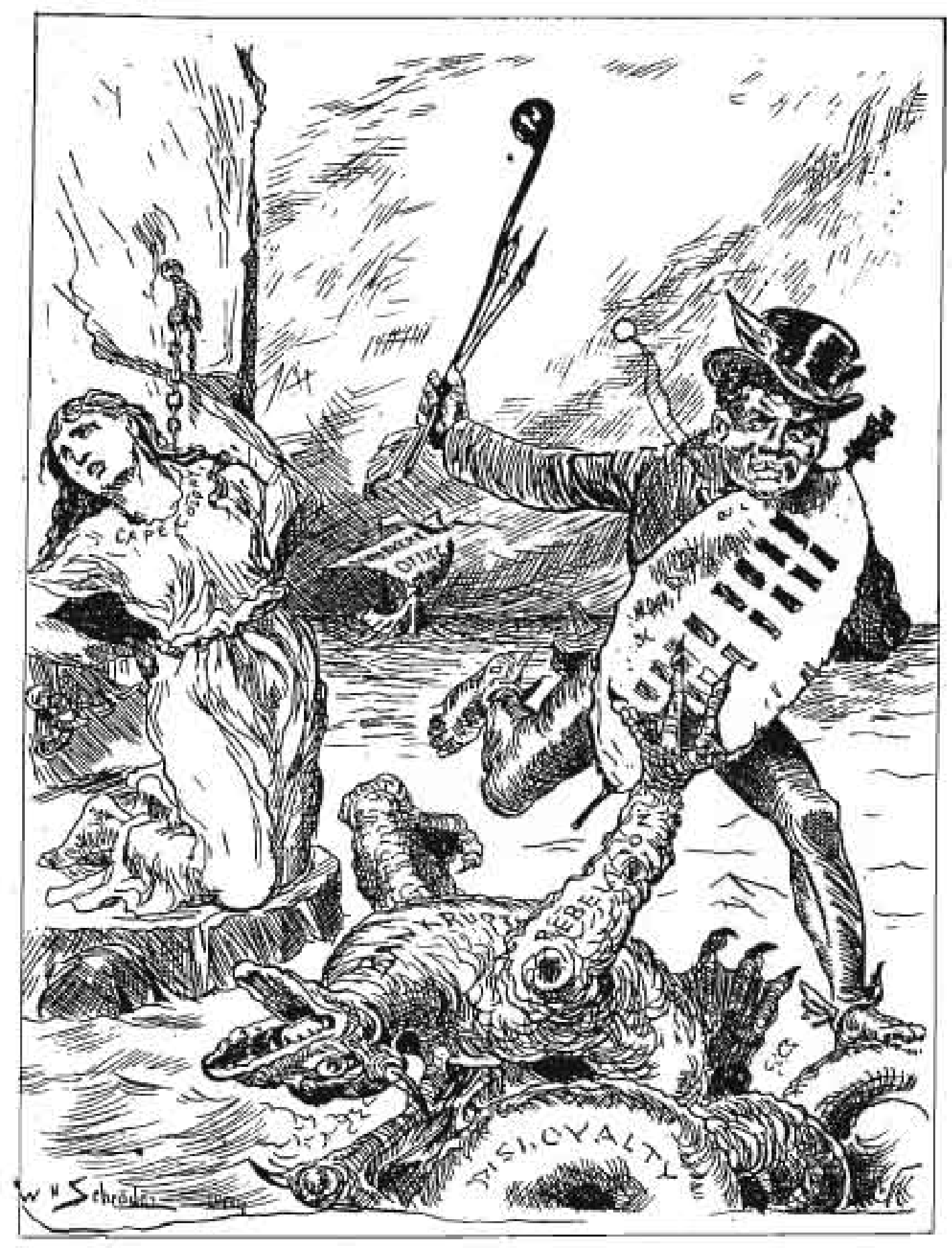

\section{FIGURE 24}

The style of the illustrations in William Schroder's self-published weekly satirical paper The Knobkeme, published in the Cape between 1884 and 1886, demonstrates that the origins of South African cartooning are part of a continuum that extends back, via the $18^{\text {th }}$ and $19^{\text {th }}$ century British tradition of satirical caricature, to the origins of European and British cartooning. (Schroder, The Knobkemie, 1884) 
literacy and growth of popular consumer magazine publishing provided the social and economic context for newspaper comics to evolve into the comic book industry.

Simultaneously, cartooning was present at the birth of motion pictures ${ }^{59}$. The invention of sound in cinema in the late 1920's was heralded by Walt Disney's early animated cartoon movies, in which a musical score provided an aural framework for the movement of animated cartoon characters across a screen, and these films are regarded as amongst the first successful combinations of sound and image in cinema (Maltin, 1980: 34). The rise of television after the Second World War began to erode the marketplace for printed comics while exponentially extending the demand for animated cartoon films. This, in turn, necessitated the invention of new forms of animation capable of being produced at the rate that television demanded, moving the centre of animated cartooning activity away from cinema to the television screen.

During the last quarter of the 20th century, in the vertically and horizontally integrated postmodern media environment in which media products are simultaneously distributed across a range of media platforms and linked into product merchandising, cartooning continued to play an important role, with the emergence of multi-mediated properties like The Simpsons and Pokemon and the translation of numerous comic book subjects into live action cinema. Simultaneously, the World Wide Web has generated completely new forms of animated cartooning by making it possible for individual artists working with HTML-based animation programmes to distribute their products globally. As Scott McCloud argues in his second book, Reinventing Comics (2000), the Web will contine to evolve as an accessible and appropriate medium for the proliferation of new types of comics making that do not depend on print for distribution, but are located in cyberspace ${ }^{60}$.

\footnotetext{
59 The first animated film, J. Stuart Blackton's 'Humorous phases of Funny Faces', was shown in 1906 (Maltin, 1980: 2).

${ }^{60}$ Rico Schacherl, the artist behind the popular South African strip Madam $\mathcal{E}$ Eve, is an inveterate Web cartoonist, interacting on an ongoing basis with like-minded artists around the world. One of the most fascinating features of this new kind of interaction amongst the 'global virtual community' of Web cartoonists are the "comics jams' where a number of artists collaborate on a single story, each doing a page at a time. Most of these ongoing strips will probably never be distributed in printed form.
} 
In the postmodern context ${ }^{61}$, where critical distinctions between high and popular art are virtually impossible to maintain, cartooning is entirely interwoven into the iconography of commodified global culture. At the same time, desktop publishing technology allows amateur and marginal publishers to produce sophisticated products, while Internet-based distribution channels allow individual creators to reach international audiences. In the context of postmodernism and what Spiegelman (1998) has called "post-literacy", the field of cartooning has fragmented into a dazzling array of genres, styles and niche market sectors. 'Post-Fordism' 62 - the idea that manufacturers are technologically enabled to target their products to smaller and more specific marketplaces - is highly applicable to cartooning at the end of the $20^{\text {th }}$ century.

\section{The Anglophone and Francophone traditions}

The origins of distinct national traditions of cartooning were already evident in the 19th century ${ }^{63}$. The bande dessinee tradition is one of high artistry in the telling of popular stories, but its exposure to English readers has generally been limited to translations of Herge's Tintin and Goscigny and Uderzo's Asterix ${ }^{64}$. In Britain, on the other hand, the tradition of political and social satire pioneered by Hogarth and Gillray has enjoyed a vigorous and unabated popularity throughout the 20 th century.

An interesting insight into the origins of the American tradition and its bifurcation from the British tradition is provided by Thomas Craven, in his 1943 celebration of American cartooning, Cartoon Cavalcade.:

\footnotetext{
61 In the postmodern context, we no longer expect art to be mimetic. We are beyond the notion that pictures should aspire to being 'copies' of reality. On the contrary, we are frequently disappointed when reality fails live up to the pictures we have seen of it. We recognise that most of the images we see in shop windows, in glossy magazine advertisements or on billboards are illusions constructed by art directors, photographers and graphic designers - constituting a constructed hyper-reality to which we are encouraged to aspire, knowing all along that it is ultimately unattainable. This knowing, cynical attitude is an essential aspect of the postmodern condition, and has often been remarked upon (for instance, see Hebdige, 1999).
}

62 The notion of 'Fordism' evidently derives from Gramsci (1971).

63 The importance ascribed by French-speaking Europeans to la bande dessinee is signified in the statue of Rudolphe Topffer (1799-1846), acknowledged as the father of the Belgian/French/Swiss tradition, that stands today in a prominent position in Geneva.

64 These products are so well known that it has not been deemed necessary to provide references for them in the Bibliography. A Google search for 'Tintin' yielded over 80000 references. 
The old-fashioned Englishman who still esteems the senescent Punch as the crowning attainment of Anglo-Saxon culture cannot be expected to appreciate the deep ventral humour of our graphic jokesters. It is too rowdy and unrefined for him, and as for our comic strips, criticism is superfluous! He thinks they are too revolting for words; and some of them are, I regret to say, and incredibly stupid into the bargain. (Craven, 1943: 5)

For Craven, it is "the indigenous quality of irreverence" that sets American cartooning apart, which in its rowdiness and lack of refinement celebrates the robust, pioneering character of the American immigrant, "born and bred with no cultural memories in his heart" (Craven, 1943: 5). The often-heard claim that Richard Fenton Outcault's The Yellow Kid, published in Joseph Pulitzer's New York World in 1896, was "the originating instance of the comics form" (Witek 1999: 9), is a primary example of the tendency amongst U.S. critics to lay claim to comics as an American invention65. But, as Roger Sabin (1993) demonstrates, this claim is spurious ${ }^{66}$. Joseph Witek theorises that the American claim of 'ownership' over the comics form is founded in an ideological imperative that required U.S. critics to valorise American popular culture as "the articulation of the hopes, dreams and opinions of the American people" and as evidence that "not all U.S. culture derives from pre-existing European models" (Witek, 1999: 10)67.

The celebration of newspaper comics as a uniquely American popular culture form by media studies theorists of the liberal-pluralist school is in sharp contrast to the denigration poured upon comic books some 20 years later in the context of McCarthyism. A hint of this is already found in Craven's comment that some comics "are too revolting for words [...] and incredibly stupid into the bargain". The way in which bande dessinee is celebrated in French-speaking Europe has no equivalent in the

\footnotetext{
${ }^{65}$ See www.reponset.com/yellowkid.
}

66 Numerous European and British antecedants have been identified. Both Hogarth (1697-1764) and Gillray (1757-1815) used sequential panels and speech balloons and Rudolph Topffer (1799-1846) used panel sequences that linked pictures and text in the 1840s. Sabin argues that Ally Sloper's Half Holiday, published in England in 1884 by Charles Dalziel, was "the first modern comic" (Sabin, 1993: 17).

67 Tony Bennett's (1982) categorisation of the "Four Traditions" of media theory corroborates this view (see Chapter One), noting that the American liberal-pluralist approach to media studies in the 1930s sought to counter the negativism of the Marxist thinkers associated with the Frankfurt School, who were extremely pessimistic about mass society, and who advanced scathing critiques of its cultural products. The U.S. tradition of media research was more optimistic, striving "to transform the phrase 'mass society' from a pejorative into a positive term" and to appropriate it in support of a liberal-pluralist position (Bennett, 1982(1): 39). 
English-speaking world, where comics have historically been denigrated by the literary and educational establishment (Spiegelman, 1998)68.

In the early years of U.S. comics scholarship, according to Witek, there was a trend to marginalise comic books from serious critical discourse, relegating them to the category of 'pulp', while famous newspaper comic strips such as Walt Kelly's Pogo were celebrated as a form of contemporary American 'folk art'. As a result of this marginalisation, a theoretical vocabulary for the formal analysis of comic books as a category of cultural production was lacking. Consequently, comic book publishers, practitioners and their supporters were unable to marshall an adequate defence when, as a result of the moral panic occasioned by the publication of Frederick Wetham's damning book Seduction of the Innocent in 1954, comic books were attacked as "a threat to the moral fibre of America's youth" (Witek 1999: 5).

\section{Mid-century anti-comics campaigns}

The reluctance to ascribe aesthetic legitimacy to comics in the English-speaking world may have been representative of prevailing critical attitudes at a time when the guardians of highbrow culture were hard-pressed to maintain critical distinctions between the traditional canons of fine art and literature and the invasive cultural forms that owed their emergence to rapid developments in mediated mass culture ${ }^{69}$. Witek (1999) notes that, historically, a sense of low self-esteem and a typically apologetic tone is discernable in the writings of many U.S. comics practitioners and commentators, who have tended either to submit to the perception that comics are a childish, lowbrow or non-serious form of cultural production, or have stridently sought to champion comics in the face of such attitudes. This sense of low esteem was exacerbated during the punitive anti-comics campaign of the 1950s that followed the publication of Dr Wertham's Seduction of the Innocent. Through a series of court actions and other measures, the reading of comic books was linked to juvenile delinquency, effectively

\footnotetext{
${ }^{68}$ Spiegelman, the Pulitzer Prize-winning creator of Maus, has this to say: "While in the U.S. being a cartoonist still carries about the same prestige as being a sign painter, European cartoonists can be as highly regarded as, say, film directors" (Spiegelman 1998: 81).

69 This view is supported by Bart Beaty's (1999) demonstration of opposition by the American educational establishment to the adaptation of 'Great 'Tradition' literature into the Classics Illustrated comics series.
} 
tarnishing the idea they might effectively be used in education, or that they might be seen to have literary or artistic merit ${ }^{70}$.

The anti-comics lobby spread to Great Britain, Australia and Canada during the $1950 \mathrm{~s}$ and there were also anti-comics campaigns in Germany, the Phillipines, Taiwan, South Korea and Japan-all countries where the American presence was very strong in the postwar period. It is evident that the kind of offensive mounted against comics in the English-speaking world, Germany and U.S.-dominated Asian countries after the Second World War, did not ocur in French-speaking countries ${ }^{71}$, confirming that completely different attitudes towards comics have traditionally existed in the Anglophone and Francophone worlds ${ }^{72}$. The stereotypical views of comics as the products of an undesirable subculture inimical to the advancement of literary appreciation, a 'secondclass' or 'Cinderella' discipline, a juvenile pursuit unfit for adult participation, or the cause of juvenile delinquency, do not appear to have the same kind of currency in nonEnglish-speaking countries.

This issue is of importance to the study of South African cartooning, because it offers a clue as to the evident differences between the Afrikaans and English cartooning traditions in South Africa. Afrikaans comics are arguably more closely linked to the European bande dessinee tradition than to the English-language traditions. Indeed, during the mid-century period, Afrikaans comics enjoyed a legitimacy that was never accorded to English-language comics. While there have been several notable Afrikaans comic strips, of which T.O. Honiball's Adoons-hulle is probably the most highly regarded, there have been few if any locally-produced English-language comics of similar stature. 73 The antipathy to comics that appears to have existed, and may still exist, amongst the

\footnotetext{
70 Pulp Demons: International Dimensions of the Postwar Anti-Comics Campaign (1999), a collection of essays edited by John Lent, details anti-comics campaigns in the U.S., Great Britair, Australia and Canada during the 1940s and 1950s.
}

71 The absence of examples of anti-comics crusades in the Francophone world is not specifically commented upon by Dr Lent (1999) or his co-authors.

72 The French BD writer-artist Pascale Rabate, a prize-winner at the Angouleme Festival in 2000, claims that graphic novels are eclipsing written novels in France today, and in France alone, 23000 copies of Volume 1 and 18000 copies of Volume 2 of his graphic novel bicus had already been sold (interview, 2003). In other non-French speaking European countries such as Italy, Spain and the Netherlands, adult comics also enjoy a positive profile (Sabin, 1993).

73 Adoons-hulle appeared in in Die Huisgenoot from 1948 until 1971 and, according to Schoonraad (1983: 100), Honiball's characters "het deel geword van die Afrikaanse volkskultuur" (became part of Afrikaner folk culture). 
black educational establishment in South Africa and southern Africa generally, may have originated in the missionary-based English-language educational system through which many African intellectuals in South Africa and other southern African countries have passed ${ }^{74}$.

The predicament in which cartooning in general (and comics in particular) finds itself in the English-speaking world has often been remarked upon, particularly by practitioners themselves, who have often complained about the poor light in which their work is regarded by the academic and cultural establishment. Spiegelman has this to say on the subject:

Throughout its more than 150-year-old history, the comic strip has been the hunchback half-witted bastard dwarf step-child of the graphic arts. It's only very recently that the medium has begun to find some legitimacy in bookstores under its new alias as the 'graphic novel'. (Spiegelman 1998: 74)

Spiegelman's cynicism is borne out by the celebrated British caricaturist, Ralph Steadman:

Illustration and cartooning remain a lowly profession, albeit an attractive one, considered by newspaper and magazine editors as a device to help sell newspapers and magazines, because, like newspapers and magazines, they are relatively cheap. Pictures attract those who do not wish to read, cannot read or just don't understand. Cartoons play a supportive role that fills an awkward space left by someone who failed to fill enough copy - the last minute scribble that captures something that maybe cannot be said in words. In its elevated position, next to the leader column, it serves as an easily digested pictorial version of the writer's elegiac wisdom, but always playing second fiddle to the writer. (Ralph Steadman, IJOCA Vol. 4, No. 2, 2002: 100)

The words of these two very famous English-language cartoonists, one talking about comics, the other about editorial cartooning, leave no doubt that, in their minds at least, cartooning has historically been regarded as "a lowly profession". But while editorial cartooning, as Steadman suggests, is "elevated" by its association with the press and the

\footnotetext{
${ }^{74}$ This idea is derived from personal observation and is cautiously presented. Research would have to be undertaken to verify it, but it is interesting to note that African cartooning traditions are strongest in the Francophone countries and weakest in the Anglophone coutrtries. The exception is Tanzania where most cartooning is in Swahili.
} 


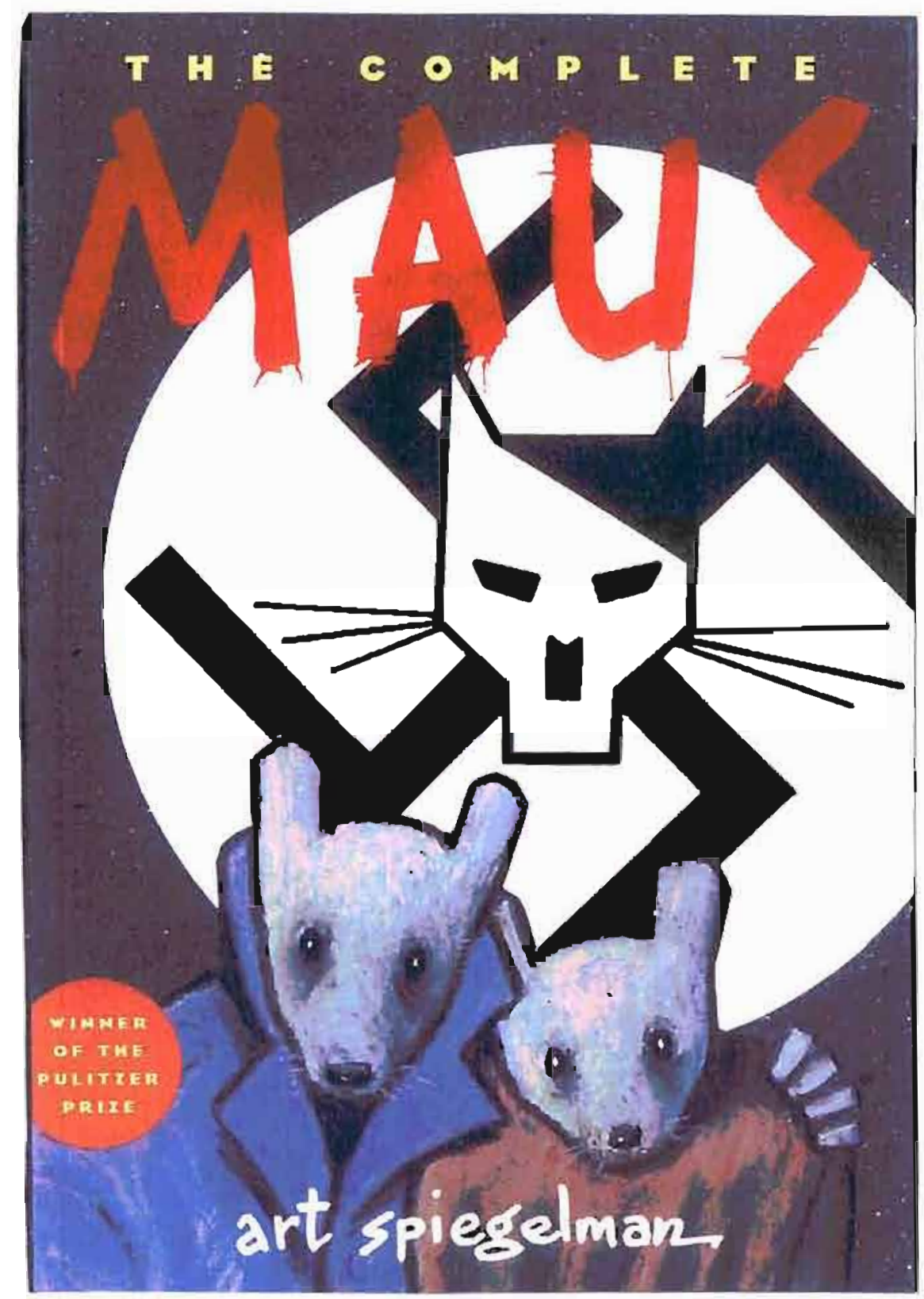

FIGURE 25

The first volume of An Spiegleman's comic strip, Maus, about his parents' experience of the holocaust, was published in 1986. Volume 2 was published in 1992, and The Complete Maus in 1996. Maus received the Pulitzer Prize for literature in 1992, in the fiction category, but following the author's intervention, the award was later reissued in the non-fiction category. 
"elegiac wisdom" of newspaper editors, comic books have historically been denigrated in the English-speaking world ${ }^{75}$.

Over the last decade and a half, spurred on by the success of Spiegelman's Pulitzer Prize-winning Maus (Figure 25), new genres of expressionist, autobiographical and socio-political graphic fiction have emerged, aimed at a mature, educated segment of the comics-buying public, from politically engaged black and white 'graphic journalism', such as Joe Sacco's Palestine, to sophisticated full-colour adult fantasy, such as Neil Gaiman's The Sandman ${ }^{76}$. The field of English-language comic book publishing at the beginning of the $21^{\text {st }}$ century is international, impressively variegated and intellectually stimulating. While it is beyond the scope of this study to deal in any kind of detail with the evolution of comics over the last 50 years, it is necessary to make some general observations that are relevant to the discussion of South African comics in the 1980s and 1990s.

\section{Some significant trends in contemporary cartooning}

During the postwar period (1945-1965), comic books in the USA and Britain celebrated the defeat of Nazism and Fascism and the rise of what Daniel Lerner (1958) called "the Great Society". To the offspring of the Allied soldies, born during the 'baby boom' (19451955), comic books were an integral part of popular culture. The comic strip heroes of this period-Superman, Batman and their imitators-remain icons of Western dominance ${ }^{77}$.

\footnotetext{
75 Will Eisner lays some of the blame for this on comics practitioners themselves: "...Sequential art has been generally ignored as a form worthy of scholarly discussion [...] I believe that the reason for this sits as much on the shoulders of the practitioner as the critic [...] unless comics address subjects of greater moment, how can they hope for serious intellectual review?" (1985:5).

${ }^{76}$ Sacco's Palestine, initially published as a series of eight comic books (1992-3) and later collected into a trade paperback with an introduction by Edward Said, "gained widespread praise for the depth of his research, the sensitivity of his handling of a volatile subject, and his virtuoso skills as a cartoonist" (Mark Nevins, ПJOCA, Vol. 4, No. 2, 2002). Gaiman's The Sandman, initially published as an ongoing comic book series by DC Comics' Vertigo imprint between 1989 and 1997, has been collected into a set of 10 graphic novels. The most recent Sandman volume, Endless Nights, was published directly to luxurious hard cover in 2003 at a cover price of $\$ 25$, without preceding comic book publication, indicating the extent to which the marketplace for sophisticated graphic literature products has matured in the U.S. over the last decade.

77 See Michael Chabor's The Amazing Adventures of Cavalier and Clory (2000) for a fictionalized evocation of the ideological role of superhero comics in the USA during World War II. Similarly, as Dorfman and Matelart (1976) have argued, Disney comics propagated U.S. imperialist ideology.
} 


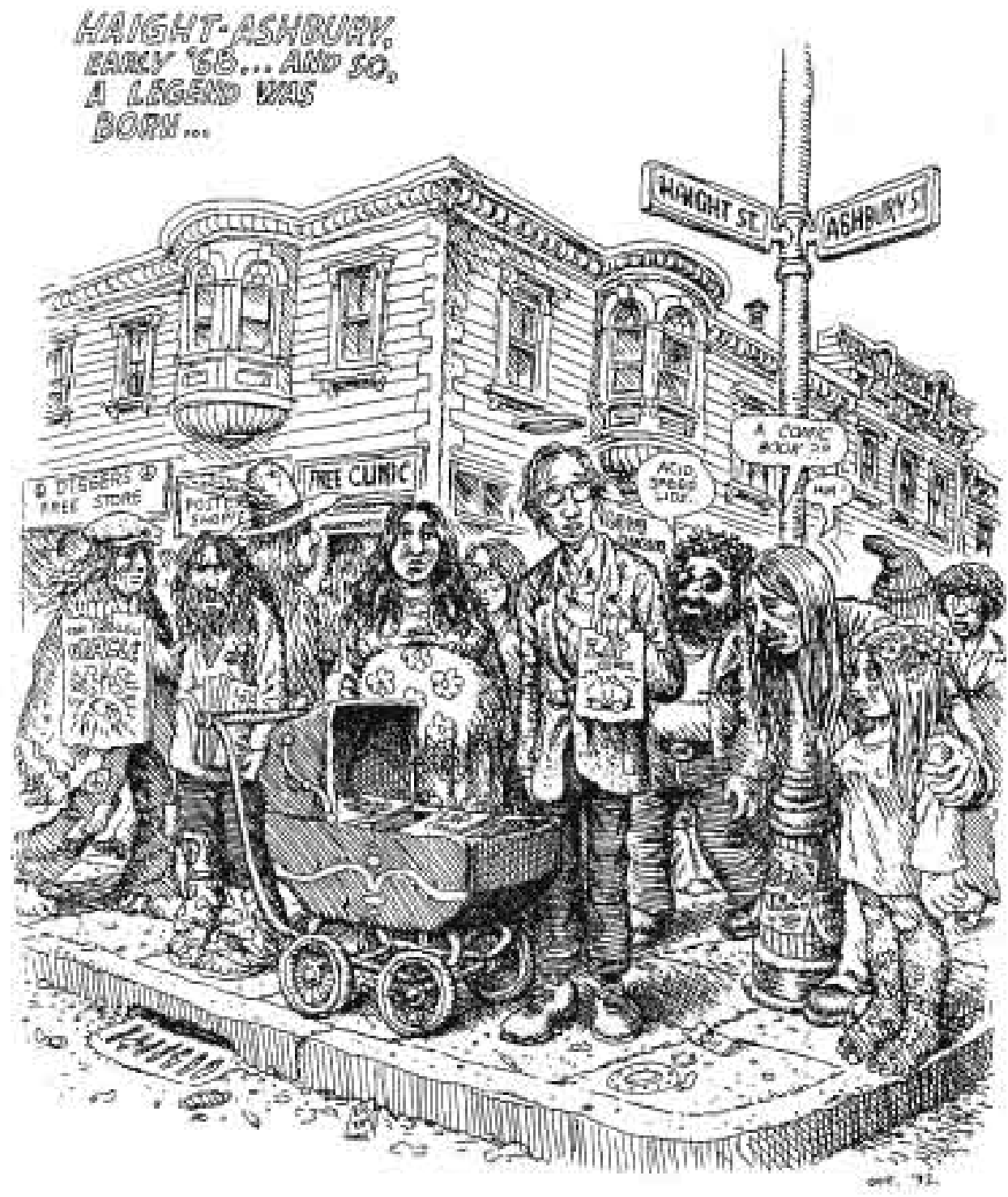

FIGURE 26

The work of artist-writers such as Robert Crumb arose in the late 1960 s and early $1970 \mathrm{~s}$ in the context of the anti-establishment counter-culture, achieving global recognition and spawning a generation of imitators. Of central importance to the underground comix movement was the relative ease with which comics could be produced outside the mainstream of capitalist-owned publishing enterprises.

(The R. Crumb Coffee Table Art Book, 1997). 
As the 'baby boomers' achieved adulthood, their coming of age was accompanied by a profound rejection of the values of their parents. The escalation of the war in Vietnam was accompanied by the rise of what came to be known as the 'counter-culture', a shortlived but intense and colourful period characterised by political dissent and the rejection of prevailing moral, cultural and aesthetic values. The 'counter-culture decade' of the mid-1960s to the mid-1970s is best remembered for the popular music it produced, but it was also a period of enormous significance for comic book publishing in the Western world 78 .

The underground comix movement was, to a significant extent, a reaction to the suffocating influence of the Comics Code Authority, established in the late 1950s to police the publishing of comic books in the USA after the publication of Frederick Wertham's Seduction of the Innocent. The establishment of the Comics Code Authority and the manipulation of distribution channels thereafter succeeded in securing the terrain for mainstream comic book publishers for at least a decade ${ }^{79}$. Against this backdrop, alternative and independent comic book publishing arose in the late 1960s and early 1970s in the context of the anti-establishment counter-culture. The work of artist-writers such as Robert Crumb achieved global recognition and spawned a generation of imitators (Figure 26). Of central importance to this renaissance was the relative ease with which comics could be produced outside the mainstream of capitalistowned publishing enterprises. Comic strips remain an area of popular media where cultural products can be produced virtually single-handed with a minimal reliance on technology. While the U.S. comics mainstream had developed a production-line approach which separated the roles of writer, editor, penciller, inker, colourist and letterer, thus ensuring a high level of editorial control over the finished product, the independent writer-artist-publisher could undertake all of these tasks single-handedly ${ }^{80}$.

\footnotetext{
78 Jay Kennedy's The Official Underground and New Wave Comix Price Guide (1982) lists thousands of comic books published by individuals and 'home grown' publishers in the USA and Canada between 1962 and 1982.

${ }^{79}$ It was argued at the time by William Gaines's EC Comics that the Comics Code Authority was a "monopolistic instrument" and, according to Nyberg, "the fact that the code left a relative handful of companies in control of the industry was no coincidence" (Nyberg, in Lent (ed) 1999: 56).

${ }^{80}$ In 1967, Robert Crumb produced a comic book entiled ZAP!, printed on a small offset printing press belonging to a "hipster poet" (Crumb 1997: 85). Five thousand copies of the comic were distributed informally on the streets of the Haight-Ashbury district of San Fransisco, at that time the epicentre of the hippie movement. This event is generally regarded as heralding the birth of the underground comix movement (The R. Crumb Coffee Table Art Book, 1997: 84).
} 
These underground comix dealt with taboo themes, largely within the 'sex, drugs and rock'n'roll' idiom, but there were examples that tackled political and socio-economic issues and others which used the medium effectively to explore deeply personal and autobiographical themes. By 1974, the 'underground' period had exhausted itself and revised obscenity laws in the USA further limited the distribution of underground comix (Kennedy 1982: 14). However, the idea that comic books could find a nonmainstream market was secure and the subsequent development of an international 'alternative' or 'independent' comics marketplace has ensured the survival to this day of socially engaged and marginal comic book publishing.

In French-speaking Europe, Spain, Italy, Germany and the Netherlands, as well as elsewhere in Europe, cartooning and graphic narrative traditions have developed along their own trajectories, which are beyond the scope of this study to examine. Likewise, in Asia, particularly in Japan and Hong Kong, comics have achieved levels of production and distribution unheard of in the English-speaking world ${ }^{81}$. The influence of the Japanese style is pervasive throughout animated cartooning on global television and there is a very high degree of convergence, intertextuality and horizontal integration between comics in print, animated films, interactive computer games and other related products.

By the 1980s, the various graphic narrative traditions had begun to converge to service a growing international adult comics marketplace ${ }^{82}$. In 1980, Art Spiegelman ${ }^{83}$ and Francois Mouly launched $R A W$, a post-modern 'graphix' magazine, in New York. $R A W$ featured work by European and Japanese writer-artists artists alongside refugees from the US mainstream and claimed to have "an $80.7 \%$ share of the Alienated and Anxious market" (Spiegelman \& Mouly, 1987) ${ }^{84 .}$

\footnotetext{
${ }^{81}$ Lent, 1992, 1993, 2000, 2003; Wai-ming Ng, 2000, 2003; Mahamood, 2003; Onada, 2003; Ogi (interview
with Shimizu Isao), 2003.

82 This marketplace has continued to grow, with 'independent publishers, such as the US Fantagraphics Books and the Canadian Drawn \& Quarterly, producing comics publications in English that rival their French counterparts in quality and sophistication. Meanwhile, this marketplace is also strongly traded by the 'adult' imprints of major publishers, such as DC's Vertigo.
}

${ }^{83}$ Spiegelman had previously edited Arcade: The Comics Revue (1974/5) and his work had appeared in a range of alternative and underground comics and magazines.

${ }^{84}$ RAW was later bought by Penguin Books and the format dramatically reduced, but did not survive beyond the early 1990s. 
In Britain, the postwar genre of weekly black \& white comics such as Beano, Lion, Tiger and Hurricane and Hotspur held sway until the late 1970s when a new sci-fi weekly, $2000 \mathrm{AD}$, began to attract a huge audience of disaffected punk-generation teenagers. From the 2000AD stable emerged Crisis (1987-1991), a short-lived but immensely influential weekly concerned itself almost exclusively with political and gender-related themes. Many of these strips were designed for republication as graphic novels (Barker, 1989; Sabin, 1993). The highly literate, irreverent and inventive strips in these publications contributed to a major re-evaluation of their own product by U.S. superhero comic publishers, Marvel and DC. By the mid-1990's, these mainstream U.S. publishers had significantly reinvented the style, content and production values of their publications. DC initiated Vertigo, an overtly adult, literary imprint and both Superman and Batman were "born again" in a new, post-modern guise ${ }^{85}$.

Like the underground comix of the ' 60 s and '70s, these more recent developments have had their impact in South Africa. Anton Kannemeyer and Conrad Botes, the creators of Bitterkomix, have been strongly influenced by international developments in alternative comics and bande dessinee, and the significance of these influences will be examined in more detail in the next two chapters. It is against this international backdrop, and in the context of the theoretical ideas advanced in the first two chapters, that key developments in South African cartooning during the $20^{\text {th }}$ century will be discussed, with a particular emphasis on the 1985-1994 period.

${ }^{85}$ A number of the most influential British writers and illustrators crossed the Atlantic to work for Marvel and DC. Of the major new names in comics authorship who emerged during this period, several of the most influential, such as Alan Moore and Neil Gaiman, were British. 


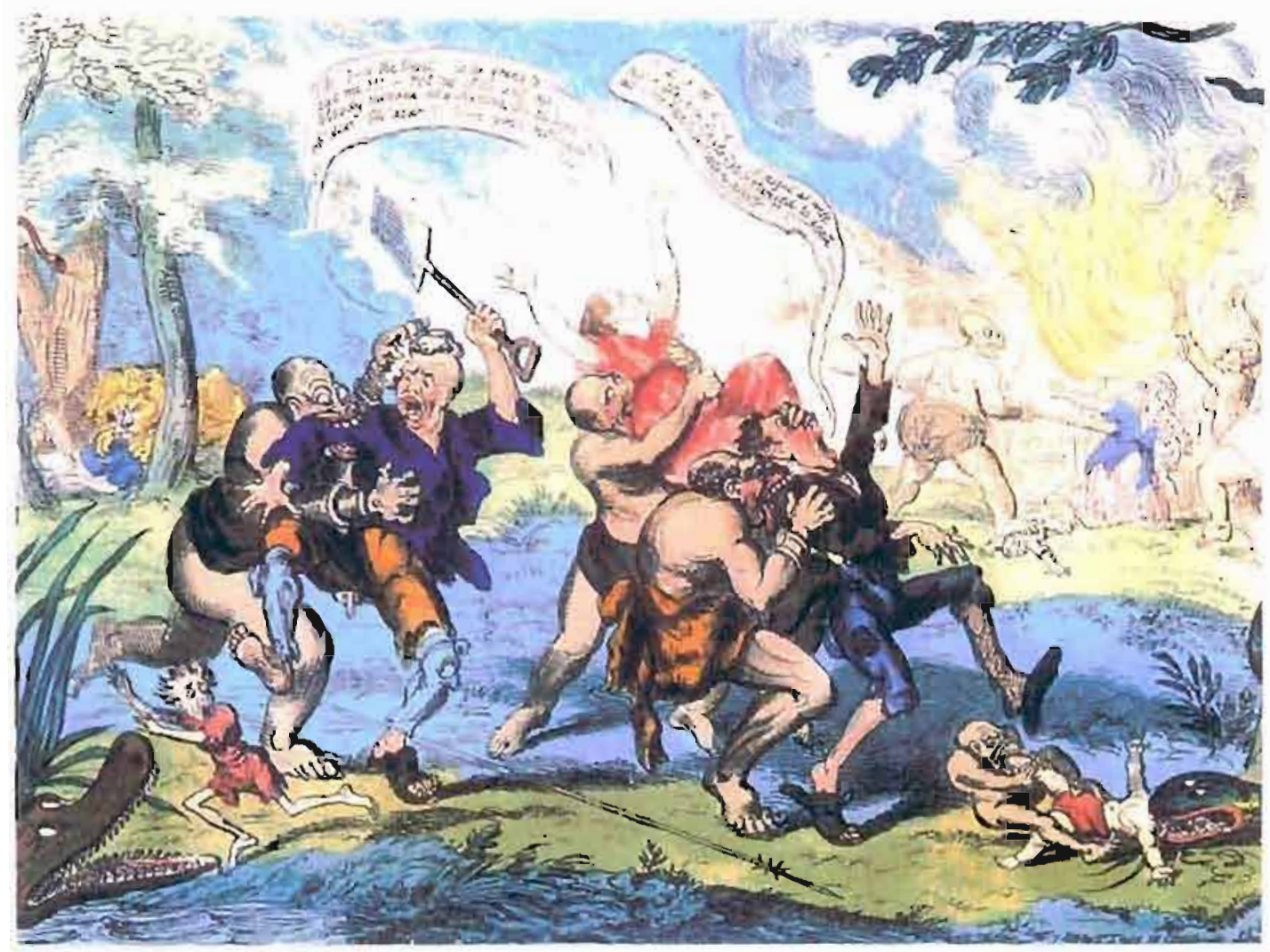

FIGURE 27

This cartoon by the celebrated British political caricaturist George Cruikshank (1792-1878), published in London in September 1819 and entitled "Blessings of Emigration to the Cape". graphically demonstrates the scepticism with which British public viewed the prospect of settlement in South Africa. (Bryer, L. \& Hunt, K.S. The 1820 Seltiers, 1984) 


\section{Chapter Four}

\section{South African Cartooning before 1984: Themes and Directions}

\section{Introduction}

A cartoon by the celebrated British political caricaturist George Cruikshank (1792-1878), published in London in September 1819 and entitled "Blessings of Emigration to the Cape", graphically demonstrates the scepticism with which the British public viewed the prospect of settlement in South Africa. The cartoon depicts a group of settlers being devoured by a crocodile, a python, a lion and a mob of cannibals (Figure 27) ${ }^{86}$. One of the settlers cries out: "Oh L-d. Oh L-d!!! I might as well have staid in England to be starved to death as come here to be eaten alive!" The humour softens the horror of a truly barbaric scene, but does not obscure the fearful and contemptuous attitude with which the British public viewed South Africa's indigenous population.

A few years later, between 1836 and 1845, an anonymous San artist or artists produced a painting on the wall of a small rock shelter below the Drakensberg, depicting a violent clash between San hunters, on foot with their bows and arrows and surrounded by cattle, and a group of mounted men (Figure 28) ${ }^{87}$. David Lewis-Williams (2003) identifies the mounted riders with their wide brimmed hats, flintlock muskets and powder horns as Boers, accompanied by other riders with feathered hats and assegais who are probably Khoekhoe or Bantu-speaking servants or slaves. In the highly detailed painting, several of the riders have dismounted and white flashes from the barrels of their muskets and flashes from the pans of their flintlocks can be made out. In the foreground, a San man lies dead.

To the right of the painting, an obscure figure, easily overlooked, denotes the presence of a San shaman in the throes of a trance dance. According to Lewis-Williams, this figure may represent an appeal to the San deity, / Kaggen, for assistance in the struggle

\footnotetext{
${ }^{86}$ George Cruikshank, 1819, reproduced in Bryer, L. \& Hunt, K.S. The 1820 Settlers, 1984: 19. According to Bryer, Cruikshank was a fierce opponent of the emigration scheme. Some of his cartoons on the subject are held in the William Fehr Collection, Cape Town.

${ }^{87}$ Reproduced and described by David Lewis-Williams in Images of Mystery: Rock Art of the Drakensberg, 2003: 119.
} 


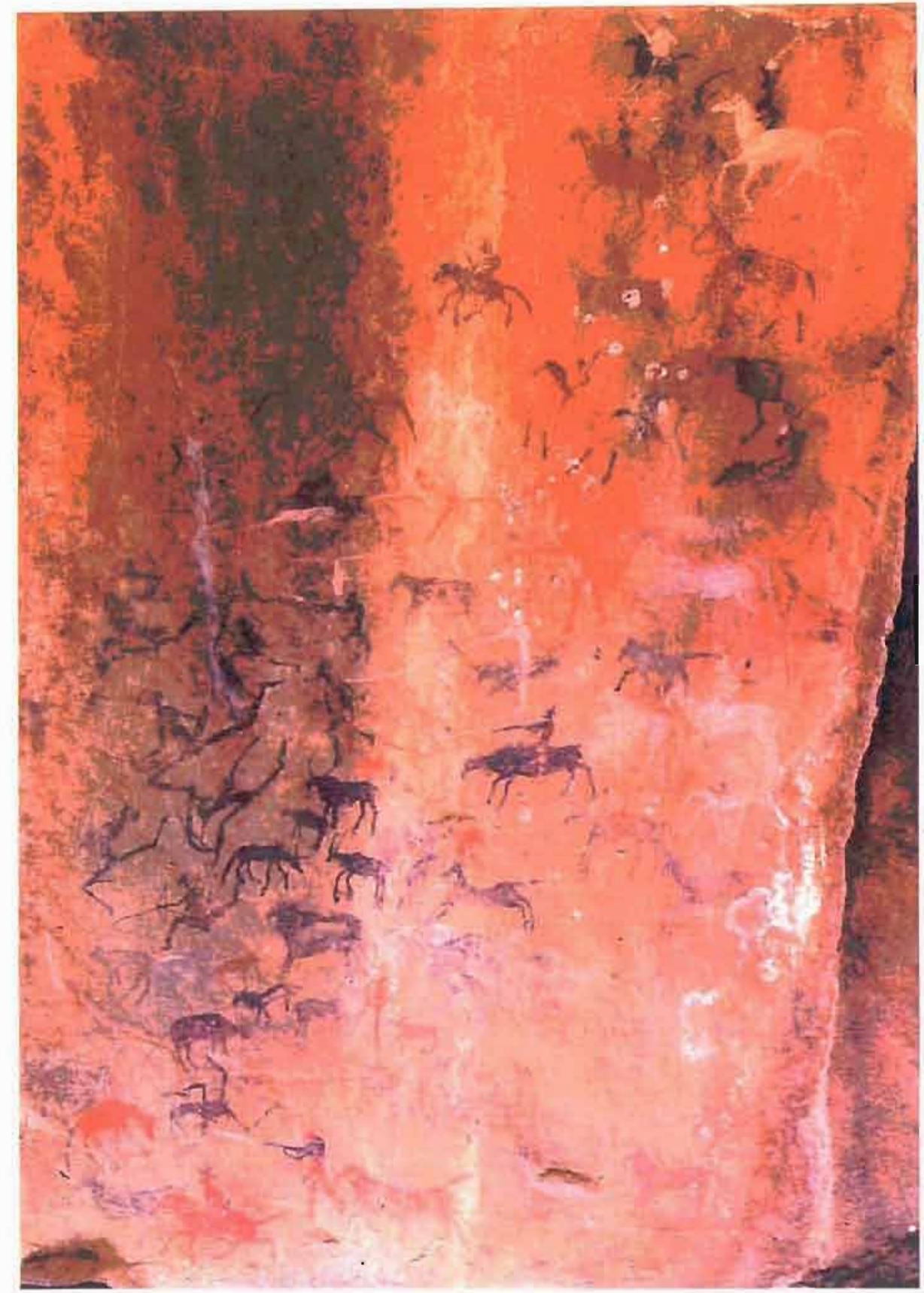

FIGURE 28

This painting, produced by an anonymous San artist or artists on the wall of a small rock shelter below the Drakensberg between 1836 and 1845, depicts a violent clash between San hunters, on foot with their bows and arrows and surrounded by cattle, and a group of mounted riders with flintlock muskets and powder horns. The riders wearing wide brimmed hats are Boers. The other riders with feathered hats and assegais are probably Khoekhoe or Bantu-speaking servants or slaves. Several of the riders have dismounted and while flashes from the barrels of their muskets and flashes from their flintlocks can be made out. In the foreground, a San man lies dead.

(Lewis-Williams, Images of Mystery: Rock Art of the Drakensberg, 2003) 
against the colonists. If so, the appeal failed, and the painting is a sobering record, not only of the defeat of a group of cattle raiders, but also of the extinction of the Drakensberg San.

While Lewis-Williams has vigorously argued that San rock art should not be seen as a form of narrative art depicting the daily life of the San, but rather as evidence of the spiritual dimensions of San culture, this painting and others like it nevertheless provide a visual record of the violent clash of cultures that launched the modern history of South Africa. They also serve as a reminder of the destruction of a culture that extended back to the dawn of human society. The reverence with which San art and culture is regarded today is a far cry from the attitudes of the Boer farmers who set out to exterminate what they perceived to be a verminous threat to their herds. And one has to look no further than Cruikshank's cartoon to see that such attitudes were not restricted to the Boers.

The comparison between these two contemporaneous works of art, so different in every way, is instructive. Both Cruikshank's cartoon and the San painting invoke a deity to protect them from 'the other', and in both the invocation is partially obscured ${ }^{88}$. Both works serve ideological purposes, but whereas the cartoon makes no effort to accurately depict its subject, the San painting is surprisingly realistic. In the cartoon, the cannibals are given huge simian teeth, capable of tearing a person apart. The lion, the python and the crocodile are also endowed with deadly teeth, suggesting that the cannibals have more in common with these dangerous animals than they have with the people they are attacking.

In the San painting, the horses and riders are depicted with a startling degree of accuracy, particularly in the depiction of their firearms. In the cartoon, by contrast, the settlers have no weapons and one of the men is trying to defend himself with a spade. This tongue-in-cheek detail reinforces Cruikshank's opinion that the settlers would be helpless victims in Africa. The truth was quite different, as demonstrated in a contemporaneous painting by F.T. I'Ons, entitled 'The young settler', in which a young man leans on his musket and presents the viewer with a fearless stare ${ }^{89}$.

\footnotetext{
${ }^{88}$ In the cartoon the invocation reads "Oh, L-d Oh, L-d!!!", as though it would be sacreligious to mention God's name in a cartoon, while in the rock painting the deity is hidden in a corner of the scene.

${ }^{89}$ Reproduced in Bryer, L. \& Hunt, K. S. The 1820 Settlers, 1984: 47.
} 
Cruikshank's cartoon is a fine example of how cartooning often lays bare the ideological underpinnings of a historical moment, providing information about prevailing attitudes that it may not have been the artist's intention to impart. It is unlikely that Cruikshank intended to satirise British racism in this cartoon: according to Bryer (1984:19), he was a fierce critic of the emigration scheme and it is more likely that his intention was to discourage the settlement of British people in what was perceived to be a dangerous and barbaric land. However, his cartoon has survived as a clear record of a racism that perceived the indigenous population of South Africa as a sub-human 'other'. And throughout the turbulent years that lay ahead, South African cartoonists would continue to expose-often in painful and unconscious ways - the conflicting ideologies and raw emotions of an emerging nation whose biggest challenge would be to accommodate diversity.

\section{The South African satirical tradition}

Cruikshank's cartoon also demonstrates the continuity between the great British satirical tradition of Hogarth, Rowlandson and Gillray and the origins of South African cartooning. Murray Schoonraad's ency clopaedic Suid-Afrikaanse Spot- en Strookprentkunstenaars (1983) and Ken Vernon's Penpricks: The Drawing of South Africa's Battlelines (2000) reveal that the early years of the Cape colony were a time of vigorous publishing activity. And, as has often been the case in South Africa since then, it was the shortlived, marginal newspapers and magazines that provided opportunities for the emergence of cartooning talent. One of these, The Zingari, a satirical weekly published from 1870 to 1875, carried cartoons by South African born William Schroder (1852-1892), described by Vernon (2000) as "the first of South Africa's great cartoonists" (2000: 20-1).

Schroder's work appeared predominantly in English-language publications like The Lantern, Cape Argus, the South African Illustrated News, Cape Punch, and The Star, but later in his life he also worked for papers sympathetic to the Afrikaner cause, Het Volksblad and The Press. Between 1884 and 1886 he published his own weekly paper, The Knobkerrie. Schroder's short but productive career epitomised the theme of conflicted identity that was to remain germinal to South African cartooning for the next 100 years. Although much of his work was for the anti-Afrikaner English language press, his real sympathies lay with the Afrikaner, symbolised by the plucky, anti-imperial stance of Transvaal president Paul Kruger ${ }^{90}$. His equivocation is wonderfully demonstrated in an

90 Shortly before his death in 1892, Schroder worked for the pro-Afrikaner Pretoria paper The Press, where he was able more easily to express his true colours (Vernon, 2000:21). Interestingly, amongst the patrons 


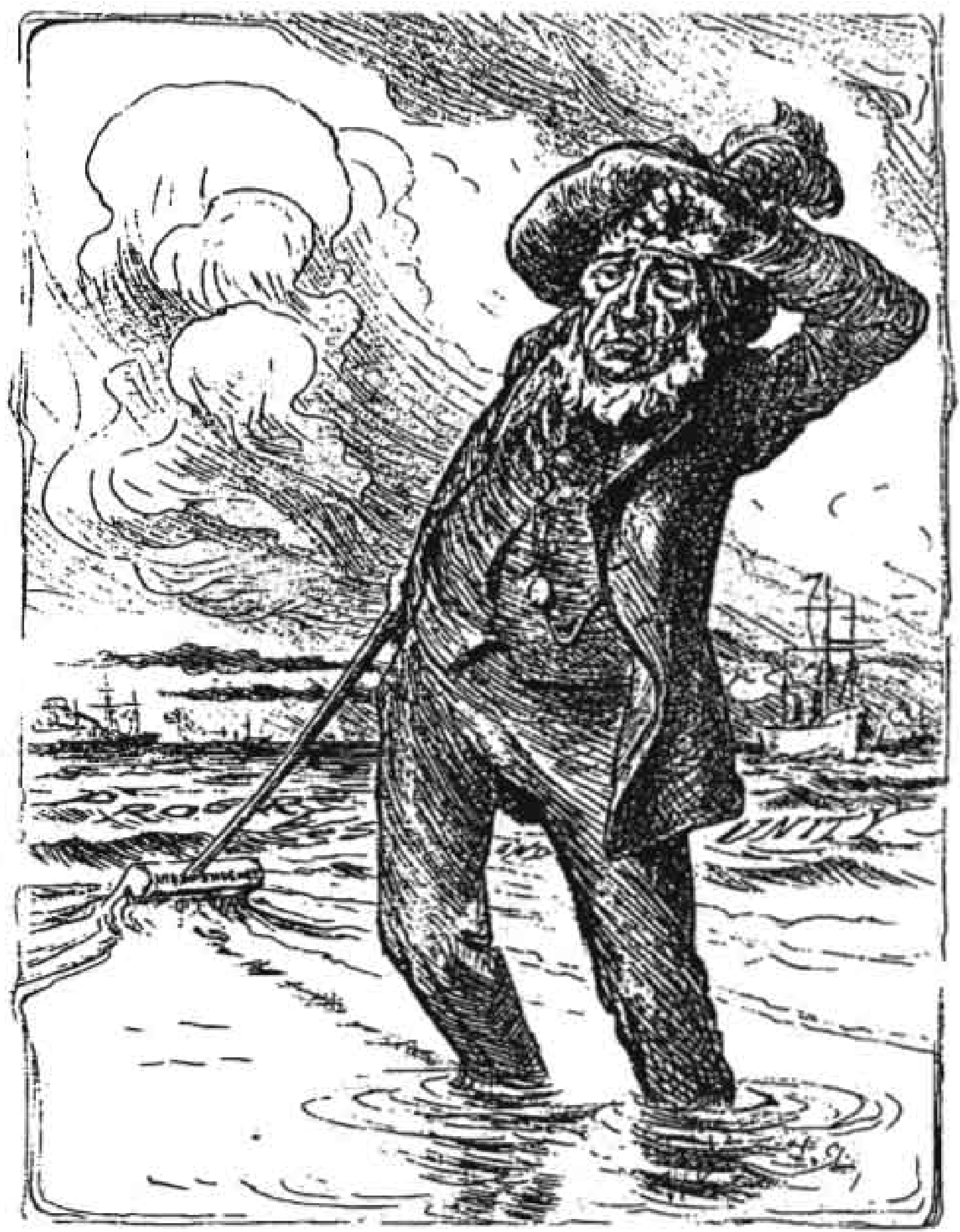

FIGURE 29

In this cartoon by Wiliam Schroder, published in The Lantem in 1887, Transvaai president Paul Kruger, armed with a broom marked 'independence', attempts to sweep back the tide of 'Progress and Unity that threatens to enguif his Boer republic. While the cartoon parodies the hopelessness of Kruger's position, the sympathetic portrat of the exhausted Kruger, his hand across his brow, betrays Schroder's true sentiments. (Vernon, 2000.) 
1887 cartoon in The Lantern depicting Kruger, armed with a broom marked 'independence', attempting to sweep back the tide of 'Progress and Unity' that threatens to engulf his Boer republic (Figure 29) ${ }^{91}$. While the cartoon parodies the hopelessness of Kruger's position, the sympathetic portrait of the exhausted Kruger, his hand across his brow, betrays Schroder's true sentiments.

South African cartooning derived from both Anglophone and Francophone origins, with the former represented in the English press and the latter more evident in the Afrikaans press. After an initial period of bilingualism during the early $1800^{\prime}$ s, the South African press bifurcated into opposing English and Afrikaans traditions and "a symbolically conflictual relationship" between papers in the two languages continued until the 1990s (Vernon, 2000: 16). According to Vernon, English-speaking readers traditionally viewed the Afrikaans papers as parochial, conservative and racist, while Afrikaans readers perceived the English press to be dangerously liberal and subversive.

The pre-eminent South African cartoonist in the early years of the century was Daniel Boonzaier (1865-1950)92, whose drawings first appeared in The Knobkerrie in 1884. It was here that Boonzaier's unschooled hand came under the influence of William Schroder, who led the young artist in the direction of political caricature (Schoonraad, 1983: 21). Boonzaier was employed as a caricaturist on The Owl during the war years of 1899-1902, and Owlographs: A collection of Cape celebrities in caricature, appeared in 1901.

In 1903, the Boer-supporting, anti-imperialist and anti-capitalist South African News announced that it had become the first South African newspaper to hire a full-time cartoonist-Daniel Boonzaier (Vernon, 2000: 31). A fervent Afrikaner nationalist, Boonzaier immediately stamped his influence on the history of South African cartooning with the creation of a character who was to become a perennial symbol on the South African political stage-- the arch-capitalist Randlord, Hoggenheimer (see

of The Schroder Art Memento: An Artistic Souvenir of South African History, published in 1893 to commemorate Schroder's death, was "His Honour S.J.P. Kruger, State President of the South African Republic". (The Memento was published by "The Press" Works, Pretoria, 1893.)

91 William Schroeder, The Lantern, 1887, reproduced in Vernon, 2000: 21.

92 Vernon describes him as "the greatest South African cartoonist ever" (2000: 31). 


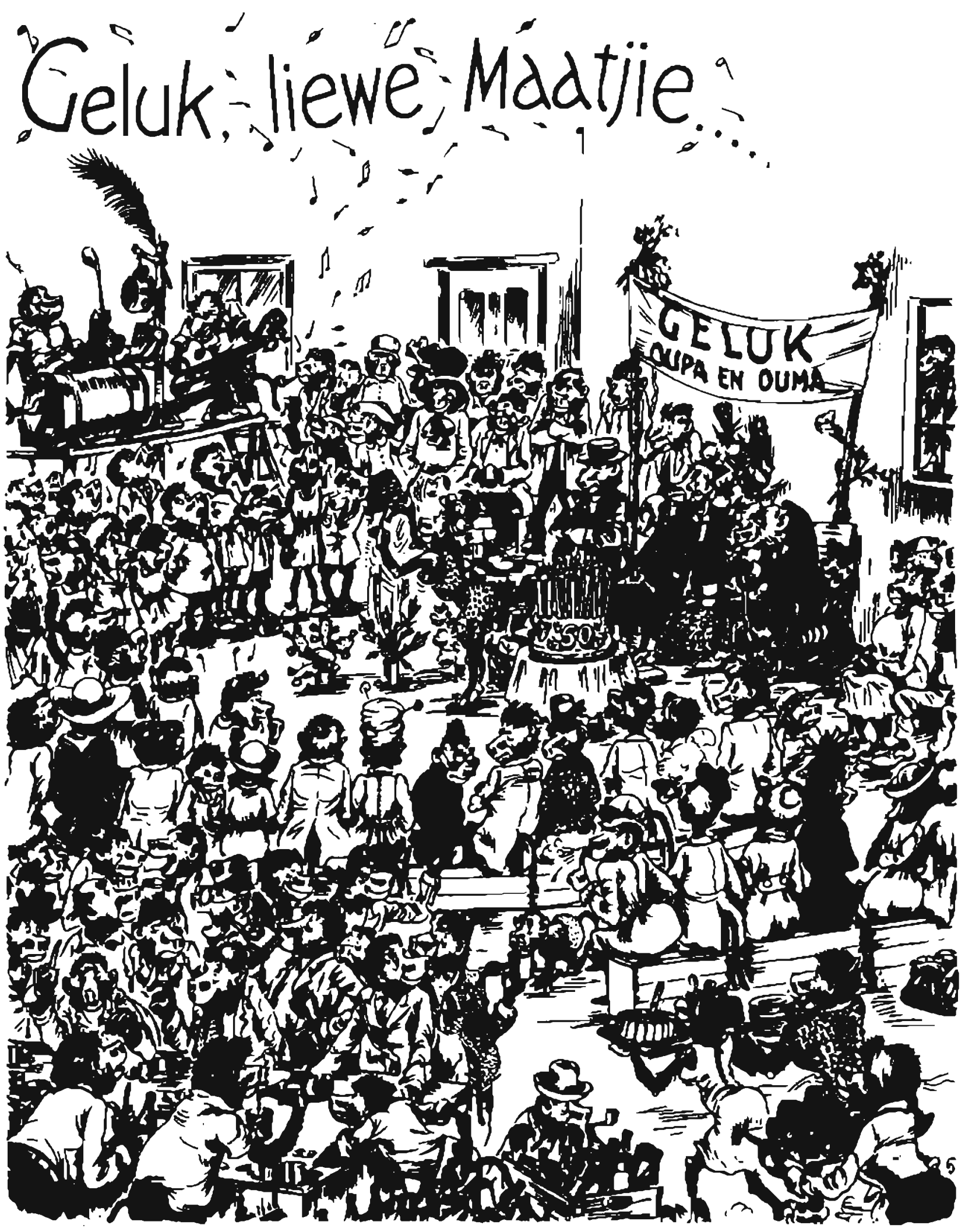

FIGURE 30

Thomas O. Honiball (1905-1991), author of the comic strips Adoons-hulle (above), as well as Oom Kaspaas and Jakkals en Wolf, was arguably the country's greatest comic strip practitioner.

The importance of his work lies in its humorous and affectionate depiction of an 'innocent' Afrikaans rural village life, not yet seriously challenged by anti-apartheid critiques. 
Figure 15, Chapter Two)93. Vernon describes Hoggenheimer as "the first truly South African symbolic stereotype ${ }^{\prime 94}$ and Hoggenheimer look-alikes continued to be produced by generations of South African cartoonists thereafter (Vernon, 2000: 34), until the character was eventually reincarnated in the 1980s in the guise of William Kentridge's Soho Eckstein.

From 1915, Boonzaier was employed as full-time cartoonist and illustrator on Die Burger, a position he held until 1941, when he was replaced by Thomas O. Honiball (1905 -1991), the creator of Afrikanerdom's most famous comic strip, Adoons-hulle (Figure 30) 95 . This continuity is some indication of the strong foundation that cartooning enjoyed in the South African press, particularly in the major Afrikaans papers.

While Boonzaier may have been South Africa's greatest cartoonist, Honiball was arguably the country's greatest comic strip practitioner. In 1941 he settled down to a long career as a full-time cartoonist at Nationale Pers in Cape Town, filling the seat vacated by Boonzaier. Until his retirement in 1974, his cartoons and comic strips appeared in Die Burger, Beeld, Rapport, Die Huisgenoot and Landbouweekblad. His first comic strip, Oom Kaspaas, appeared in Die Burger in 1939, and his work with animal characters in the Afrikaans children's magazine Die Jongspan led to Adoons-hulle, published in Die Huisgenoot from 1948 to 1971. The baboons and other animal characters in these comic strips became part of Afrikaans 'volkskultuur' (folk culture) (Schoonraad, 1989: 100). Ideologically, Honiball was secure within his milieu and his caricatures of African and 'coloured' characters appear unselfconscious. Although he was well known for his political cartoons in Afrikaans papers, he was, according to Schoonraad, less comfortable with political cartooning than with the illustrated folk tales and animal stories for which he is so famous. The importance of his work lies in its humorous and affectionate depiction of an 'innocent' Afrikaans rural village life, not yet seriously challenged by anti-apartheid critiques.

\footnotetext{
93 Hoggenheimer is portrayed, opposite Joseph Chamberlain, in the South African News in 1903 (reproduced in Vernon, 2000: 27). In a later portrayal in. Die Burger in 1917, he is shown manipulating the English press (Vernon, 2000: 47).
}

94 The original name and and figure of Hoggenheimer came from a popular theatrical production of the time, but "it was Boonzaier who turned the figure into the bloated, cigar-smoking, arrogant parasite portrayed as sucking the life-blood out of the people and the land of South Africa" (Vernon, 2000: 32).

${ }^{95}$ T.O. Honiball, Adoons-hulle , 1966, reproduced in Schoonraad, 1983: 101. 


\section{HY LEI ... MAAR WAARHEEN?}

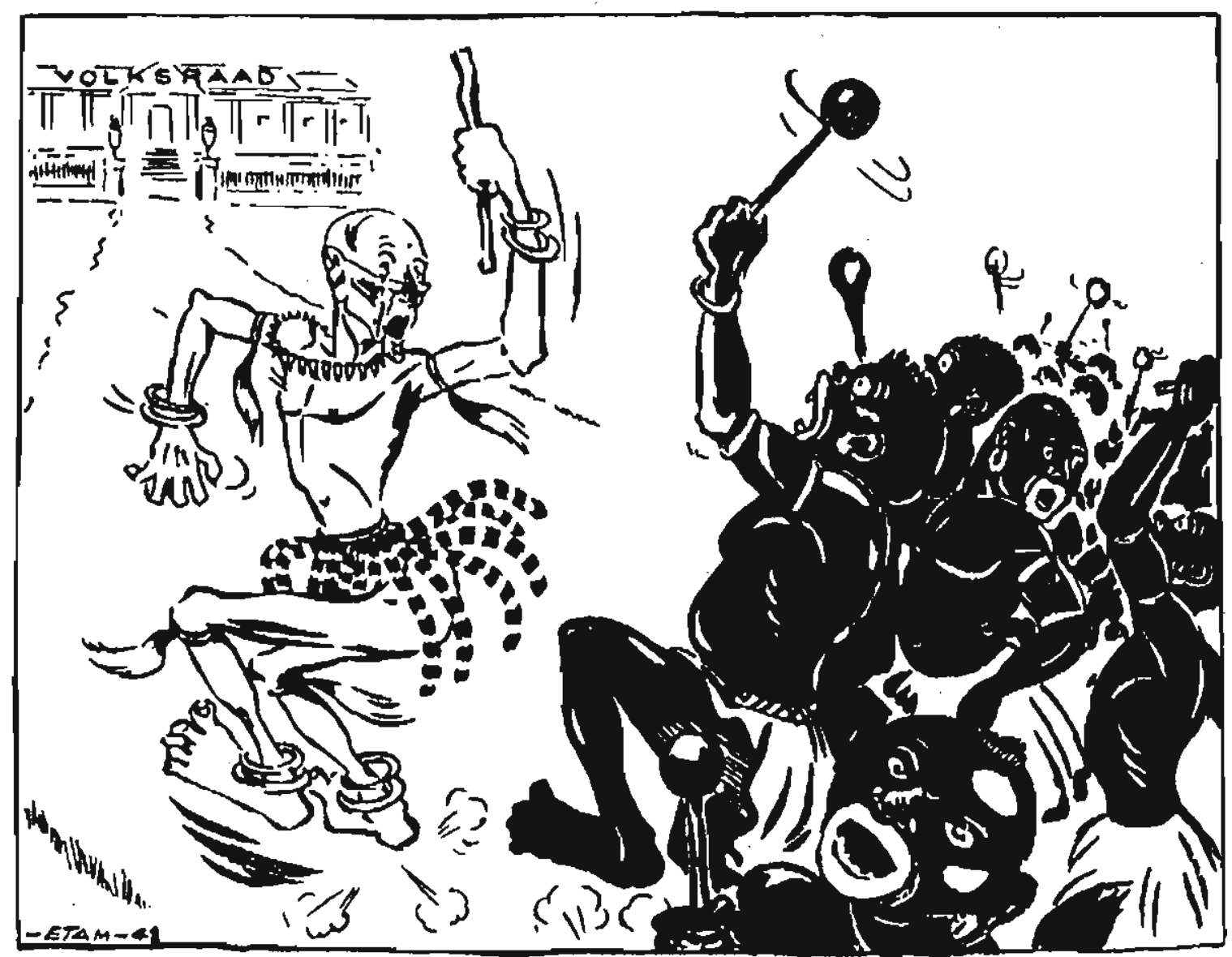

FIGURE 31

The work of political cartoonists like Eric Thamm at Die Transvaler and Victor Ivanhoff at Vaderiand demonstrated a high degree of ideological congruency with the policies of the Nationalist government, with Thamm's work in particular (above) exhibiting a virulent racism. (Eric Thamm, Die Transvaler, 1948, reproduced in Vemon, 2000.) 

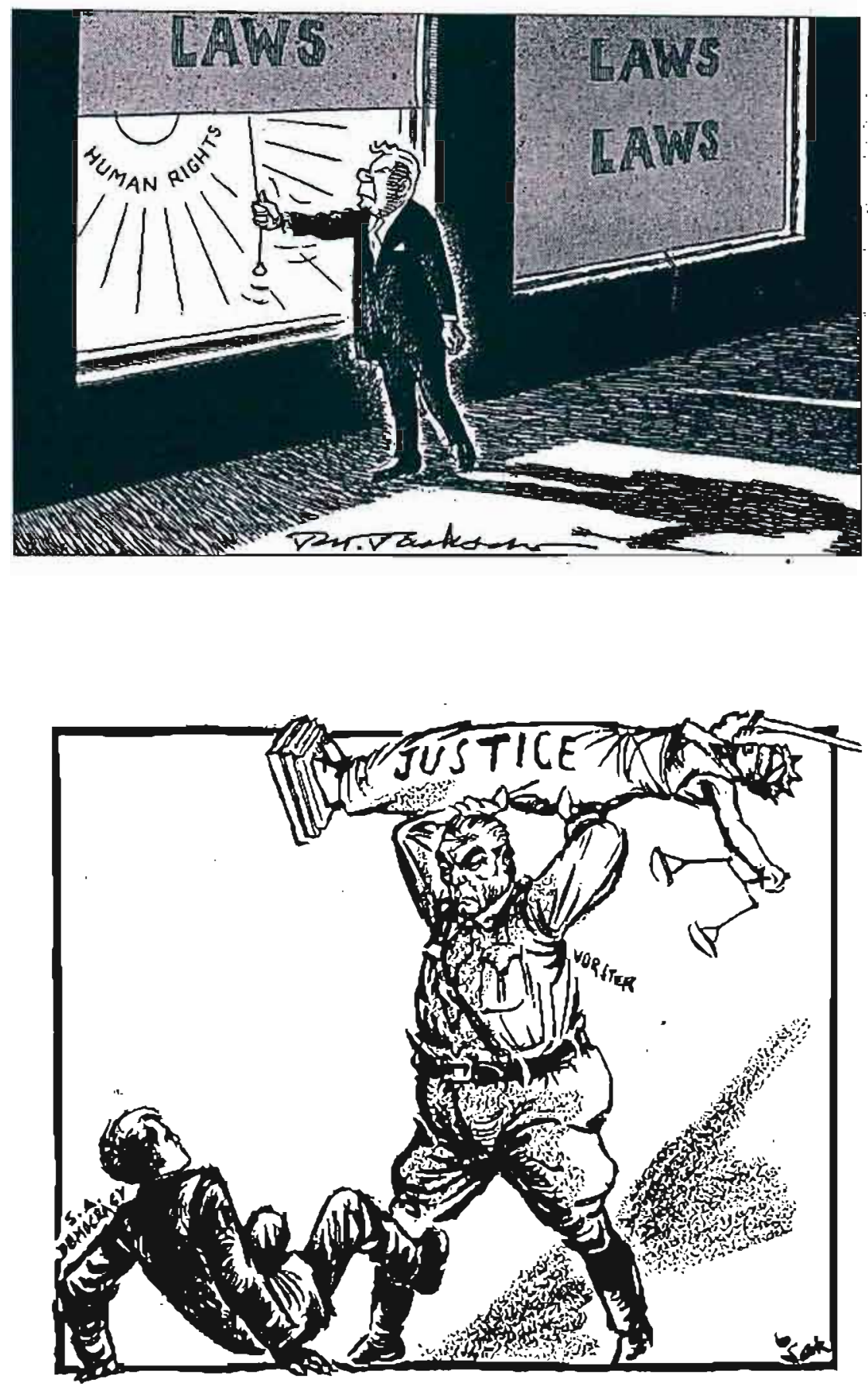

FIGURE 32

Between the 1950s and the 1970s, liberal cartoonists in the English press like Abe Berry, Bob Connolly, John Jackson (above), David Marais, Jock Leyden and Len Sak (below) all produced fine examples of biting satire rooted in angry sentiments.

(John Jackson, 1958; Len Sak, 1961, both reproduced in Dugmore et al, 1999). 


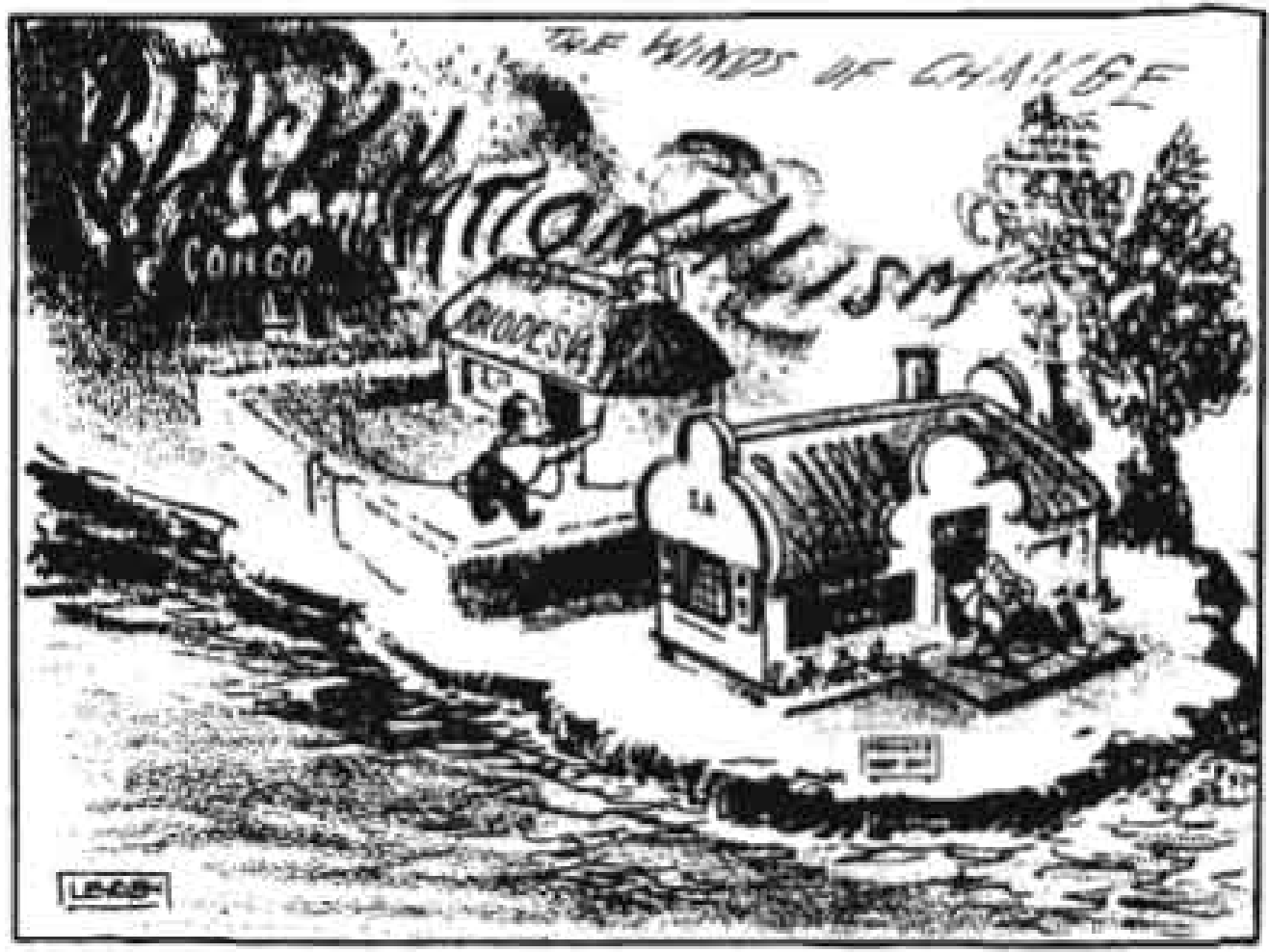

FIGURE 33

In this 1960 cartoon drawn in response to the farnous 'Wind of Change' speech by British prime minister Harold Mamillan, Jock Leyden shows Rhodesian prime minister Roy Welensky hosing down the thatched roof of his cottage in a vain attempt to protect it against the fiery blaze of African nationalism sweeping down from the Congo, while below him, at the southern tip, the architect of grand apartheid, Hendrik Venwoerd, suns himself unconcernedly outside his Cape Dutch house. (Vemon, 2000.) 
Small-town Afrikaner culture provided a nesting ground for several unremarkable cartoon strips, the best known of which was Ben Babsie en Familie by Keith and Lorna Stevens, published in various magazines and newspapers from 1955 (Schoonraad, 1983). In this strip, and in their Flip Foster and T) strips, this couple adroitly gave expression to the myopic parochialism of the white South African lifestyle during the apartheid decades. Meanwhile the work of political cartoonists like Eric Thamm at Die Transvaler and Victor Ivanhoff at Vaderland demonstrated a high degree of ideological congruency with the policies of the Nationalist government, with Thamm's work in particular exhibiting a virulent racism (Figure 31)\%6.

\section{The contradictions of liberalism}

The liberal English newspaper cartoonists of the mid-century period were less comfortably located within their ideological milieu than their Afrikaans counterparts. Ken Vernon refers to their position as one of "confusion and equivocation" - mirroring the conundrum of a press that was opposed in principle to the Nationalist government, but whose readers tacitly supported the government's apartheid policies (Vernon, 2000: 118). The contradictory situation of the South African English-speaking intellectual, as both critic and beneficiary of the country's political system, is evident in the work of several major English newspaper cartoonists. These contradictions were stretched to breaking point after 1976, but between the 1950s and the 1970s, Abe Berry, Bob Connolly, John Jackson, David Marais, Jock Leyden and Len Sak (Figure 32) ${ }^{97}$ all produced fine examples of biting satire rooted in angry sentiments.

The cartoons of Jock Leyden, a Scotsman who drew for Durban's Daily News from 1939 until 1981, provide many classic expressions of the liberal dilemma. In a 1960 cartoon, drawn in response to the famous 'Wind of Change' speech by British prime minister Harold Mamillan ${ }^{98}$, Leyden shows Rhodesian prime minister, Roy Welensky, hosing down the thatched roof of his cottage in a vain attempt to protect it against the fiery blaze of African nationalism sweeping down from the Congo, while below him, at the southern tip, the architect of grand apartheid, Hendrik Verwoerd, suns himself

\footnotetext{
96 Eric Thamm, Die Transvaler, 1948, reproduced in Vernon, 2000: 92.

97 John Jackson, 1958; Len Sak, 1961. Both reproduced in Dugmore et al, 1999: 24).

98 In a 1960 address to the South African parliament, Macmillan referred to the irresistable 'wind of change' blowing through the African continent (Vernon 2000: 106).
} 

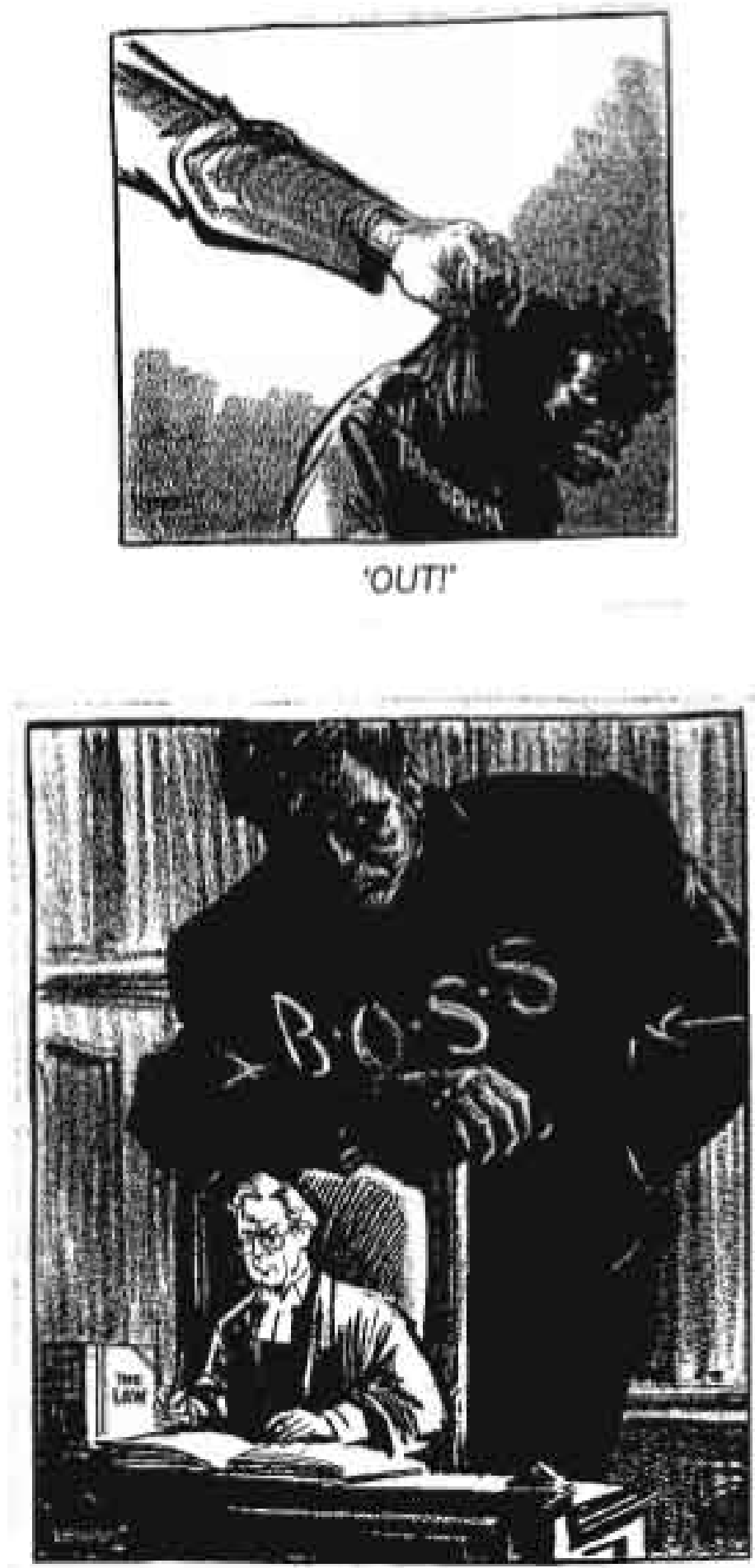

FIGURE 34

(Above) In this cartoon (1967), Leyden clearly expresses approval for the government's antiterrorism legislation, but in the cartoon below (1969) he depicts the government's Burbau of State Security (BOSS) as a shadowy and ominous figure looming over the South African judiciary These two cartoons suggest that liberals experienced a sense of being hemmed in by extremism on both sides of the political spectrum. (Vernon, 2000.) 

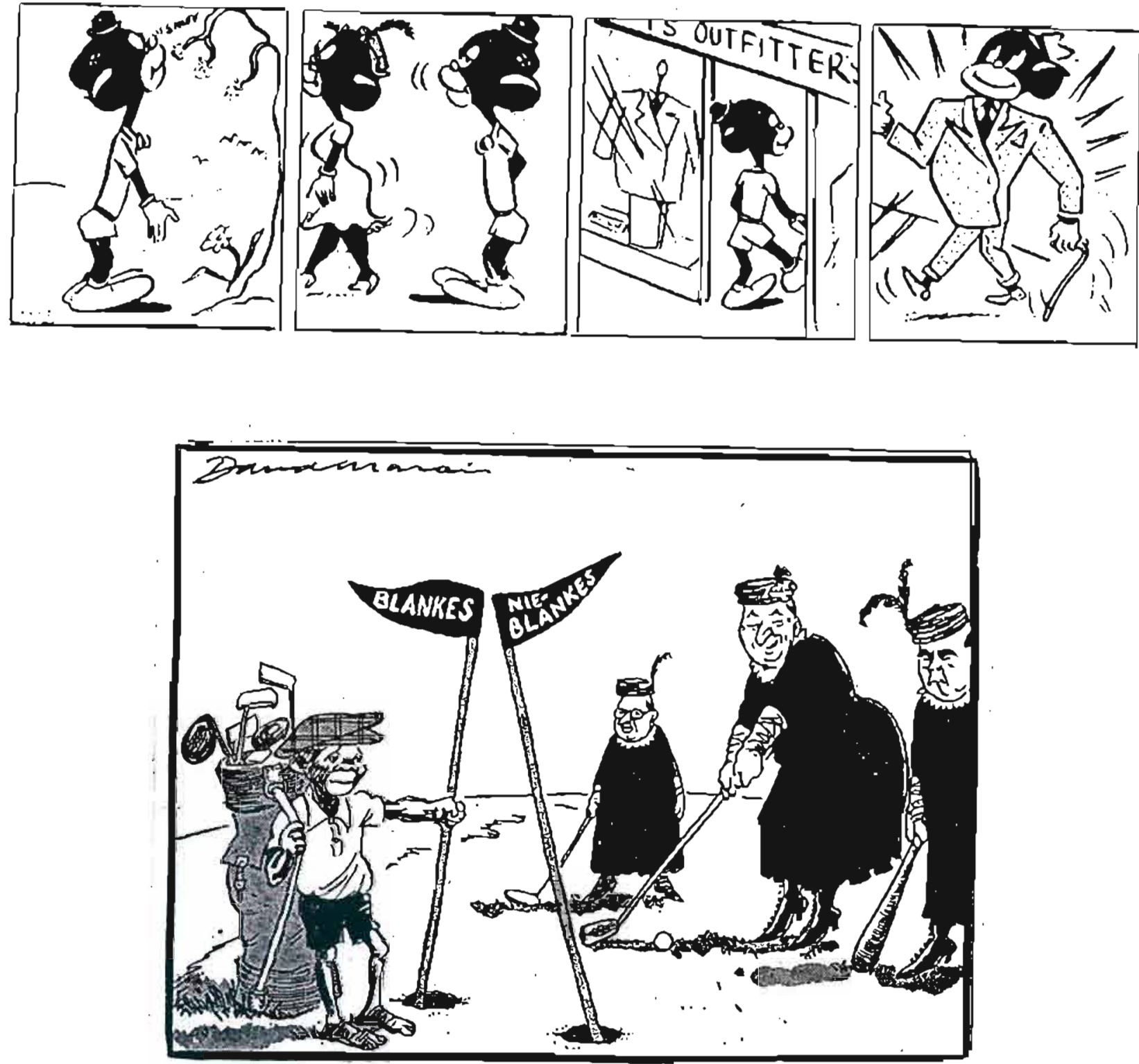

FIGURE 35

The conventions of stereotypical racist caricature, with African characters portrayed as 'coons' (the upper part of the face rendered in black and the lower part, including the cheeks and lips, rendered in white) are visible in the work of many white South African cartoonists, Afrikaner and English alike. Phaki by G.L. Norton (above), published in a Chamber of Mines publication for mineworkers, circa 1950, is unselfconsciously racist, but even David Marais (below), a committed opponent of the human rights abuses of the Nationalist government (Vemon, 2000: 105), slipped into this usage from time to time, as in this 1963 cartoon. (Vernon, 2000.) 


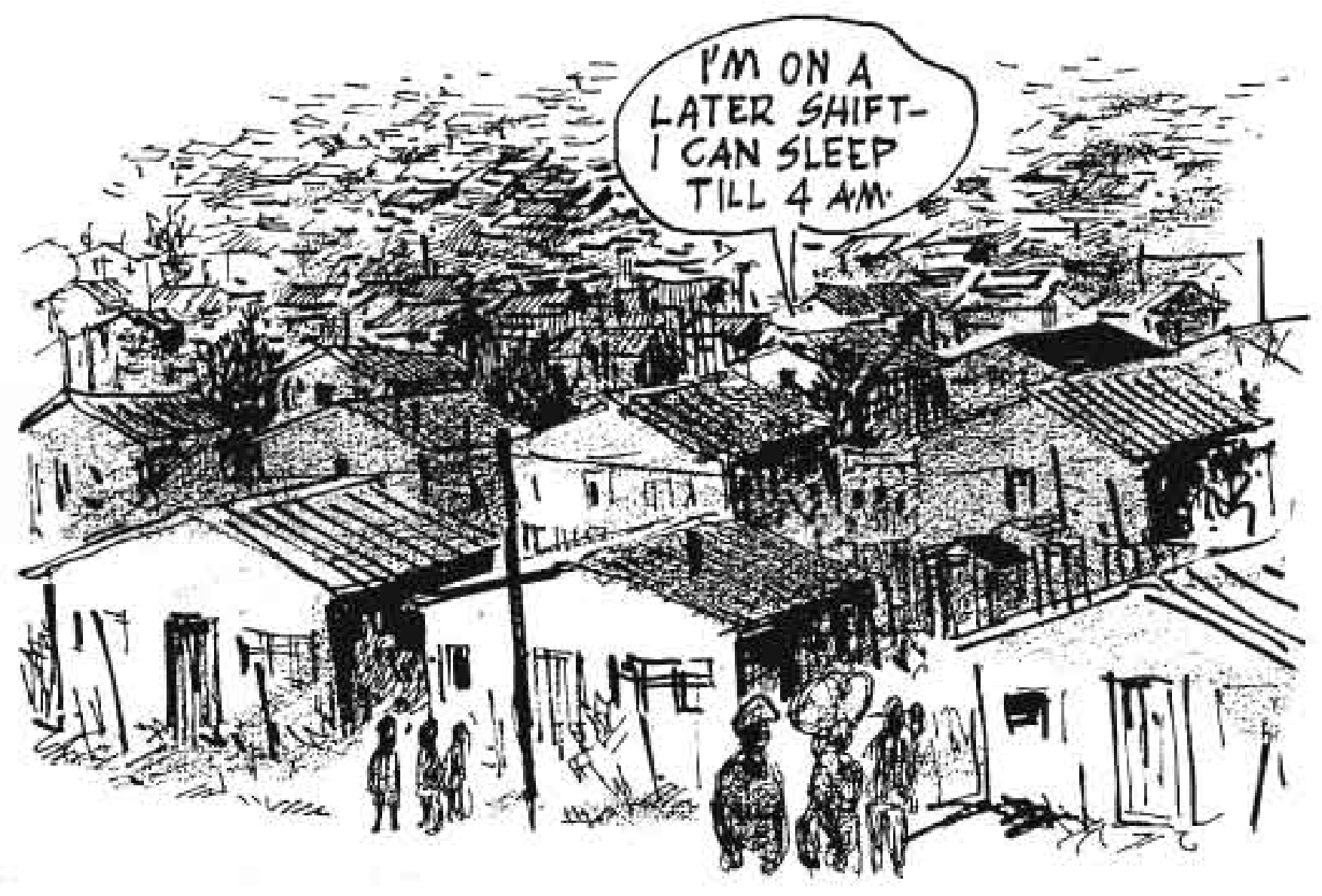

FIGURE 36

Abe Berry (1911-198-), who drew for the Johannesburg Star and other English papers from the 1950 s until the 1980s, had the unusual ability amongst liberal cartoonists to sympathetically portray the tribulations of ordinary African people entangled in the web of apartheid legislation.

(Berry. Abe Berry's South Africa and How it Works, 1989.) 
unconcernedly outside his Cape Dutch house (Figure 33) ${ }^{99}$. This cartoon was clearly intended as a warning to white South Africans.

Vernon suggests that Leyden "bought hook, line and sinker the Nationalist line that Africans fighting for political equality were "terrorists"'(2000: 118). In a 1967 cartoon, Leyden depicts the long arm of the law firmly grasping a grimacing black man labelled 'terrorism', with a caption that simply reads: "Out!"100 In this cartoon Leyden clearly expresses approval for the government's anti-terrorism legislation. But in a 1969 cartoon he depicts the government's Bureau of State Security (BOSS) as a shadowy and ominous figure looming over the South African judiciary (Figure 34) ${ }^{101}$. In their expression of distaste for black political activism on the one side and secret police activity on the other, these cartoons reveal an important aspect of South African liberalism: a sense of being hemmed in by extremism on both sides of the political spectrum. But, as the political crisis deepened, the ambivalent 'wishy-washy liberal' 102 position expressed in these cartoons would become increasingly untenable.

\section{The semiotics of racial caricature}

The portrayal of black South Africans in cartoons and comic strips by white artists provides some insight into the ideological underpinnings of apartheid society during the middle years of the century. There are many examples of South African cartoons that exhibit the conventions of stereotypical racist caricature, with African characters portrayed as 'coons' (the upper part of the face rendered in black and the lower part, including the cheeks and lips, rendered in white) (Figure 35)103.

Most white cartoonists concentrated their critiques on the white political arena, seldom attempting to give expression to the realities of black life under apartheid104. Even

\footnotetext{
99 Jock Leyden, Daily News, 1960, reproduced in Vernon, 2000: 108.

100 Jock Leyden, Daily News, 1967, reproduced in Vernon, 2000: 117.

101 Jock Leyden, Daily News, 1969, reproduced in Vernon, 2000: 114.

102 A common expression at the time.
}

103 See Phaki by G.L. Norton, circa 1950; C.W. Shackleton, circa 1940; Monty Wilson, The Natal Daily News, circa 1941; Eric Thamm, Die Transzaler, 1948; and E.A.Groves, Cape Argus, 1952 (all in Schoonraad, 1983). Much later, in the 1990s, Bitterkomix artist Joe Dog was to exploit this convention in his satires.

104 See, for example, David Marais, Europeans Only (1960) and John Jackson, Running Comentary (1967). 


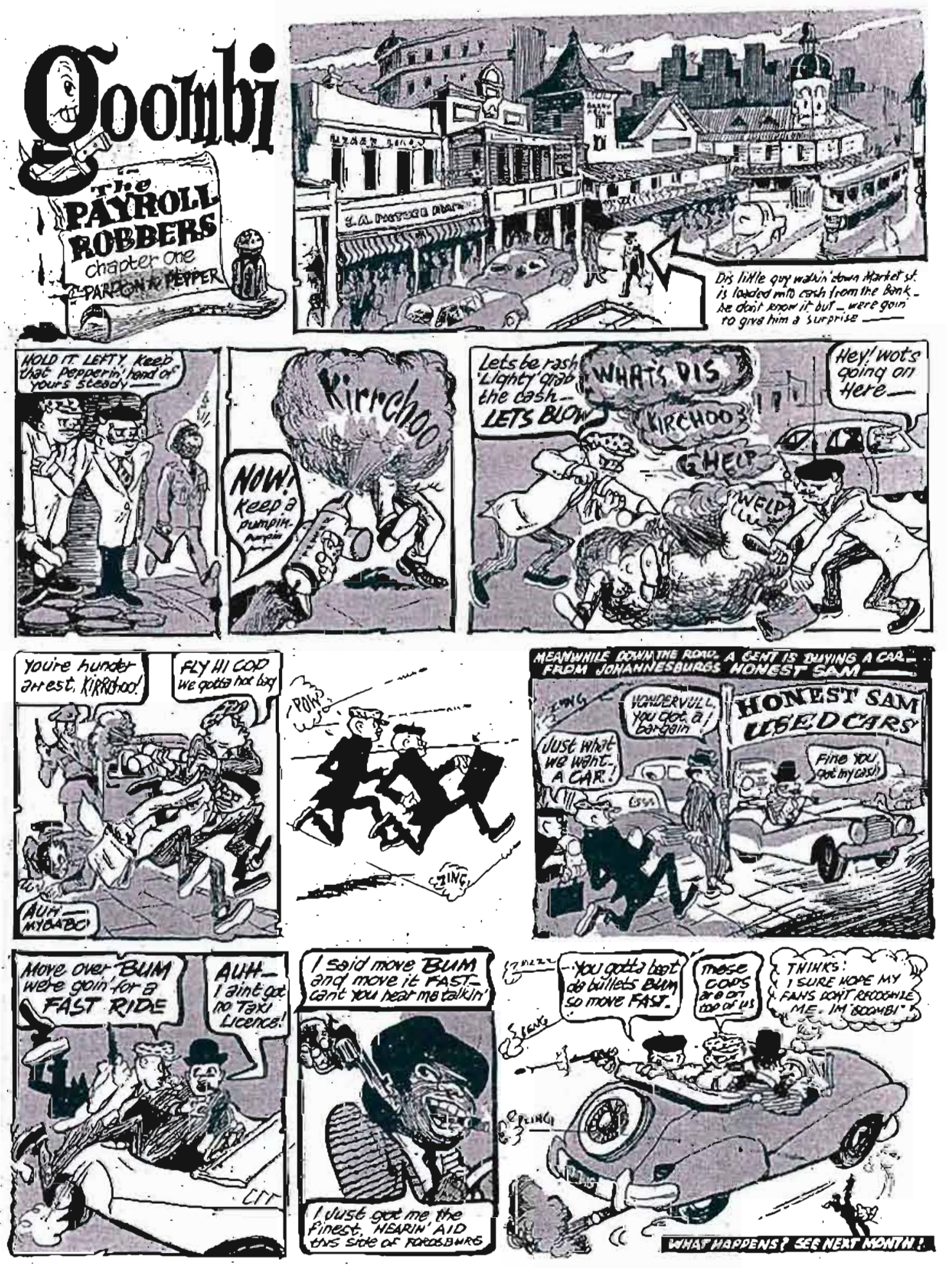

FIGURE 37

The anonymous strip Goombi-Private Detective, which appeared in Drum in the late 1950s, was notable for its dialogue - an interesting combination of township vemacular and the 'hard-boiled' Americanese of detective novels-as well as its faithful depiction of street life and local architecture. (Drum, 1958.) 
where the cartoonists were critical of apartheid, their portrayals of black people often, if unconsciously, displayed the conventions of racial caricature-in particular the telltale thick white lips of the 'coon' stereotype (see Figure 35) ${ }^{105}$. It is arguable that the use of these stereotypical conventions - even where they were not specifically intended to denigrate the African - signified that the cartoonists concerned were unable to identify with the people that they were drawing, but saw them as 'the other'.

There were exceptions, however. Abe Berry (1911-198-), for example, who drew for the Johannesburg Star and other English papers from the 1950s until the 1980s, had the ability to sympathetically portray the tribulations of ordinary African people entangled in the web of apartheid legislation (Figure 36) ${ }^{106}$. His Day By Day strip in The Star often portrayed political developments in South Africa through the eyes of black people (Schoonraad, 1983: 18).

In 1956-7, an intriguing comic strip, entitled Goombi-Private Detective, began appearing in Drum. No artist or writer was credited, but the strip nevertheless had several merits, including its dialogue, a combination of township vernacular and the 'hard-boiled' Americanese of detective novels, and its faithful depiction of street life and architecture in a variety of locations-Sophiatown, Vrededorp, Alexandra, Cape Town's District Six and Malay Quarter, and Durban's 'Casbah'. The authors obviously intended to reinforce the strip's local flavour by locating the action around popular landmarks of black culture (Figure 37)107. This strip, though anonymously produced and short-lived, is important because it was an attempt to combine the hard-bitten detective genre of comics with the legendary lifestyle of Sophiatown during the Drum era.

Amongst the white cartoonists whose work appeared in the black press, the most dedicated and influential has undoubtedly been Len (Lennie) Sak. Born in 1931 in Port Elizabeth, Sak became a freelance cartoonist in 1956, working variously and sometimes

\footnotetext{
105 David Marais, Cape Times, 1958, reproduced in Marais, 1960. Even though Marais was a committed opponent of the human rights abuses of the Nationalist government (Vernon, 2000: 105), he slipped into this usage from time to time.

106 Abe Berry, Abe Berry's South Africa and How it Works, 1980

${ }^{107}$ A 1957 story begins with the attempted kidnapping of a singer called Dolly (Rathebe) at the famous Bantu Men's Social Centre. Other actual locations featured in the strip include the Odin Cinema in Sophiatown, the Victory Lounge in Durban, the Crescent Restaurant in Vrededorp, the Alabama Cinema in Cape Town's Malay Quarter and Hanover (spelt "Hangover") Street in District Six.
} 


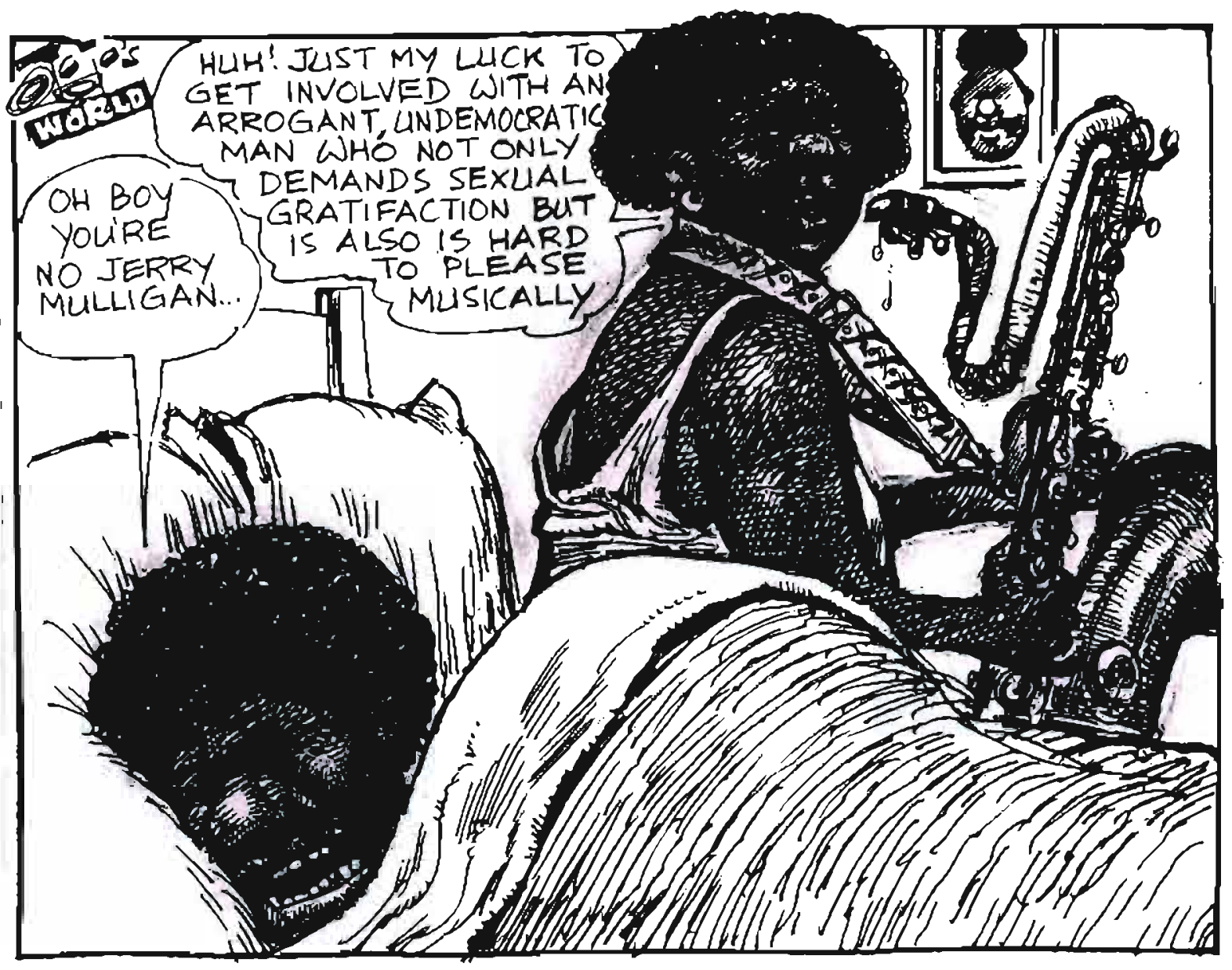

FIGURE 38

Len Sak developed a uniquely 'black' cartooning style which came to be associated with the stable of black newspapers in which his cartoons appeared. Although by his own admission he seldom ventured into the black townships where his cartoons were set, his work completely transcended the limitations of his personal experience and succeeded in documenting the social, cultural and political life of black South Africans for more than four decades. (Sak, Jojo's World: Celebrating 33 years of a Township Cartoon Hero, 1991.) 
simultaneously for The South African Jewish Frontier, Die Brandwag, Bantu World, Golden City Post, Drum, The Cape Argus and the Sunday Times. His character Jojo appeared in Drum for the first time in 1959, and went on to appear regularly in The World, Weekend World, Post, Sunday Post and later the Sowetan until the end of the century. In 1965 he began working as a full-time cartoonist and illustrator for The World and Weekend World, also drawing at that time for Post, Sunday Post and Financial Mail. Cherrie, a lifestyle strip with a black female lead character, appeared in The World and Weekend World from 1968 to 1980 (Schoonraad 1983: 207-211). By 1970 his style had become definitive-a uniquely 'black' cartooning style associated with the stable of black newspapers in which it appeared (Figure 38) ${ }^{108}$. Although he led a somewhat cloistered existence and, by his own admission, seldom ventured into the black townships where his cartoons were set $^{109}$, his work completely transcended the limitations of his personal experience. Through the force of a remarkable imagination and extraordinary powers of empathy for the common man, he succeeded in documenting the social, cultural and political life of black South Africans for more than four decades. Like several other leading South African cartoonists, Sak is Jewish, of eastern European origin, and his experiences of marginalisation as a child growing up in wartime Port Elizabeth contributed to his powers of empathy ${ }^{110}$. Although he has been criticised for his moralising and sermonising tendencies ${ }^{111}$, his ability, as a white artist, to produce and sustain credible black cartoon characters, to portray township life in an authentic way and to demonstrate a sense of style and a perspective with which black readers were able to identify, over such a long period, is a remarkable achievement.

The most intriguing aspect of Sak's ouvre is his character, Jojo, arguably the most popular black South African cartoon character of the $20^{\text {th }}$ century. Jojo originated in 1958 as the result of a commission by the editor of Drum, Tom Hopkinson, who asked Sak to invent a South African cartoon strip whose hero was to be "a little guy living in a typical black dormitory township... [who would] transcend hardships, mishaps and disasters

108 From Jojo's World: Celebrating 33 years of a Township Cartoon Hero, Sowetan/Operation Hunger, 1991.

109 Interview, 2001.

110 Interview, 2002.

111 According to Vernon (2000: 171), "Sak was not the cartoonist to go for the jugular in any circumstance, being inclined to moralise and preach rather than attack". 


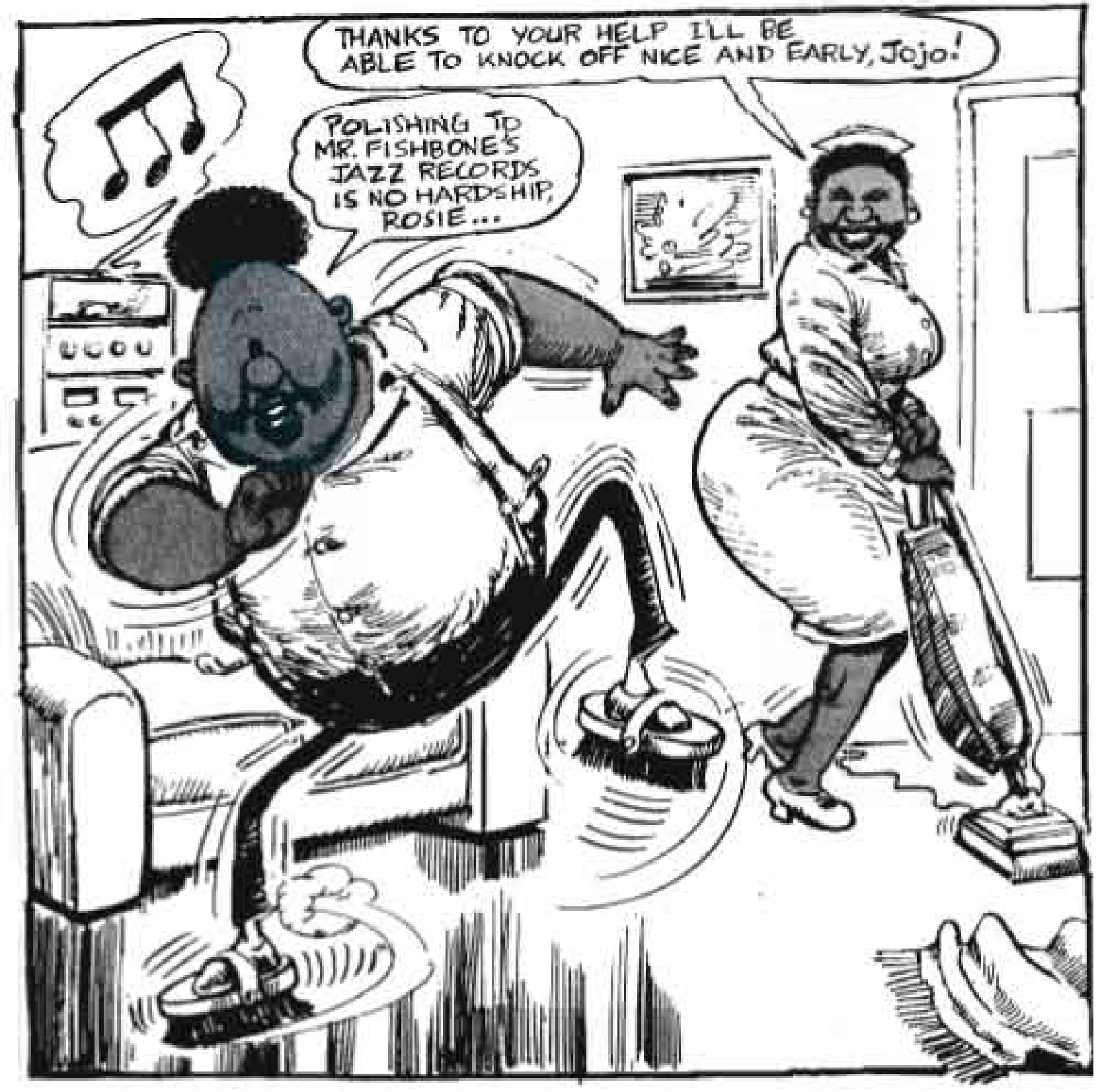

FIGURE 39

What is remarkable about Len Sak's Jojo is that, despite the thick lips, button nose, and exaggerated tuft of frizzy hair-all well-known conventions of racial stereotyping-jojo was not regarded by readers of the papers in which he appeared as a racial stereotype, but rather as a much-loved icon of the struggie of ordinary black people (Sak, Jojo's World: Celebrating 33 years of a Township Cartoon Hero, 1991.) 
with dignity and humour-and have the last laugh." 112 What is remarkable about Sak's treatment of this character, is that, despite Jojo's thick lips, button nose, and exaggerated tuft of frizzy hair-all well-known conventions of racial stereotyping-Jojo was not regarded by readers of the papers in which he appeared as a racial stereotype, but rather as a much-loved icon of the struggle of ordinary black people (Figure 39)113. According to Sowetan editor Aggrey Klaaste (1991), "Jojo was held in awe by young and old people. Jojo became a part of black life, and by the rhythms that guide the artistic impulse, of all life." 114 And, according to Sak, "I am told that Black people who know Jojo find it hard to believe that the cartoons are done by a non-black" (Sorrell, 1987).

The anomaly of Len Sak's Jojo poses some intriguing questions about the semiotics that lie behind the conventions of racial stereotyping. According to semiotic theory ${ }^{115}$, visual images are complex encoded combinations of iconic, indexical and symbolic signs. The iconic aspects of the image are those that are denotative or visually self-evident, but there is no such thing as a purely denotative image. All images are connotative to some extent, and none so much as cartoons and caricatures. In semiotic terms, the character of Jojo is a code made up of visual signs, arranged according to well-understood conventions. The egg-shaped head and body, the partially undone braces, the spindly legs, the open-necked white shirt with its rolled-up sleeves and the chubby arms and hands, combined with the exaggerated facial features, connote the attributes of goodnaturedness, resilience in the face of hardship, and a richness of spirit despite material poverty.

What lifts the character of Jojo above the limitations of racial caricature is, simply, the intention of the artist. Sak set out not to satirise black people but to create an empathetic black 'Everyman' to represent a populist viewpoint with which the readers of Drum would be able to identify. Most interesting are Jojo's eyes. Set very close together, they are presented as unvarying oval dots with no indication of pupils, eyelids or eyebrows.

112 From the introduction to Jojo's World: Celebrating 33 years of a Township Cartoon Hero, Sowetan/Operation Hunger, 1991.

${ }^{113}$ Len Sak's Jojo, from Jojo's World: Celebrating 33 years of a Township Cartoon Hero, Sowetan/Operation Hunger, 1991.

114 Aggrey Klaaste, Introduction to Jojo's World: Celebrating 33 years of a Township Cartoon Hero, Sowetan/Operation Hunger, 1991.

115 Contemporary semiotic theory adds to Saussure's linguistic semiology the triad of icon, index and symbol derived from Peirce. See Chapters One and Two. 
By maintaining the eyes in an unchanged form through all the character's various expressions, the artist effectively removes the eyes from the chain of signification and the character's expression is reliant solely on the shape of the mouth and cheeks. In cartooning this is not unusual, and some of the world's most famous cartoon characters, such as Herge's Tintin and Charles Schultz's Charlie Brown, have simple dots for eyes. The effect of this technique, according to Scott McCloud's theory of iconic abstraction (1993: 29-53), is to generalise the character, to increase the level of reader identification, and to allow the reader to 'inhabit' the character ${ }^{116}$.

The conventions of racial stereotyping have continued to be employed by subsequent cartoonists, even those highly critical of apartheid and sympathetic to the black liberation struggle, and it may be fair to argue in their defence that the use of stereotypical conventions in itself does not imply racist tendencies, but that it is in the visible intention of the artist, inasmuch as this is discernable, that the presence or absence of racism is signified. Nevertheless, as many cartoonists have demonstrated, it is quite possible to portray black people in cartoons without resorting to racial stereotypes ${ }^{117}$. Therefore, the use of the conventions of racial stereotyping is ideologically significant and serves as a signifier of the complex ideological position of the artist.

An equivocal and sometimes contradictory ideological position is signalled in this way in the work of Richard Smith, one of the most versatile and highly skilled cartoonists of the apartheid era, whose cartooning career began in the late 1960s. Born in Scotland in 1947, Smith was strongly influenced by the British tradition of satirical caricature, and in particular by the work of Ralph Steadman and Gerald Scarfe ${ }^{118}$. Unlike those of Berry or Sak, Smith's cartoons did not investigate the lives of black South Africans in any depth and his renditions of the African man-in-the-street tended towards the thick-lipped 'coon' stereotype ${ }^{119}$. His contribution lay rather in his introduction into the South African press of the extreme forms of caricature for which the British satirical magazine Private Eye was infamous. According to Vernon, Smith's most important contribution

${ }^{116}$ McCloud's theory of iconic abstraction is discussed in some detail in Chapter Two.

117 Sak's ouevre provides many examples of naturalistically drawn black characters, which sometimes appear alongside racial caricatures in the same panel (see Figure 50).

118 Interview, 2000. Smith also cites Ronald Searle and Saul Steinberg amongst his many influences.

${ }^{119}$ See Richard Smith, The Best of Smith \& Abbot Ink, 1973. 


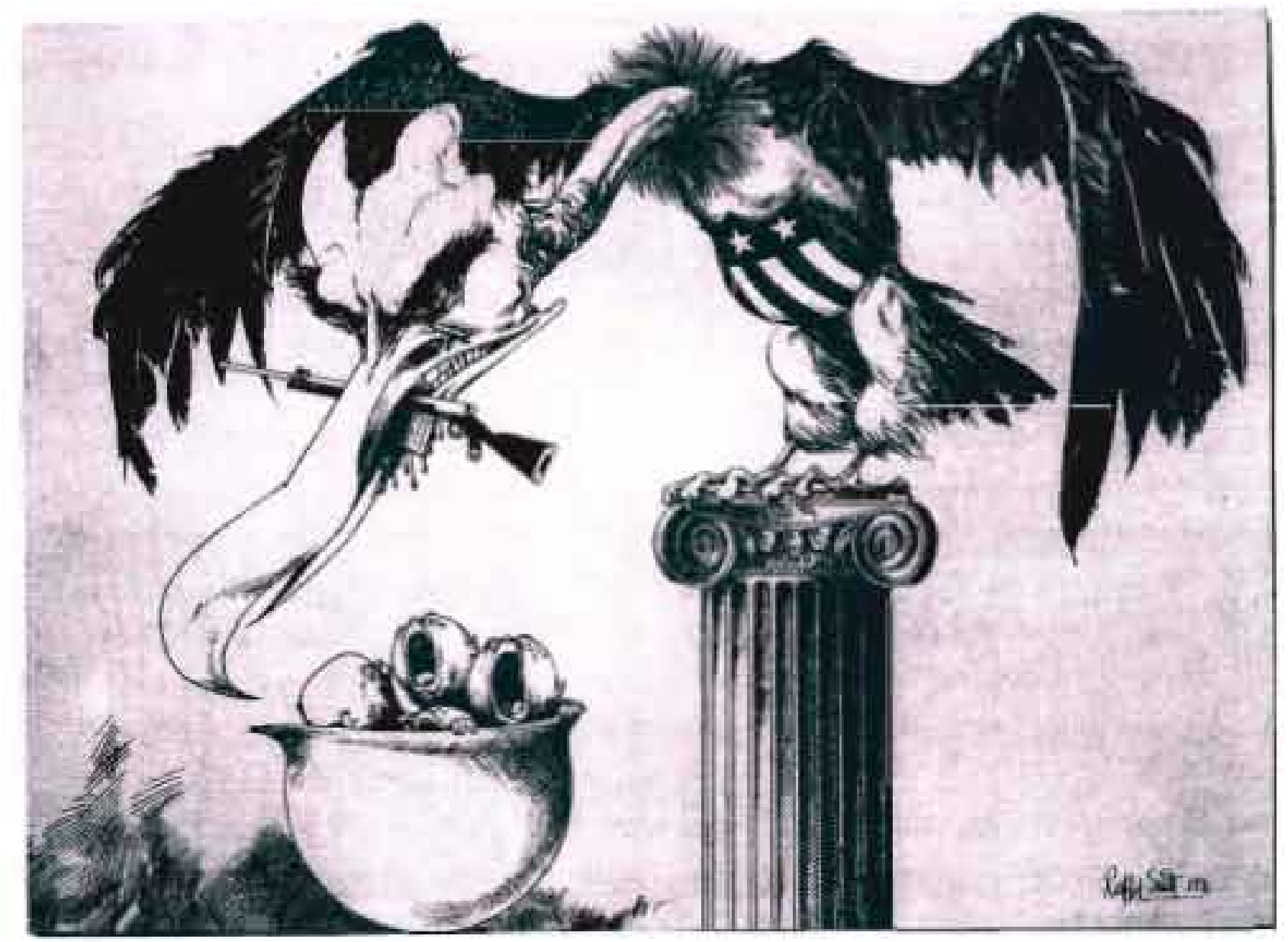

FIGURE 40

Richard Smith's early work was particularly grotesque, as befitted the iconoclastic zeitgeist of the late $1960 \mathrm{~s}$ and early $1970 \mathrm{~s}$, and was often international in subject matter, as in this superb 1971 caricature of Nixon, one of the best of his early works. (Black 4 Whife in ink exhibition catalogue, 2002.) 
was "to scribble a new moustache on the staid face of cartooning in the English press" (2000: 118). His early work was particularly grotesque, as befitted the iconoclastic zeitgeist of the late $1960^{\prime} \mathrm{s}$, but was also international in subject matter, revealing the young artist's desire to succeed internationally (Figure 40).

Smith emigrated to South Africa with his parents at the age of 11 and joined the Sunday Times as full-time cartoonist 11 years later, after a cartoon strip satirising the famous South African heart transplant surgeon, Dr Chris Barnard, in the 1968 edition of the Wiwatersrand University's student RAG magazine, Wits Wits, created a public furore. His rise to fame was rapid and the following year he was invited to submit work to an international exhibition in the USA ${ }^{120}$. In 1970 he moved to Britain where he attempted to make a career for himself in the British press, producing drawings for Punch, Time Out and BBC Television, but in 1972 he returned to South Africa and teamed up with writer David Barrit to create the political cartoon strip Smith $\mathcal{E}$ Abbot Ink, which appeared in the Rand Daily Mail from 1972 until 1976. This extremely important strip turned the leading politicians of the time-Prime Minister BJ Vorster, his Cabinet colleagues, opposition politicians, such as de Villiers Graaf and Helen Suzman, as well as international figures, such as Margaret Thatcher and Ronald Reagan-into cute puppet figures with large heads and stunted bodies (see Figure 41). The strip was a kind of political Punch and Judy show, each three or four panel episode ending with a witticism in which the characters would invariably be hoisted on the petard of their own insincere and contradictory behaviour.

Although Smith was often fiercely critical of the apartheid government, and despite the sharp wit that he frequently displayed, his work, like that of the liberal cartoonists who preceded him, generally concentrated on the internecine conflicts of the white political arena and did not align itself with the liberation struggle. While purporting to criticise the politicians of that era, Smith $\mathcal{E}$ Abbot Ink actually satirised them in a rather gentle way ${ }^{121}$. In 1976, the strip, entitled Richard Smith Ink, after the departure of Barrit for the USA in 1974, came to an end, and thus never engaged with the increasingly polarised post-1976 political climate.

120 "Mice that Roar", an international exhibition of cartoons at the University of Wisconsin (interview, 2002).

121 It is interesting to compare Smith's approach to that satirical dramatist Pieter Dirk Uys, who produced similarily affectionate satires of the same politicians on the stage. 

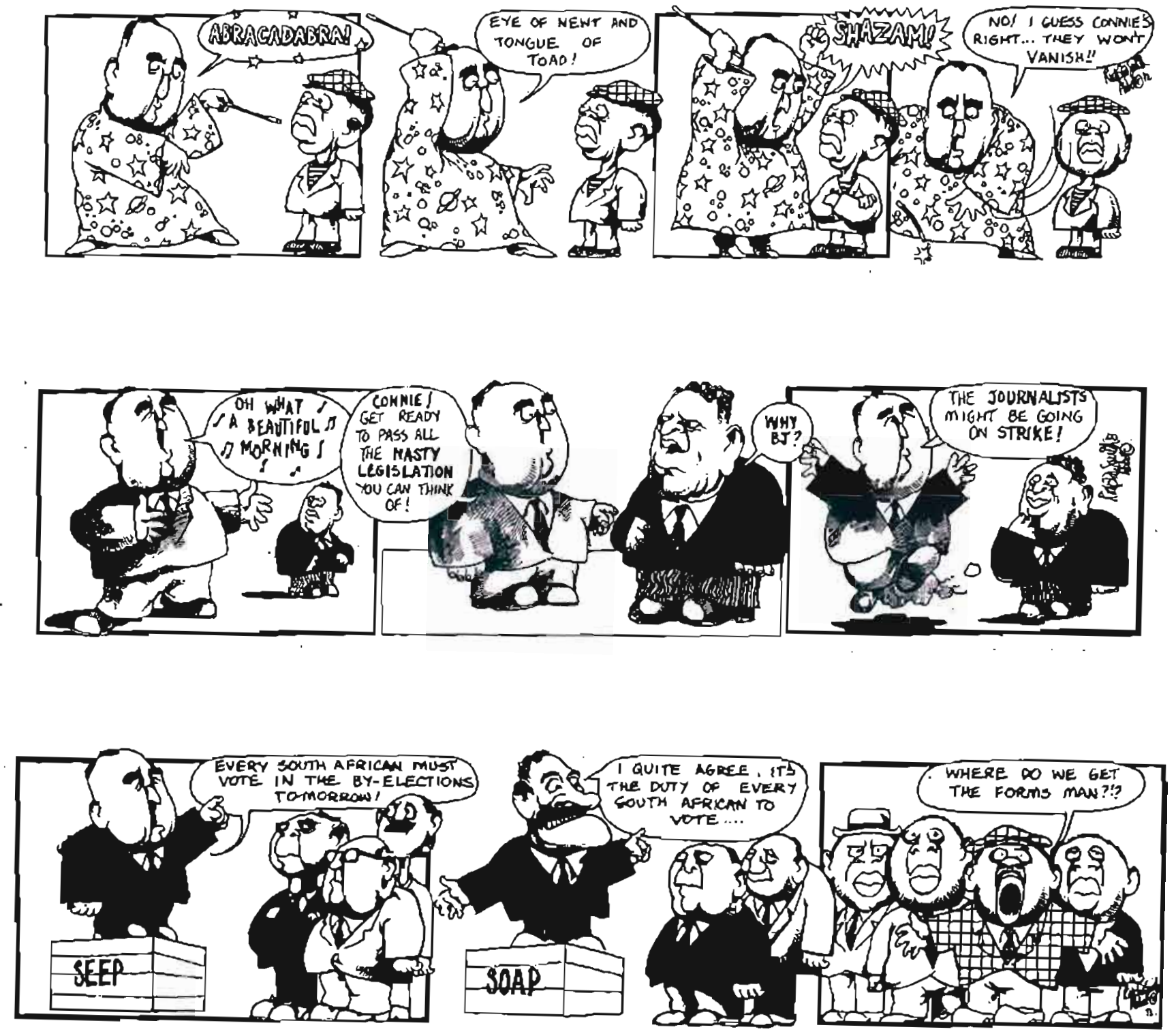

FIGURE 41

In his cartoon strip Smith and Abbot Ink, Richard Smith caricatured apartheid politicians in a rather affectionate way, while his portrayal of African characters tended towards the thick-lipped 'coon' slereotype. (Smith \& Abbot's Greatest Hits, 1973) 
Just as their employment of the conventions of racial caricature tells us something about the English-speaking liberal cartoonists of the 1950s-1970s, so does their treatment of the politicians of the day. Caricatures do not always vilify or demonise: as demonstrated in Smith $\&$ Abbot Ink, they can also be rather affectionate in the quality of the satire they present. In his early work, Smith produced brutally caricatured satires of local and international politicians, such as B.J.Vorster, Albert Herzog and Richard Nixon, that revealed an iconoclastic distaste for everything they stood for. But in Smith \& Abbot Ink, and in his more mature work for the Sunday Times, Sunday Express, Leadership, Optima, Financial Mail and other publications of the mainstream opposition press, Smith's caricatures tended to humanise rather than demonise their subjects and the laughter they provoke is not unsympathetic (Figure 41). The element of complicity that is revealed here, and the limitation of the artist's sphere of interest to the white political and cultural arena, are familiar features of the work of the mid-century liberal cartoonists, and in a sense Smith's work can be seen as a bridge between them and the cartoonists of the darker, more harrowing post-1976 period ${ }^{122}$.

The demonisation of apartheid's political elite in his early work earns Richard Smith a place amongst the country's important opposition cartoonists, even though he claimed to be "not that interested" in political topics ${ }^{123}$. And although his mature caricatures are more successful in capturing the human qualities of their subjects than in expressing a clear political viewpoint, his early work planted an iconoclastic seed in the landscape of South African cartooning that was to bear fruit in the work of Derek Bauer and Zapiro. Smith's first important published work, the vicious attack on Chris Barnard in the 1968 edition of Wits Wits, is a case in point. In this strip, Smith depicted Barnard as a vampire, eager to rip the heart from his next donor so that he could continue his grisly work. The white South African public was horrified by this denigration of a national icon. This strip is important because it introduced several new themes into South African cartooning. First, as has been said, Smith's vicious style liberated South African cartooning from the restraints of decorum and ushered in a new era of political caricature. Second, it revealed the existence of an important sub-genre of cartooning amongst student activists, who published their own campus newspapers and whose alliances with non-governmental organisations (NGOs), the emerging trade union movement and radical ecumenical bodies were to result in the growth of a vigorous

122 Smith continued to produce editorial cartoons for the Sunday Times, Financial Mail, Ftontline, Leadership and many other publications into the $1990 \mathrm{~s}$.

${ }^{123}$ News/Check, April 1970. 
alternative press. Third, by targeting a 'non-political' public figure, Smith revealed opportunities for satire beyond the political arena, in the very tissue of South African life, where ideological assumptions and social norms provided great opportunities for satirical dissection. The surgical disembowelment of South African life has remained a feature of South African student and underground comix, as exemplified in the challenging Afrikaans Bitterkomix of Anton Kannemeyer and Conrad Botes.

Over the years Smith's style evolved, becoming more finely tuned and delicate, and the watercolour caricatures he produced for the Sunday Times, Financial Mail and Leadership are some of the finest South African works in this genre. However, as his artistic technique became more refined, the satirical edge of his earlier work gradually blunted and his political wit failed to keep pace with the rapid transformations in South African society. In 1998 he was unceremoniously dumped by Sunday Times editor Mike Robertson, who represented the new post-apartheid regime at Times Media Ltd ${ }^{124}$, in favour of Zapiro. Smith's desire to succeed as a fine artist had meanwhile become increasingly predominant throughout the 1990s and he began to concentrate on oil painting, accepting several major portrait commissions. By 2002 he had abandoned cartooning altogether and he and his wife moved to the Greek island of Cephalonia, where he concentrated on landscape painting ${ }^{125}$.

Smith's cartooning career demonstrates the evolution of the liberal viewpoint across several time zones in South Africa's political history, and provides some sense of the international political and artistic context in which it was located. Whereas his early work was radical for its time, both in content and technique, in Smith's more mature work the expression of rather broad and unspecific liberalism ${ }^{126}$ is articulated against a desire to achieve artistic excellence and refinement for its own sake. But by 1998, when Smith was replaced at the Sunday Times by Zapiro, South Africa had changed irrevocably. Zapiro is a creature of the new South Africa and his considerable skill as a caricaturist has played humble servant to the dictates of an unequivocal radicalism. This 'changing of the guard' at the country's most influential Sunday paper is

124 In 1996 Times Media Ltd was sold to a black-led consortium headed by Cyril Ramaphosa, who had been the ANC's senior negotiator during the transition period (Vernon, 2000: 177).

125 In 2003 Smith accepted the position of curator of the prestigious Brett Kebble Awards art competition and exhibition and returned to South Africa to undertake this project.

126 In a 1974 interview with the Sunday Times, Smith claimed not to be "partisan in any way" (Schoonraad, 1983: 234), a position that he continued to maintain throughout his cartooning career. 


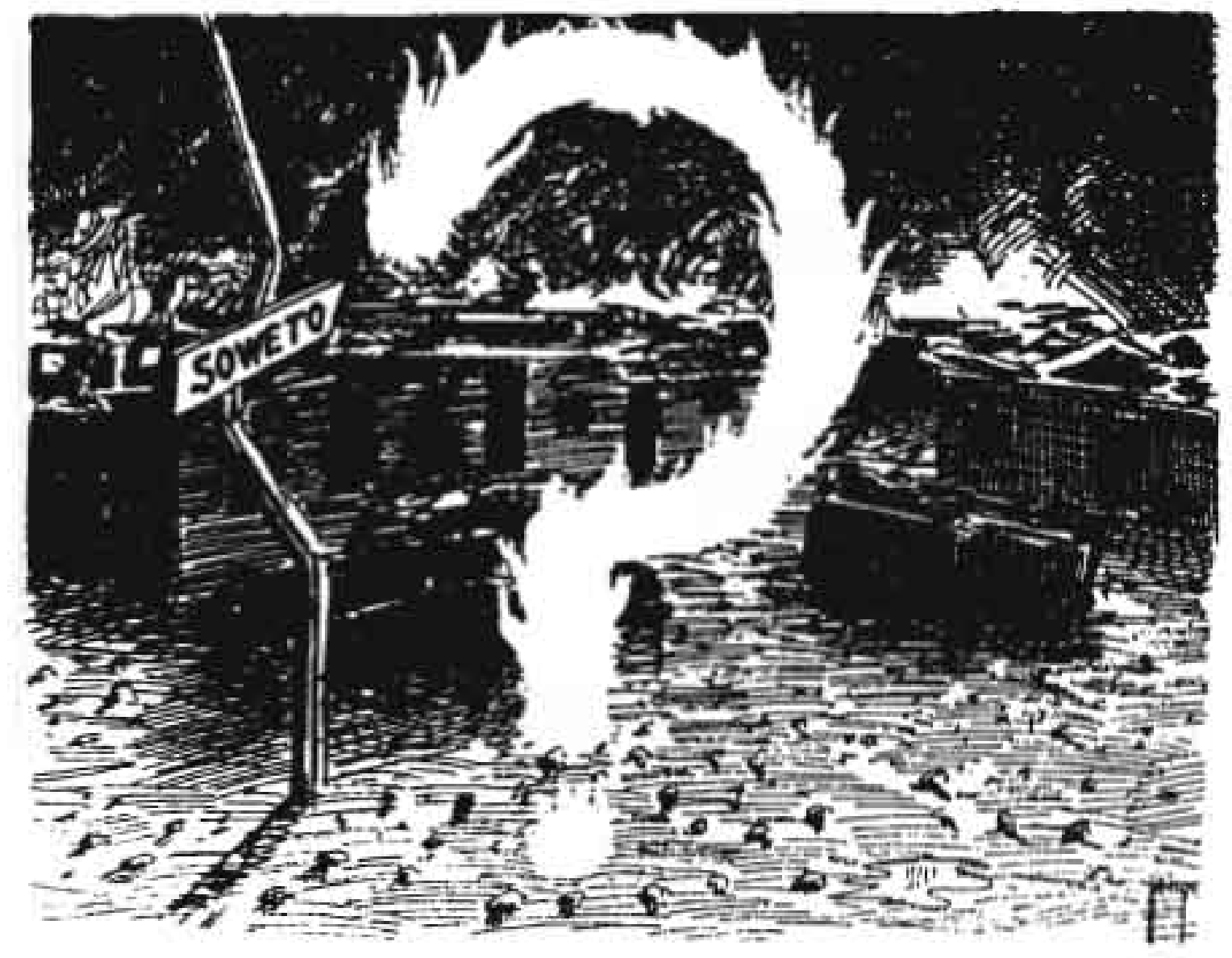

FIGURE 42

In this telling cartoon, Donald Kenyon, the cartoonist at East London's Daily Despatch, reveais that most white South Africans, led by state propaganda to believe that black political activity was orchestrated by sinister Soviet-led forces, simply had no idea what the Soweto uprising of 16 June 1976 was really about. (Vernon, 2000.) 
emblematic of the enormous changes that took place at every level of South African life after 1976.

\section{The challenge of Soweto}

According to Hein Marais (1998), the resurgence of popular resistance against the apartheid state in the 1970 s was propelled by four developments. The first was the economic crisis, accompanied by rising levels of unemployment amongst black workers, leading to a wave of strikes. The second was the successful achievement of independence by Mozambique and Angola, both in 1975, signalling the possibility of national liberation in southern Africa and contributing to "a growing sense of of seige among whites and immeasurably boosting courage and resolve among blacks" (1998: 39). The third was the rise of Black Consciousness (BC) ideology and the emphasis on psychological liberation amongst black intellectuals, as exemplified in the writings of Steve Biko. The fourth was a more heightened and widespread politicisation of the oppressed that tended to attribute all forms of discrimination, deprivation and oppression to the apartheid system.

As membership of anti-apartheid organisations multiplied and a wave of resistancerelated activity spread through all sectors of society ${ }^{127}$, the cosy ambivalence of the liberal position lost all legitimacy in the face of a hardening ideological resolve that saw reform and revolution as mutually exclusive and argued that "apartheid cannot be reformed" (Marais, 1998: 40). The traumatic events of 1976 threw white South Africa into a state of ideological disarray as liberals and verkramptes alike attempted to come to grips with the meaning of June 16. As Vernon (2000: 132-3) shows in his analysis of their responses to the shock of Soweto, white cartoonists such as Donald Kenyon and Bob Connolly, preoccupied as they were with white party politics, demonstrated a painful lack of understanding of the history and dy namics of popular resistance in South Africa. The crisis of Soweto revealed a vast existential and ideological chasm between black and white. In a particularly telling cartoon, Donald Kenyon, the cartoonist at East London's Daily Despatch, depicted a flaming white question mark superimposed over the burning township (Figure 42)128. Most white South Africans, led by state propaganda to believe that black political activity was orchestrated by sinister

127 This activity was not restricted to black townships, but expressed itself in white society too, especially on the English-language university campuses, in the ecumenical sector and in the rapidly proliferating non-governmental organisation (NGO) sector.

128 Donald Kenyon, Daily Despatch, 1976. Reproduced in Vemon, 2000: 132. 
Soviet-led forces bent on a total onslaught against their way of life, simply had no idea what the Soweto uprising was really about.

It was necessary for cartooning, as it was for society in general, to delve beneath the symptomatic episodes of social unrest to establish their structural causes. But, despite their often vehement expressions of distaste for the visible excesses of the apartheid system, the liberal cartoonists of the mid-century period generally lacked the necessary levels of political insight and informed opinion to be able to transcend the arena of white party politics and engage with or give expression to the mass democratic movement and the liberation struggle. A new generation of opposition cartoonists would be required to tackle the social and political crisis brought so sharply to light by the Soweto uprising. 


\section{Cartooning in the Last Decade of Apartheid}

\section{Introduction}

The first section of this chapter will show that the South African intellectual environment of the mid-1980s, exemplified in the critical writing of the period, was characterised by argumentative, frequently hostile exchanges between an exhausted liberalism attempting to reinvigorate itself in a postmodern guise, and an aggressive tendentiousness that aligned itself with the liberation struggle. In the field of political cartooning, similar tendencies expressed themselves, as the comforts of liberalism disappeared and with them went the so-called 'old guard' of South African liberal cartooning-John Jackson, David Marais, Donald Kenyon, Bob Connolly and Jock Leyden-most of whom had reached or were reaching the end of their careers (Vernon, 2000: 135; 165).

Simultaneously, the hegemonic dominance of the major English and Afrikaans newspaper groups with their strong ties to the political establishment was being eroded by an unexpected irritant: the emerging 'alternative' press. The new weekly newspapers and monthly magazines that arose in the $1980 \mathrm{~s}^{129}$, mostly funded by donor agencies or governments sympathetic to the international anti-apartheid movement, were to provide many opportunities for the emergence of new cartooning talent. Likewise, a range of educational projects initiated by externally-funded change organisations-who had ambitious educational and propaganda agendas with regard to the voteless masses but were excluded from access to radio and television-led to an interest in the potential of cartooning as a conscientising and mobilising tool.

Thus, by 1985, a new phase in South African publishing - matched by a new phase of cartooning-was already underway. This chapter demonstrates how the dominant themes that characterised the intellectual environment at large expressed themselves in the various kinds of cartooning that emerged at this time, and how the stylistic and

\footnotetext{
129 These included, inter alia, Grassroots (Cape Town), The Eye (Pretoria), Speak (Lenasia), Umthoyama (Port Elizabeth), Ukusa (Durban) and Saamstaan (Oudshoorn) in the early 1980s, followed in the mid-1980s by the weekly newspapers South (Western Cape), Umafrika (Durban), New Nation (Johannesburg), Vrye Weekblad (Johannesburg) and the Weekly Mail (Johannesburg) (Berger, 2000: 79). The alternative press of the 1980s saw itself, according to Berger, as "the new dominant press in waiting" (2000: 80).
} 
artistic dimensions of these cartooning products were shaped and influenced by the political, ideological and economic imperatives of the day.

The key proposition of this study is that between 1985 and 1994, oppositional cartooning gave visual signification to discourses of resistance that existed in the democratic movement at that time. In doing so, oppositional cartooning contributed to the disruption of the hegemony of the apartheid state and the legitimation of the antiapartheid struggle by providing jokes, stories, symbols and icons that ordinary South Africans were able to relate to and utilise in their own attempts to make sense of what was happening around them.

Obviously, these discourses of resistance were not invented by the cartoonists themselves - they were already present within the democratic movement and those sectors of society, both in the country and internationally, who were sympathetic to it. The first section of this chapter looks very briefly at how these discourses expressed themselves in one area of South African intellectual life-the field of literary criticism. The purpose of this brief excursion into the critical writing of the period is to reveal what Thompson (1990) calls the 'doxa' of the 'ethnographic moment' - the prevailing "opinions, beliefs and understandings" (1990: 279) that existed in the democratic movement at that particular moment in South Africa's history. Given that it is impossible, in a historical study such as this, to inhabit the ethnographic moment itself ${ }^{130}$, evidence about it has to be derived from an examination of other cultural and media products of the period, sourced from the fields of literature, fine art, journalism or popular culture. The limitations of this study proscribe more than a very brief investigation of one of these fields of cultural production ${ }^{131}$. Admittedly, the critical writings of literary figures might seem to be a rather academic and elevated source of ethnographic doxa, but in the absence of primary data, these writings do have the virtue

\footnotetext{
130 Thompson (1990: 279) suggests using interviews, participant observation and other kinds of ethnographic research to "reconstruct the ways in which symbolic forms are interpreted and understood" at the moment of reception, but these suggestions obviously have to be modified for a historical study such as this. In this case, my own memories of the period, during which I was actively involved in alternative publishing and underground cartooning, constitute a kind of participant observation in retrospect. The interviews I have undertaken with cartoonists active in the period corroborate this personal experience, to which is added the examination of critical writing by politically engaged writers and cultural activists produced during the period.
}

131 There have been several important studies of related areas of cultural practice, notably by Gavin Younge (1988) and Sue Williamson (1989) on township and resistance art, Morris Smithers (1991) on poster art and Sabine Marschall (2002) on community mural art. 
of penetrating in a very direct way to the nub of the the issues that dominated the resistance discourses of the period.

\section{The intellectual milieu}

Writing in 1947, Jean-Paul Sartre described how French writers of the 1930's felt themselves to be abruptly 'situated' by the impending Second World War: "The detachment which our predecessors were so fond of practising had become impossible [...] History flowed in upon us [...] we had no choice but to produce a literature of a historical character "(1967: 157). Similarly, many artists and writers in South Africa during the last two decades of the apartheid era felt themselves to be swept along by powerful moral and ideological imperatives. It was as if, like Sartre, they 'had no choice'; as if 'history' itself had demanded that they abandon notions of artistic detachment and become embroiled in the unfolding drama of the anti-apartheid struggle.

This struggle saw a coming together of a range of political tendencies across a broad left wing, liberal, ethical and ecumenical consensus. Communists stood shoulder to shoulder with churchgoers, Muslim radicals with Jewish intellectuals, in the carnpaign to rid South Africa of the hated apartheid system. In this highly charged political atmosphere, artists and writers working in a variety of disciplines felt called upon to make some sort of contribution, however limited it might be, to the liberation struggle. This resulted in a remarkable flowering of art against apartheid and led, within the arts community, to a vigorous debate about artistic quality versus commitment, aesthetics versus propaganda ${ }^{132}$.

However, this is not to suggest that all white South African intellectuals heeded, or even heard, the call to political engagement. In white literary and artistic circles, there were many who either opposed the liberation struggle, or who deplored the use of art and literature for 'propagandistic' purposes ${ }^{133}$. And for the majority of the country's whites, the South African struggle was about maintaining their western, democratic way of life against the looming threat of communism. In a 1988 cartoon published in The Star, Andy (Dave Anderson) provides a compelling visual metaphor of how the state seemed able

132 See Younge's Art of the South African Townships (1988) and Sue Williamson's Resistance Art in South Africa (1989).

${ }^{133}$ As a student at the University of Natal during the 1970 s, I often observed this tendency amongst some of my lecturers. 

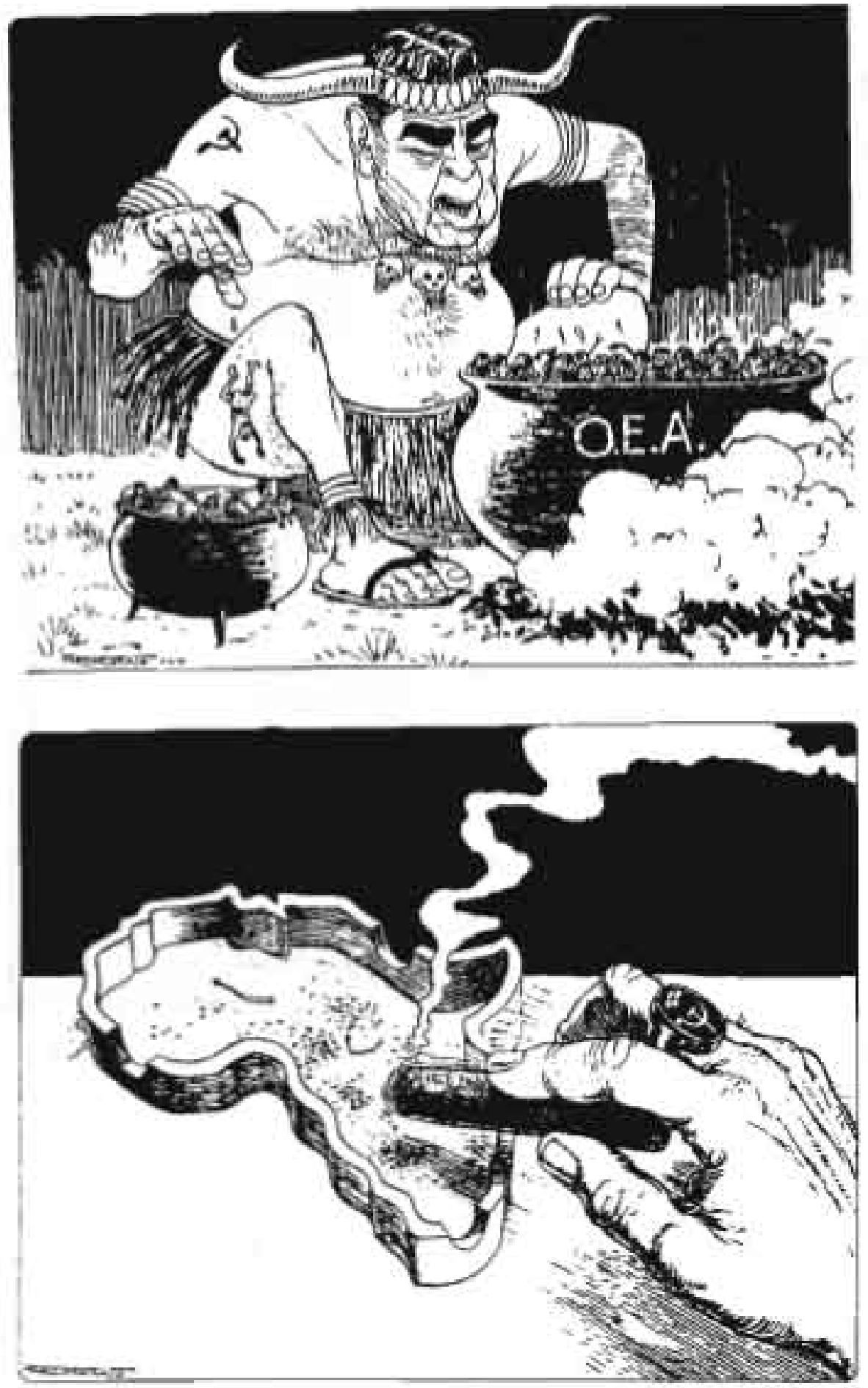

FGGURE 43

These two Mouton cartoons from Die Burger bniliantly express key aspects of the ideology propagated by the state during the 1970s-and accepted as 'common sense' by the majority of white South Africans. In the first (1975). Russian president Leonid Brezhnev is portrayed as a withchdoctor manipulating African leaders, while in the second (1976) a Soviet hand taps ash from a Cuban cigar into an Africa-shaped ash tray. (Vernon, 2000) 
to link all its critics, even the Christian ones, to Soviet communism (see Figure 53) ${ }^{134}$. In a 1986 cartoon in Die Burger, Fred Mouton depicts a hooded ANC puppeteer manipulating doll-like figures representing the anti-government UDF (United Democratic Front), 'boendoe howe' (kangaroo courts), 'onrus plegers' (rioters) and 'linkse meerlopers' (leftists) (see Figure 6, Chapter 2)135. This is a brilliant example of how the state (well served here by the cartoonist) invoked the anti-communist rhetoric of the Cold War in support of the government's 'total strategy' to combat the 'total onslaught' by Soviet communism against Western democratic values in South Africa. Black political unrest was portrayed as orchestrated by sinister 'instigators' linked to the Soviet quest for world dominance and the real causes of political unrest were displaced from their source in the lived experience of black people, who were depicted as faceless pawns in an international power struggle.

The dominant ideology propagated by the state during the 1970 s-and accepted as 'common sense' by the majority of white South Africans-is well expressed in Mouton's cartoons, produced for Die Burger where he worked from 1970 onwards. In a series of 1975 cartoons he depicts Russia's Leonid Brezhnev as a withchdoctor manipulating African leaders, or arming Fidel Castro lookalikes with AK 47 rifles to invade Angola136, while in a 1976 cartoon a Soviet hand taps ash from a Cuban cigar into an Africa-shaped ash tray (Figure 43) ${ }^{137}$. In a 1977 cartoon, Mouton celebrates the post-1976 security crackdown that resulted in the detention, interrogation and torture of thousands of activists and culminated in the death of Steve Biko and the banning of all Black Consciousness organisations. The cartoon shows a giant white fist squeezing the life out of a mockingly caricatured, stick waving black activist, indicating that the government has the situation securely 'in hand' ${ }^{\prime 38}$.

The events of 1976 revealed that blacks and whites lived on opposite banks of a deep existential chasm and that the realities of their lives were completely different. While domestic work provided black men and women with intimate insights into the bourgeois world of their employers, there were no similar opportunities for white

\footnotetext{
134 Anderson, D. The Cartoons of Andy, 1989: 33.

135 Mouton, F. Die Oosterlig, 1986. Reproduced in Vernon, 2000: 158.

136 Mouton, F. Die Burger, 1975. Reproduced in Vemon, 2000: 124-5.

137 Mouton, F. Die Burger, 1976. Reproduced in Vernon, 2000: 138.

138 Mouton, F. Die Burger, 1977. Reproduced in Vernon, 2000: 134.
} 
people to experience the realities of township life. This physical and social separation dwelt heavily on the minds of many of the country's intellectuals, and, as has happened often enough in history, it was the petit bourgeois writers, artists, students and activists who first bridged the gap, venturing into a contested ideological space where issues of identity were paramount ${ }^{139}$.

The need to offer up one's creative talents to 'the struggle', and in so doing achieve a reconstruction of identity, was a key theme for many white artists and writers. For those who worked alongside black activists, problems of credibility and legitimacy, of the need for 'permission' to speak on behalf of the oppressed black masses, were often a crucial issue, especially in the period between the rise of Black Consciousness organisations in the mid-1970s and the emergence of the non-racial ethos of the Mass Democratic Movement in the 1980s. While they rejected the hegemonic ideology of white South Africa, white intellectuals nevertheless often felt themselves to be excluded from the possibility of authentic identification with and acceptance by their black counterparts. This malaise was exacerbated by the tone of much Black Consciousness writing, which had little sympathy for the white liberal predicament:

[T]he young black writer cannot afford the 'white' luxury of indulging himself in art for art's sake as if it were a game for selfish, self-centered and hermit-like persons... because what we are involved in is something much bigger: the freedom of humanity. And that humanity is personified in the voteless masses of South Africa. (Mutloatse, 1980: 1-5).

This quotation is from the introduction to Forced Landing, a groundbreaking collection of Black Consciousness writings published in 1980 and edited by Mothobi Mutloatse. The stereotype that it presents of the white writer as selfish, self-centred, out of touch with the real world and obsessed with the notion of 'art for art's sake', is indicative of the racial hostility that existed within the literary environment at that time ${ }^{140}$.

139 In Writing and Being (1995), Nadine Gordimer describes this process: "We broke the easiest taboos first. Black musicians, teachers, journalists, aspirant writers met their white counterparts to talk, drink and dance- the two latter rituals standard as the preparation for many different kinds of human intercourse" (1995: 129).

${ }^{140}$ Mutloatse, a journalist at The Voice, a radical newspaper published by the South African Council of Churches, was a director of Ravan Press, a founding member of Staffider magazine, and a vocal member of the Johannesburg branch of PEN, the international writers' organisation. PEN meetings were held at the Staffrider offices in Johannesburg and attended by such luminaries of South African letters as Nadine Gordimer, Lionel Abrahams and Mike Kirkwood, alongside emerging writers of the 'Staffrider generation' such as Mutloatse, Ingoapele Madingoane, Jaki Seroke and Matsemela Manaka. This meeting of minds was brought to an end in 1982 when Mutloatse led a breakaway from PEN to form the African writers' 


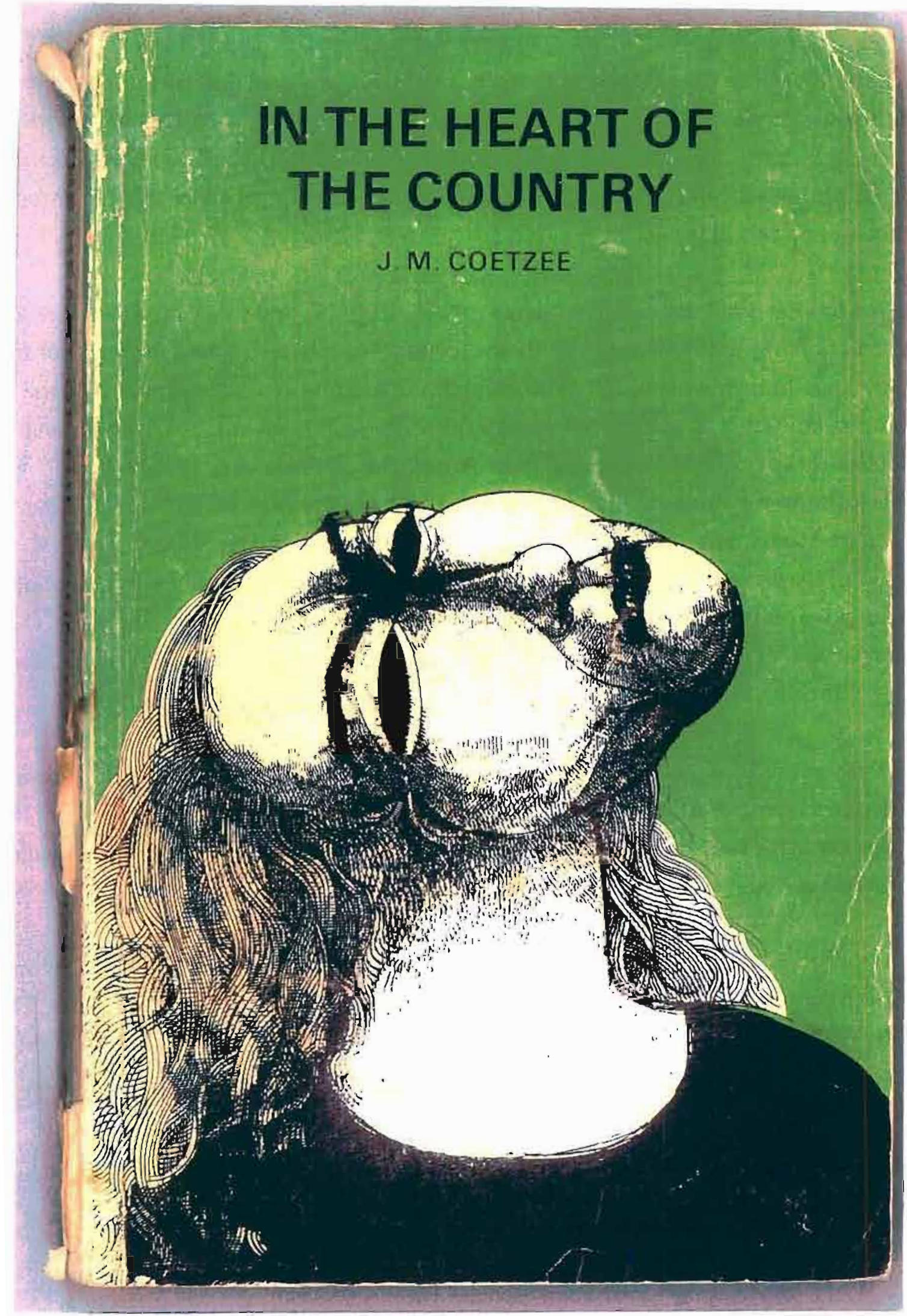

FIGURE 44

Richard Smith's cover illustration for J. M. Coelzee's in the Heart of the Country, published by Ravan Press in 1978 , portrays the novel's protagonist, Magda-a barren, melancholy spinster trapped in a bleak, symbolic landscape. 
In direct contrast to the aggressive posture adopted by Mutloatse as a spokesman for the Black Consciousness position is the anguished tone of Nadine Gordimer's (1995) description of the predicament of the white writer during the late apartheid period. Gordimer clearly envied the 'unproblematic' authenticity of the 'committed' black writer. Reviewing the work of Mongane Wally Serote, she wrote: "His revolutionary activity and his poetry are conditional for existence each upon the other as flesh is to blood" (1995: 38). By contrast, she saw herself as "one who belongs nowhere" (1995: 120). Having turned her back on the identity stamped upon her in "the white minority's fenced garden", she found herself without a coherent identity to replace the one she had spurned: "I had no lineal connection with the past around me" (1995: 118). Her complicity in apartheid, simply by virtue of being a white South African, was, she felt, aptly described by the poet-activist Jeremy Cronin as "this thing stuck to my feet" (1995: 37).

Gordimer felt that she could not be free to practice her craft while freedom was denied to others. For her, the project of reformulating a new South African identity to replace her racially-defined white identity involved "taking responsibility for what was being done in the name of white skin: what was implied was the obligation to oppose and destroy the power of racism in its seat of government" (1995: 133). But not all white intellectuals possessed Gordimer's steely resolve. What Parry (1998: 150) calls "the uneasy but timid white South African liberal consciousness" was prey to a complex mixture of emotional, moral, political and artistic concerns that varied widely from one artist or writer to the next. While some were motivated by the sense of a historical imperative or injunction to suspend artistic freedom in service of a higher goal, others were paralysed by the complex issues of identity and commitment they faced.

On the cover of J. M. Coetzee's In the Heart of the Country, published by Ravan Press in 1978, appears a compelling illustration by cartoonist Richard Smith (Figure 44) ${ }^{141}$. It is a portrait of the novel's protagonist, Magda-a barren, melancholy spinster trapped in a bleak, symbolic landscape. On the back cover of the book is an indistinct photographic portrait of the author-a disheveled, haunted figure, his face half in shadow. The prose of the novel, celebrated by literary critics as signifying "the instability of language and

Association (AWA) and from Ravan to establish Skotaville publishers, "a truly independent Black printing and publishing house based in Soweto" (Mutloatse 1999, quoted by Cloete 2000: 51).

${ }^{141}$ Richard Smith, cover illustration: Coetzee, J.M. In the Heart of the Country, 1978. 
the unreliability of narration" (Parry 1998: 150), is equally stylised and unsettling, reflecting on "an encounter in which the legacies of European modernism and modern linguistics enter the turbulent waters of colonialism and apartheid" (Coetzee, quoted by Parry 1998: 149).

First published in 1976, in the year of the Soweto uprising, just as the fabric of white South African dominance began irreversibly to fragment, In the Heart of the Country is an excruciating enunciation of the anomie and impotence of the white intellectual, caught, like a rodent in headlights, in an indeterminate interregenum:

My life is not past, my art cannot be the art of memory. What will happen to me has not happened. I am a blind spot hurtling with both eyes open into the maw of the future, my password 'And then?'. And if at this instant I do not look as if I am hurtling, it is only because I dither for a while in the empty house, feeling the comfort of the sunlight glancing off the same rows of copperware it glanced off before I was born into this world. (Coetzee, 1976: 43)

Like Gordimer, Coetzee's Magda is afflicted with the negation of identity, the sense of a hollow centre that cannot be filled: she sees herself as "a hole with a body draped around it... a hole crying to be whole" (1976: 41). But unlike Gordimer, she has no recourse to remake herself anew. There is no hope, just a spiralling descent into obsession and despair. It is a vivid portrait of the end of an era, of an unraveling hegemony.

This sense of indeterminacy, of a "suspension of vision", is noted by Elleke Boehmer (1988: 44) as a characteristic of South African English fiction in the last phase of apartheid. She speaks of "a cultural or artistic pessimism", of a "loss of will", of "a closing down or narrowing of possibility." The fictions of the 1980s appeared to "shut down on tomorrow", unable to take upon themselves the burden of prediction. "Hopedfor but as-yet-inconceivable, the long-delayed moment of liberation, too, forced its own particular hiatus at the end of the South African narrative. What lay beyond that moment was a gap, a space of which it was impossible to imagine the shape" (1998: 45).

Borrowed from Gramsci (1971), the notion of an interregenum, of a moment of history when the past has withered but the future has yet to take shape, was strongly prevalent in the early 1980s, a time when it seemed to many that the fortress of the apartheid state would take decades to breach. Boehmer describes the South African cultural landscape at this time as "[a] parched place, a society of dead-ends, closures, multiple restrictions 


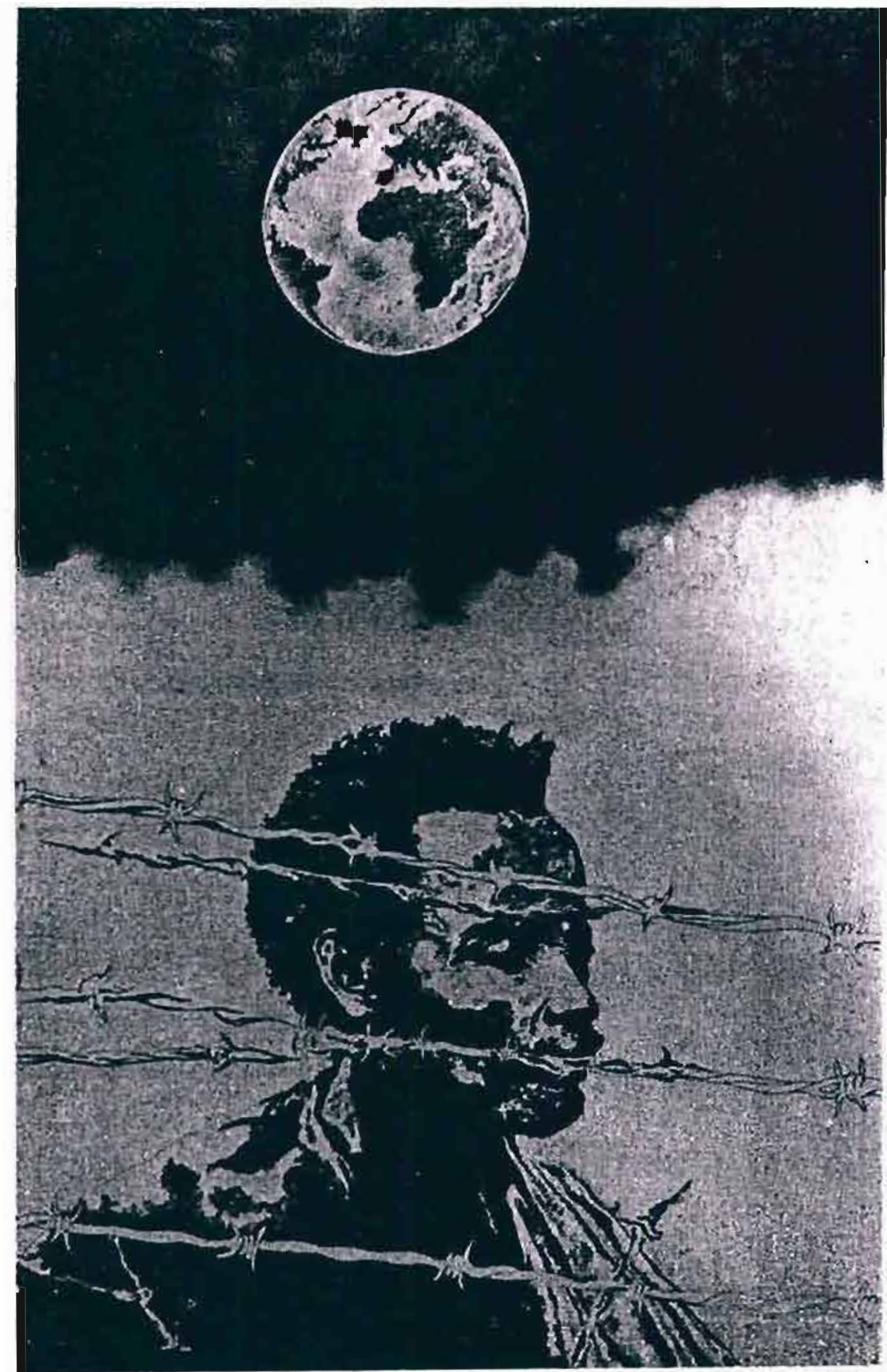

FIGURE 45

Staffrider, the most influential oppositional arts publication of the period, saw writers and artists as the voices of dispossessed communities. The art published in the magazine brought a visually symbolic dimension to the rhetoric of Black Consciousness, as in this drawing by Dikobe Marins, entitled "For My Brothers in Exile". (Ten Years of Staffinder, 1988). 
on speech and movement, blockages of every kind, spiritual and political" . She speaks of "States of Emergency of the mind, both those attributable to the state, and those created within the liberation movement itself' $(1988: 52)$.

Suspended in the "bloody interregenum" between past and future (Parry 1998: 163), unsettled by "the instability of language and the unreliability of narration", the highly stylised, reflexive liberal narrative is characterised by "paradox and impasse, gaps and silences" which are "the planned strategy of a highly self-conscious practice which displays the materials and techniques of its own process of production" (Parry, 1998: 150).

By contrast, the black writing of the period was characterised by "[t]he preference for a responsible realism aimed at reclaiming black history and registering black agency" and the expectation that black writers would "commit themselves to developing a purposeful, expressive, and accessible literature depicting oppression, illuminating the struggle, and serving to raise consciousness" (Parry, 1998: 160).

For black artists and writers, the problems of identity and legitimacy, of the need for permission to speak on behalf of the oppressed black masses, did not exist. They often saw themselves as 'voices of the people'. The notion of the writer as a spokesperson, a conduit for the feelings and aspirations of oppressed communities, underlay the ethos of Staffrider, the most influential oppositional arts publication of the period (Figure 54). According to the magazine's founder, Mike Kirkwood, "It used to be suggested, in the pages of Staffrider, that a writer was in some sense the voice of a distinct community, which 'spoke' to other communities via the network of those interlocutors and their readers" (Kirkwood 1988: 4).

In an essay published in Staffrider, Thamsanqa Mnyele (1988) argued that black artists should remain faithful to the utilitarian principles of traditional African art, overlaid with a kind of socialist realism:

That principle that governs traditional art is still valid today; i.e. that art must have a function: a walking song, the sculpture that serves as a chair, the majestically decorated houses of the Ndebelespeaking communities. The subject matter is drawn from the actual activities of the people in their living surroundings, the source and supreme function of art. We may go further and say that the actual act of creating the visual imagery is informed by the community and nourished by it, consciously or unconsciously, and that it is the community which will and must act as audience. (Mnyele 1988: 298). 
Like Mutloatse, Mnyele drafts the black artist into service on behalf of the oppressed African communities, from whose daily lives the subject matter and inspiration of the artist must be drawn and to whom the end product must be redirected. Mnyele goes on to deplore the parlous state of 'committed' African art in the country during the 1980s, calling for "a new calibre of cultural worker":

We must now create this new man and woman whose visuals and songs will be informed by the most pressing needs and demands of their time, place and circumstances: they ought to be articulate but simple so as to be accountable to their work and with clear political insight, a skilled hand and firm revolutionary sentiment. (Mnyele 1988: 299)

This kind of rhetoric, while representing a strong tendency within the militant cultural movement in the early 1980s, was not the only position adopted by black writers and artists. Chris van Wyk, who took over as editor of Staffrider in 1982, gave voice to a more critical, less racially exclusive position and an increasing concern with aesthetic issues (van Wyk 1988: 166). Whereas Mutloatse, writing in 1980, had declared war on establishment modes of criticism and literary norms, promising to "pee, shit and spit on literary convention" (1980: 5), by 1988 Njabulo Ndebele was advancing the position that the "high level of explicit political preoccupation" in black writing had led to a "superficiality" resulting from "the tendency to produce fiction that is built around the interaction of surface symbols" and thus, "instead of clarifying the tragic human experience of oppression [...] becomes grounded in the very negation it seeks to transcend" (Ndebele 1988: 329).

Two clear tendencies, linked to two opposing sets of formal and stylistic concerns, thus emerge from this troubled interregenum. On the one hand is the militant, optimistic school of 'committed' art and writing, associated initially with Black Consciousness, but becoming less racially biased as the 1980 s progressed. On the other hand is the anguished and contradictory white liberal position, suspended between identities and associated with the postmodern metafictions of J.M. Coetzee. To match the former with the stylistic concerns of mimetic realism, and the latter with the fractured, shifting forms of postmodernism, as though realism was black and revolutionary, while postmodernism was white and liberal, would be an oversimplification. But, in effect, this is precisely what Lewis Nkosi, a writer of some stature whose career stretches back to the days of Drum, suggests in an essay entitled "Postmodernism and black writing in South Africa" (1998): 
My purpose is to insist that in South Africa there exists an unhealed - I will not say incurable - split between black and white writing, between on the one side an urgent need to document and to bear witness and on the other the capacity to go on furlough, to loiter, and to experiment. (Nkosi, 1998: 75).

The contrasting tendencies that have been identified in the literature of the period are also visible in oppositional cartooning between 1985 and 1994. Evident in the cartooning of the period is the parallel existence of, on the one hand, the mimetic preoccupations of realism or naturalism, especially where comics have been used for conscientisation and political education, and on the other, the reflexive strategies of postmodernism, often invoked in the angry satire and confessional self-analysis of the 'underground' genres. Although there have been far fewer black cartoonists than black writers and artists, and although most of the cartoonists of the time were white, the opposing tendencies identified by Nkosi nevertheless express themselves across the field of South African cartooning.

Whereas Nkosi has suggested that these opposing literary tendencies may be linked to issues of race, in cartooning this bifurcation relates more to the ideological function of the texts in which these tendencies are visible than to the skin colour of the authors. Neo-realistic or naturalistic styles tended to be adopted where cartoons or comics were used for didactic purposes, whereas reflexive, postmodern approaches were generally associated with anti-establishment satirical cartooning. However, this is less than a rule than an observation, and there are many exceptions. The tension between what could be termed 'didactic realism' on the one side, and 'satirical experimentalism' on the other will frequently be observed in the analysis of South African cartoons and comics produced between 1985 and 1994. In some cases both tendencies may be shown to exist side by side in the same work. A couple of examples are advanced here to elucidate this point, but more will be presented during the course of the next two chapters.

Two educational comics published in the late 1980s, both of which have a clearly defined purpose and function-to educate the reader and produce the political effect of conscientisation---demonstrate the principle of engagement enunciated by Thamsanqa Mnyele in his Staffrider article: both are aimed at the dispossessed black community and both display "a firm revolutionary sentiment" (1988: 299). The first is a comic booklet 


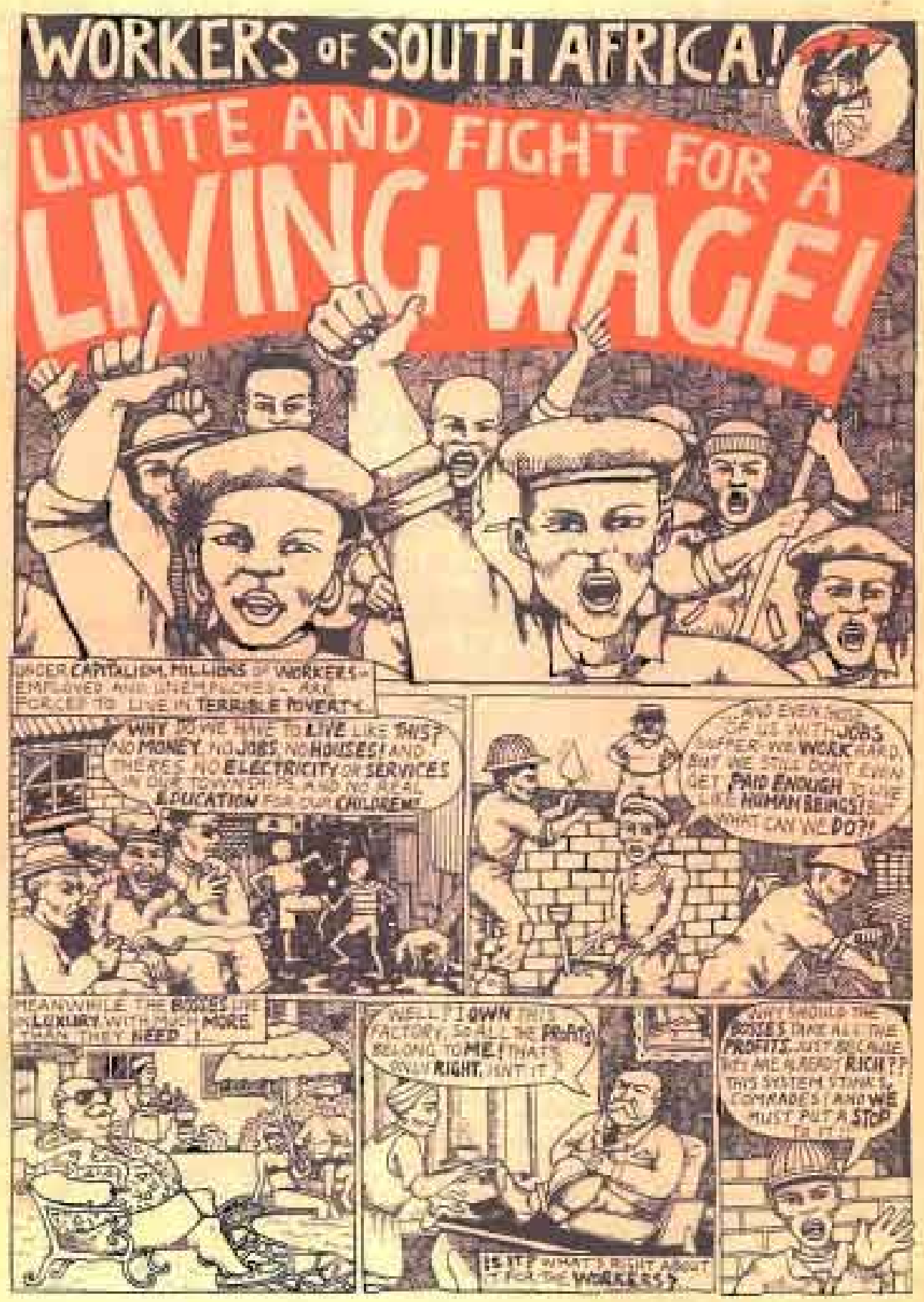

FIGURE 46

An unsigned and undated comic booklet published by Cosatu in 1989 combines a naive cartoon style with a didactic text, resulting in a product that functions primarily as a piece of propaganda

The comic employs several easily-recognisable South African cartooning codes- the Hoggenheimer code' satirises the white 'bosses', while what could be called 'the bantustan code' employs the convention of a shiry Mercedes roaring through an impoverished bantustan to satirise 'homeland leaders who collaborated with the apartheid regime. The black workers are shown as militant, nardworking and dignified, while the white bosses, policemen and politicians are viciously caricatured. 
published by Cosatu in 1989 (Figure 46) ${ }^{142}$ and the second is a comic book published by Sached ${ }^{143}$ in 1988 (Figure 47) ${ }^{144}$. In the first example, a cartoony style is combined with a tendentious didacticism, resulting in a product that functions primarily as a piece of propaganda. In the second, the preoccupations of fine art, and particularly those of naturalistic representation, result in a more complex product, the function of which is to conscientise and educate the reader while at the same time providing an aesthetically rewarding reading experience.

The Cosatu comic, which combines a naive cartooning style with revolutionary rhetoric exhorting workers to support demands for unemployment benefits, housing, a 40-hour week, job security, education, maternity leave and a living wage, employs several easilyrecognisable South African cartooning codes. What could be called 'the Hoggenheimer code' exhibits the conventions introduced 100 years ago by Daniel Boonzaier- suit, bald head, moustache and cigar-to satirise the white 'bosses', while what could be called 'the bantustan code' employs the convention of a shiny Mercedes roaring through an impoverished bantustan to satirise 'homeland' leaders who collaborated with the apartheid regime. Throughout, the black workers are shown as militant, hardworking and dignified, while the white bosses, policemen and politicians are viciously caricatured.

In the Sached comic, entitled Equiano: The Slave Who Fought to be Free, the artist, Rick Andrew, employs a studied naturalism deemed to be suitable for the story ${ }^{145}$, the seriousness of which is heralded by its portentous title. Prior to beginning work on the project, the artist was instructed by the editors to refrain from a satirical approach to the script: "[ $t$ he comic is aimed at black readers (9-14 years old) and we require a realistic, not ironic or satirical treatment of the story" (Andrew, 1988: 56) ${ }^{146}$. This instruction

\footnotetext{
142 Unsigned and undated, this pamphlet bearing the Cosatu logo makes reference to events that indicate that it was published in 1989.

143 The South African Council of Higher Education, a leading NGO in the educational sector.

144 Ozynski, J. and Perlman, H. Illustrated by Rick Andrew. Equiano: The slave who fought to be free, People's College Comics, Sached Trust, 1988.

145 Andrew (1988) produced this comic in partial fulfillment of his higher diploma in Graphic Design, and his record of the process provides valuable insights into the thinking of the comic's editors, who were extremely concerned with issues of political correctness.

146 Andrew subsequently employed a model to sit for him and "[m] ost of the figures and heads of Equiano were drawn directly to the page from life" (1988: 56).
} 


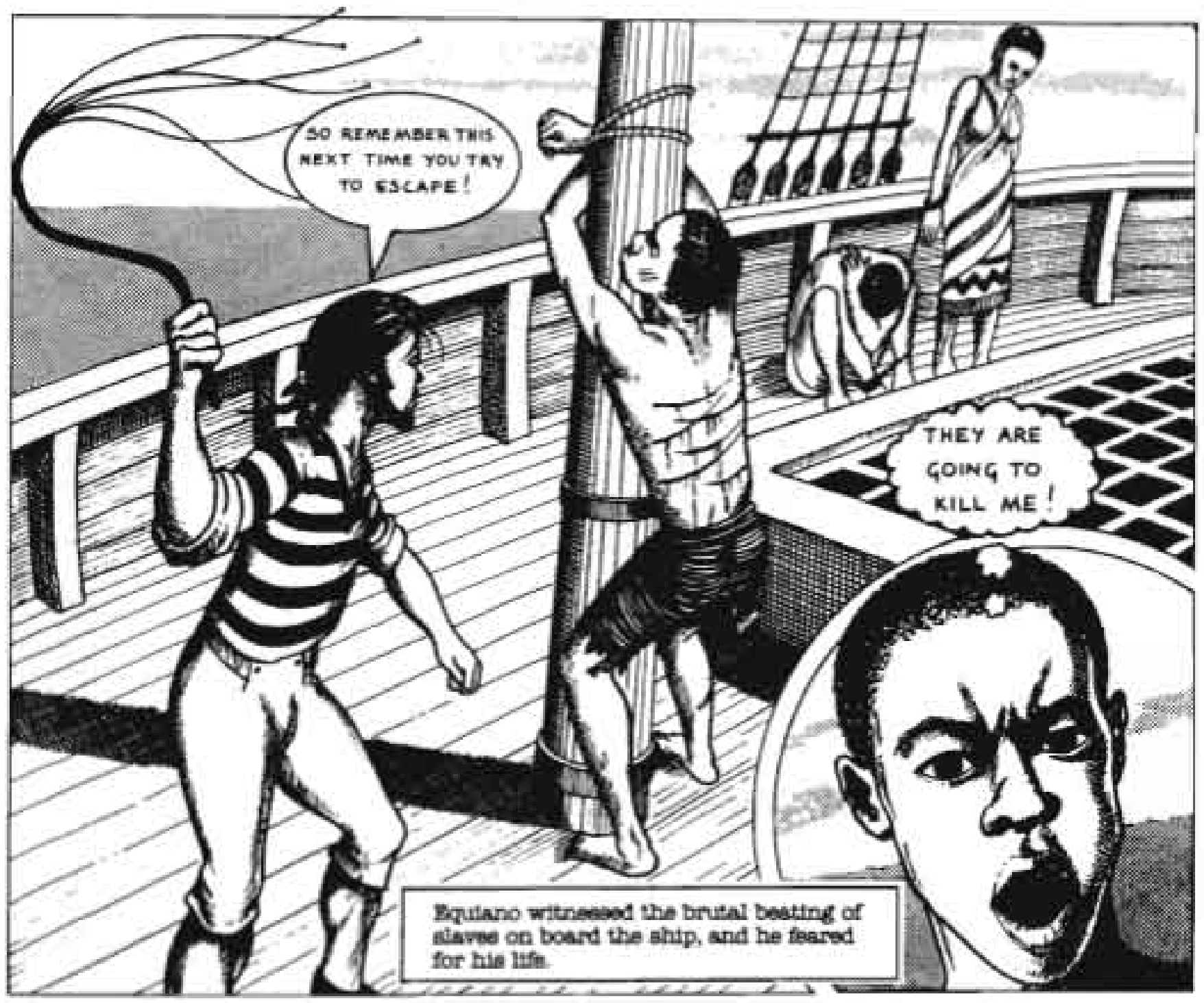

FIGURE 47

A page from Equiano: The Siave Who Fought to be Free, a comic book published by Sached in 1988 as one of a planned series of People's College Comics, and illustrated by Rick Andrew. Here the preoccupations of naturaliatic representation result in a more complex product, the function of which is to conscientise and educate the reader while at the same time providing an aesthetically rewarding reading experience. (Sached Trust, 1988.) 


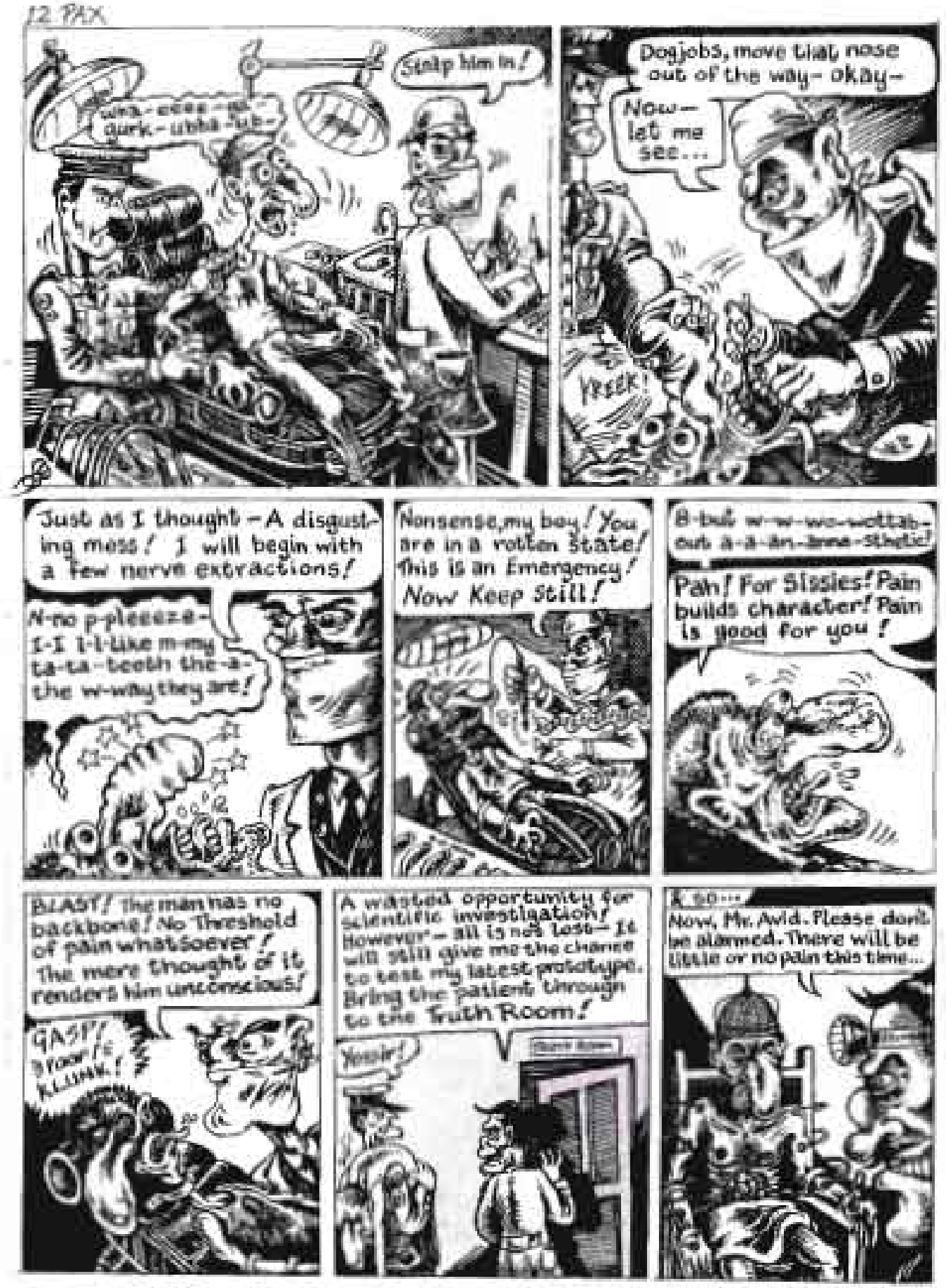

FGURE 48

A page from The Big Chïlum by N.D. Mazin (19e5-6), The protagonist (a neurotic philosophy lecturer) is subjected to a ludicrous interrogation by a demented security policeman.

(PAX. Pre-Azanian Comix, 1986) 


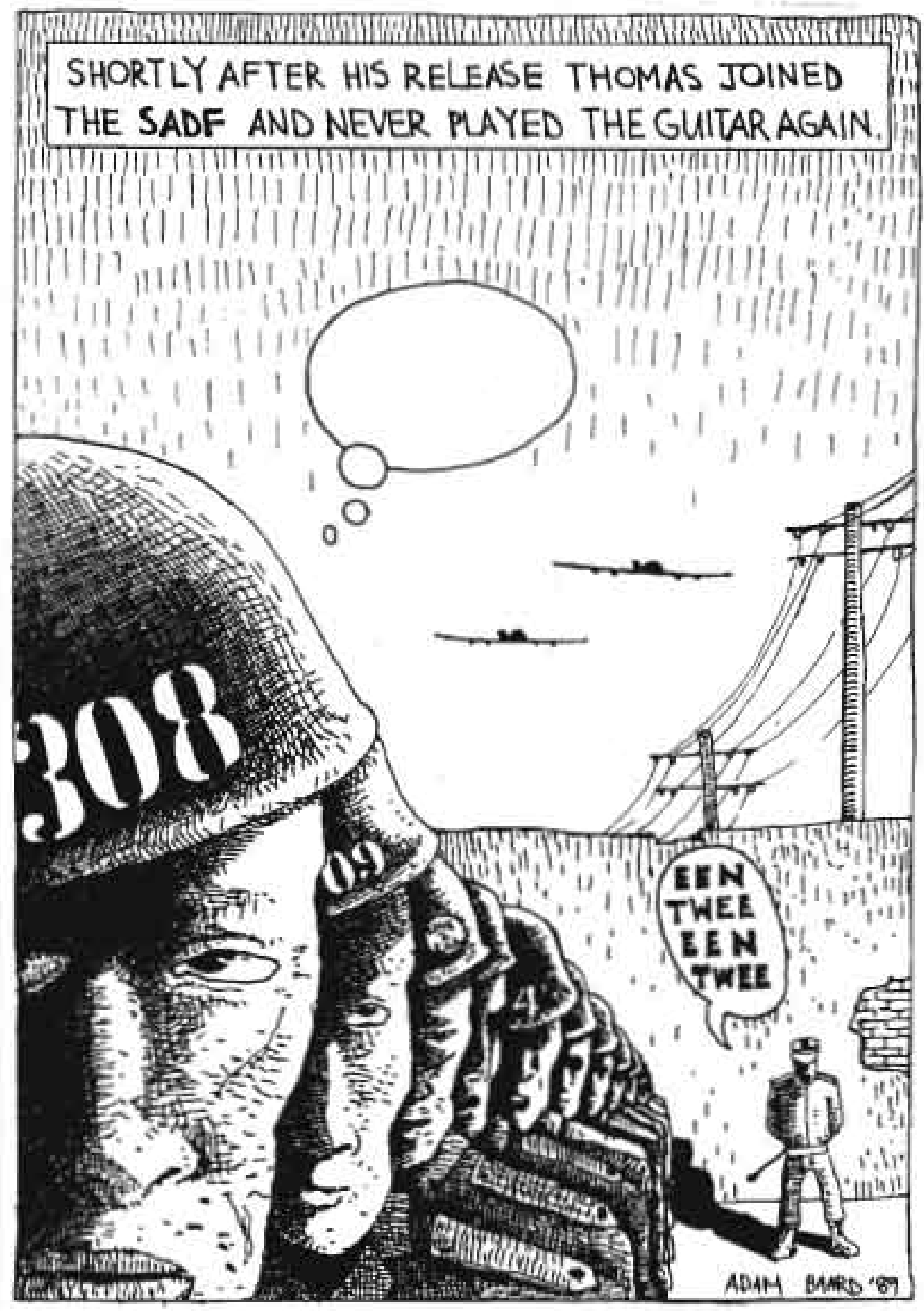

FIGURE 49

A page from Case No. 308 by Joe Dog (Anton Kannemeyer) and Adam Baard (Conrad Botes) (1989), published in the first issue of Bitterkomix in 1992 . The protagonist, an 'unstable' tock. gutarist, is brainwashed and reprogrammed into a compliant zombio by an evil military 
implies a conceptual link between the age and race of the targeted readers and the need for a realistic, rather than ironic or satirical treatment of the story, as though irony and satire might not be suitable for 9-14 year-old black children. This sombre viewpoint, which eschews irony or satire in the pursuit of pedagogical efficacy, has been a familiar one in the field of South African educational comic production and has resulted in some of the dullest comic strips ever produced in this country ${ }^{147}$. In this case, the instructions by the editors to the artist, and the constraints that these evidently placed upon him, resulted in a somewhat wooden, humourless treatment that is fortunately redeemed by the lyricism and appealing clarity of Andrews' drawings. Without recourse to humour or exaggeration-two of the most important defining characteristics of cartooning-Equiano is nevertheless a readable story with a strong visual signature that gives some sense of the history of slavery and the excitement of exploration and discovery in the $18^{\text {th }}$ century.

In contrast to these exemplary attempts to mobilise the comics form in service of social and political change are two examples of underground comix produced in the mid- to late 1980s. In "The Big Chillum" by N.D. Mazin (1985-6)148, the protagonist (a neurotic philosophy lecturer) is subjected to a ludicrous interrogation by a demented security policeman (Figure 48), while in "Case No. 308" (1989) by Joe Dog (Anton Kannemeyer) and Adam Baard (Conrad Botes), published in the first issue of Bitterkomix in 1992, the protagonist (an "unstable" rock guitarist) is brainwashed and reprogrammed into a compliant zombie by an evil military psychologist (Figure 49)149. Both strips are anguished expositions of white alienation, displaying Kafka-esque feelings of powerlessness, an inordinate fear of punishment at the hands of the apartheid state's security establishment, a self-immolating satirical tone, and a level of self-consciousness that tends towards the reflexive ${ }^{150}$.

${ }^{147}$ This topic will be presented in more detail in the discussion of educational comics in Chapter Six.

148 The strip was serialised in PAX (PreAzanian Comix) in 1985 and 1986. N.D. Mazin is a psuedonym used by myself.

149 Bitterkomix, No.1, 1992. The strip, the first produced by Kannemeyer and Botes, was drawn between 1998 and 1991. Botes uses several psuedonyms, most commonly 'Konradski'.

150 In Kannemeyer's more mature work, reflexivity becomes a predominant strategy and his work often"displays the materials and techniques of its own process of production" (Parry, 1998: 150). 


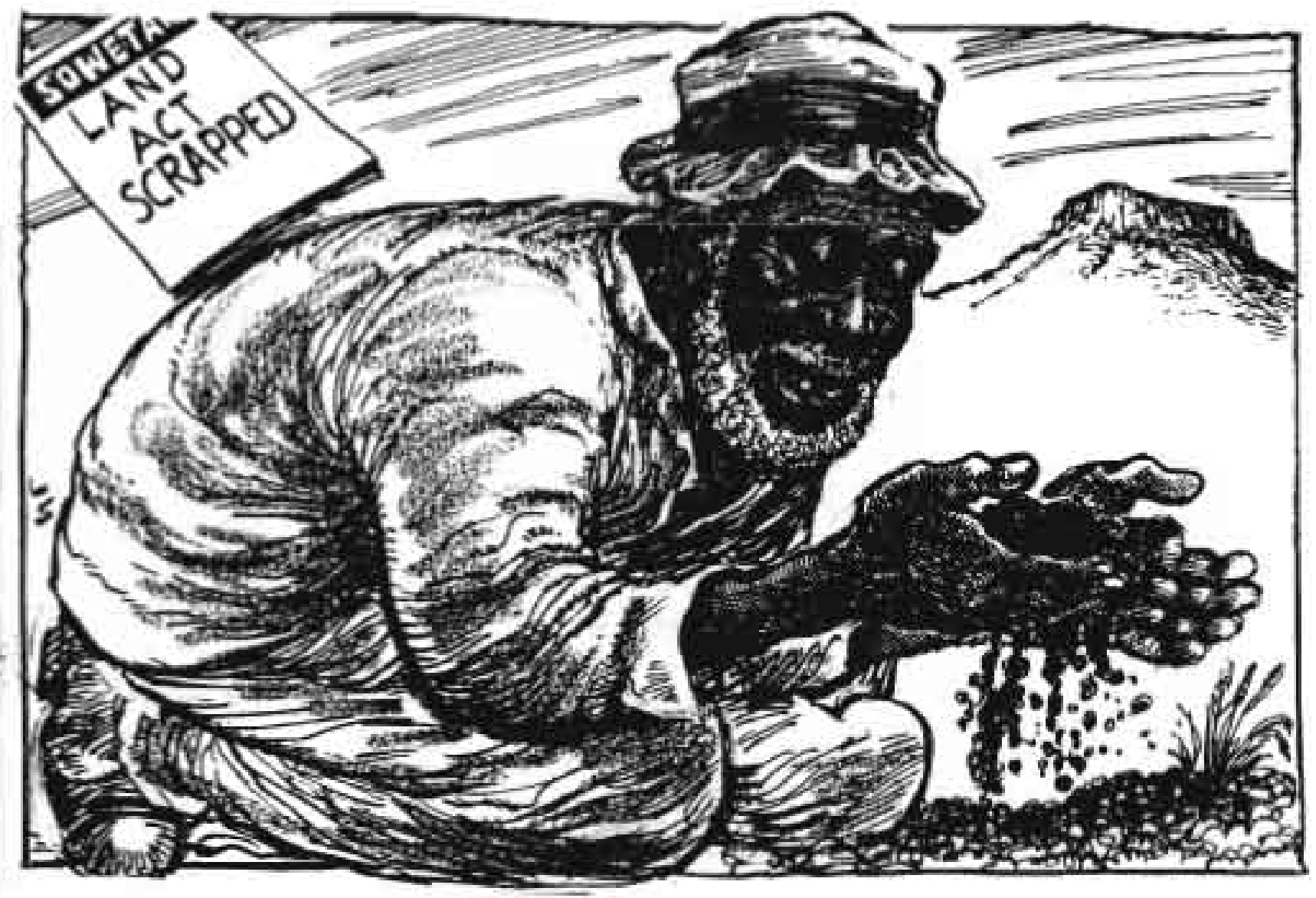

FIGURE 50

In Len SaK's Sowetan cartoons a mildly satirical but avowedly didactic tone is combined with varying degrees of realism and caricature, depending on the subject matter and mood the cartoonist aimed to achieve. (Sak, Jojo's World: Celebrating 33 years of of a Township Cartoon Hero, 1991.) 


\section{Key themes in oppositional cartooning}

The most important theme to emerge from these examples-and from the many to follow-is that during this particular period of South Africa's cultural history, cartooning practitioners, like practitioners in other disciplines, were passionately engaged with the political process. Cultural activists who would otherwise not have become involved in cartooning turned to cartoons and comics because they believed that these forms could valuably be used to communicate with semi-literate readers. However, the social, racial and class positions of individual cartooning practitioners were varied, and the responses of individuals to the challenges of resistance took a number of different forms, ranging from mild protest to fearless activism.

Because cartooning depends largely on the press for its distribution, the activism of individual cartoonists was channeled and to some extent defined by the publications in which their work appeared ${ }^{151}$. Alongside the stringent censorship laws in force during the period, a further element of self-censorship prevailed, as mainstream newspaper editors, publishers and their legal advisors sought to evade the consequences of publishing material that might result in banning or other legal action against them ${ }^{152}$. In 1982 the government consolidated its press laws under the Internal security Act ${ }^{153}$ and published a continually updated list of people who could not be quoted, which by 1990 amounted to some 500 names (Vernon, 2000: 157). In July 1985 the government declared a state of emergency over certain parts of the country which was momentarily lifted in 1986 and then immediately re-imposed to cover the entire country, lasting for the rest of the decade (Vernon, 2000: 160). Under these circumstances publishing in general and journalism in particular were extremely risky occupations. The sheer risk associated with openly expressing support for the liberation movement and committing anti-

\footnotetext{
151 Interestingly, Berger argues that during this period it is difficult to speak about a 'mass media' in South Africa, since the white press serviced white readers, the black press black readers, and the alternative media "reached little more than a leadership layer in the resistance" (2000: 73-4).

152 The more daring alternative papers had to face the consequences of their outspokenness. For instance, the ANC-supporting New Nation was repeatedly banned and its editor, Zwelakhe Sisulu, subjected to a three-year barring order and long periods of imprisonment, including eight months in solitary confinement (Vemon, 2000: 156).
}

153 The Act empowered the government to demand a deposit of up to R40 000 from newspapers if it believed it might be necessary to ban them at any time (Vernon, 2000: 156). 


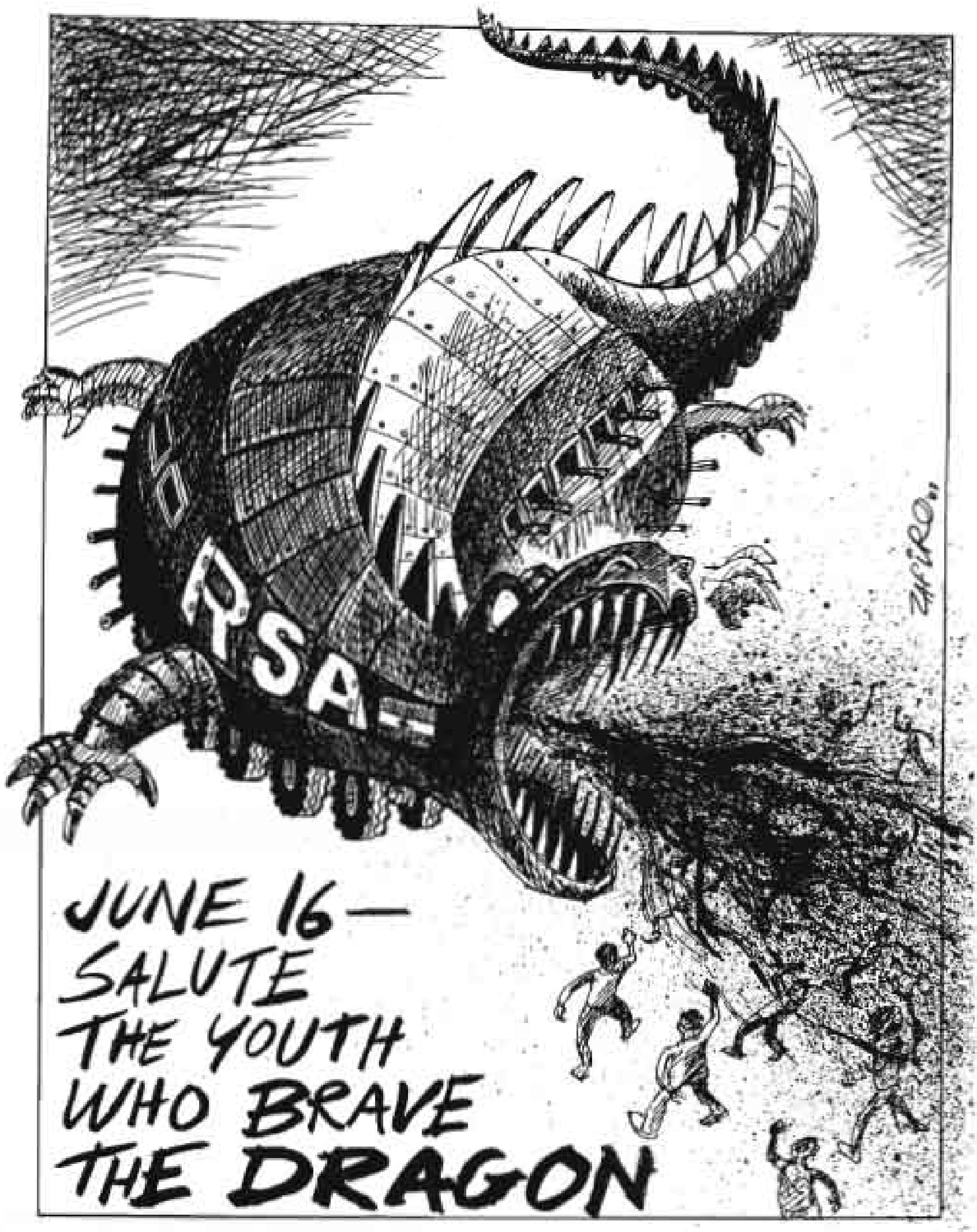

FIGURE 51

The work of the new political cartoonists to emerge in the late 1980s demonstrates a sense of passionate engagement, not only in the choice of subject matter, but in the actual physical vehemence with which these cartoonists committed their images to paper. (Above) A Zapiro cartoon from South, 1987 (Supplied by the cartoonist) 
apartheid sentiments and images to paper was undoubtedly one of the most important contextual factors that influenced the cartooning of the period ${ }^{154}$.

\section{Political cartooning in the mainstream opposition press}

In the country's mainstream opposition newspapers, the hardening of South Africa's political arteries after 1976 was signalled by a toughening up of cartooning activity, particularly at the extremities of the body politic. The editorial cartooning of the period displays the tension between didactic realism and satirical experimentalism in some interesting ways. In Len Sak's Sowetan cartoons a mildly satirical but avowedly didactic tone is combined with varying degrees of realism and caricature, depending on the subject matter and mood the cartoonist aimed to achieve (Figure 50) ${ }^{155}$. The work of Dov (David) Fedler in The Star and Andy (Dave Anderson) in the Rand Daily Mail and The Star ${ }^{156}$ was characterised by sombre tones, pessimistic black humour and the evolution of a tendentious inkiness that seemed entirely appropriate to a nation embroiled in conflict and acrimony (see Figure 52).

The lack of equanimity visible in the early caricatures of Richard Smith reasserts itself in several of Fedler's cartoons (see Figure 57) in the late 1980s and early 1990s. But it is in the work of the two most important new political cartoonists to emerge in the late 1980s, Derek Bauer and Zapiro (Figure 51) ${ }^{157}$, that the sense of passionate engagement is at its most visible, expressed not only in the choice of subject matter, but in the actual physical vehemence with which these cartoonists committed their images to paper. Borrowed from Ralph Steadman, but given a peculiarly morbid relevance in the South African context, is a recurrent tendency to splatter ink, as if it were blood, across the surface of the drawing.

\footnotetext{
154 Cartoonists who were detained in connection with their cartooning activities during the 1980 s included Zapiro (interview, 2001) and Mogorosi Motshumi (interview, 2001). Dikobe Martins, who is one of four black cartoonists listed by Schoonraad (1983), but was also a writer and political activist, was detained for a considerable period. Nanda Soobben describes how he was forced to leave the country as a result of his cartooning activities (interview, 2002).
}

155 Len Sak, from Jojo's World: Celebrating 33 years of of a Township Cartoon Hero, 1991.

156 Anderson also drew for The Natal Witness, The Evening Post and the Sunday Times.

157 There are two distinct phases to Zapiro's career: the first when he worked for the alternative Cape Town weekly, South, in the late 1980s, and the second following his return from his studies in New York in 1991. 


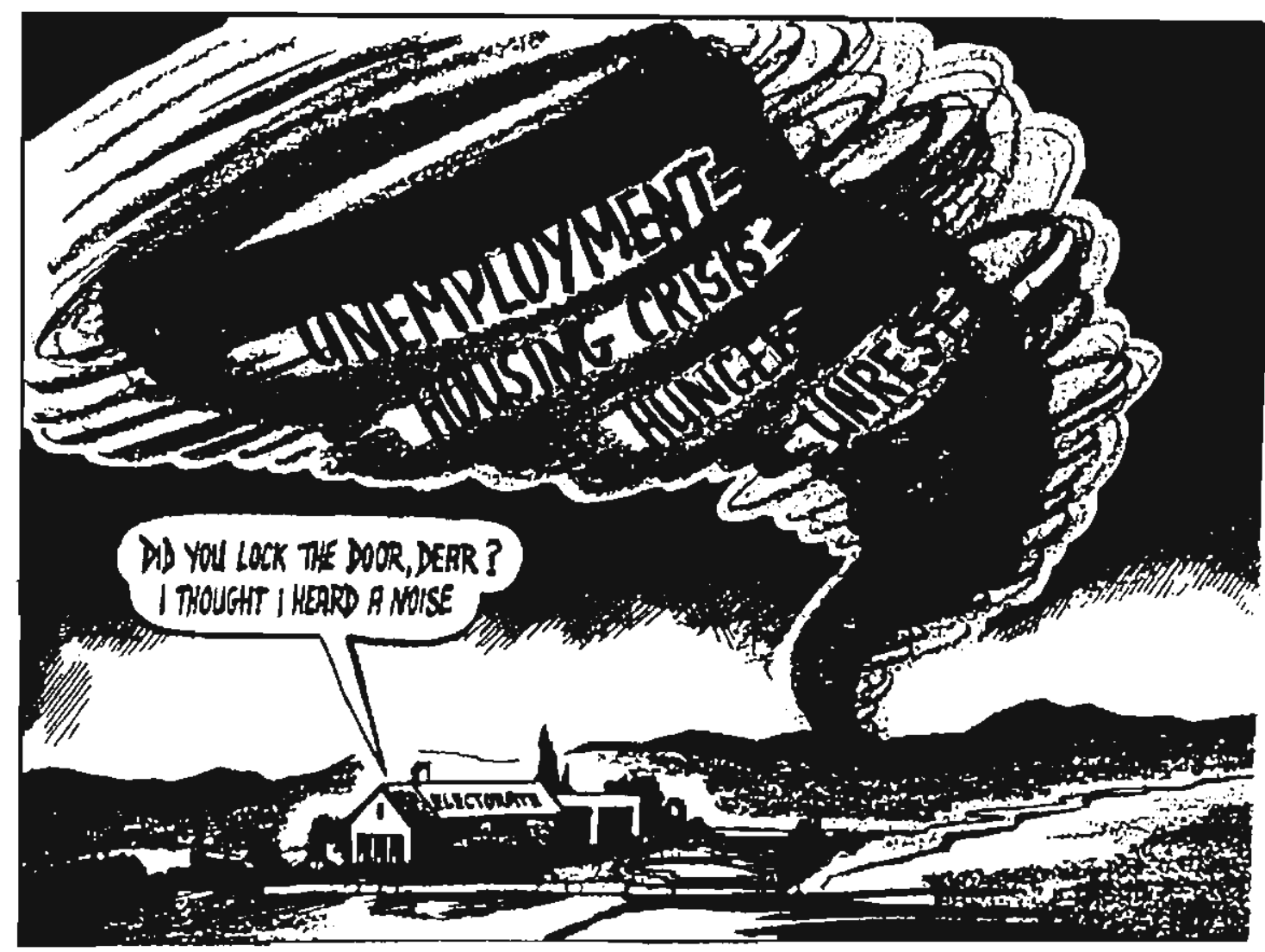

\section{FIGURE 52}

In this 1982 cartoon, reminiscent of Leyden's 1960 'Wind of Change 'cartoon (see Figure 39, Chapter 4). Andy (Dave Anderson) portrays the isolation of South Africa's white electorate as it languishes in blinkered trepidation in the path of the oncoming storm.

(Vemon, 2000.) 
When discussing the South African press in the 1980 s and 1990s, there are definitional problems that need to be clarified. Following Berger (2000), the distinction between 'mainstream opposition press' and 'alternative press', has been adopted, rather than the racial classifiction of the press into the 'white press' and the 'black press'. Berger's definition of the 'alternative press' is also followed, with the rider that any newspapers not included under this definition are regarded as being situated in the mainstream opposition press. Under this definition, black papers like Sowetan and Post Natal are defined as mainstream opposition papers, whereas New Nation and Umafrica are defined as 'alternative'. Several cartoonists, notably Len Sak, Derek Bauer, Zapiro and Stephen Francis and Rico of Madam E Eve, worked in both the mainstream and alternative press, while at least one newspaper, the Weekly Mail (which became the Weekly Mail $\mathcal{E}$ Guardian and later the Mail $\mathcal{E}$ Guardian), changed status from alternative to mainstream $^{158}$.

Leading cartoonists who were already working in the mainstream opposition press in 1985 included Richard Smith (Sunday Times), Len Sak (Sowetan), Andy (Dave Anderson) (Rand Daily Mail, The Star), Tony Grogan (Cape Times), Dov Fedler (The Star), and Nanda Soobben (Post Natal). Of these, Sak and Smith are discussed in Chapter Four. The leading cartoonists who began working in the alternative press but moved into the new mainstream were Derek Bauer, Zapiro (Jonathan Shapiro), (Alastair) Findlay, and Stephen Francis and Rico (Ricardo Schacherl) of Madam \& Eve. They will be discussed in Chapter Six in the context of the alternative press where their work began.

The sombre, heavily inked cartooning of Andy (Dave Anderson evocatively captures the politically-charged atmosphere of the harrowing mid-1980s period. In a 1982 cartoon, reminiscent of Leyden's 1960 'Wind of Change ' cartoon (see Figure 39, Chapter 4), Andy portrays the isolation of South Africa's white electorate as it languishes in blinkered trepidation in the path of the oncoming storm (Figure 52) ${ }^{159}$. Anderson began his cartooning career at the Natal Witness, followed by a stint as full-time cartoonist on the Pretoria News from 1979 until 1981. He then worked briefly for the Sunday Times

\footnotetext{
${ }^{158}$ Berger points out that that the Mail $\&$ Guardian was the only one of the alternative weeklies to survive into the new South Africa (2000: 94).

159 Andy, Evening Post, 1982, reproduced in Vernon, 2000: 147. Vernon identifies a subsequent cartoon by Fred Mouton, published in Die Burger in 1988, that follows up on the same theme (reproduced in Vernon 2000: 162).
} 


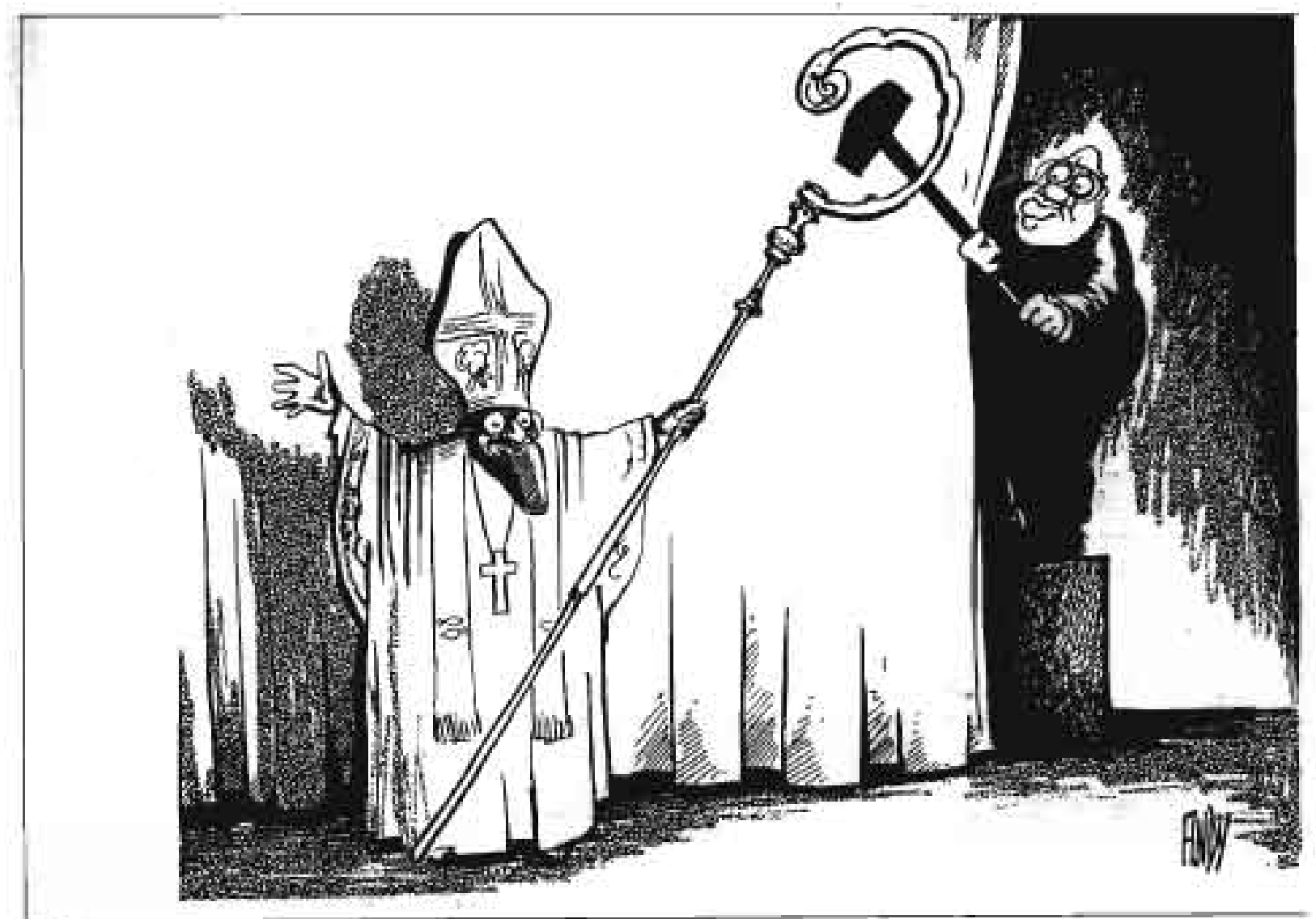

FIGURE 53

In this cartoon published in The Star, 21 March 1988, Andy (Dave Anderson) provides a compelling visual metaphor of how the state seemed able to link all its critics, even the Christian ones, to Soviet communism. (Anderson, The Cartoons of Andy, 1989.) 


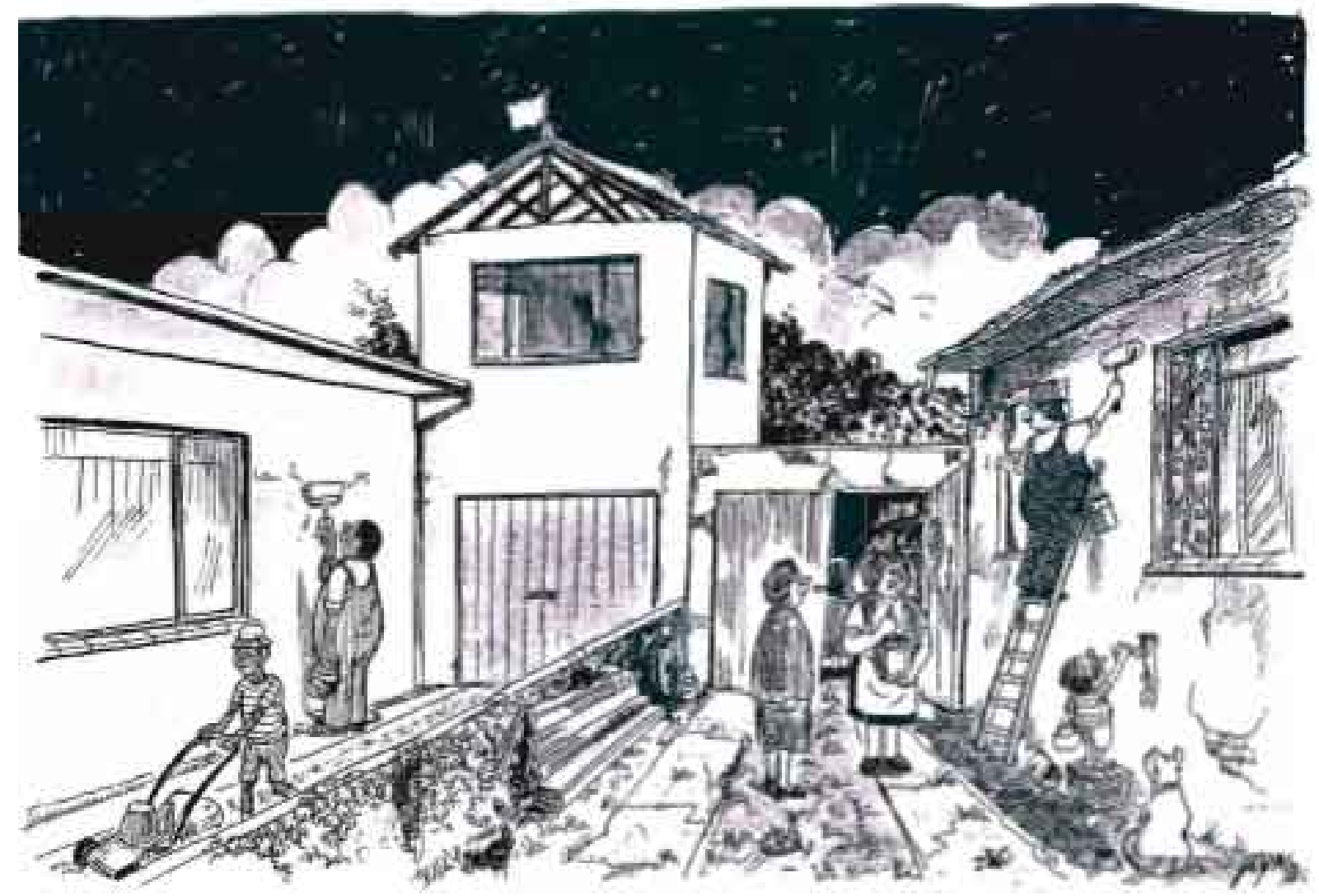

FIGURE 54

In direct contrast to the weightiness of Anderson's work is the much lighter and witter cartooning of Tony Grogan, who often produces beautifully sketched scenes in the Gies style.

(Cape Times, March 1993, reproduced in Grogan's 100 Best Cartoons, 2003.) 


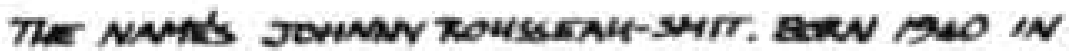

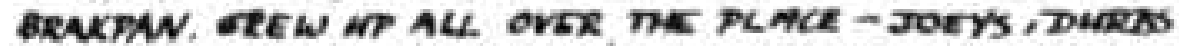

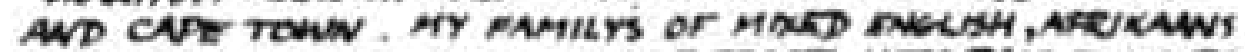

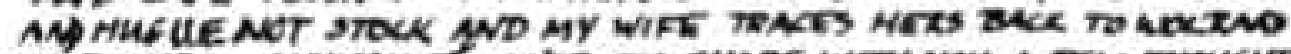

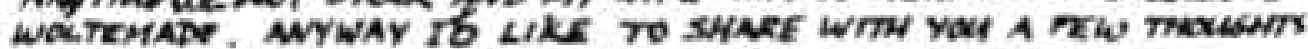
ABOUT TNIS CESAT LAND OF BLUE SXIES, BRAMIVLES, ZONECTHS. TRICANERAL TNELINANES, ROCKETINE PRKCES, STATES OF EMERGENCY, BIER AND RUEBY...

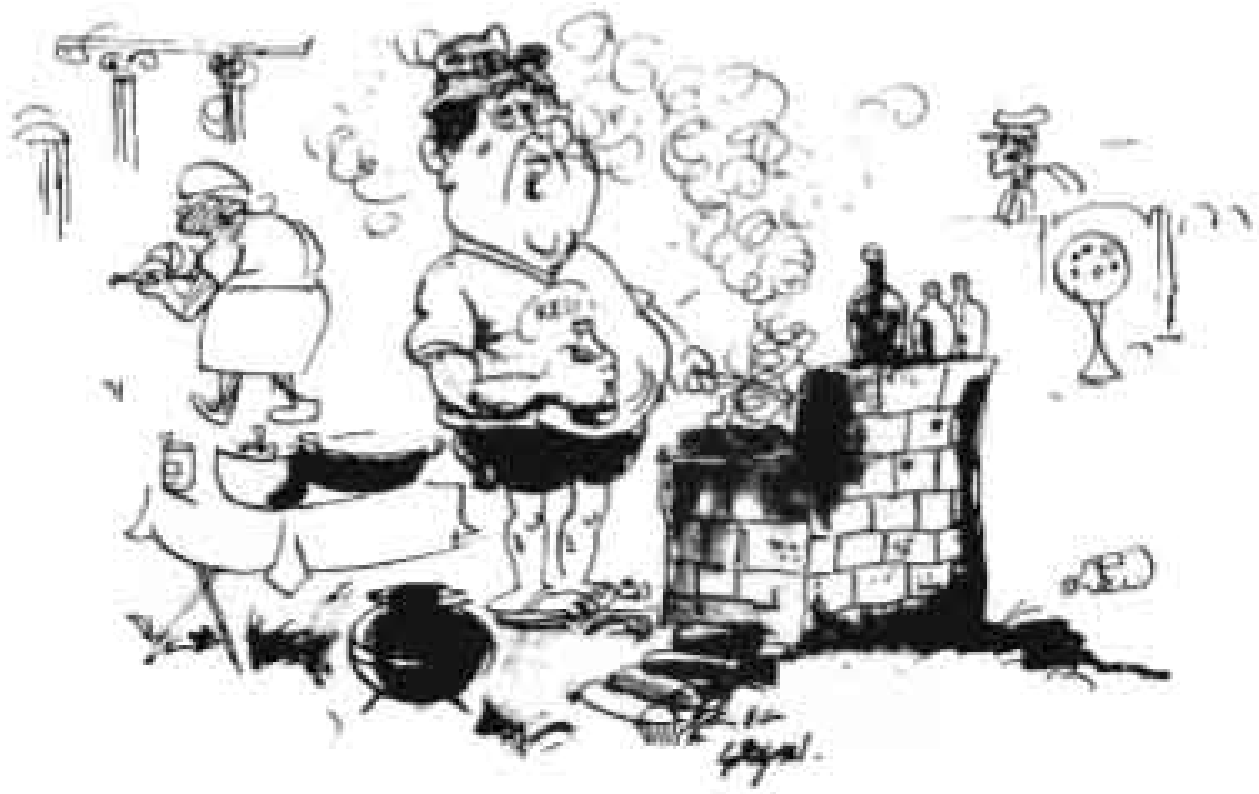

FIGURE 55

Grogan's character Johnny Rosseau-Smit is a typical" white South African male who exemplifies the zeitgeist of white middie-class South Africa in the late 1980 s. The viewpoint that the character articulates is conservative but remarkably tolerant of social change.

(From Grogan's South Africa, 1989). 
before taking up the cartoonist's position (previously held by Bob Connolly) at the Rand Daily Mail in 1982. After that paper's demise in 1985, he drew for The Star and Sunday Star. As Vernon points out, Anderson was quintessentially of the post-1976 school, not only in terms of his style, but also in terms of his political critique, which transcended the limitations of white party politics (Vernon, 2000: 140). According to Vernon, Anderson's cartoons were often "minor artistic masterpieces" that "revealed an intuitive political insight, a sharp and cutting wit, allied to a sense of social justice" (2000: 140) (Figure 53). His caricatures of the politicians of the day were "brilliantly cruel" and he "pioneered a new trend in cartooning [...] attacking not so much the NP per se as the ideology of apartheid itself" (2000: 140).

In direct contrast to the weightiness of Anderson's work is the much lighter and wittier cartooning of Tony Grogan, who began drawing for the Cape Times in 1974 and continues in that position to this day. Although Grogan's style was initially very inconsistent, ranging from beautifully sketched scenes in the Giles style (Figure 54) ${ }^{160}$ to messy scribbles, his robust sense of humour and political acumen have produced a large number of excellent political cartoons ${ }^{161}$. Grogan's cartoons of the period often adeptly revealed the logical absurdities that underlay the pronouncements and actions of National Party politicians.

In 1989 Grogan produced an unusual little book entitled Grogan's South Africa, which, despite its mediocre production values, provides a remarkable insight into the zeitgeist of middle class white South Africa at that moment in history ${ }^{162}$. The protagonist, Johhny Rosseau-Smit, a 'typical' white South African male (born in Brakpan, of mixed English, Afrikaans and Huguenot descent) offers the reader "a few thoughts about this great land of blue skies, braaivleis, boycotts, tricameral parliaments, rocketing prices, states of emergency, beer and rugby..." (Figure 55) ${ }^{163}$. The 'thoughts' attributed to Rosseau-Smit are a collection of standard white South African jokes, fables and urban legends mixed with Grogan's wry and often very astute observations of the political process,

${ }^{160}$ Grogan, Cape Times, March 1993. Reproduced in Grogan's 100 Best Cartoons, 2003.

161 Like Dave Marais before him, Grogan was a journalist before becoming a full-time cartoonist (Vernon 2000: 139).

162 This book provides exactly the kind of desirable ethnographic doxa of which Thompson (1990: 279) speaks.

163 Tony Grogan, Grogan's South Africa., 1989. 
interwoven with a racy potted history of the country since 1652 . The viewpoint that emerges is conservative but remarkably tolerant of social and political change. Just as "rocketing prices, states of emergency, beer and rugby" are grouped together in the introductory speech as though they were all of equal status, a wide range of issues that concerned white voters at the time-economic sanctions, sport boycotts, the government's political machinations, crime and violence, the collapsing economy and fear of the ANC-are thrown together in a kind of 'mixed grill' that the average middleclass white South African had no choice but to swallow, or emigrate. The sophistication of Grogan's humour lies in the self-deprecating realism with which the thoughts of his protagonist are presented.

More than any other South African cartoonist, Dov Fedler bridges the gap between postwar liberalism and the new radicalism of the post-1976 period. Fedler is a notoriously irascible cartooning professional whose sheer brilliance as a political commentator has often been eclipsed by his often-heard claim that he hates politics and only draws political cartoons because he has to ${ }^{164}$. Possibly as a result of this, Fedler has been wrongly typecast as "a cartooning mercenary" and an "uncommitted observer" of the South African political scene (Vernon, 2000: 120). But Fedler is a complicated, multilayered character and his self-deprecating stance is somewhat disingenuous. In his introduction to a 1991 collection of Fedler's cartoons, Arnold Benjamin, then assistant editor at The Star, made reference to "moods, temperament and bouts of introspection as well as periodic strokes of genius" and described Fedler as "a larger than life character". Adding to the Fedler legend, Benjamin claimed that, in his early years as a cartoonist on The Star, Fedler "didn't read the papers", sourcing his ideas instead from conversations with his newspaper colleagues, "drawing as he talked", either in his "gloriously cluttered" city centre studio or in a local delicatessen, where "he would rough out his ideas on a plastic table amid milk shakes and Mrs Plotkin's pastrami sandwiches"165.

In the introduction to a 2001 collection of his cartoons, Fedler describes how in the Lithuanian village where his father was born, the street lamps were still lit with oil. He also describes how, when he began cartooning in the late 1960s, he was "the new kid on the block, contending with giants such as Bob Connolly, Jock Leyden, Abe Berry and

\footnotetext{
${ }^{164}$ Schoonraad's opinion, based on interviews conducted in 1970, 1981 and 1982, is that Fedler had scant interest in politics, politicians or political cartooning (1983: 73). And, despite all the evidence to the contrary, Fedler told me the same thing during an interview in 2002.
}

165 Arnold Benjamin, intraduction to The Season of Violins, a 1991 collection of Fedler's cartoons. 


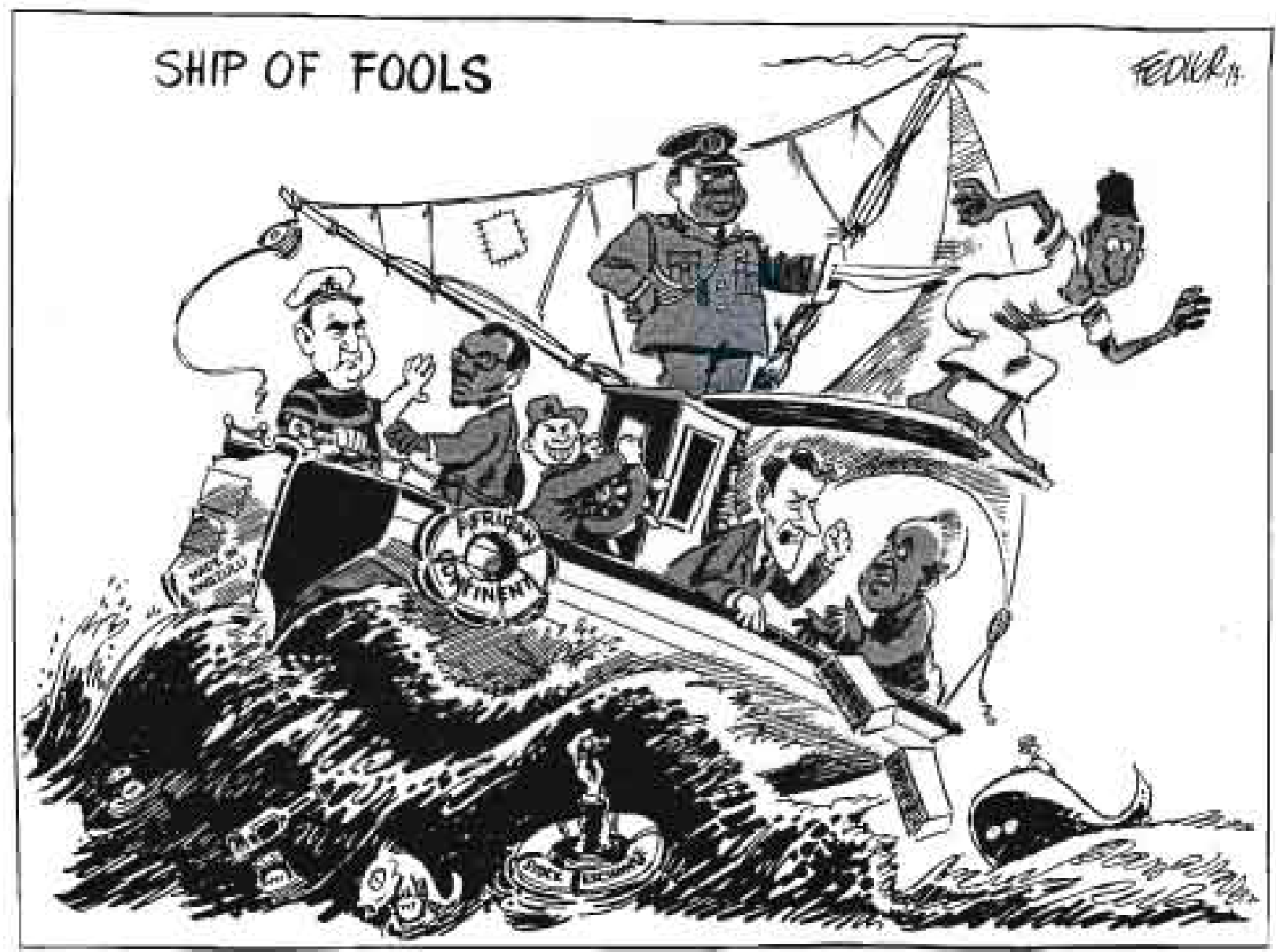

FIGURE 56

The precision of Fedler's early work, as in this 1973 cartoon, is contrasted against the vehemence of his work during the "ink and blood" period of the early 1990s (see Figure 67), but nevertheless displays his ingrained distrust of politicians.

(Fedler, My Son the Cartoonist, 1974) 
David Marais"166. These two statements situate Fedler concisely. Born in Johannesburg in 1940, he began drawing at an early age ${ }^{167}$ and as a young cartoonist identified strongly with the highly influential Jewish American comics creators of the postwar period such as Will Eisner (The Spirit), Jerry Siegel and Joe Schuster (Superman), Bob Kane (Batman), and Jack Davis and Mort Drucker of Mad Magazine, whose influence is strongly evident in his easy, accomplished style (interview, 2002). Fedler jokes that instead of "taking a left turn at Gibraltar", his parents should have gone on to New York, where he would have felt more truly at home ${ }^{168}$. Further evidence of this hankering after mid-century American popular culture are his satirical paintings featuring an imaginary fourth Marx brother, and his unpublished graphic novel, Gagman, set in New York ${ }^{169}$.

Fedler's first published cartoon appeared in Wits Wits, the Wits University student rag magazine ${ }^{170}$, in 1958 . Thereafter he worked in advertising, and began producing cartoons and illustrations for the Rand Daily Mail, the Sunday Chronicle and The Star. From 1963 his editorial cartoons began appearing regularly in The Star and a range of other papers (Schoonraad, 1983: 74)171 (Figure 56).

In the context of resistance cartooning in South Africa, Fedler's importance lies not in his espousal of a particular political tendency or his support for the liberation struggle. It lies rather in his curmudgeonly outrage against the vicissitudes of a socio-political environment stalked by corrupt politicians, incompetent public servants, rapacious businessmen and ruthless criminals. The viewpoint of white bourgeois South African society is clearly expressed in his work, but it is less the viewpoint of a political group

\footnotetext{
166 Dov Fedler, Introduction to Follow my Leaders, 2001.

167 According to Schoonraad (1983: 73), Fedler began drawing cartoons (of Hitler) when he was three years old.

168 Interview, 2002.

169 Fedler's Gagman is an unpublished illustrated manuscript, undated and unfinished.

170 Richard Smith's first cartoons also appeared in this publication.

171 Schoonraad (1983) lists, alongside those already mentioned, Sondagstem, New Nation, Scope, Signatures, Garden and Home, Zionist Recorder, The Motorist, Radio en TV Dagboek, Famioly Radio and TV and Medical Chronicle. He has also produced several serialised comic strips and illustrated a number of books. He continues to divide his time between newspaper cartooning and commercial work for advertising agencies, consumer magazines and corporate clients.
} 


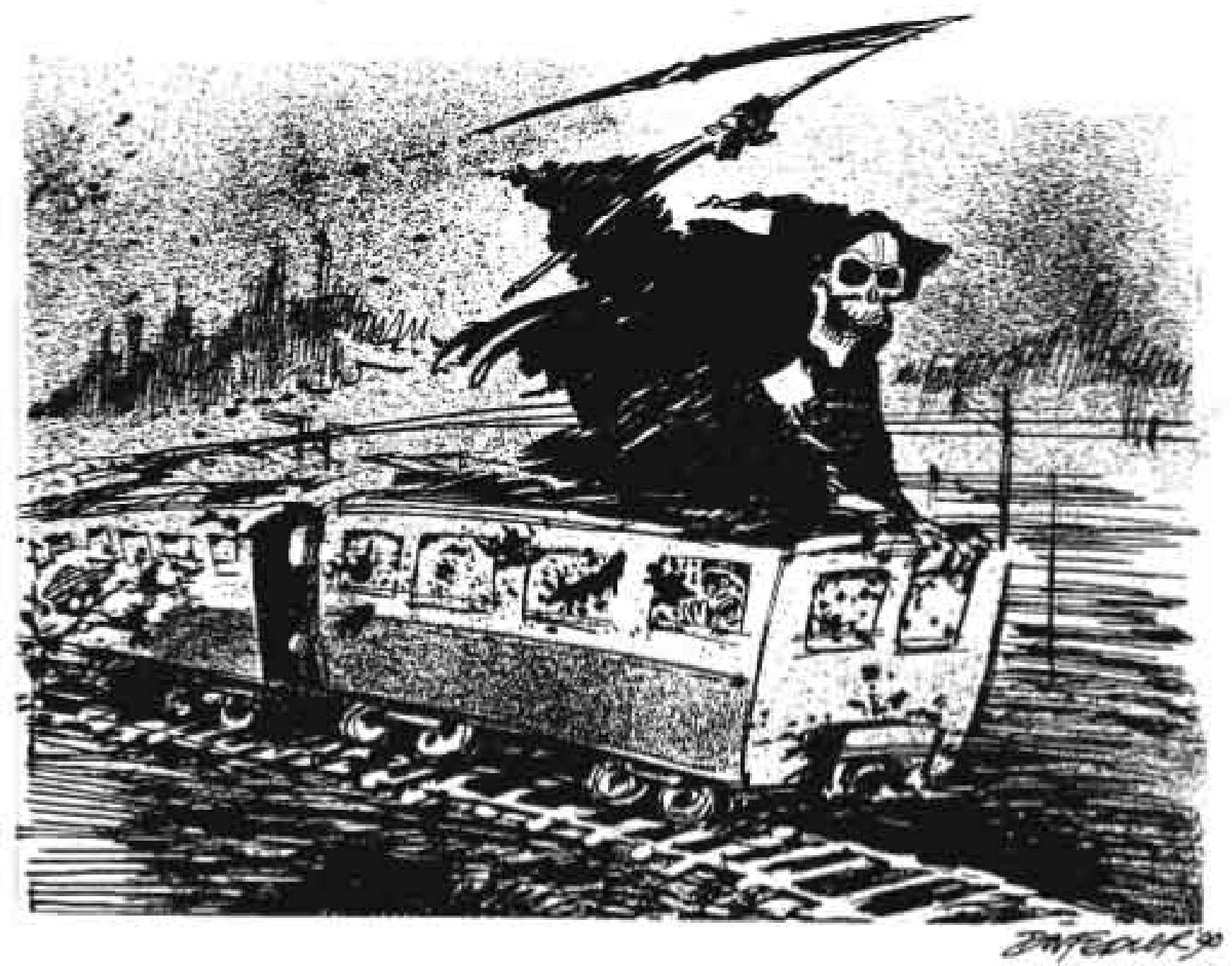

FIGURE 57

Like Bauer and Zapiro, Fedier gave expression to the bloody period of the early 1990 s with a string of ink-spattered cartoons crying out against the apparently meaningless violence that appeared to be tearing the country apart on the eve of the transition. It later emerged that this violence was the work of the state-sponsored 'third force'.

(Fedier, The Season of Violins, 1991) 
intent of maintaining power than the expression of a thoroughy modern angst: the alienation of the individual caught up in a Kafka-esque world that is almost impossible to understand or influence for the better. Paradoxically, unlike the world of the American cartoonists whom he so admired--and despite the pervasive pessimism of the $1980 s^{172}$ Fedler's world ultimately proved itself to be open to the influence of the collective will of individuals determined to change it for the better. The powerful emotional forces brought into being by the South African transition disallowed, if only momentarily, the distanced scepticism that perhaps more comfortably would have been Fedler's metier (and which he regained after 1994)173. There are numerous examples of his work from the late 1980 s and early 1990 s that display the passion of the politically engaged cartoonist (Figure 58), and in this respect, Fedler holds a special position amongst the opposition cartoonists of the post-1976 period.

It was in the pages of Post (Natal) that one of the first of South Africa's new generation cartoonists-and the first black cartoonist to make an impact in the mainstream opposition press-emerged. Born in 1954, Nanda Soobben studied art at the M.L. Sultan Technikon in Durban and began submitting cartoons to the Sunday Times Extra, a supplement aimed at black readers, in 1975 . In 1980 he began producing a regular weekly cartoon for Post ( $N a t a l)$, a paper targeted at Indian readers, dealing mainly with the "lighter side of news and sports"174. From his first cartoons, the young Soobben began bringing a more serious edge to his brief, often examining how the entrenched racism of South African life impacted on the Indian community. In one 1980 cartoon 175 an Indian golfer dresses up as a waiter so that he can collect his prize in the racially exclusive Greytown country club, while another ${ }^{176}$ bemoans the fact that after 120 years

\footnotetext{
172 The "cultural or artistic pessimism" of the 1980s is noted by Elleke Boehmer (1988: 44).

173 Vernon (2000: 120) presents the opinion that Fedler "remains at heart an uncommitted observer, a fatal drawback for the political cartoonist".
}

174 Post Natal, 18 October, 1980. Prior to 1980, Soobben had contributed cartoons to the Sunday Times Extra, where his first published cartoon appeared in 1975.

175 Post Natal, 16 November, 1980. This cartoon refers to the famous case of Papwa Sewgolum, an Indian golfer who had been forced to receive his trophy in the rain because blacks were not allowed into the clubhouse of the Royal Durban Golf Club.

176 Post Natal, 26 October, 1980. 


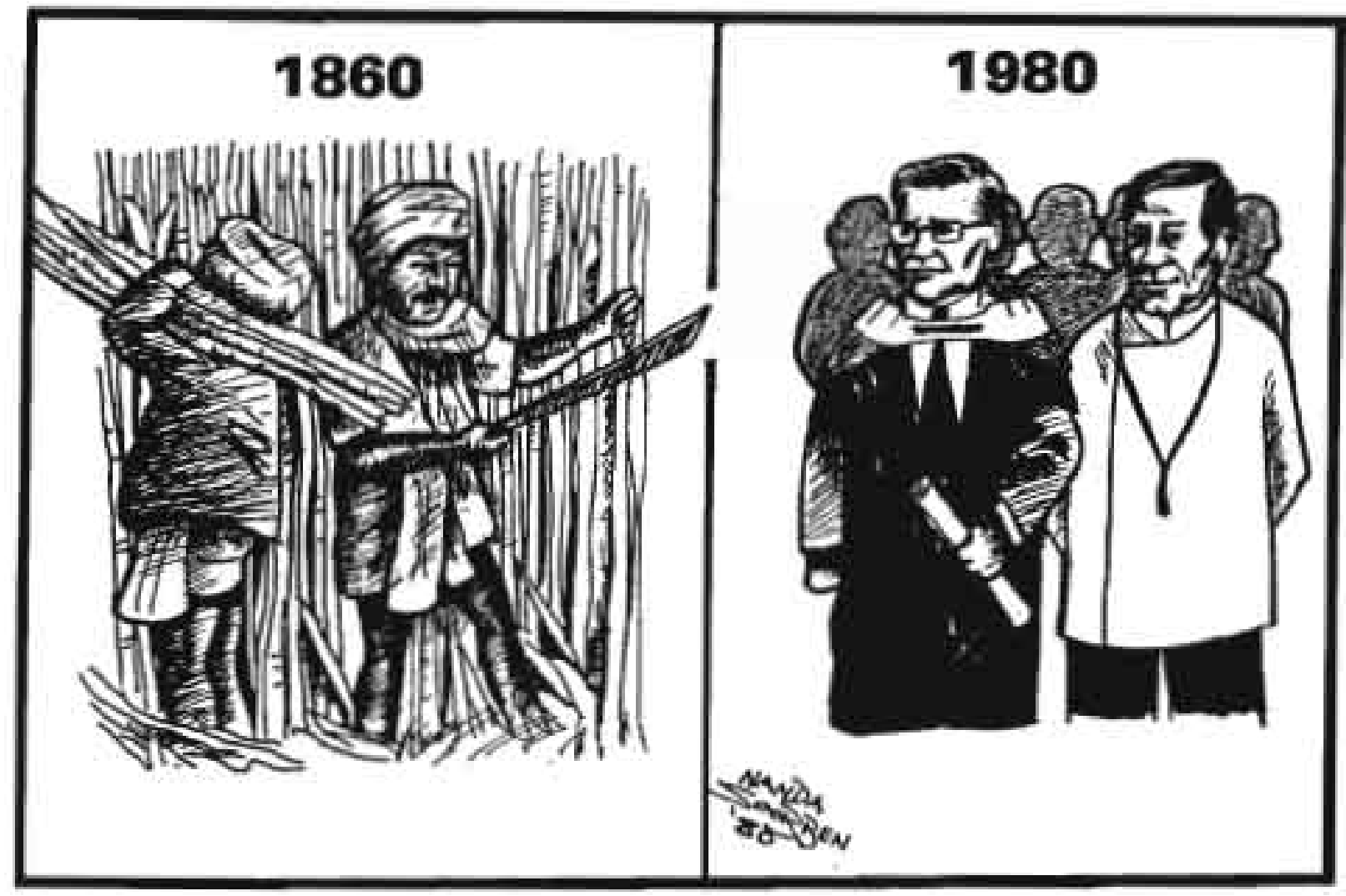

120 years of progress and stIII 2 nd class...

FIGURE 58

Nanda Soobben's cartoons in Post Natal often examined how the entrenched racism of South African life impacted on the Indian community. (Post Natal, 1980.) 
in the country, despite their achievements and contributions to South African life, Indians were still regarded by the state as second class citizens (Figure 58).

Soobben continued to draw for the Post until 2000, but with several interruptions. In 1986, finding the political situation intolerable, he left the country and experienced the peripatetic life of the political exile, living in Brazil in 1986-7 and in the USA from 1990 to 1993. In 1990 he exhibited his watercolours at the University of North Carolina, where he was profiled as an anti-apartheid cartoonist (interview 2002), and in New York, where he subsequently began studying computer animation at the Parson's School of Design. Returning to South Africa in 1993, he resumed drawing for the Post, and launched an independent graphic design, fine art and animation academy in 1995.177

Although he was absent from South Africa for most of the 1985-1994 period, Soobben is nevertheless important because his personal struggle is evocative of the kinds of difficulties faced by the few black artists who attempted to make a career out of cartooning in the 1980s. After his initial period as a weekly cartoonist at the Post, between 1980 and 1986, the continuity of his work was disrupted by the periods he spent out of the country, and it was not until the late 1990s that his career as South Africa's first mainstream black cartoonist was resumed in earnest. In 1998 he was appointed as political cartoonist on Durbar's newly established weekly newspaper, Independent on Saturday, and his full-colour cartoons and caricatures, in a bold and unusual style that combines strong outlines with cross-hatching and pencil crayon colouring, were prominently displayed in this paper. In the following year he was appointed to the Daily News, making him the first black cartoonist to be appointed to a mainstream South African daily newspaper. Sadly, the prominence accorded to his work in the weekly was not carried through into the daily, and the space provided for his cartoon was progressively reduced. Although Soobben's later work-particularly the full colour cartoons in the Independent on Saturday-is more polished, it lacks the wit and energy of his early work in the Post, where although his style was somewhat rough and inconsistent, his cartoons often exhibited the passionate engagement of the politically committed cartoonist.

\footnotetext{
177 Today Soobben's Centre For Fine Art, Animation and Design is a reputable academic institution from which several noteworthy young cartoonists have emerged.
} 


\section{Chapter Six}

\section{From Rebels at the Gate \\ to Icons of the New Mainstream: \\ South Africa's New Cartooning, 1985-1994}

\section{Introduction}

Within the severely constrained publishing environment of the 1980 s, there were three main streams through which oppositional cartooning was channeled: the mainstream opposition press, the alternative press (including NGO-based educational publications) and underground comix (including cartooning in the student press). Across these three streams, oppositional cartooning can be divided into three functional categories:

- counter-hegemonic satire and ridicule of the establishment;

- pro-social iconography supportive of the transition; and

- education and conscientisation around social and political issues.

The previous chapter looked at editorial and political cartooning in the mainstream opposition press against the background of the embattled ideological milieu of the 1980s. This chapter examines, against the same background, how the three functional categories of oppositional cartooning expressed themselves in the alternative press and in underground and student comix. It also looks at the structural and ideological conditions under which alternative press cartoonists and underground cartoonists operated, and how these dictated or influenced important aspects of their practice.

This chapter will demonstrate that the new phase of political cartooning in the alternative press after 1985 was characterised by much more extreme forms of counterhegemonic satire than had previously existed in South Africa, as well as the emergence of new forms of cartoon iconography that functioned to valorise the transition to democracy. While political cartooning was strongly satirical, the educational comic strips that emerged from the NGO sector in the 1980 s $^{178}$, were generally concerned with

\footnotetext{
178 These comics developed in the early 1990 s into a substantial, if momentary, publishing stream supported by social responsibility funding from the private sector and the state during the transition period.
} 
education and conscientisation around social and political issues, the preparation of people for democracy and the provision of pro-transitional iconography, and their publishers tended to eschew satire in favour of naturalistic styles and the expression of politically correct discourses. The link between these educational comic publishing intitiatives and the subsequent emergence of 'edutainment' television after 1994 is noted.

The chapter then examines the role played by the underground comix of the period. Because the alternative press was generally (though not always) located within the context of the emerging NGO sector, and thus subject to ideological constraints, some artists, inspired by the U.S. underground comix publishing movement of the late $1960 \mathrm{~s}$ and 1970s, chose to produce their own comix rather than position their work within the ideological parameters of existing alternative press publications. These underground comix were counter-cultural, giving voice to the fears and insecurities of increasingly marginalised white intellectuals, but not always counter-hegemonic, particularly with regard to their portrayal of gender issues, which in some cases tended to reproduce rather than challenge existing relations of domination in society. While oppositional political cartooning was generally pro-social in the sense that it supported the transition to the new South Africa, some underground comix had nihilistic, anti-social tendencies. These were not necessarily resistent to change, however, and could be seen as expressions of the psychic instability experienced by white South Africans during the traumatic early phase of the transition period.

In examining the functional categories of South African cartooning, it is necessary to draw attention once again to the relative lack of black cartoonists during the period, and in fact, across the entire 20th century. Amongst the 380 cartoonists included in Murray Schoonraad's encyclopaedic survey of South African cartooning, first published in Afrikaans in 1983, only four black cartoonists appear-Newell Goba, Mogorosi Motshumi, Dikobe wa Mogale (Dikobe Martins) and Nanda Soobben-whereas Ken Vernon mentions none in his review of newspaper cartooning in the $20^{\text {th }}$ century ${ }^{179}$. The

179 Schoonraad, 1983; Vernon, 2000. The rubric 'black' is used to include cartoonists of African, Indian and so-called 'coloured' extraction. 
black cartoonists of the period whose work is discussed in this study are Nanda Soobben, Mogorosi Motshumi, Mzwakhe Nhlabati and Percy Sedumedi. While it lies beyond the scope of this study to investigate in any detail the reasons for the relative non-emergence of black cartooning in South Africa in comparison with other African countries, it is nevertheless suggested that this lack was the result of the absence of an enabling environment ${ }^{180}$. A very brief argument will be advanced that draws upon comparisons between South Africa and other African countries where vigorous indigenous traditions of cartooning have evolved in the latter decades of the $20^{\text {th }}$ century as an integral aspect of locally produced popular culture ${ }^{181}$. It will be argued that the relative lack of black cartoonists to illustrate those products aimed at communicating educational messages to ordinary black people in the townships, informal settlements and 'homeland' areas, impacted negatively on the effectiveness of those initiatives.

\section{Ink and blood in the alternative press}

As has been observed above, the new, radical generation of South African cartoonists came not from the mainstream press with its long history of complicity in the economic system that underpinned the apartheid state ${ }^{182}$, but from the newly emergent 'alternative' press 183 . A relatively short-lived phenomenon, lasting from the late 1970 s to the mid 1990s, the alternative press consisted of a range of oppositional newspapers, magazines and publishing projects that were financially supported by external funding agencies and were an expression of both the broad-based international anti-apartheid movement and the internal democratic movement. Berger uses the term 'alternative

\footnotetext{
180 See Mason, A. (2001.1) "Black \& White in Ink: Discourses of Resistance in South African Cartooning" (African and Asian Studies, Vol. 1, No. 4, 2002) for a fuller treatment of this topic. This article, which also appeared in Africa Mediterraneo No. 38, 2002, contains several regretable errors, particularly the incorrect spelling of the names Nhlabati and Soobben. These errors are corrected in this study.
}

181 See Packalen 1999; Packalen \& Odoi, 1999; Nyamjoh, 1999; Mason 2001.2.

182 According to Berger, the privately-owned liberal press was regarded as "an arm of big business, interested primarily in making profits rather than in democracy or serving the people" and "stood accused of actively sheltering whites from knowing the full human cost of apartheid and -even worse-charged with disorganising and disinforming members of the oppressed who read them" (2000: 78).

183 This aspect of South Africa's press history has been extensively researched and documented. See Louw and Tomaselli (1991), Berger (2000) and Cloete (2000). 
press" to refer to publications "which were pitted in direct opposition to racism in South African society, including opposition to racist publications" (2000: 73). He points out that the alternative press is not the same as the black press, since, as discussed in the previous chapter, there were several mainstream black papers.

In many cases, external donor funding sheltered the alternative press and the NGO publishing sector of the 1980s and early 1990 s from the imperatives of economic sustainability, allowing them to become the site of a variety of bold experiments in popular communication for social change, including the use of cartoons and comic strips for political education and conscientisation. Many of these publications and publishers were repeatedly targetted by the state and subjected to bannings and other forms of harrassment, but their flexibility, strong community links, political acumen and access to funds gave them a guerrilla-like elusiveness and tenacity that fitted perfectly into the embattled political environment of the 1980s.

Unfortunately, the strengths that enabled alternative publications to survive in the turbulent last decade of apartheid proved to be less useful in the mercenary postapartheid period, where the lack of business skills amongst their managers and editors contributed, with one or two notable exceptions, to their demise. In addition, according to Cloete (2000: 68): "[t]he dispersal of intellectuals and activists into the State accompanied by a kind of political exhaustion played a major role in the collapse of the alternative publishing sector", along with "the transfer of international donor funding to State institutions during the transition period". The most successful of the alternative publications, the Weekly Mail, survived by virtue of its strategic amalgamation with the British Guardian (Berger, 2000: 94).

It was in the alternative press and particularly in the pages of the Weekly Mail that South African political cartooning began to find a new voice in the 1980s. The equivocal stance of the so-called 'liberal' papers was aggressively challenged by the Weekly Mail in its early years, creating an environment for a more vigorous cartooning to emerge. This new energy was exemplified in the unrestrained ink-spattered cartoons of Derek Bauer, who, even more than the early Richard Smith, was stylistically influenced by Ralph Steadman and Gerald Scarfe. In Bauer's work, ink often stood for blood, in which many 


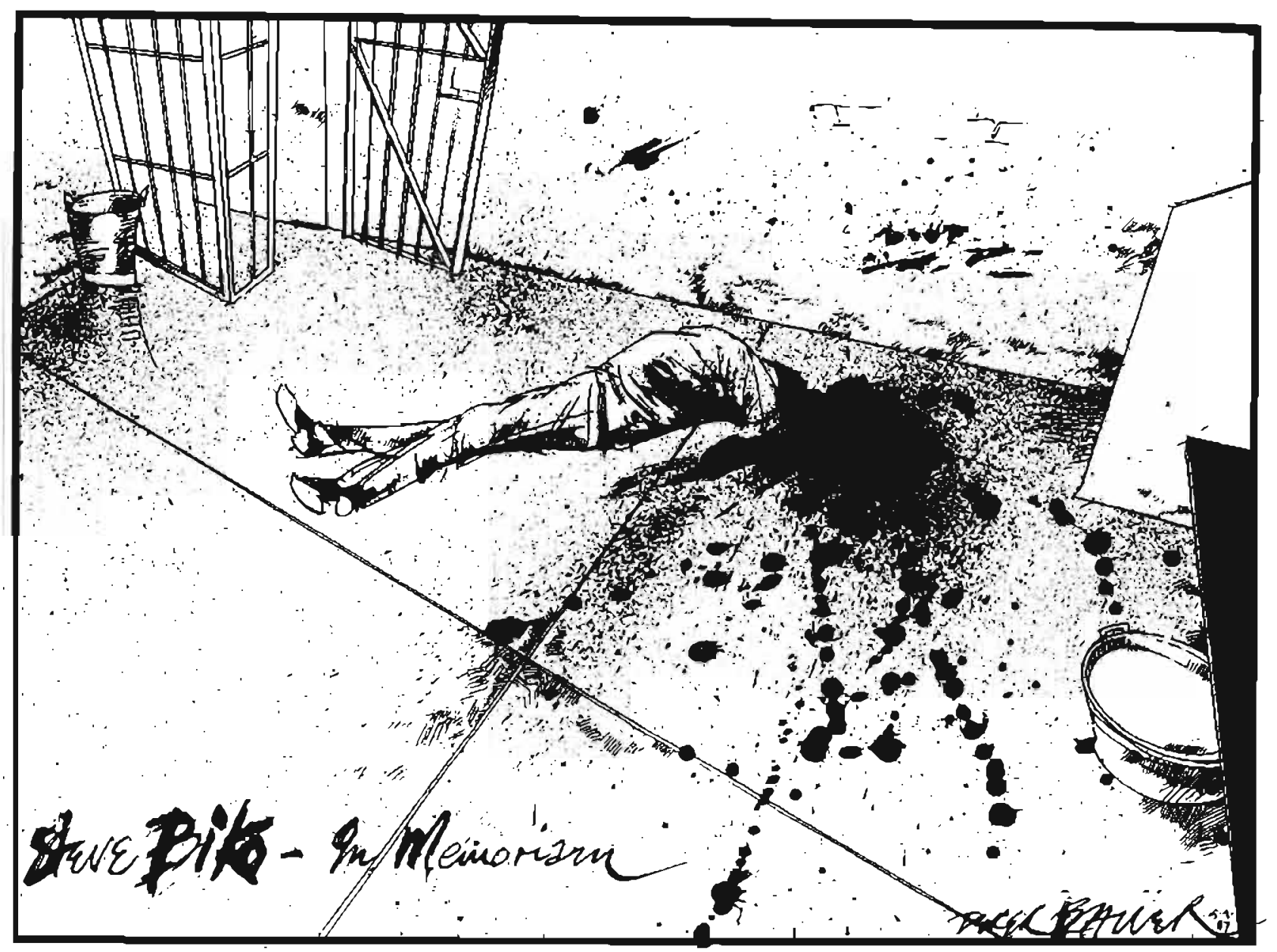

FIGURE 59

In Bauer's work, ink often stood for blood, in which many of his cartoons were drenched as South African society began to tear itself apart. This 1987 commemoration of ten years since the death in detention of Steve Biko provided an opportunity for Bauer to use the ink spatter technique in an extreme statement of horror and revulsion. The rigid, stylised prison bars add to the horror of the scene by providing a contrast to the apparently unrestrained inkblots.

(Bauer, S. A. Flambe and Other Recipes for Disaster, 1989.) 


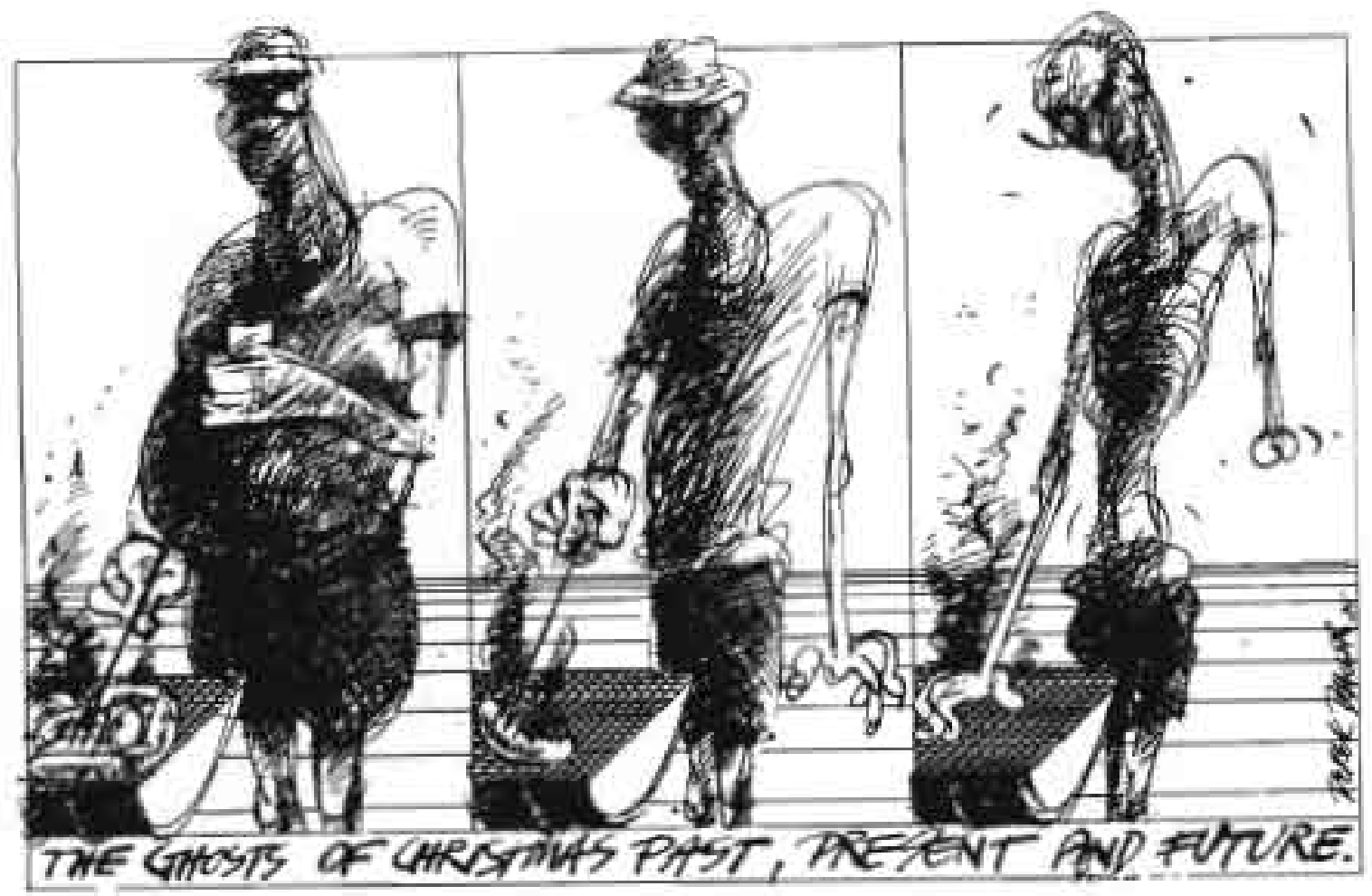

FIGURE 60

Bauer's cartoon "The Ghosts of Christmas Past, Present and Future", a metamorphosis that transforms the familiar iconic code of a white man cooking meat at a braar into a sizzling carcass that evokes the necklace murders of the period, is also reminiscent of the work of the celebrated British painter Francis Bacon. (Bauer, S. A. Flambe and Other Recipes for Disaster, 1989) 


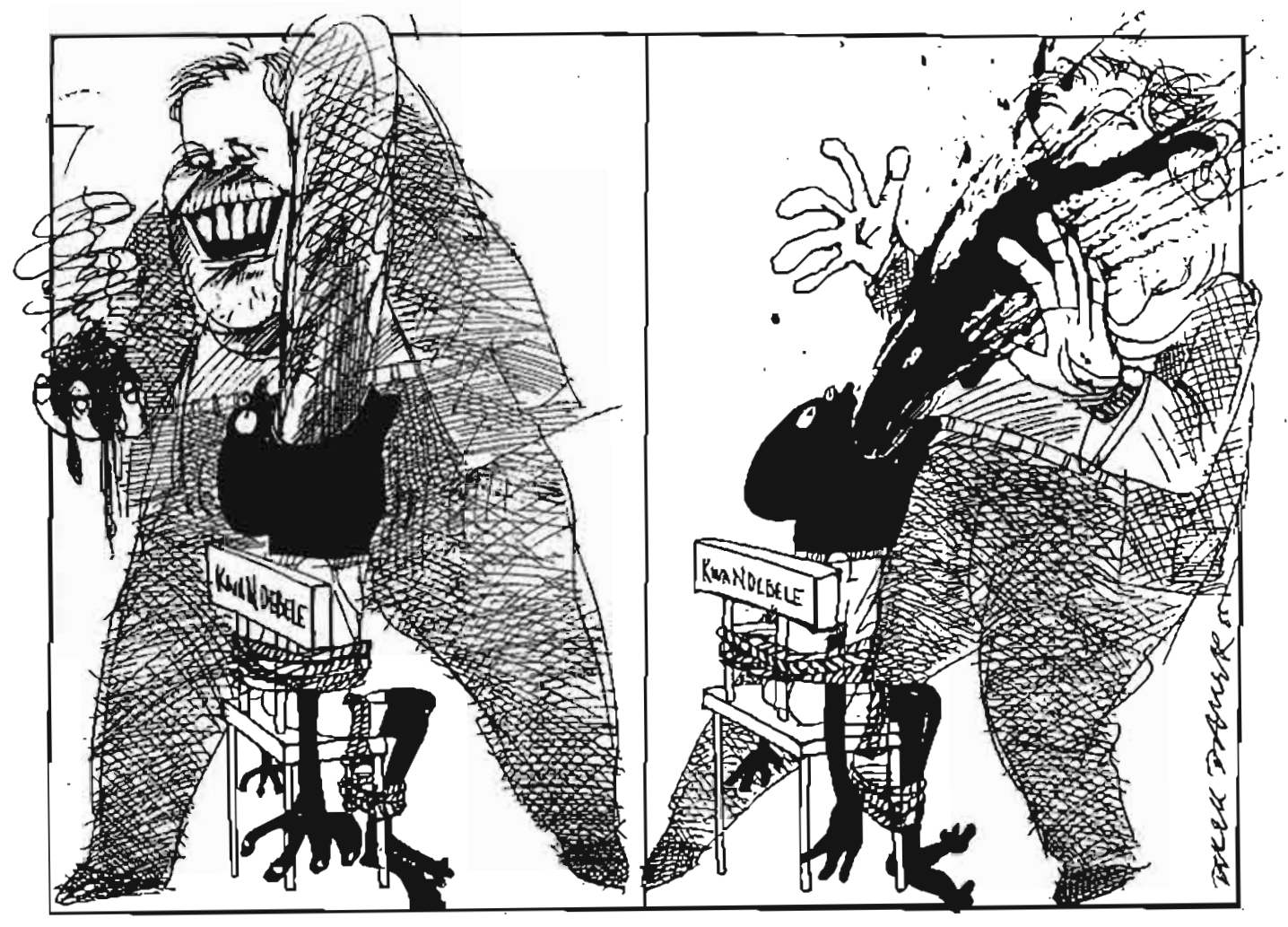

FIGURE 61

The National Party's Chris Heunis shoves the 'gift' of independence down the throat of the KwaNdebele 'homeland' in this vicious cartoon. The spurious substance, brilliantly depicted in an evil spray of ink, is vomited straight back at the politician.

(Bauer, S.A. Flambe and Other Recipes for Disaster, 1989.) 
of his cartoons were appropriately drenched as South African society began to tear itself apart (Figure 59)184.

Born in East London in 1955, Bauer became the Weekly Mail's political cartoonist in 1985 and immediately unleashed a barrage of blistering satire that went far beyond anything produced by Smith, even at his most extreme. Like Smith, Bauer had strong fine art tendencies and was adept at a brutal pen and ink style that, combined with savage facial and anatomical distortions, not only suggest the obvious comparison with Steadman and Scarfe, but also recalls the caricatures of the German satirical expressionist Georg Grosz. Bauer's cartoon "The Ghosts of Christmas Past, Present and Future" (Figure $60)^{185}$, a metamorphosis that transforms the familiar iconic code of a white man cooking meat at a braai ${ }^{186}$ into a sizzling carcass that evokes the necklace murders of the period, is also reminiscent of the work of the celebrated British painter Francis Bacon.

Closer to home, Bauer's work demonstrates links with a South African fine arts tradition that has often turned to caricature and anatomical distortion to convey strong emotions of anger and revulsion against the brutality of institutionalised racism ${ }^{187}$. J.M. Coetzee has recognised in William Kentridge's work an indebtedness to Georg Grosz and Weimar-age satire (Cameron, 1999: 85) and a comparison between Bauer's cartoons and Grosz's pen and ink drawings is equally merited. But perhaps the comparison should be taken further, to extend into the socio-political and artistic milieux of Germany before the First World War and South Africa in the last decade of apartheid. There are visible similarities in the iconic codes to which the artists in both periods had recourse: for instance, Groscz's capitalist, "the now familiar fat man with a curled moustache and thin beard, wearing a pince-nez" or his militarist, "angular, with a jutting aggressive jaw" (Kranzfelder 1994: 46), are familiar codes in South African cartooning. The pessimistic sense of impending apocalypse that arises out of Grosz's paintings "The City" (1917) and "To Oskar Panizza" (1918) ${ }^{188}$, with their apparently chaotic but highly

\footnotetext{
184 Derek Bauer, 1987, reproduced in S.A. Flambe and Other Recipes for Disaster, 1989.

185 Derek Bauer, SA Flambe and Other Recipes for Disaster, 1989: 49.

${ }^{186}$ See Grogan's character Johnny Rosseau-Smit (Figure 64).

${ }^{187}$ See, for instance, the work of Jane Alexander and Robert Hodgins in Sue Williamson's Resistance Art in South Africa, 1989.
}

${ }^{188}$ See Kranzfelder, 1994: 14; 25. 


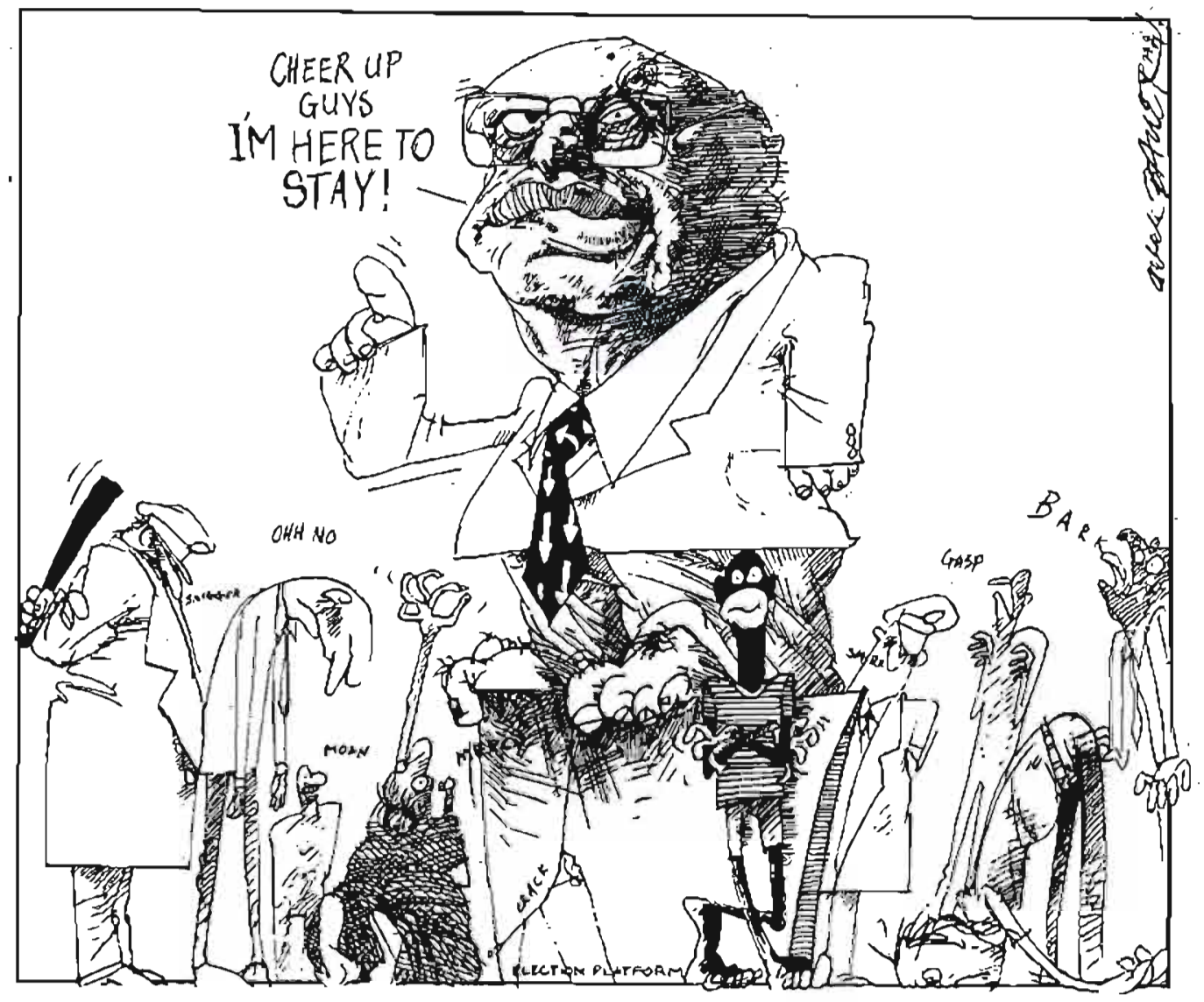

FIGURE 62

Bauer's drawings frequently subverted political correctness, as in this use of the racist 'black Sambo' code. His use of the codes and conventions of cartooning was often bafflingly inconsistent, as in his application here of the convention of barefootedness (see Figures 63 and 65) to P.W. Botha. (Bauer, S.A. Flambe and Other Recipes for Disaster, 1989.) 
formailsed compositions and their sense of a decadent interregenum about to be torn apart by brutal conflict, is echoed in paintings of the mid- to late 1980s by Penny Siopis, William Kentridge and Norman Catherine ${ }^{189}$.

It has been said that the unrestrained vehemence of Grosz's satire was fueled by hatred and pessimism (Kranzfelder 1994), and a similarly unrestrained vehemence is characteristic of Bauer's work in the mid- to late 1980s. The work of both artists demonstrates a sadistic enjoyment of the unspeakable, but Bauer's humour tends to trivialise as it excoriates: if there is psychic anguish there, it is battened down. A 1986 cartoon in which the National Party's Chris Heunis shoves the 'gift' of independence down the throat of a black man bound in a chair labelled "KwaNdebele" is a case in point. Heunis, the apartheid minister of constitutional development, is literally shown with a pile of shit in one hand, while the other is viciously rammed down the black figure's throat. The spurious substance, brilliantly depicted in an evil spray of ink, is vomited straight back at the politician (Figure 61)190.

Bauer's flippancy, as much as his style, is an attribute that secures him for cartooning rather than the fine arts. His opinions were as impudent as his style was unrestrained and his frequent subversion of political correctness, particularly in his use of the racist 'black Sambo' code (Figure 62)191, speaks of an irreverent iconoclasm unconcerned with consequences. Bauer's work was well suited to the violent 1980s, a time when extreme opinions and confrontational actions were the order of the day. But the very attributes that made him the ideal cartoonist to document this period counted against him in the 1990 s, when the rapid evolution of new discourses and alliances demanded a much more nuanced and analytical approach. By 1994, when he was replaced at the Weekly Mail by Zapiro, Bauer's restless intellect had taken him on to new interests and activities, and his contribution to South African cartooning carne to an end. He was tragically killed in a car crash in December $2001^{192}$.

\footnotetext{
189 See examples in Williamson, 1989.

190 Bauer, SA Flambe and Other Recipes for Disaster, 1989: 31

191 Another bold iconoclast, Joe Dog (Anton Kannemeyer) was to use the same code in the 1990s, but in a less offensive, more calculated way.

192 Bauer's work has only been assembled into one collection, SA Flambe and Other Recippes for Disaster, published in 1989 , but a major posthumous volume is currently in preparation.
} 


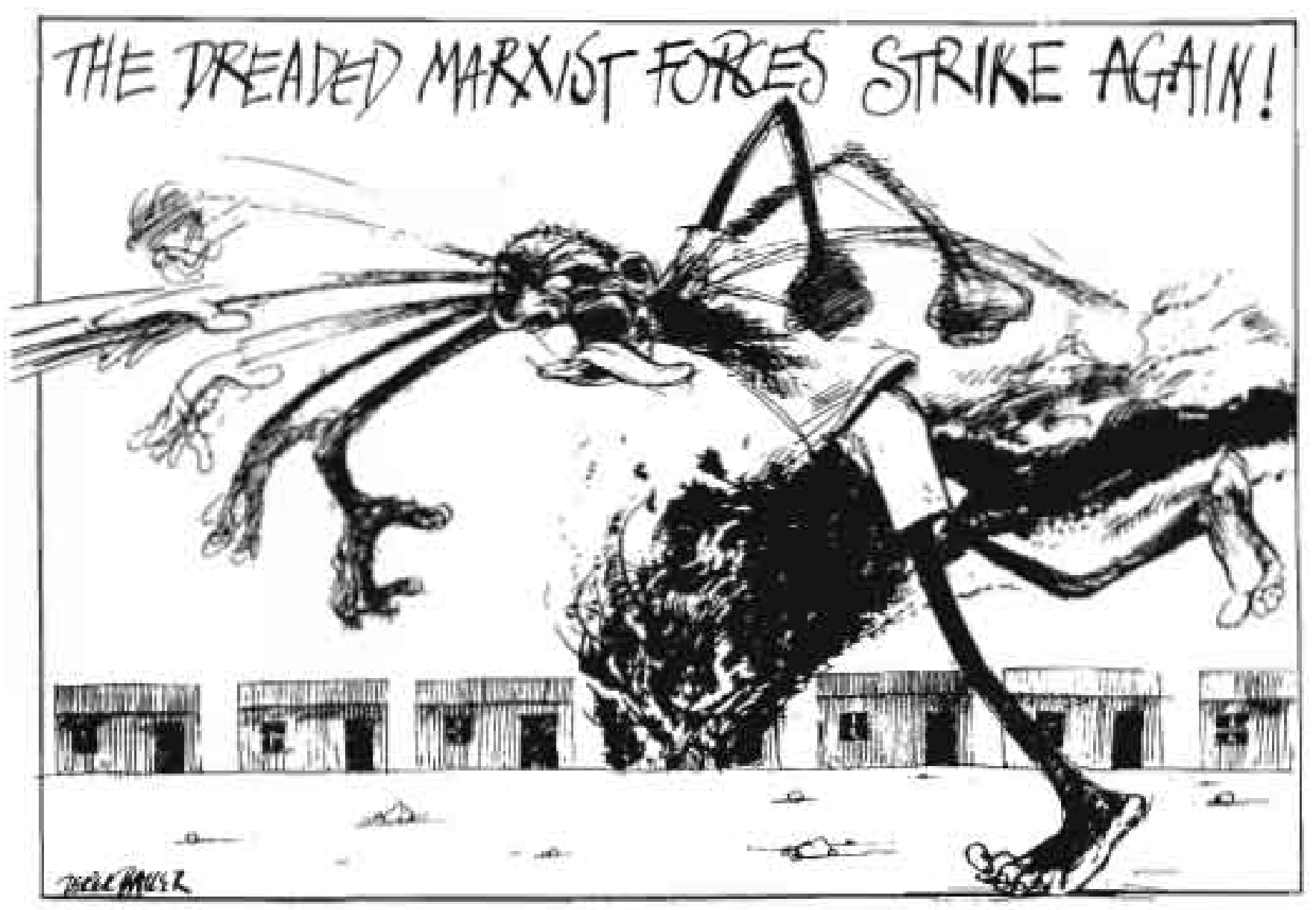

FIGURE 63

In Bauer's cartoons black people are frequently depicted as the other, as in this undated emergency era cartoon. While the text critiques the state's strategy of labeling every act of popular resistance as a communist act, the code embedded in the drawing belitties the rioting youth, and by implication, popular resistance itself.

(Bauer, SA Flambe and Other Recipes for Disaster) 
Bauer's reputation as one of the most influential cartoonists of the late apartheid period is secure, but it is worth interrogating how this importance is constituted. Bauer is important not only because he gave graphic expression to a bloodstained period in South Africa's political history, but also from a theoretical point of view. Because it is so extreme, his work vividly demonstrates the indivisibility of the formal qualities of an artistic work from its content, and how this indivisible gestalt can never be anything but the expression of a particular time and place, influenced by what has gone before and what currently prevails in both art and life.

In the case of cartooning, as in other areas of creative activity, the existence of a certain form of expression may be dependent upon the existence of a vehicle that is able to carry it, and the symbiosis between Bauer and the early Weekly Mail is an excellent example of this principle. The early years of the Weekly Mail were characterised by a fearless insouciance that brought a student press sensibility into the mainstream of South African newspaper publishing-indeed, the Weekly Mail's early editions looked rather like a student newspaper. While playing a dangerous cat and mouse game with the authorities, the Weekly Mail's editors taunted the established press, revealing them by comparison to be timid and constrained by structural linkages that prevented them from shouldering the burden of genuine resistance to the apartheid regime. The reckless, iconoclastic and angry stance that characterised the early Weekly Mail was appropriately captured in Bauer's work, which evolved in the space provided into a powerful statement of resistance.

Nevertheless, Bauer was a white cartoonist of the apartheid era, and his work still demonstrates stronger links with the tradition that it brings to an end than with the tradition that would emerge in the post-apartheid period. In terms of the opposition identified in the previous chapter between 'reflexive postmodernism' and 'responsible realism', Bauer's work adheres to the former and pays no lip service to the latter. But the "uneasy but timid white South African liberal consciousness" (Parry, 1998: 150) is nowhere to be found in Bauer's cartoons, which are consistently confrontational, unapologetic and controversial. Of the three main functions of South African oppositional cartooning identified at the beginning of this chapter, Bauer's work ably fulfills the first (satire and ridicule of the apartheid regime and its collaborators), seldom fulfills the second (pro-social iconography supportive of the transition), and seldom, if ever, fulfills the third (the use of cartooning for educational or conscientising purposes). 


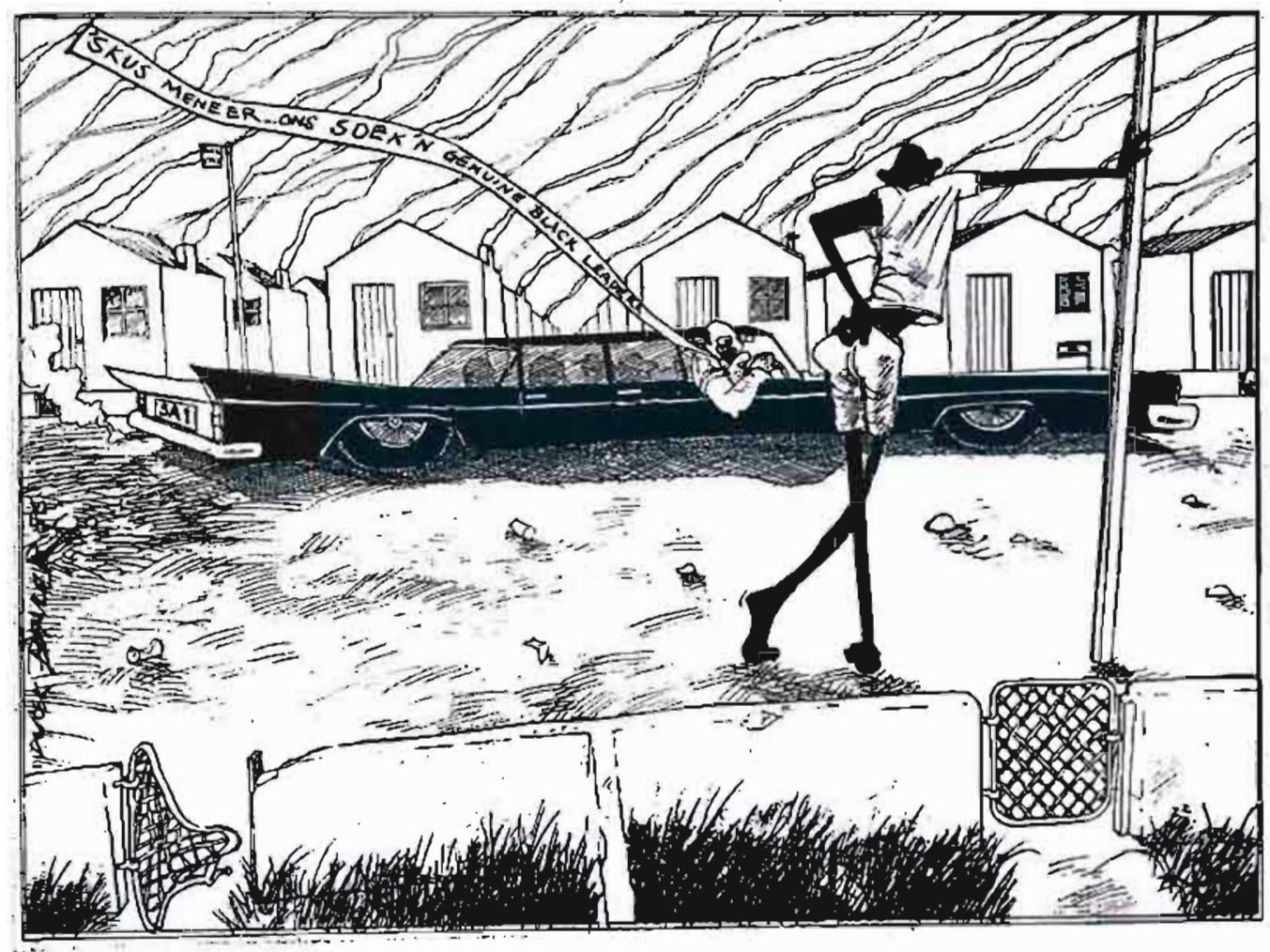

FIGURE 64

"Excuse me Sir, we seek a genuine black leader." This 1986 cartoon mocks the desperation of the P.W. Botha government to acquire the collaboration of credible black leaders and their ignorance of how to go about it. The use of the word "Meneer" (Sir) is intentionally ironic. (Bauer, SA Flambe and Other Recipes for Disaster, 1989.) 
In terms of the earlier discussion of the semiotics of racial caricature, Bauer's treatement of black politicians, and black people generally, is interesting. Like the white liberal cartoonists who preceded him, Bauer concentrated mainly on the white party political arena and his cartoons-certainly those collected in SA Flambe and Other Recipes for Disaster (1989)_demonstrate no deep understanding of or real affinity for the politics of the townships or the mass democratic movement. Black people are frequently depicted as 'the other'. For example, in an undated emergency era cartoon entitled "The dreaded Marxist forces strike again!" (Figure 63)193, a heavily caricatured black youth is shown hurling a rock or stone while a township shack burns in the background. While the text critiques the state's strategy of labelling every act of popular resistance as a communist act, the code embedded in the drawing tells a more interesting story. From the caricatured face with its bulbous nose hangs a long doglike tongue, while the figure is shown barefoot, bringing to mind a 1976 Soweto cartoon by Fred Mouton in which the rioters are all shown barefoot. Vernon suggests that the depiction of barefootedness in Mouton's cartoon functions "to emphasise that they are a rabble" (2000: 133), and the same interpretation may be advanced about this drawing. Certainly, barefootedness is a convention used extensively in the racist mid-century cartoons of Eric Thamm ${ }^{194}$ to represent the uncivilised primitiveness of African people, and it has been suggested that this convention is fairly common amongst Western cartoonists in the depiction of African leaders ${ }^{195}$.

In a 1986 Bauer cartoon the driver of a limousine cruising through a township confronts a barefoot black youth: "Skus Meneer... ons soek 'n genuine black leaderr (sic)" (Excuse me, Sir...we seek a genuine black leader (Figure 64)196. The cartoon mocks the desperation of the P.W. Botha government to acquire the collaboration of credible black leaders and their ignorance of how to go about it, and the use of the word "Meneer" is intentionally ironic. In this cartoon the township scene is convincingly depicted, while the barefoot black youth is portrayed as a featureless silhouette, and the limousine and its shadowy passengers representing the security establishment are ridiculously caricatured. In another cartoon of the same period, PW Botha is shown ramming the

\footnotetext{
${ }^{193}$ Derek Bauer, SA Flambe and Other Recipes for Disaster , 1989: 2.

194 See Vernon, 2000: 90; 91; 92; 104.

195 Zapiro, interview, 2003.

196 Bauer, 1989: 25.
} 


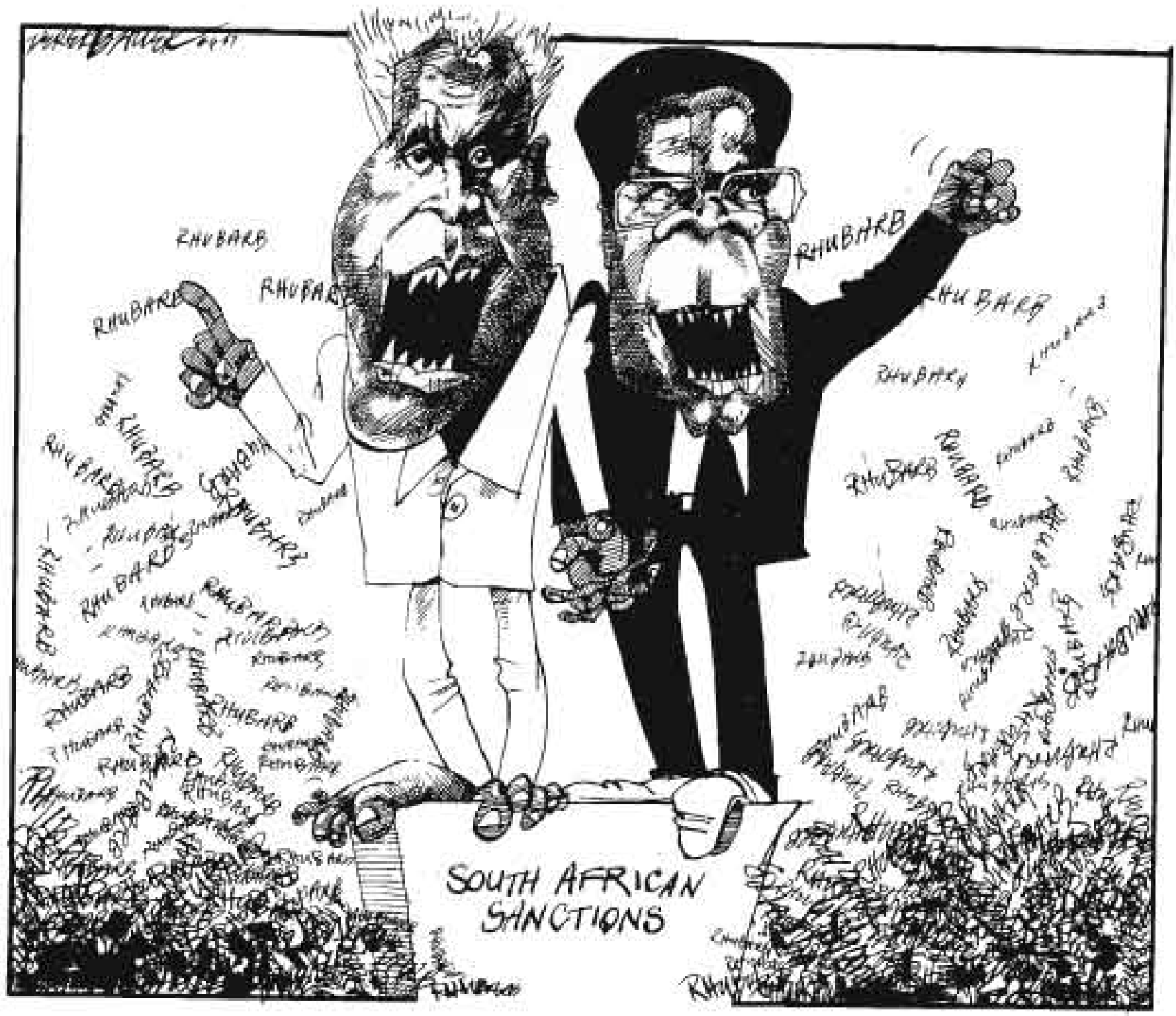

FIGURE 65

in this 1987 cartoon about sanctions, the word "rhubarb" piling up around Kenneth Kaunda and Robert Mugabe suggests that their opinions about anti-South African sanctions are worthless platitudes. This cartoon expresses a mainstream white viewpoint and depicts Kaunda and Mugabe with sharp dangerous teet, while Kaunda is shown barefoot, with apelike, curting toes.

(Bauer, SA Flambe and Other Recipes for Disaster, 1989.) 
elongated muzzle of a handgun up the nose of a black man, while another black man lies across the table with a knife in his back. Botha's words are: "Luister kaffir, I only talk with moderate black leaders"197. In these and other cartoons, the machiavellian brutality of the regime is unequivocally portrayed, but the black characters are subjected to the same kind of savage caricature as the white characters. In this sense, Bauer's caricature is very even-handed.

In a 1987 cartoon about sanctions (Figure 65) ${ }^{198}$, Kenneth Kaunda and Robert Mugabe are shown holding hands and standing on a soapbox, the word "rhubarb" coming from their mouths and piling up around them, suggesting that their opinions about antiSouth African sanctions are worthless platitudes. This cartoon is not only interesting because it expresses a mainstream white viewpoint, but also because of its depiction of Kaunda and Mugabe. Both are given sharp dangerous teeth (reminiscent of the teeth of the Hottentots in Cruikshank's cartoon of a century before), while Kaunda is shown barefoot, his toes curling, apelike, around the edges of the soapbox. In terms of the convention of barefootedness discussed above, this could be interpreted as racist, but Bauer's use of codes and conventions is often bafflingly inconsistent-in the 1987 cartoon of PW Botha mentioned above (see Figure 62), Botha is given the same simian feet as the 'back Sambo' figure below him, thus subverting the convention. Such inconsistencies make it very difficult to form an opinion about the intentionality that underlay Bauer's use of iconic codes, conventions and symbols.

The spontaneity and directness of Bauer's work suggests that his approach might have been less analytic than visceral, and that sometimes the pure enjoyment of drawing might have eclipsed the primacy of the political message. It is undoubtedly in its instinctiveness that the power of Bauer's work inheres, and if he is not one of the most analytic of South Africa's great cartoonists, his images are certainly amongst the most vehement and memorable ever produced in this country.

197 Bauer, 1989: 24.

198 Bauer, 1989: 52. 


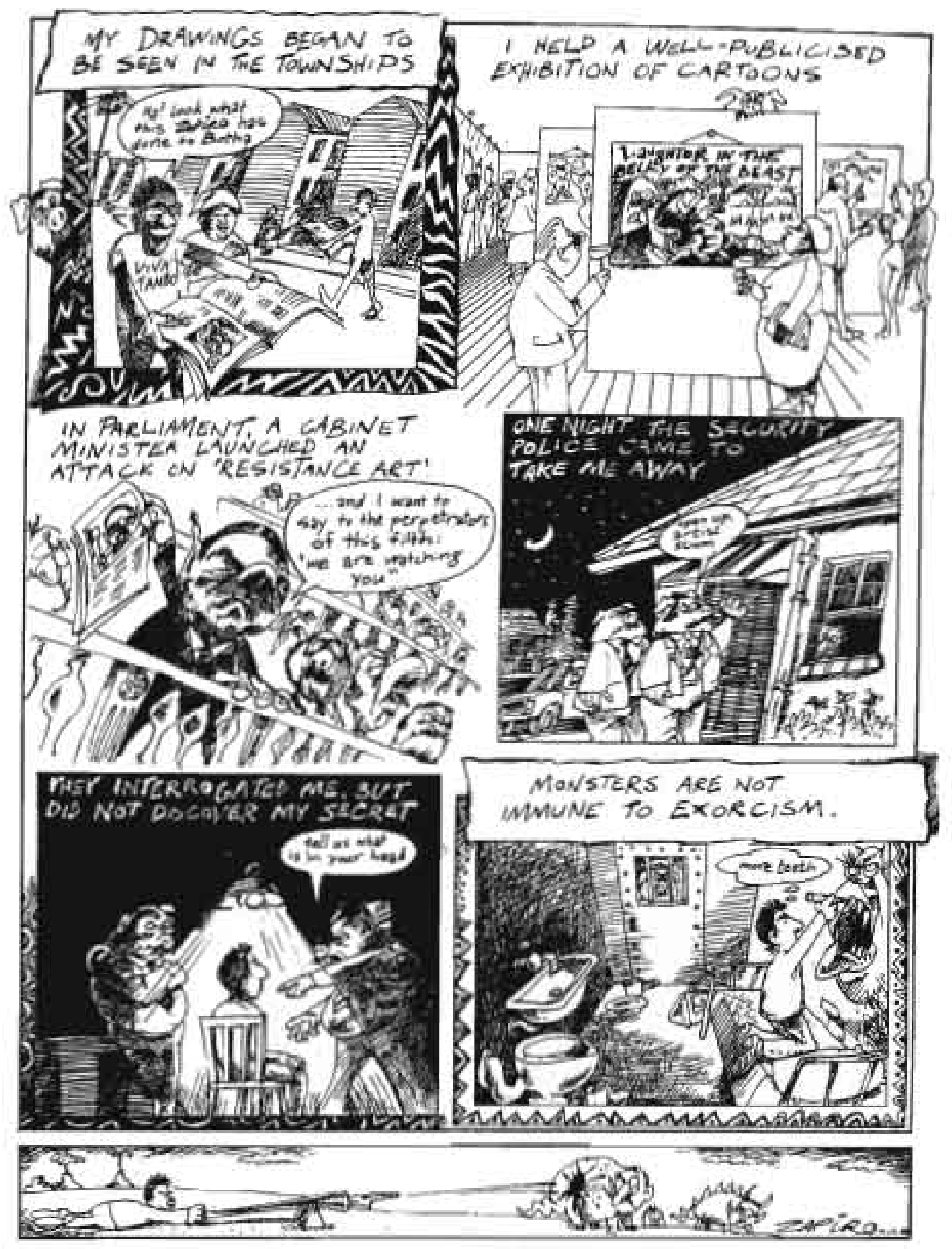

FIGURE 66

Page 2 of a two-page autobiographical comic strip, produced by Zapiro soon after he was released from detention in 1988. (Reproduced in Mamba Comix No.1, 2003). 


\section{Zapiro: Cartooning the transition}

Born in Cape Town in 1958 into a politically active family, Jonathan Shapiro began drawing at an early age. In a 1988 autobiographical comic strip (Figure 66$)^{199}$, he describes how at age four, with his mother's encouragement, he began drawing pictures of monsters to exorcise them from his dreams: "The monsters disappeared but I didn't stop drawing... At twenty nine, I was still drawing...there were other monsters to draw" - a reference to PW Botha and other right-wing 'monster' politicians of the apartheid era. The strip goes on to describe how his cartoons began appearing in alternative newspapers distributed in the townships, how he achieved prominence with a 1988 exhibition of cartoons entitled "Laughter in the Belly of the Beast", how he was warned against subversive artistic activity by a cabinet minister in parliament, and how he was arrested, interrogated and detained by the security police later that year.

The first major cartoon to appear with the now familiar Zapiro signature on the bottom right hand corner was a large full-colour illustration dated 1986 and produced for a 1987 calendar published by the Western Cape branch of the United Democratic Front (UDF) (Figure 67). It remains one of Zapiro's most important works. Drawn in a deceptively cute style reminiscent of the Belgian cartoonist Mordillo, the cartoon depicts a street scene outside a UDF office in a coloured suburb on the Cape Flats. It is packed with detail and incident and contains direct visual references to UDF personalities, as well as many other indirect or coded references, describing the atmosphere of that particular time and place with a very high level of realism, despite the cartoony style.

In the foreground of the drawing is a military 'Buffel' on which three confused looking soldiers are placed. One of the soldiers is holding an End Conscription Campaign (ECC) leaflet, while another has a dummy in his mouth. The third winces as a township kid shoots him in the nose with a pea shooter. The white soldiers are portrayed as 'babies', placed there against their will, whereas the police, several of whom are depicted as 'pigs', are portrayed as well-armed professionals, albeit a bit clumsy (the water cannonwielding riot policeman is spraying another policeman on the street) ${ }^{200}$. Between the

\footnotetext{
199 Zapiro, 1988. "Behind the Barbed Wire Curtain', Mamba Comix No.1, 2003.

200 Zapiro toid the audience at a 2004 exhibition opening that when he was detained, subsequent to the publication of the calendar, the police asked him: "Why do you draw us as pigs?" His retort: "I draw what
I see!".
} 


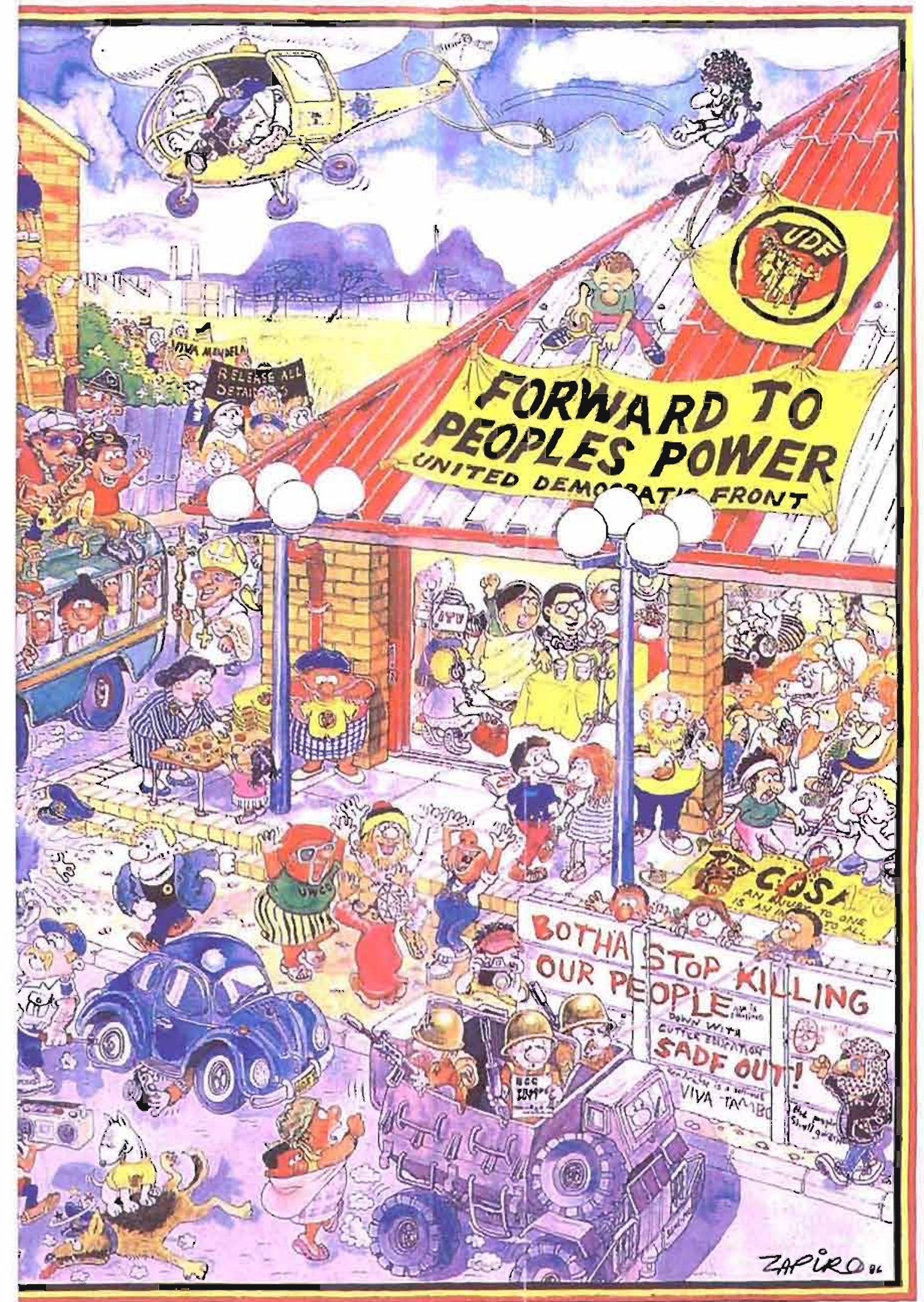

FIGURE 67

ie 1987 UDF calendar illustration was Zapiro's first major cartoon and remains one of his most important. (Reproduced in Mamba Comix No.1. 2003) 
policemen is a black man with a white cloth labelled "witdoek" around his head201. On the street a police sergeant with a sjambok surreptitiously punctures the tyre of a Volkswagen Beetle parked in the street, signifying the kinds of vindictive 'dirty tricks' to which the security establishment was prone: the press photographer under the Beetle signifies the role of the press in exposing these. In the sky above is a police helicopter manned by two 'pigs', one videotaping the scene below ${ }^{202}$. On the adjoining street the horse-drawn vegetable wagon, with both the driver and the horse giving black power salutes, signifies that popular support for the UDF amongst ordinary members of society, in this case a traditional small trader, is deep rooted. A housewife on a landing behind and a child on the stairs of the building, as well as and two doves on the telephone wire, all demonstrate the clenched fist salute, reinforcing the sense of popular support. The bus, not quite in ANC colours ${ }^{203}$, is packed with UDF supporters bussed in from one of the African townships for a meeting, a common occurrence at the time.

Cultural participation in the struggle is signified by the musicians on top of the bus. The military fatigues and red boots worn by the activist on top of the bus signify militaristic tendencies within the UDF. The old white-bearded man on top of the bus, also throwing the clenched fist salute, signifies the continuity of the struggle.

Behind the bus is Archbishop Desmond Tutu, leading a march of activists carrying banners with the inscriptions "RELEASE ALL DETAINEES" and "VIVA MANDELA", while in the UDF office a meeting is being addressed by struggle icons Fatima Meer and Alan Boesak, filmed by an ITV crew. In the audience are people of all races, including a ginger haired woman knitting and a Jewish man wearing a yarmulke ${ }^{204}$. An Indian man in a struggle shirt is arguing with a plainclothes 'pig', who is reaching for a revolver in a holster at his waist, indicating that UDF meetings were often attended by armed plainclothes policemen. Outside the office stands a ginger-bearded fat man in a UDF tee-shirt with a mobile telephone, signifying the logistic capabilities of the UDF, and next to him a coloured woman and a white man work together on a COSATU poster,

201 The "witdoeke" were police collaborators.

202 Police videotaping of demonstrations or meetings to assist in identifying 'ringleaders' was common practice.

${ }^{203}$ Zapiro was advised by the UDF's lawyers to remove a black stripe from the bus, as it was illegal at the time to diplay the ANC colours. However, a small ANC flag above the march in the background was retained (interview, 2004).

204 Like many of the figures in the drawing, these characters are based on real people. Other characters are composites of the attributes of several people (interview, 2004). 
signifying the close relationship between the UDF and COSATU. On the roof of the building a Jewish-looking activist ${ }^{205}$ in a vest and striped pants lassooes the tail of the police heliocopter with a rope onto which is attached a yellow banner displaying the UDF logo.

On the pavement and on the street are several other significant figures. A group consisting of a coloured woman in a UWCO (United Women's Congress) tee-shirt, a black man in a worker's overall, a white hippie man and a Muslim man dance on the pavement, suggesting that there is something joyous about the UDF's non-racial ethos. The figure of the determined-looking white priest advancing towards the police vehicles demonstrates the important role played by ecumenical activists in presenting a moral challenge to the authorities. In the right hand bottom corner is a Muslim radical with an Arafat-like scarf wrapped around his head. The leather jacket and dark glasses give this figure a sinister air.

On the street amongst the vehicles are several white activists and students, a rastafrarian with a joint and a radio, a young white man with a baby in a sling on his chest and a black woman with a baby on her back, portraying the range of people who involved themselves in the activities of the UDF. The young white radicals with their hippie clothes are typical of student activists of the day. Holding up the police 'Ratel' is a woman with a black sash over her shoulder, signifying the UDF's relationship with The Black Sash, an esteemed women's organisation. Autobiographical elements in the drawing include the cartoonist's mother, smartly dressed in a striped suit, selling UDF buttons on the pavement, while his sister crosses the street clutching a sheaf of computer printouts labelled UDF, and Zapiro himself, holding hands with his wife to be, Karina Turok, enters the building with a sheaf of drawings.

Clearly, an enormous amount of information is encoded into the drawing, which operates on several levels. On the most obvious level it is a celebration of people's power and political organisation. All the activists are very cheerful and the struggle is portrayed as being great 'fun'. On a deeper level, disguised by the cheerfulness of the characters and the bright colours of the drawing, is a recognition of the sombre reality of the political situation, signified by the slogans on a prefabricated wall in the foreground:

205 This character represents leading UDF activist, Jojo Shapiro. Confusion between the two Shapiros led to Zapiro's arrest and detention in connection with a planned Mandela birthday celebration the following year (interview, 2004). 


\section{“BOTHA STOP KILLING OUR PEOPLE; MK IS COMING; DOWN WTTH GUTTER EDUCATION, SADF OUT!; HENDRICKSE IS A SELL-OUT; VIVA TAMBO; THE} PEOPLE SHALL GOVERN". Zapiro confirms that he intentionally used the "cute sort of cartooning style" of this drawing "to do things that were profoundly political and deeply subversive" 206 .

The cartoon demonstrates an optimistic revolutionary spirit based on the conviction that the racist Nationalist government would soon fall under the combined onslaught of the internal democratic movement and the externally driven armed struggle. The attitude expressed in the cartoon is in direct contrast to the pessimism of the white liberal writers of the 1980s who felt themselves to be trapped in an interminable interregenum and could picture no coherent future for themselves in a new South Africa ${ }^{207}$.

The UDF calendar cartoon was undoubtedly a courageous and risky piece of work, produced at an extremely troubled and dangerous time in South African history, and could only have been seen by the security establishment as a brazen affront to their authority. Certainly, from that moment, if not already, Zapiro was a marked man in the eyes of the security police. On the other hand, the UDF was the most potent nongovernmental force in the land ${ }^{208}$, and the sense that his actions were buoyed by the tide of history undoubtedly strengthened Zapiro's resolve. The production of this image would prove to be one of the most important-and astute-interventions of his career.

Both Zapiro's mother and sister were detained by the security police in the late 1980s. His own political involvement began when, like so many young white men of his day, he was faced with conscription. He had completed four years of architectural study at the University of Cape Town, but had decided that architecture was not for him. He

\footnotetext{
206 Francis Nyamnjoh, interview with Zapiro, IJOCA, Vol. 2 No. 2, 2000.

207 See Chapter Five.
}

208 In 1985 the UDF had adopted the ANC's call for South Africa to be "rendered ungovernable", unleashing a phase of broad-based insurrectionary activity that was met by the 1985 state of emergency and the second state of emergency in 1986 (Marais, 1998: 53). The ANC now occupied the "symbolic center of resistance": the political struggle within the country and the armed struggle outside it were strategically linked, although the UDF was organizationally independent of the ANC. While the ANC did not actually direct resistance activities on the ground, "its growing authority at the ideological and symbolic levels did enable it to strongly influence the overal] terms in which resistance actions were couched-hence the formidable resonance of its calls for 'ungovernability' and a 'people's war' "(Marais, 1998: 54). 

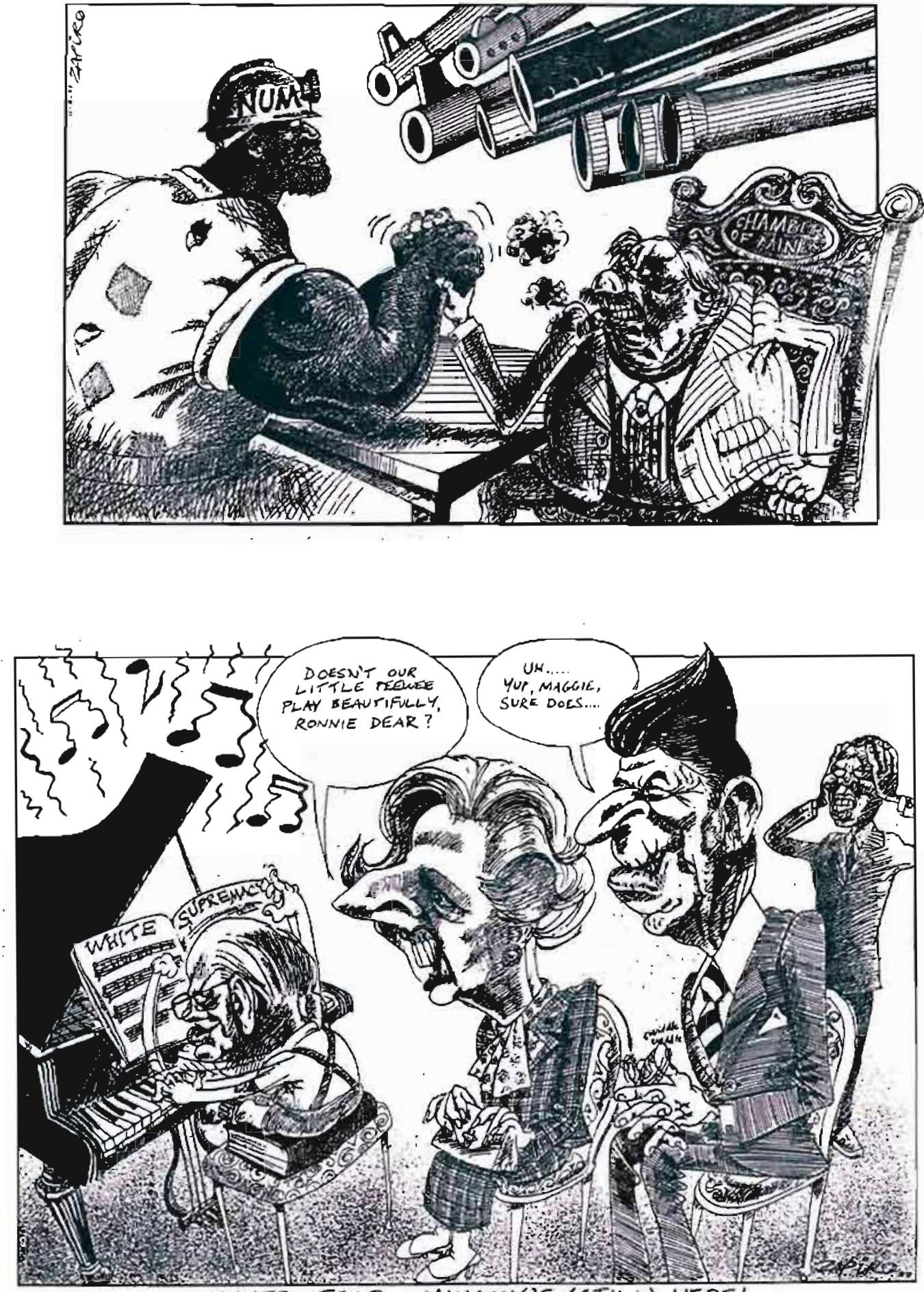

NEVER FEAR, MUMMY'S (STILL) HERE!

FIGURE 68

The South cartoons perfectly demonstrate the lineage in South African cartooning from the British Privale Eye tradition of Steadman and Scarfe, through Richard Smith, to Bauer and then Zapiro.

(South, 1987) 
travelled overseas at the end of 1982, and visited Herge's studios in Brussels and Uderzo in Paris. It was an inspirational moment. On his return, he decided to register for graphic design at the University of Cape Town, but the military authorities charged that he had changed courses in an illegal attempt to avoid national service. After repeated attempts to put his case before the Conscription Board, he was conscripted and spent two years in the army, during which he was openly supportive of the UDF, refused to carry a gun and became involved in the activities of the End Conscription Campaign (ECC). Despite his openly confrontational approach, his graphic design and illustration abilities were recognized and he spent the second year of his military service working as a draughtsman in a naval office in Simonstown on the Cape Peninsula, and was allowed to go home after work. During this period he openly participated in activities organised by the Western Cape branch of the UDF and was arrested for taking part in a UDF motorcade demonstration in 1983. This arrest led to his being investigated by the military authorities. During this time he assiduously practised his drawing, having resolved to take up cartooning as a career.

At the end of his military service he began drawing for a variety of organisations, but it was the request by the Western Cape branch of the UDF for the calendar illustration that presented him with his first major opportunity. The finished product was highly prized by the organisation and its adherents, and drew the attention both of the security establishment and of media activists throughout the country, announcing as it did the arrival of a major new talent in service of the democratic movement. In 1987 Zapiro was commissioned by South, a paper founded "in the wake of the 1980 s youth rebellion in the coloured community in the Western Cape" (Berger, 2000: 91), to produce a weekly cartoon. South was one of the new alternative weekly newspapers, sometimes referred to as "the Grassroots generation of papers", linked to the UDF209.

The approximately 60 drawings produced by Zapiro for South in 1987 and 1988 are not well known, since the paper had a limited circulation, and to date they have not been republished, although they were exhibited at the Baxter Theatre in 1988. They reveal the blossoming of a prodigious talent, and in some ways are more exciting from an artistic

\footnotetext{
${ }^{209}$ According to Berger (2000: 79-80) the Grassroots generation of papers made an important contribution to building the coalition of some 400 organisations that became the UDF in August 1983, and saw themselves as "the new dominant press in waiting".
} 


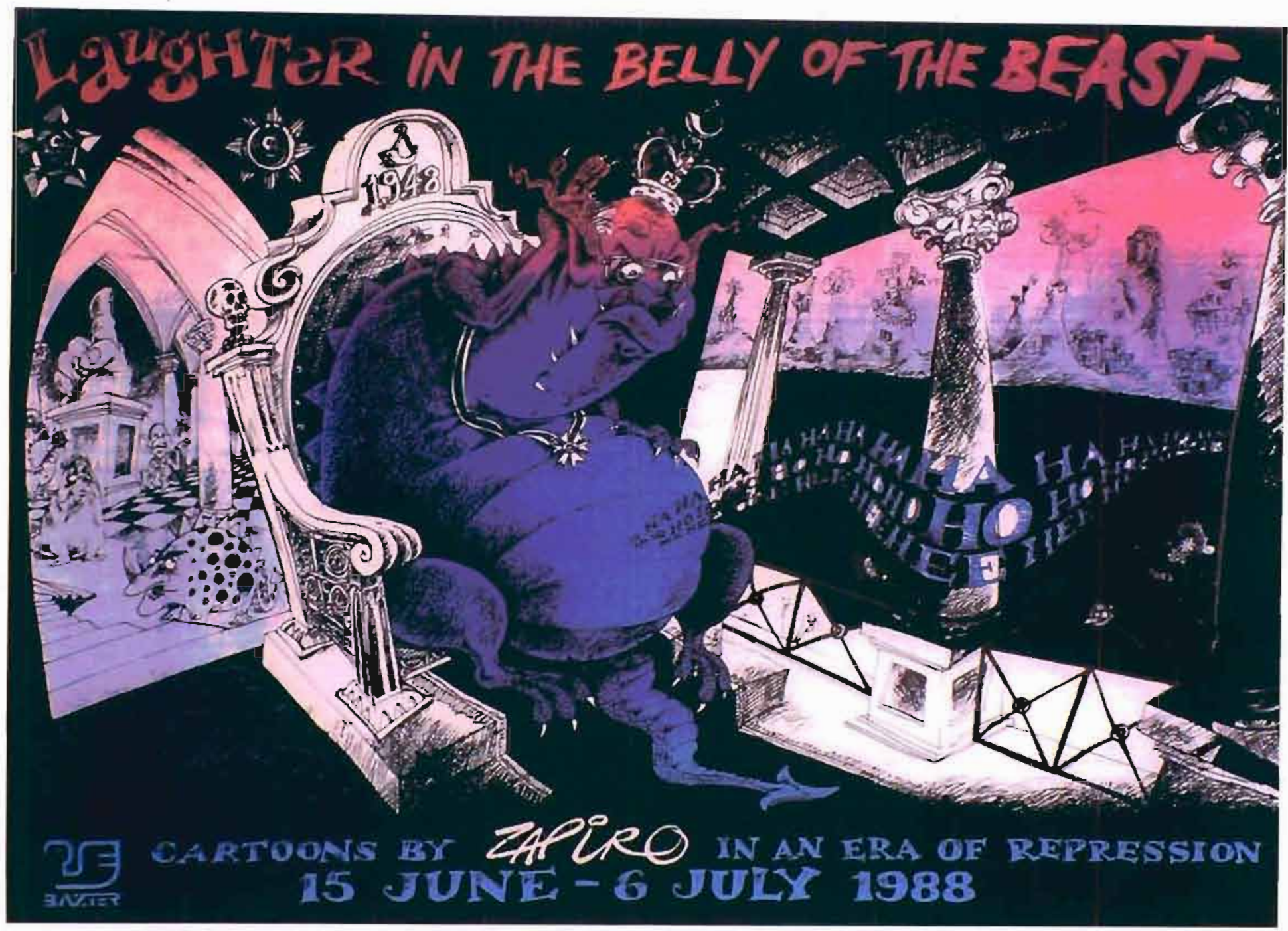

FIGURE 69

The poster for the 1988 exhibition of Zapiro's political work, entitled "Laughter in the Belly of the Beast", held at Cape Town's prestigious Baxter Theatre. The exhibition was followed by Zapiro's detention for 11 days, including five days in solitary confinement.

(Reproduced in Mamba Comix No.1, 2003) 
point of view than the products of the more disciplined and workmanlike approach adopted by Zapiro from 1994. The South cartoons perfectly demonstrate the lineage in South African cartooning from the British Private Eye tradition of Steadman and Scarfe, through Richard Smith, to Bauer and then Zapiro (Figure 68). They also reveal two of Zapiro's strongest attributes as a political cartoonist-a natural talent for caricature and an incisive political acumen. These often ink-spattered cartoons provide evidence of the stylistic debt to Bauer (see Figure 51), but perhaps more importantly, they demonstrate how much Zapiro's work differed from that of Bauer in terms of content and analysis. Unlike any previous major South African cartoonist, Zapiro was able to see the struggle from the point of view of the mass democratic movement, and to give expression to a style of political analysis that represented prevailing opinions in the movement and arose out of close contact with informed people within it. Throughout his career since then, Zapiro has assiduously maintained the opinion base provided by these contacts, and has had frequent recourse to advice from an extensive network of political activists, analysts and academics, especially when ideologically complex situations have arisen (interview, 2002).

Zapiro has frequently said that at this time he was fully committed to the national liberation struggle, and saw his work as an instrument in the furtherance of the ideals of that struggle. As has been noted, both his sister and his mother were detained in Pollsmoor prison for their political activities, the former for six weeks in 1985, and the latter for seven weeks the following year, and many friends and comrades had spent months in detention. Zapiro's early cartooning for South thus took place in a context in which activists were prepared to expose themselves to high levels of risk for the sake of their ideals.

In addition to his work for South, Zapiro also produced illustrations and covers for the radical Afrikaans magazine Die Suid Afrikaan and posters and pamphlets for political organisations. In 1987, partly on the basis of his political work, Zapoiro secured a Fullbright Scholarship to study at the School of Visual Arts in New York, a world famous centre of cartooning, the following year. In 1988, before he left for the USA, he mounted the exhibition of his political work, entitled "Laughter in the Belly of the Beast" at Cape Town's prestigious Baxter Theatre (Figure 69). The exhibition was followed by a visit from the security police, and then detention, along with seven other people who were involved in organising activities around Nelson Mandela's 70th Birthday. Zapiro spent 11 days in detention, including five days in solitary confinement. 


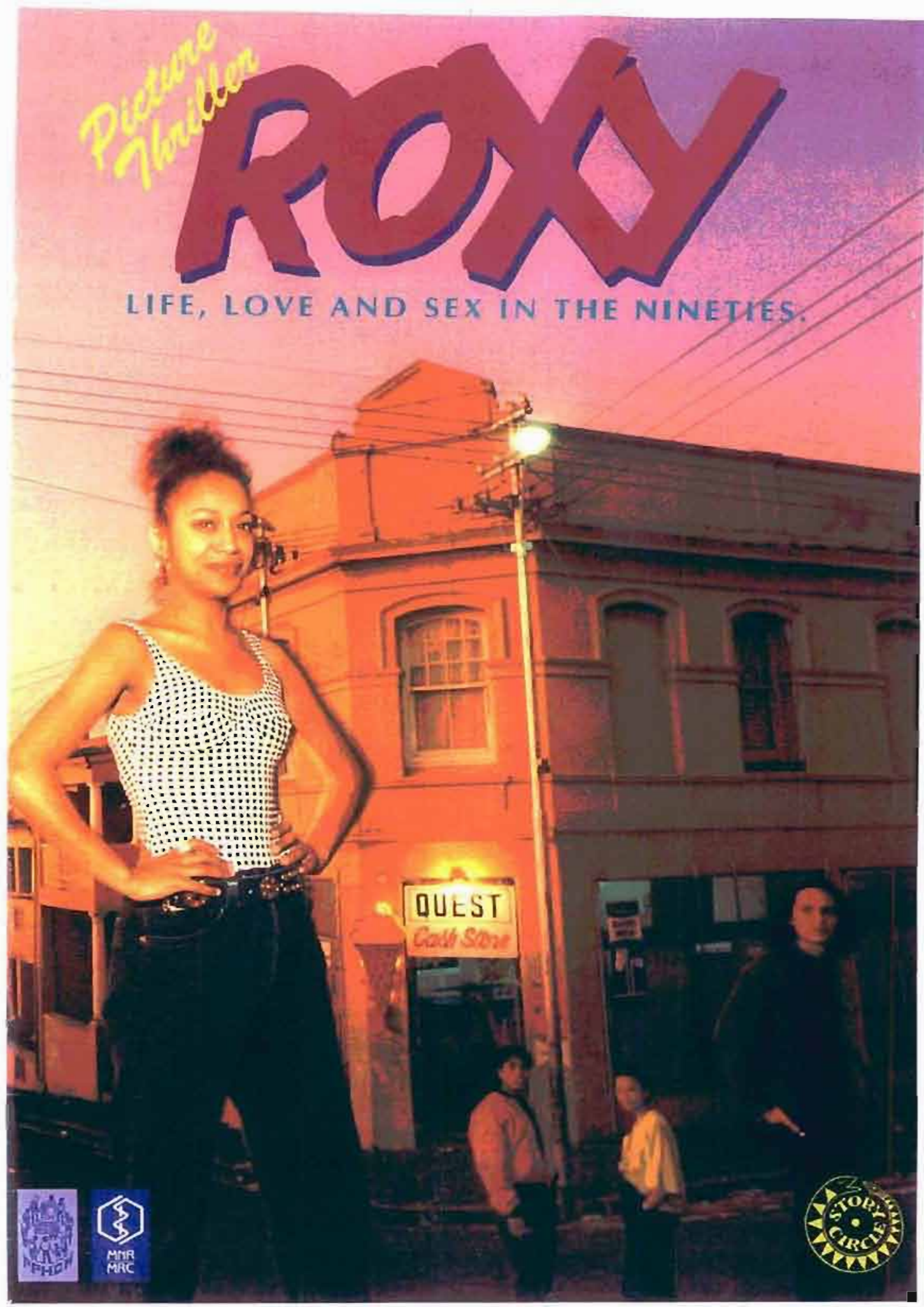

FIGURE 70

The cover of the highly acclaimed Aids education publication Roxy: Life, Love and Sex in the Nineties, produced by Zapiro's Story Circle group in 1991. Most of the publications produced by the Story Circle were photo-comics. (Story Circle/Progressive Primary Health Care Network/Medical Research Council, 1991) 
It later transpired that the security police had confused him with fellow activist Jojo Shapiro, one of the activists portrayed in the UDF calendar cartoon.

On his release, Zapiro left immediately for the USA, where he threw himself vigorously into the study of drawing, etching, visual communication, comics (under Will Eisner) and satirical cartooning (under Harvey Kurtzman). A year later he was able to study the graphic novel under Art Spiegelman. This personal history of commitment to the idealistic agenda of the liberation struggle, combined with his subsequent education at a world-renowned centre of cartooning under some of the most famous names in the field, formed Zapiro into a cartoonist uniquely suited to record the drama and euphoria of South Africa's transition.

Zapiro returned to South Africa in 1991, but his return to political cartooning was not immediate. Somewhat unsettled by the radically changed political environment, he was reluctant to plunge back immediately into the highly visible realm of political cartooning, and has described this intense and violent period in the nation's history as "a difficult time" during which he had had "a weird feeling" about the politics of the day $^{210}$. In particular he was troubled by the disbanding of the UDF after the unbanning of the ANC., worrying that the strong spirit of non-racialism that had characterized the UDF, particularly in the Western Cape, might be lost 211.

With several other young media activists, Zapiro formed the Story Circle212, a media production group that produced a number of educational photo-comics, the most important of which was the highly acclaimed Aids education publication Roxy: Life, Love and Sex in the Nineties (Figure 70). That Zapiro chose to use his real name, Jonathan Shapiro, rather than his nom de plume in the publication's credits, and that he chose to work with photo-comics rather than his chosen medium, indicate that the period

210 Francis Nyamnjoh, interview with Zapiro, IJOCA, Vol. 2 No. 2, 2000.

211 Interview, 2004.

212 The name of this group indicates the influence of Neil Napper and Peter Esterhuysen's Storyteller Group, which by that time had already produced several ground-breaking South African comic publications. 


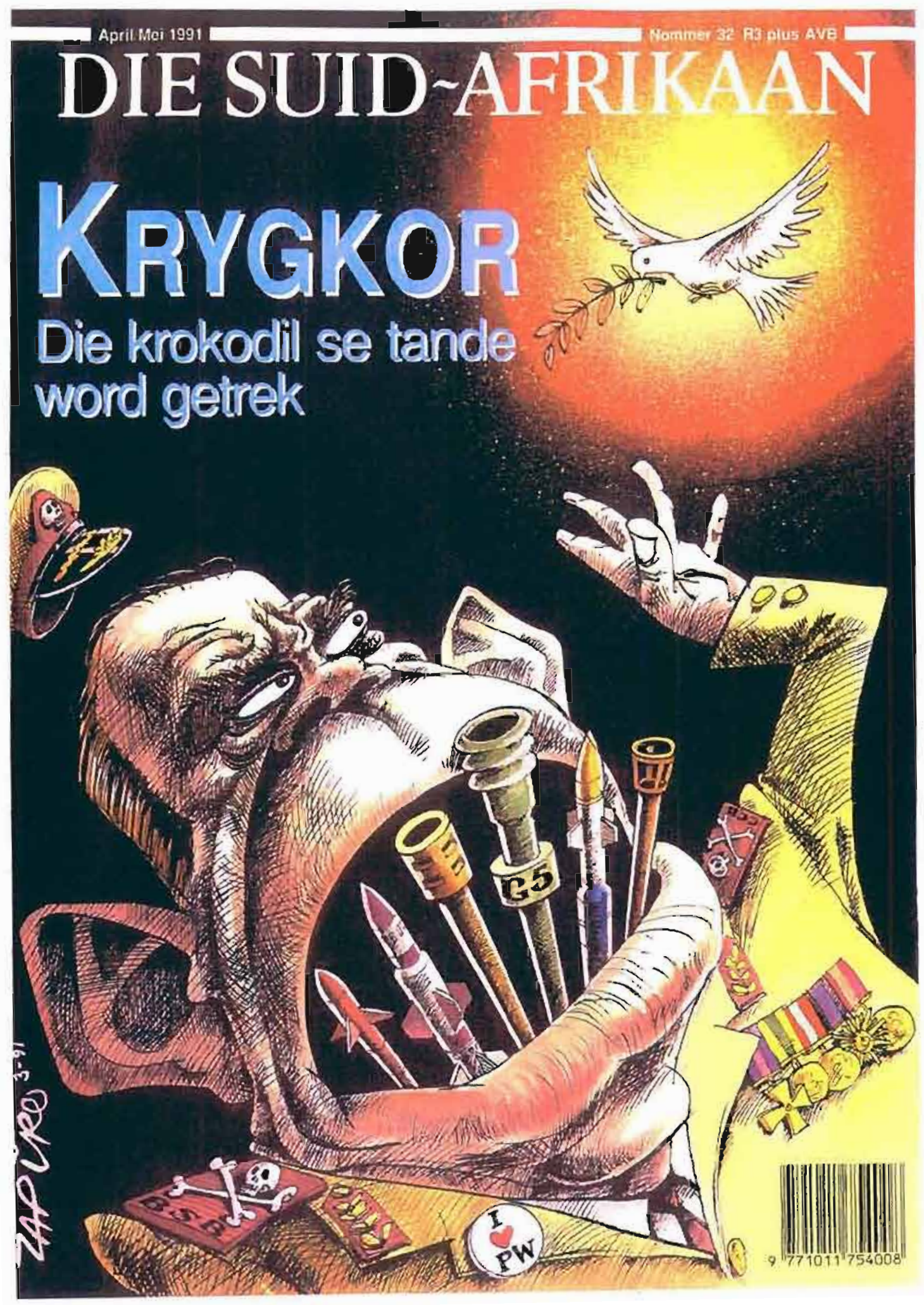

\section{FIGURE 71}

After his return to South Africa in 1991, Zapiro produced several covers for Die Suid-Afrikaan, which he considers to be amongst his best work (interview, 2004) (Cover design, Die Suid-Afrikaan No. 32, 1991.) 
immediately following his return to South Africa was a period of readjustment to the changing political realities of the 1990s, during which his inclination was to experiment with various options before returning to the demanding world of political cartooning. At this time he also produced several covers for Die Suid-Afrikaan, which he considers to be amongst his best work (interview, 2004) (Figure 71).

Amongst the reasons for choosing the photo-comic medium for Roxy was the consideration that photo-comics had been a popular form of pulp literature in South Africa from the 1960s to the 1980s, whereas there was no indigenous comics tradition in South Africa. It was felt that in this context a photo-comic would have more impact because it would seem more real to the young readers at whom it was aimed, whereas fears were expressed that a drawn comic might trivialise the subject matter ${ }^{213}$. Roxy was highly acclaimed amongst Aids activists and public health educators, and two more photo-comics were produced by the Story Circle, Tomorrow People, about human rights and democracy, and A Trolley Full of Rights, about child abuse prevention.

Zapiro's return to political cartooning in 1994 was sudden and prolific: in February of that year he replaced Derek Bauer on the Weekly Mail \& Guardian and in July joined the Sowetan as its daily editorial cartoonist, displacing Len Sak. Within the year he had established his authority as the country's leading political cartoonist, and since then his work has become the benchmark against which all cartooning activity in South Africa will be measured for decades to come.

What sets Zapiro apart from the white cartoonists who preceded him are the 'struggle credentials' conferred upon him as a result of his involvement as an activist in the democratic movement in general, and in particular in the local activities of the UDF in Cape Town. He has often mentioned how important these credentials have been in securing his credibility and legitimacy amongst the country's emerging political elite. In a sense his political involvement gave him a license after 1994 to harshly criticise powerful ANC leaders when he believed that the occasion demanded it, not least

\footnotetext{
213 (Interview, 2004.) The fear that translation into comic strip form will inherently trivialise serious subject matter is a common theme and is discussed in Chapter 3 . It is also noted in Chapter 5 in the case of the Sached comic Equiano's Travels.
} 


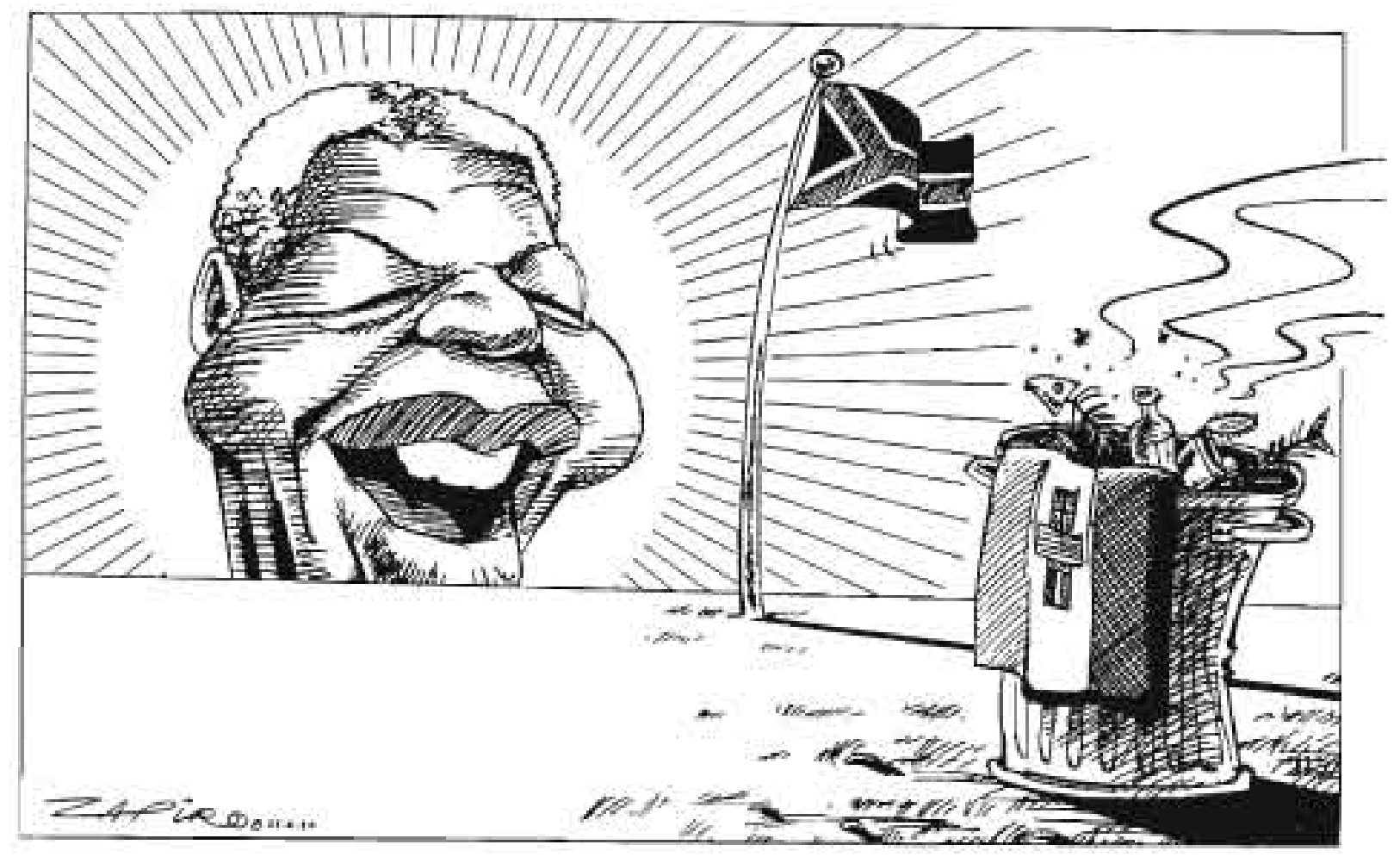

FIGURE 72

Zapiro's election day cartoon, published on 27 April 1994, is more complex than it looks. The thesis of the cartoon-Mandela as the rising sun-is a fairly conventional idea, while the antithesis, the old South African flag relegated to 'the dustbin of history', plays on a well-known left-wing cliché. The new South African flag, fluttering in a fresh breeze in the centre of the drawing, represents the synthesis of the old and the new. Less obvious is the placement of Mandela on the left, the dustbin on the right, and the new flag at the centre, suggesting that the new government would of necessity have to move towards the centre of the poiltical spectrum. 
amongst whom has been Thabo Mbeki. This license was secured early on, and the 1987 UDF calendar cartoon is very important in this respect, because its messages were directed not so much at the state, or at the myopic white electorate at large, but at the democratic movement itself. One of the cartoon's key messages is that of non-racialism, from which stems the need to secure recognition for the role of white activists in South Africa's political struggle.

In a very real sense, Zapiro sees his cartoons as interventions in the political process, as a way of giving expression to ideas that he feels need to be aired in the public domain, cutting across the bluster and spin-doctoring of the politicians who are his targets. As the political situation changed, his role matured from one in which he echoed the sentiments of the liberation movement, to one in which he was often required to identify the moral centre of a political quagmire.

Of particular interest, in the case of a cartoonist so closely associated with a political movement, are questions of artistic freedom in the face of the demands of propaganda. Zapiro has given voice to the difficulties he experienced in the immediate postapartheid period, when his allegiance to the ANC was challenged by unconscionable political behavior within the new government: "...it's a completely different situation now. It couldn't be more different [...] it's harder now to know who your enemies are, because the enemy sometimes comes from within [...] things like nepotism and abuse of power start creeping in [...] it's easier to do a cynical cartoon than an idealistic cartoon. All those things make it more difficult right now"214.

In a 1997 review of one of Zapiro's books ${ }^{215}$, Jeremy Cronin refers to the cartoonist's "double vision", in which the tragic and the comic appear side by side, and which enables him "to illustrate, up front, two or more conflicting value systems withir the same cartoon". This dialectical quality-the ability to include antithetical points of view within a single drawing, and bring them into some sort of synthesis - is a familiar characteristic of Zapiro's work in general.

\footnotetext{
${ }^{214}$ Interview with Francis Nyamnjoh, JOCA Vol. 2, No. 2, 2000.

215 Jeremy Cronin, Stunday Independent, 1997. Review of Zapiro's The Hole Truth.
} 


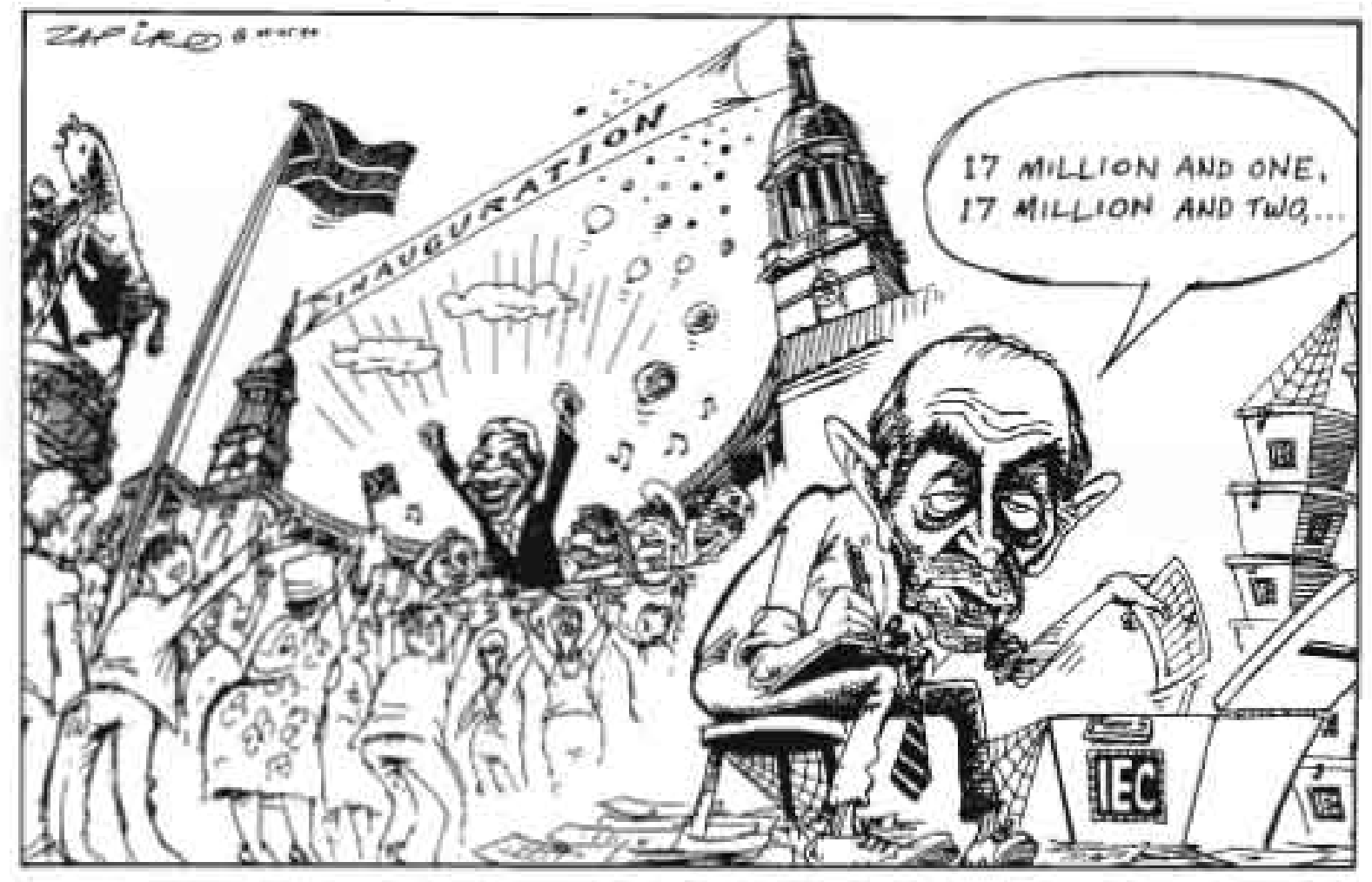

FIGURE 73

In his portrayal of Mandela's inauguration. Zapiro presents thesis (a successful election) and antithesis (administrative problems) in the same drowing. but this time leaves the oulcome hanging as a question in the reader's mind: will the new South Africa be able to overcome the enormous problems it has inherited? (Zapiro, 1996.) 
Zapiro's best cartoons from 1994 and 1995 are collected in Zapiro: The Madiba Years (1996). It was an epic year in which to begin the second phase of his career as a political cartoonist, and this year produced several major images that were to become canonical. Of these, some were purely celebratory, but even at their most unequivocal, they are often dialectical in composition and intent. A good example is the cartoon celebrating election day, $27^{\text {th }}$ April 1994, at first glance a simple declaratory image (Figure 72). The thesis of the cartoon-Mandela as the rising sun-is a fairly conventional idea, while the antithesis, the old South African flag relegated to 'the dustbin of history', plays on a well-known left-wing cliché. The new South African flag, fluttering in a fresh breeze in the centre of the drawing, represents the synthesis of the old and the new. Less obvious is the placement of Mandela on the left, the dustbin on the right, and the new flag at the centre, suggesting perhaps that the new government would of necessity have to move towards the centre of the political spectrum.

A few days later, as the nation waited impatiently for the final ballot figures from the Independent Electoral Commission (IEC), the new government decided to proceed with Mandela's inauguration. In his portrayal of this moment, Zapiro again presents thesis (a successful election) and antithesis (administrative problems) in the same drawing, but this time leaves the outcome hanging as a question in the reader's mind: will the new South Africa be able to overcome the enormous problems it has inherited? (Figure 73.)

Zapiro's cartooning epitomises the three main functions of South African oppositional cartooning during the last decade of the apartheid period: satire and ridicule of the apartheid regime; the creation of pro-social iconography supportive of the transition; and the use of cartooning for educational or conscientising purposes. But in a sense, Zapiro's work also goes beyond these, because of his ability to enter into dialogue with the new dispensation, and to be simultaneously supportive and critical of it. Unfortunately, the most vivid examples of this role occur after 1994, and therefore lie outside of the scope of this study, but it is clear that Zapiro's 'struggle credentials' -his close involvement with and understanding of the ideology and politics of the liberation movement-provided him with unique qualities that enabled him so effectively to document South Africa's transition.

As the daily editorial cartoonist for the mass circulation black daily newspaper, Sowetan, and for the intellectual, left-leaning weekly, Mail \& Guardian, (and later for the mass 

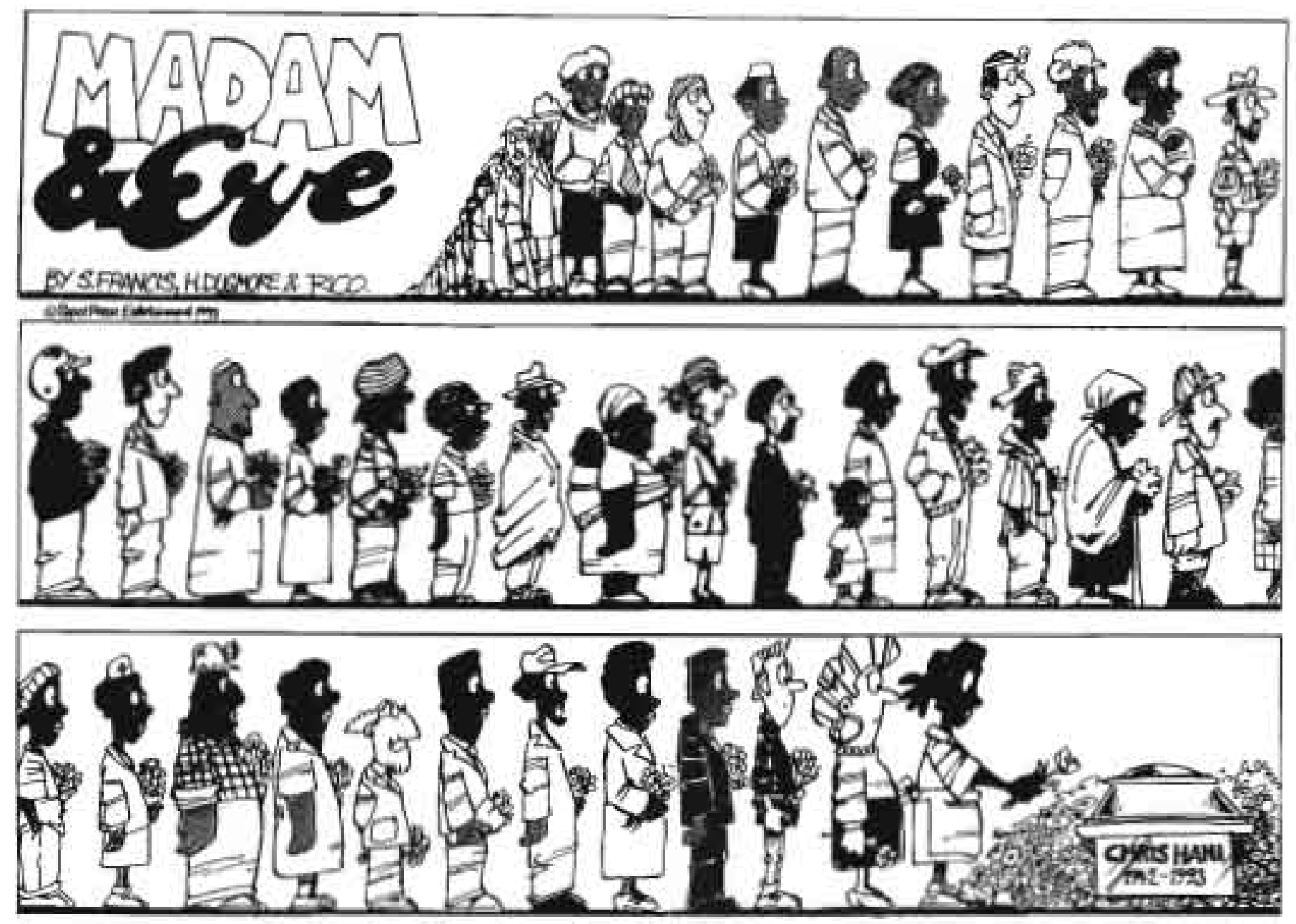

FIGURE 74

In this 1993 strip, Madam 8 Eve's main characters, Gwen Anderson and Eve Sisulu, stand in a long line of South Africans of every race and class, assembled to moum the passing of Chris Hani, the ANC leader assassinated by rightwing activists. The cartoon uncarnily prefigures the queues of voters who would line up around the country to cast their ballots the following year.

(Francis, Dugmore \& Rico, 1993.) 
circulation weekly, Sunday Times) Zapiro is the first South African cartoonist to draw for an audience that could be described as 'national'. His ability to communicate with readers across the social and political spectrum speaks not only of his personal genius, but also of South Africa's profound transformation from an embattled and fragmented society to a unitary nation.

\section{Madam \& Eve: Reinventing social relationships}

Alongside the emergence of Zapiro, the publication in 1992 of the first Madam \& Eve strips in the Weekly Mail \& Guardian, should be viewed as one of the most significant developments in the history of South African cartooning. In a 1993 strip (Figure 74)216, Madam \& Eve's main characters, Gwen Anderson and Eve Sisulu, are included in a long line of people stretching down through the three tiers of the strip. At the end of the line is the coffin of Chris Hani, the ANC leader assassinated by rightwing activists. In the line stand South Africans of every hue and persuasion, a national consensus assembled to mourn the passing of a revolutionary hero and potential successor to Nelson Mandela. It was a moment of deep crisis for the nation, threatening to disrupt the fragile negotiation process that would ultimately lead to the election of 27 April 1994. The queue of mourners in this cartoon prefigures the queues of voters who would line up around the country to cast their ballots the following year. This strip is historic not only because it visually prefigures the voter queues of 27 April 1994, but also because it would not have been possible a few years earlier. It is perhaps not unreasonable to suggest that, in a very profound way, the emergence of a national idiom in South African cartooning could only occur in the context of a national consensus.

The first Madam \& Eve strips appeared in theWeekly Mail \& Guardian in 1992, created by the team of Stephen Francis, Harry Dugmore and Rico (Schacherl). In a cartoon dating from the strip's earliest days, Madam is at confession. "Father, forgive me, for I have sinned," she says to the invisible priest behind the grille. "For ten years I have been paying my maid Eve only ten rand for a full day's work." There is a pregnant pause before the priest replies: "Is she available Tuesdays?" (Figure 75.)217

216 Reproduced in Francis, Dugmore \& Rico, The Madam \& Eve Collection, 1993: 83.

217 Reproduced in Francis, Dugmore \& Rico, The Madam \& Eve Collection, 1993: 7. 

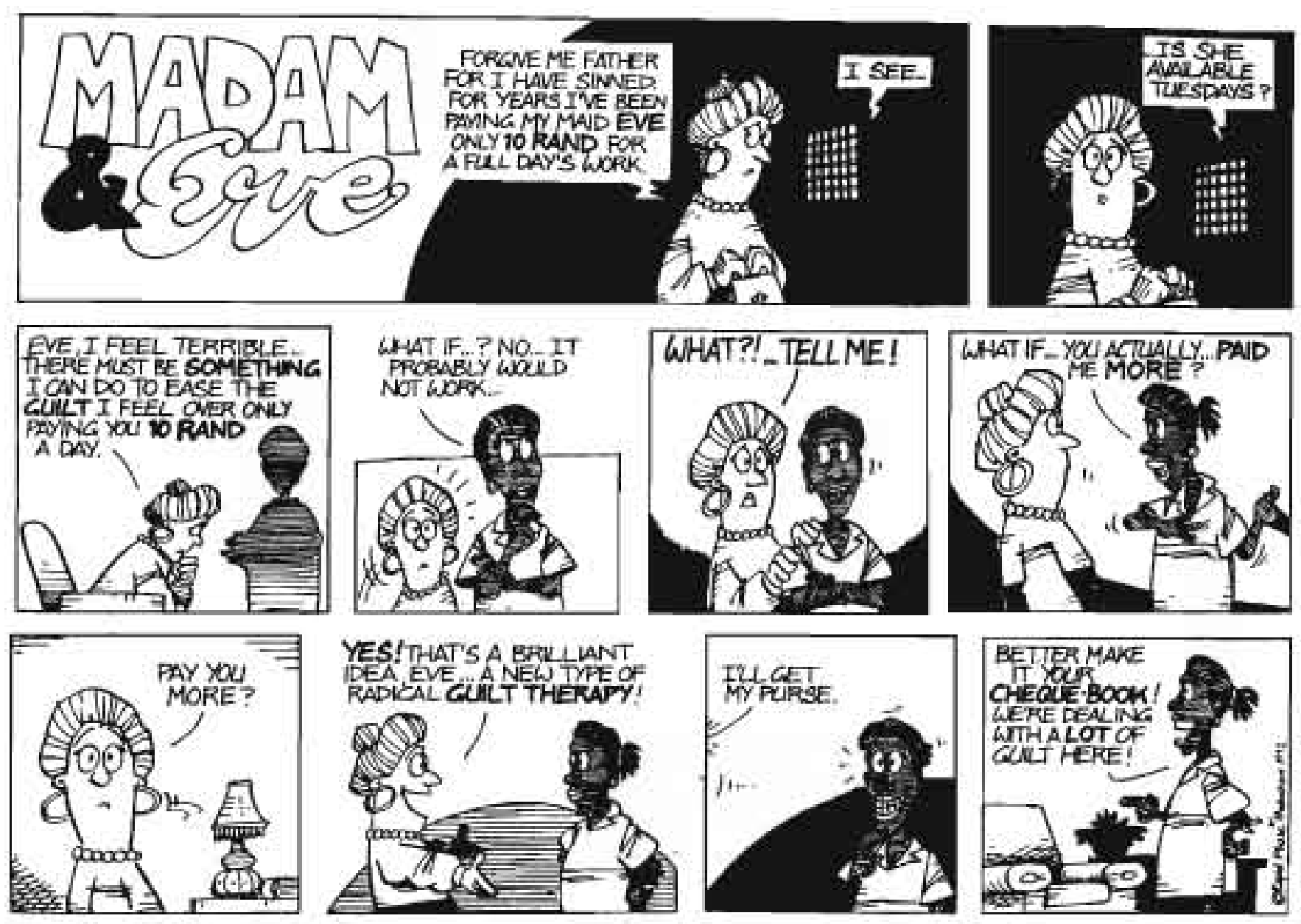

FIGURE 75

One of the earliest Madam \& Reve cartoons, published in 1992. In this witty riposte, the essential components of a brilliant cartoon strip that struck at the heart of the South African condition were assembled, setting the tone for a steady stream of jokes that has made Madam \& Eve the most successful strip in the history of South African cartooning. (Francis, Dugmore \& Rico, 1993.) 
In this witty riposte, the essential components of a brilliant cartoon strip that struck at the heart of the South African condition were assembled, setting the tone for a steady stream of jokes that has made Madam $\mathcal{E}$ Eve the most successful strip in the history of South African cartooning. It is syndicated in a dozen newspapers and several magazines in the country, and enjoys exposure in France, Denmark and Sweden. Prior to 1994, the implementation of the Nationalist government's apartheid policies had been so effective that it was impossible to speak of a unitary South African culture: the sense of 'South Africanism' that the country's citizens now share is only ten years old. Madam $\mathcal{E}$ Eve was the first South African cartoon strip to tap into this new zeitgeist.

While Rico, the artist of the strip, is a South African of Austrian birth, the writer, Stephen Francis, is an American who had moved to South Africa for marital reasons shortly before their collaboration began. He has often said that it was his position as a non-South African outsider, observing the close and complex relationship between his mother-in-law, after whom the strip's Gwen Anderson is named, and her domestic servant, that enabled him to see the humorous potential of a situation that most white South Africans were too close to, and too conflicted about, to see clearly (interview, 2001).

At the time when they originated the concept for the strip, Francis and Rico were out-ofwork humorists, struggling to make a living after an inauspicious publishing debut. Along with humour writers Gus Silber, Arthur Goldstuck and Harry Dugmore, and cartoonists Bill O'Connor, Stidy (Anthony Stidolph), Derek Bauer and others, they had been employed by Laughing Stock, a short-lived humour magazine published in the late 1980s. After the collapse of the magazine, they were employed to write and illustrate a series of egregious 'gift books' with titles like The Barf Book, 101 Uses for a Dead Yuppie, 30 Great Reasons to Keep Smoking and All Men Are Bastards ${ }^{218}$. While the content of these publications was generally execrable, this venture had a very important effect. It gathered together a group of humorists who were to bring a refreshingly absurdist tone into the South African popular publishing mainstream at a time when the intellectual milieu was characterised, as has been demonstrated above, by anxiety and pessimism.

218 All published by Laughing Stock Publishers, Johannesburg/ Bath, 1989-90. 


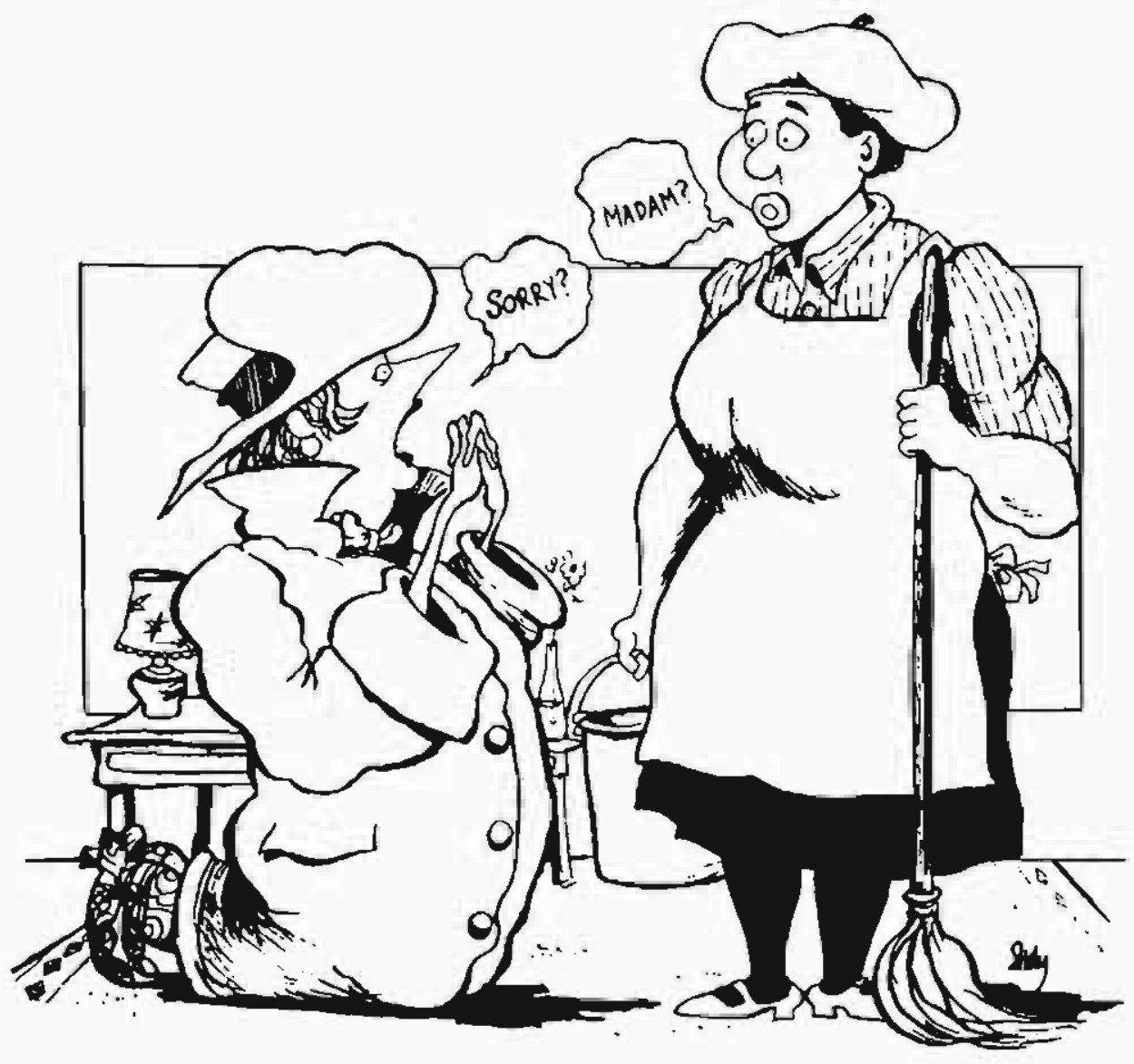

FIGURE 76

Gus Silber's It Takes Two to Toyi-Toyi: A Survival Guide to the New South Africa, illustrated by Stidy (Anthony Stidolph), anticipates many of the themes that were to be explored in Madam and Eve. (Silber, 1991.) 
Gus Silber and Arthur Goldstuck, who both wrote for the Weekly Mail, moved on to successful editorial careers, and Harry Dugmore joined with Francis and Rico, cowriting Madam \& Eve in the early years and playing a central role in the establishment of Rapid Phase Entertainment, the media company that grew out of their activities. In 1991, Gus Silber wrote It Takes Two to Toyi-Toyi: A Survival Guide to the New South Africa, a satirical treatment of the impending socio-political transition, illustrated by Stidy. The tone of this book, and the themes it deals with, are very similar to those of the early Madam \& Eve strips. The tone is resigned, cynical, but not bitter-very similar to the stoical acceptance of one disaster after another that had been exhibited by Tony Grogan's character Johnny Rosseau-Smit in Grogan's South Africa (see Chapter Five). The themes are familiar: pervasive crime, ridiculous security arrangements, lethal minibus taxis, affirmative action taken to its extremes and radically changed relationships between white housewives and their domestic servants. One of Stidy's illustrations shows a 'madam' kneeling down to apologise to her domestic worker (Figure 76)219, anticipating the kind of relationship issues that were to be explored in Madam and Eve.

A less funny but equally revealing book is Going, Going, Gone: The ultimate auction sale for the New South Africa (1992), written by Arthur Goldstuck and illustrated by Rico Schacherl. Premised on the idea that the institutions and cultural relics of the old South Africa should be auctioned off to make way for the new, the book was Rico's first major outing as an illustrator, providing an opportunity for him to hone the deceptively simple style that would be employed, a few months later, in the origination of Madam \& Eve.

While Madam \& Eve might have seemed to the average newspaper reader of the time to have arrived from nowhere, it was in fact the product of a gestation period in which a new generation of South African humour writers and cartoonists, centered around the Weekly Mail \& Guardian, Laughing Stock and other publications, had been exploring tones and registers of humour, looking for an appropriate way to address the imminent arrival of the new political dispensation. White South Africa in the early 1990s,

${ }^{219}$ Gus Silber, It Takes Two to Toyi Toyi, illustrated by Anthony Stidolph, 1991: 42. 

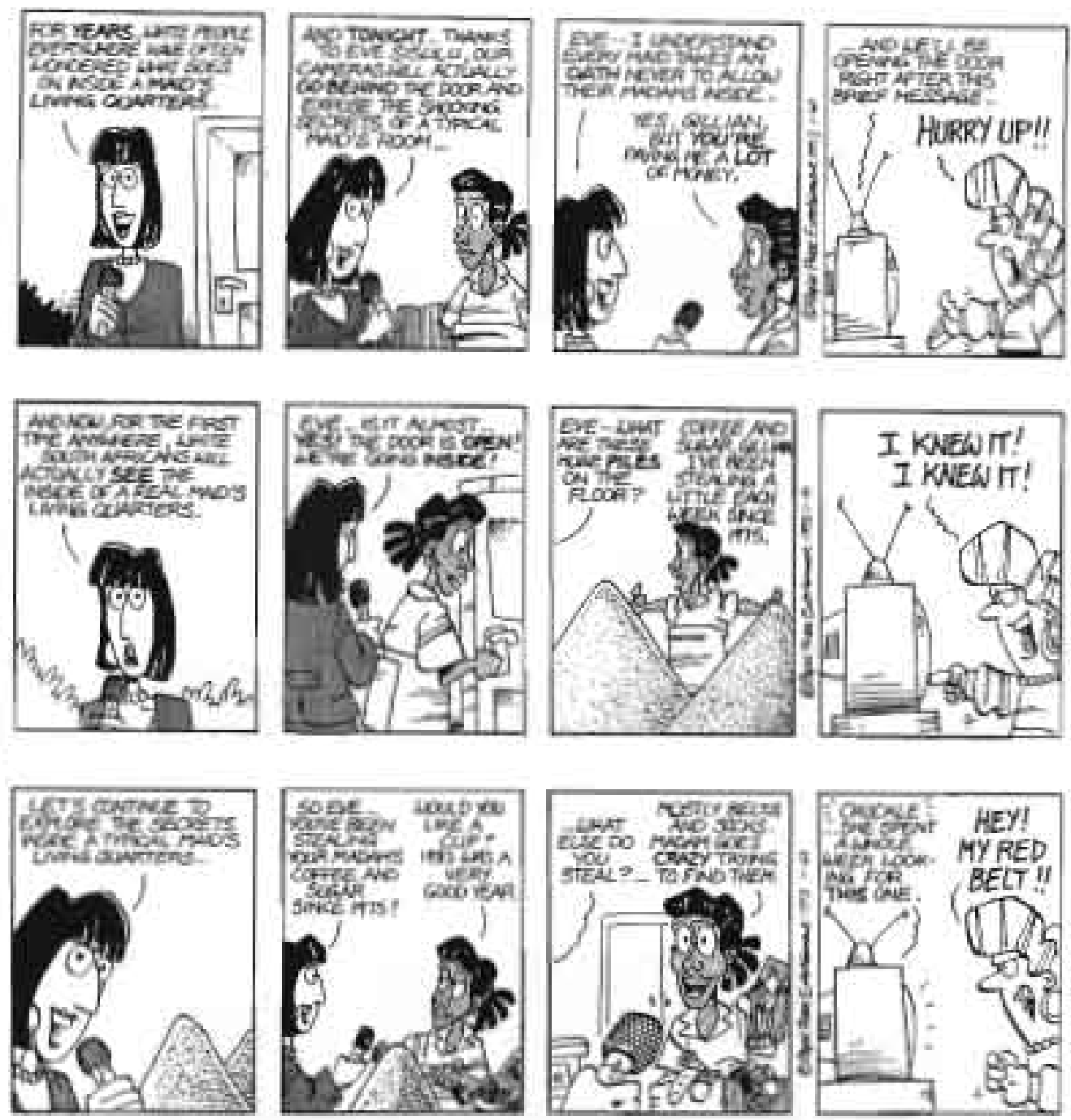

FIGURE 77

In its eariy years, Madam and Eve relentlessiy milked the stereotypical fears and entrenched phobias of white South Africa, ranging from the fear of eventual retribution in the form of property confiscation and land grabs, to the pilfering of the sugar. (Francis, Dugmore \& Rico, 1993.) 


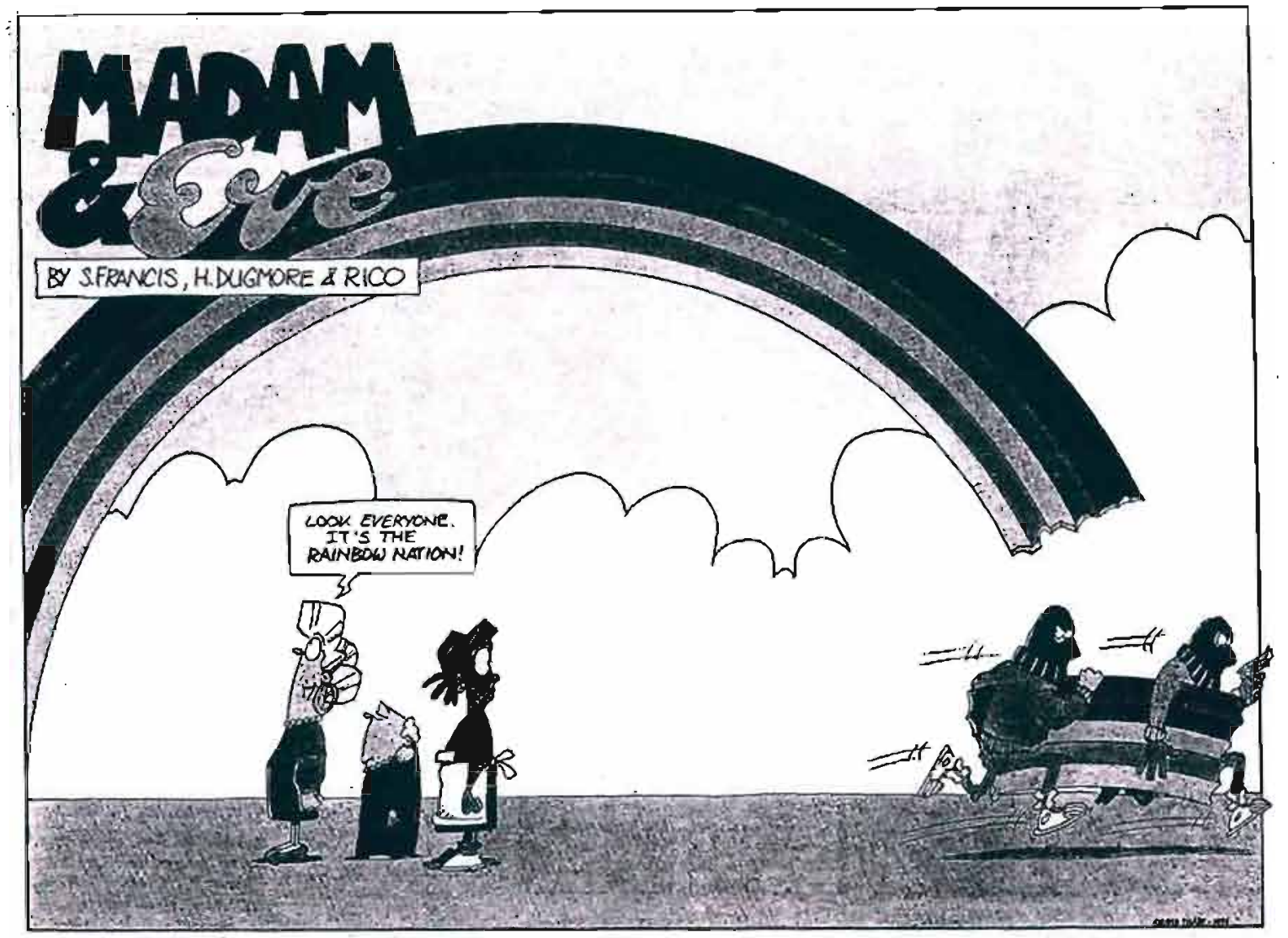

FIGURE 78

This famous 1994 cartoon of a pair of balaclava-hooded gangsters making off with a piece of the Rainbow Nation's iconic rainbow is a recognition that high levels of crime are likely to be endemic to South African society for some time to come. (Francis, Dugmore \& Rico, 1998) 

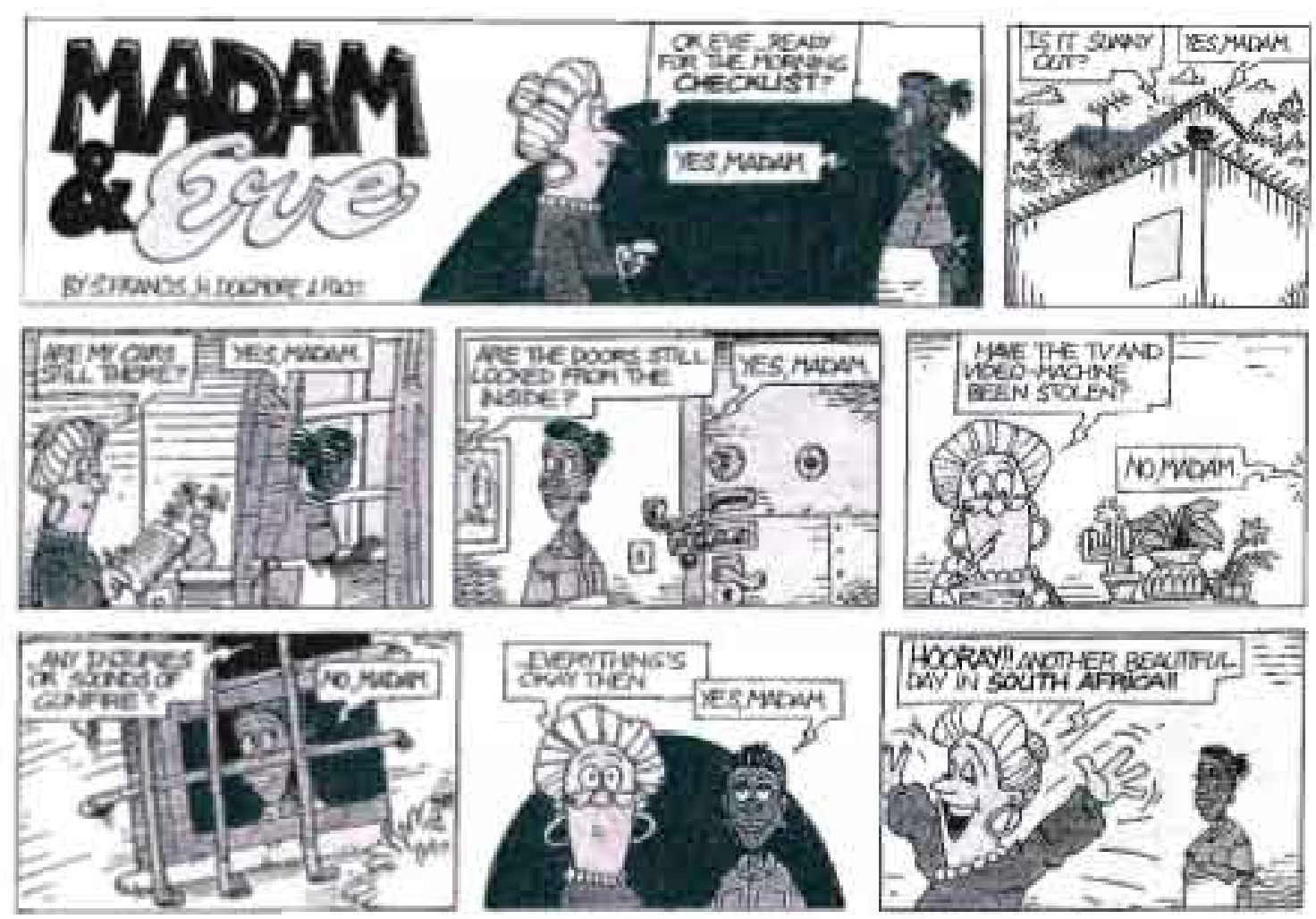

FIGURE 79

In this very early strip from 1992, the excessive security arrangements required for safe urban living in South Africa are naturalised in the phrase "Everything's okay then".

(Francis, Dugmore \& Rico, 1993) 
apprehensive about the future and exhausted by the relentlessly unfolding sociopolitical drama, was an extremely tense society, and the kind of humour explored by Silber, Goldstuck, Francis, Dugmore and the cartoonists they worked with acted as a pressure valve, allowing white South Africans to laugh at themselves. But perhaps more importantly, it allowed a number of painful and hard-to-talk-about issues to come to the surface, disguised as jokes.

The immediate success of Madam $\mathcal{E} E v e$, in a country where comic strips had previously been targeted at ethnic or culturally specific readerships, lay in its ability to cross over ideological and cultural boundaries. It did this by virtue of its irreverent approach to the intimate domestic employment relationship, one that has universal resonance for South Africans of all races and classes, either as employers or employees, or as members of the families of one or the other. The highly skewed economic relations that have prevailed since colonial times have enabled almost all white South African families to employ servants, thus according even the most economically and intellectually challenged whites the status and power of employers.

These long-standing relationships had given rise to stereotypical fears and entrenched patterns of behavior, ranging from the fear of eventual retribution in the form of property confiscation and land grabs, to the pilfering of the sugar (Figure 77) 220 . In the early years of Madam \& Eve, Francis, Dugmore and Rico relentlessly milked these phobias and stereotypes in a succession of repetitive jokes and witty rejoinders that combined social commentary with a clever use of visual puns, incongruous juxtapositions, role reversals and other conventions of cartoon humour.

Interestingly, it may have been the strip's lack of a clearly identifiable political position that made it so marketable. Unlike Zapiro, who would often risk alienating readers by adopting an angry posture or controversial position, Francis and Rico tended to adopt the same detached, bemused and slightly cynical view of all tendencies across the South African body politic. The early Madam \& Eve strips were remarkably non-judgemental and even-handed, adopting the same tone in their treatment of gangsters and petty

${ }^{220}$ Reproduced in Francis, Dugmore \& Rico, The Madam \& Eve Collection, 1993: 80 

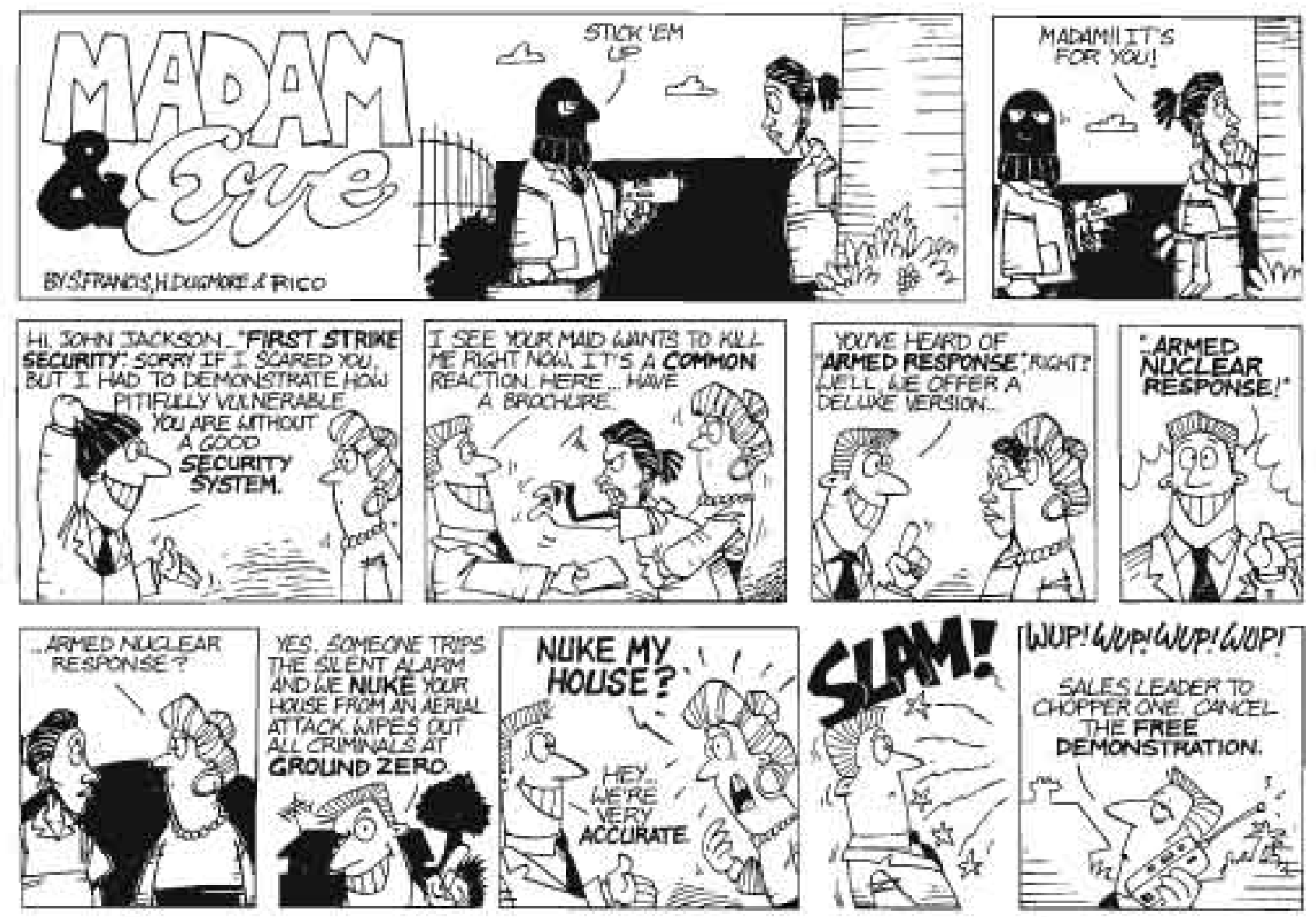

FIGURE 80

In another early strip, the country's security industry is shown to be guilty of cynically exploiting white fears of crime for their own enrichment, thus reducing themselves to the same status as the criminals against whom they purport to be fighting. (Francis, Dugmore \& Rico,1993) 
crooks as they did with law-abiding characters. The effect was to naturalise the situation of widespread crime, as exemplified in the famous cartoon of a pair of balaclava-hooded gangsters making off with a piece of the Rainbow Nation's iconic rainbow (Figure 78)221. This strip is as much a complaint about high levels of crime as it is a recognition that these high levels of crime are likely to be endemic to South African society for some time to come. In a 1992 strip on a similar theme, the excessive security arrangements required for safe urban living in South Africa are naturalised in the phrase "Everything's okay then" (Figure 79)222. The humour derives from the recognition by the reader that these precautions may be extreme, but they are something that people are going to have to live with. In another early strip, the country's security industry is shown to be guilty of cynically exploiting white fears of crime for their own enrichment, thus reducing themselves to the same status as the criminals they purport to be fighting against (Figure 80) 223 .

Their refusal to take an authentically critical stance has been criticised, as has its reproduction of racial and gender stereotypes 224 , but Francis and Rico have tended to sidestep such criticisms. Their most disarming gambit is to affect a disingenuous naivety and an unbridled commercialism in the purveying of their products, which have in the past included a range of Madam $\mathcal{E}$ Eve merchandise such as caps, clothing, stationery items and other gimmicks. Given the limited syndication opportunities that exist in South Africa, the Madam $\mathcal{E}$ Eve team have not been able to rely solely on income generated by the Madam $\mathcal{E}$ Eve strip itself, and have diversified their activities to include a range of media interventions and consultancy services 225 .

\footnotetext{
221 Reproduced in Francis, Dugmore \& Rico, Madam E Eve's Greatest Hits, 1998: 178

222 Reproduced in Francis, Dugmore \& Rico, The Madam \& Eve Collection, 1993: 55

223 Reproduced in Francis, Dugmore \& Rico, The Madam \& Eve Collection, 1993: 9

224 See Britten, S. (2000). MA dissertation.
}

225 Other projects have included the live-action TV sitcom, which has met with mixed reception but has nevertheless proved durable, and Vern and Dern, a deadpan cartoon strip about a pair of hillbillies that makes no reference to the South African situation. Rico also produces several Internet-based on-line comics in collaboration with like-minded cartoonists in other countries, and is involved in a variety of illustration work, while Francis is similarly involved in a range of writing and television projects, including working as a stand-up comedian. 


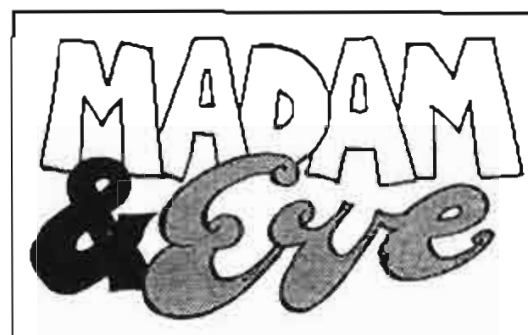

BV SRRANCS, RDUGMORE \& RKCO
THIS IS MORIFYNG! IMAGWE.
BEING HELD UP BY THE
EASTER BLNNY.

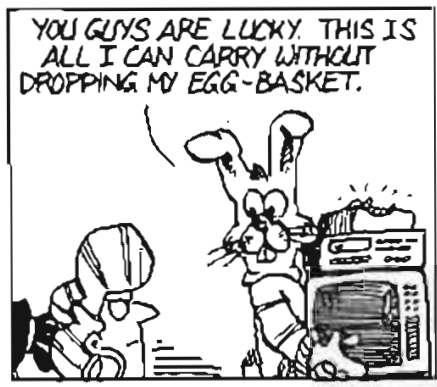

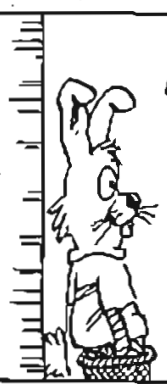
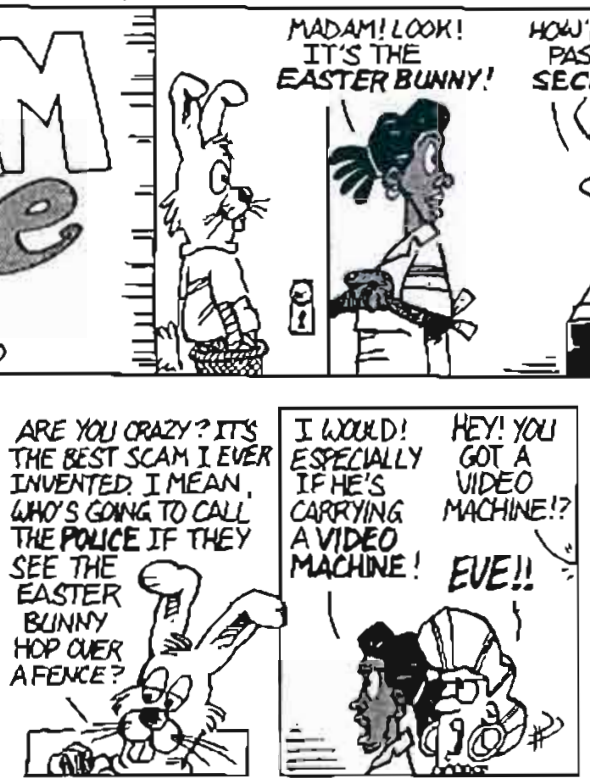

HOW'D HE GET

SECURITY?
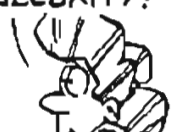

To

donos
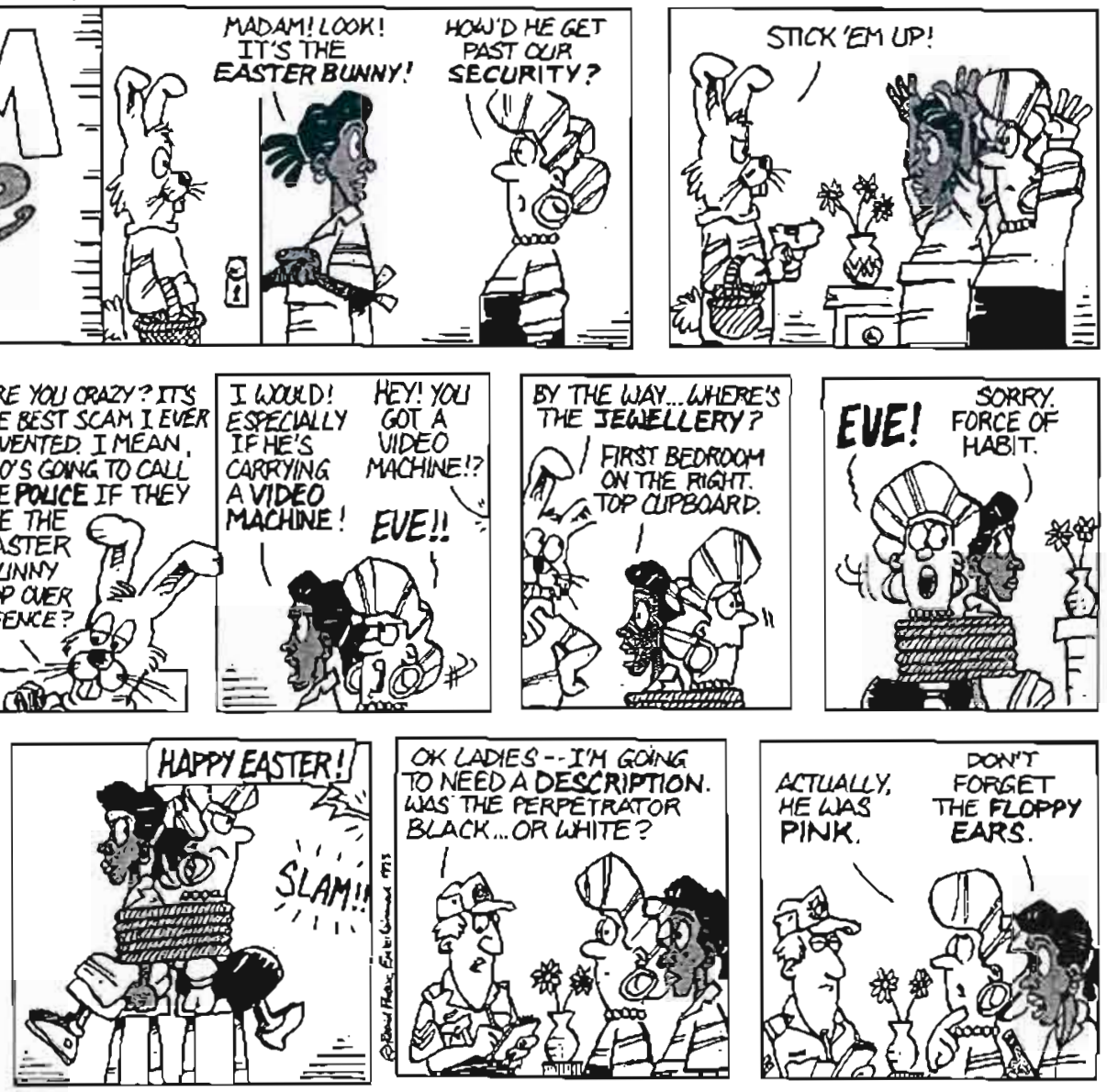

FIGURE 81

Gwen Anderson and Eve Sisulu are presented as co-dependent, and when faced with an external threat to their closed domestic world, in the form of crime, there is a sense of solidarity, of being 'in this together. (Francis, Dugmore \& Rico, 1993)
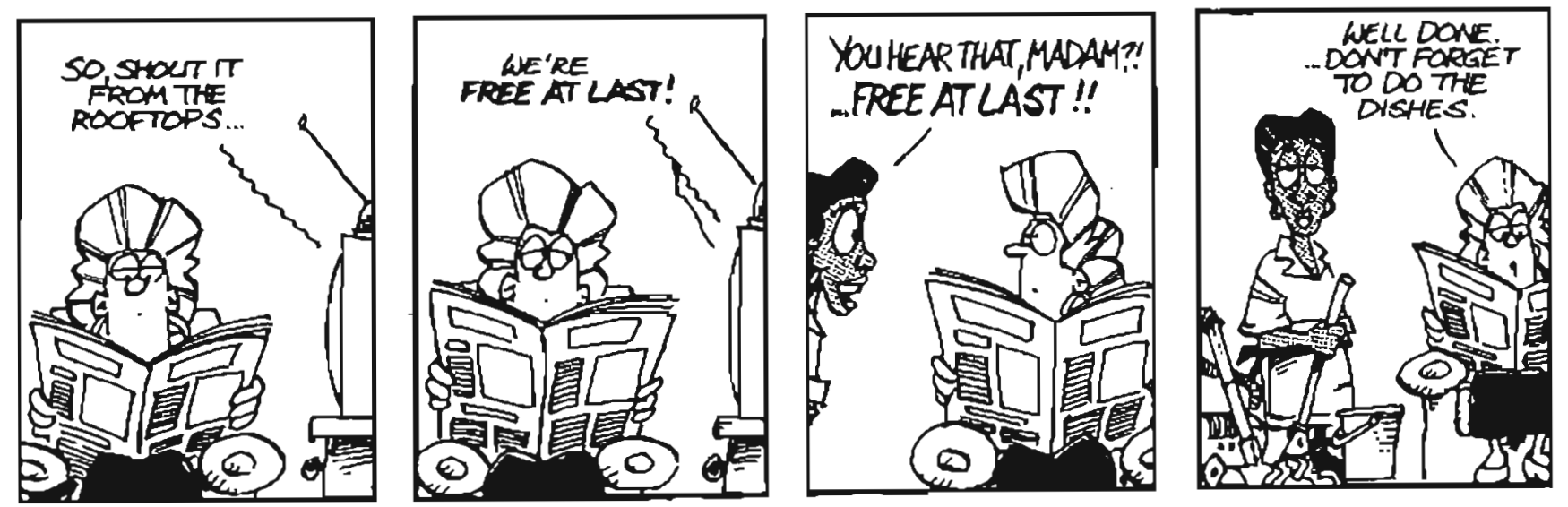

FIGURE 82

In this brief exchange, the continuation and maintenance of South Africa's exploilative ecomomic system, despite political change, is acknowledged. (Francis, Dugmore \& Rico, 1994) 
Madam \& Eve contains a mix of ingredients palatable to a wider range of South African tastes than any newspaper comic strip had previously been able to satisfy. It is perhaps unsurprising that such a strip should have emerged simultaneously with the new political dispensation it celebrates. Perhaps Francis and Rico's greatest achievement has been to create a set of characters who, while ostensibly functioning to comment on the unfolding social and political process in South Africa, have each accumulated a strong sense of individuality in their own right. In particular, the character of Eve is well individuated. While she is represented as being lazy, this characteristic is not pejoratively articulated: it is seen as her right to seek opportunities to escape work by lying on the ironing board, since the work she is required to do (ironing and cleaning) is tiresome and repetitive. She is often given a witty rejoinder or the last laugh, and is often presented as cleverer or more streetwise than her employer. The two of them are set up against each other in an interminable, but not unaffectionate battle: Eve to escape her responsibilities as an employee (work); Madam to escape her responsibilities as an employer (paying a living wage). Nevertheless they are presented as co-dependent, and when faced with an external threat to their closed domestic world in the form of crime, there is a sense of solidarity, of being 'in this together' (Figure 81)22.

The humour stimulated by the strip is often the humour of recognition, through which the reader gains some insight into his or her own behaviour. In this sense, the strip turns its readers into the subjects of its satirical discourse. The early Madam \& Eve strips were less concerned with satirising the apartheid regime itself than with poking fun at ordinary people who occupied various positions within the social order. In this sense their critique was often more socio-economic and structural than political. A good example is the 1994 strip where Eve cries: "You hear that, Madam?!... Free at last!" her employer looks up briefly from the newspaper to reply: "Well done... Don't forget to do the dishes." (Figure 82)227 In this brief exchange, the maintenance and continuation of South Africa's exploitative economic system, despite political change, is acknowledged.

The strips often reproduce or exaggerate stereotypical perceptions for satirical effect: domestic workers, symbolised by Eve, are by nature lazy and would rather sleep on the

226 Francis, Dugmore \& Rico, The Madam \& Eve Collection, 1993: 79. 
ironing board than iron on it, while employers, symbolised by Gwen Anderson, are naturally stingy and will instinctively avoid paying a living wage. Recognition and satirical exaggeration of these behaviours does not necessarily legitimate them, but it does make them more transparent. Holding up a mirror in which readers can see parodies of their own behaviours thus serves a pro-social, educative function.

While Rapid Phase Entertainment have allowed the Madam \& Eve characters to be used from time to time for educational purposes, education and conscientisation has not been their primary purpose. Their most obvious contribution, in terms of the three functions of oppositional cartooning identified earlier, has been to provide pro-social iconography supportive of the transition to a new South Africa. Ironically, this iconography has generally dwelt on the well-known wrongs and limitations of South African life-crime and corruption, internalised racism and economic inequality. But by bringing these subjects to the forefront of popular discourse in a humorous way, the strip has arguably served to assist its readers in developing insights that help them make sense of, or at least come to terms with, the changed reality they now inhabit. If the creators of Madam E Eve have had a conscious developmental strategy-and it is unlikely that they would own up to such a thing - it has been to promote the values of tolerance and acceptance of diversity amongst the nation's population. These values amount to survival strategies in the new South Africa and are essential if ordinary people, especially those who were socially, economically and politically advantaged under apartheid, are to integrate themselves in a positive manner into the new, non-racial social order that it is the avowed task of the so-called 'Rainbow Nation' to construct.

Like Zapiro, Madam \& Eve has the distinction of being born at the right time: both began in the alternative press in the dying years of apartheid and were on hand to witness and celebrate the transition to the new South Africa. In doing so, they imprinted themselves on the national psyche by providing humorous visual images and accessible texts that their readers could identify with and, like Mandela and the ANC, were themselves transformed from rebels outside the citadel into icons of the new mainstream.

227 Francis, Dugmore \& Rico, Free at Last: The Second Madam \& Eve Collection, 1994: 1 


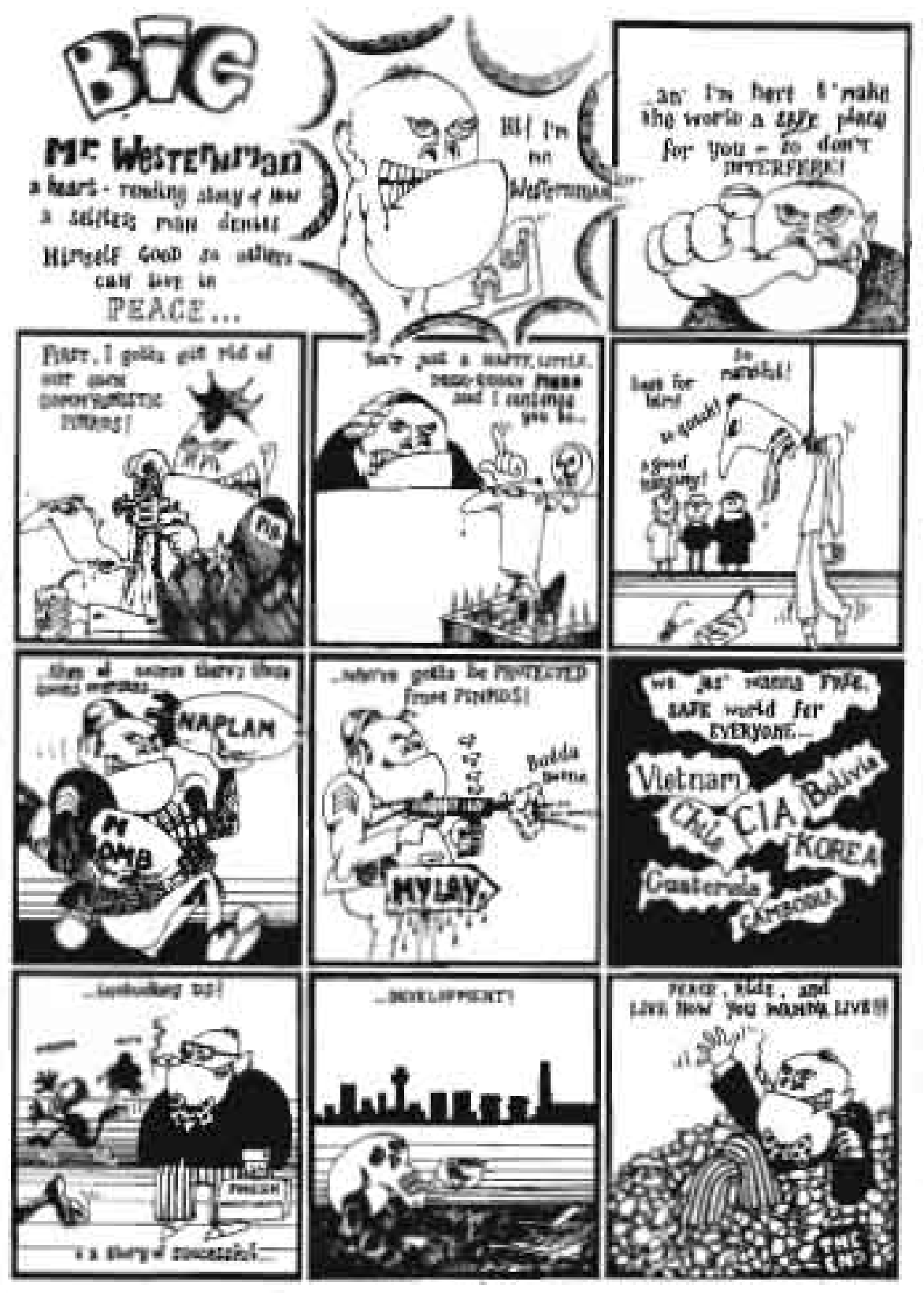

FIGURE 83

Student cartoonists in the early 1970s, like Wits University's Franco Frescura, Costas and Olly (above), critiqued South African society from a radicat New Left perspective.

(Real name unknown, Wits Student, circa 1972) 


\section{wits student}

WITWATEASAANO UNIVERSITY STUOENT NEWSPAPEA

FanAY IU SEPTEMAGR: IVI

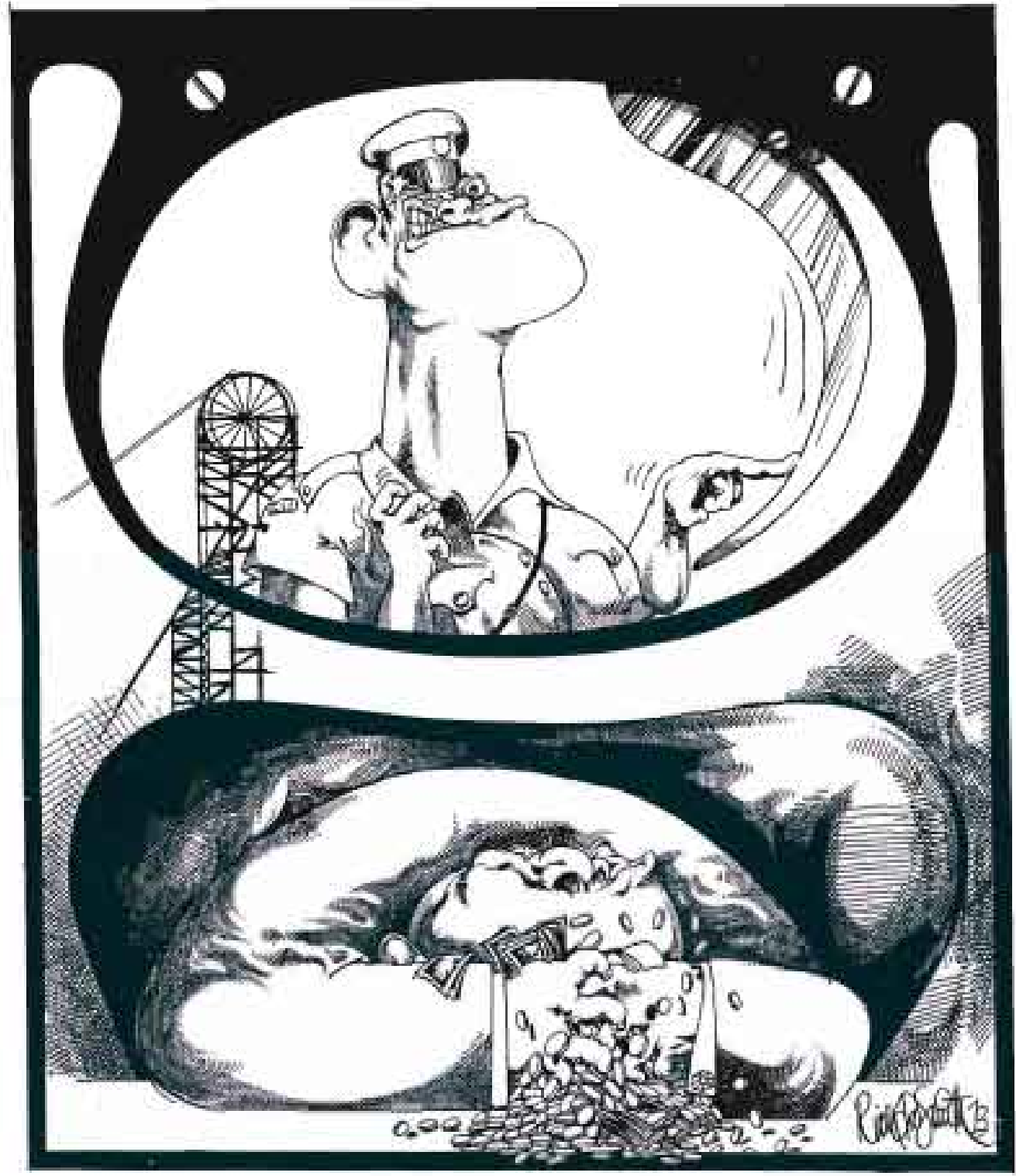

FIGURE 84

Richard Smith produced several memorable covers and cartoons for Wits Student and other student publications, demonstrating here the radical form that distinguished the early years of his career. This cartoon demonstrates strikingly similar sentiments to one of Zapiro's 1987 South cartoons (see Figure 68). (Smith, Wits Student, circa 1972) 

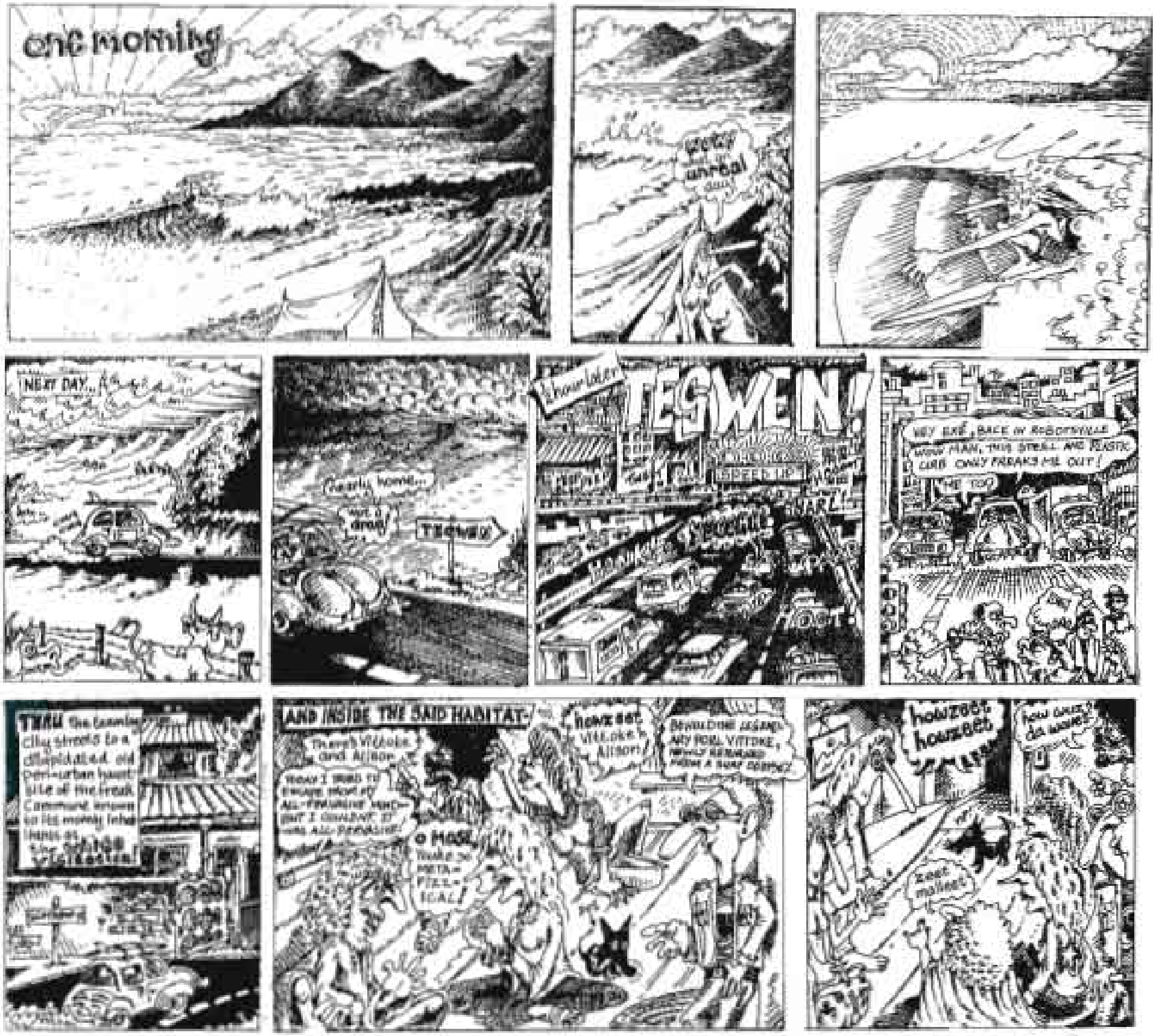

FIGURE 85

Working under the nom de phume 'Pooh', the present writer published a satire about student radicals and hippies entitied Viftoke in Azania, very derivative of Gibert Shelton's Fabulous Fumy Freak Brothers, in the student press at the Universates of Natal and Cape Town (Pooh, 1979) 


\section{Cartooning for social change:}

\section{Educational comics and the Storyteller Group}

In South Africa during the 1970s, during the heyday of the National Union of South African Students (NUSAS), the student press at the English-speaking universities was relatively strong and highly politicised, and supported an active cartooning subculture strongly influenced by U.S. underground comix. Student comix from this period demonstrated the influence of underground cartoonists like Gilbert Shelton and Robert Crumb. Student cartoonists of the early 1970s, like Wits University's Franco Frescura, Costas and Olly (Figure 83)228, critiqued South African society from a radical New Left perspective, and Richard Smith produced several memorable covers and cartoons for Wits Student and other student publications (Figure 84)229. At the University of Natal, the present writer, working under the nom de plume 'Pooh', produced a satire about student radicals and hippies entitled Vittoke in Azania, published in the student press at the Universities of Natal and Cape Town (Figure 85)230.

As has been observed above, interest in cartooning was being expressed at that time amongst media activists in the broad-based anti-apartheid movement. The publication in 1976 of Marx for Beginners by the legendary Brazilian activist and cartoonist, Rius231, had sparked interest in the use of cartooning for revolutionary educational purposes. As has been observed above, South African media activists hoped that the combination of words and pictures might assist in conveying relatively complex messages to people in peri-urban and rural settings where low levels of literacy were the norm. In 1980 the

\footnotetext{
${ }^{228}$ Comix by Olly (real name and exact date unknown) appeared in the Wits University student paper, Wits Student, in the early 1970 s.

229 Richard Smith, cover of Wits Student, (University of the Witwatersrand Students' Representative Council, circa 1972).
}

230 Vittoke in Azania appeared in Dome (University of Natal, Durban Students' Representative Council) and Varsity (University of Cape Town Students' Representative Council) in 1978 and 1979.

231 The publication of this book, which sold over a million copies in 12 languages, was the beginning of the "Beginners" series, covering an extraordinary range of historical, philosophical, literary and scientific 


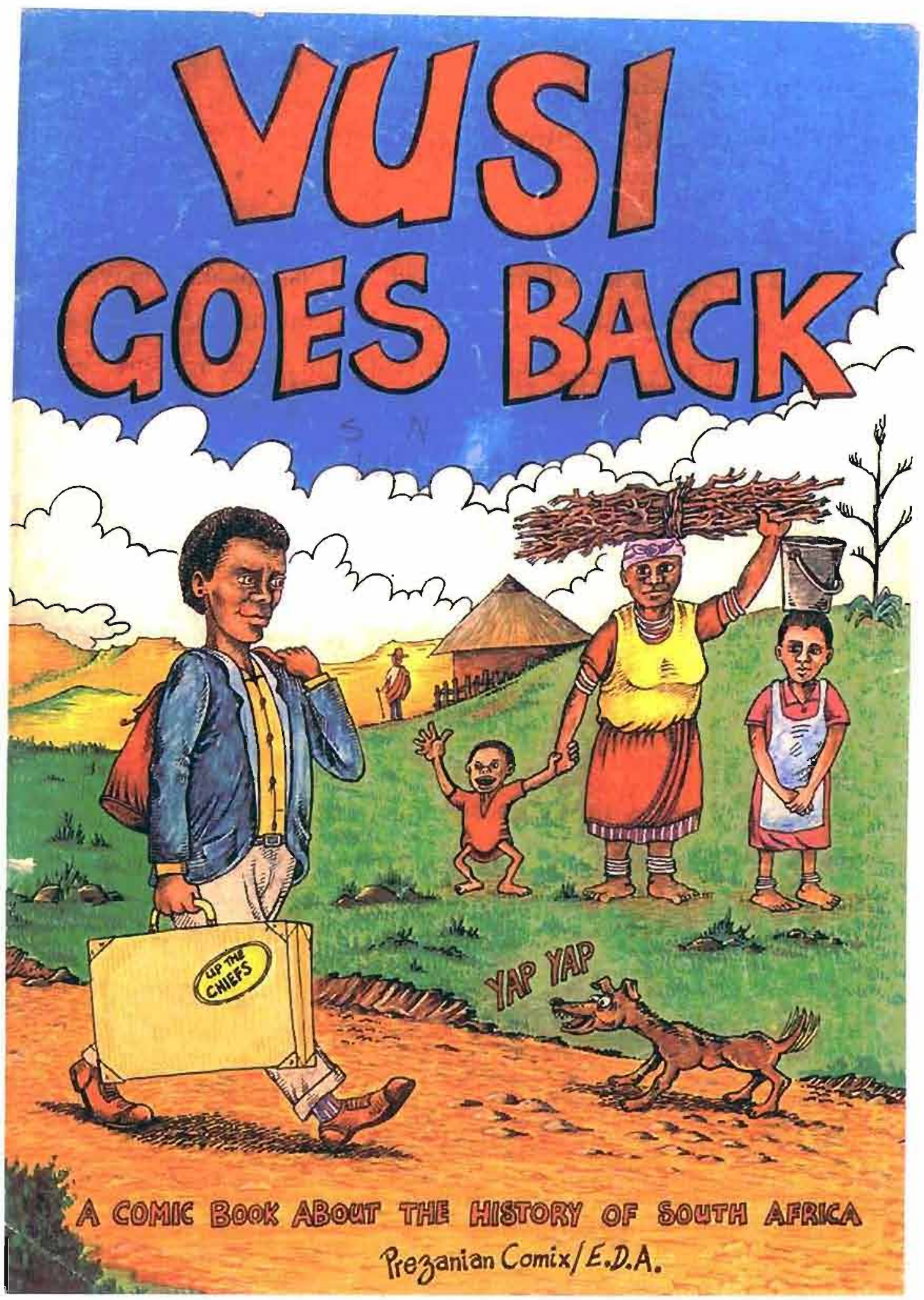

FIGURE 86

Vusi Goes Back: A Comic about the History of South Africa, written by Dick Cloete and Robert Berold and illustrated by Andy Mason, served as the introduction to People's Workbook, published by EDA in 1981. The portrayal of whites and Africans in the slrip is interesting, with the former caricatured, while the latter are presented more naturalistically. (Cloete and Mason, 1981). 
present writer was commissioned by the Johannesburg-based Environmental and Development Agency (EDA) to collaborate with Dick Cloete and Robert Berold in the creation of a comic strip about the history of South Africa, seen from a rural perspective, which was to serve as the introduction to People's Workbook, published by EDA in 1981. The outcome of this collaboration was Vusi Goes Back: A Comic about the History of South Africa (Figure 86) 232 .

People's Workbook, a self-help manual aimed at assisting impoverished communities in the 'bantustans' to achieve some measure of self-sufficiency, was said to have been inspired by the U.S. counter-culture survival manual, The Whole Earth Catalogue. Rick Andrew observed that the style of Vusi Goes Back"is an interesting conglomerate of caricature and 'naturalism' with the intention of giving dignity to the African while satirizing the European"233, while Schoonraad described the strip's artwork as "weak and naive", remarking that it nevertheless did not succeed in giving the impression that it had been produced by a black person 234 .

Following on from Vusi Goes Back, the present writer (under the psuedonym N.D.Mazin) undertook several other cartooning projects, including the comic strip Sloppy, initially co-authored with Mogorosi Motshumi, which first appeared in 1981 in Learn and Teach, an NGO-funded monthly magazine with a Frereian approach to literacy education. Sloppy dealt with issues of township life in a humorous way, and was often used by the publication's editors as a vehicle for the transmission of advice about such subjects as

topics (now published in the UK as the 'Introducing..." series). (Rius, originally Marx for Beginners, 1976; now entitled Introducing Marx, 1998).

232 Vusi Goes Back waspublished as an independent comic book by Prezanian Comix in 1982.

233 Rick Andrew, unpublished dissertation, 1988. Andrew's observation anticipates this study's argument about 'responsible realism' vs 'satirical experimentalism' (see Chapter Five). From an authorial point of view, I can say that the difference in the rendering of white and black people, noticed by Andrew, probably arose out of a fear of portraying black people in a racist way, while whites were freely caricatured.

234 Schoonraad, 1983: 157: “Die strookprent word gekenmerk deur 'n swak en naiewe tekentegniek wat nie daarin slaag om die produk soos die sketswerk van 'n Swartman te laat lyk nie." While it was never my intention to make the comic look like it had been produced by a black person, Schoonraad's assumption is nevertheless instructive in view of the discussion in this study of the representation of black subjects by white cartoonists. 

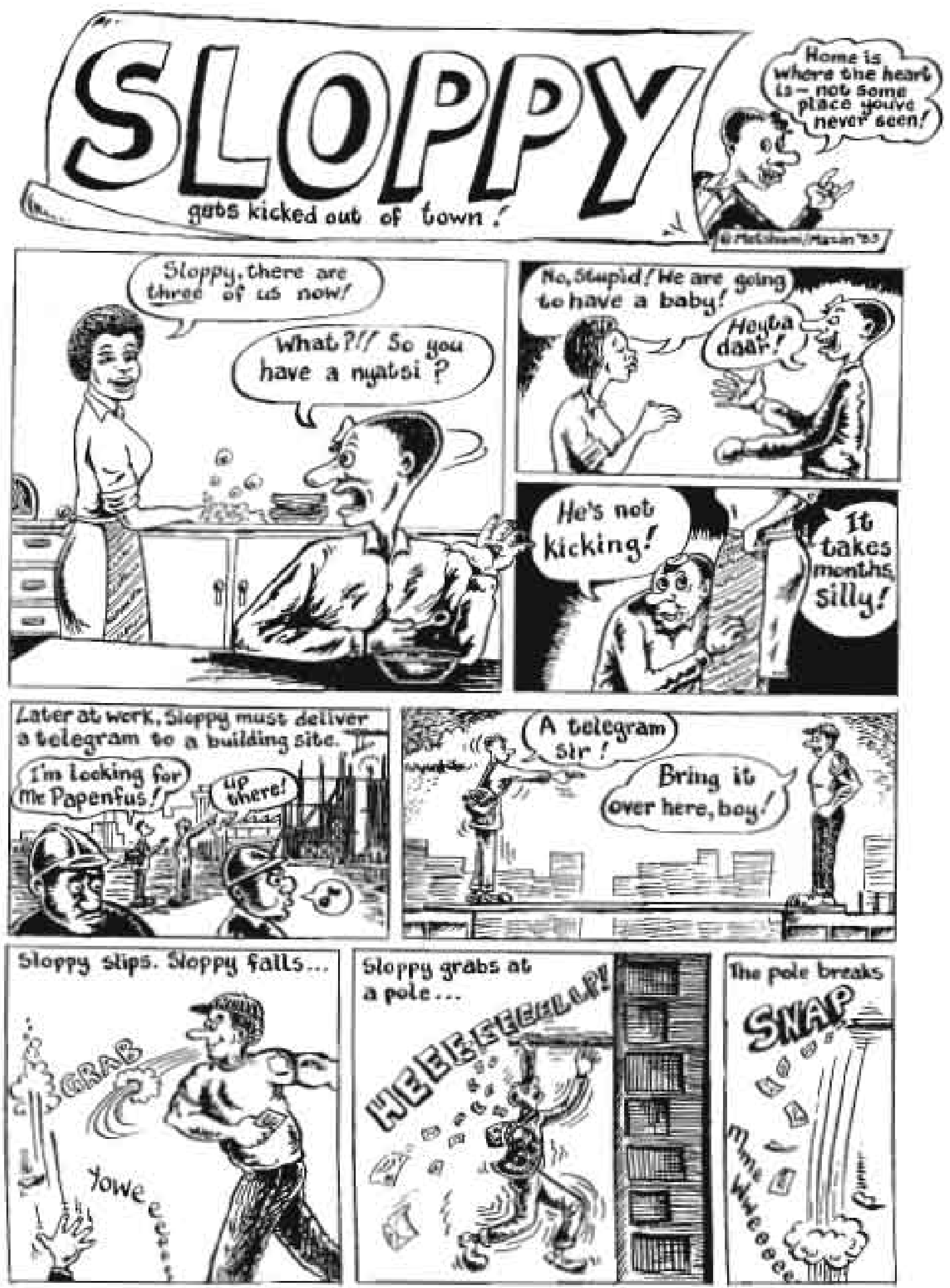

FIGURE 87

Siloppy, by Mogorosi Motshumi (initially co-authored with N D. Mazin), dealt with township life and often provided advice to readers. (Mogorosi Motshumi and N. D. Mazin 1883) 
higher purchase agreements and employment contracts (Figure 87)235. After the first three years of joint authorship, Motshumi continued to write and draw the strip for the rest of the decade.

Motshumi, who today works as a freelance cartoonist, was one of very few black cartoonists to be regularly published in the late apartheid period ${ }^{236}$. Alongside his work on Sloppy, he also produced editorial illustrations for Learn and Teach and other publications emanating from Johannesburg's NGO sector. Born in 1955, Motshumi grew up in Batho township near Bloemfontein. His first published cartoons appeared in The Friend in 1978, until he was detained by the security police for two weeks after the publication of a cartoon they found offensive. The newspaper refused to take him back after his release and he moved to Johannesburg, where he began working for The Voice, a radical ecumenical newspaper, and submitted drawings to Staffrider magazine. His comic strip In the Ghetto ran for about a year until The Voice closed down. He was then recruited by the Learn and Teach literacy organisation to illustrate and lay out literacy booklets, where he joined another black illustrator, Goodman Mabote. Sloppy began in the first issue of Learn and Teach, the organisation's monthly magazine, and is the most sustained comic strip about township life to have emerged from the late apartheid period. Its characters were humble proletarian folk living in Soweto, and Motshumi took them through an endless succession of misadventures over the next ten years, with frequent recourse to autobiographical themes. It is a tragedy of South African cartooning that Sloppy was allowed to fall by the wayside when Learn and Teach collapsed, along with other alternative press publications, in the mid-1990s. Motshumi continues to work as a freelance cartoonist.

Another black comic strip creator worthy of mention is Percy Sedumedi, a talented street artist, poet and painter who has since vanished into obscurity, but who in the 1980s self-published several issues of a comic entitled Travels of the Free Spirit. This freeflowing metaphysical romp with martial arts theme matched the personality of this enigmatic character. Several of Sedumedi's stories were republished in PAX (Pre-

235 Sloppy, by Mogorosi Motshumi and N.D.Mazin, Learn and Teach, 1983.

236 Interviews, 2002, 2003. 


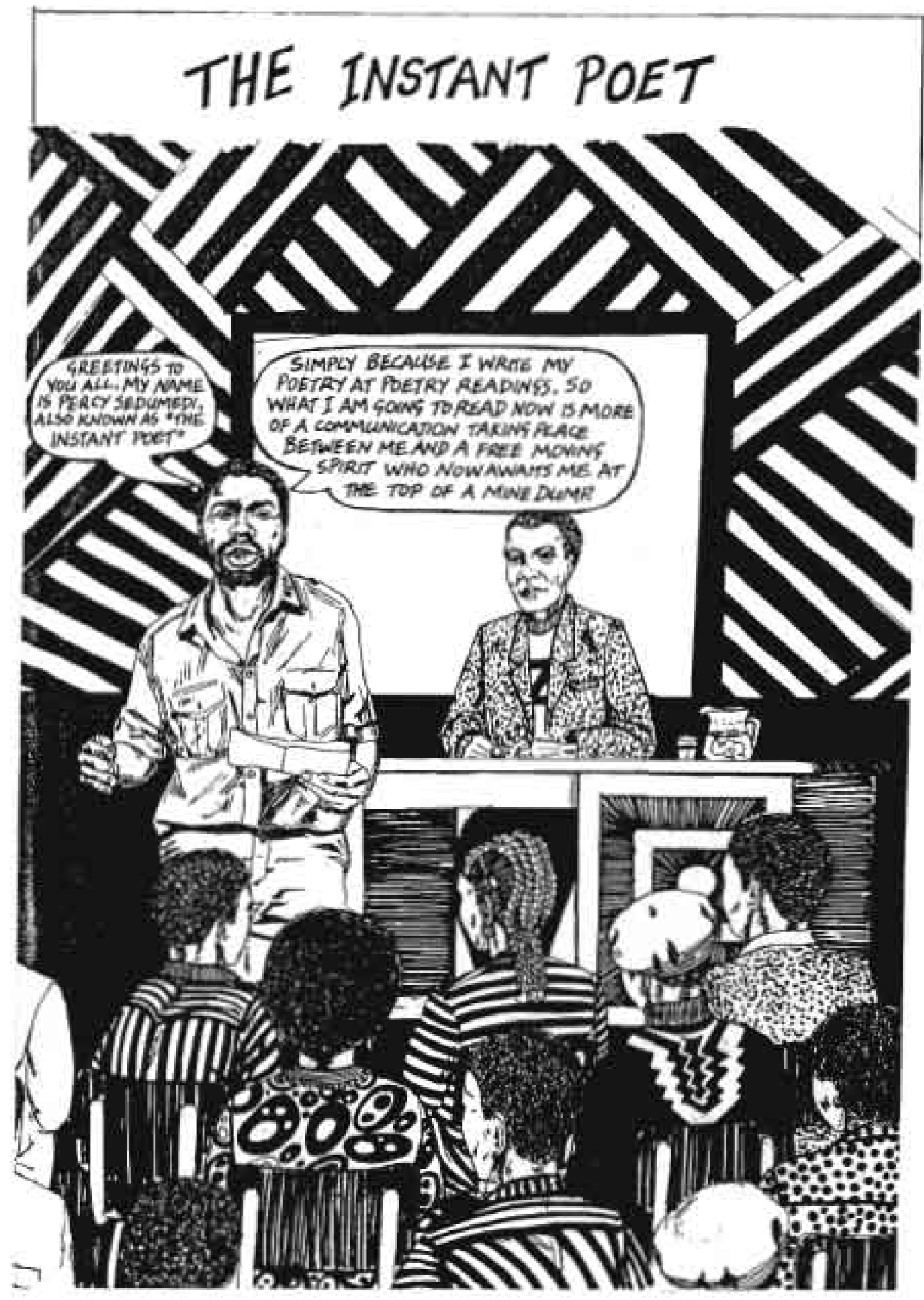

FIGURE 88

Percy Sedumed, a taiented street artist, poet and painter, self-published several issues of a comic entited Travels of the Free Spint in the 1980s. Several of Sedumedi's stories were republished in PAX (Pre-Azanian Comix) in 1987-8, including this strip. entitiod "The Instant Poet". (Sedumedi, 1987) 
Azanian Comix) in 1987-8 (Figure 88 )237 and a strip by Sedumedi (date unknown) also appeared briefly in Drum magazine. Sedumedi also worked briefly with the Storyteller Group.

Educational comic strips were also published by the South African Council for Higher Education (Sached) in its monthly magazine for school children, Upbeat 238 . One of first of these strips, based on the 1959 autobiographical novel Down Second Avenue by Ezekiel Mphahlele, was illustrated by Mzwakhe Nhlabati and appeared in Upbeat in 1981 (Figure 89). It was was later published as a comic book, the first in a planned series of educational People's College Comics, in 1988. Of interest are the 14 pages of exercises at the back of the comic book, clearly demonstrating the intention to utilise the comic strip form as an educational tool. Over the next two years Sached published two more People's College Comics titles: Equiano: The Slave Who Fought To Be Free, illustrated by Rick Andrew; and Mhudi, a comic book based on the novel by Sol T. Plaatjie, illustrated by Grant Cresswell, published in association with the Storyteller Group.

A highly skilled illustrator with a distinctive style, Nhlabati worked in the Sached art department and provided illustrations for Upbeat and other Sached publications in the 1980s. His work also appeared regularly in Staffrider magazine, for which he also produced occasional covers. He produced the cover illustration for the first edition of Call Me Not a Man by Mtutuzeli Matshoba, an important fictional work in the black consciousness tradition, published by Ravan Press in 1982. During the course of his involvement with Upbeat, he illustrated several serialised comic strips of an educational nature in the magazine, including Romance at Riverdale High, written by acclaimed novelist Chris van Wyk.

Following the publication by Sached of the People's College Comics there was a renewed interest in the use of comic books for educational purposes amongst progressive media activists. The most influential and sustained educational comics

\footnotetext{
237 Percy Sedumedi, "The Instant Poet, PAX (Pre-Azanian Comix) No. 1, 1987).

238 I worked as assistant editor of this publication in 1981 and 1982, and was involved in a number of cartooning projects for the magazine during these and subsequent years, including adaptations of Weep Not Child by Ngugi Wa'Thiongo (illustrated by Mzwakhe Nhlabati), A Message in The Wind by Chris van Wyk, and the strip Stella Starfinder's Encyclopaedia of the Universe.
} 

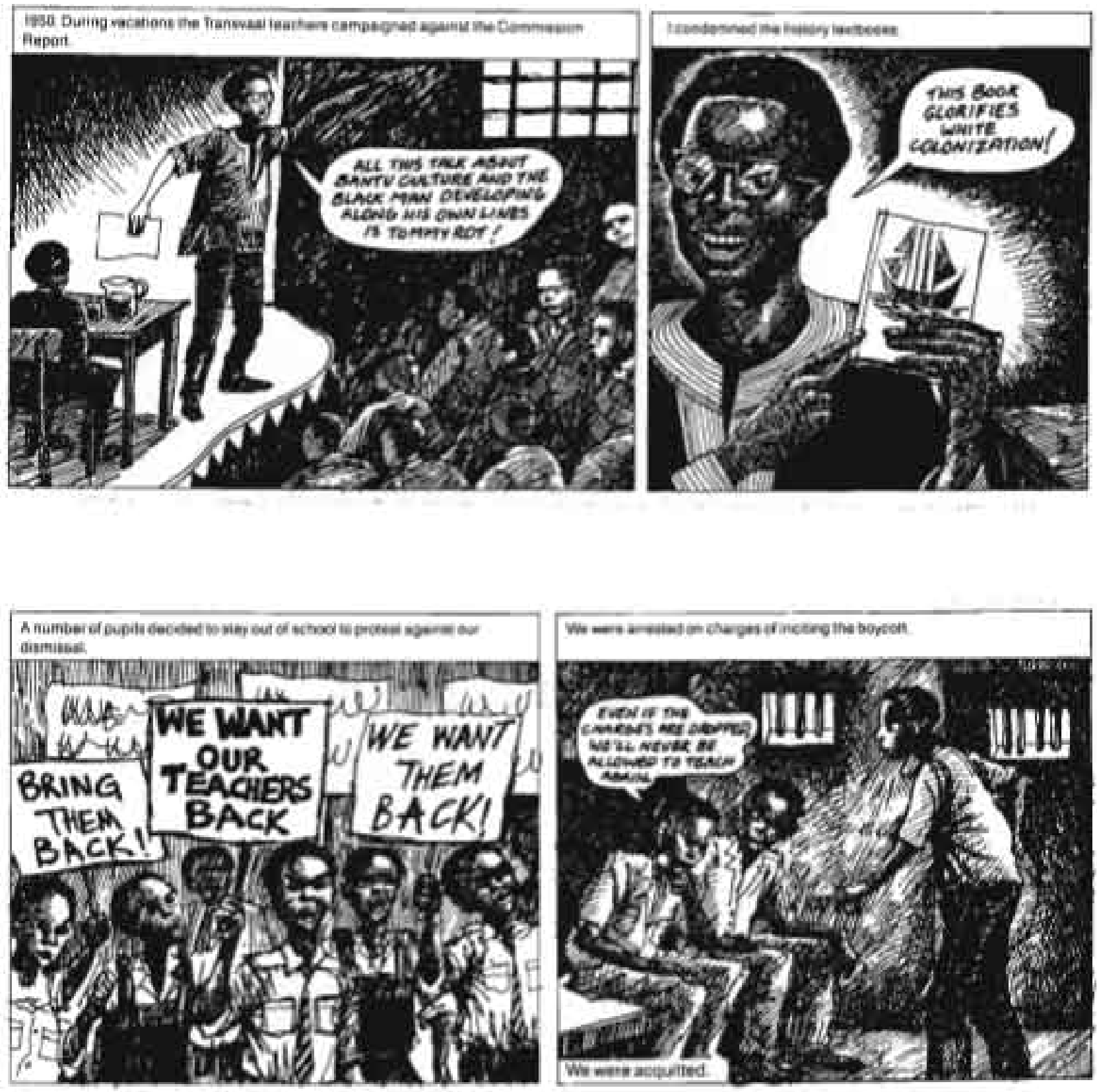

FIGURE 89

This comic strip, based on the 1959 autobiographical novel Down Second Avenue by Ezekiel Mphahleie and illustrated by Mzwakhe Nhlabati, appeared in Upbeat in 1981 and was later published as the first of Sached's People's Colege Comics series, in 1988.

(Lawson, Nhlabati of $w$, Upbeat magazine, 1981) 
publishing initiative of this period was the Storyteller Group, founded by Neil Napper and Peter Esterhuysen. These two visionaries pioneered the idea of kick-starting a 'reading culture' by providing 'popular visual literature' to disadvantaged communities who had been structurally deprived of appropriate texts and reading opportunities ${ }^{239}$. In 1990, working with illustrator Carlos Carvalho, they created 99 Sharp Street, a comic strip series aimed at teenagers. The strip was serialised in a mass circulation promotional magazine distributed by a leading clothing retailer to black consumers.

99 Sharp Street's use of colloquial language and the local references in the artwork proved very popular and, based on this response, the Storyteller Group embarked upon an ambitious research and publishing programme that drew in schools, tertiary institutions, literacy organisations, libraries, newspapers, NGOs and corporate funders. More than a quarter of a million copies of the resulting comic book, A River of Our Dreams, were distributed via the New Nation newspaper and by participating institutions and organisations (Figure 90)240.

The Storyteller Group was extremely active between 1991 and 1996, and at one stage employed more than 20 full-time staff members 241 . The group produced a number of titles and distributed millions of comic books free of charge to black communities ${ }^{242}$. These comics covered a range of social and educational themes from HIV/Aids to environmental conservation and entrepreneurship. The group used several cartoonists, notably Carlos Carvalho and Alastair Findlay, and their comics were generally printed in full colour. They favoured the Belgian 'clear line' approach to illustration, reminiscent of Herge's Tintin, in preference to the cross-hatched black and white 'underground' styles that had tended to characterise Sached's Upbeat and People's College Comics.

\footnotetext{
${ }^{239}$ Two interviews with Neil Napper and one with Peter Esterhuysen were undertaken between 2001 and 2003. The Storyteller Group also produced several important documents outlining the theoretical principles behind their approach. (See Bibliography.)

240 Storyteller Group, A River of Our Dreams, 1991.

241 Interview with Neil Napper, 2001.

242 See the Bibliography for a list of some of the Storyteller Group's publications.
} 


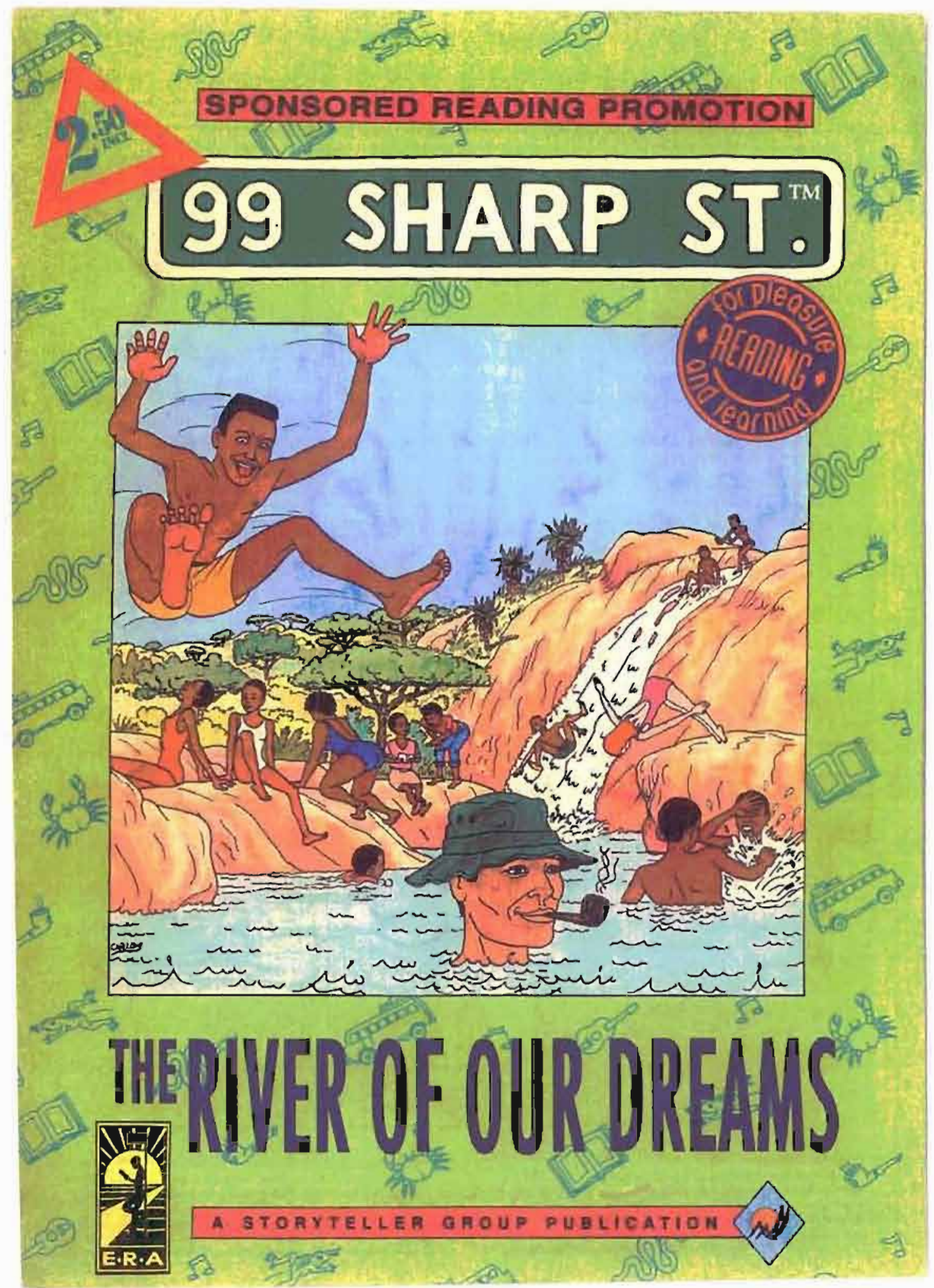

FIGURE 90

More than a quarter of a million copies of the Storyteller Group's 1991 comic book, A River of Our Dreams, were distributed via the New Nation newspaper and by participating institutions and organisations. (Storytelier Group, 1991) 
Most of the Storyteller Group's comics were either sponsored or commissioned, and they were generally distributed free of charge. As a result, each comic bore the imprint of the financial relationships that underlay its existence. Thus a 1990 comic commissioned by the Soweto Civic Association was designed to inform township residents of the negotiations leading up to the signing of the Soweto Accord, according to which residents would resume paying for rent and services 243 , while a 1991 comic book sponsored by the International Red Cross sought to convince township residents of the organisation's neutrality in order to ensure the protection of Red Cross medics working in areas of unrest ${ }^{244}$.

In each case, a highly participatory creative process, involving 'democratic' participation by representatives of the target group to ensure the validity and credibility of the stories told, was employed. In this way the kind of problems faced by white cultural activists in all disciplines - problems of credibility, legitimacy and the 'right to speak' for others-were addressed by a collaborative process. At the same time, talented young black illustrators were identified and trained to produce comic strips in a new, indigenous idiom.

The collaborative and affirmative nature of the Storyteller Group's process tended to demand the involvement of large numbers of participants, facilitators and consultants, and the costs incurred were ultimately unsustainable. Sadly, this visionary attempt to kickstart a reading revolution in South Africa based around comics texts was undermined by the economic relations within which it was trapped. While the potential readership was envisioned as millions of South Africans who lacked appropriate reading materials and would benefit from the production of easy-to-read comics produced especially for them, the publishers were financially dependent on a few funders and large companies to finance these experiments as part of their social responsibility spending. Another consequence was that, because the comics were given away free of charge, it was impossible to gauge how popular they really were.

\footnotetext{
243 Voice of the SCA: The Soweto Accord. A Victory for Residents. Soweto Civic Association, 1990.

244 Red Cross: Helping People in Need. International Committee of the Red Cross, 1991.
} 

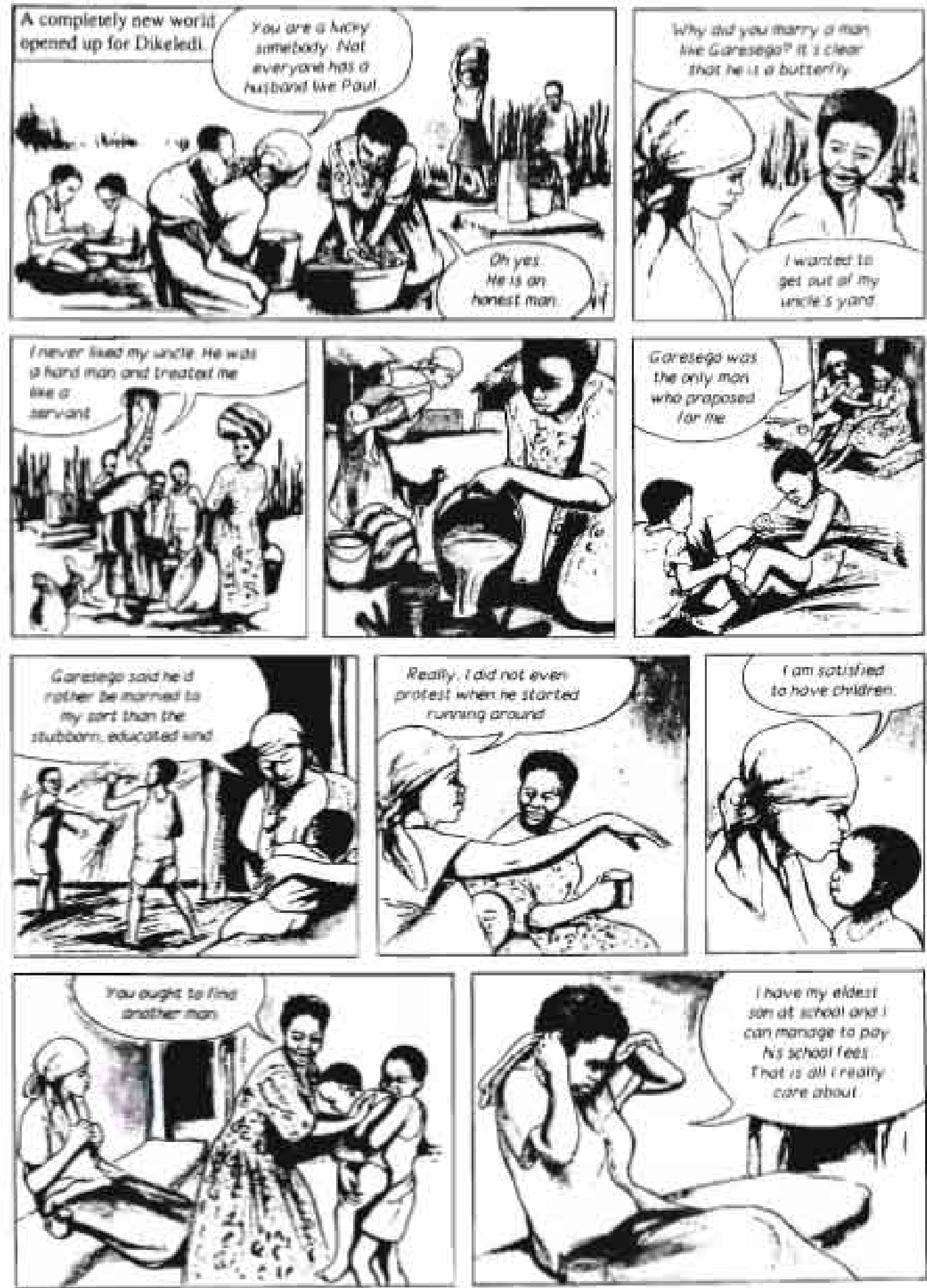

FIGURE 91

In 1993 the Storyteller Group published Deep Cuts, containing 'graphic adaptations' of short stories by Can Themba (ilustrated by Grant Cressweli), Alex La Guma (illuatrated by Janine Cornieise and Carole Howes), and Bessie Head (illustrated by Lois Head, above).

(Maskew Miler Longmanstoryteller Group, 1993) 
In 1993 the Storyteller Group published Deep Cuts ${ }^{245}$, a beautifully produced little book containing 'graphic adaptations' of short stories by black South African writers, illustrated by Grant Cresswell, Janine Cornielse and Carole Howes, and Lois Head (Figure 91). The measured, unhurried rhythms of these graphic narratives, rendered very artistically in grey scale, each in a different style, with painstaking authenticity of location and characterisation, more than do justice to the rather oblique stories from which they are derived. The book was ahead of its time, akin in style and presentation to contemporary graphic novels published in France and the U.S., but it was aimed at a public that had no previous experience of this kind of literature. Despite a very low cover price, it was unable to secure effective distribution, and, out of context and lost between genres, it sank, virtually without trace.

One of the most important comics produced by the Storyteller Group was Heart to Heart: From Dream Love to True Love (1994), an innovative treatment of sexual themes that reflects on the process of its own creation to such an extent that it literally rewrites itself an alternative ending in front of the reader's gaze (Figure 92) ${ }^{246}$. The comic works as a reflexive text which interrogates the problems of its own existence and demands a similar introspection of the reader.

The comic was based on a collaborative story-making process initiated by Patricia Watson, a teacher in a rural school in the Mpumalanga province who had previously worked for the Storyteller Group as a researcher on Deep Cuts ${ }^{247}$. Watson invited a group of youngsters together to workshop a story for a comic about sex, Aids and gender issues. But on completion of this process, the Storyteller Group had serious misgivings about continuing the project because "existing patriarchal relations of domination in the community were naturalized" in the workshop process (Watson Shariff and Janks, 2001). The resulting story, in which "a beautiful young woman had to

\footnotetext{
245 Storyteller Group, Deep Cuts: Graphic Adaptations of Stories by Can Themba, Alex la Guma and Bessie Head,
1993.

246 Storyteller Group, Heart to Heart: From Dream Love to True Love, 1994.

${ }^{247}$ See Watson Shariff and Janks, 2001. "Changing Stories: The Making and Analysis of a Critical Literacy Romance comic", in IJOCA, Vol. 3, No. 2, 2001.
} 


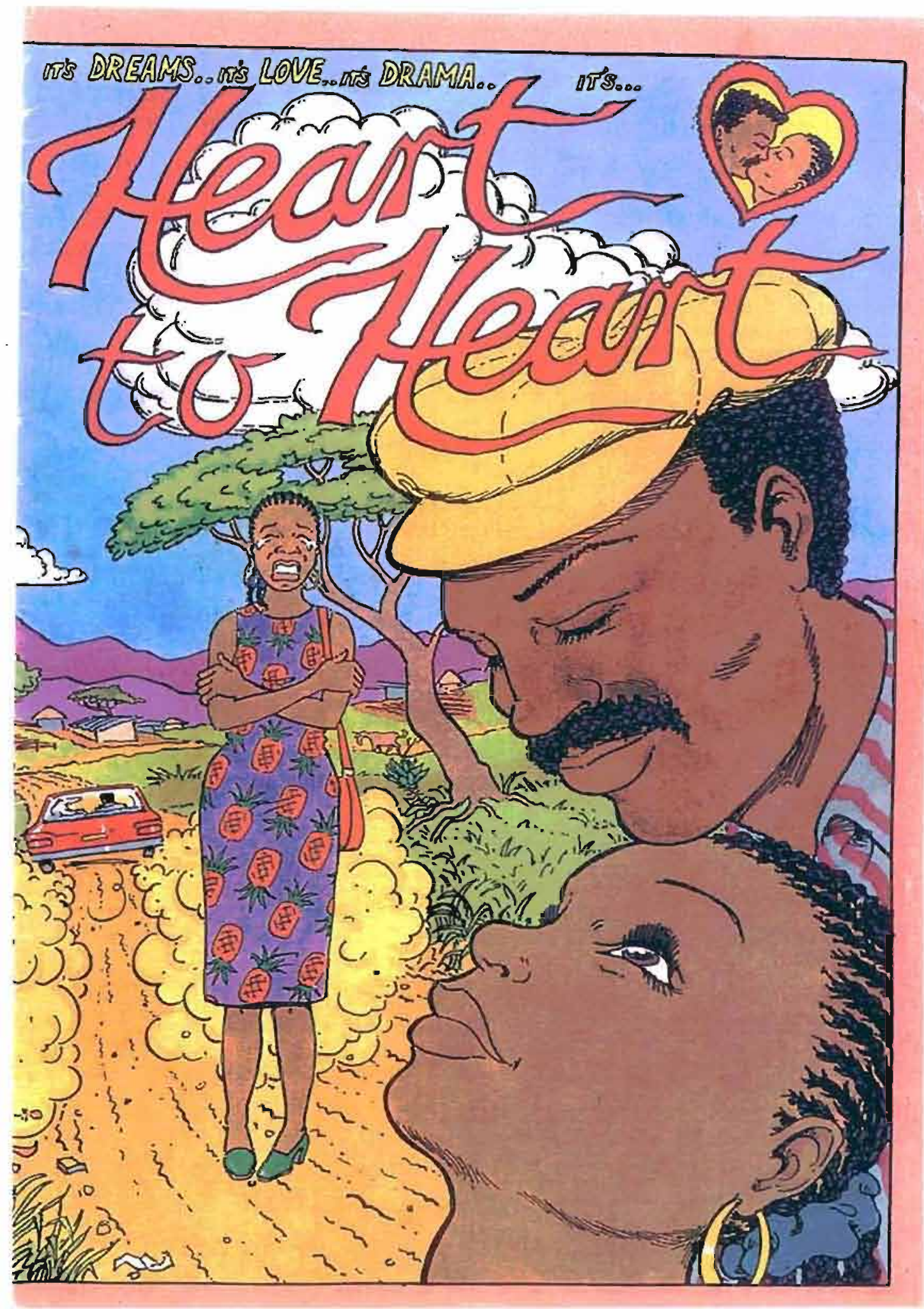

FIGURE 92

One of the most important comics produced by the Storyteller Group was Heart to Heart: From Dream Love to True Love (1994), an innovative treatment of sexual themes that presents the reader with two alternative endings. (Storyleller Group, 1994) 
let go of her career dreams to marry a man who was not faithful to her" (Storyteller Group, 1991), was considered to be "trivial and unpublishable" (Watson Shariff and Janks, 2001). Esterhuysen's response was typical of his process: he encouraged Watson to take the story back to the workshop group so that they could interrogate it and rewrite a more suitable ending.

The entire process was then recorded in comic strip form, and the comic ended up with two alternative endings linked by a 'meta-narrative' in which the participants themselves are shown in discussion about which ending is the more realistic and appropriate. Of great interest here is the editors' decision to use a naturalistic style to tell the fictional stories, and a hip, wittily postmodern style for the reflexive discourse on method. Extraordinarily, Heart to Heart contains and resolves, within the very structuration of its stylistic elements, the dichotomy between the contrasting tendencies of 'responsible realism' and 'reflexive postmodernism' identified earlier in this chapter.

Neil Napper and Peter Esterhuysen parted company after 1994, Esterhuysen to pursue the writing opportunities that were opening up in the newly 'liberated' electronic media, while Napper continued with educational comic work. It has been observed earlier in this chapter that, during the 1980s, anti-apartheid media activists, barred from access to the electronic media, saw comics as another way of getting their educational material to mass audiences ${ }^{248}$. Some of these comics were successful, but others ended up as static, unreadable rows of talking heads, causing some activists to lose interest in comics as an educational medium. One argument within the NGO media sector was that comic books were not the best medium for this kind of communication, simply because were not a familiar medium to the majority of South Africans. This point of view is justifiable to some extent, and it is true that the Story Circle's decision to produce comics using photos rather than drawings resulted in some of the most effective educational comics of the period ${ }^{249}$. Nevertheless, this does not diminish the

\footnotetext{
248 Alongside the Storyteller Group, both the Story Circle and the Rapid Phase Group were commissioned to produce popular educational comics on democracy-related themes during the transitional period. See Rapid Phase's Ken Jou Regte / Know Your Rights (undated), a comic about the new constitution, and the Story Circle's More se Mense / Tomorrow People (1994), a photo-comic about the "countdown to democracy".

249 See page 139 for discussion of the Story Circle's Roxy.
} 
fact that comic strips and comic books can be excellent educational media, if utilised properly. In any event, once the possibility of working in television and radio presented itself, many media activists lost interest in comics and turned their full attention to the new opportunities offered by the electronic media.

Peter Esterhuysen was one of a new generation of television writers who saw opportunities to devise new forms of authorial process appropriate to the challenges facing South Africa's new public broadcasting sector. Esterhuysen subsequently worked on Soul City, which dealt with social, relationship and health issues, and experimented with intertextual strategies that included the extension of the television stories into interactive comic books (interview, 2002). Thereafter, he participated in the origination and writing of Yizo Yizo, a gritty, violent and highly controversial series about conditions in township schools and their surrounding neighbourhoods.

Sadly, Esterhuysen tragically passed away shortly before the time of writing. At his memorial service, Angus Gibson, the producer of Yizo Yizo, acknowledged that Esterhuysen had played an important role in bringing the participatory strategies pioneered by the Storyteller Group to bear on the authorial process of this groundbreaking educational television series.

\section{Reviling the father: Bitterkomix and the white underground}

Nowhere in the history of South African cartooning is the relationship between the style of drawing and the subject matter of the work articulated more knowingly or more challengingly than in the Afrikaans Bitterkomix of Anton Kannemeyer (Joe Dog) and Conrad Botes (Konradski) ${ }^{250}$. These artists have brought a new, transgressive edge to South African cartooning, not only in terms of their content, which is consistently outrageous and confrontational, but also stylistically, in their elision of the categories of fine art, pop culture and public toilet graffiti. Over the last decade, the more cerebral Kannemeyer has developed a range of styles, which he uses to achieve different results, from a careful, clear line that parodies the Kuifie (Tintin) comics of his youth, to an

\footnotetext{
${ }^{250}$ Most of the biographical and contextual information for this section was derived from a series of interviews with Kannemeyer and Botes between 2001 and 2003. Kannemeyer also provided me with a meticulously compiled dossier of press cuttings and critical reviews.
} 


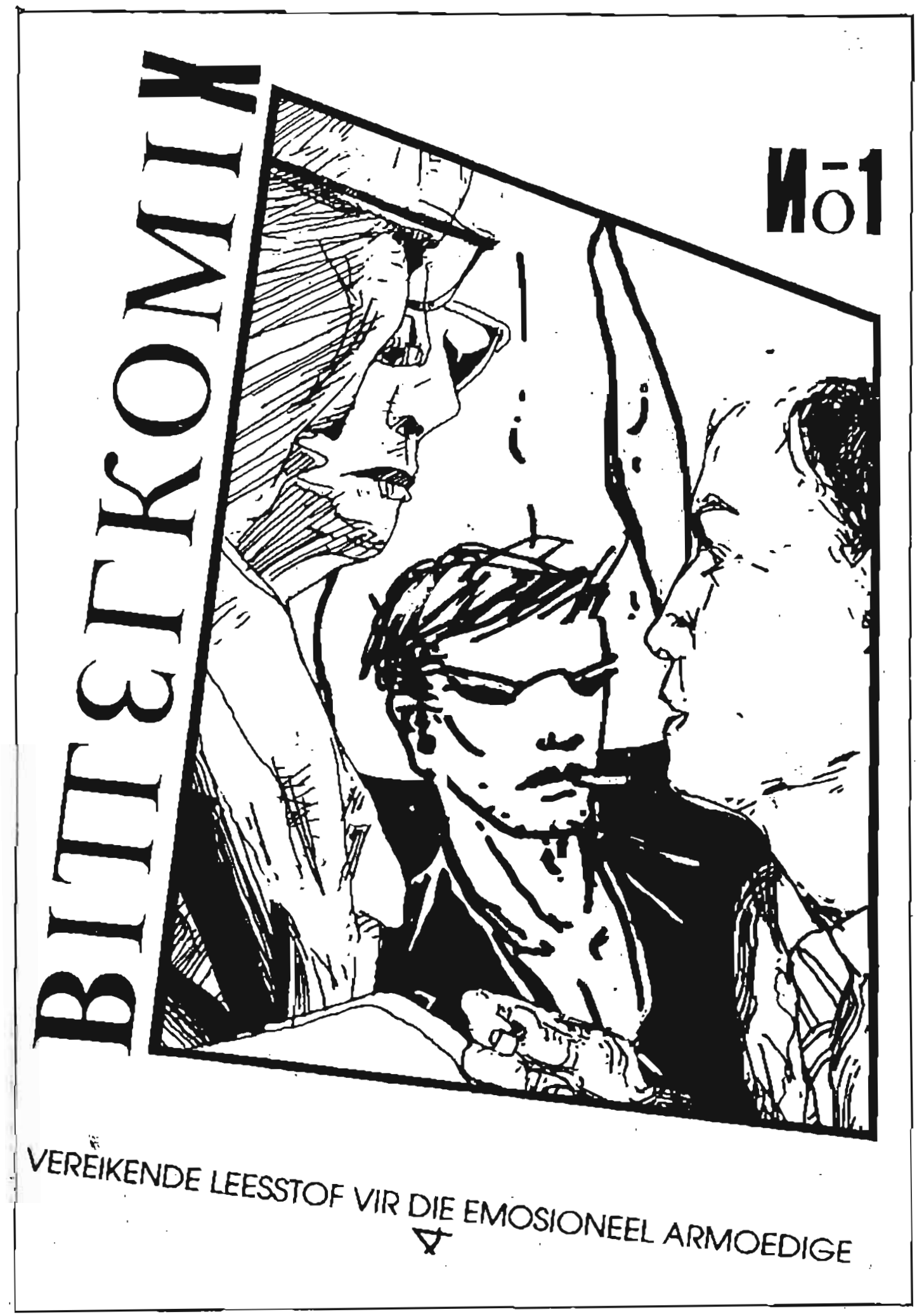

FIGURE 93

The first edition of Bitterkomix, published in 1991, contained sludent work by Anton Kannemeyer and Conrad Botes, demonstrating influences as diverse as France's Moebius, Britain's 2000AD. the U.S. underground's R. Crumb and S. Clay Wilson, and international expressionist comix.

(Bitterkomix, 1991) 
intentionally clumsy, 'bad art' style that is entirely appropriate to the scatological content that it is employed to convey. An inveterate bibliophile, Kannemeyer has submerged himself in the study of comics, comix and bande dessinee, and is equally versed in the Anglophone and Francophone traditions. If he is somewhat Apollonian in his approach to his material, Botes is Dionysian by comparison. Now recognized as a major South African artist ${ }^{251}$, Botes employs a loose, somewhat heavy and brilliantly accomplished brush line in the rendition of his dark and torrid graphic narratives.

But these stylistic differences were not yet clearly evident in the work produced by the two young iconoclasts before the end of 1994. In the first edition of Bitterkomix, published in 1991, but containing student work produced between 1989 and 1991, it is often difficult to distinguish their work from each other, so strong was their mutual influence as they explored the terrain of contemporary comics making (Figure 93). Their first issue demonstrates influences as diverse as France's Moebius, Britain's 2000AD, the U.S. underground's R. Crumb and S. Clay Wilson, and the international expressionist comix and 'graphix' published in Art Spiegelman and Francois Mouly's Raw. The discussion of these artists will of necessity be truncated, since their major work falls outside the scope of this study. However, certain dominant themes are already present in work accomplished by 1994. A few observations about their subsequent work are advanced to assist in the interpretation of this material.

Bitterkomix fits securely into the underground comix tradition that began in San Fransisco with the publication of Robert Crumb's Zap! in 1967 and rapidly spread to other countries, including South Africa, where illicit copies of U.S. underground comix began surfacing in the early 1970s. South African underground comix were thus not an offshoot from or reaction to the mainstream comics industry in this country, since there wasn't one, but were a direct imitation of the American and European underground and expressionist genres. As noted earlier in this chapter, underground-style comics had been published in the student press in the 1970s, and the same artists remained active in the 1980s, producing a number of underground comix titles that had extremely limited distribution, during the course of the decade. One of these was PAX (Pre-Azanian

251 Conrad Botes won the Volkskas Atelier award, one of the country's most prestigious fine art prizes, in 2004. 


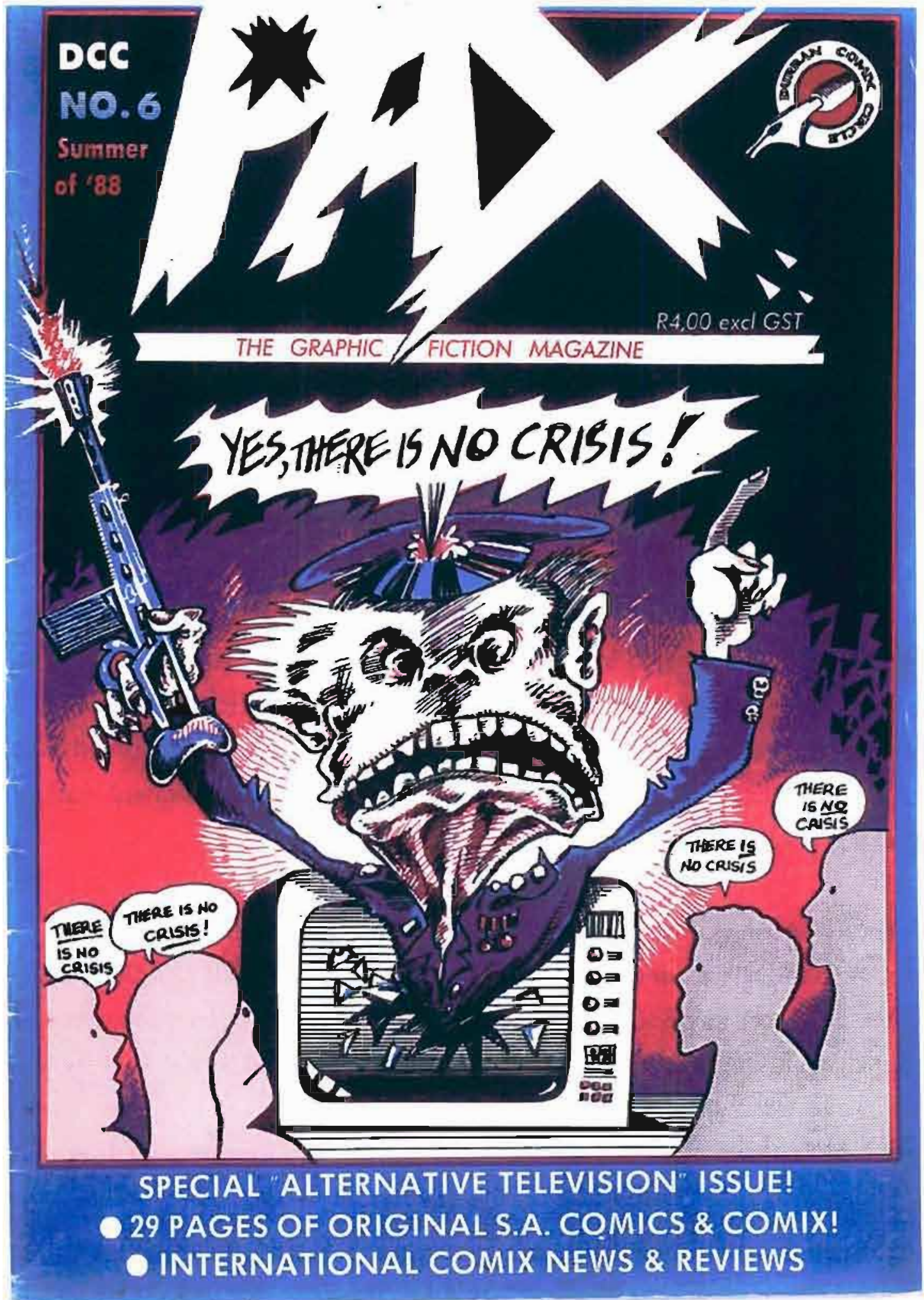

\section{FIGURE 94}

The cover of PAX (Pre-Azanian Comix) No. 6. Published by the Durban Comix Circle and coedited by Andy Mason and Chris Moon, PAX appeared six times between 1985 and 1987, and featured the work of, amongst others, Rick Andrew, Jeff Rankin, Rodney Prynne, N.D. Mazin, Wild Beast, Chris Moon and Rico Schacherl. (Durban Comix Circle,1987) 
Comix), published by the Durban Comix Circle and co-edited by the present writer and fellow underground comix enthusiast Chris Moon (Figure 94). PAX appeared six times between 1985 and 1987, and featured the work of, amongst others, Rick Andrew, Jeff Rankin, Rodney Prynne, N.D. Mazin, Wild Beast, Chris Moon and Rico Schacherl. The stories published in PAX speak, often unwittingly, of the complex ideological position occupied by disaffected young white intellectuals, who aspired to the metropolitan cultures of London, New York and Sydney, but were hemmed in by the realities of an increasingly repressive local regime (which, amongst other things, did not allow the possession of underground comix). They were also subjected to ideological imperatives that demanded some form of commitment to the anti-apartheid struggle (see Chapter Five). The PAX cartoonists expressed both a sense of outrage and a sense of impotence in the face of the power of the apartheid state. They also expressed a sense of alienation and exclusion from international metropolitan culture-an appropriate response given the international cultural boycott against South Africa. Some of the material in the publication was gratuitously counter-cultural and anti-establishment, and some was escapist and derivative of international genres, but PAX cartoonists did produce several strips that engaged authentically with the ideological challenges of the period (Figure 95) ${ }^{252}$. In this sense the publication's content was consistent with the ideological milieu described in Chapter Five. Another underground comix publication was Johannesburg's Icy Blind (Figure 96) ${ }^{253}$, featuring the work of the enigmatic Peter Rapp. This obscure, anonymously produced publication, though very rudimentary in form and with a negligible circulation, was notable for the quality of its satirical drawings, presenting a distorted nightmare world that in some inchoate way expressed the psychic turmoil of alienated white South African youth at that time. Several underground cartoonists of the period-notably Wild Beast (Joanne Bloch), whose work appeared in both PAX and Icy Blind-also self-published their own underground comix (Figure 97)254.

The impact of the few underground titles that appeared in the 1980s was not, in itself, very great, but they nevertheless had a place within the counter-hegemonic discourses

\footnotetext{
252 Rick Andrew, a page from “Urban Blues”, PAX: Pre-Azanianian Comix, No. 1, 1985.

253 Icy Blind: The Final Kak, circa 1988.

254 Wild Beast self-published at least two comix, In the Jungle (circa 1990) and Slurpy Durpy (circa 1991).
} 


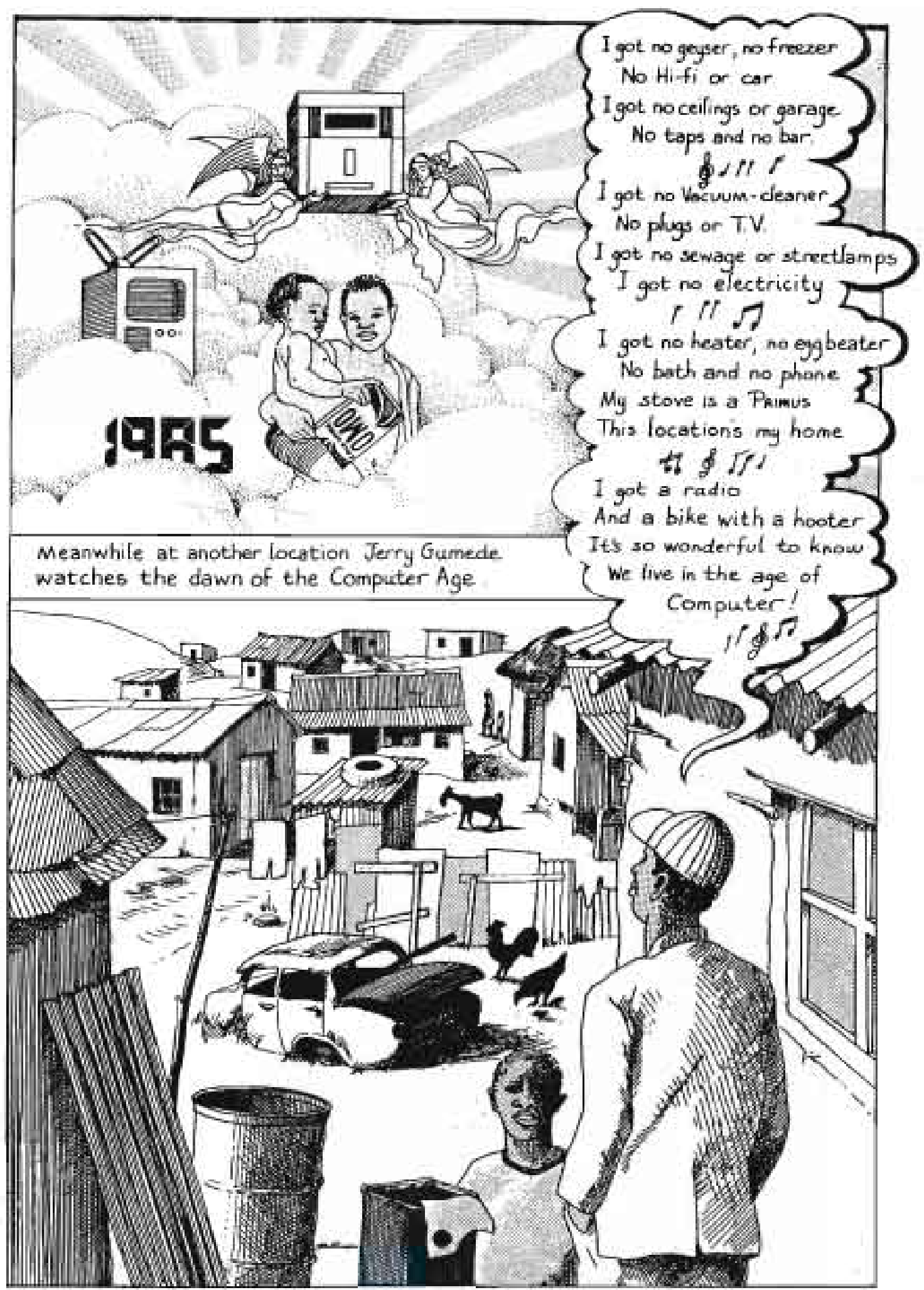

FIGURE 95

Some of the material in PAX was gratuitously counter-cultural and anti-establishment, and some was escapist and derivative of international genres, but the magazine did produce several strips that engaged authentically with the ideological challenges of the period, such as the strip "Urban Blues' by Rick Andrew. (Durban Comix Circle, 1987) 


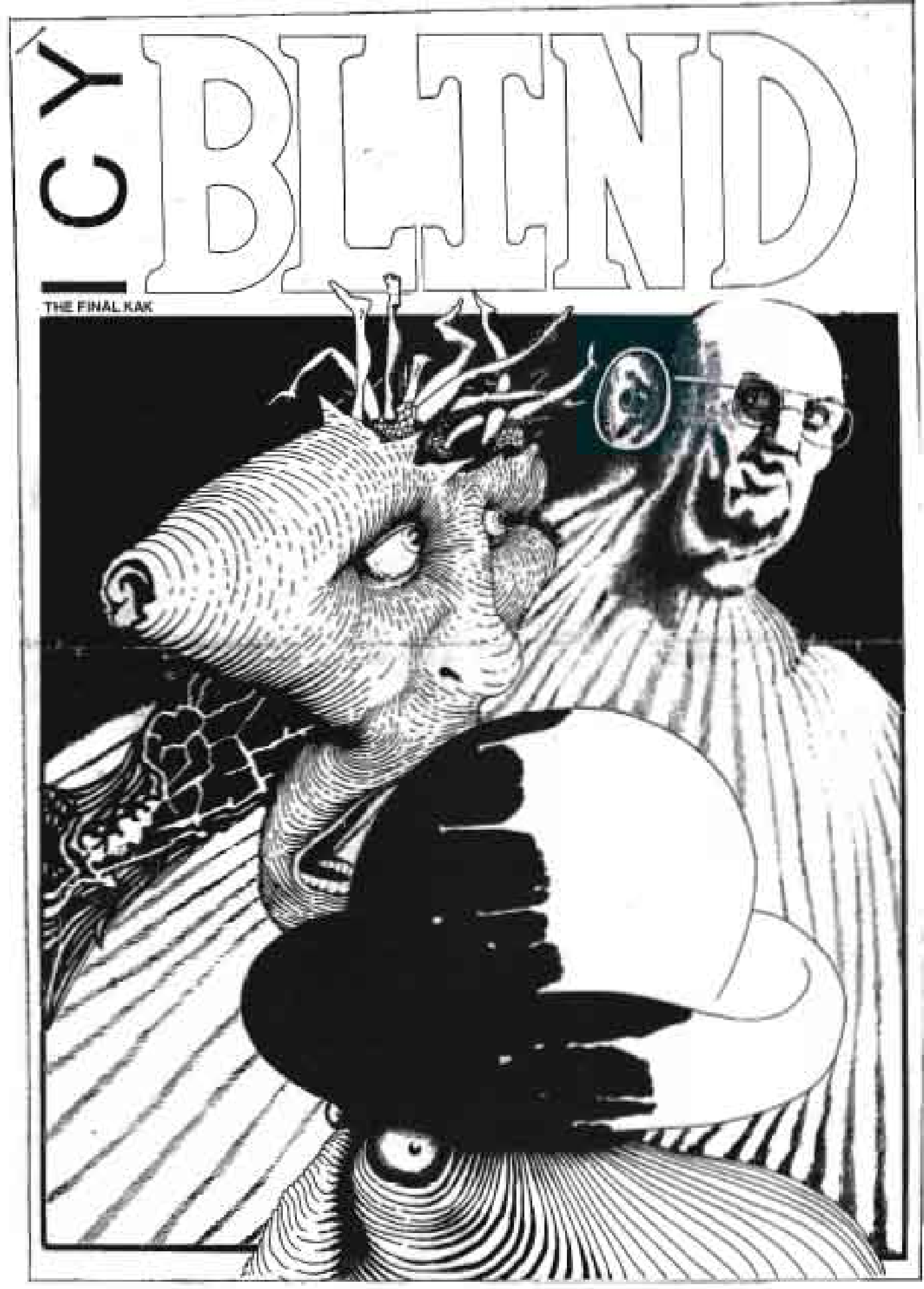

FIGURE 96

The obscure and anonymously produced Johannesburg underground comix publication, lcy Blind (I see blind), though very rudimentary in form and with a negligible circulation, was notable for the satirical comix of Peter Rapp, presenting a distorted nightmare worid that expressed the psychic turmoil of alienated white South African youth. (/cy Blind, undated, circa 1985-1988) 


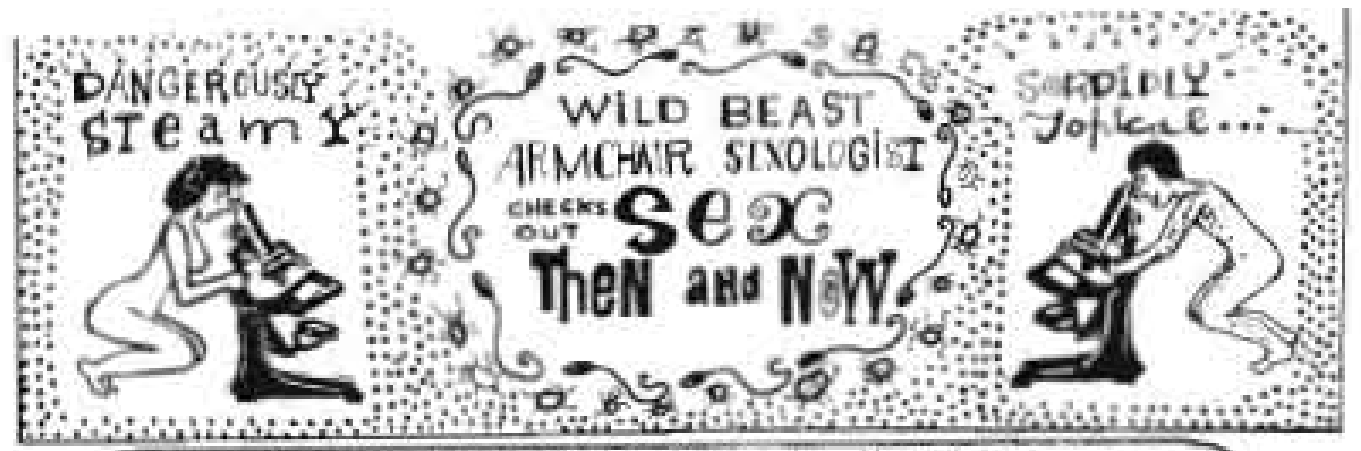

ThE NIGHTMARE THAT SEX IS TODAY IS HIGHLIGHTED BY TIE IDLE DREAM THAT IT WAS YESTERDAY

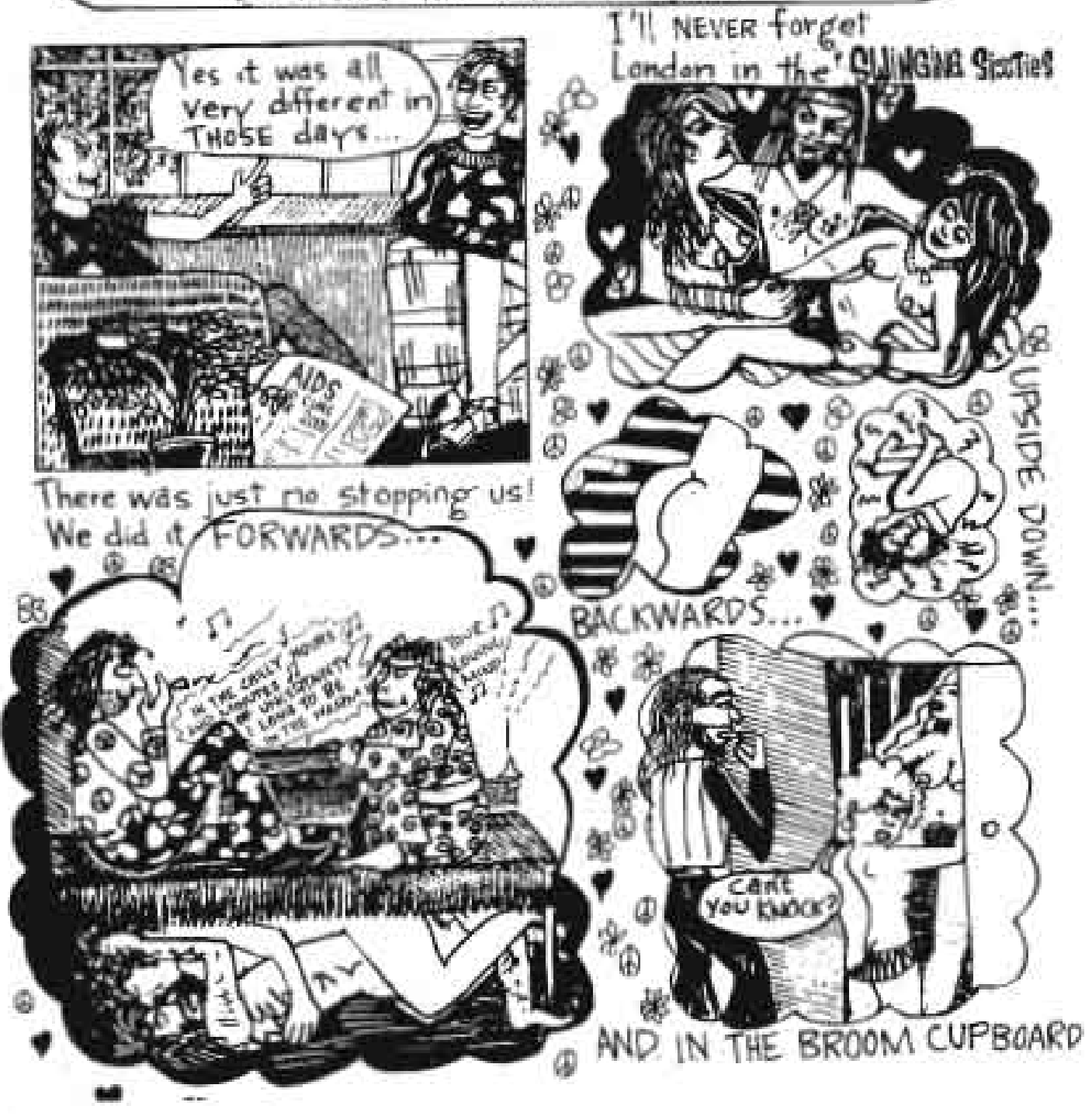

FIGURE 97

A comic page by Wild Beast (Joanne Bloch), whose punk-style comix appeared in both PAX and icy Bind, and who self-published the underground comix in the Jungle (circa 1990) and Slurpy Durpy (circa 1991). (Wild Beast, 1991) 
that sought to undermine the dominant ideology of the apartheid state. Linked to or supportive of the political forces challenging the South African state during the late apartheid period were a broad range of anti-establishment social and cultural tendencies, linked to the concerns of the international counter-culture movement. This movement had emerged in the west in the 1960s in the context of the Vietnam war, and alongside the political manifestations of the antiwar and civil rights movements in the U.S. and Europe, it found expression in folk, protest and rock music, in the journalism of 'alternative' periodicals, in literature and fine art, and of course, in comix.

It was not until the early 1990s that South African underground cartooning was to come to the notice of the general public in any significant way, with the publication of the first issue of Bitterkomix in 1992 (see Figure 93) ${ }^{255}$. Consisting of work produced by Anton Kannemeyer and Conrad Botes, who were at that time postgraduate fine art students and part-time lecturers at the University of Stellenbosch, the magazine immediately drew the attention of the local Afrikaans press, who hailed it as a demonstration of a new spirit of inventiveness in Afrikaans art and letters, noting that Kannemeyer's father was a prominent figure in Afrikaner literary circles 256 .

There was nothing particularly threatening about the first issue of Bitterkomix, which portrayed its young protagonists as "swartskape "(black sheep)—outsiders, intellectuals and trouble-makers who opposed military conscription and rejected the conformity of white South African, and particularly Afrikaans, culture. Kannemeyer and Botes exhibited some of the original artwork from the magazine in a group show at Cape Town's prestigious Baxter Theatre complex, to critical acclaim ${ }^{257}$. The staging of exhibitions featuring work from the magazine subsequently became a key promotional strategy, serving to bring their work to the attention of the wider public through reviews in the arts pages of the local press. The quality of their drawings and their

255 Bitterkomix No. 1, 1992.

256 Die Matie (Stellenbosch), 20 August, 1992; Die Burger, 31 August, 1992.

257 Die Burger, 30 September, 1992. This was the same venue where Zapiro's “Laughter in the Belly of the Beast" exhibition had been held four years before. 

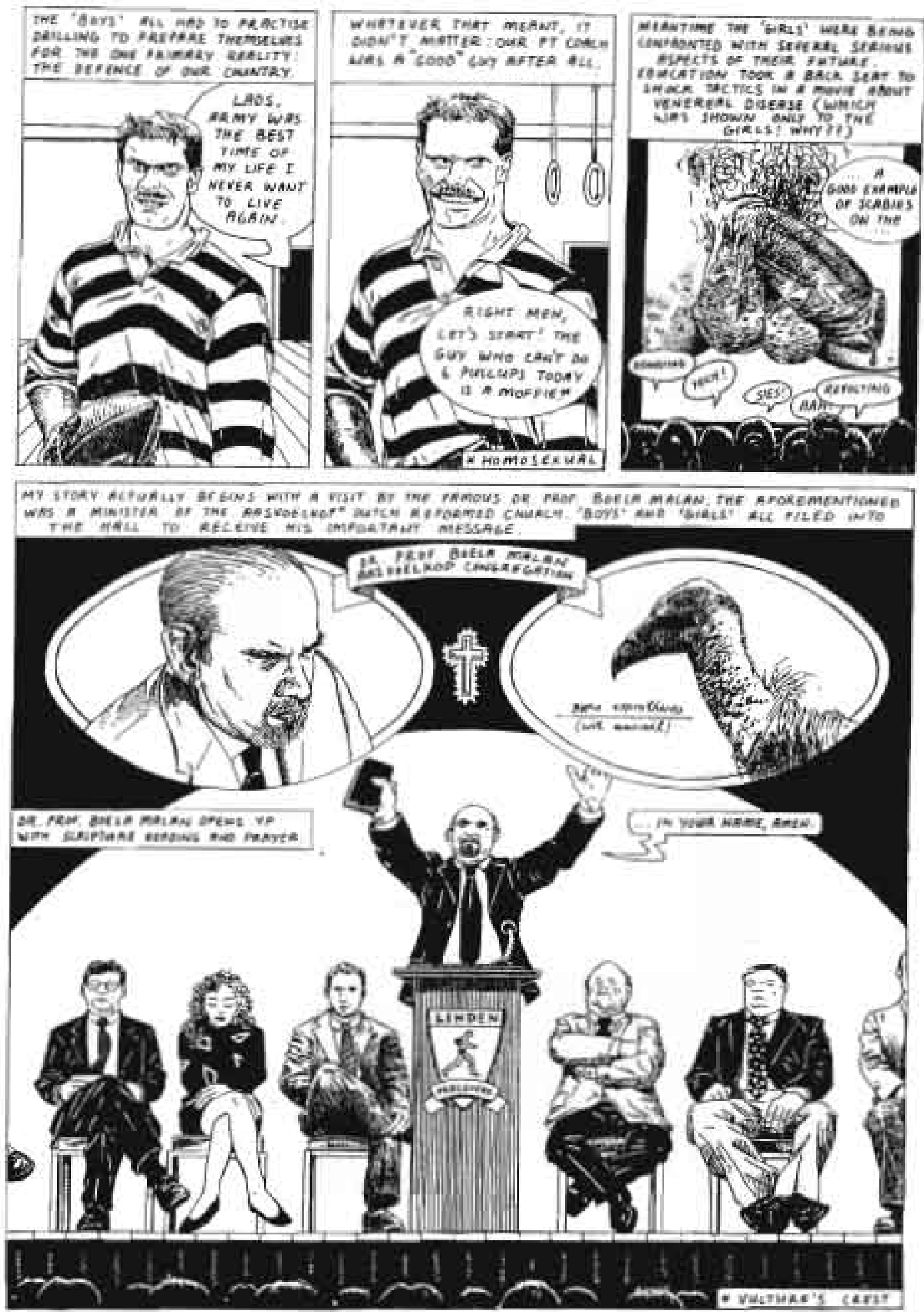

FIGURE 98

"Jeugweerbaarheid" (youth preparedness) (here seen in its 1998 English translation), by Joe Dog (Anton Kannemeyer) is an early example of the autobiographical and confessional style that would become increasingly characteristic of his work. (Bitterkomix, 1993) 
position as award-winning young artists at one of the country's leading art schools ensured that their work was taken seriously by art critics.

The second and third issues of Bitterkomix, both published in 1993, were more focused and confrontational, featuring nudity, sex and violence, impassioned attacks on aspects of Afrikaner culture such as corporal punishment in schools and the Broederbond, as well as several excursions into the low-life world of alcohol, drugs and prostitution that would become a dominant theme in Conrad Botes' mature work. The publication continued to receive the stamp of approval from the arts establishment, and a second Bitterkomix exhibition was held at the University of Stellenbosch's art gallery, opened by the renowned Afrikaner poet and journalist Antjie Krog. Krog followed up with an article in the alternative Afrikaans paper, Vrye Weekblad, that linked Bitterkomix to Asterix and Titntin ${ }^{258}$. In Bitterkomix No.3 there are several uncomfortable, challenging pieces, such as "Do a Dance For Daddy", by Joe Dog and Claudette Schreuders, in which the theme of sexual abuse within the family first emerges. It is also in this issue that the transgressive, phallocentric drawings of Lorcan White (Mark Kannemeyer) first make their appearance.

By the fourth issue, published in early 1994, Bitterkomix had found a distinctive voice-a searing attack on Afrikaner morality that confronted the establishment and exposed taboos in a series of powerful graphic narratives. The themes of outrage and resistance to the dominant cultural ideology of obedience and sexual repression are powerfully evident. Kannemeyer, by this issue of the magazine, had begun to evolve a versatile, incisive style of social criticism, strongly informed by autobiographical themes. Particularly noteworthy are his "A Short \& Feeble Affair" and "Jeugweerbaarheid" (youth preparedness) (Figure 98)259, in which the confessional approach characteristic of his later work is already evident. Conrad Botes' "Kanker" (cancer) and "Siembamba", by contrast, are heavily inked in his characteristic free-flowing brush style, revealing this artist to be a graphic storyteller of note, whose images of anguish, disillusionment,

\footnotetext{
258 Antjie Krog: "Watter soort mens is jy: 'n kuifie of 'n asterix?" Vrye Weekblad, 8 July, 1993.

${ }^{259}$ Anton Kannemeyer, "Jeugweerbaarheid", Bitterkomix No. 4, 1994.
} 

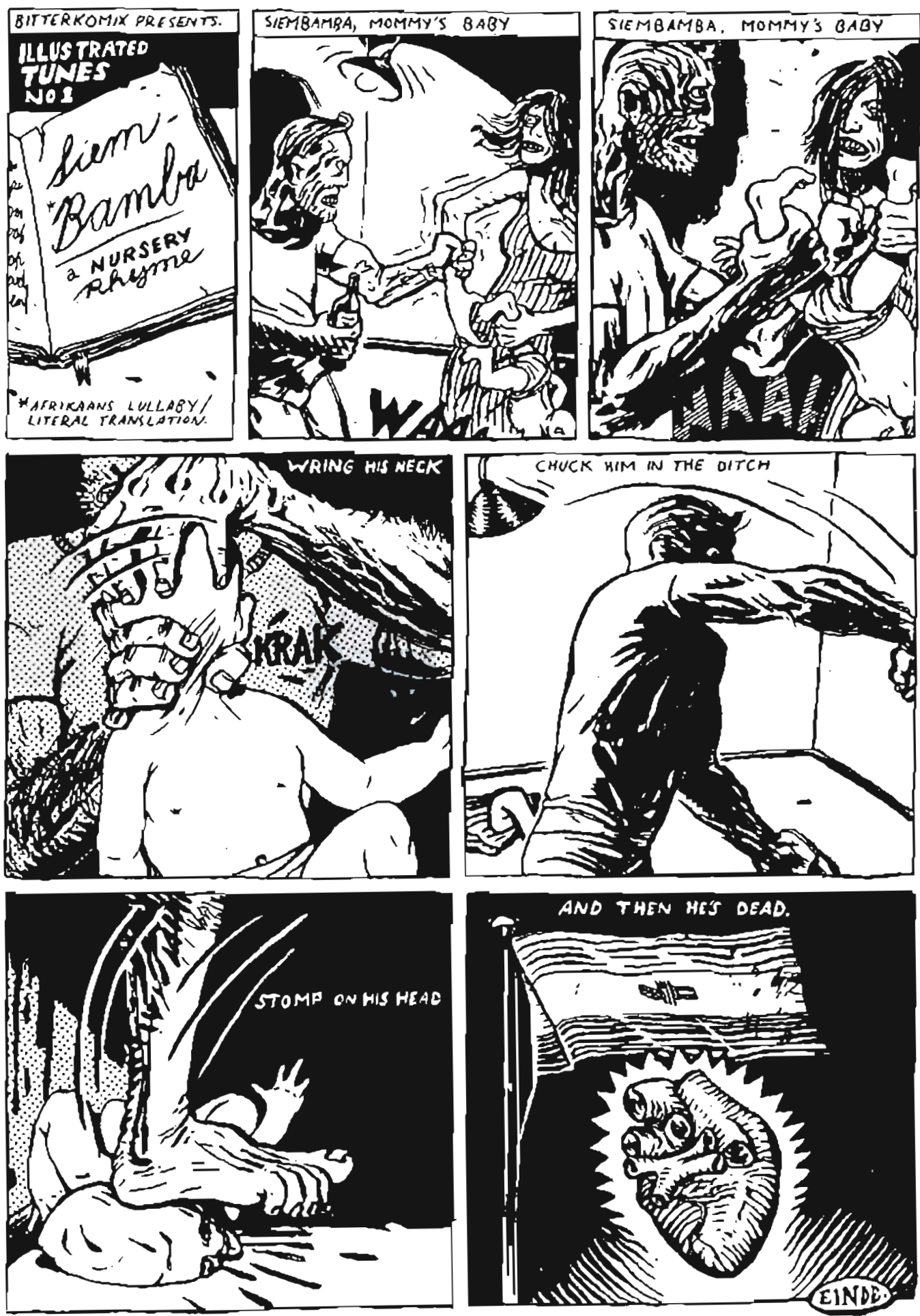

FIGURE 99

"Siembamba" by Conrad Botes (here seen in the 1998 English translation) demonstrates his characteristic free-flowing brush styie, and reveals him to be a graphic storyteller of note, whose images of anguish, disillusionment, death and loss evoke strong feelings (Bitterkomix, 1998) 

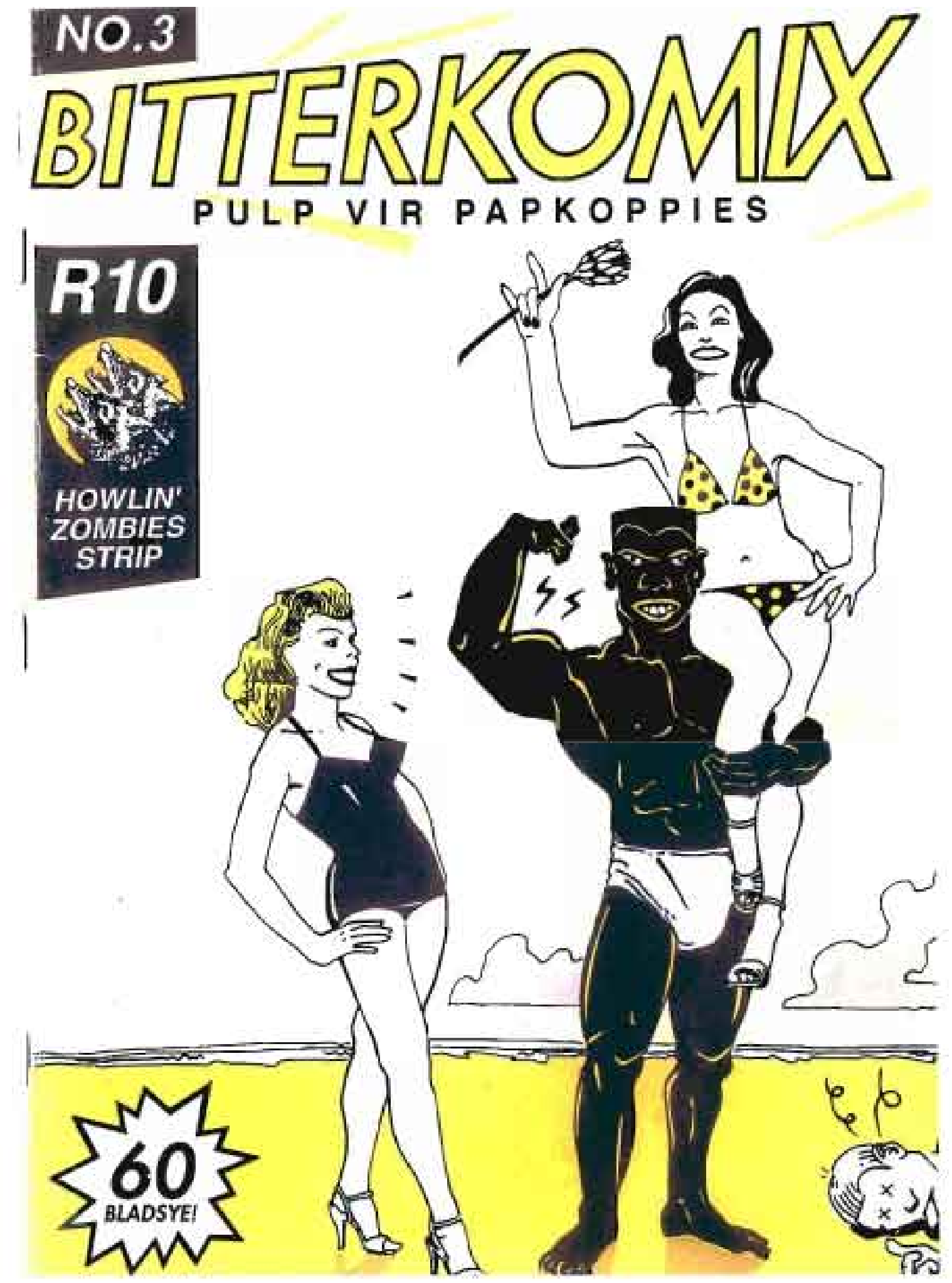

FIGURE 100

The cover of Bitterkomix No. 3(1994), by Anton Kannemeyer, exploits the theme of white male sexual (and political) inadequacy contrasted against black potency, in a briliant parody of the advertisements for body building products found in U.S comic books. (Bitterkomix, 1993) 

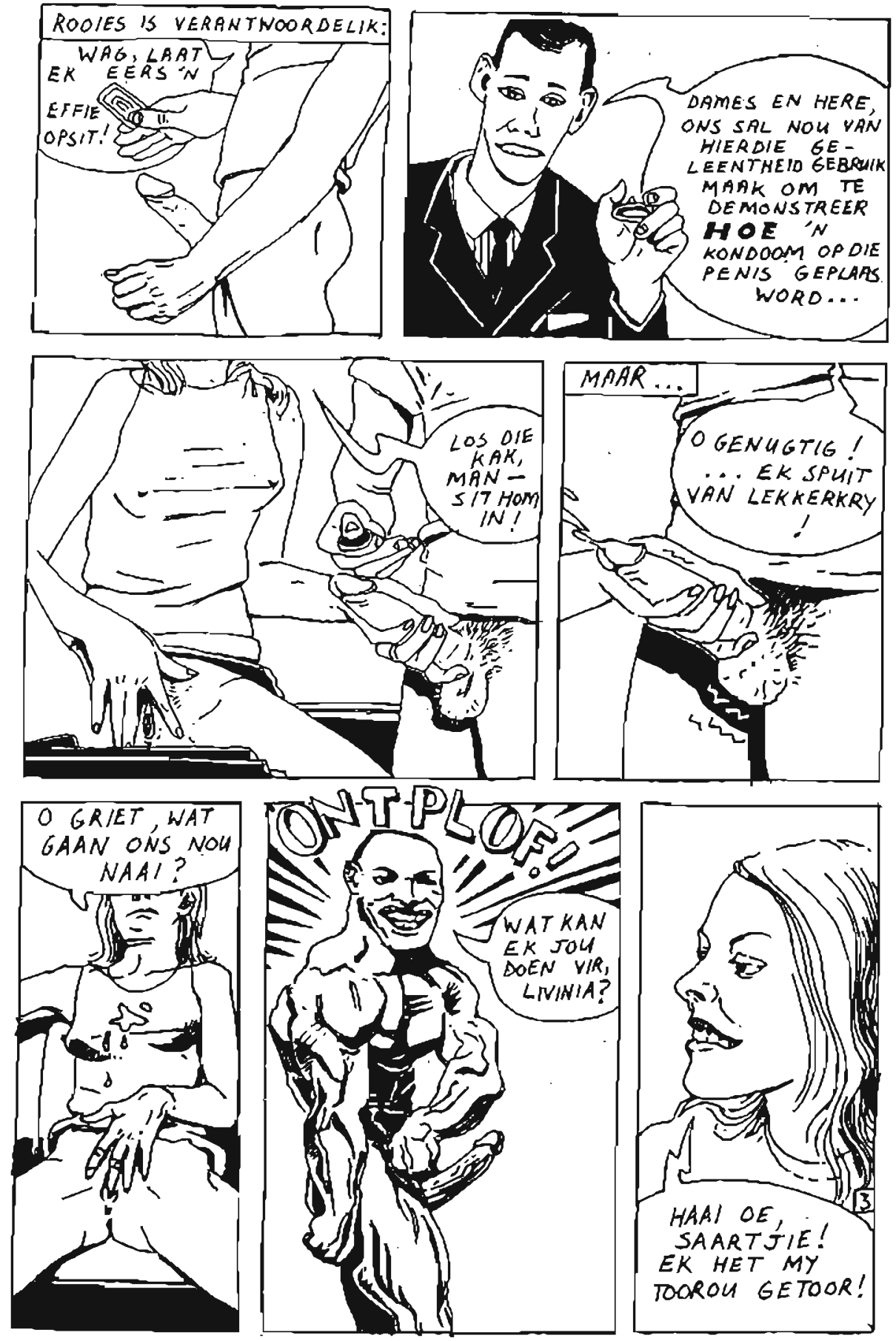

FIGURE 101

The theme of white male sexual inadequacy contrasted against black potency is laken to its logical extreme in the strip "Liefde en Missiele" (Love and Rockets), which parodies a well-known Afrikaans children's television programme, Lewe Heksie. Griet, the dependable horse, who in the

TV show is always standing by to help Levinia in times of trouble, is Iransformed here into a wellendowed black man. (Bitterkomix, 1994) 
death and loss evoke strong feelings (Figure 99) ${ }^{260}$. The magazine was given added weight by the inclusion of heavy, painful autobiographical narratives by Ina van Zyl.

In the same year, Bitterkomix, in collaboration with Hond, a marginal Afrikaans publisher, published Gif: Afrikaner Sekskomix" (Poison: Afrikaner sex comix), an unrestrained exploration of male sexual obsession. This blatantly confrontational publication represented a turning point, releasing Bitterkomix from the approbation of the Afrikaner literary establishment. An exhibition of work from the publication at the Stellenbosch University gallery sent shockwaves through the local community, and the university authorities were drawn into a public debate about freedom of expression.

The decision to produce Gif as a separate publication from Bitterkomix appeared to give the artists an even greater license-here the satire is uncompromisingly pornographic and confrontational. The artists evidently took great pleasure in the intricate depiction of sexual organs in states of full arousal, and the taboo sight of penile penetration of mouth, vagina and anus is explored from a variety of angles. The theme of white male sexual inadequacy contrasted against black potency, brilliantly parodied on the cover of Bitterkomix No. 3 (Figure 100)261, is taken to its logical extreme in the strip "Liefde en Missiele" (Love and Rockets), which parodies a well-known Afrikaans children's television programme. In the strip, Griet, the dependable horse from Liewe Heksie, who is always standing by to help Levinia in times of trouble, is transformed into a wellendowed black man whose large penis is shown penetrating Levinia as she tells the two inadequate white "plaasjapies"(farm boys) to "fokkof" (fuck off) (Figure 101)262. The strip also parodies the sequential illustrations used in $\mathrm{HIV} /$ Aids awareness publications and condom packet inserts, with a mock description of how to put a condom on the penis. There are two feminine strips in Gif, both by Ina van Zyl, but these are completely submerged under a tirade of violently male-oriented, phallocentric imagery whose satirical intent does little to moderate its impact.

\footnotetext{
${ }^{260}$ Conrad Botes, "Siembamba: 'n' kleuter liedjie", Bitterkomix No. 4, 1994.

261 Cover of Bitterkomix No 3., 1993.

262 Gif: Afrikaner Sekskomix, 1994: 3.
} 
Unsurprisingly, Gif was greeted with public outrage. Whereas the Bitterkomix artists had previously been celebrated for their bold cultural critique, they now became the target of critical censure from all sides of the ideological spectrum. But the vitriol that rained down on the creators of Gif did not appear to dampen public enthusiasm for their work. Instead, Kannemeyer and Botes were rocketed to prominence and feted by fans and media at the various public events they organised to promote their work. Newspapers and magazines latched onto the story, with a number of critics using the opportunity to advance their own opinions about pornography, satire and social critique. In the Weekly Mail \& Guardian (14 October, 1994), the paper's critic, Shaun De Waal263 argued that Gif was not pomography: "... it is willing to titillate, but it is not afraid of being grotesque, disconcerting or blackJy farcical". Jan van Zyl, writing in Die Matie (11 August, 1994) humorously described it as "'n boek wat nie sonder ' $n$ kondom gelees kan word nie" (a book that should not be read without a condom).

Kannemeyer included the original art of the strip "Liefde en Missiele" in an exhibition by University of Stellenbosch Creative Arts Department staff members in September 1994. Variously described in the press and the gallery's visitor's book as "a disgusting cancer", "pure pornography", "afstootlik", "pervers", "skokkend" (vile, perverse, shocking) and so on, the piece attracted large numbers of visitors to the exhibition ${ }^{264}$. Exploiting the moment, Kannemeyer invited concerned members of the public to a discussion in the gallery where the work was roundly condemned by a Christian group but defended by philosophy lecturer Dr. Willie van der Merwe who argued that, on the basis of his experiences as a military chaplain in Angola, he found it true to life. Kannemeyer was given the opportunity to defend his work: "Ek gebruik die seksuele bloot net as medium om verskeie sosiale kwessies aan te spreek" (Eikestadnuus, 23 September, 1994) 265 .

\footnotetext{
263 Shaun De Waal subsequently produced several comic strips about the gay lifestyle, published in Bitterkomix. Jack Marks, a comic book about his schooldays, was published by Bitterkomix and Hond in 1998.
}

264 Die Matie (Stellenbosch), 15 September 1994; Die Burger, 20 September, 1994; Eikestadnuus, (Stellenbosch), 23 September, 1994.

265 "I use the sexual merely as a medium to address various social issues." 
Gif represented an intentional strategy of outrage: its aim was to outrage the public by transgressing the norms of morality, while simultaneously expressing outrage against what its authors believed to be a society enmired in deceit. Thereafter, the Bitterkomix artists consciously pursued a double-edged strategy, using Dada-esque tactics to generate controversy and bring their concerns to the notice of a wider public, while simultaneously working very seriously with narratives that interrogated and exposed the anxieties and taboos of Afrikaner society and history. Kannemeyer continued to place transgressive works in public spaces and a pair of screen prints entitled "Afrikaner Meisies hou van Pielsuig" (Afrikaans girls love cock-sucking) created further controversy when it was exhibited at a group show in Durban in 1995. The work in question is one of Kannemeyer's most radical and challenging. Consisting of two silkscreen prints, it depicts a number of close-up views of the faces of young women ecstatically engaged in fellatio with anonymous penises. Beneath each picture is a caption giving the woman's name: "Johanna", "Mev. Willem Barnard", "Die dogter van Dr. Malan" (the daughter of Dr. Malan), "Die vrou en moeder van Cornelius en Louis Visagie" (the wife and mother of Cornelius and Louis Visagie) and so on. By linking the women in the pictures to their social and family circumstances, these titles suggest that "pielsuig" is a popular and highly regarded Afrikaner activity, effectively subverting the taboos around sexual practices that underlie the notion of respectability.

Once again, as Shaun de Waal (above, page 154) pointed out with regard to Gif, it is the work's ability to titillate the viewer that makes it so challenging. The sense of voyeurism and sexual arousal that the viewer experiences while examining the pictures is likely to be deeply shocking to those to whom pornography is anathema. The power of the work resides in the intensely contradictory feelings it produces.

While Bitterkomix No. 5 lies strictly outside of the scope of this study, in that it was published in 1995, this issue of the magazine is very important because it contains several seminal pieces of work that are in a sense the outcome of the experimentation that went before. These pieces demonstrate a deeply considered and finely honed usage of comix as a serious literary form, and set the tone for what was to come as the artists began to reach maturity. 

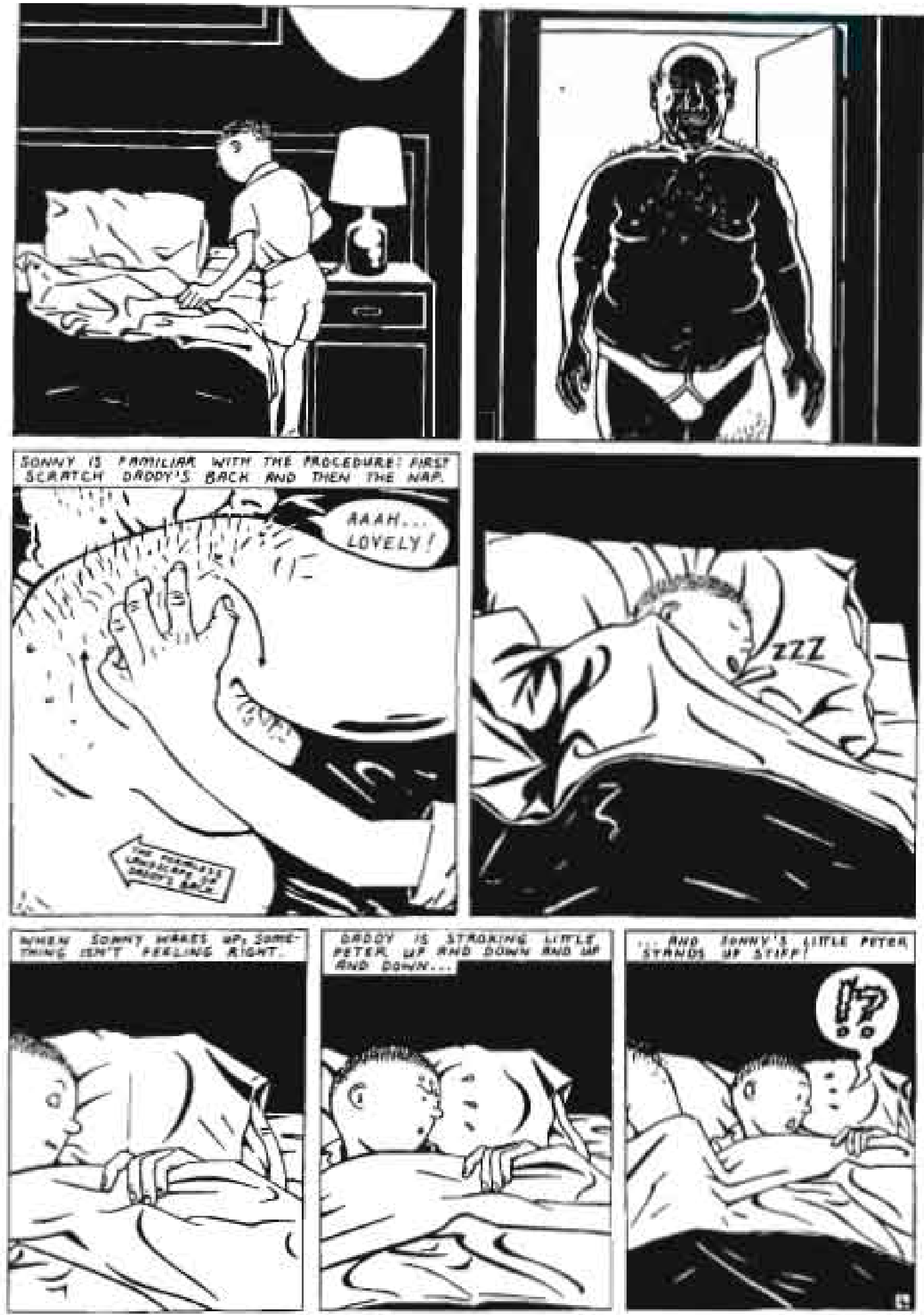

FIGURE 102

In 'Boetie' (Sonny), Kannemeyer tells a dispassionate, agonising and clearly autobiographical story about the sexual molestation of a boy by his father, in which the source of his concern with the issue of sexual abuse within the family is forcefully brought to light (Bitterkomix, 2005) 
In a patriarchal, conservative society adherent to strong group values, vilification of the father figure constitutes a serious transgression. This theme is evident in Bitterkomix from the first issue, in a number of strips that deal with the father figure both in the intimate family context, and on the broader social and symbolic level of "die vaderland" (the fatherland) and God as "onse Vader" (our Father). In "Boetie" (Sonny), Kannemeyer tells a dispassionate, agonising and clearly autobiographical story about the sexual molestation of a boy by his father, in which the source of his concern with the issue of sexual abuse within the family is forcefully brought to light (Figure 102) ${ }^{266}$.

Kannemeyer introduces "Boetie" with two drawings of himself as Joe Dog, dishevelled and drinking a bottle of vodka, clearly on the brink of a major personal revelation. The first two panels are drawn in a loose, underground style, but then the artist reverts to a very neat, clear-line style for the story he has gathered the courage to tell. Every blade of grass on the lawn, every hair on the father's back and every detail of the furnishings in the father's sitting room are dispassionately rendered, as if the artist was impelled by a need to achieve absolute clarity and truth in his depiction of the incident. The story, in which the father instructs the boy to get into bed and then strokes his penis, is told with economy and restraint. It is this very restraint that makes it so disturbing and powerful, especially when compared to the unrestrained, gratuitous approach of Botes' heavily inked and rather lurid retelling of the historic battle of Blood River, which appears in the same issue (Figure 103)267. This battle is a celebrated moment in Afrikaner mythology in which, it was believed, the embattled Voortrekkers received divine aid that enabled them to repulse a Zulu army, killing so many that the river turned red with blood. These investigations thus operate on two levels-at the intimate level of the guilty secrets that lurk behind the precast concrete walls of the Afrikaner suburbs, and at the level of Afrikaner history and mythology.

Undoubtedly, the reason that Bitterkomix has had such a disproportionate cultural impact, despite its limited distribution, is because its vivid, searing imagery emanates from so deep within Afrikaner culture, exposing anxieties and taboos that few writers have had the courage to speak about so honestly. At the same time, its bold,

\footnotetext{
266 Joe Dog, a page from "Boetie", Bitterkomkix No. 5, 1995.

267 Conrad botes, "Bloedrivier", Bitterkomkix No. 5, 1995.
} 

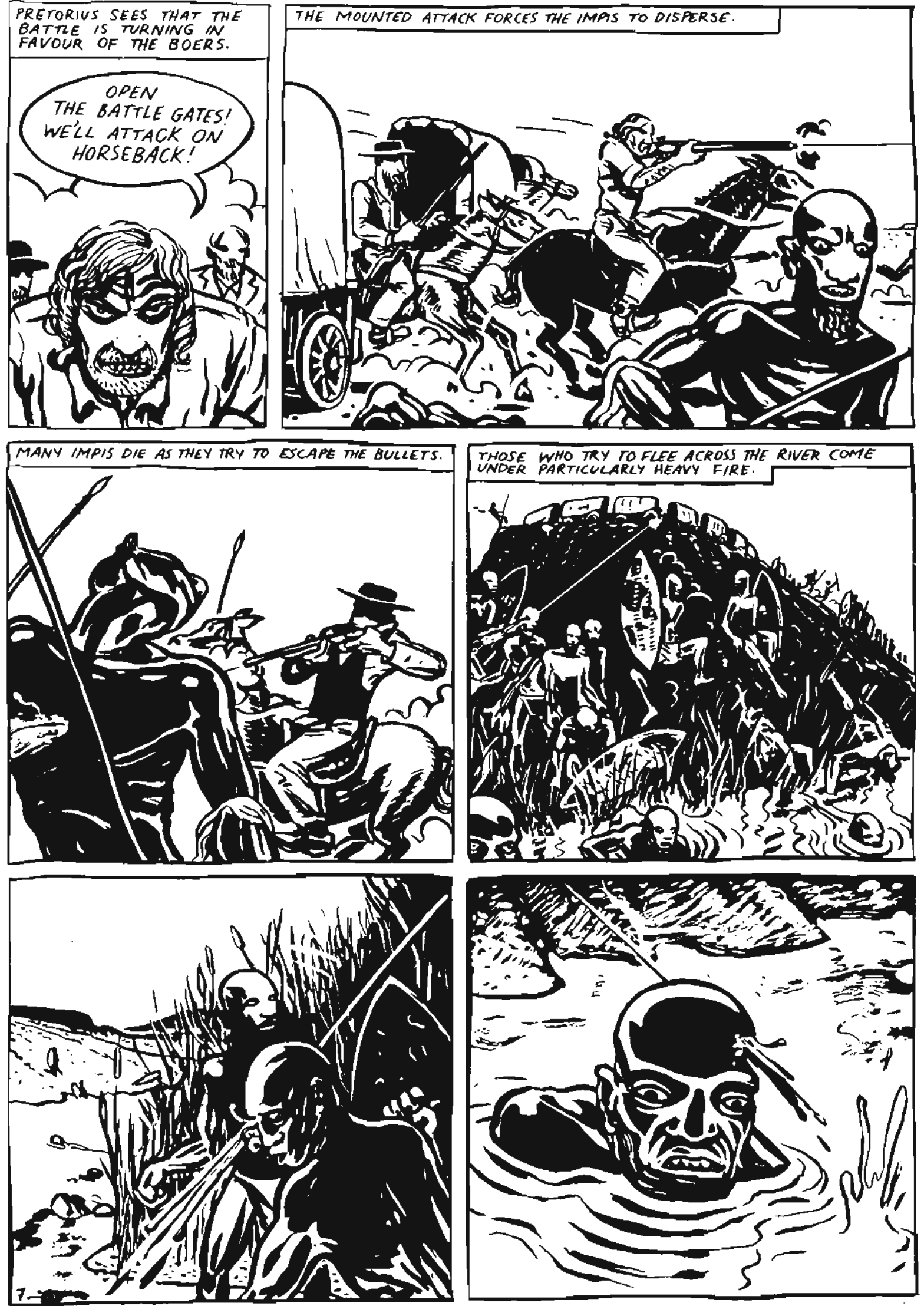

FIGURE 103

A page from Conrad Botes' lurid retelling of the historic battle of Blood River, a celebrated moment in Afrikaner mythology. (Bitterkomix, 1995) 
uncompromising graphic style and its development of an evocative visual lexicon drawn from Afrikaner culture, Roman Catholic iconography, toilet wall graffiti, pornographic magazines and a variety of other sources in pop culture and the fine arts, give it a cutting-edge, street culture appeal that is highly regarded (and often borrowed) by art directors in advertising and magazine publishing.

Bitterkomix, as an expression of resistance discourses in South Africa, also has relevance in terms of the relaxation by the new South African government of the strict censorship controls that had been enforced under the apartheid regime. The loosening of political and ideological control over the media was accompanied by a loosening of control over sexually explicit material, and one of the manifestations of this was an explosion of pornographic literature. One such product was the Afrikaans pornographic magazine, Loslyf, launched in May 1995, in which cartoons and a comic strip by the Bitterkomix artists appeared (see Figure 2, Chapter 2 )268. However, this aspect of their work falls outside of the scope of this study.

It is nevertheless necessary to grapple with the implications of the Bitterkomix strategy of using pornography as a tactic to engage with and challenge public morality. The publication of Gif in 1994 represented the beginning of a sustained attack on public morals in the pages of Bitterkomix, which continued for several years, reaching a climax in Bitterkomix No. 8 (1998), and easing off thereafter. In this issue, some of the Bitterkomix artists' most transgressive works appeared, including Botes' "Vaalseun" (Dullboy), and Kannemeyer's "Onse Vader" (Our Father) and "Hemel Help Ons (Twee)" (Heaven Help Us, Part 2). The difficulty that these works present hinges on the extent to which the use of pornography as a strategy to provoke public debate is manipulative and gratuitous. Gif and the "pielsuig" artworks generated heated critical debate in academic circles and in the press, bringing several important issues into focus. A key issue is the role of satire (to challenge and if necessary offend) versus the prevention of hate speech and its equivalent in the visual arts. Extreme misogyny is a form of hate, and its presence in art is highly problematic. The Bitterkomix artists have been accused of this, and there are several instances, particularly in the non-consensual sex scenes (of which there are several in the Bitterkomix ouevre) where the material is difficult, if not impossible, to 
defend. Imagery that depicts violence against women and thus reproduces gendered relations of domination will always be highly problematic, especially in a society plagued by sexual violence, such as ours.

These concerns notwithstanding, Bitterkomix is important within the context of counterhegemonic cartooning in South Africa, particularly in the links that it draws between the abuse of political power and sexual deviance. By working in a very personal, confessional way with these concerns, the Bitterkomix artists have produced a series of documents that delve further and deeper into the psyche of apartheid than any other South African cartoon or comic strip has ever done, and probably cut as deeply as any work produced in other disciplines. Crucially, they make no attempt to speak for or on behalf of anyone but themselves, and this gives their work a searing authenticity.

In this sense, Bitterkomix stands in stark contrast to those South African publishing projects that have attempted to utilize the various forms of cartooning in service of specific social and political goals. The ideological problems of identity and legitimacy that relate to the artist's right to speak for others have no purchase upon the work of artists who speak only of their own condition. Paradoxically, by refusing to speak for others, and by focusing their attention on the minutae of their own experience, Kannemeyer and Botes have ensured that their art remains uncompromised. Because of this, its universal significance, confirmed by intense interest in the Bitterkomix artists amongst the international art community, is assured.

268 "The end of Afrikaans prudery is naai" (no writer credited) in Weekly Mail \& Guardian, April 21-27, 1995. 


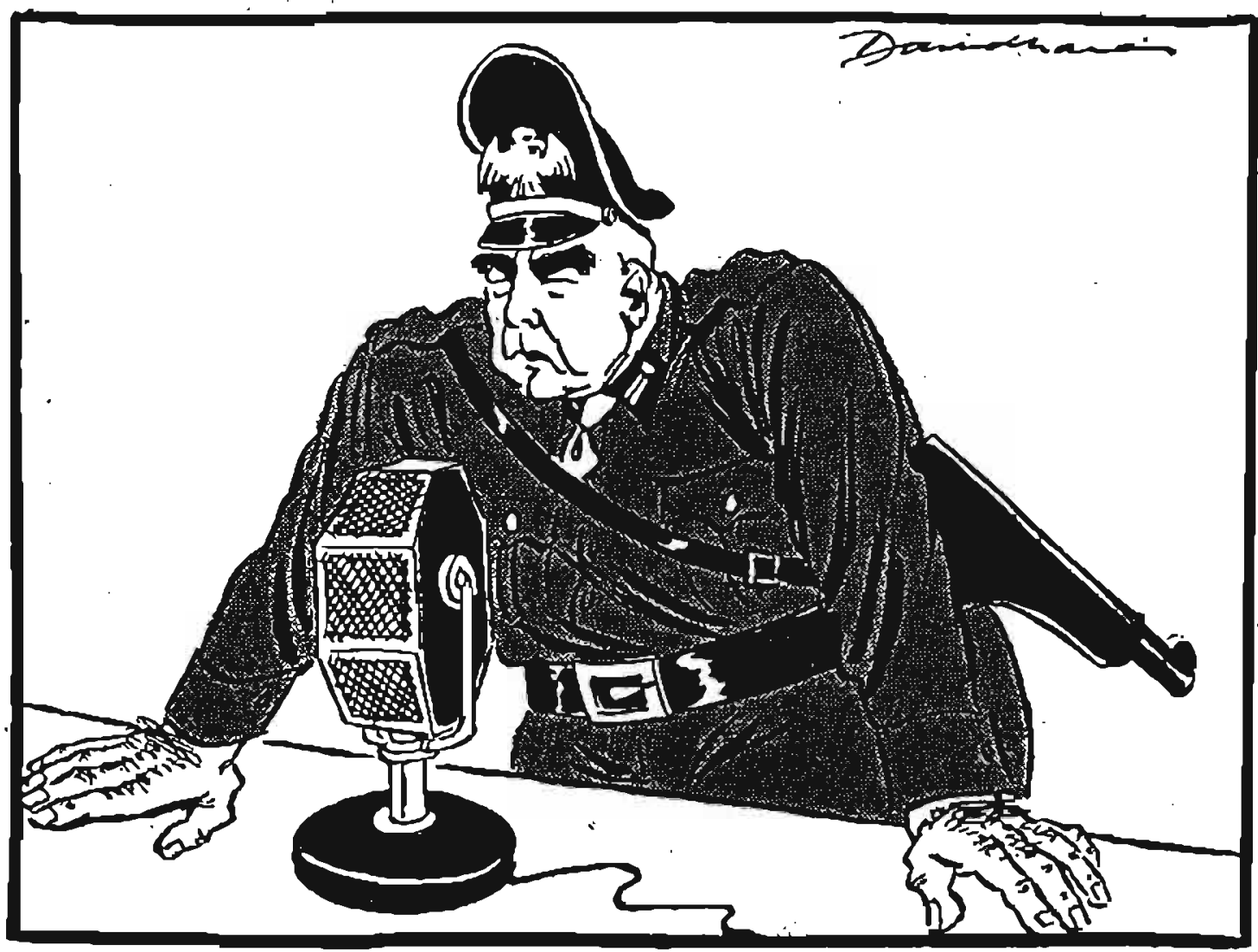

"Here is the deadly, devastating revelation about Nusas-No Nusas is good Nusas." 29 September 1900

FIGURE 104

David Marais enjoyed depicting prime minister B.J. Vorster in Nazi-style uniform. He also depicted Vorster's cabinet ministers in Klu Klux Klan cloaks and hoods. (M arais, 1963) 


\section{Conclusion}

\section{Review of key arguments}

Chapters Five and Six of this study demonstrate that in the last decade of apartheid (1985-1994), South African cartoonists gave visual expression to discourses of resistance that existed in the democratic movement at that time. Occasionally, as in Zapiro's UDF calendar illustration, they were at the forefront of these discourses, or as in Bitterkomix's Gif (1994), they went beyond these into transgressive areas of discourse. The first and most obvious expression of resistance discourse was the vilification of apartheid politicians and their policies. As Chapter Four shows, the first cartoonists to actively critique the repressive apartheid state were the liberal cartoonists of the post-war period. These cartoonists critiqued the government's authoritarian posture and racist policies-depicting prime ministers Hedrik Verwoerd and B.J. Vorster in Nazi-style uniforms or their cabinet ministers in Klu Klux Klan cloaks and hoods (Figure 104) —but, as the selected cartoons by Jock Leyden demonstrate, this criticism was shot through by the dilemma of liberalism-a sense of being caught between extreme political tendencies to both the right and the left.

While the liberal cartoonists were able to critique the repressive tendencies of the state, their position with regard to the second aspect of resistance discourse- the legitimation of the liberation struggle-was more complex and tenuous. They were fearful of the politics of black resistance and were generally unable to distance themselves from the prevailing ideology of the day, which linked the black liberation struggle to the threat of communism. In the pro-government white press-and particularly in the Afrikaans press, as demonstrated in the cartoons of Fred Mouton - the liberation movement was depicted as an evil force manipulated by cynical Soviet interests. The liberal cartoonists were unable to critique this position in any substantive way, were slow to valorise the liberation movement, and generally did not demonstrate any in-depth understanding of black liberation politics.

The continuation of these liberal tendencies into the transition period has been demonstrated in the work of Richard Smith. His early work was characterised by vicious caricatures of Nationalist politicians, but over time his caricatures lost their bite 
and, unlike the more tenacious Dov Fedler, he was unable to negotiate the transition to a viable post-apartheid cartooning practice. Likewise, the work of Len Sak, while it secured the approval of leading black pressmen like Aggrey Klaaste, and was evidently well-loved by black readers, was enmired in a moralising humanism that became increasingly untenable in the politically sophisticated post-apartheid context. It may also have been that the depiction of Sak's character Jojo, which demonstrates the conventions of racial caricature, was no longer appropriate in this racially hypersensitive period.

In the cartoons of Derek Bauer, the contradiction between the persistent tendencies of liberalism and the demands of history becomes vividly evident. Bauer's visceral, unrestrained cartooning provides stark evidence of the internal conflict within the white liberal consciousness, still locked into the stereotypes of the past, but cogniscant of the need for a new mindset.

It is perhaps no coincidence that all three of these great liberal cartoonists-Smith, Sak and Bauer-were replaced by the same man. Zapiro proved to be the only South African political cartoonist with the stature and acumen to adequately represent the complex politics of the transitional period across the mainstream press (Sunday Times), the black press (Sowetan) and the left wing, previously alternative press (Weekly Mail \& Guardian). As has been demonstrated, his direct political involvement in the activities of the UDF in Cape Town during the 1980s provided him with political 'credentials' that allowed him to both champion and criticise the politics of the ANC. He was the first white South African cartoonist to consistently represent the ideology of the liberation struggle. His work admirably performed the three main functions of resistance cartooning in South Africa: denigration of apartheid, valorisation of the liberation movement, and education and conscientisation for social change.

It was during the traumatic transition period that a new role emerged for South African cartooning: to assist ordinary South Africans, black and white, to cope with the challenges of rapid social and political change. The presentation of iconic ANC figures in a positive light, as in the Madam $\mathcal{E}$ Eve cartoon commemorating the death of Chris Hani, was one function of this role. Another was the provision of pro-transformation symbols and icons that had widespread appeal. Zapiro's much-loved Madiba icon is an obvious example of this, but his work also had a dialectical element that celebrated the positive aspects of change while remaining mindful of problems and dangers. In Madam $\mathcal{E}$ Eve, the provision of pro-transformation iconography is equally subtle and complex, 
as exemplified in the cartoon that shows two hoodlums running off with a section of the Rainbow Nation's rainbow. Here, the cartoon functions paradoxically to naturalise social disorder by making a joke about it: it points out that the transition to the new South Africa is likely to be accompanied by a variety of social ills that South Africans have to accept as the (hopefully temporary) price of transition. This resigned, humorous and stoical stance advocates tolerance and the acceptance of diversity as prerequisites for a successful transition. This sense of stoical resignation is not unfamiliar: it is present, for example, in many of Dov Fedler's cartoons, in Grogan's South Africa and in It Takes Two To Toyi Toyi by Gus Silber and Stidy.

The third main function of resistance cartooning in the 1985-1994 period was the provision of information to low-literacy communities to prepare them for their new role as citizens in a new democratic South Africa, and in some cases, to mobilse them around social and political issues. In this area of cartooning, as in newspaper cartooning, the absence of black South African cartoonists had profound consequences, in that there were very few artists who could authentically portray the life circumstances of the impoverished black majority. Media activists who sought to use cartooning for popular education and conscientisation were often unable to overcome this problem and their work suffered as a result. Some developed innovative responses to this predicament, of which collaboration between skilled white technical practitioners and black content providers was the most effective. Perhaps the most sophisticated response to this dilemma was provided by the Storyteller Group: they developed an inclusive participatory story telling strategy and trained up a cadre of young black illustrators. However, the scale of operations required by this methodology was unsustainable, particularly as the Storyteller Group comics were given away, not sold, and thus the entire operation relied on the provision of corporate social responsibility, government and NGO funding. Interestingly, a new variation of the model has recently emerged in the form of Supa Strikas, a commercial comic publishing enterprise based around soccer, where corporate funding is secured through the innovative stratagem of placing product logos into the cartoon panels themselves.

\section{Theoretical and methodological issues}

A key concern of this study has been theoretical. The study of cartooning to this day is plagued by imprecise language and the lack of clearly defined methodological procedures, and this study attempts to speak to this predicament. It is argued that the study of cartooning is the province of media and cultural studies, and that the central 
theoretical principles hammered out by media and cultural studies theorists over the last fifty years are appropriate to the study of cartooning. However, as Hall and Evans (1999) note, media and cultural studies theorists have been more effective in developing a body of theory around audio-visual culture than they have been in developing a language for the analysis of textual-visual culture. In the relative absence of a wellformulated body of theory around cartooning, this study has turned to the writings of cartooning practitioners themselves, in particular Scott McCloud, to investigate the process of cartooning. It is argued that while McCloud's theoretical insights are valuable and in some cases profound, his work is limited because his findings are not integrated into the existing body of media and cultural studies theory. In some cases his idiosyncratic usage of concepts and terminology directly contradicts accepted usage within media and cultural studies discourse. This study has attempted to express some of McCloud's insights in the language of media and cultural studies.

Using very basic concepts derived from the discourses of media and cultural studies, this study has also attempted to suggest a broad theoretical position from which to embark upon the critical analysis of cartoons and comics as forms of popular visual culture. A key concept is that cartooning (or comic art) is a cluster of signifying practices that express themselves in a variety of forms designed to be transmitted via the print media. The formulation of cartooning as a cluster of signifying practices, expressed as cartooning forms, and transmitted via the media, overcomes the common tendency to refer to comics as media, which they are not. It also combats the common impulse to draw a line between comics and cartoons, as though they were the products of fundamentally different practices, which they clearly are not. Having made these distinctions, the study offers some suggestions as to the defining characteristics, or 'way of signifying', that the various forms of cartooning share.

Finally, the study argues for the utilisation of a very logical and pragmatic approach to interpretation that views the individual textual unit within its socio-historical and discursive contexts. A useful theoretical exposition of this approach is provided by Thompson's (1990) methodology for the interpretation of symbolic forms. This study has hopefully demonstrated the efficacy of Thompson's approach by applying it to South African cartooning between 1985 and 1994. The study sets the cartooning of this period against the socio-historical background of the South African transition and the discursive background provided by the theorisation of cartooning as a cluster of signifying practices. It has attempted to show how those cartoonists who gave expression to discourses of resistance responded to the political and ideological 

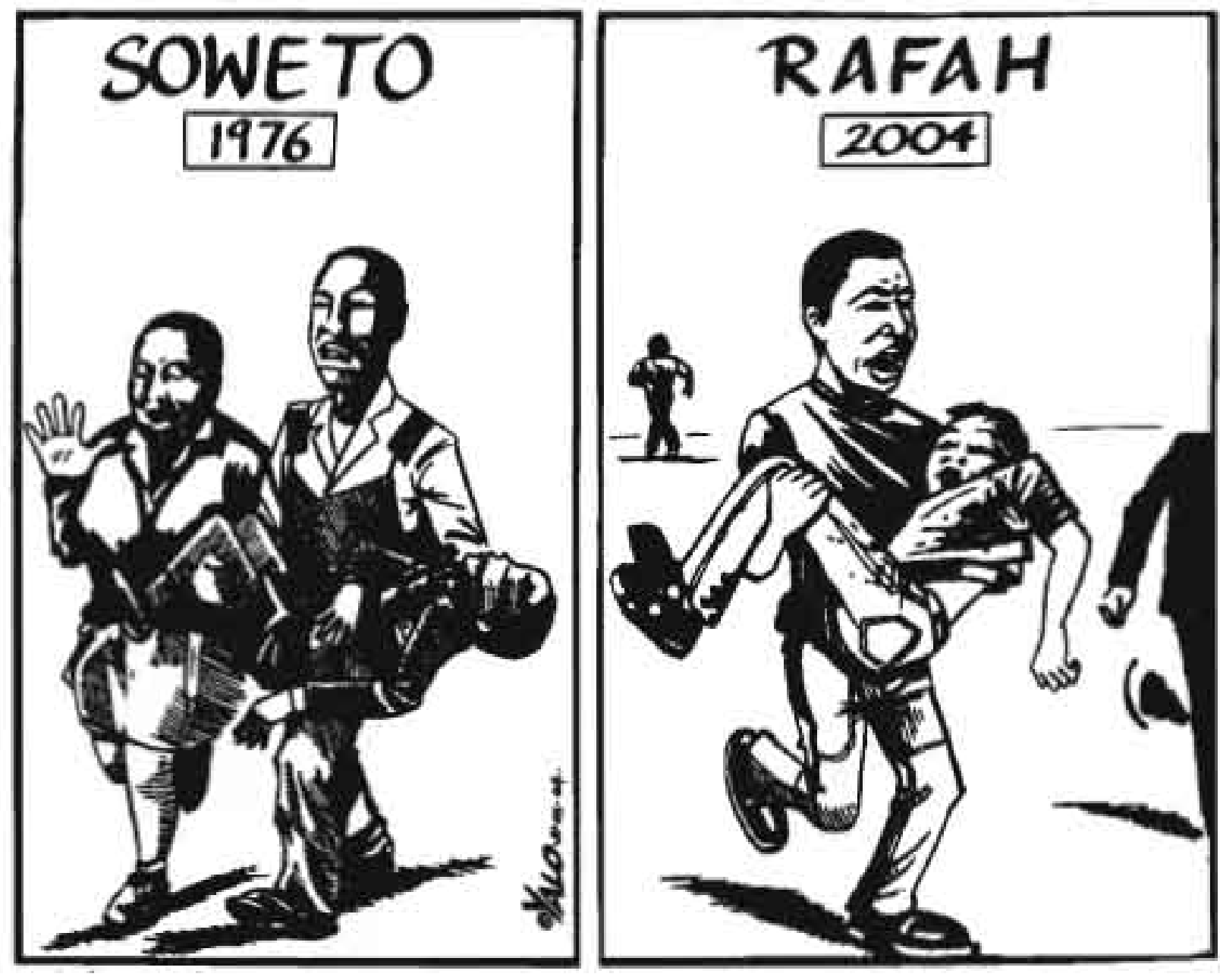

FIGURE 105

Sifiso Yalo, the staff cartoonist at ThisDay, has maturod from an unknown ant student to orve of the country's respected cartooning voices in a matter of wo years, proving himself capable of commenting on both domestic and international politics. (ThisDay, 2004) 
demands of their socio-historical context, how their work was defined and shaped by the publishing processes via which it was mediated, and how it gave expression to ideological content of which the cartoonists themselves were often unaware. The unintended messages that we are able to derive from the re-interpretation of these preinterpreted symbolic forms provide valuable information about the prevailing attitudes of the day. They also bear testimony to the power of cartooning, which enables the gifted cartoonist, armed with nothing more than a drawing instrument and a piece of paper, to crystalise the political, ideological, emotional and psychic dimensions of a complex historical moment into a witty sketch accompanied by a few lines of pithy text.

\section{Postscript: the way forward}

The story of South African cartooning certainly does not stop in 1994, although, clearly, that date represents the turning of a page and the starting of a new chapter. Ten years later, South African cartooning is in good shape, although the transition to postapartheid realities has had a profound effect on the milieu of out which the most important cartooning of the last decade of apartheid emerged. Hopefully, the nature of these changes has been sufficiently described in the body of this study, but a few key observations perhaps need to be re-emphasised.

The work of Zapiro, still the most important South African political cartoonist, is a case in point. He has made the transition from a cartoonist closely aligned to the liberation movement and the ANC, to one who now fulfills the exemplary 'watchdog' role of the ideologically independent political cartoonist, operating from an ethical rather than a party political base. He has mounted sustained attacks on aspects of government policy of which he does not approve, and notable targets have been the corruption of some elected officials, the government's stance with regard to neighbouring Zimbabwe, its position on the HIV / Aids issue and its tardy roll-out of anti-retroviral medication. Zapiro has become a renowned commentator on South African affairs and is frequently invited by the mass media to comment on current issues. He has also been invited to participate in a number of international events, in both in the cartooning and more broadly political arenas. This change in the status of the country's leading cartoonist-from a rebel outside the citadel to an icon of the new mainstream-exemplifies the change in status that the nation as a whole has enjoyed 

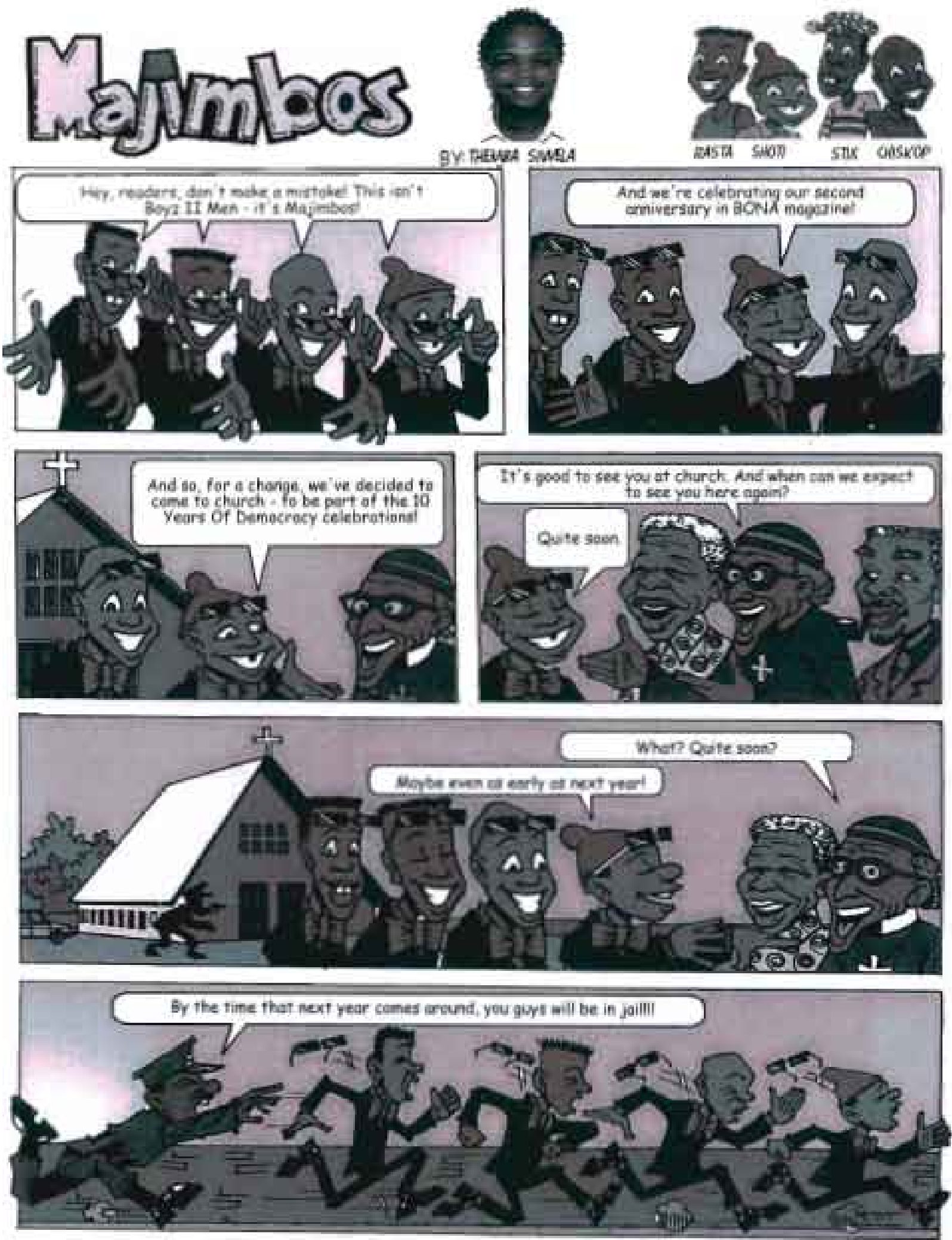

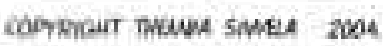

BONA-AUGUST 2004

FIGURE 106

Themba Siwela, previously an uniknown township artist, now enjoys prominence as the author of the poputar cartoon strip Majumbos, which appears monthly in the multilingual black consumer magazine, Bona. (Bona. 2004) 


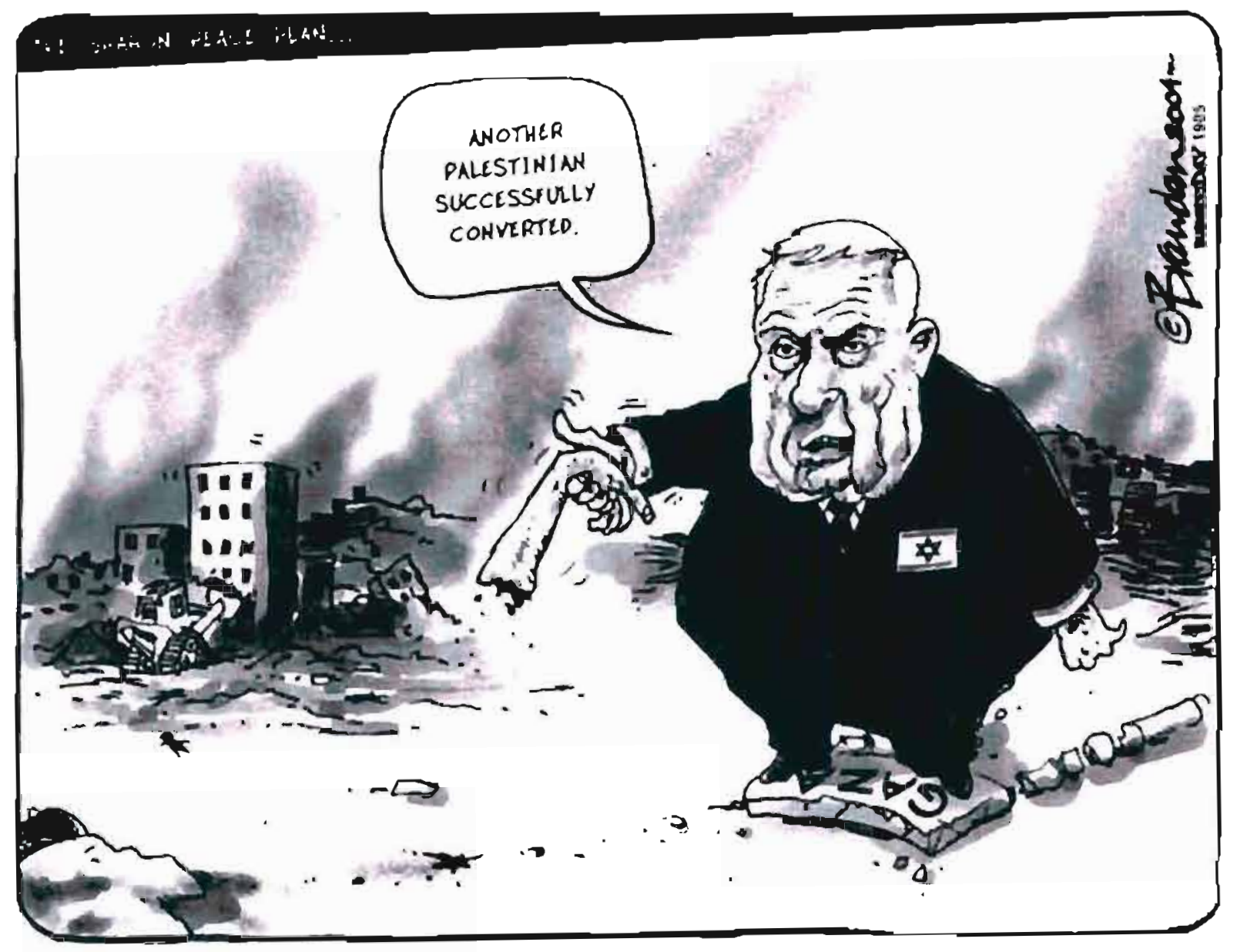

FIGURE 107

Brandan Reynolds, who grew up in a disadvantaged Coloured community on the Cape Flats, holds the prestigious daily editorial cartoon slot at Business Day, providing a daily commentary on domestic and international issues, such as this sharp criticism of Israeli Prime Minister Arial Sharon's approach to the Palestinian question. (Business Day, 2004) 
over the last ten years. While most of the key problems that faced South Africa a decade ago have not gone away, there is one major difference: the country is no longer politically unstable. However, as the cartoonists themselves are often the first to point out, the sense of security and material well-being enjoyed by the beneficiaries of the new dispensation does not necessarily extend to the 20 million or more people who still live in dire poverty, plagued by the HIV/Aids pandemic and without hope of gainful employment in the near future.

The new stability, social security and relative prosperity enjoyed by those South Africans whose lives have materially improved in the last decade has had positive effects in the field of South African cartooning, some of which still have to be felt. A key area of innovation, to which the country's cartooning community has not yet adequately responded, is the emergence of a number of new publications that provide potential opporturities for emerging cartoonists. In particular, the new tabloids such as the Daily Sun, the Sunday Sun and the Sowetan Sunday World have proved to be very open to publishing the work of emerging cartoonists. Two young black cartoonists-neither of whom is old enough to have been active before 1994 -Bethuel Mangena (Sowetan Sunday World) and Siphiwo Sobopha (Sunday Sun), are publishing regularly in these publications. Sifiso Yalo, the staff cartoonist for the new national daily newspaper, ThisDay, has matured from an unknown art student to one of the country's respected cartooning voices in a matter of two years, proving himself capable of commenting on both domestic and international politics (Figure 105). Themba Siwela, previously an unknown township artist, now enjoys prominence as the author of the popular cartoon strip Majimbos, which appears monthly in the multilingual black consumer magazine, Bona (Figure 106). Meanwhile, Brandan Reynolds, who grew up in a disadvantaged Coloured community on the Cape Flats, holds the prestigious daily editorial cartoon slot at Business Day (Figure 107). Several training and development intiatives are underway to identify and train new South African cartoonists from previously disadvantaged backgrounds, and the country's art schools now view cartooning as a viable career option for their students. At the same time, there is a vast area of potential illustration work in wait for those with the talent to explore it, in the form of school textbooks that still have to be rewritten and illustrated in the new, non-racial, South African idiom. 


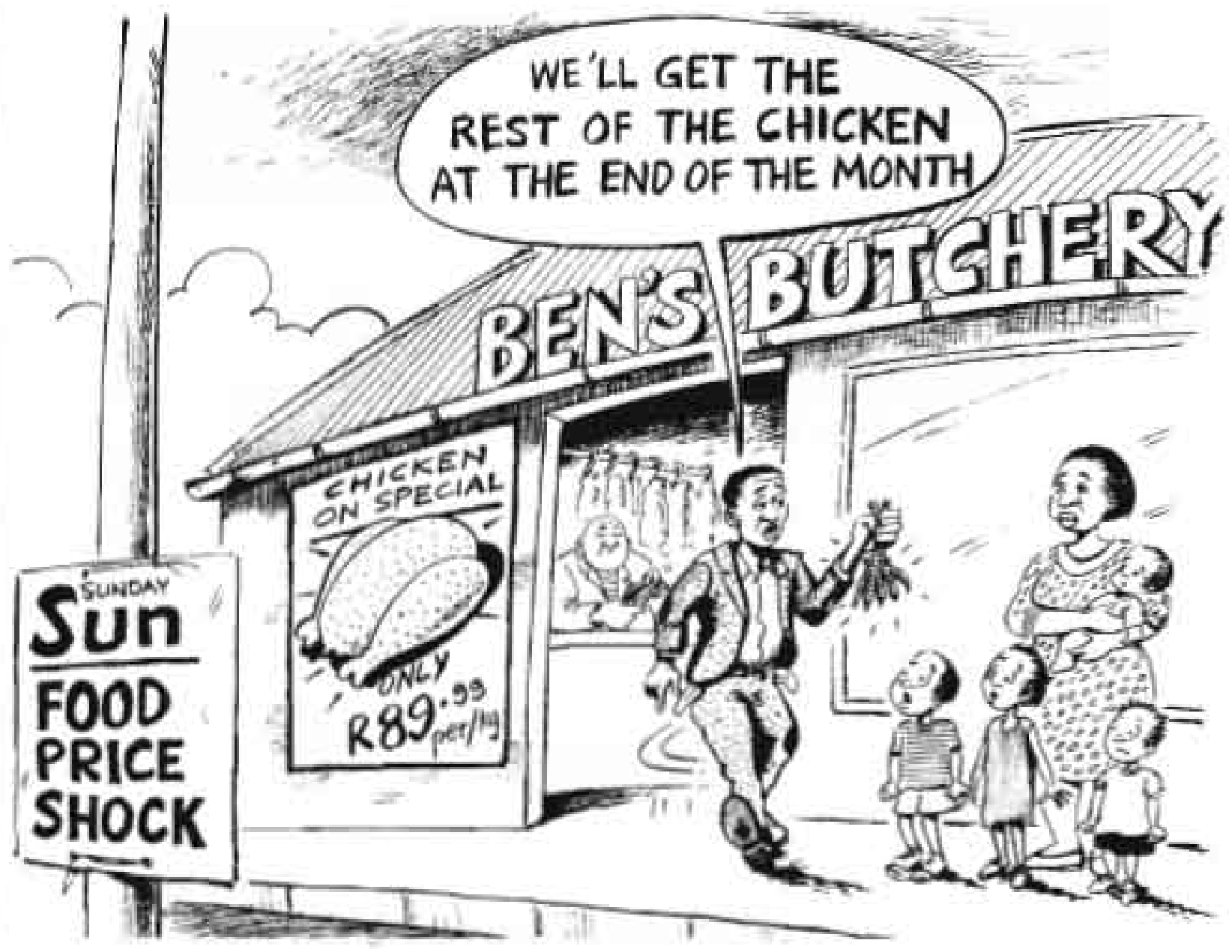

FIGURE 108

Alastair Findlay, whose work as a political cartoonist began in the Vrye Weekblad in the late 1980s, now appears weekly in City Press and the Sunday Sun. (Sunday Sun, 2002) 
In addition to the prescribed limitations of the research that underlies this study, are many gaps and under-researched areas of which the writer is painfully aware. These will no doubt be obvious to the informed reader. However there is a dearth of critical material on cartooning in South Africa and it is hoped that some of the forays made in this study will be taken up and either extended, rejected or improved upon by subsequent researchers. One of the pitfalls of a broad survey such as this is that the important contributions of some practitioners will invariably be under-represented, and there are several cartoonists who have received insufficient attention, not because they are less important, but simply because of limitations imposed by time and other practical considerations.

Amongst the cartoonists whose contributions are not sufficiently acknowledged in this study are Alastair Findlay, whose work as a political cartoonist began in Vrye Weekblad in the late 1980s, and now appears weekly in City Press and the Sunday Sun (Figure 108). Findlay is both a prolific freelance cartoonist and a fine artist, and produces illustrations and strips for a range of publications. He was closely involved in the work of the Storyteller Group, and collaborated with Peter Esterhuysen and Neil Verlaque-Napper on some of their most important and groundbreaking projects. He also earned himself the distinction of illustrating the first Afrikaans graphic novel, Hemel op Aarde (Heaven on Earth) (1997), in collaboration with writer Tinus Horn, reinforcing the ascendancy of Afrikaans writers and artists in the field of graphic literature in South Africa.

Another daily cartoonist who is not included, and has generally received less critical attention that he should have, is Stidy (Anthohny Stidolph) who has been the editorial cartoonist on The Witness (previously the Natal Witness) since 1991. The Witness is one of South Africa's few independent regional papers, and as a result, Stidy's work has not been widely circulated beyond the Pietermaritzburg area of KwaZulu-Natal. However his work has recently begun to receive wider recognition following the publication of a collection of his cartoons to commemorate 10 years of democracy (Figure 109)269.

269 Stidy, Over the Rainbow: The first 10 years of South Africa's democracy in cartoons, 2004. 


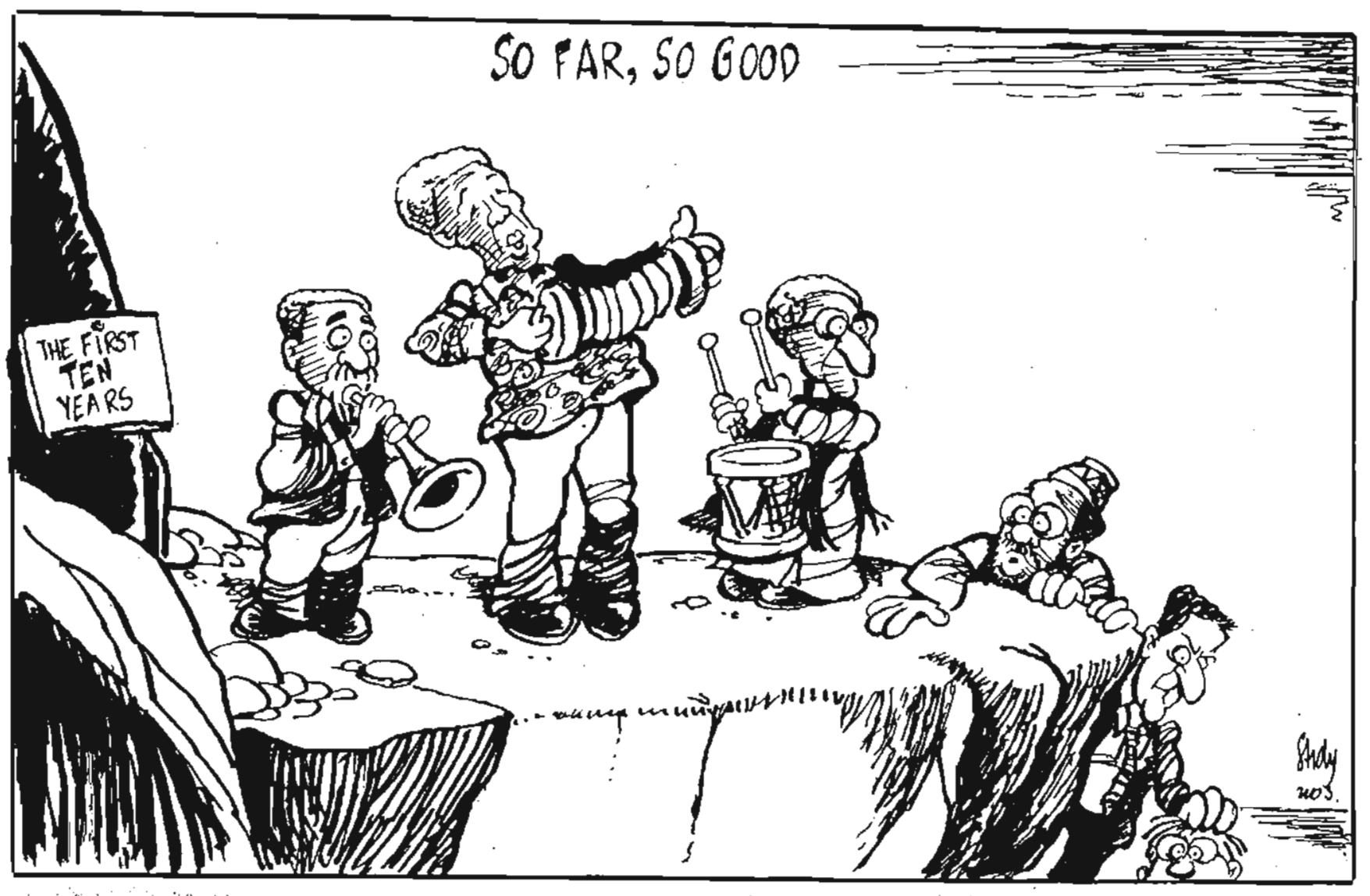

FIGURE 109

The work of Stidy (Anthohny Stidolph), editorial cartoonist on The Witness (previously the Natal Witness) since 1991, is less well known than it should be, but has recently begun to receive wider recognition following the 2004 publication of a collection of his cartoons to commemorate 10 years of democracy. (Stidy, 2004) 
The work of Brandan Reynolds, who has been producing the daily editorial cartoon for Business Day since October 2002, needs to be acknowledged, although his active period as a working cartoonist began in 1995 and thus falls outside of the scope of this study. Working under the name Brandan, he has emerged as a leading South African political cartoonist, but is not widely recognised as such by the broader public because Business Day, obviously, is specifically targeted at the business community. Prior to his present post, he worked for Personal Finance, a financial supplement that appeared in several major newspapers. His style has evolved dramatically in recent years, from detailed, full colour work on Personal Finance to a sparser, harder hitting, but nevertheless sophisticated black and white editorial cartoon style that uses digitally-generated midtones to excellent effect (see Figure 107) ${ }^{270}$.

With regard to South Africa's emerging generation of young black cartoonists, it is too early to predict how they will approach the demanding road that lies ahead. While it was extremely difficult, if not virtually impossible, for a black cartoonist to make a successful career out of cartooning before 1990, the present climate is one in which, according to ThisDay's Sifiso Yalo, there is abundant demand for new cartooning talent ${ }^{271}$. Several training and mentoring initiatives involving well-known cartoonists have been established, and cartooning projects such as the Cape Town-based Igubu Group, Stellenbosch's Stripshow, and the Durban Cartoon Project's Mamba Comix, provide opportunities for new signatures to reveal themselves.

The recent publication of Joe Daly's graphic novella, The Red Monkey, by an innovative Cape Town publisher 272 has set a precedent that is likely to stimulate more activity in this genre. This strip originally appeared in $S L$, a youth-oriented consumer magazine,

\footnotetext{
270 Reynolds registered at the University of Cape Town's prestigious Michaelis School of Fine Art in 1988, where he was the only student of colour, but left after one year, finding a more comfortable environment at the Ruth Prowse Art School in the inner city suburb of Woodstock, where he enjoyed the support of lecturers who encouraged him to explore his natural cartooning talent. In 1995 he began submitting a weekly cartoon to the Cape Argus, and produced a monthly cartoon for Democracy in Action, published by Idasa. In 1997 he joined Personal Finance as full-time illustrator and graphic artist. In 2000 he and his wife relocated to the U.S. for two years, where he worked as a graphic artist on the Atlanta Journal Constitution. On his return to South Africa in 2002 he joined Business Day.
}

271 Interview, 2004.

272 Joe Daly, The Red Monkey: The Leaking Cello Case, was published by Double Storey Books, 2003. Double Storey also published collections by Zapiro and Grogan in 2003. 
and several other youth culture magazines are beginning to allocate space to graphic narratives of various kinds.

One of the most intriguing developments on the South African comics publishing scene has been the success of Supa Strikas, a full-colour soccer comic that is distributed as a monthly supplement to the Sunday Times ${ }^{273}$. The publishers, Strika Entertainment, have expanded their distribution into other African countries, reformatting the South African comics to local requirements, and claim to be reaching millions of readers with a range of comic book products ${ }^{274}$. Interestingly, these comics are mostly distributed free of charge, not sold, and revenue is generated by the placement of advertisers' product names within the comic panels themselves, particularly on the shirts of the players and on the hoardings around the fields where much of the action takes place. In terms of the free distribution of their comics, Strika Entertainment are utilising a well-organised adaptation of the model pioneered by the Storyteller Group. This may indeed be the most appropriate and cost-effective way to introduce a comics reading and comics buying habit into the potentially massive South African popular culture marketplace, which has never before had any recreational comics of this kind designed specifically for it.

It remains to be seen whether South Africa's struggling black working-class consumers can be persuaded on any significant scale to part with their hard-earned cash for the pleasure of a hour or two's fun with a comic book for themselves and their offspring. If they can, there is no reason, as the Storyteller Group's Neil Verlaque-Napper ${ }^{275}$ has been arguing for more than a decade, why South Africa's massive urban populations shouldn't get into the habit of buying and reading popular visual literature at a scale that is sufficient to float a sustainable South African comic book industry. Perhaps the dream of a South African comics renaissance is not so far fetched after all.

\footnotetext{
273 It also appears as a weekly strip in the Sunday Times Magazine and is collected into an annual that is sold commercially.

${ }^{274}$ Interview with Supa Strikas publisher Oliver Power, 2003.

275 For most of the period under study, Neil Verlaque-Napper used the name Neil Napper, and this usage has been retained for historical reasons, since it appears in most of the publications he contributed to during the period under study.
} 
Through the use of humour and satire, South African cartoonists made a contribution to the process whereby the attitudes of ordinary people, black and white, were transformed from hatred and hostility to tolerance and acceptance of diversity, to the extent that they demonstrably have been able to cope with the profound emotional and psychic demands to which they have been exposed over the past decade. This process is ongoing and South African cartoonists will continue to be critical of the government of the day, pointing the way to desirable outcomes, and suggesting ways in which ordinary people can cope with an often incomprehensible macrocosm. Hopefully, too, our country's cartoonists will continue to enjoy the benefits of freedom of expression, now entrenched in the South African constitution.

21 October 2004

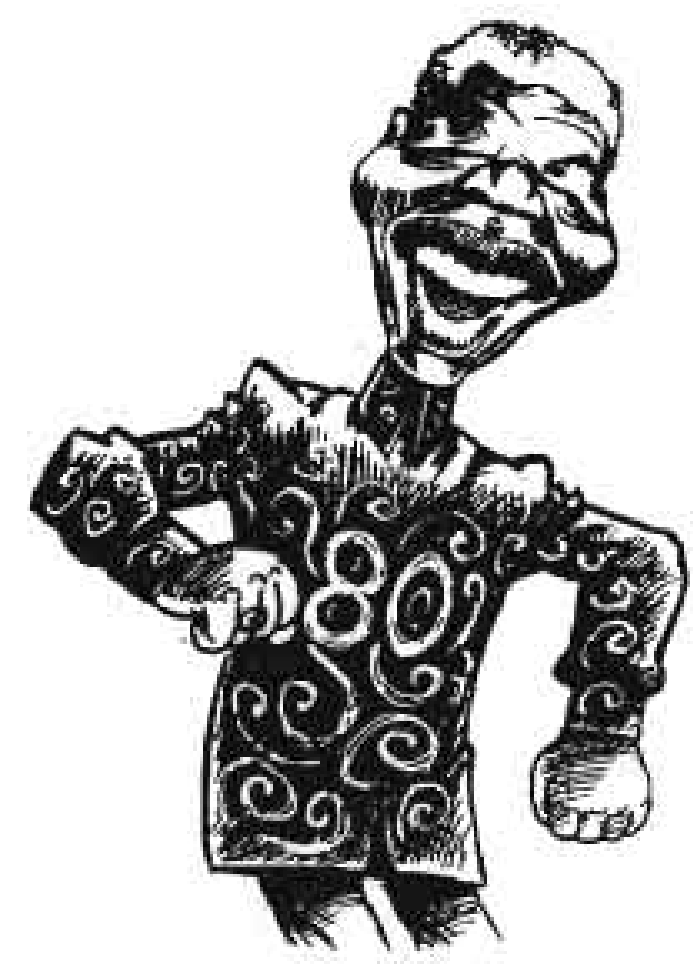




\section{Bibliography}

\section{Style Notes:}

(i) In order to display continuity, where there are multiple titles under an author or authorial group, the titles are listed chronologically.

(ii) Where an artist uses a psuedonym, the pseudonym is used in the reference. The artist's real name is given in brackets the first time it is listed, and where necessary thereafter. Where the artist/author reverts to his or her real name, the real name is listed.

(iii) Where real names are used, the surname is listed first, but pseudonyms are listed as presented.

(iv) Where a work is produced collaboratively under a group name, the group name is listed first, followed by the artist and author names.

(v) Artists are listed first, followed by authors or editors.

(vi) Where a work exists both as a comic strip in a larger publication and a selfstanding comic book, it is listed in both Section 1 and Section 2.

\section{Cartoons, comic strips, illustrations and art works cited}

Alexander, Jane. 1989. Art works reproduced in Williamson, 1989: 42-45.

Andy (Anderson, Dave). 1988. "The government views the Archbishop's roadshow as pro-communist propaganda". Cartoon, The Star. Reproduced in Anderson, 1989: 33.

Andy. 1982. Cartoon portraying the isolation of South Africa's white electorate, Evening Post. Reproduced in Vernon, 2000: 147.

Andrew, Rick. 1987. "Urban Blues". Comic strip, PAX (Pre-Azanian Comix), No. 1. Anonymous. 1958. "Goombi - Private Detective". Serialised comic strip, Drum. Bauer, Derek. 1989. Cartoon about sanctions, depicting Kaunda and Magube. Reproduced in Bauer, 1989: 52.

Bauer, Derek. 1989. Cartoon showing KwaNdebele refusing the gift of independence. Cartoon, reproduced in Bauer, 1989: 31. 
Bauer, Derek. 1989. "Cheer up guys I'm here to stay!" Cartoon, reproduced in Bauer, 1989: 61.

Bauer, Derek. 1989. "The dreaded Marxist forces strike again”. Cartoon, reproduced in Bauer, 1989: 2.

Bauer, Derek. 1989. "The ghosts of Christmas past, present and future". Cartoon, reproduced in Bauer, 1989: 49.

Bauer, Derek. 1989. "'Skus meneer ... ons soek 'n genuine black leader". Cartoon, reproduced in Bauer, 1989: 25.

Bauer, Derek. 1989. "Steve Biko: In Memoriam". Cartoon, reproduced in Bauer, 1989: 70. Beyer, Mark. 1979. "Dead Things". Comic strip, reproduced in Spiegelman and Mouly (eds), 1987: 26-27.

Biox, Richard. 1921. "DA-DA" (New York Dada Group). Cartoon, reproduced in Naumann, 1996: 10.

Boonzaier, Daniel. 1917. Cartoon of Hoggenheimer manipulating the English press, Die Burger. Reproduced in Vernon 2000: 47.

Boonzaier, Daniel. 1903. Cartoon of Hoggenheimer opposite Joseph Chamberlain, South African News. Reproduced in Vernon 2000: 31.

Botes, Conrad. 1994. "Kanker". Comic strip, Bitterkomix No. 4, 1994.

Botes, Conrad. 1994. "Siembamba". Comic strip, Bitterkomix No. 4., 1994. Translated into English in The Best of Bitterkomix Vol. 1., 1998.

Botes, Conrad. 1995. "Bloedrivier". Comic strip, Bitterkomix No. 5., 1995. Translated into English as "Blood River" in The Best of Bitterkomix Vol. 1., 1998.

Botes, Conrad. 1998. "Vaalseun". Comic strip, Bitterkomix, No. 8, 1998. Translated into English as "Dullboy" in The Best of Bitterkomix Vol. 1., 1998.

Connolly, Bob. 1966. Cartoon depicting the Afrikaner 'bogeyman', Rand Daily Mail. Reproduced in Vernon, 2000: 119.

Cruikshank, George. 1819. "Blessings of Emigration to the Cape". Cartoon, reproduced in Bryer and Hunt, 1984: 19.

Fedler, Dov. 1991. "Fancy meeting you here". Cartoon about AIDS and violence, The Star. Reproduced in Fedler, 1991.

Fedler, Dov. 1990. Cartoon about train massacres, The Star. Reproduced in Fedler, 1991. Findlay, Alastair. 2002. Cartoon about rocketing food prices, Sunday Sun. Reproduced in Mason, 2002: 21. 
Francis, Stephen; Dugmore, Harry and Rico (Schacherl, Ricardo). 1992. "Is she available Tuesdays?" Cartoon reproduced in Francis et al, 1993: 7.

Francis, S., Dugmore H. and Rico. 1993. Cartoon milking the stereotypical fears and entrenched phobias of white South Africa. Reproduced in Francis et al, 1993: 80.

Francis, S., Dugmore H. and Rico. 1993. Cartoon showing the country's security industry cynically exploiting white fears of crime for their own enrichment. Reproduced in Francis et al, 1993: 9.

Francis, S., Dugmore H. and Rico. 1993. Cartoon presenting Madam and Eve as codependent when faced with an external threat. Reproduced in Francis et al, 1993: 79.

Francis, S., Dugmore H. and Rico. 1993. Cartoon depicting the funeral of Chris Hani. Reproduced in Francis et al, 1993: 83.

Francis, S., Dugmore H. and Rico. 1994. "Free at last!" Cartoon reproduced in Francis et al, 1994: frontispiece.

Francis, S., Dugmore H. and Rico. 1996. Cartoon of a pair of balaclava-hooded gangsters making off with a piece of the Rainbow Nation's rainbow. Reproduced in Francis et al, 1998: 178.

Olly (real name unknown). c.1972. "Big Mr. Westernman". Comic strip, Wits Student. Reproduced in Mason, 2002: 13.

Grogan, Tony. 1993. Cartoon about mixed suburbs, Cape Times. Reproduced in Grogan, 2003.

Grogan, Tony. 1989. Frontispiece cartoon of the character Johnny Rousseau-Smit, Grogan, 1989.

Grosz, Georg. 1918. To Oskar Panizza. Painting, reproduced in Kranzfelder, 1994: 25.

Grosz, Georg. 1917. The City. Painting, reproduced in Kranzfelder, 1994: 14. Hodgins, Robert. Paintings, reproduced in Williamson, 1989: 52-53.

Honiball, T.O. 1966. "Adoons-hulle". Comic strip, Huisgenoot. Reproduced in Schoonraad, 1983: 101.

Hulsing, Milan.1998. "Museum of Archeology". Comic strip, Lapin No. 25, 1999. Jackson, John. 1958. Cartoon depicting the loss of human rights, reproduced in Dugmore et al, 1999: 20. 
Joe Dog (Kannemeyer, Anton) and Adam Baard (Botes, Conrad). 1989. “Case No. 308”. Comic strip, Bitterkomix No. 1, 1992.

Joe Dog. 1994. "Jeugweerbaarheid". Comic strip, Bitterkomix No. 4, 1994. Translated into English in The Best of Bitterkomix, Vol. 1, 1998.

Joe Dog and Schreuders, Claudette. 1993. "Do a Dance for Daddy". Comic strip,

Bitterkomix No. 3, 1993.

Joe Dog. 1993. Cover illustration, Bitterkomix No. 3, 1993.

Joe Dog. 1994. "Liefde en Missiele”. Comic strip, Gif: Afrikaner Sekskomix.

Joe Dog. 1995. Cartoon about penile amputation, Loslyf.

Joe Dog. 1995. "Boetie". Comic strip, Bitterkomix No. 5, 1995. Translated into English as "Sonny" in The Best of Bitterkomix Vol. 1, 1988.

Joe Dog. 1998. "Hemel Help Ons (Twee)". Comic strip, Bitterkomix No.8, 1998.

Joe Dog. 1998. “Onse Vader”. Comic strip, Bitterkomix No. 8, 1998.

Kenyon, Donald. 1976. Cartoon about June 16, Daily Despatch. Reproduced in Vernon, 2000: 132.

Leyden, Jock. 1969. Cartoon depicting BOSS as an ominous figure looming over the South African judiciary. Daily News. Reproduced in Vernon, 2000: 114.

Leyden, Jock. 1967. “Out!". Cartoon about terrorism, Daily News. Reproduced in Vernon, 2000: 117.

Leyden, Jock. 1960. "The Winds of Change". Cartoon about African nationalism, Daily News. Reproduced in Vernon, 2000: 106.

Marais, David. 1963. Cartoon depicting prime minister B.J. Vorster in Nazi-style uniform, Cape Times. Reproduced in Marais, 1963.

Marais, David. 1958. "Blankes, Nie-blankes". Cartoon about beach apartheid, Cape Times. Reproduced in Marais, 1960.

Mason, Andy (artist). Cloete, Dick (writer); Berold, Robert (editor); 1981. “Vusi Goes Back". Comic strip, Berold and Caine (eds), 1981: 9.

N.D. Mazin (Mason, Andy). 1985-86. "The Big Chillum". Comic strip serialised in PAX (PreAzanian Comix), Issues 1-4, 1985-6.

Motshumi, Mogorosi (with N.D. Mazin, 1981-3). 1983. "Sloppy". Comic strip serialised in Learn and Teach Magazine, 1981-1993.

Mouton, Fred. 1986. "Punch and Burn 'em Show". Cartoon demonising the ANC, Die Oosterlig. Reproduced in Vernon, 2000: 158. 
Mouton, Fred. 1982. Cartoon portraying the isolation of South Africa's white electorate, Die Burger. Reproduced in Vernon 2000: 162.

Mouton, Fred. 1976. Cartoon showing a Soviet hand tapping ash from a Cuban cigar into an Africa-shaped ash tray, Die Burger. Reproduced in Vernon, 2000: 138.

Mouton, Fred. 1975. Cartoon portraying Russian president Leonid Brezhnev as a witchdoctor manipulating African leaders. Die Burger. Reproduced in Vernon, 2000: 124 .

Muelen, Ever. 1987. "What's wrong with this picture?" and "The girl can't help it". Comic strips reproduced in Spiegelman and Mouly, 1987.

Nhlabati, Mzwakhe. 1982. Cover illustration, Call Me Not a Man by Mtutuzeli Matshoba.

Nhlabati, Mzwakhe. 1982. "Romance at Riverdale High". Comic strip illustration, story written by Chris Van Wyk, Upbeat, c. 1984.

Nhlabati, Mzwakhe. 1982. "Weep Not Child". Comic strip illustration, adaptation of the novel by Ngugi Wa'Thiongo, Upbeat. c.1982.

Nhlabati, Mzwakhe. 1981. "Down Second Avenue". Comic strip illustration, adaptation of the novel by Es'kia Mphahlele, Upbeat, 1981. (Published as a comic book in the People's College Comics series, 1988).

Norton, G.L. c.1950. "Phaki". Comic strip displaying racial caricature, Umteteli wa Bantu (Chamber of Mines), reproduced in Schoonraad, 1983: 173.

Panter, Gary. 1987. "Jimbo is Running Sore". Comic strip, reproduced in Spiegelman and Mouly (eds), 1987: 51.

Pooh (Mason, Andy). 1978-9. "Vittoke in Azania". Comic strip serialised in Dome (University of Natal) and Varsity (University of Cape Town), 1978-9.

Reynolds, Brandan. 2004. Cartoon criticizing Israel's approach to the Palestinian question, Business Day.

Sak, Len. 1961. Cartoon of B.J. Vorster hurling down the statue of Justice, reproduced in Dugmore et al, 1999: 24.

Sak, Len. n.d. Jojo's World cartoon on gender issues, reproduced in Sak, 1991.

Sak, Len. n.d. Jojo's World cartoon on domestic labour, reproduced in Sak, 1991.

Satrapi, Marjane. 1999. "Le Foulard". Comic strip, Lapin No. 25, 1999.

Schroder, William. 1887. Cartoon of Transvaal President Paul Kruger, The Lantern. Reproduced in Vernon, 2000: 21. 
Sedumedi, Percy. 1987. "The Instant Poet". Comic strip, PAX (Pre-Azanian Comix) No. 1, 1987.

Sedumedi, Percy. n.d. Travels of the Free Spirit. Self-published comic book, circa 1985-7.

Shapiro, Jonathan. See Zapiro.

Siwela, Themba. 2004. Majimbos. Monthly comic strip in Bona, 2003-4.

Smith, Richard. 1985. Cover illustration of P.W. Botha, Leadership (issue no. not known).

Smith, Richard. 1978. Cover illustration: Coetzee, J.M. In the Heart of the Country.

Johannesburg: Ravan Press.

Smith, Richard. 1973. Cover illustration, Wits Student.

Smith, Richard. 1971. "Richard Nixon". Reproduced in Mason, 2002: 8.

Soobben, Nanda. 1980. "120 years of progress and still 2nd class". Cartoon about the achievements of the Indian community, Post Natal.

Stidy (Stidolph, Anthony). 2004. "So far, so good". Cartoon about South Africa's first ten years, reproduced in Stidy, 2004.

Stidy. 1991. Cartoon, anticipating Madam E Eve, in Silber, 1991: 42.

Stieglitz, Alfred. 1917. Photograph of Marcel Duchamp's, Fountain. In Naumann. 1996:

90.

Swarte, Joost. 1987. "The Clock Strikes" and "The Last Word in Fashion". Comic strips reproduced in Spiegelman and Mouly, 1987.

Thamm, Eric. 1948. "Hy lei ... maar waarheen?" Cartoon about General Smuts, Die

Transvaler. Reproduced in Vernon, 2000: 92.

Yalo, Sifiso. 2004. Cartoon comparing Soweto, 976 to Rafah, 2004. ThisDay.

Zapiro (Shapiro, Jonathan). 1987. "Never fear, Mummy's (still) here!” Cartoon about

support for the apartheid regime by Thatcher and Reagan, South.

Zapiro. 1987. Cartoon showing National Union of Mineworkers arm-wrestling with the Chamber of Mines, South.

Zapiro. 1988. "Behind the Barbed Wire Curtain". Comic strip, Mamba Comix No. 1, 2003.

Zapiro. 1987. "June 16: Salute the Youth Who Brave the Dragon". Cartoon, South.

Zapiro. 1987. UDF calendar illustration. Reproduced in Mamba Comix No. 1, 2003.

Zapiro. 1988. Poster for 1988 exhibition of Zapiro's work Laughter in the Belly of the Beast. Reproduced in Mamba Comix, No. 1, 2003. 
Zapiro. 1991. Cover illustration depicting Magnus Malan, Die Suid-Afrikaan. Reproduced in Mamba Comix, No. 1, 2003.

Zapiro, 1994. Election day cartoon. Reproduced in Zapiro 1996: 11.

Zapiro, 1994. Cartoon portraying Mandela's inauguration. Reproduced in Zapiro, 1996: 12.

Zapiro. 1997. "The brilliant career of Eugene Terreblanche". Cartoon, Mail E Guardian. Reproduced in Zapiro, 1997.

Zapiro. 2000. Cartoon critiquing the government's approach to the implementation of antiretroviral treatment to people with Aids, Sowetan. Reproduced in Zapiro, 2001: 108.

Zapiro). 2000. Cartoon showing senior press figures licking the shoes of government minister Essop Pahad, Mail \& Guardian. Reproduced in Zapiro, 2001: 22.

Zapiro. 2001. Cartoon depicting Trevor Manuel and Jeff Radebe dancing to the tune of the IMF and World Bank, Sunday Times. Reproduced in Zapiro, 2001: 152.

Zapiro. 2001. Cartoon about rising medical expenses, Sowetan. Reproduced in Zapiro, 2001: 51 .

\section{Collections of cartoons and comic strips, graphic novels, comic books and comic magazines}

Anderson, Dave (Andy). 1989. The Cartoons of Andy. Johannesburg: Jonathan Ball. Andrew, Rick (artist); Ozynski, Joyce and Perlman, Harriet (writers). 1988. Equiano: The Slave Who Fought to be Free. Johannesburg: Peoples's College Comics/Sached Trust. Andrew, Rick (artist) and Harbour, Rodney (writer). 1987. Bonginkosi. Durban:

Appropriate Housing Technology Unit, University of Natal.

Anonymous. c. 1987-9. Icy Blind: Free Comic No. 42. Honeydew.

Anonymous. c. 1987-9. Icy Blind: The Final Kak. Honeydew.

Anonymous. c. 1985-7. Icy Blind: Free. Honeydew.

Anonymous. c. 1985-7. Icy Blind: Free No. 5. Honeydew.

Anonymous. c. 1985-7. Icy Blind: Autumn Sampler. Honeydew.

Bauer, Derek. 1989. S.A. Flambe and Other Recipes for Disaster. Text by Susan DeVilliers.

Cape Town: David Philip. 
Berry, Abe. 1980. Abe Berry's South Africa and How it Works. Johannesburg: Jonathan Ball.

Botes, Conrad and Kannemeyer, Anton (eds). 1992. Bitterkomix No 1: Vereikende Leestof vir Emosioneel Armoedige. Stellenbosch: Bitterkomix.

Botes, Conrad and Kannemeyer, Anton (eds). 1993. Bitterkomix No. 2: Leestof on Graag

Mee te Ontspan!. Stellenbosch: Bitterkomix.

Botes, Conrad and Kannemeyer, Anton (eds). 1993. Bitterkomix No. 3. Pulp vir Papkoppies.

Stellenbosch: Bitterkomix.

Botes, Conrad and Kannemeyer, Anton (eds). 1994. Bitterkomix No.4. Verkoop Jou Siel vir Security. Stellenbosch: Bitterkomix.

Botes, Conrad and Kannemeyer, Anton (eds). 1995. Bitterkomix No 5. Komieks deur SuidAfrikanersvVir Suid-Afrikaners. Stellenbosch: Bitterkomix.

Botes, Conrad and Kannemeyer, Anton (eds). 1996. Bitterkomix No 6.Afrikaners is Plesierig. Stellenbosch: Bitterkomix.

Botes, Conrad and Kannemeyer, Anton (eds). 1997. Bitterkomix No 7. Op die randjie van 'n dieper insig. Stellenbosch: Bitterkomix Pulp.

Botes, Conrad and Kannemeyer, Anton (eds). 1998. Bitterkomix No 8. Stellenbosch:

Bitterkomix Pulp.

Botes, Conrad and Kannemeyer, Anton (eds). 1999. Bitterkomix No 9. Stellenbosch:

Bitterkomix Pulp.

Botes, Conrad and Kannemeyer, Anton (eds). 2000. Bitterkomix No 10. Stellenbosch:

Bitterkomix Pulp.

Botes, Conrad and Kannemeyer, Anton (eds). 2001. Bitterkomix No 11. Deur My gaan die Ewige Ellende Binne. Stellenbosch: Bitterkomix Pulp.

Botes, Conrad and Kannemeyer, Anton (eds). 2002. Bitterkomix No 12. In die Uur van die Donker Dors. Stellenbosch: Bitterkomix Pulp.

Botes, Conrad and Kannemeyer, Anton (eds). 2004. Bitterkomix No 13. Testosteroon

Uitgawe: Satiereise Beeldverhaal vir Lewenslustige Dames.. Stellenbosch: Bitterkomix Pulp.

Botes, Conrad and Kannemeyer, Anton (eds). 2002. Bitterkomix: Special 2002 Edition.

Stellenbosch: Bitterkomix Pulp.

Botes, Conrad and Kannemeyer, Anton (eds). 1994. Gif: Afrikaner Sekskomix.

Stellenbosch: Bitterkomix/Hond. 
Botes, Conrad and Kannemeyer, Anton (eds). 1998. The Best of Bitterkomix, Vol 1.

Stellenbosch: Bitterkomix Pulp.

Botes, Conrad and Kannemeyer, Anton (eds). 2002. The Best of Bitterkomix, Vol 2.

Stellenbosch: Bitterkomix Pulp.

Burns, Charles. 1988. Hard-Boiled Defective Stories. New York/London: Raw/Penguin.

COSATU. 1989. Workers of South Africa! Unite and Fight for a Living Wage! No artist credited. Johannesburg: COSATU.

Crumb, Robert. 1997. The R. Crumb Coffee Table Art Book. London: Kitchen Sink Press/Bloomsbury.

Daly, Joe. 2003. The Red Monkey: The Leaking Cello Case. Johannesburg: Double Storey Books.

De Waal, Shaun. 1998. Jack Marks. Stellenbosch: Hond/Bitterkomix.

Dugmore, Harry, Francis, Stephen and Rico (Schacherl, Ricardo) 1994. Ken Jou Regte /

Know Your Rights. Johannesburg: Human Rights Commission/ Rapid Phase Group.

Dugmore, Harry, Francis, Stephen and Rico (eds). 1999. Nelson Mandela: A life in cartoons.

Johannesburg: David Philip.

Eisner, Will. 1985. A Contract with God and Other Tenement Stories. Princeton: Kitchen

Sink Press.

Fedler, Dov. 1973. My son the cartoonist. Johannesburg: Peter Brown.

Fedler, Dov. 1991. The Season of Violins. Johannesburg: B \& D Printers.

Fedler, Dov. 2001. Follow my leaders. Johannesburg: Ravan Press.

Fedler, Dov. n.d. Gagman. Unfinished and unpublished illustrated manuscript.

Findlay, Alastair (artist) and Horn, Tinus (writer). 1997. Hemel op Aarde. Cape Town:

Queillerie

Francis, Stephen, Dugmore, Harry, and Rico (Schacherl, Ricardo). 1993. The Madam \&

Eve Collection. Johannesburg: Weekly Mail \& Guardian Publishing/Rapid Phase Group.

Francis, S., Dugmore H. and Rico. 1994. Madam \& Eve:Free at Last.

London/Johannesburg: Penguin Books.

Francis, S., Dugmore H. and Rico. 1995. Madam E Eve: All aboard for the Graoy Train.

London/Johannesburg: Penguin Books.

Francis, S., Dugmore H. and Rico. 1996. Madam \& Eve: Somewhere over the Rainbow Nation. London/Johannesburg: Penguin Books. 
Francis, S., Dugmore H. and Rico. 1997. Madams are from Mars, Maids are from Venus. London/Johannesburg: Penguin Books.

Francis, S., Dugmore H. and Rico. 1998. Madam \& Eve's Greatest Hits.

London/Johannesburg: Penguin Books.

Francis, S., Dugmore, H. and Rico. 1999. International Maid of Mystery. Cape

Town/Johannesburg: David Philip Publishers.

Francis, S., Dugmore H. and Rico. 2000. The Madams are Restless: A New Madam E' Eve

Collection. Johannesburg: Rapid Phase.

Francis, Stephen, and Rico. 2001. Crouching Madam Hidden Maid: A New Madam \& Eve Collection. Johannesburg: Rapid Phase.

Francis, Stephen, and Rico. 2003.Madam \& Eve: 10 Wonderful Years. Johannesburg: Rapid Phase Group.

Francis, Stephen, and Rico. 2003. The Maidtrix. Johannesburg: Rapid Phase.

Gaiman, Neil. 2003. The Sandman: Endless Nights. New York: DC Comics.

Goldstuck, Arthur and Schacherl, Rico. 1992. Going, Going, Gone: The Ultimate

AuctionSsale for the New South Africa. London/Johannesburg: Penguin Books

Goldstuck, Arthur; Dugmore, Harry and O'Connor, Bill. 1989. 30 Great Reasons to Keep

Smoking. Bath: Laughing Stock Publishing Limited.

Grogan, Tony, 1986. Up the Creek. Cape Town: Chameleon Press.

Grogan, Tony. 1989. Grogan's South Africa. Cape Town: Chameleon Press.

Grogan, Tony. 2003. Grogan's 100 Best Cartoons. Johannesburg: Double Storey Books.

Hernandez, Gilbert. 1987. Heartbreak Soup. London: Titan Books.

Hernandez, Jaime. 1987. Love \& Rockets. London: Titan Books.

Hofmekler, Ori. 1982. Hofmekler's People. Kent: New English Library.

Honiball, T.O. 1975. Oom Kaspaas Maak ' $n$ ' Plan. Cape Town: Tafelberg.

Honiball, T.O. 1978. Jakkals en Wolf in die pekel. Cape Town: Tafelberg.

Honiball, T.O. 1961. Adoons-Hulle No 5. Port Elizabeth: Nasionale Boekhandel.

Jackson, John. 1967. Running Commentary. Cape Town: Howard Timmins.

Kalarytis, Costas (artist); Francis, Stephen and Goldstuck, Arthur (writers). 1990. All

Men Are Bastards. Bath: Laughing Stock Publishing Ltd.

Kelly, Walt. 1954. The Incompleat Pogo. New York: Simon and Schuster.

Leyden, Jock. 1944. There's a Thing. Durban: John Singleton \& Williams Ltd.

Marais, David. 1960. Europeans Only. Cape Town: Jan Botha Publishers. 
Marais, David.1963. I Like it Here. Cape Town: Jan Botha Publishers.

Marais, David. 1963. Out of This World. Cape Town: Books of Africa.

Marais, David. 1964. This is a Hi-jack. Cape Town: Howard Timmins.

Mason, Andy (artist), Cloete, Dick (writer), Berold, Robert (editor). 1982. Vusi Goes Back: A Comic about the History of South Africa. Johannesburg: Pre-Azanian Comix.

Mason, Andy (ed). 2003. Mamba Comix No. 1: Graphic Fiction with a Lethal Byte. Durban: Durban Cartoon Project.

N.D. Mazin (Mason, Andy) (ed). 1985. PAX No. 1: Pre-Azanianian Comix. Durban: Durban Comix Circle.

N.D. Mazin (ed). 1986. PAX No. 2: Man, this country really needs some harmony. Durban: Durban Comix Circle.

N.D. Mazin (ed). 1986. PAX No. 3: Durban's Homegrown Comix Magazine. Durban: Durban Comix Circle/Graphic Equalizer.

N.D. Mazin (ed). 1986. PAX No. 4: Lemming Issue. Durban: Durban Comix Circle/Graphic Equalizer.

N.D. Mazin and Moon, Chris (eds). 1987. PAX No. 5: The Graphic Fiction Magazine.

Durban: Durban Comix Circle/Graphic Equalizer.

N.D. Mazin and Moon, Chris (eds). 1988. PAX No. 6:Special "Alternative Television" Issue.

Durban: Durban Comix Circle/Graphic Equalizer.

O'Connor, Bill (artist); Goldstuck, Arthur and Dugmore, Harry (writers). 1989. 30 Great

Reasons to Start Smoking. Bath: Laughing Stock Publishing Ltd.

O'Connor, Bill (artist); Goldstuck, Arthur and Francis, Stephen (writers). 1989. So You

Think You're Depressed? Bath: Laughing Stock Publishing Ltd.

Ottomo, Katsuhiro. 2000. Akira, Book One. Tokyo: Mash-Room Co. Ltd/Milwaukie:

Dark Horse Comics.

Sacco, Joe. 2003. Palestine. London: Jonathan Cape.

Sak, Len. 1991. Jojo's World: Celebrating 33 years of a Township Cartoon Hero. Johannesburg:

Sowetan/Operation Hunger.

Scarfe, Gerald. 1993. Scarfe Face. London: Sinclair-Stevenson.

Schroder, William. (ed/publisher) 1884-1886. The Knobkerrie. Cape Town. (Special

collection of South African comic art, University of Stellenbosch.)

Shapiro, Jonathan. See Zapiro. 
Silber, Gus. 1991. It Takes Two to Toyi-Toyi: A Survival Guide to the New South Africa.

Mlustrated by Stidy (Anthony Stidolph). London/Johannesburg: Penguin Books. Smith, Richard. 1973. Smith \& Abbot's Greatest Hits. Johannesburg: Rand Daily Mail. South African Airways. n.d. Safety on Board/Veiligheid aan Boord: B737. South African Airways instruction card.

Spiegelman, Art. 1987. Maus: A Survivor's Tale, Book 1. London, Penguin.

Spiegelman, Art. 1996. The Complete Maus. London: Penguin.

Spiegelman, Art and Mouly, François (eds). 1987. Read yourself RAW. New York:

Pantheon.

Spiegelman, Art. 1998. Comix, Essays, Graphics and Scraps. Palermo: Sellerio Ediotore-La

Centrale dell'Arte.

Stidy (Stidolph, Anthony). 2003. Over the Rainbow: The first 10 years of South Africa's

democracy in cartoons. Pietermaritzburg: The Witness.

Story Circle. Shapiro, Jonathan, et al. 1991. Roxy: Life, Love and Sex in the Nineties. Cape

Town: Story Circle Group/Progressive Primary Health Care Network/Medical

Research Council.

Story Circle. Shapiro, Jonathan, et al. 1994. Môre se Mense / Tomorrow's People. Cape

Town: Story Circle.

Storyteller Group. Carvalho, Carlos (artist) Esterhuysen, Peter and Napper, Neil

(writers/editors). 1990. 99 Sharp Street: The River of Our Dreams. Johannesburg:

Storyteller Group.

Storyteller Group. 1990. Voice of the SCA: The Soweto Accord. Johannesburg: Storyteller

Publications/Soweto Civic Association.

Storyteller Group. 1991. Red Cross: Helping People in Need. Johannesburg: Storyteller

Publications / International Committee of the Red Cross.

Storyteller Group. Cresswell, Grant (artist); Adler, Michelle and Mason, Andy (writers);

Behr, Mary Anne and van Wyk, Chris (editors). 1991. Mhudi: Sol Plaatjie's classic tale

of love and war in the time of Mzilikazi. Johannesburg: Storyteller Group/Sached.

Storyteller Group. Cresswell, Grant, Cornielse, Janine, Howes, Carole and Head, Lois

(artists); Esterhuysen, Peter (editor). 1993. Deep Cuts: Graphic Adaptations of stories

by Can Themba, Alex La Guma \& Bessie Head. Cape Town: Maskew Miller

Longman/Storyteller Group. 
Storyteller Group. Wells, Justin and --- (artists); Watson, Patricia and Esterhuysen, Peter (writers/ editors). 1994-95. Heart to Heart: From Dream Love to true Love.

Johannesburg: Storyteller Publications.

Storyteller Group. Findlay, Alistair and Malindi, Vusi (artists). Van Zyl, Paul;

Esterhuysen, Peter (writers/editors). n.d. Truth and Reconciliation: An Introduction to the Truth and Reconciliation Commission. Johannesburg: Centre for the Study of

Violence and Reconciliation/The Storyteller Group.

Supa Strikas. Bonani, Bonisa, Peter Woodbridge, Ndevu, Mfundo and de Wet Rial

(artists); Legg, Bruce (story editor). 2002. Supa Strikas Annual 2002. Strika Entertainment (Pty) Ltd.

Supa Strikas. Bonani, Bonisa, Peter Woodbridge, Ndevu, Mfundo and de Wet Rial, Sean

Verster and Saaid Rahbeeni (artists); Legg, Bruce (story editor). 2003. Supa Strikas Annual 2003. Strika Entertainment (Pty) Ltd.

Trudeau, G.B. 1981. The People's Doonesbury: Notes from Underfoot: 1978-1980. London:

Wilwood House.

Van Staden, Leonora and Louw, Nicolene (eds). 2003. Stripshow, No. 1. Stellenbosch:

Strip Art Project.

Wild Beast (Bloch, Joanne) c.1990. In the Jungle. London: self-published.

Wild Beast (Bloch, Joanne) c.1991. Slurpy Durpy. London: self-published.

Zapiro (Shapiro, Jonathan). 1996. The Madiba Years. Cape Town/Johannesburg: David Philip Publishers.

Zapiro. 1997. The Hole Truth. Cape Town/Johannesburg: David Philip Publishers.

Zapiro. 1999. Call Mr Delivery. Cape Town/Johannesburg: David Philip Publishers.

Zapiro. 2000. The Devil Made Me Do It! Cape Town/Johannesburg: David Philip Publishers.

Zapiro, 2001: The ANC Went in 4 X4. Cape Town/Johannesburg: David Philip Publishers. 


\section{Articles, Books and Dissertations}

Adorno, Theodor W. and Horkheimer, Max. 1979. "The culture industry: enlightenment as mass deception". In During (ed), 1993.

Althusser, Louis. 1969. "Ideology and ideological state apparatuses". In Evans and Hall, 1999.

Andrew, Richard. 1988. Narrative Strip Illustration for Print, with an Emphasis on the

Human Figure. Technikon Natal: Unpublished Higher Diploma dissertation.

Attridge, Derek and Jolly, Rosemary (eds). 1998. Writing South Africa: Literature, apartheid, and democracy, 1970-1995. Cambridge: Cambridge University Press.

Barker, Martin. 1989. Comics: Ideology, power and the critics. Manchester/New York:

Manchester University Press.

Barnett, Clive. "Media, Scale and Democratisation". In Tomaselli and Dunn (eds), 2001. Barthes, Roland. 1973. “Rhetoric of the Image”. In Evans \& Hall (eds), 2000: 33-40.

Beaty, Bart. 1999. "Featuring Stories by the World's Greatest Authors: Classics

Mlustrated and the 'middlebrow problem' in the postwar era". IJOCA Vol. 1, No. 1. Benjamin, Arnold. 1991. Introduction to Fedler, 1991.

Benjamin, Walter. 1968. "The Work of Art in the Age of Mechanical Reproduction". In Evans \& Hall (eds), 2000: 72-79.

Bennett, Tony. 1982 (1). "Theories of the media, theories of society". In Gurevitch, et al (eds), 1982.

Bennett, Tony. 1982 (2). "Media, reality, signification". In Gurevitch, et al (eds), 1982.

Bennett, Tony. 1982 (3). "Popular Culture and 'the turn to Gramsci'". In Boyd-Barrett \& Newbold,(eds), 1995.

Berger, John. 1972. Ways of Seeing. UK: British Broadcasting Corporation/Penguin Books.

Berger, Guy. 2000. "Publishing for the People: The Alternative Press 1980-1999". In Evans \& Seeber (eds), 2000.

Boehmer, Elleke. 1988. "Endings and new beginnings: South African fiction in transition". In Attridge \& Jolly (eds), 1998.

Botes, Conrad. 1998. Fallosentrisme in Suid-Afrikaanse Fotoverhale: ' $n$ Ondersoek in geslag en ras as stelsels van dominasie. University of Stellenbosch: Unpublished MA dissertation. 
Boyd-Barrett, Oliver and Newbold, Chris. (eds) 1995. Approaches to Media: A Reader.

London: Arnold.

Britten, Sarah. 2000. Conflicting Humours: Race, Gender and National Identity in Madam \&

Eve. University of the Witwatersrand: Unpublished MA thesis.

Bryer, Lynne and Hunt, Keith S. 1984. The 1820 Settlers. Cape Town: Don Nelson.

Bryson, Norman. 1983. "The Natural Attitude". In Evans and Hall, 1999.

Cameron, Dan; Christov-Bakargiev and Coetzee, J.M. 1999. William Kentridge. London:

Phaidon Press Limited.

Campaign. 1999. The 100 best posters of the century. London: Campaign/Profile Books.

Chabon, Michael. 2000. The Amazing Adventures of Kavalier \& Clay. New York: Picador.

Chandler, Daniel. 1996 Semiotics for Beginners: Codes.

http:/ / www.aber.ac.uk/media/Documents/S4B/sem08.html.

Cloete, Dick. 2000. "Alternative Publishing in South Africa in the 1970s and 1980s". In

Evans \& Seeber, 2000.

Coetzee, J.M. 1976. In the Heart of the Country. Johannesburg: Ravan Press.

Couperie, Pierre. 1972. "Antecedents and Definition of the Comic strip". Graphis Volume

28, No. 159.

Craven, Thomas. 1943. Cartoon Cavalcade. New York: Simon \& Schuster.

Cronin, Jeremy. 1997. Review of Zapiro's The Hole Truth. Sunday Independent.

Curran, James; Morley, David and Walkerdine, Valerie. (eds) 1996. Cultural Studies and

Communications. London: Arnold.

De Waal, Shaun. 1994. Review of Gif: Afrikaner Seks Comix (Bitterkomix/Hond), Mail \& Guardian, 14 October.

Dorfman, Ariel and Mattelart, Armand. 1975. How to Read Donald Duck: Imperialist

ideology in the Disney comic. New York: International General.

During, Simon. (ed) 1993. The Cultural Studies Reader. London: Routledge.

Eisen, Jonathan. (ed). 1969. The Age of Rock. New York: Vintage Books.

Eisner, Will. 1985. Comics and Sequential Art. Florida: Poorhouse Press.

Evans, Jessica. 1999. "Cultures of the visual: Introduction". In Evans \& Hall, 1999.

Evans, Jessica and Hall, Stuart (eds). 1999. Visual Culture: The Reader. London: Sage

Publications.

Evans, Nicholas and Seeber, Monica. 2000. The Politics of Publishing in South Africa.

London: Holger Ehling Publishing/Pietermaritzburg: University of Natal Press. 
Ferguson, Marjorie and Golding, Peter. (eds). 1997. Cultural Studies in Question. London: Sage Publications Ltd.

Foucault, Michel. 1970. The Order of Things. London: Routledge.

Freire, Paulo. 1972. Pedagogy of the Oppressed. UK: Sheed \& Ward.

Friedman, Hazel. 2000. Norman Catherine. Johannesburg: Goodman Gallery.

Gitlin, Todd. 1997. "The Anti-political Populism of Cultural Studies". In Ferguson, \& Golding, 1997: p25-38.

Goldscheider, Ludwig. 1975. Michaelangelo: Paintings, Sculptures, Architecture (5 $^{\text {th }}$ edition). London: Phaidon.

Gordimer, Nadine. 1995. Writing and Being. Cambridge, Massachusetts: Harvard University Press.

Gramsci, Antonio (edited by Smith, G. N. \& Hoare, Q. ). 1971. Selections from the Prison Notebooks. London: Lawrence \& Wishart Ltd.

Gurevitch, Michael; Bennett, Tony; Curran, James and Woollacott, Janet. (eds) 1982.

Culture, Society and the Media. London: Methuen \& Co. Ltd.

Hall, Stuart. 1980. "Cultural Studies: two paradigms". In Boyd-Barrett and Newbold (eds), 1995.

Hall, Stuart. 1982. "The Rediscovery of Ideology: Return of the repressed in media studies". In Gurevitch, et al (eds), 1982.

Hall, Stuart. 1985. "Signification, Representation, Ideology: Althusser and the poststructuralist debates". In Curran et al (eds), 1996.

Hall, Stuart. 1993. "Encoding, decoding". In During (ed), 1993.

Hebdige, Dick. 1985. "The bottom line on planet one: squaring up to The Face". In Evans \& Hall. 1999.

Hendricksen, J. 1994. Lichtenstein. Koln: Taschen.

Herman, Edward S. and McChesney, Robert W. 1997. The Global Media: the new missionaries of corporate capitalism. London and Washington: Cassell.

Herman, Edward S. and Chomsky, Noam. 1988. Manufacturing Consent: The Political Economy of the Mass Media. New York: Pantheon Books.

Honour, Hugh and Fleming, John. 1991. A World History of Art, 3rd edition. London: Laurence King Ltd.

Hopkins, David. 2000. After Modern Art. Oxford: Oxford University Press. 
Hughes, Robert. 1991. The Shock of the New: Art and the Century of Change. London: Thames \& Hudson Ltd.

Jamal, Ashraf. 2004. The Rat in Art: Conrad Botes, Pop and the Posthuman. Cape Town:

Erdmann Contemporary.

Johnson, Paul. 2003. Art: A New History. London: Weidenfeld \& Nicolson.

Kannemeyer, Anton. 1997. "Die Ikonoklastiese Strip, Polemiek en Bitterkomix. University of Stellenbosch: Unpublished MA dissertation..

Kellner, Douglas. 1997. “Overcoming the Divide: Cultural Studies and Political

Economy". In Ferguson \& Golding, 1997: p102-120.

Kennedy, Jay. 1982. The Official Underground and Newave Comix Price Guide.

Wisconsin/Massachusetts: Kitchen Sink Press/Boatner Norton Press.

Kirkwood, Mike. 1988. "Remembering Staffrider". In Oliphant et al, 1988.

Klingender, F.D. 1944. Hogarth and English Caricature. London/New York: Transatlantic

Arts Ltd.

Kranzfelder, Ivo. 1994. George Grosz 1893-1959. Köln: Benedikt Taschen.

Krog, Antjie. 1993. "Watter soort mens is jy: 'n kuifie of 'n asterix?" Vrye Weekblad, 8

July.

Kwon, Jae-Woong. 2002. "Korean cartoonists' reactions to Bush's 'Axis of Evil"”. IJOCA, Vol. 4. No. 2: pp276-286.

Kunzle, David. 1973. The Early Comic Strip. London: University of California Press Ltd. Laing, Dave. 1978. The Marxist Theory of Art: An Introductory Survey. Sussex: The

Harvester Press Limited/New Jersey: Humanities Press Inc.

Lanier, 2003. "The Eric Drooker Interview". The Comics Journal, No. 253.

Lent, John A. 1991. "Lively Cartooning in the Land of Castro". The Comics Journal, September 1991.

Lent, John A. 1992. "Chinese Comic Art: Historical and contemporary perspectives".

Asian Culture, Vol XX No 4. Asian-Pacific Cultural Centre, Taipei.

Lent, John A. 1993. "The historical and global dimensions of comic art". Phillipines

Communication Journal, Vol II No 8, University of the Phillipines, Quezon.

Lent, John A. 1993. "South East Asian cartooning: Comics in Phillipines, Singapore and Indonesia". Asian Culture, Vol XXI No 4. Asian-Pacific Cultural Centre, Taipei.

Lent, John A. 1995. "They are more than just Pow! Wham! or Ha! Ha!". The Journal of Development Communication, Vol 6 No 2. 
Lent, John A. 1995. "Asian Comic Strips: Old Wine in New Bottles". Asian Culture, Vol XXUI No 4. Asian-Pacific Cultural Centre, Taipei.

Lent, John A. 1999. "The Horrors of Cartooning in Slim's Algeria". IJOCA, Vol 1 No. 1.

Lent, John A. (ed). 1999. Pulp Demons: International Dimensions of the Postwar Anti-Comics Campaign. USA/London/Canada: Associated University Presses, Inc.

Lent, John A. 2002. "Fear [of] and Loafing [with] Ralph Steadman in Turkey". IJOCA,

Vol 4 No 2 .

Leymarie, Jean. 1972. Picasso: Artist of the Century. London: Macmillan.

Lewis-Williams, David. 2003. Images of Mystery: Rock Art of the Drakensberg. Cape Town:

Double Storey.

Louw, Eric and Tomaselli, Keyan G. (eds). The Alternative Press in South Africa. Bellville:

Anthropops/London: James Currey.

Lukács, Georg. 1962. The Historical Novel. UK: Merlin Press.

Maltin, Leonard. 1980. Of Mice and Magic: A History of American Animated Cartoons. New York: New American Library.

Marais, Hein. 1998. South Africa: Limits to Change: The political economy of transformation. London/New York: Zed Books Ltd/Cape Town: University of Cape Town Press. Mason, Andy. 2001. "The Role of Civil Society in the Development of Democratic Media in Southern Africa": The Namibian Example. In Tomaselli and Dunn (eds). 2001.

Mason, Andy. 2001. "Cartooning journalism in Africa puts political power into perspective". Media Development, Vol. XLVIII 2/2001.

Mason, Andy. 2001. “Africa Ink: Cartoonists Working Group, Towards an Association of African Cartoonists: Report of an International Workshop on Cartoon Journalism and Democratisation in Southern Africa". IJOCA, Vol. 4, No. 2.

Mason, Andy. 2002. "Black and White in Ink: Discourses of Resistance in South African Cartooning". African and Asian Studies, Vol. 1, No 4. (Also in Africa e Mediterranero, No. 38.)

Mason, Andy. 2002. Black \& White in Ink. Under the Skin of South African Cartooning. Exhibition catalogue. Durban: NSA Gallery/Artworks Communications. Marschall, Sabine. 2002. Community Mural Art in South Africa. Pretoria: Unisa Press. McQuail, Denis, 1994. Mass Communication Theory, third edition. London: Sage Publications Ltd.

McCloud, Scott. 1993. Understanding Comics: The Invisible Art. New York: HarperCollins. 
McCloud, Scott. 2000. Reinventing Comics. New York: Paradox Press.

McLuhan, Marshall. 1964. Understanding Media. London: Abacus.

McRobbie, Angela. 1996. "More! New Sexualities in Girls' and Women's Magazines". In Curran et al (eds), 1996.

Mnyele, Thamsanqa. 1988. "Thoughts on Bongiwe and Role of Revolutionary Art". In Oliphant et al (eds), 1988.

Mosco, Vincent. 1996. The Political Economy of Communication. London: Sage Publications. Mphahlele, Ezekiel. 1959. Down Second Avenue. London: Faber and Faber.

Murdock, Graham. 1997. "Base Notes: The Conditions of Cultural Practice”. In Ferguson \& Golding (eds), 1997.

Mutloatse, Mothobi. (ed). 1980. Forced Landing. Staffrider Series, No. 3. Johannesburg: Ravan Press.

Nevins, Mark David. 2002. "'Drawing from Life': An Interview with Joe Sacco". IJOCA, Vol. 4. No. 2.

Nyamnjoh, Francis. 2000. "Zapiro and South African Political Cartooning". IJOCA, Vol. 2 No. 2.

Nyberg, Amy K. 1999. "Comic Book Censorship in the United States". In Lent (ed), 1999. Oliphant, Andries Walter and Vladislavic, Ivan. (eds) 1988. Ten Years of Staffrider 19781988. Johannesburg: Ravan Press.

O'Sullivan, Tim; Hartley, John; Saunders, Danny; Montgomery, Martin and Fiske, John. 1994. Key Concepts in Communication and Cultural Studies, 2nd edition. London and New York: Routledge.

Packalén, Leif and Odoi, Frank. 1999. Comics with an Attitude ... A Guide to the Use of Comics in Deoelopment Information. Helsinki: Ministry for Foreign Affairs of Finland. Parry, Benita. 1998. "Speech and Silence in the Fictions of J.M. Coetzee". In Attridge \& Jolly.

Pearsall, Judy et al (eds). 1999. The Concise Oxford Dictionary, $10^{\text {th }}$ edition. Oxford: Oxford University Press.

Perry, George and Aldridge, Alan. 1971. The Penguin Book of Comics (revised edition).

London: Penguin Books.

Rius, 1996 Marx for Beginners. Republished in 1998 as Introducing Marx. Cambridge: Icon Books. 
Ruhrberg, Karl; Schneckenburger, Manfred; Fricke, Christiane and Honnel, Klaus. (Ed) Ingo F. Walther. 2000. Art of the 20th Century. Koln/London: Taschen.

Sabin, Roger. 1988. Adult Comics: An Introduction. London/New York: Routledge. Sartre, Jean-Paul. 1947. What is Literature? London: Methuen \& Co. Ltd.

Schoonraad, Murray and Elzabé. 1983. Suid-Afrikaanse Spot-en Strookprent-kunstenaars.

Roodepoort: CUM-Boeke.

Simon, Roger, 1982. Gramsci's Political Thought: An Introduction. London: Lawrence \& Wishart.

Sontag, Susan. 1978. "The Image World". In Evans \& Hall, 1999.

Sorrell, Jennifer.1987. "Lennie Sak: Creator of Cartoon Character Jojo", ADA: Art Design Architecture No. 3.

Sweetman, David. Toulouse;Lautrec and the Fin de Siecle. 1999. London: Hodder \& Stoughton.

The Posterbook collective of the South African History Archive. 1991. Images of Defiance:

South African Resistance Posters of the 1980s. Johannesburg: Ravan Press.

The Press Works. 1893. The Schröder Art memento: An Artistic Souvenir of South African

History: Fig 28. Pretoria: "The Press" Works, SAR.

Thompson, John B. 1990. Ideology and Modern Culture. Cambridge: Polity Press.

Thompson, John B. 1995. The Media and Modernity. London: Polity Press.

Tomaselli, Keyan G. 1996. Appropriating Images: The Semiotics of Visual Representation.

Denmark: Intervention Press.

Tomaselli, Keyan G. and Dunn, Hopeton (eds). 2001. Media, Democracy and Renewal in Southern Africa. Colorado Springs: International Academic Publishers Ltd.

Towle, Benjamin F. 2003. "An Examination of Historiography in the Comics Medium". IJOCA, Vol 5 No 2.

Van Zyl, Jan. 1994. Review of Gif: Afrikaner Seks Comix, Die Matie, 11 August.

Vernon, Ken. 2000. Penpricks: The Drawing of South Africa's Political Battlelines. Cape

Town: The Spearhead Press.

Watson Shariff, Patricia and Janks, Hilary. 2001. "Changing Stories: The Making and Analysis of a Critical Literacy Romance Comic". IJOCA, Vol. 3, No. 2.

Weekly Mail \& Guardian. April 21-27, 1995. Anon."The end of Afrikaans prudery is naai". Williams, Raymond. 1976. Keywords: A vocabulary of culture and society. London: Fontana Press. 